\title{
Transition-Metal and Solvent-Free Oxidative C-H Fluoroalkoxylation of Quinoxalinones with Fluoroalkyl Alcohols
}

Jun Xu, Huiyong Yang, Heng Cai, Hanyang Bao, Wanmei Li* and Pengfei Zhang*

College of Material Chemistry and Chemical Engineering, Hangzhou Normal University, Hangzhou 310036, China.

Email: liwanmei@hznu.edu.cn (Li, W.); chxyzpf@ hotmail.com (Zhang, P.)

\section{Supporting Information}

\section{Table of contents}

General Information

1. Experimental Section 3

2. Characterization of the products 8

3. X-ray Crystal Data for $\mathbf{3 l}$ and 7d 32

4 References $\quad 34$

5. Copies of ${ }^{1} \mathrm{H},{ }^{13} \mathrm{C}$ and ${ }^{19} \mathrm{~F}$ NMR Spectra 35

6. Copies of IR Spectra 141 


\section{General Information}

All reagents and deuterated solvents were commercially available and used without further purification. All products were separated by silica gel (200-300 mesh) column chromatography with petroleum ether (PE) $\left(60-90^{\circ} \mathrm{C}\right)$ and ethyl acetate (EA). ${ }^{1} \mathrm{H},{ }^{13} \mathrm{C}$ and ${ }^{19} \mathrm{~F}$ NMR spectra were recorded on a Bruker Advance 500 spectrometer at ambient temperature with $\mathrm{CDCl}_{3}$ and $\mathrm{CD}_{3} \mathrm{SOCD}_{3}$ as solvent and tetramethylsilane (TMS) as the internal standard. Fourier transform infrared spectroscopy (FTIR) spectra were recorded on a Nicolet iS 5 in attenuated total reflectance (ATR) mode and reported with wave number $\left(\mathrm{cm}^{-1}\right)$. Melting points were determined on an X-5 Data microscopic melting point apparatus. Analytical thin layer chromatography (TLC) was performed on Merk precoated TLC (silica gel 60 F254) plates. Compounds for HRMS were analyzed by positive mode electrospray ionization (ESI) using Agilent 6530 QTOF mass spectrometer.

\section{Table S1. Optimization of Reaction Conditions ${ }^{a, b}$}

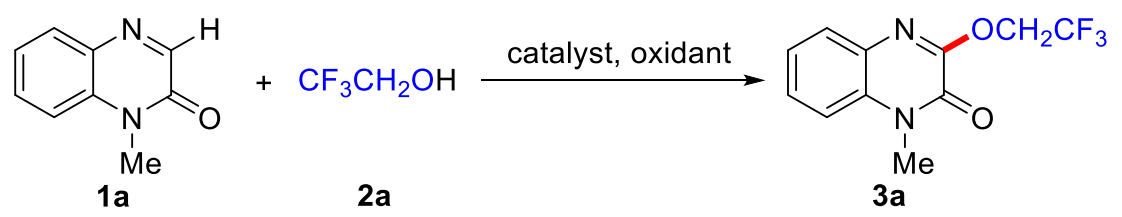

$\begin{array}{ccccc}\text { entry } & \text { photocatalyst } & \text { oxidant } & \text { solvent } & \text { yield }(\%)^{b} \\ 1 & \text { eosin Y } & \mathrm{PhI}(\mathrm{TFA})_{2} & \mathrm{MeCN} & 65 \\ 2 & \mathrm{Ir}(\mathrm{ppy})_{3} & \mathrm{PhI}(\mathrm{TFA})_{2} & \mathrm{MeCN} & 63 \\ 3 & \text { rhodamine B } & \mathrm{PhI}(\mathrm{TFA})_{2} & \mathrm{MeCN} & 60 \\ 4 & - & \mathrm{PhI}(\mathrm{TFA})_{2} & \mathrm{MeCN} & 64 \\ 5^{c} & - & \mathrm{PhI}(\mathrm{OPiv})_{2} & \mathrm{MeCN} & 42 \\ 6^{c} & - & \mathrm{PhI}(\mathrm{OAc})_{2} & \mathrm{MeCN} & 19 \\ 7^{c} & - & \mathrm{PhI}(\mathrm{OH}) \mathrm{OTs} & \mathrm{MeCN} & \text { trace } \\ 8^{c} & - & \mathrm{K}_{2} \mathrm{~S}_{2} \mathrm{O}_{8} & \mathrm{MeCN} & 0 \\ 9^{c} & - & \mathrm{TBHP} & \mathrm{MeCN} & 0 \\ 10^{c} & - & \mathrm{DTBP} & \mathrm{MeCN} & 0 \\ 11^{c} & - & \mathrm{DDQ} & \mathrm{MeCN} & 0 \\ 12^{c} & - & - & \mathrm{MeCN} & 0 \\ 13^{c} & - & \mathrm{PhI}(\mathrm{TFA})_{2} & \mathrm{DCE} & \text { trace } \\ 14^{c} & - & \mathrm{PhI}(\mathrm{TFA})_{2} & \text { dioxane } & \text { trace } \\ 15^{c} & - & \mathrm{PhI}(\mathrm{TFA})_{2} & \mathrm{THF} & \text { trace } \\ 16^{c} & - & \mathrm{PhI}(\mathrm{TFA})_{2} & \text { toluene } & 17 \\ 17^{c} & - & \mathrm{PhI}(\mathrm{TFA})_{2} & \mathrm{DMF} & \text { trace } \\ 18^{c} & - & \mathrm{PhI}(\mathrm{TFA})_{2} & \mathrm{DMSO} & \text { trace } \\ 19^{c} & - & \mathrm{PhI}(\mathrm{TFA})_{2} & \mathrm{H})_{2} \mathrm{O} & 0 \\ 20^{c} & - & \mathrm{PhI}(\mathrm{TFA})_{2} & \text { acetone } & 42 \\ 21^{c} & - & \mathrm{PhI}(\mathrm{TFA})_{2} & \text { EtOAc } & 23\end{array}$

${ }^{\text {a }}$ Reaction conditions: $\mathbf{1 a}(0.2 \mathrm{mmol}), \mathbf{2 a}$ (1.5 equiv), catalyst (1 mol \%), oxidant (1.5 equiv), solvent $(0.2 \mathrm{~mL})$, blue LED, open flask, $1 \mathrm{~h} .{ }^{\mathrm{b}}$ Isolated yields. ${ }^{\mathrm{c}}$ Without light and stirred at $35^{\circ} \mathrm{C}$. 


\section{Experimental Section}

\subsection{General procedure for the synthesis of products 3-9}

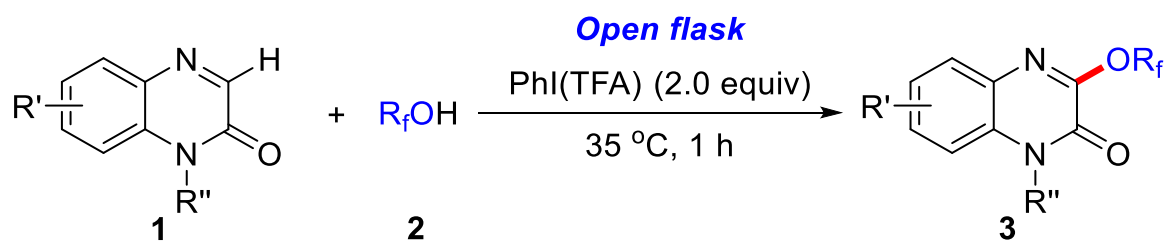

A mixture of quinoxalinones (1) (0.2 mmol), fluoroalkyl alcohols (2) (3.0 equiv) and $\mathrm{PhI}(\mathrm{TFA})_{2}$ (2.0 equiv) in a $10 \mathrm{~mL}$ tube was stirred at $35{ }^{\circ} \mathrm{C}$ for 1 hour. After the completion (as indicated by TLC), the reaction was quenched with saturated $\mathrm{NaHCO}_{3}$ solution. The mixture was then extracted with EtOAc and the collected organic layer was washed with brine, dried with $\mathrm{MgSO}_{4}$. The solvent was removed under reduced pressure, and the obtained residue was further purified by silica gel column chromatography (200-300 mesh silica gel, PE/EA = 5:1).

\subsection{General procedure for the synthesis of product $3 \mathrm{~b}$ on 1 mmol scale}

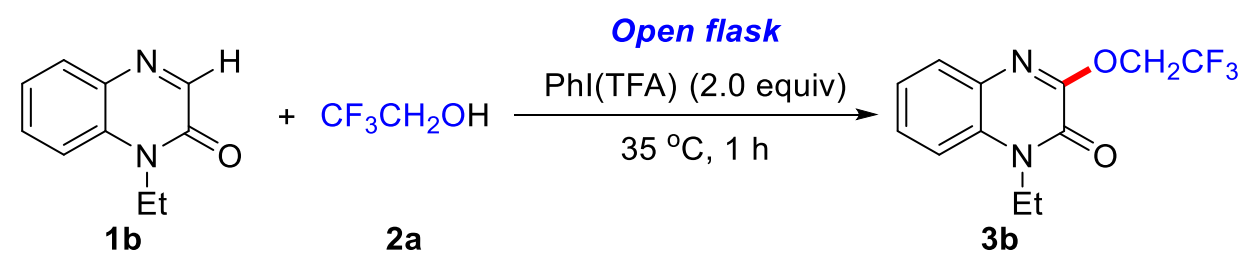

A mixture of quinoxalinone (1b) $(1.0 \mathrm{mmol})$, trifluoroethanol (2a) (3.0 equiv) and $\mathrm{PhI}(\mathrm{TFA})_{2}$ (2.0 equiv) in a $10 \mathrm{~mL}$ tube was stirred at $35{ }^{\circ} \mathrm{C}$ for 1 hour. After the completion (as indicated by TLC), the reaction was quenched with saturated $\mathrm{NaHCO}_{3}$ solution. The mixture was then extracted with EtOAc and the collected organic layer was washed with brine, dried with $\mathrm{MgSO}_{4}$. The solvent was removed under reduced pressure, and the obtained residue was further purified by silica gel column chromatography (200-300 mesh silica gel, PE/EA = 5:1).

\subsection{General procedure for the synthesis of product $3 \mathrm{~h}$ by using 1aa as substrate}

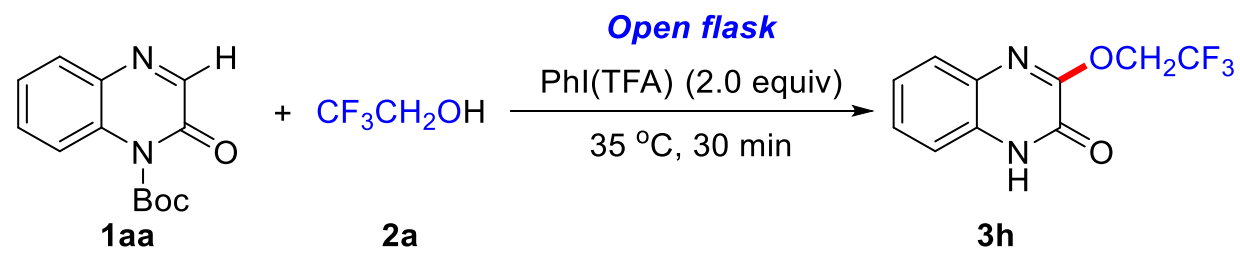

A mixture of quinoxalinones (1aa) $(0.2 \mathrm{mmol})$, trifluoroethanol (2a) (3.0 equiv) and $\mathrm{PhI}(\mathrm{TFA})_{2}$ (2.0 equiv) in a $10 \mathrm{~mL}$ tube was stirred at $35{ }^{\circ} \mathrm{C}$ for $30 \mathrm{~min}$. After the completion (as indicated by TLC), the reaction was quenched with saturated $\mathrm{NaHCO}_{3}$ solution. The mixture was then extracted with EtOAc and the collected organic layer was washed with brine, dried with $\mathrm{MgSO}_{4}$. The solvent was removed under reduced pressure, and the obtained residue was further purified by silica gel column chromatography (200-300 mesh silica gel, PE/EA = 5:1). The yield of $\mathbf{3 h}$ was $75 \%$. 


\subsection{General procedure for gram-scale synthesis of products $3 \mathrm{~h}$}

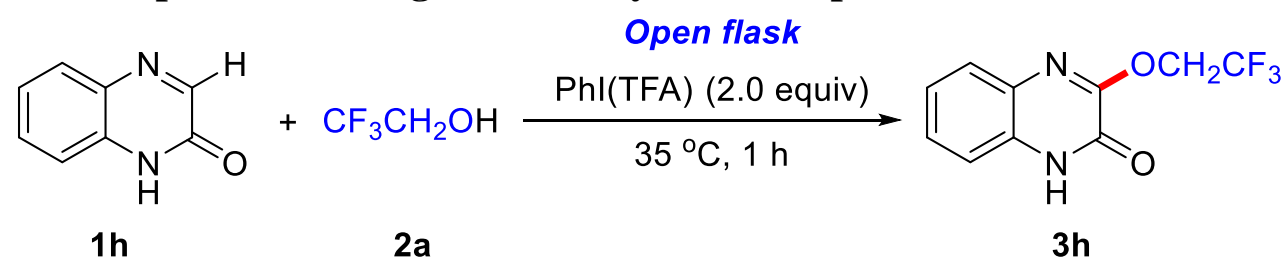

A mixture of quinoxalinone (1h) $(6.0 \mathrm{mmol})$, trifluoroethanol (2a) $(3.0$ equiv) and $\mathrm{PhI}(\mathrm{TFA})_{2}$ (2.0 equiv) in a $25 \mathrm{~mL}$ round-bottom flask was stirred at $35{ }^{\circ} \mathrm{C}$ for 1 hour. After the completion (as indicated by TLC), the reaction was quenched with saturated $\mathrm{NaHCO}_{3}$ solution. The mixture was then extracted with EtOAc and the collected organic layer was washed with brine, dried with $\mathrm{MgSO}_{4}$. The solvent was removed under reduced pressure, and the obtained residue was further purified by silica gel column chromatography (200-300 mesh silica gel, PE/EA = 5:1).

\subsection{General procedure for chlorination of product $3 \mathrm{a}^{1}$}

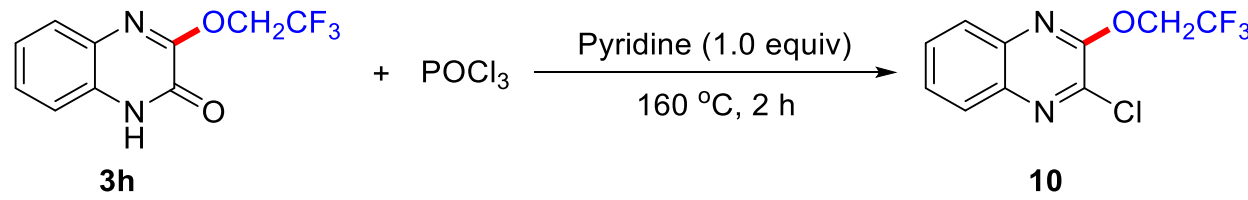

A mixture of trifluoroethyl ether $(\mathbf{3 h})(5.0 \mathrm{mmol}), \mathrm{POCl}_{3}(1.2$ equiv) and pyridine (1.0 equiv) in a $15 \mathrm{~mL}$ pressure tube was stirred at $160{ }^{\circ} \mathrm{C}$ for 2 hours. After the completion (as indicated by TLC), the reaction was quenched with saturated $\mathrm{NaHCO}_{3}$ solution. The mixture was then extracted with EtOAc and the collected organic layer was washed with brine, dried with $\mathrm{MgSO}_{4}$. The solvent was removed under reduced pressure, and the crude product was further purified by silica gel column chromatography (200-300 mesh silica gel, PE/EA = 10:1) to afford product $\mathbf{1 0}$.

\subsection{General procedure for Suzuki coupling of chlorinated trifluoroethyl ether ${ }^{1}$}

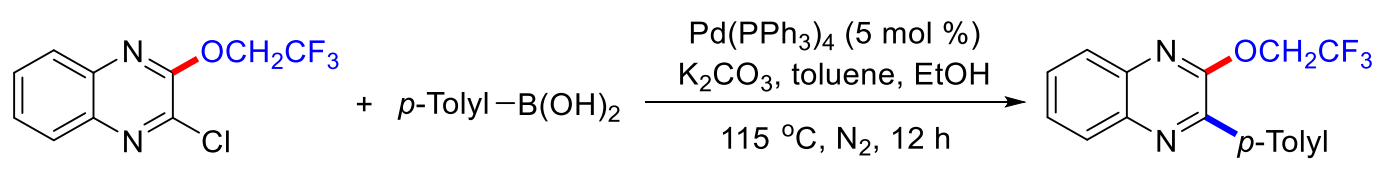

10

$11 \mathrm{a}$

A mixture of chlorinated trifluoroethyl ether (10) $(0.2 \mathrm{mmol}), p$-Tolylboronic acid (1.5 equiv), $\mathrm{Pd}\left(\mathrm{PPh}_{3}\right)_{4}(5 \mathrm{~mol} \%), \mathrm{K}_{2} \mathrm{CO}_{3}(2.0 \mathrm{M}$ in water, $1.1 \mathrm{~mL})$, toluene $(1.5 \mathrm{~mL})$, EtOH $(0.5 \mathrm{~mL})$ in a $15 \mathrm{~mL}$ pressure tube was stirred at $115{ }^{\circ} \mathrm{C}$ under a nitrogen atmosphere for 12 hours. After the completion (as indicated by TLC), the mixture was then extracted with EtOAc and the collected organic layer was washed with brine, dried with $\mathrm{MgSO}_{4}$. The solvent was removed under reduced pressure, and the crude product was further purified by silica gel column chromatography (200-300 mesh silica gel, $\mathrm{PE} / \mathrm{EA}=10: 1)$ to afford product 11a.

\subsection{General procedure for Sonogashira coupling of chlorinated trifluoroethyl ether $^{2}$}




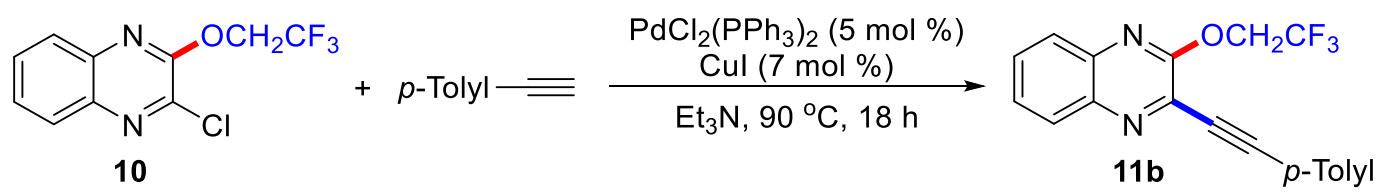

A mixture of chlorinated trifluoroethyl ether (10) $(0.2 \mathrm{mmol}), p$-Tolylacetylene $(1.2$ equiv), $\mathrm{PdCl}_{2}\left(\mathrm{PPh}_{3}\right)_{2}(5 \mathrm{~mol} \%), \mathrm{CuI}(7 \mathrm{~mol} \%), \mathrm{Et}_{3} \mathrm{~N}(1.0 \mathrm{~mL})$ in a $15 \mathrm{~mL}$ pressure tube was stirred at $90{ }^{\circ} \mathrm{C}$ under a nitrogen atmosphere for 18 hours. After the completion (as indicated by TLC), the reaction was quenched with $\mathrm{H}_{2} \mathrm{O}$. The mixture was then extracted with EtOAc and the collected organic layer was washed with brine, dried with $\mathrm{MgSO}_{4}$. The solvent was removed under reduced pressure, and the crude product was further purified by silica gel column chromatography (200-300 mesh silica gel, $\mathrm{PE} / \mathrm{EA}=10: 1$ ) to afford product $\mathbf{1 1 b}$.

\subsection{General procedure for amination of chlorinated trifluoroethyl ether ${ }^{3}$}

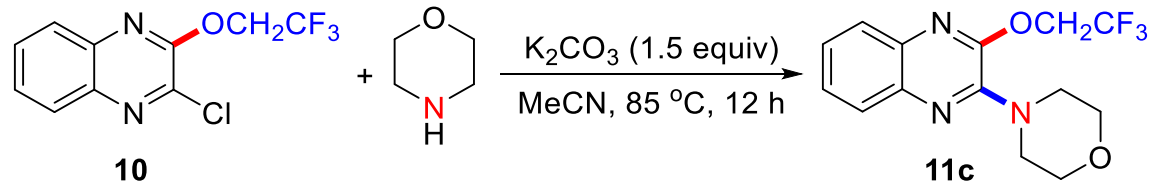

A mixture of chlorinated trifluoroethyl ether (10) $(0.2 \mathrm{mmol})$, morpholine (1.5 equiv), $\mathrm{K}_{2} \mathrm{CO}_{3}$ (1.5 equiv) and $\mathrm{MeCN}(1.5 \mathrm{~mL})$ in a $15 \mathrm{~mL}$ pressure tube was stirred at $85{ }^{\circ} \mathrm{C}$ for 12 hours. After the completion (as indicated by TLC), the reaction was quenched with $\mathrm{H}_{2} \mathrm{O}$. The mixture was then extracted with EtOAc and the collected organic layer was washed with brine, dried with $\mathrm{MgSO}_{4}$. The solvent was removed under reduced pressure, and the crude product was further purified by silica gel column chromatography $(200-300$ mesh silica gel, $\mathrm{PE} / \mathrm{EA}=10: 1)$ to afford product 11c.

\subsection{General procedure for methoxylation of chlorinated trifluoroethyl ether ${ }^{1}$}

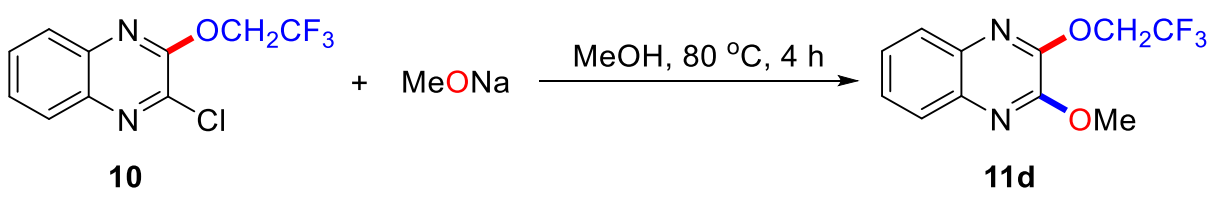

A mixture of chlorinated trifluoroethyl ether (10) $(0.2 \mathrm{mmol}), \mathrm{MeONa}$ (5.0 equiv) and $\mathrm{MeOH}(1.5 \mathrm{~mL})$ in a $15 \mathrm{~mL}$ pressure tube was stirred at $80{ }^{\circ} \mathrm{C}$ for 4 hours. After the completion (as indicated by TLC), the reaction was quenched with $\mathrm{H}_{2} \mathrm{O}$. The mixture was then extracted with EtOAc and the collected organic layer was washed with brine, dried with $\mathrm{MgSO}_{4}$. The solvent was removed under reduced pressure, and the crude product was further purified by silica gel column chromatography (200-300 mesh silica gel, $\mathrm{PE} / \mathrm{EA}=10: 1$ ) to afford product 11d.

\subsection{General procedure for phosphorization of chlorinated trifluoroethyl ether ${ }^{4}$}

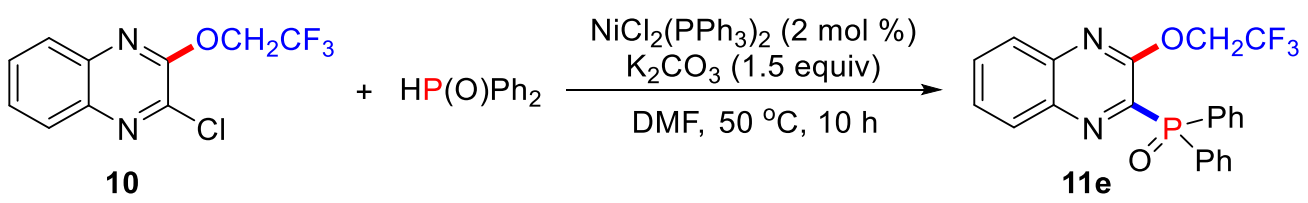


A mixture of chlorinated trifluoroethyl ether (10) $(0.2 \mathrm{mmol})$, Diphenylphosphine oxide (1.2 equiv), $\mathrm{NiCl}_{2}\left(\mathrm{PPh}_{3}\right) 2(2 \mathrm{~mol} \%), \mathrm{K}_{2} \mathrm{CO}_{3}(1.5$ equiv), $\mathrm{DMF}(1.0 \mathrm{~mL})$ in a 10 $\mathrm{mL}$ tube was stirred at $50{ }^{\circ} \mathrm{C}$ under a nitrogen atmosphere for 10 hours. After the completion (as indicated by TLC), the reaction was quenched with $\mathrm{H}_{2} \mathrm{O}$. The mixture was then extracted with EtOAc and the collected organic layer was washed with brine, dried with $\mathrm{MgSO}_{4}$. The solvent was removed under reduced pressure, and the crude product was further purified by silica gel column chromatography (200-300 mesh silica gel, $\mathrm{PE} / \mathrm{EA}=10: 1)$ to afford product 11e.

\subsection{General procedure for thioetherification of chlorinated trifluoroethyl ether ${ }^{5}$}

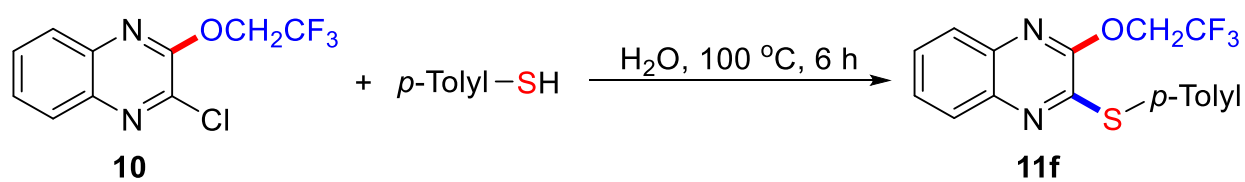

A mixture of chlorinated trifluoroethyl ether (10) $(0.2 \mathrm{mmol}), p$-methylthiophenol (1.1 equiv) and $\mathrm{H}_{2} \mathrm{O}(1.0 \mathrm{~mL})$ in a $15 \mathrm{~mL}$ pressure tube was stirred at $100{ }^{\circ} \mathrm{C}$ for 6 hours. After the completion (as indicated by TLC), the mixture was then extracted with EtOAc and the collected organic layer was washed with brine, dried with $\mathrm{MgSO}_{4}$. The solvent was removed under reduced pressure, and the crude product was further purified by silica gel column chromatography (200-300 mesh silica gel, PE/EA = 10:1) to afford product $\mathbf{1 1 f}$.

\subsection{General procedure for sulfonylation of chlorinated trifluoroethyl ether ${ }^{6}$}

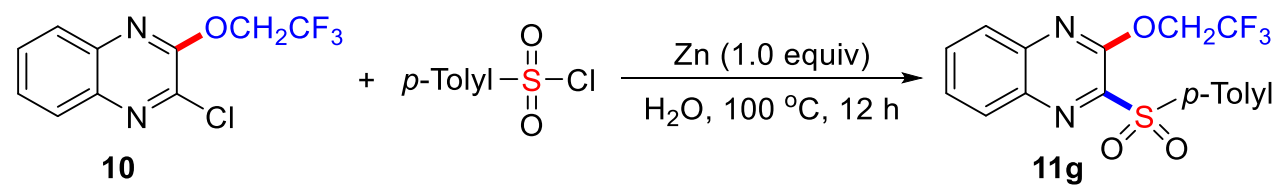

A mixture of chlorinated trifluoroethyl ether (10) $(0.2 \mathrm{mmol})$, Tosyl chloride (2.0 equiv), znic powder (1.0 equiv) and $\mathrm{H}_{2} \mathrm{O}(1 \mathrm{~mL})$ in a $15 \mathrm{~mL}$ pressure tube was stirred at $100{ }^{\circ} \mathrm{C}$ for 12 hours. After the completion (as indicated by TLC), the mixture was then extracted with EtOAc and the collected organic layer was washed with brine, dried with $\mathrm{MgSO}_{4}$. The solvent was removed under reduced pressure, and the crude product was further purified by silica gel column chromatography (200-300 mesh silica gel, $\mathrm{PE} / \mathrm{EA}=5: 1$ ) to afford product $\mathbf{1 1 g}$.

\subsection{General procedure for homocouplings of chlorinated trifluoroethyl ether ${ }^{7}$}

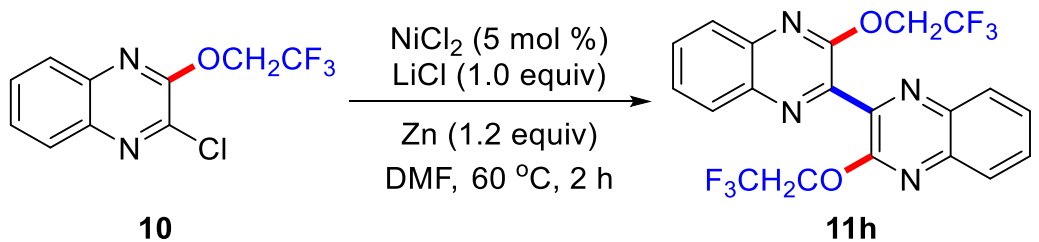

A mixture of chlorinated trifluoroethyl ether (10) $(0.2 \mathrm{mmol}), \mathrm{NiCl}_{2} \cdot 6 \mathrm{H}_{2} \mathrm{O}(5 \mathrm{~mol} \%)$, $\mathrm{LiCl}$ (1.0 equiv), zinc dust (1.2 equiv) and DMF $(1.0 \mathrm{~mL})$ in a $15 \mathrm{~mL}$ pressure tube was heated to $50{ }^{\circ} \mathrm{C}$, then, a grain of iodine crystal and two drops of acetic acid were 
added to the mixture. The mixture was stirred at $60{ }^{\circ} \mathrm{C}$ for 2 hours. After the completion (as indicated by TLC), the reaction was quenched with $\mathrm{H}_{2} \mathrm{O}$. The mixture was then extracted with EtOAc and the collected organic layer was washed with brine, dried with $\mathrm{MgSO}_{4}$. The solvent was removed under reduced pressure, and the crude product was further purified by silica gel column chromatography (200-300 mesh silica gel, $\mathrm{PE} / \mathrm{EA}=10: 1$ ) to afford product $\mathbf{1 1 h}$.

\subsection{General procedure for amination of products $3^{8}$}<smiles>[R]n1c(=O)c(OCC(F)(F)F)nc2ccccc21</smiles>

3a, $\mathrm{R}=\mathrm{Me}$

3h, $\mathrm{R}=\mathrm{H}$<smiles>[R]n1c(=O)c(N2CCOCC2)nc2ccccc21</smiles>

12a, $\mathrm{R}=\mathrm{Me}, 77 \%$

12b, $\mathrm{R}=\mathrm{H}, 72 \%$

A mixture of trifluoroethyl ether (3) $(0.2 \mathrm{mmol})$, morpholine (1.5 equiv), $\mathrm{Et}_{3} \mathrm{~N}$ (1.5 equiv) and DMSO $(1.0 \mathrm{~mL})$ in a $15 \mathrm{~mL}$ pressure tube was stirred at $120{ }^{\circ} \mathrm{C}$ for 12 hours. After the completion (as indicated by TLC), the reaction was quenched with $\mathrm{H}_{2} \mathrm{O}$. The mixture was then extracted with EtOAc and the collected organic layer was washed with brine, dried with $\mathrm{MgSO}_{4}$. The solvent was removed under reduced pressure, and the crude product was further purified by silica gel column chromatography (200-300 mesh silica gel, $\mathrm{PE} / \mathrm{EA}=5: 1)$ to afford product $\mathbf{1 2}$.

\subsection{General procedure for the synthesis of product $13^{8}$}

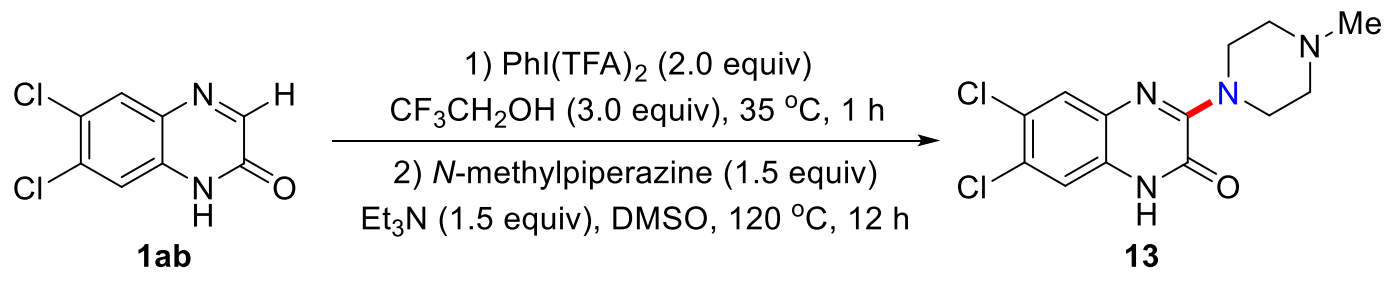

histamine -4 receptor antagonist

A mixture of quinoxalinones (1ab) $(0.5 \mathrm{mmol})$, trifluoroethanol (2a) (3.0 equiv) and $\mathrm{PhI}(\mathrm{TFA})_{2}$ (2.0 equiv) in a $10 \mathrm{~mL}$ tube was stirred at $35{ }^{\circ} \mathrm{C}$ for 1 hour. After the completion (as indicated by TLC), the reaction was quenched with saturated $\mathrm{NaHCO}_{3}$ solution. The mixture was then extracted with EtOAc and the collected organic layer was washed with brine, dried with $\mathrm{MgSO}_{4}$. After the solvent was removed, $\mathrm{N}$-methylpiperazine (1.5 equiv), $\mathrm{Et}_{3} \mathrm{~N}$ (1.5 equiv) and DMSO $(2.0 \mathrm{~mL})$ were added and then stirred at $120{ }^{\circ} \mathrm{C}$ for 12 hours. After the completion (as indicated by TLC), the reaction was quenched with $\mathrm{H}_{2} \mathrm{O}$. The mixture was then extracted with EtOAc and the collected organic layer was washed with brine, dried with $\mathrm{MgSO}_{4}$. The solvent was removed under reduced pressure, and the crude product was further purified by silica gel column chromatography $(200-300$ mesh silica gel, PE/EA $=5: 1)$ to afford product 13. 


\section{Characterization of the products}

1-Methyl-3-(2,2,2-trifluoroethoxy)quinoxalin-2(1H)-one (3a)<smiles>Cn1c(=O)c(OCC(F)(F)F)nc2ccccc21</smiles>

Obtained as a white solid (45 mg, 88\% yield); M.p. 156-157 ${ }^{\circ} \mathrm{C} .{ }^{1} \mathrm{H}$ NMR $(500 \mathrm{MHz}$, $\left.\mathrm{CDCl}_{3}\right) \delta 7.65(\mathrm{dd}, J=7.9,1.1 \mathrm{~Hz}, 1 \mathrm{H}), 7.51-7.46(\mathrm{~m}, 1 \mathrm{H}), 7.34(\mathrm{dd}, J=11.2,4.1$ $\mathrm{Hz}, 1 \mathrm{H}), 7.30(\mathrm{~d}, J=8.3 \mathrm{~Hz}, 1 \mathrm{H}), 4.91(\mathrm{q}, J=8.3 \mathrm{~Hz}, 2 \mathrm{H}), 3.75(\mathrm{~s}, 3 \mathrm{H}) ;{ }^{13} \mathrm{C}$ NMR $\left(126 \mathrm{MHz}, \mathrm{CDCl}_{3}\right) \delta 152.04,150.23,132.12,129.97,128.17,127.88,124.25,123.15$ $(\mathrm{q}, J=277.2 \mathrm{~Hz}), 113.83,62.80(\mathrm{q}, J=36.5 \mathrm{~Hz}), 29.63 ;{ }^{19} \mathrm{~F}$ NMR $\left(471 \mathrm{MHz}, \mathrm{CDCl}_{3}\right)$ $\delta$-72.90; ATR-FTIR $\left(\mathrm{cm}^{-1}\right)$ : 2961, 1675, 1449, 1264, 1158, 1034; HRMS (ESI+): Calculated for $\mathrm{C}_{11} \mathrm{H}_{9} \mathrm{~F}_{3} \mathrm{~N}_{2} \mathrm{O}_{2} \mathrm{H}:[\mathrm{M}+\mathrm{H}]^{+}$259.0689, Found 259.0689.

\section{1-Ethyl-3-(2,2,2-trifluoroethoxy)quinoxalin-2(1H)-one (3b)}<smiles>CCn1c(=O)c(OCC(F)(F)F)nc2ccccc21</smiles>

Obtained as a white solid (231 mg, 85\% yield); M.p. 124-125 ${ }^{\circ} \mathrm{C} .{ }^{1} \mathrm{H}$ NMR (500 MHz, $\left.\mathrm{CDCl}_{3}\right) \delta 7.65(\mathrm{dd}, J=8.2,1.4 \mathrm{~Hz}, 1 \mathrm{H}), 7.49-7.45(\mathrm{~m}, 1 \mathrm{H}), 7.32(\mathrm{dd}, J=8.0,5.8 \mathrm{~Hz}$, $2 \mathrm{H}), 4.89$ (q, $J=8.4 \mathrm{~Hz}, 2 \mathrm{H}), 4.36(\mathrm{q}, J=7.2 \mathrm{~Hz}, 2 \mathrm{H}), 1.39(\mathrm{t}, J=7.2 \mathrm{~Hz}, 3 \mathrm{H}) ;{ }^{13} \mathrm{C}$ NMR $\left(126 \mathrm{MHz}, \mathrm{CDCl}_{3}\right) \delta 152.04,149.69,131.01,130.32,128.15,128.12,124.00$, $123.16(\mathrm{q}, J=277.2 \mathrm{~Hz}), 113.65,62.81(\mathrm{q}, J=36.5 \mathrm{~Hz}), 37.84,12.34 ;{ }^{19} \mathrm{~F}$ NMR $(471$ $\left.\mathrm{MHz}, \mathrm{CDCl}_{3}\right) \delta$-73.02; ATR-FTIR $\left(\mathrm{cm}^{-1}\right): 2979,1659,1451,1282,1164,1016$; HRMS (ESI+): Calculated for $\mathrm{C}_{12} \mathrm{H}_{11} \mathrm{~F}_{3} \mathrm{~N}_{2} \mathrm{O}_{2} \mathrm{H}:[\mathrm{M}+\mathrm{H}]^{+} 273.0846$, Found 273.0845.

\section{1-Butyl-3-(2,2,2-trifluoroethoxy)quinoxalin-2(1H)-one (3c)}<smiles>O=C(c1ccccc1)n1c(=O)c(OCC(F)F)nc2ccccc21</smiles>

Obtained as a white solid (49 mg, 82\% yield); M.p. $118-119{ }^{\circ} \mathrm{C} .{ }^{1} \mathrm{H}$ NMR $(500 \mathrm{MHz}$, $\left.\mathrm{CDCl}_{3}\right) \delta 7.65(\mathrm{dd}, J=7.9,1.4 \mathrm{~Hz}, 1 \mathrm{H}), 7.49-7.44(\mathrm{~m}, 1 \mathrm{H}), 7.34-7.28(\mathrm{~m}, 2 \mathrm{H})$, $4.89(\mathrm{q}, J=8.3 \mathrm{~Hz}, 2 \mathrm{H}), 4.32-4.25(\mathrm{~m}, 2 \mathrm{H}), 1.80-1.72(\mathrm{~m}, 2 \mathrm{H}), 1.53-1.45(\mathrm{~m}$, 2H), $1.00(\mathrm{t}, J=7.4 \mathrm{~Hz}, 3 \mathrm{H}) ;{ }^{13} \mathrm{C}$ NMR $\left(126 \mathrm{MHz}, \mathrm{CDCl}_{3}\right) \delta 152.04,149.94,131.28$, $130.28,128.12,128.06,123.99,123.15$ (q, $J=277.2 \mathrm{~Hz}), 113.84,62.82$ (q, $J=36.5$ $\mathrm{Hz}), 42.61,29.25,20.22,13.76 ;{ }^{19} \mathrm{~F}$ NMR $\left(471 \mathrm{MHz}, \mathrm{CDCl}_{3}\right) \delta-73.01$; ATR-FTIR $\left(\mathrm{cm}^{-1}\right)$ : 2961, 1672, 1468, 1271, 1166; HRMS (ESI+): Calculated for $\mathrm{C}_{14} \mathrm{H}_{15} \mathrm{~F}_{3} \mathrm{~N}_{2} \mathrm{O}_{2} \mathrm{H}$ : $[\mathrm{M}+\mathrm{H}]^{+}$301.1159, Found 301.1164. 
1-(Cyclohexylmethyl)-3-(2,2,2-trifluoroethoxy)quinoxalin-2(1H)-one (3d)<smiles>Cn1c(=O)c(OCC(F)(F)F)nc2ccccc21</smiles>

Obtained as a white solid (60 mg, 88\% yield); M.p. $144-145{ }^{\circ} \mathrm{C} .{ }^{1} \mathrm{H}$ NMR $(500 \mathrm{MHz}$, $\left.\mathrm{CDCl}_{3}\right) \delta 7.64(\mathrm{dd}, J=7.9,1.3 \mathrm{~Hz}, 1 \mathrm{H}), 7.48-7.43(\mathrm{~m}, 1 \mathrm{H}), 7.30(\mathrm{dd}, J=14.9,8.0$ $\mathrm{Hz}, 2 \mathrm{H}), 4.89$ (q, $J=8.3 \mathrm{~Hz}, 2 \mathrm{H}), 4.17$ (d, $J=7.3 \mathrm{~Hz}, 2 \mathrm{H}), 1.92(\mathrm{dd}, J=10.4,3.8 \mathrm{~Hz}$, $1 \mathrm{H}), 1.76-1.63(\mathrm{~m}, 5 \mathrm{H}), 1.24-1.14(\mathrm{~m}, 5 \mathrm{H}),{ }^{13} \mathrm{C} \mathrm{NMR}\left(126 \mathrm{MHz}, \mathrm{CDCl}_{3}\right) \delta 152.07$, $150.39,131.69,130.21,128.09,127.93,123.95,123.16$ (q, $J=277.2 \mathrm{~Hz}), 114.31$, $62.85(\mathrm{q}, J=36.5 \mathrm{~Hz}), 48.55,36.48,30.85,26.13,25.75 ;{ }^{19} \mathrm{~F} \mathrm{NMR}\left(471 \mathrm{MHz}, \mathrm{CDCl}_{3}\right)$ $\delta$-72.97; ATR-FTIR $\left(\mathrm{cm}^{-1}\right)$ : 2923, 1679, 1667, 1462, 1273, 1008; HRMS (ESI+): Calculated for $\mathrm{C}_{17} \mathrm{H}_{19} \mathrm{~F}_{3} \mathrm{~N}_{2} \mathrm{O}_{2} \mathrm{H}:[\mathrm{M}+\mathrm{H}]^{+}$341.1472, Found 341.1479.

Methyl 2-(2-oxo-3-(2,2,2-trifluoroethoxy)quinoxalin-1(2H)-yl)acetate (3e)<smiles></smiles>

Obtained as a white solid (50 mg, 79\% yield); M.p. $136-137{ }^{\circ} \mathrm{C} .{ }^{1} \mathrm{H}$ NMR $(500 \mathrm{MHz}$, $\left.\mathrm{CDCl}_{3}\right) \delta 7.66(\mathrm{dd}, J=7.9,1.1 \mathrm{~Hz}, 1 \mathrm{H}), 7.46-7.41(\mathrm{~m}, 1 \mathrm{H}), 7.33(\mathrm{t}, J=7.6 \mathrm{~Hz}, 1 \mathrm{H})$, $7.06(\mathrm{~d}, J=8.3 \mathrm{~Hz}, 1 \mathrm{H}), 5.07(\mathrm{~s}, 2 \mathrm{H}), 4.90(\mathrm{q}, J=8.3 \mathrm{~Hz}, 2 \mathrm{H}), 3.78(\mathrm{~s}, 3 \mathrm{H}) ;{ }^{13} \mathrm{C} \mathrm{NMR}$ $\left(126 \mathrm{MHz}, \mathrm{CDCl}_{3}\right) \delta 167.28,151.79,149.95,131.26,130.03,128.37,128.21,124.54$, $123.08(\mathrm{q}, J=277.2 \mathrm{~Hz}), 113.23,62.96(\mathrm{q}, J=36.5 \mathrm{~Hz}), 52.91,43.74 ;{ }^{19} \mathrm{~F}$ NMR (471 $\left.\mathrm{MHz}, \mathrm{CDCl}_{3}\right) \delta$-73.01; ATR-FTIR $\left(\mathrm{cm}^{-1}\right): 2960,1744,1668,1614,1417,1249,1155$, 1086; HRMS (ESI+): Calculated for $\mathrm{C}_{13} \mathrm{H}_{11} \mathrm{~F}_{3} \mathrm{~N}_{2} \mathrm{O}_{4} \mathrm{H}$ : $[\mathrm{M}+\mathrm{H}]^{+}$317.0744, Found 317.0749 .

tert-Butyl 2-(2-oxo-3-(2,2,2-trifluoroethoxy)quinoxalin-1(2H)-yl)acetate (3f)<smiles></smiles>

Obtained as a white solid (59 mg, 83\% yield); M.p. $124-125{ }^{\circ} \mathrm{C} .{ }^{1} \mathrm{H}$ NMR $(500 \mathrm{MHz}$, $\left.\mathrm{CDCl}_{3}\right) \delta 7.66(\mathrm{~d}, J=8.0 \mathrm{~Hz}, 1 \mathrm{H}), 7.44(\mathrm{dd}, J=11.4,4.2 \mathrm{~Hz}, 1 \mathrm{H}), 7.33(\mathrm{t}, J=7.6 \mathrm{~Hz}$, $1 \mathrm{H}), 7.05(\mathrm{~d}, J=8.3 \mathrm{~Hz}, 1 \mathrm{H}), 4.97(\mathrm{~s}, 2 \mathrm{H}), 4.90(\mathrm{q}, J=8.3 \mathrm{~Hz}, 2 \mathrm{H}), 1.46(\mathrm{~s}, 9 \mathrm{H}) ;{ }^{13} \mathrm{C}$ NMR $\left(126 \mathrm{MHz}, \mathrm{CDCl}_{3}\right) \delta 165.76,151.84,149.96,131.39,130.00,128.21,128.15$, $124.39,123.09$ (q, $J=277.2 \mathrm{~Hz}), 113.32,83.40,62.93(\mathrm{q}, J=36.5 \mathrm{~Hz}), 44.54,27.96$; ${ }^{19} \mathrm{~F}$ NMR (471 MHz, $\left.\mathrm{CDCl}_{3}\right) \delta$-73.00; ATR-FTIR $\left(\mathrm{cm}^{-1}\right): 3011,1724,1673,1613$, 1420, 1203, 1007; HRMS (ESI+): Calculated for $\mathrm{C}_{16} \mathrm{H}_{17} \mathrm{~F}_{3} \mathrm{~N}_{2} \mathrm{O}_{4} \mathrm{H}:[\mathrm{M}+\mathrm{H}]^{+} 359.1213$, Found 359.1214 .

1-Allyl-3-(2,2,2-trifluoroethoxy)quinoxalin-2(1H)-one (3g)<smiles>C=CCn1c(=O)c(OCC(F)(F)F)nc2ccccc21</smiles> 
Obtained as a white solid (40 mg, 71\% yield); M.p. $112-113{ }^{\circ} \mathrm{C} .{ }^{1} \mathrm{H}$ NMR $(500 \mathrm{MHz}$, $\left.\mathrm{CDCl}_{3}\right) \delta 7.65(\mathrm{dd}, J=8.0,1.2 \mathrm{~Hz}, 1 \mathrm{H}), 7.47-7.41(\mathrm{~m}, 1 \mathrm{H}), 7.32(\mathrm{dd}, J=11.1,4.2$ $\mathrm{Hz}, 1 \mathrm{H}), 7.28(\mathrm{~d}, J=8.4 \mathrm{~Hz}, 1 \mathrm{H}), 5.94(\mathrm{ddd}, J=22.4,10.4,5.2 \mathrm{~Hz}, 1 \mathrm{H}), 5.29(\mathrm{~d}, J=$ $10.4 \mathrm{~Hz}, 1 \mathrm{H}), 5.21(\mathrm{~d}, J=17.2 \mathrm{~Hz}, 1 \mathrm{H}), 4.94(\mathrm{~d}, J=5.2 \mathrm{~Hz}, 2 \mathrm{H}), 4.90$ (q, $J=8.3 \mathrm{~Hz}$, $2 \mathrm{H}) ;{ }^{13} \mathrm{C}$ NMR $\left(126 \mathrm{MHz}, \mathrm{CDCl}_{3}\right) \delta 152.04,149.85,131.33,130.32,130.14,128.07$, $127.98,124.19,123.10$ (q, $J=277.2 \mathrm{~Hz}), 118.50,114.41,62.89$ (q, $J=36.5 \mathrm{~Hz}$ ), 44.98; ${ }^{19} \mathrm{~F}$ NMR $\left(471 \mathrm{MHz}, \mathrm{CDCl}_{3}\right) \delta$-72.99; ATR-FTIR $\left(\mathrm{cm}^{-1}\right): 3080,2923,1683$, 1614, 1473, 1248, 1168, 989, 919; HRMS (ESI+): Calculated for $\mathrm{C}_{13} \mathrm{H}_{11} \mathrm{~F}_{3} \mathrm{~N}_{2} \mathrm{O}_{2} \mathrm{H}$ : $[\mathrm{M}+\mathrm{H}]^{+}$285.0846, Found 285.0855.

\section{3-(2,2,2-Trifluoroethoxy)quinoxalin-2(1H)-one (3h)}<smiles>O=c1[nH]c2ccccc2nc1OCC(F)(F)F</smiles>

Obtained as a white solid (39 mg, 80\% yield); M.p. $192-193{ }^{\circ} \mathrm{C} .{ }^{1} \mathrm{H}$ NMR (500 MHz, DMSO) $\delta 12.57(\mathrm{~s}, 1 \mathrm{H}), 7.56(\mathrm{~d}, J=8.0 \mathrm{~Hz}, 1 \mathrm{H}), 7.44-7.39(\mathrm{~m}, 1 \mathrm{H}), 7.31-7.24(\mathrm{~m}$, 2H), $5.06(\mathrm{q}, J=8.9 \mathrm{~Hz}, 2 \mathrm{H}) ;{ }^{13} \mathrm{C}$ NMR $(126 \mathrm{MHz}, \mathrm{DMSO}) \delta 153.26,150.24,131.31$, $129.73,128.17,126.76,124.22(\mathrm{q}, J=277.2 \mathrm{~Hz}), 123.93,115.65,62.70(\mathrm{q}, J=36.5$ $\mathrm{Hz}) ;{ }^{19} \mathrm{~F}$ NMR (471 MHz, DMSO) $\delta$-71.94; ATR-FTIR $\left(\mathrm{cm}^{-1}\right): 2912,1681,1614$, 1420, 1305, 1171, 1033; HRMS (ESI+): Calculated for $\mathrm{C}_{10} \mathrm{H}_{7} \mathrm{~F}_{3} \mathrm{~N}_{2} \mathrm{O}_{2} \mathrm{H}$ : $[\mathrm{M}+\mathrm{H}]^{+}$ 245.0533, Found 245.0533.

\section{1-Benzyl-3-(2,2,2-trifluoroethoxy)quinoxalin-2(1H)-one (3i)}<smiles>O=c1c(OCC(F)F)nc2ccccc2n1Cc1ccccc1</smiles>

Obtained as a white solid (53mg, 79\% yield); M.p. $129-130{ }^{\circ} \mathrm{C} .{ }^{1} \mathrm{H}$ NMR $(500 \mathrm{MHz}$, $\left.\mathrm{CDCl}_{3}\right) \delta 7.65(\mathrm{dd}, J=7.9,1.4 \mathrm{~Hz}, 1 \mathrm{H}), 7.37-7.31(\mathrm{~m}, 2 \mathrm{H}), 7.29(\mathrm{dd}, J=8.4,5.4 \mathrm{~Hz}$, $4 \mathrm{H}), 7.26-7.24(\mathrm{~m}, 2 \mathrm{H}), 5.52(\mathrm{~s}, 2 \mathrm{H}), 4.92(\mathrm{q}, J=8.3 \mathrm{~Hz}, 2 \mathrm{H}) ;{ }^{13} \mathrm{C}$ NMR $(126 \mathrm{MHz}$, $\left.\mathrm{CDCl}_{3}\right) \delta 152.10,150.45,134.90,131.43,130.22,128.98,128.16,128.00,127.87$, $127.03,124.28,123.11(\mathrm{q}, J=277.2 \mathrm{~Hz}), 114.68,62.98(\mathrm{q}, J=36.5 \mathrm{~Hz}), 46.42 ;{ }^{19} \mathrm{~F}$ NMR (471 MHz, $\left.\mathrm{CDCl}_{3}\right) \delta$-72.93; ATR-FTIR $\left(\mathrm{cm}^{-1}\right)$ : 2958, 1667, 1609, 1489, 1322, 1188, 750, 699; HRMS (ESI+): Calculated for $\mathrm{C}_{17} \mathrm{H}_{13} \mathrm{~F}_{3} \mathrm{~N}_{2} \mathrm{O}_{2} \mathrm{H}$ : $[\mathrm{M}+\mathrm{H}]^{+}$335.1002, Found 335.1009.

\section{1-(4-Methylbenzyl)-3-(2,2,2-trifluoroethoxy)quinoxalin-2(1H)-one (3j)}<smiles>Cc1ccc(Cn2c(=O)c(OCC(F)(F)F)nc3ccccc32)cc1</smiles> 
Obtained as a white solid (56 mg, 81\% yield); M.p. $177-178{ }^{\circ} \mathrm{C} .{ }^{1} \mathrm{H}$ NMR (500 MHz, $\left.\mathrm{CDCl}_{3}\right) \delta 7.63(\mathrm{dd}, J=8.2,1.4 \mathrm{~Hz}, 1 \mathrm{H}), 7.36-7.31(\mathrm{~m}, 1 \mathrm{H}), 7.28-7.25(\mathrm{~m}, 2 \mathrm{H})$, $7.17(\mathrm{~d}, J=8.1 \mathrm{~Hz}, 2 \mathrm{H}), 7.11(\mathrm{~d}, J=8.0 \mathrm{~Hz}, 2 \mathrm{H}), 5.47(\mathrm{~s}, 2 \mathrm{H}), 4.91(\mathrm{q}, J=8.3 \mathrm{~Hz}$, 2H), $2.29(\mathrm{~s}, 3 \mathrm{H}) ;{ }^{13} \mathrm{C} \mathrm{NMR}\left(126 \mathrm{MHz}, \mathrm{CDCl}_{3}\right) \delta 152.12,150.44,137.63,131.92$, 131.46, 130.22, 129.62, 128.11, 127.96, 127.08, 124.20, 123.16 (q, $J=277.2 \mathrm{~Hz}$ ), 114.70, $62.96(\mathrm{q}, J=36.5 \mathrm{~Hz}), 46.20,21.09 ;{ }^{19} \mathrm{~F} \mathrm{NMR}\left(471 \mathrm{MHz}, \mathrm{CDCl}_{3}\right) \delta-72.93$; ATR-FTIR $\left(\mathrm{cm}^{-1}\right)$ : 2927, 1681, 1614, 1473, 1268, 1180, 785; HRMS (ESI+): Calculated for $\mathrm{C}_{18} \mathrm{H}_{15} \mathrm{~F}_{3} \mathrm{~N}_{2} \mathrm{O}_{2} \mathrm{H}:[\mathrm{M}+\mathrm{H}]^{+} 349.1159$, Found 349.1163.

\section{1-(4-Fluorobenzyl)-3-(2,2,2-trifluoroethoxy)quinoxalin-2(1H)-one (3k)}<smiles>O=c1c(OCC(F)(F)F)nc2ccccc2n1Cc1ccc(F)cc1</smiles>

Obtained as a white solid (54 mg, 76\% yield); M.p. $132-133{ }^{\circ} \mathrm{C} .{ }^{1} \mathrm{H}$ NMR (500 MHz, $\left.\mathrm{CDCl}_{3}\right) \delta 7.65(\mathrm{dd}, J=7.9,1.4 \mathrm{~Hz}, 1 \mathrm{H}), 7.40-7.35(\mathrm{~m}, 1 \mathrm{H}), 7.32-7.28(\mathrm{~m}, 2 \mathrm{H})$, $7.28-7.22(\mathrm{~m}, 2 \mathrm{H}), 7.00(\mathrm{t}, J=8.6 \mathrm{~Hz}, 2 \mathrm{H}), 5.48(\mathrm{~s}, 2 \mathrm{H}), 4.92(\mathrm{q}, J=8.3 \mathrm{~Hz}, 2 \mathrm{H})$; ${ }^{13} \mathrm{C} \mathrm{NMR}\left(126 \mathrm{MHz}, \mathrm{CDCl}_{3}\right) \delta 162.31(\mathrm{~d}, J=247.0), 152.07,150.40,131.27,130.69$ $(\mathrm{d}, J=3.8 \mathrm{~Hz}), 130.25,128.96(\mathrm{~d}, J=8.8 \mathrm{~Hz}), 128.20,128.13,124.39,123.13(\mathrm{q}, J=$ $277.2 \mathrm{~Hz}), 115.93(\mathrm{~d}, J=21.4 \mathrm{~Hz}), 114.42,62.99(\mathrm{q}, J=36.5 \mathrm{~Hz}), 45.74 ;{ }^{19} \mathrm{~F}$ NMR $\left(471 \mathrm{MHz}, \mathrm{CDCl}_{3}\right) \delta$-72.96, -114.07; ATR-FTIR $\left(\mathrm{cm}^{-1}\right): 2961,1676,1616,1511$, 1272, 1166, 752; HRMS (ESI+): Calculated for $\mathrm{C}_{17} \mathrm{H}_{12} \mathrm{~F}_{4} \mathrm{~N}_{2} \mathrm{O}_{2} \mathrm{H}:[\mathrm{M}+\mathrm{H}]^{+} 353.0908$, Found 353.0910.

1-(4-Chlorobenzyl)-3-(2,2,2-trifluoroethoxy)quinoxalin-2(1H)-one (31)<smiles>O=c1c(OCC(F)(F)F)nc2ccccc2n1Cc1ccc(Cl)cc1</smiles>

Obtained as a white solid (53 mg, 72\% yield); M.p. $187-188{ }^{\circ} \mathrm{C} .{ }^{1} \mathrm{H}$ NMR (500 MHz, $\left.\mathrm{CDCl}_{3}\right) \delta 7.66(\mathrm{dd}, J=7.9,1.5 \mathrm{~Hz}, 1 \mathrm{H}), 7.39-7.34(\mathrm{~m}, 1 \mathrm{H}), 7.29(\mathrm{~d}, J=8.7 \mathrm{~Hz}, 3 \mathrm{H})$, $7.21(\mathrm{dd}, J=15.1,4.8 \mathrm{~Hz}, 3 \mathrm{H}), 5.48(\mathrm{~s}, 2 \mathrm{H}), 4.92(\mathrm{q}, J=8.3 \mathrm{~Hz}, 2 \mathrm{H}) ;{ }^{13} \mathrm{C}$ NMR $(126$ $\left.\mathrm{MHz}, \mathrm{CDCl}_{3}\right) \delta 152.04,150.39,133.80,133.42,131.21,130.23,129.17,128.52$, $128.23,128.14,124.45,123.12$ (q, $J=277.2 \mathrm{~Hz}), 114.40,63.00$ (q, $J=36.5 \mathrm{~Hz}$ ), 45.79; ${ }^{19} \mathrm{~F}$ NMR (471 MHz, $\left.\mathrm{CDCl}_{3}\right) \delta$-72.94; ATR-FTIR $\left(\mathrm{cm}^{-1}\right): 2959,1671,1613$, 1490, 1269, 1125, 798; HRMS (ESI+): Calculated for $\mathrm{C}_{17} \mathrm{H}_{12} \mathrm{ClF}_{3} \mathrm{~N}_{2} \mathrm{O}_{2} \mathrm{H}:[\mathrm{M}+\mathrm{H}]^{+}$ 369.0612, Found 369.0619. 


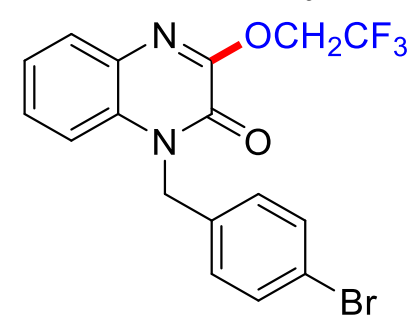

Obtained as a white solid (64 mg, 78\% yield); M.p. 208-209 ${ }^{\circ} \mathrm{C} .{ }^{1} \mathrm{H}$ NMR (500 MHz, $\left.\mathrm{CDCl}_{3}\right) \delta 7.66(\mathrm{dd}, J=7.9,1.5 \mathrm{~Hz}, 1 \mathrm{H}), 7.44(\mathrm{~d}, J=8.5 \mathrm{~Hz}, 2 \mathrm{H}), 7.39-7.34(\mathrm{~m}, 1 \mathrm{H})$, $7.30(\mathrm{td}, J=7.7,1.2 \mathrm{~Hz}, 1 \mathrm{H}), 7.19(\mathrm{dd}, J=8.3,1.0 \mathrm{~Hz}, 1 \mathrm{H}), 7.16(\mathrm{~d}, J=8.4 \mathrm{~Hz}, 2 \mathrm{H})$, $5.46(\mathrm{~s}, 2 \mathrm{H}), 4.92(\mathrm{q}, J=8.3 \mathrm{~Hz}, 2 \mathrm{H}) ;{ }^{13} \mathrm{C} \mathrm{NMR}\left(126 \mathrm{MHz}, \mathrm{CDCl}_{3}\right) \delta$ 152.03, 150.39, $133.95,132.13,131.20,130.23,128.83,128.23,128.15,124.45,123.11$ (q, $J=265.9$ $\mathrm{Hz}), 121.87,114.39,63.02(\mathrm{q}, J=36.5 \mathrm{~Hz}), 45.86 ;{ }^{19} \mathrm{~F}$ NMR $\left(471 \mathrm{MHz}, \mathrm{CDCl}_{3}\right) \delta$ -72.95; ATR-FTIR $\left(\mathrm{cm}^{-1}\right)$ : 2962, 1671, 1616, 1491, 1240, 1182, 796; HRMS (ESI+): Calculated for $\mathrm{C}_{17} \mathrm{H}_{12} \mathrm{BrF}_{3} \mathrm{~N}_{2} \mathrm{O}_{2} \mathrm{H}$ : $[\mathrm{M}+\mathrm{H}]^{+}$413.0107, Found 413.0114.

\section{1-(4-Nitrobenzyl)-3-(2,2,2-trifluoroethoxy)quinoxalin-2(1H)-one (3n)}<smiles>O=c1c(OCC(F)(F)F)nc2ccccc2n1Cc1ccc([N+](=O)[O-])cc1</smiles>

Obtained as a white solid (52 mg, 69\% yield); M.p. 190-191 ${ }^{\circ} \mathrm{C} .{ }^{1} \mathrm{H}$ NMR (500 MHz, $\left.\mathrm{CDCl}_{3}\right) \delta 8.18(\mathrm{~d}, J=8.8 \mathrm{~Hz}, 2 \mathrm{H}), 7.69(\mathrm{dd}, J=7.8,1.6 \mathrm{~Hz}, 1 \mathrm{H}), 7.44(\mathrm{~d}, J=8.8 \mathrm{~Hz}$, $2 \mathrm{H}), 7.39-7.35(\mathrm{~m}, 1 \mathrm{H}), 7.33(\mathrm{td}, J=7.6,1.4 \mathrm{~Hz}, 1 \mathrm{H}), 7.12(\mathrm{dd}, J=8.2,1.2 \mathrm{~Hz}, 1 \mathrm{H})$, $5.61(\mathrm{~s}, 2 \mathrm{H}), 4.94(\mathrm{q}, J=8.3 \mathrm{~Hz}, 2 \mathrm{H}) ;{ }^{13} \mathrm{C} \mathrm{NMR}\left(126 \mathrm{MHz}, \mathrm{CDCl}_{3}\right) \delta 151.95,150.36$, $147.63,142.21,130.98,130.26,128.43,128.38,127.87,124.77,124.26,123.07$ (q, $J$ $=277.2 \mathrm{~Hz}), 114.07,63.08(\mathrm{q}, J=36.5 \mathrm{~Hz}), 45.84 ;{ }^{19} \mathrm{~F}$ NMR $\left(471 \mathrm{MHz}, \mathrm{CDCl}_{3}\right) \delta$ -72.96; ATR-FTIR $\left(\mathrm{cm}^{-1}\right)$ : 2957, 1701, 1616, 1521, 1352, 1272, 1167, 773; HRMS (ESI+): Calculated for $\mathrm{C}_{17} \mathrm{H}_{12} \mathrm{~F}_{3} \mathrm{~N}_{3} \mathrm{O}_{4} \mathrm{H}:[\mathrm{M}+\mathrm{H}]^{+}$380.0853, Found 380.0858 .

\section{1-(3-Methylbenzyl)-3-(2,2,2-trifluoroethoxy)quinoxalin-2(1H)-one (3o)}<smiles>Cc1cccc(Cn2c(=O)c(OCC(F)(F)F)nc3ccccc32)c1</smiles>

Obtained as a white solid (56 mg, 80\% yield); M.p. $131-132{ }^{\circ} \mathrm{C} .{ }^{1} \mathrm{H}$ NMR (500 MHz, $\left.\mathrm{CDCl}_{3}\right) \delta 7.69-7.60(\mathrm{~m}, 1 \mathrm{H}), 7.37-7.32(\mathrm{~m}, 1 \mathrm{H}), 7.30-7.24(\mathrm{~m}, 2 \mathrm{H}), 7.23-7.17$ (m, 1H), 7.07 (d, $J=7.4 \mathrm{~Hz}, 3 \mathrm{H}), 5.48(\mathrm{~s}, 2 \mathrm{H}), 4.92(\mathrm{q}, J=8.3 \mathrm{~Hz}, 2 \mathrm{H}), 2.30(\mathrm{~s}, 3 \mathrm{H})$; ${ }^{13} \mathrm{C}$ NMR $\left(126 \mathrm{MHz}, \mathrm{CDCl}_{3}\right) \delta 152.12,150.48,138.82,134.84,131.50,130.22$, $128.82,128.66,128.16,127.95,127.60,124.24,124.07,123.17$ (q, $J=277.2 \mathrm{~Hz}$ ), $114.74,62.98(\mathrm{q}, J=36.5 \mathrm{~Hz}), 46.46,21.42 ;{ }^{19} \mathrm{~F} \mathrm{NMR}\left(471 \mathrm{MHz}, \mathrm{CDCl}_{3}\right) \delta-72.92$; 
ATR-FTIR $\left(\mathrm{cm}^{-1}\right)$ : 2925, 1676, 1616, 1449, 1240, 1155, 759, 654; HRMS (ESI+): Calculated for $\mathrm{C}_{18} \mathrm{H}_{15} \mathrm{~F}_{3} \mathrm{~N}_{2} \mathrm{O}_{2} \mathrm{H}$ : $[\mathrm{M}+\mathrm{H}]^{+} 349.1159$, Found 349.1169.

1-(3-Chlorobenzyl)-3-(2,2,2-trifluoroethoxy)quinoxalin-2(1H)-one (3p)<smiles>O=c1c(OCC(F)(F)F)nc2ccccc2n1Cc1cccc(Cl)c1</smiles>

Obtained as a white solid (54 mg, 73\% yield); M.p. 153-154 ${ }^{\circ} \mathrm{C} .{ }^{1} \mathrm{H}$ NMR $(500 \mathrm{MHz}$, $\left.\mathrm{CDCl}_{3}\right) \delta 7.66(\mathrm{dd}, J=7.9,1.4 \mathrm{~Hz}, 1 \mathrm{H}), 7.40-7.35(\mathrm{~m}, 1 \mathrm{H}), 7.33-7.28(\mathrm{~m}, 1 \mathrm{H})$, $7.25(\mathrm{~d}, J=5.0 \mathrm{~Hz}, 3 \mathrm{H}), 7.21-7.17(\mathrm{~m}, 1 \mathrm{H}), 7.16(\mathrm{dd}, J=5.3,2.9 \mathrm{~Hz}, 1 \mathrm{H}), 5.49$ (s, $2 \mathrm{H}), 4.93(\mathrm{q}, J=8.3 \mathrm{~Hz}, 2 \mathrm{H}) ;{ }^{13} \mathrm{C} \mathrm{NMR}\left(126 \mathrm{MHz}, \mathrm{CDCl}_{3}\right) \delta 152.02,150.38,136.93$, $134.96,131.23,130.29,130.23,128.30,128.20,128.15,127.11,125.20,124.48$, 123.12 (q, $J=277.2 \mathrm{~Hz}), 114.39,63.04$ (q, $J=36.5 \mathrm{~Hz}), 45.90 ;{ }^{19} \mathrm{~F}$ NMR $(471 \mathrm{MHz}$, $\left.\mathrm{CDCl}_{3}\right) \delta$-72.94; ATR-FTIR $\left(\mathrm{cm}^{-1}\right):$ 2990, 1678, 1621, 1474, 1329, 1240, 1170, 754, 689; HRMS (ESI+): Calculated for $\mathrm{C}_{17} \mathrm{H}_{12} \mathrm{ClF}_{3} \mathrm{~N}_{2} \mathrm{O}_{2} \mathrm{H}$ : $[\mathrm{M}+\mathrm{H}]^{+}$369.0612, Found 369.0619 .

1-(3-Bromobenzyl)-3-(2,2,2-trifluoroethoxy)quinoxalin-2(1H)-one (3q)<smiles>O=c1c(OCC(F)(F)F)nc2ccccc2n1Cc1cccc(Br)c1</smiles>

Obtained as a white solid (62 mg, 75\% yield); M.p. $146-147{ }^{\circ} \mathrm{C} .{ }^{1} \mathrm{H}$ NMR (500 MHz, $\left.\mathrm{CDCl}_{3}\right) \delta 7.66(\mathrm{dd}, J=7.9,1.3 \mathrm{~Hz}, 1 \mathrm{H}), 7.45-7.39(\mathrm{~m}, 2 \mathrm{H}), 7.37(\mathrm{dd}, J=11.4,4.2$ $\mathrm{Hz}, 1 \mathrm{H}), 7.30$ (dd, $J=11.1,4.0 \mathrm{~Hz}, 1 \mathrm{H}), 7.22-7.16(\mathrm{~m}, 3 \mathrm{H}), 5.48$ (s, 2H), 4.93 (q, $J$ $=8.3 \mathrm{~Hz}, 2 \mathrm{H}) ;{ }^{13} \mathrm{C} \mathrm{NMR}\left(126 \mathrm{MHz}, \mathrm{CDCl}_{3}\right) \delta 152.02,150.38,137.20,131.22,131.15$, $130.55,130.23,129.99,128.31,128.15,125.67,124.49,123.12$ (q, $J=277.2 \mathrm{~Hz}$ ), 123.10, 114.38, $63.04(\mathrm{q}, J=36.5 \mathrm{~Hz}), 45.85 ;{ }^{19} \mathrm{~F} \mathrm{NMR}\left(471 \mathrm{MHz}, \mathrm{CDCl}_{3}\right) \delta-72.93$; ATR-FTIR $\left(\mathrm{cm}^{-1}\right)$ : 2925, 1676, 1616, 1472, 1273, 1168, 753, 702; HRMS (ESI+): Calculated for $\mathrm{C}_{17} \mathrm{H}_{12} \mathrm{BrF}_{3} \mathrm{~N}_{2} \mathrm{O}_{2} \mathrm{H}$ : $[\mathrm{M}+\mathrm{H}]^{+}$413.0107, Found 413.0110.

1-(3-Nitrobenzyl)-3-(2,2,2-trifluoroethoxy)quinoxalin-2(1H)-one (3r)<smiles>O=c1c(OCC(F)(F)F)nc2ccccc2n1Cc1cccc([N+](=O)[O-])c1</smiles>

Obtained as a white solid (45 mg, 60\% yield); M.p. $182-183{ }^{\circ} \mathrm{C} .{ }^{1} \mathrm{H}$ NMR (500 MHz, $\left.\mathrm{CDCl}_{3}\right) \delta 8.16(\mathrm{dd}, J=4.5,2.4 \mathrm{~Hz}, 2 \mathrm{H}), 7.70(\mathrm{dd}, J=7.9,1.5 \mathrm{~Hz}, 1 \mathrm{H}), 7.61(\mathrm{~d}, J=$ $8.1 \mathrm{~Hz}, 1 \mathrm{H}), 7.52(\mathrm{dd}, J=8.7,7.8 \mathrm{~Hz}, 1 \mathrm{H}), 7.42-7.37(\mathrm{~m}, 1 \mathrm{H}), 7.34(\mathrm{td}, J=7.7,1.3$ 
$\mathrm{Hz}, 1 \mathrm{H}), 7.17(\mathrm{dd}, J=8.3,1.1 \mathrm{~Hz}, 1 \mathrm{H}), 5.61(\mathrm{~s}, 2 \mathrm{H}), 4.94(\mathrm{q}, J=8.3 \mathrm{~Hz}, 2 \mathrm{H}) ;{ }^{13} \mathrm{C}$ NMR $\left(126 \mathrm{MHz}, \mathrm{CDCl}_{3}\right) \delta 151.99,150.38,148.62,137.07,133.16,131.02,130.29$, 130.19, 128.45, 128.41, 124.74, 123.12, 123.10 (q, $J=277.2 \mathrm{~Hz}), 122.08,113.99$, 63.09 (q, $J=36.5 \mathrm{~Hz}), 45.74 ;{ }^{19} \mathrm{~F}$ NMR $\left(471 \mathrm{MHz}, \mathrm{CDCl}_{3}\right) \delta$-72.95; ATR-FTIR $\left(\mathrm{cm}^{-1}\right)$ : 2922, 1681, 1624, 1506, 1444, 1327, 1155, 756, 654; HRMS (ESI+): Calculated for $\mathrm{C}_{17} \mathrm{H}_{12} \mathrm{~F}_{3} \mathrm{~N}_{3} \mathrm{O}_{4} \mathrm{H}$ : $[\mathrm{M}+\mathrm{H}]^{+}$380.0853, Found 380.0862 .

\section{1-(2-Fluorobenzyl)-3-(2,2,2-trifluoroethoxy)quinoxalin-2(1H)-one (3s)}<smiles>O=c1c(OCC(F)(F)F)nc2ccccc2n1Cc1ccccc1F</smiles>

Obtained as a white solid (54 mg, 77\% yield); M.p. $145-146{ }^{\circ} \mathrm{C} .{ }^{1} \mathrm{H}$ NMR (500 MHz, $\left.\mathrm{CDCl}_{3}\right) \delta 7.65(\mathrm{dd}, J=7.9,1.4 \mathrm{~Hz}, 1 \mathrm{H}), 7.40-7.35(\mathrm{~m}, 1 \mathrm{H}), 7.30(\mathrm{dd}, J=11.4,3.9$ $\mathrm{Hz}, 1 \mathrm{H}), 7.27-7.23(\mathrm{~m}, 2 \mathrm{H}), 7.14-7.06(\mathrm{~m}, 2 \mathrm{H}), 7.05-7.00(\mathrm{~m}, 1 \mathrm{H}), 5.58(\mathrm{~s}, 2 \mathrm{H})$, 4.93 (q, $J=8.3 \mathrm{~Hz}, 2 \mathrm{H}) ;{ }^{13} \mathrm{C}$ NMR $\left(126 \mathrm{MHz}, \mathrm{CDCl}_{3}\right) \delta 160.29(\mathrm{~d}, J=247.0), 152.02$, $150.62,131.15,130.17,129.66(\mathrm{~d}, J=8.8 \mathrm{~Hz}), 128.71(\mathrm{~d}, J=2.5 \mathrm{~Hz}), 128.36,128.03$, $124.76(\mathrm{~d}, J=3.8 \mathrm{~Hz}), 124.43,123.13(\mathrm{q}, J=277.2 \mathrm{~Hz}), 122.00(\mathrm{~d}, J=13.9 \mathrm{~Hz})$, $115.59(\mathrm{~d}, J=21.4 \mathrm{~Hz}), 114.26(\mathrm{~d}, J=2.5 \mathrm{~Hz}), 63.00(\mathrm{q}, J=36.5 \mathrm{~Hz}), 39.83(\mathrm{~d}, J=$ $5.0 \mathrm{~Hz}) ;{ }^{19} \mathrm{~F}$ NMR $\left(471 \mathrm{MHz}, \mathrm{CDCl}_{3}\right) \delta$-72.95, -118.26; ATR-FTIR $\left(\mathrm{cm}^{-1}\right): 3039$, 1674, 1615, 1339, 1239, 1128, 754; HRMS (ESI+): Calculated for $\mathrm{C}_{17} \mathrm{H}_{12} \mathrm{~F}_{4} \mathrm{~N}_{2} \mathrm{O}_{2} \mathrm{H}$ : $[\mathrm{M}+\mathrm{H}]^{+}$353.0908, Found 353.0918.

\section{1-(2-Chlorobenzyl)-3-(2,2,2-trifluoroethoxy)quinoxalin-2(1H)-one (3t)}<smiles>O=c1c(OCC(F)(F)F)nc2ccccc2n1Cc1ccccc1Cl</smiles>

Obtained as a white solid (53 mg, 72\% yield); M.p. $154-155{ }^{\circ} \mathrm{C} .{ }^{1} \mathrm{H}$ NMR (500 MHz, $\left.\mathrm{CDCl}_{3}\right) \delta 7.67(\mathrm{dd}, J=7.8,1.6 \mathrm{~Hz}, 1 \mathrm{H}), 7.45(\mathrm{dd}, J=8.0,1.0 \mathrm{~Hz}, 1 \mathrm{H}), 7.37-7.32(\mathrm{~m}$, $1 \mathrm{H}), 7.30(\mathrm{td}, J=7.6,1.3 \mathrm{~Hz}, 1 \mathrm{H}), 7.22(\mathrm{td}, J=7.9,1.3 \mathrm{~Hz}, 1 \mathrm{H}), 7.11(\mathrm{td}, J=7.7,0.9$ $\mathrm{Hz}, 1 \mathrm{H}), 7.03(\mathrm{dd}, J=8.2,1.2 \mathrm{~Hz}, 1 \mathrm{H}), 6.81(\mathrm{~d}, J=7.7 \mathrm{~Hz}, 1 \mathrm{H}), 5.61(\mathrm{~s}, 2 \mathrm{H}), 4.94(\mathrm{q}$, $J=8.3 \mathrm{~Hz}, 2 \mathrm{H}) ;{ }^{13} \mathrm{C} \mathrm{NMR}\left(126 \mathrm{MHz}, \mathrm{CDCl}_{3}\right) \delta 152.02,150.47,132.63,131.92$, $131.15,130.18,129.83,128.99,128.40,128.01,127.39,127.00,124.50,123.13$ (q, $J$ $=277.2 \mathrm{~Hz}), 114.59,63.02(\mathrm{q}, J=36.5 \mathrm{~Hz}), 44.00 ;{ }^{19} \mathrm{~F} \mathrm{NMR}\left(471 \mathrm{MHz}, \mathrm{CDCl}_{3}\right) \delta$ -72.91; ATR-FTIR ( $\left.\mathrm{cm}^{-1}\right)$ : 3035, 1675, 1614, 1490, 1325, 1175, 1069, 751; HRMS (ESI+): Calculated for $\mathrm{C}_{17} \mathrm{H}_{12} \mathrm{ClF}_{3} \mathrm{~N}_{2} \mathrm{O}_{2} \mathrm{H}:[\mathrm{M}+\mathrm{H}]^{+}$369.0612, Found 369.0615. 
<smiles>O=c1c(OCC(F)(F)F)nc2ccccc2n1Cc1ccc(F)cc1F</smiles>

Obtained as a white solid (48 mg, 65\% yield); M.p. $145-146{ }^{\circ} \mathrm{C} .{ }^{1} \mathrm{H}$ NMR $(500 \mathrm{MHz}$, $\left.\mathrm{CDCl}_{3}\right) \delta 7.66(\mathrm{dd}, J=7.9,1.4 \mathrm{~Hz}, 1 \mathrm{H}), 7.43-7.37(\mathrm{~m}, 1 \mathrm{H}), 7.34-7.29(\mathrm{~m}, 1 \mathrm{H})$, $7.26-7.22(\mathrm{~m}, 1 \mathrm{H}), 7.14(\mathrm{td}, J=8.6,6.4 \mathrm{~Hz}, 1 \mathrm{H}), 6.91-6.84(\mathrm{~m}, 1 \mathrm{H}), 6.82-6.74$ $(\mathrm{m}, 1 \mathrm{H}), 5.52(\mathrm{~s}, 2 \mathrm{H}), 4.92(\mathrm{q}, J=8.3 \mathrm{~Hz}, 2 \mathrm{H}) ;{ }^{13} \mathrm{C} \mathrm{NMR}\left(126 \mathrm{MHz}, \mathrm{CDCl}_{3}\right) \delta 162.64$ $(\mathrm{dd}, J=250.7,12.6 \mathrm{~Hz}), 160.38(\mathrm{dd}, J=248.2,12.6 \mathrm{~Hz}), 151.97,150.62,131.01$, $130.19,130.06$ (dd, $J=10.1,5.0 \mathrm{~Hz}), 128.41,128.14,124.55,123.11$ (q, $J=277.2$ $\mathrm{Hz}), 118.03(\mathrm{dd}, J=14.2,3.9 \mathrm{~Hz}), 114.02(\mathrm{~d}, J=2.5 \mathrm{~Hz}), 112.10(\mathrm{dd}, J=21.4,3.8$ $\mathrm{Hz}), 104.08(\mathrm{t}, J=25.2 \mathrm{~Hz}), 62.98(\mathrm{q}, J=36.5 \mathrm{~Hz}), 39.39(\mathrm{~d}, J=3.8 \mathrm{~Hz}) ;{ }^{19} \mathrm{~F}$ NMR $\left(471 \mathrm{MHz}, \mathrm{CDCl}_{3}\right) \delta-72.97,-109.79(\mathrm{~d}, J=7.9 \mathrm{~Hz}),-113.84(\mathrm{~d}, J=7.9 \mathrm{~Hz})$; ATR-FTIR $\left(\mathrm{cm}^{-1}\right)$ : 3041, 1676, 1615, 1291, 1199, 1165, 1094, 862, 792; HRMS (ESI+): Calculated for $\mathrm{C}_{17} \mathrm{H}_{11} \mathrm{~F}_{5} \mathrm{~N}_{2} \mathrm{O}_{2} \mathrm{H}:[\mathrm{M}+\mathrm{H}]^{+} 371.0814$, Found 371.0809.

\section{1,6-Dimethyl-3-(2,2,2-trifluoroethoxy)quinoxalin-2(1H)-one (3v)}<smiles>Cc1ccc2c(c1)nc(OCC(F)(F)F)c(=O)n2C</smiles>

Obtained as a white solid (44 mg, 81\% yield); M.p. 165-166 ${ }^{\circ} \mathrm{C} .{ }^{1} \mathrm{H}$ NMR $(500 \mathrm{MHz}$, $\left.\mathrm{CDCl}_{3}\right) \delta 7.52(\mathrm{~d}, J=8.1 \mathrm{~Hz}, 1 \mathrm{H}), 7.15(\mathrm{~d}, J=8.1 \mathrm{~Hz}, 1 \mathrm{H}), 7.09(\mathrm{~s}, 1 \mathrm{H}), 4.88(\mathrm{q}, J=$ $8.3 \mathrm{~Hz}, 2 \mathrm{H}), 3.73$ (s, 3H), $2.50(\mathrm{~s}, 3 \mathrm{H}) ;{ }^{13} \mathrm{C} \mathrm{NMR}\left(126 \mathrm{MHz}, \mathrm{CDCl}_{3}\right) \delta 151.46,150.34$, $138.64,131.95,127.88,127.56,125.40,123.11(\mathrm{q}, J=277.2 \mathrm{~Hz}), 114.05,62.72(\mathrm{q}, J$ $=36.5 \mathrm{~Hz}), 29.56,21.88 ;{ }^{19} \mathrm{~F}$ NMR $\left(471 \mathrm{MHz}, \mathrm{CDCl}_{3}\right) \delta-73.03$; ATR-FTIR $\left(\mathrm{cm}^{-1}\right)$ : 2958, 1674, 1620, 1466, 1302, 1165, 1124, 1070; HRMS (ESI+): Calculated for $\mathrm{C}_{12} \mathrm{H}_{11} \mathrm{~F}_{3} \mathrm{~N}_{2} \mathrm{O}_{2} \mathrm{H}:[\mathrm{M}+\mathrm{H}]^{+}$273.0845, Found 273.0856.

\section{6-Methoxy-1-methyl-3-(2,2,2-trifluoroethoxy)quinoxalin-2(1H)-one (3w)}<smiles>COc1ccc2c(c1)nc(OCC(F)(F)F)c(=O)n2C</smiles>

Obtained as a white solid (48 mg, 83\% yield); M.p. $172-173{ }^{\circ} \mathrm{C} .{ }^{1} \mathrm{H}$ NMR $(500 \mathrm{MHz}$, DMSO) $\delta 7.43(\mathrm{~d}, J=9.0 \mathrm{~Hz}, 1 \mathrm{H}), 7.11(\mathrm{dt}, J=7.7,2.8 \mathrm{~Hz}, 2 \mathrm{H}), 5.07$ (q, $J=8.9 \mathrm{~Hz}$, $2 \mathrm{H}), 3.82(\mathrm{~s}, 3 \mathrm{H}), 3.61(\mathrm{~s}, 3 \mathrm{H}) ;{ }^{13} \mathrm{C} \mathrm{NMR}(126 \mathrm{MHz}, \mathrm{DMSO}) \delta 156.23,152.72$, $149.39,130.56,126.53,124.19$ (q, $J=277.2 \mathrm{~Hz}), 116.38,116.16,110.07,62.70$ (q, $J$ $=36.5 \mathrm{~Hz}), 56.01,29.98 ;{ }^{19} \mathrm{~F}$ NMR $(471 \mathrm{MHz}, \mathrm{DMSO}) \delta-71.94$; ATR-FTIR $\left(\mathrm{cm}^{-1}\right)$ : 2962, 1678, 1606, 1514, 1407, 1295, 1157, 1070; HRMS (ESI+): Calculated for $\mathrm{C}_{12} \mathrm{H}_{11} \mathrm{~F}_{3} \mathrm{~N}_{2} \mathrm{O}_{3} \mathrm{H}:[\mathrm{M}+\mathrm{H}]^{+}$289.0795, Found 289.0799. 
7-Chloro-1-methyl-3-(2,2,2-trifluoroethoxy)quinoxalin-2(1H)-one (3x)<smiles>Cn1c(=O)c(OCC(F)F)nc2ccc(Cl)cc21</smiles>

Obtained as a white solid (42 mg, 72\% yield); M.p. $150-151{ }^{\circ} \mathrm{C} .{ }^{1} \mathrm{H}$ NMR (500 MHz, $\left.\mathrm{CDCl}_{3}\right) \delta 7.56(\mathrm{~d}, J=9.1 \mathrm{~Hz}, 1 \mathrm{H}), 7.29(\mathrm{dd}, J=6.6,2.2 \mathrm{~Hz}, 2 \mathrm{H}), 4.88(\mathrm{q}, J=8.3 \mathrm{~Hz}$, 2H), $3.71(\mathrm{~s}, 3 \mathrm{H}) ;{ }^{13} \mathrm{C}$ NMR $\left(126 \mathrm{MHz}, \mathrm{CDCl}_{3}\right) \delta 152.04,149.93,133.94,132.94$, $128.85,128.51,124.52,123.01$ (q, $J=277.2 \mathrm{~Hz}), 113.95,62.92$ (q, $J=36.5 \mathrm{~Hz}$ ), 29.75; ${ }^{19} \mathrm{~F}$ NMR (471 MHz, $\left.\mathrm{CDCl}_{3}\right) \delta$-73.02; ATR-FTIR $\left(\mathrm{cm}^{-1}\right): 2922,1681,1613$, 1463, 1280, 1167, 1005; HRMS (ESI+): Calculated for $\mathrm{C}_{11} \mathrm{H}_{8} \mathrm{ClF}_{3} \mathrm{~N}_{2} \mathrm{O}_{2} \mathrm{H}:[\mathrm{M}+\mathrm{H}]^{+}$ 293.0299, Found 293.0300.

7-Bromo-1-methyl-3-(2,2,2-trifluoroethoxy)quinoxalin-2(1H)-one (3y)<smiles>Cn1c(=O)c(OCC(F)F)nc2ccc(Br)cc21</smiles>

Obtained as a white solid (52 mg, 78\% yield); M.p. $150-151{ }^{\circ} \mathrm{C} .{ }^{1} \mathrm{H}$ NMR $(500 \mathrm{MHz}$, $\left.\mathrm{CDCl}_{3}\right) \delta 7.50(\mathrm{dd}, J=7.9,1.0 \mathrm{~Hz}, 1 \mathrm{H}), 7.47-7.41(\mathrm{~m}, 2 \mathrm{H}), 4.88(\mathrm{q}, J=8.3 \mathrm{~Hz}, 2 \mathrm{H})$, $3.71(\mathrm{~s}, 3 \mathrm{H}) ;{ }^{13} \mathrm{C}$ NMR $\left(126 \mathrm{MHz}, \mathrm{CDCl}_{3}\right) \delta 152.19,149.88,133.14,129.06,128.90$, $127.41,123.00(\mathrm{q}, J=277.2 \mathrm{~Hz}), 121.75,116.90,62.94(\mathrm{q}, J=36.5 \mathrm{~Hz}), 29.75 ;{ }^{19} \mathrm{~F}$ NMR (471 MHz, $\left.\mathrm{CDCl}_{3}\right) \delta$-73.02; ATR-FTIR $\left(\mathrm{cm}^{-1}\right)$ : 2923, 1675, 1611, 1453, 1176 , 1124, 1002; HRMS (ESI+): Calculated for $\mathrm{C}_{11} \mathrm{H}_{8} \mathrm{BrF}_{3} \mathrm{~N}_{2} \mathrm{O}_{2} \mathrm{H}:[\mathrm{M}+\mathrm{H}]^{+}$336.9794, Found 336.9791.

\section{1,6,7-Trimethyl-3-(2,2,2-trifluoroethoxy)quinoxalin-2(1H)-one (3z)}<smiles>Cc1cc2nc(OCC(F)(F)F)c(=O)n(C)c2cc1C</smiles>

Obtained as a white solid (45 mg, 79\% yield); M.p. 139-140 ${ }^{\circ} \mathrm{C} .{ }^{1} \mathrm{H}$ NMR $(500 \mathrm{MHz}$, $\left.\mathrm{CDCl}_{3}\right) \delta 7.39(\mathrm{~s}, 1 \mathrm{H}), 7.03(\mathrm{~s}, 1 \mathrm{H}), 4.86(\mathrm{q}, J=8.4 \mathrm{~Hz}, 2 \mathrm{H}), 3.69(\mathrm{~s}, 3 \mathrm{H}), 2.39$ (s, $3 \mathrm{H}), 2.33(\mathrm{~s}, 3 \mathrm{H}) ;{ }^{13} \mathrm{C} \mathrm{NMR}\left(126 \mathrm{MHz}, \mathrm{CDCl}_{3}\right) \delta 151.51,150.18,137.57,133.10$, $129.98,128.05,127.97,123.21$ (q, $J=277.2 \mathrm{~Hz}), 114.44,62.64$ (q, $J=36.5 \mathrm{~Hz}$ ), 29.48, 20.30, 19.17; ${ }^{19} \mathrm{~F}$ NMR $\left(471 \mathrm{MHz}, \mathrm{CDCl}_{3}\right) \delta$-73.02; ATR-FTIR $\left(\mathrm{cm}^{-1}\right): 2955$, 1667, 1622, 1499, 1328, 1243, 1170, 1149, 1064; HRMS (ESI+): Calculated for $\mathrm{C}_{13} \mathrm{H}_{13} \mathrm{~F}_{3} \mathrm{~N}_{2} \mathrm{O}_{2} \mathrm{H}:[\mathrm{M}+\mathrm{H}]^{+}$287.1002, Found 287.1009.

6,7-Dichloro-1-methyl-3-(2,2,2-trifluoroethoxy)quinoxalin-2(1H)-one (3aa)<smiles>Cn1c(=O)c(OCC(F)F)nc2cc(Cl)c(Cl)cc21</smiles>

Obtained as a white solid (48 mg, 74\% yield); M.p. 163-164 ${ }^{\circ} \mathrm{C} .{ }^{1} \mathrm{H}$ NMR $(500 \mathrm{MHz}$, $\left.\mathrm{CDCl}_{3}\right) \delta 7.72(\mathrm{~s}, 1 \mathrm{H}), 7.38(\mathrm{~s}, 1 \mathrm{H}), 4.87(\mathrm{q}, J=8.2 \mathrm{~Hz}, 2 \mathrm{H}), 3.70(\mathrm{~s}, 3 \mathrm{H}) ;{ }^{13} \mathrm{C} \mathrm{NMR}$ $\left(126 \mathrm{MHz}, \mathrm{CDCl}_{3}\right) \delta 152.79,149.61,132.10,131.52,129.21,128.68,127.98,122.89$ 
$(\mathrm{q}, J=277.2 \mathrm{~Hz}), 115.32,63.08(\mathrm{q}, J=36.5 \mathrm{~Hz}), 29.90 ;{ }^{19} \mathrm{~F}$ NMR $\left(471 \mathrm{MHz}, \mathrm{CDCl}_{3}\right)$ $\delta$-73.01; ATR-FTIR $\left(\mathrm{cm}^{-1}\right): 2964,1691,1618,1459,1243,1156,1014$; HRMS (ESI+): Calculated for $\mathrm{C}_{11} \mathrm{H}_{7} \mathrm{Cl}_{2} \mathrm{~F}_{3} \mathrm{~N}_{2} \mathrm{O}_{2} \mathrm{H}:[\mathrm{M}+\mathrm{H}]^{+}$326.9910, Found 326.9912 .

\section{3-(2-Fluoroethoxy)-1-methylquinoxalin-2(1H)-one (4a)}<smiles>Cn1c(=O)c(OCCF)nc2ccccc21</smiles>

Obtained as a white solid (38 mg, 86\% yield); M.p. $134-135{ }^{\circ} \mathrm{C} .{ }^{1} \mathrm{H}$ NMR $(500 \mathrm{MHz}$, $\left.\mathrm{CDCl}_{3}\right) \delta 7.62(\mathrm{~d}, J=7.9 \mathrm{~Hz}, 1 \mathrm{H}), 7.43(\mathrm{t}, J=7.8 \mathrm{~Hz}, 1 \mathrm{H}), 7.30(\mathrm{t}, J=7.6 \mathrm{~Hz}, 1 \mathrm{H})$, $7.28-7.25(\mathrm{~m}, 1 \mathrm{H}), 4.91-4.87(\mathrm{~m}, 1 \mathrm{H}), 4.81-4.78(\mathrm{~m}, 1 \mathrm{H}), 4.78-4.75(\mathrm{~m}, 1 \mathrm{H})$, $4.74-4.69(\mathrm{~m}, 1 \mathrm{H}), 3.72(\mathrm{~s}, 3 \mathrm{H}) ;{ }^{13} \mathrm{C}$ NMR $\left(126 \mathrm{MHz}, \mathrm{CDCl}_{3}\right) \delta 153.47,150.82$, 131.77, 130.73, 127.64, 127.40, 124.02, 113.69, $81.01(\mathrm{~d}, J=171.4 \mathrm{~Hz}), 66.08(\mathrm{~d}, J=$ $21.4 \mathrm{~Hz}), 29.53 ;{ }^{19} \mathrm{~F}$ NMR $\left(471 \mathrm{MHz}, \mathrm{CDCl}_{3}\right) \delta-224.20$; ATR-FTIR $\left(\mathrm{cm}^{-1}\right): 1668$, 1637, 1301, 1243, 1050; HRMS (ESI+): Calculated for $\mathrm{C}_{11} \mathrm{H}_{11} \mathrm{FN}_{2} \mathrm{O}_{2} \mathrm{H}:[\mathrm{M}+\mathrm{H}]^{+}$ 223.0878, Found 223.0870.

\section{1-Ethyl-3-(2-fluoroethoxy)quinoxalin-2(1H)-one (4b)}

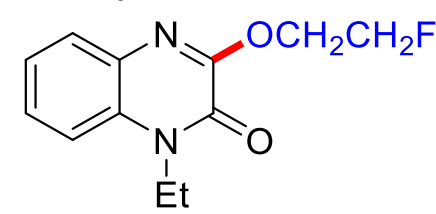

Obtained as a white solid (39 mg, $82 \%$ yield); M.p. $148-149{ }^{\circ} \mathrm{C} .{ }^{1} \mathrm{H}$ NMR $(500 \mathrm{MHz}$, $\left.\mathrm{CDCl}_{3}\right) \delta 7.63(\mathrm{~d}, J=7.8 \mathrm{~Hz}, 1 \mathrm{H}), 7.43(\mathrm{t}, J=7.8 \mathrm{~Hz}, 1 \mathrm{H}), 7.30(\mathrm{dd}, J=7.8,4.3 \mathrm{~Hz}$, $2 \mathrm{H}), 4.94-4.86(\mathrm{~m}, 1 \mathrm{H}), 4.84-4.78(\mathrm{~m}, 1 \mathrm{H}), 4.78-4.73(\mathrm{~m}, 1 \mathrm{H}), 4.74-4.64(\mathrm{~m}$, $1 \mathrm{H}), 4.35(\mathrm{q}, J=7.2 \mathrm{~Hz}, 2 \mathrm{H}), 1.39(\mathrm{t}, J=7.2 \mathrm{~Hz}, 3 \mathrm{H}) ;{ }^{13} \mathrm{C} \mathrm{NMR}\left(126 \mathrm{MHz}, \mathrm{CDCl}_{3}\right) \delta$ $153.47,150.35,131.11,130.61,127.96,127.40,123.84,113.56,81.01$ (d, $J=171.4$ $\mathrm{Hz}), 66.04(\mathrm{~d}, J=21.4 \mathrm{~Hz}), 37.72,12.37 ;{ }^{19} \mathrm{~F}$ NMR $\left(471 \mathrm{MHz}, \mathrm{CDCl}_{3}\right) \delta-224.17$; ATR-FTIR $\left(\mathrm{cm}^{-1}\right)$ : 2960, 1663, 1608, 1491, 1336, 1261, 1222, 1070; HRMS (ESI+): Calculated for $\mathrm{C}_{12} \mathrm{H}_{13} \mathrm{FN}_{2} \mathrm{O}_{2} \mathrm{H}:[\mathrm{M}+\mathrm{H}]^{+}$237.1034, Found 237.1034.

\section{3-(2-Fluoroethoxy)quinoxalin-2(1H)-one (4c)}<smiles>O=c1[nH]c2ccccc2nc1OCCF</smiles>

Obtained as a white solid (32 mg, 76\% yield); M.p. $170-171{ }^{\circ} \mathrm{C} .{ }^{1} \mathrm{H}$ NMR $(500 \mathrm{MHz}$, $\left.\mathrm{CDCl}_{3}\right) \delta 11.89(\mathrm{~s}, 1 \mathrm{H}), 7.54(\mathrm{~d}, J=7.9 \mathrm{~Hz}, 1 \mathrm{H}), 7.33-7.30(\mathrm{~m}, 2 \mathrm{H}), 7.23$ (ddd, $J=$ 8.5, 5.9, $2.8 \mathrm{~Hz}, 1 \mathrm{H}), 4.86-4.83(\mathrm{~m}, 1 \mathrm{H}), 4.75(\mathrm{dt}, J=6.4,4.0 \mathrm{~Hz}, 2 \mathrm{H}), 4.70-4.67$ $(\mathrm{m}, 1 \mathrm{H}) ;{ }^{13} \mathrm{C}$ NMR $\left(126 \mathrm{MHz}, \mathrm{CDCl}_{3}\right) \delta 152.77,151.24,129.85,128.43,126.53$, 125.78, 123.52, 114.76, $79.99(\mathrm{~d}, J=171.4 \mathrm{~Hz}), 65.25(\mathrm{~d}, J=21.4 \mathrm{~Hz}) ;{ }^{19} \mathrm{~F}$ NMR $\left(471 \mathrm{MHz}, \mathrm{CDCl}_{3}\right) \delta$-224.16; ATR-FTIR $\left(\mathrm{cm}^{-1}\right): 2906,1685,1616,1577,1488,1277$, 1108, 1054; HRMS (ESI+): Calculated for $\mathrm{C}_{10} \mathrm{H}_{9} \mathrm{FN}_{2} \mathrm{O}_{2} \mathrm{H}:[\mathrm{M}+\mathrm{H}]^{+}$209.0721, Found 209.0719 . 


\section{6-Bromo-3-(2-fluoroethoxy)-1-methylquinoxalin-2(1H)-one (4d)}<smiles>Cn1c(=O)c(OCCF)nc2cc(Br)ccc21</smiles>

Obtained as a white solid (48 mg, 80\% yield); M.p. $177-178{ }^{\circ} \mathrm{C} .{ }^{1} \mathrm{H}$ NMR $(500 \mathrm{MHz}$, $\left.\mathrm{CDCl}_{3}\right) \delta 7.77(\mathrm{~d}, J=2.2 \mathrm{~Hz}, 1 \mathrm{H}), 7.52(\mathrm{dd}, J=8.8,2.3 \mathrm{~Hz}, 1 \mathrm{H}), 7.13(\mathrm{~d}, J=8.9 \mathrm{~Hz}$, $1 \mathrm{H}), 4.88(\mathrm{dd}, J=4.9,3.8 \mathrm{~Hz}, 1 \mathrm{H}), 4.79(\mathrm{dd}, J=4.9,3.6 \mathrm{~Hz}, 1 \mathrm{H}), 4.76-4.74(\mathrm{~m}, 1 \mathrm{H})$, $4.70(\mathrm{dd}, J=5.0,3.6 \mathrm{~Hz}, 1 \mathrm{H}), 3.70(\mathrm{~s}, 3 \mathrm{H}) ;{ }^{13} \mathrm{C} \mathrm{NMR}\left(126 \mathrm{MHz}, \mathrm{CDCl}_{3}\right) \delta 154.16$, $150.47,131.79,130.94,130.12,116.60,115.03,80.85$ (d, $J=171.4 \mathrm{~Hz}), 66.37$ (d, $J=$ $21.4 \mathrm{~Hz}$ ), 29.68; ${ }^{19} \mathrm{~F}$ NMR (471 MHz, $\left.\mathrm{CDCl}_{3}\right) \delta-224.20$; ATR-FTIR $\left(\mathrm{cm}^{-1}\right): 2955$, 1666, 1607, 1490, 1300, 1249, 1127, 1064; HRMS (ESI+): Calculated for $\mathrm{C}_{11} \mathrm{H}_{10} \mathrm{BrFN}_{2} \mathrm{O}_{2} \mathrm{H}:[\mathrm{M}+\mathrm{H}]^{+}$300.9983, Found 300.9980.

\section{1-Benzyl-3-(2-fluoroethoxy)quinoxalin-2(1H)-one (4e)}<smiles>O=c1c(OCCF)nc2ccccc2n1Cc1ccccc1</smiles>

Obtained as a white solid (43 mg, 72\% yield); M.p. 162-163 ${ }^{\circ} \mathrm{C} .{ }^{1} \mathrm{H}$ NMR $(500 \mathrm{MHz}$, $\left.\mathrm{CDCl}_{3}\right) \delta 7.62(\mathrm{dd}, J=7.8,1.6 \mathrm{~Hz}, 1 \mathrm{H}), 7.32-7.30(\mathrm{~m}, 1 \mathrm{H}), 7.28(\mathrm{dd}, J=9.5,1.9 \mathrm{~Hz}$, $3 \mathrm{H}), 7.27-7.20(\mathrm{~m}, 4 \mathrm{H}), 5.52(\mathrm{~s}, 2 \mathrm{H}), 4.94-4.90(\mathrm{~m}, 1 \mathrm{H}), 4.82(\mathrm{dd}, J=5.0,3.6 \mathrm{~Hz}$, $1 \mathrm{H}), 4.81-4.78(\mathrm{~m}, 1 \mathrm{H}), 4.76-4.72(\mathrm{~m}, 1 \mathrm{H}) ;{ }^{13} \mathrm{C} \mathrm{NMR}\left(126 \mathrm{MHz}, \mathrm{CDCl}_{3}\right) \delta 153.55$, $151.05,135.12$, 131.08, 130.99, 128.91, 127.78, 127.75, 127.38, 127.01, 124.04, $114.56,81.01(\mathrm{~d}, J=171.4 \mathrm{~Hz}), 66.21(\mathrm{~d}, J=21.4 \mathrm{~Hz}), 46.30 ;{ }^{19} \mathrm{~F}$ NMR $(471 \mathrm{MHz}$, $\left.\mathrm{CDCl}_{3}\right) \delta$-224.05; ATR-FTIR $\left(\mathrm{cm}^{-1}\right): 2930,1663,1606,1444,1304,1250,1195$, 1048, 761, 704; HRMS (ESI+): Calculated for $\mathrm{C}_{17} \mathrm{H}_{15} \mathrm{FN}_{2} \mathrm{O}_{2} \mathrm{H}$ : $[\mathrm{M}+\mathrm{H}]^{+}$299.1191, Found 299.1199.

\section{3-(2-Fluoroethoxy)-1-(4-methylbenzyl)quinoxalin-2(1H)-one (4f)}<smiles>COc1nc2ccccc2n(Cc2ccc(C)cc2)c1=O</smiles>

Obtained as a white solid (46 mg, 74\% yield); M.p. $146-147{ }^{\circ} \mathrm{C} .{ }^{1} \mathrm{H}$ NMR $(500 \mathrm{MHz}$, $\left.\mathrm{CDCl}_{3}\right) \delta 7.61(\mathrm{dd}, J=8.1,1.5 \mathrm{~Hz}, 1 \mathrm{H}), 7.31-7.27(\mathrm{~m}, 1 \mathrm{H}), 7.24(\mathrm{dd}, J=10.5,4.9$ $\mathrm{Hz}, 2 \mathrm{H}), 7.17(\mathrm{~d}, J=8.0 \mathrm{~Hz}, 2 \mathrm{H}), 7.10(\mathrm{~d}, J=8.0 \mathrm{~Hz}, 2 \mathrm{H}), 5.47(\mathrm{~s}, 2 \mathrm{H}), 4.94-4.88$ $(\mathrm{m}, 1 \mathrm{H}), 4.83-4.80(\mathrm{~m}, 1 \mathrm{H}), 4.80-4.77(\mathrm{~m}, 1 \mathrm{H}), 4.75-4.71(\mathrm{~m}, 1 \mathrm{H}), 2.29(\mathrm{~s}, 3 \mathrm{H})$; ${ }^{13} \mathrm{C}$ NMR $\left(126 \mathrm{MHz}, \mathrm{CDCl}_{3}\right) \delta 153.56,151.05,137.48,132.12,131.10,130.98$, $129.56,127.74,127.35,127.04,123.98,114.58,81.03$ (d, $J=171.4 \mathrm{~Hz}), 66.19$ (d, $J=$ $21.4 \mathrm{~Hz}), 46.08,21.07 ;{ }^{19} \mathrm{~F}$ NMR $\left(471 \mathrm{MHz}, \mathrm{CDCl}_{3}\right) \delta-224.00$; ATR-FTIR $\left(\mathrm{cm}^{-1}\right)$ : 
2956, 1680, 1610, 1470, 1333, 1298, 1245, 1190, 1067, 804; HRMS (ESI+): Calculated for $\mathrm{C}_{18} \mathrm{H}_{17} \mathrm{FN}_{2} \mathrm{O}_{2} \mathrm{H}:[\mathrm{M}+\mathrm{H}]^{+}$313.1347, Found 313.1342.

\section{3-(2,2-Difluoroethoxy)-1-methylquinoxalin-2(1H)-one (5a)}<smiles>Cn1c(=O)c(OCC(F)F)nc2ccccc21</smiles>

Obtained as a white solid (35 mg, 72\% yield); M.p. 131-132 ${ }^{\circ} \mathrm{C} .{ }^{1} \mathrm{H}$ NMR (500 MHz, $\left.\mathrm{CDCl}_{3}\right) \delta 7.65(\mathrm{dd}, J=7.9,1.4 \mathrm{~Hz}, 1 \mathrm{H}), 7.49-7.44(\mathrm{~m}, 1 \mathrm{H}), 7.33(\mathrm{td}, J=8.0,1.2 \mathrm{~Hz}$, $1 \mathrm{H}), 7.29(\mathrm{dd}, J=8.4,0.8 \mathrm{~Hz}, 1 \mathrm{H}), 6.26(\mathrm{tt}, J=55.4,4.4 \mathrm{~Hz}, 1 \mathrm{H}), 4.68(\mathrm{td}, J=13.0$, $4.4 \mathrm{~Hz}, 2 \mathrm{H}), 3.74(\mathrm{~s}, 3 \mathrm{H}) ;{ }^{13} \mathrm{C} \mathrm{NMR}\left(126 \mathrm{MHz}, \mathrm{CDCl}_{3}\right) \delta 152.68,150.51,131.98$, 130.31, 127.90, 127.83, 124.20, 113.77, $112.93(\mathrm{t}, J=240.7 \mathrm{~Hz}), 65.35(\mathrm{t}, J=31.5$ $\mathrm{Hz}), 29.60 ;{ }^{19} \mathrm{~F}$ NMR $\left(471 \mathrm{MHz}, \mathrm{CDCl}_{3}\right) \delta$-125.02; ATR-FTIR $\left(\mathrm{cm}^{-1}\right): 2930,1673$, 1606, 1454, 1302, 1252, 1122, 1083; HRMS (ESI+): Calculated for $\mathrm{C}_{11} \mathrm{H}_{10} \mathrm{~F}_{2} \mathrm{~N}_{2} \mathrm{O}_{2} \mathrm{H}$ : $[\mathrm{M}+\mathrm{H}]^{+}$241.0783, Found 241.0782.

\section{3-(2,2-Difluoroethoxy)-1-ethylquinoxalin-2(1H)-one (5b)}<smiles>CCn1c(=O)c(OCC(F)F)nc2ccccc21</smiles>

Obtained as a white solid (35 mg, 69\% yield); M.p. 106-107 ${ }^{\circ} \mathrm{C} .{ }^{1} \mathrm{H}$ NMR $(500 \mathrm{MHz}$, $\left.\mathrm{CDCl}_{3}\right) \delta 7.66(\mathrm{dd}, J=8.3,1.2 \mathrm{~Hz}, 1 \mathrm{H}), 7.48-7.43(\mathrm{~m}, 1 \mathrm{H}), 7.32(\mathrm{t}, J=7.5 \mathrm{~Hz}, 2 \mathrm{H})$, $6.26(\mathrm{tt}, J=55.4,4.4 \mathrm{~Hz}, 1 \mathrm{H}), 4.68(\mathrm{td}, J=13.0,4.4 \mathrm{~Hz}, 2 \mathrm{H}), 4.36(\mathrm{q}, J=7.2 \mathrm{~Hz}, 2 \mathrm{H})$, $1.39(\mathrm{t}, J=7.2 \mathrm{~Hz}, 3 \mathrm{H}) ;{ }^{13} \mathrm{C} \mathrm{NMR}\left(126 \mathrm{MHz}, \mathrm{CDCl}_{3}\right) \delta 152.67,149.98,130.85$, 130.65, 128.12, 127.87, 123.99, 113.63, $112.97(\mathrm{t}, J=240.7 \mathrm{~Hz}), 65.34(\mathrm{t}, J=31.5$ $\mathrm{Hz}$ ), 37.80, 12.37; ${ }^{19} \mathrm{~F}$ NMR (471 MHz, $\left.\mathrm{CDCl}_{3}\right) \delta$-124.97; ATR-FTIR $\left(\mathrm{cm}^{-1}\right): 2989$, 1671, 1611, 1466, 1276, 1137, 1063; HRMS (ESI+): Calculated for $\mathrm{C}_{12} \mathrm{H}_{12} \mathrm{~F}_{2} \mathrm{~N}_{2} \mathrm{O}_{2} \mathrm{H}$ : $[\mathrm{M}+\mathrm{H}]^{+}$255.0940, Found 255.0940.

\section{3-(2,2-Difluoroethoxy)quinoxalin-2(1H)-one (5c)}<smiles>O=c1[nH]c2ccccc2nc1OCC(F)F</smiles>

Obtained as a white solid (30 mg, 66\% yield); M.p. $132-134{ }^{\circ} \mathrm{C} .{ }^{1} \mathrm{H}$ NMR $(500 \mathrm{MHz}$, DMSO) $\delta 12.49(\mathrm{~s}, 1 \mathrm{H}), 7.55(\mathrm{~d}, J=7.9 \mathrm{~Hz}, 1 \mathrm{H}), 7.41-7.37(\mathrm{~m}, 1 \mathrm{H}), 7.28(\mathrm{~d}, J=8.1$ $\mathrm{Hz}, 1 \mathrm{H}), 7.25(\mathrm{t}, J=7.6 \mathrm{~Hz}, 1 \mathrm{H}), 6.47(\mathrm{tt}, J=54.4,3.5 \mathrm{~Hz}, 1 \mathrm{H}), 4.65(\mathrm{td}, J=14.9,3.5$ $\mathrm{Hz}, 2 \mathrm{H}) ;{ }^{13} \mathrm{C}$ NMR $(126 \mathrm{MHz}, \mathrm{DMSO}) \delta 154.05,150.48,131.15,130.06,127.86$, 126.72, 123.86, 115.58, $114.12(\mathrm{t}, J=240.7 \mathrm{~Hz}), 64.83(\mathrm{t}, J=31.5 \mathrm{~Hz}) ;{ }^{19} \mathrm{~F}$ NMR (471 MHz, DMSO) $\delta$-125.52; ATR-FTIR $\left(\mathrm{cm}^{-1}\right)$ : 2910, 1686, 1581, 1501, 1437, 1300, 1232, 1076, 1015; HRMS (ESI+): Calculated for $\mathrm{C}_{10} \mathrm{H}_{8} \mathrm{~F}_{2} \mathrm{~N}_{2} \mathrm{O}_{2} \mathrm{H}:[\mathrm{M}+\mathrm{H}]^{+}$ 227.0627, Found 227.0621. 


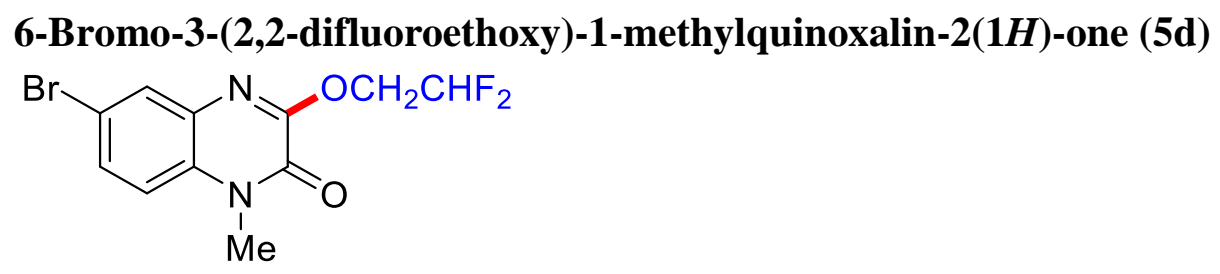

Obtained as a white solid (38 mg, 60\% yield); M.p. 136-137 ${ }^{\circ} \mathrm{C} .{ }^{1} \mathrm{H}$ NMR $(500 \mathrm{MHz}$, $\left.\mathrm{CDCl}_{3}\right) \delta 7.80(\mathrm{~d}, J=2.2 \mathrm{~Hz}, 1 \mathrm{H}), 7.55(\mathrm{dd}, J=8.8,2.2 \mathrm{~Hz}, 1 \mathrm{H}), 7.15(\mathrm{~d}, J=8.9 \mathrm{~Hz}$, $1 \mathrm{H}), 6.24(\mathrm{tt}, J=55.3,4.3 \mathrm{~Hz}, 1 \mathrm{H}), 4.67(\mathrm{td}, J=13.0,4.3 \mathrm{~Hz}, 2 \mathrm{H}), 3.71(\mathrm{~s}, 3 \mathrm{H}) ;{ }^{13} \mathrm{C}$ NMR $\left(126 \mathrm{MHz}, \mathrm{CDCl}_{3}\right) \delta 153.36,150.14,131.32,131.11,130.66,130.30,116.81$, $115.12,112.75(\mathrm{t}, J=240.7 \mathrm{~Hz}), 65.50(\mathrm{t}, J=31.5 \mathrm{~Hz}), 29.75 ;{ }^{19} \mathrm{~F}$ NMR $(471 \mathrm{MHz}$, $\left.\mathrm{CDCl}_{3}\right) \delta$-125.06; ATR-FTIR $\left(\mathrm{cm}^{-1}\right)$ : 3105, 1670, 1605, 1489, 1295, 1123, 1081; HRMS (ESI+): Calculated for $\mathrm{C}_{11} \mathrm{H}_{9} \mathrm{BrF}_{2} \mathrm{~N}_{2} \mathrm{O}_{2} \mathrm{H}:[\mathrm{M}+\mathrm{H}]^{+}$318.9888, Found 318.9981.

1-Methyl-3-(2,2,3,3,3-pentafluoropropoxy)quinoxalin-2(1H)-one (6a)<smiles>Cn1c(=O)c(OCC(F)(F)F)nc2ccccc21</smiles>

Obtained as a white solid (54 mg, 87\% yield); M.p. $102-103{ }^{\circ} \mathrm{C} .{ }^{1} \mathrm{H}$ NMR (500 MHz, $\left.\mathrm{CDCl}_{3}\right) \delta 7.65(\mathrm{dd}, J=7.9,1.1 \mathrm{~Hz}, 1 \mathrm{H}), 7.51-7.46(\mathrm{~m}, 1 \mathrm{H}), 7.34(\mathrm{t}, J=7.6 \mathrm{~Hz}, 1 \mathrm{H})$, $7.30(\mathrm{~d}, J=8.3 \mathrm{~Hz}, 1 \mathrm{H}), 4.97(\mathrm{t}, J=12.9 \mathrm{~Hz}, 2 \mathrm{H}), 3.74(\mathrm{~s}, 3 \mathrm{H}) ;{ }^{13} \mathrm{C}$ NMR $(126 \mathrm{MHz}$, $\left.\mathrm{CDCl}_{3}\right) \delta 152.06,150.12,132.14,129.91,128.21,127.87,124.23,118.61$ (qt, $J=$ 286.0, $34.8 \mathrm{~Hz}), 113.82,112.44,61.72(\mathrm{t}, J=27.2 \mathrm{~Hz}), 29.58 ;{ }^{19} \mathrm{~F}$ NMR $(471 \mathrm{MHz}$, $\left.\mathrm{CDCl}_{3}\right) \delta$-83.68, -122.91; ATR-FTIR $\left(\mathrm{cm}^{-1}\right):$ 2952, 1683, 1611, 1477, 1304, 1215, 1100, 1008; HRMS (ESI+): Calculated for $\mathrm{C}_{12} \mathrm{H}_{9} \mathrm{~F}_{5} \mathrm{~N}_{2} \mathrm{O}_{2} \mathrm{H}:[\mathrm{M}+\mathrm{H}]^{+}$309.0657, Found 309.0650 .

1-Ethyl-3-(2,2,3,3,3-pentafluoropropoxy)quinoxalin-2(1H)-one (6b)<smiles>CCn1c(=O)c(OCC(F)(F)F)nc2ccccc21</smiles>

Obtained as a white solid (55 mg, 85\% yield); M.p. $108-109{ }^{\circ} \mathrm{C} .{ }^{1} \mathrm{H}$ NMR $(500 \mathrm{MHz}$, $\left.\mathrm{CDCl}_{3}\right) \delta 7.66(\mathrm{dd}, J=8.3,1.5 \mathrm{~Hz}, 1 \mathrm{H}), 7.50-7.45(\mathrm{~m}, 1 \mathrm{H}), 7.33(\mathrm{dd}, J=7.7,6.8 \mathrm{~Hz}$, $2 \mathrm{H}), 4.96(\mathrm{t}, J=12.9 \mathrm{~Hz}, 2 \mathrm{H}), 4.36(\mathrm{q}, J=7.2 \mathrm{~Hz}, 2 \mathrm{H}), 1.40(\mathrm{t}, J=7.2 \mathrm{~Hz}, 3 \mathrm{H}) ;{ }^{13} \mathrm{C}$ NMR $\left(126 \mathrm{MHz}, \mathrm{CDCl}_{3}\right) \delta 152.08,149.61,131.04,130.27,128.17,128.16,124.02$, 118.63 (qt, $J=286.0,34.8 \mathrm{~Hz}), 113.67,112.49,61.77$ (t, $J=27.2 \mathrm{~Hz}$ ), 37.87, 12.36; ${ }^{19} \mathrm{~F} \mathrm{NMR}\left(471 \mathrm{MHz}, \mathrm{CDCl}_{3}\right) \delta$-83.66, -122.90; ATR-FTIR $\left(\mathrm{cm}^{-1}\right)$ : 2990, 1673, 1615, 1492, 1198, 1106, 1001; HRMS (ESI+): Calculated for $\mathrm{C}_{13} \mathrm{H}_{11} \mathrm{~F}_{5} \mathrm{~N}_{2} \mathrm{O}_{2} \mathrm{H}:[\mathrm{M}+\mathrm{H}]^{+}$ 323.0813, Found 323.0814.

\section{3-(2,2,3,3,3-Pentafluoropropoxy)quinoxalin-2(1H)-one (6c)}<smiles>O=c1[nH]c2ccccc2nc1OCC(F)(F)F</smiles> 
Obtained as a white solid (46 mg, 79\% yield); M.p. 161-162 ${ }^{\circ} \mathrm{C} .{ }^{1} \mathrm{H}$ NMR $(500 \mathrm{MHz}$, DMSO) $\delta 12.59(\mathrm{~s}, 1 \mathrm{H}), 7.57(\mathrm{~d}, J=7.8 \mathrm{~Hz}, 1 \mathrm{H}), 7.42(\mathrm{dd}, J=11.2,4.0 \mathrm{~Hz}, 1 \mathrm{H}), 7.28$ $(\mathrm{dd}, J=16.6,8.0 \mathrm{~Hz}, 2 \mathrm{H}), 5.15(\mathrm{t}, J=13.7 \mathrm{~Hz}, 2 \mathrm{H}) ;{ }^{13} \mathrm{C}$ NMR $(126 \mathrm{MHz}, \mathrm{DMSO}) \delta$ $153.23,150.15,131.34,129.63,128.26,126.78,123.95,118.83$ (qt, $J=286.0,34.8$ $\mathrm{Hz}), 115.66,113.33,61.48(\mathrm{t}, J=27.2 \mathrm{~Hz}) ;{ }^{19} \mathrm{~F}$ NMR $(471 \mathrm{MHz}, \mathrm{DMSO}) \delta-82.84$, -122.03; ATR-FTIR $\left(\mathrm{cm}^{-1}\right)$ : 2914, 1689, 1615, 1580, 1307, 1208, 1145, 1047; HRMS (ESI+): Calculated for $\mathrm{C}_{11} \mathrm{H}_{7} \mathrm{~F}_{5} \mathrm{~N}_{2} \mathrm{O}_{2} \mathrm{H}:[\mathrm{M}+\mathrm{H}]^{+}$295.0500, Found 295.0501.

\section{7-Bromo-1-methyl-3-(2,2,3,3,3-pentafluoropropoxy)quinoxalin-2(1H)-one (6d)}<smiles>Cn1c(=O)c(OCC(F)(F)F)nc2ccc(Br)cc21</smiles>

Obtained as a white solid (60 mg, 78\% yield); M.p. 121-122 ${ }^{\circ} \mathrm{C} .{ }^{1} \mathrm{H}$ NMR (500 MHz, $\left.\mathrm{CDCl}_{3}\right) \delta 7.50(\mathrm{~d}, J=9.0 \mathrm{~Hz}, 1 \mathrm{H}), 7.47-7.41(\mathrm{~m}, 2 \mathrm{H}), 4.94(\mathrm{t}, J=12.8 \mathrm{~Hz}, 2 \mathrm{H}), 3.71$ $(\mathrm{s}, 3 \mathrm{H}) ;{ }^{13} \mathrm{C} \mathrm{NMR}\left(126 \mathrm{MHz}, \mathrm{CDCl}_{3}\right) \delta 152.22,149.78,133.17,129.06,128.84$, $127.41,121.81,119.24$ (qt, $J=286.0,34.8 \mathrm{~Hz}), 116.90,114.80,61.87(\mathrm{t}, J=27.2 \mathrm{~Hz})$, 29.72; ${ }^{19} \mathrm{~F}$ NMR $\left(471 \mathrm{MHz}, \mathrm{CDCl}_{3}\right) \delta-83.67,-122.92$; ATR-FTIR $\left(\mathrm{cm}^{-1}\right): 2922,1687$, 1612, 1462, 1408, 1307, 1155, 1036; HRMS (ESI+): Calculated for $\mathrm{C}_{12} \mathrm{H}_{8} \mathrm{BrF}_{5} \mathrm{~N}_{2} \mathrm{O}_{2} \mathrm{H}$ : $[\mathrm{M}+\mathrm{H}]^{+}$386.9762, Found 386.9761.

\section{1,6,7-Trimethyl-3-(2,2,3,3,3-pentafluoropropoxy)quinoxalin-2(1H)-one (6e)}<smiles>Cc1cc2nc(OCC(F)(F)F)c(=O)n(C)c2cc1C</smiles>

Obtained as a white solid (54 mg, 81\% yield); M.p. $136-137{ }^{\circ} \mathrm{C} .{ }^{1} \mathrm{H}$ NMR (500 MHz, $\left.\mathrm{CDCl}_{3}\right) \delta 7.40(\mathrm{~s}, 1 \mathrm{H}), 7.04(\mathrm{~s}, 1 \mathrm{H}), 4.94(\mathrm{t}, J=13.4 \mathrm{~Hz}, 2 \mathrm{H}), 3.70(\mathrm{~s}, 3 \mathrm{H}), 2.39(\mathrm{~s}$, $3 \mathrm{H}), 2.33(\mathrm{~s}, 3 \mathrm{H}) ;{ }^{13} \mathrm{C} \mathrm{NMR}\left(126 \mathrm{MHz}, \mathrm{CDCl}_{3}\right) \delta 151.57,150.13,137.61,133.10$, $130.03,128.08,127.95,118.64$ (qt, $J=286.0,34.8 \mathrm{~Hz}), 114.46,112.15,61.56(\mathrm{t}, J=$ $27.2 \mathrm{~Hz}), 29.48,20.31,19.17 ;{ }^{19} \mathrm{~F} \mathrm{NMR}\left(471 \mathrm{MHz}, \mathrm{CDCl}_{3}\right) \delta$-83.70, -122.92; ATR-FTIR $\left(\mathrm{cm}^{-1}\right):$ 2958, 1679, 1624, 1477, 1414, 1305, 1205, 1190, 1100, 1028; HRMS (ESI+): Calculated for $\mathrm{C}_{14} \mathrm{H}_{13} \mathrm{~F}_{5} \mathrm{~N}_{2} \mathrm{O}_{2} \mathrm{H}:[\mathrm{M}+\mathrm{H}]^{+}$337.0971, Found 337.0974.

\section{1-(4-Chlorobenzyl)-3-(2,2,3,3,3-pentafluoropropoxy)quinoxalin-2(1H)-one (6f)}<smiles>O=c1c(OCC(F)(F)F)nc2ccccc2n1Cc1ccc(Cl)cc1</smiles>

Obtained as a white solid (63 mg, 75\% yield); M.p. 121-122 ${ }^{\circ} \mathrm{C} .{ }^{1} \mathrm{H}$ NMR $(500 \mathrm{MHz}$, $\left.\mathrm{CDCl}_{3}\right) \delta 7.66(\mathrm{dd}, J=7.9,1.5 \mathrm{~Hz}, 1 \mathrm{H}), 7.39-7.35(\mathrm{~m}, 1 \mathrm{H}), 7.32-7.27(\mathrm{~m}, 3 \mathrm{H})$, $7.21(\mathrm{t}, J=8.9 \mathrm{~Hz}, 3 \mathrm{H}), 5.48(\mathrm{~s}, 2 \mathrm{H}), 4.99(\mathrm{t}, J=12.9 \mathrm{~Hz}, 2 \mathrm{H}) ;{ }^{13} \mathrm{C} \mathrm{NMR}(126 \mathrm{MHz}$, $\left.\mathrm{CDCl}_{3}\right) \delta 152.08,150.28,133.81,133.43,131.26,130.18,129.18,128.53,128.26$, $128.14,124.43,118.60$ (qt, $J=286.0,34.8 \mathrm{~Hz}), 114.39,112.43,61.95(\mathrm{t}, J=27.2 \mathrm{~Hz})$, 45.80; ${ }^{19}$ F NMR (471 MHz, $\left.\mathrm{CDCl}_{3}\right) \delta-83.63,-122.88$; ATR-FTIR $\left(\mathrm{cm}^{-1}\right): 3035,1674$, 
1614, 1447, 1277, 1176, 1034, 799; HRMS (ESI+): Calculated for $\mathrm{C}_{18} \mathrm{H}_{12} \mathrm{ClF}_{5} \mathrm{~N}_{2} \mathrm{O}_{2} \mathrm{H}$ : $[\mathrm{M}+\mathrm{H}]^{+} 419.0580$, Found 419.0583.

1-(3-Bromobenzyl)-3-(2,2,3,3,3-pentafluoropropoxy)quinoxalin-2(1H)-one $(6 \mathrm{~g})$<smiles>O=c1c(OCC(F)(F)F)nc2ccccc2n1Cc1cccc(Br)c1</smiles>

Obtained as a white solid (65 mg, 70\% yield); M.p. 153-154 ${ }^{\circ} \mathrm{C} .{ }^{1} \mathrm{H}$ NMR $(500 \mathrm{MHz}$, $\left.\mathrm{CDCl}_{3}\right) \delta 7.67(\mathrm{dd}, J=7.9,1.3 \mathrm{~Hz}, 1 \mathrm{H}), 7.43-7.35(\mathrm{~m}, 3 \mathrm{H}), 7.31(\mathrm{t}, J=8.1 \mathrm{~Hz}, 1 \mathrm{H})$, $7.19(\mathrm{~d}, J=6.1 \mathrm{~Hz}, 3 \mathrm{H}), 5.48(\mathrm{~s}, 2 \mathrm{H}), 4.99(\mathrm{t}, J=12.9 \mathrm{~Hz}, 2 \mathrm{H}) ;{ }^{13} \mathrm{C}$ NMR $(126 \mathrm{MHz}$, $\left.\mathrm{CDCl}_{3}\right) \delta 152.06,150.27,137.20,131.26,131.16,130.55,130.17,130.00,128.34$, $128.16,125.67,124.48,123.10,118.74$ (qt, $J=286.0,34.8 \mathrm{~Hz}), 114.38,112.13$, $61.98(\mathrm{t}, J=27.2 \mathrm{~Hz}), 45.85 ;{ }^{19} \mathrm{~F}$ NMR $\left(471 \mathrm{MHz}, \mathrm{CDCl}_{3}\right) \delta$-83.62, -122.87; ATR-FTIR $\left(\mathrm{cm}^{-1}\right)$ : 2960, 1683, 1621, 1477, 1300, 1280, 1220, 1150, 1043, 756, 706; HRMS (ESI+): Calculated for $\mathrm{C}_{18} \mathrm{H}_{12} \mathrm{BrF}_{5} \mathrm{~N}_{2} \mathrm{O}_{2} \mathrm{H}:[\mathrm{M}+\mathrm{H}]^{+}$463.0075, Found 463.0079 .

1-Methyl-3-(2,2,3,3-tetrafluoropropoxy)quinoxalin-2(1H)-one (7a)<smiles>Cn1c(=O)c(OCC(F)F)nc2ccccc21</smiles>

Obtained as a white solid (42 mg, 73\% yield); M.p. $129-130{ }^{\circ} \mathrm{C} .{ }^{1} \mathrm{H}$ NMR (500 MHz, $\left.\mathrm{CDCl}_{3}\right) \delta 7.66(\mathrm{dd}, J=8.0,1.3 \mathrm{~Hz}, 1 \mathrm{H}), 7.50-7.46(\mathrm{~m}, 1 \mathrm{H}), 7.36-7.32(\mathrm{~m}, 1 \mathrm{H})$, $7.30(\mathrm{~d}, J=8.3 \mathrm{~Hz}, 1 \mathrm{H}), 6.15(\mathrm{tt}, J=53.0,5.0 \mathrm{~Hz}, 1 \mathrm{H}), 4.85(\mathrm{t}, J=12.2 \mathrm{~Hz}, 2 \mathrm{H}), 3.74$ $(\mathrm{s}, 3 \mathrm{H}) ;{ }^{13} \mathrm{C} \mathrm{NMR}\left(126 \mathrm{MHz}, \mathrm{CDCl}_{3}\right) \delta 152.14,150.20,132.07,130.08,128.15$, 127.91, 124.27, 114.40 (tt, $J=249.8,27.9 \mathrm{~Hz}), 113.81,109.04$ (tt, $J=250.3,36.2 \mathrm{~Hz}$ ), $62.88(\mathrm{t}, J=29.3 \mathrm{~Hz}), 29.58 ;{ }^{19} \mathrm{~F} \mathrm{NMR}\left(471 \mathrm{MHz}, \mathrm{CDCl}_{3}\right) \delta-124.57(\mathrm{t}, J=11.5 \mathrm{~Hz})$, $-139.29\left(\mathrm{~d}, J=53.0 \mathrm{~Hz}\right.$ ); ATR-FTIR $\left(\mathrm{cm}^{-1}\right): 2957,1676,1611,1473,1306,1225$, 1115, 1091, 1004; HRMS (ESI+): Calculated for $\mathrm{C}_{12} \mathrm{H}_{10} \mathrm{~F}_{4} \mathrm{~N}_{2} \mathrm{O}_{2} \mathrm{H}:[\mathrm{M}+\mathrm{H}]^{+}$291.0751, Found 291.0753.

1-Ethyl-3-(2,2,3,3-tetrafluoropropoxy)quinoxalin-2(1H)-one (7b)<smiles>CCn1c(=O)c(OCC(F)F)nc2ccccc21</smiles>

Obtained as a white solid (40 mg, 65\% yield); M.p. $124-125{ }^{\circ} \mathrm{C} .{ }^{1} \mathrm{H}$ NMR $(500 \mathrm{MHz}$, $\left.\mathrm{CDCl}_{3}\right) \delta 7.67(\mathrm{~d}, J=8.0 \mathrm{~Hz}, 1 \mathrm{H}), 7.50-7.45(\mathrm{~m}, 1 \mathrm{H}), 7.33(\mathrm{t}, J=7.9 \mathrm{~Hz}, 2 \mathrm{H}), 6.16$ (tt, $J=53.0,5.0 \mathrm{~Hz}, 1 \mathrm{H}), 4.84(\mathrm{t}, J=12.1 \mathrm{~Hz}, 2 \mathrm{H}), 4.35(\mathrm{q}, J=7.2 \mathrm{~Hz}, 2 \mathrm{H}), 1.40(\mathrm{t}, J$ $=7.2 \mathrm{~Hz}, 3 \mathrm{H}) ;{ }^{13} \mathrm{C} \mathrm{NMR}\left(126 \mathrm{MHz}, \mathrm{CDCl}_{3}\right) \delta 152.12,149.70,130.94,130.43,128.20$, $128.13,124.07,115.93$ (tt, $J=249.8,27.9 \mathrm{~Hz}), 113.66,109.03(\mathrm{tt}, J=250.3,36.2 \mathrm{~Hz}$ ), $62.90(\mathrm{t}, J=29.3 \mathrm{~Hz}), 37.86,12.36 ;{ }^{19} \mathrm{~F} \mathrm{NMR}\left(471 \mathrm{MHz}, \mathrm{CDCl}_{3}\right) \delta-124.62(\mathrm{t}, J=$ $11.5 \mathrm{~Hz}),-139.35(\mathrm{~d}, J=53.0 \mathrm{~Hz})$; ATR-FTIR $\left(\mathrm{cm}^{-1}\right)$ : 2956, 1673, 1612, 1489, 1373 , 
1299, 1133, 1097; HRMS (ESI+): Calculated for $\mathrm{C}_{13} \mathrm{H}_{12} \mathrm{~F}_{4} \mathrm{~N}_{2} \mathrm{O}_{2} \mathrm{H}:[\mathrm{M}+\mathrm{H}]^{+} 305.0908$, Found 305.0909.

3-(2,2,3,3-Tetrafluoropropoxy)quinoxalin-2(1H)-one $(7 \mathrm{c})$<smiles>O=c1[nH]c2ccccc2nc1OCC(F)F</smiles>

Obtained as a white solid (34 mg, 62\% yield); M.p. 193-194 ${ }^{\circ} \mathrm{C} .{ }^{1} \mathrm{H}$ NMR (500 MHz, DMSO) $\delta 12.54(\mathrm{~s}, 1 \mathrm{H}), 7.56(\mathrm{~d}, J=7.9 \mathrm{~Hz}, 1 \mathrm{H}), 7.40(\mathrm{dd}, J=11.2,4.0 \mathrm{~Hz}, 1 \mathrm{H}), 7.27$ (dd, $J=16.0,7.8 \mathrm{~Hz}, 2 \mathrm{H}), 6.66(\mathrm{tt}, J=51.9,5.2 \mathrm{~Hz}, 1 \mathrm{H}), 4.94(\mathrm{t}, J=14.2 \mathrm{~Hz}, 2 \mathrm{H})$; ${ }^{13} \mathrm{C}$ NMR (126 MHz, DMSO) $\delta$ 153.61, 150.32, 131.28, 129.80, 128.09, 126.74, 123.90, 115.61, 115.27 (tt, $J=249.8,27.9 \mathrm{~Hz}), 109.76$ (tt, $J=250.3,36.2 \mathrm{~Hz}), 62.29$ $(\mathrm{t}, J=29.3 \mathrm{~Hz}) ;{ }^{19} \mathrm{~F}$ NMR $(471 \mathrm{MHz}, \mathrm{DMSO}) \delta-124.40(\mathrm{t}, J=11.5 \mathrm{~Hz}),-138.65(\mathrm{~d}, J$ $=53.0 \mathrm{~Hz})$; ATR-FTIR $\left(\mathrm{cm}^{-1}\right):$ 2900, 1688, 1616, 1306, 1131, 1038, 1004; HRMS (ESI+): Calculated for $\mathrm{C}_{11} \mathrm{H}_{8} \mathrm{~F}_{4} \mathrm{~N}_{2} \mathrm{O}_{2} \mathrm{H}:[\mathrm{M}+\mathrm{H}]^{+}$277.0595, Found 277.0593.

7-Bromo-1-methyl-3-(2,2,3,3-tetrafluoropropoxy)quinoxalin-2(1H)-one $(7 \mathrm{~d})$<smiles>Cn1c(=O)c(OCC(F)F)nc2ccc(Br)cc21</smiles>

Obtained as a white solid (46 mg, 63\% yield); M.p. $131-132{ }^{\circ} \mathrm{C} .{ }^{1} \mathrm{H}$ NMR (500 MHz, $\left.\mathrm{CDCl}_{3}\right) \delta 7.51(\mathrm{~d}, J=9.0 \mathrm{~Hz}, 1 \mathrm{H}), 7.48-7.40(\mathrm{~m}, 2 \mathrm{H}), 6.13(\mathrm{tt}, J=53.0,4.8 \mathrm{~Hz}, 1 \mathrm{H})$, $4.83(\mathrm{t}, J=12.2 \mathrm{~Hz}, 2 \mathrm{H}), 3.70(\mathrm{~s}, 3 \mathrm{H}) ;{ }^{13} \mathrm{C} \mathrm{NMR}\left(126 \mathrm{MHz}, \mathrm{CDCl}_{3}\right) \delta 152.30,149.87$, $133.11,129.10,129.02,127.44,121.72,116.88,115.46$ (tt, $J=249.8,27.9 \mathrm{~Hz}$ ), 109.04 (tt, $J=250.3,36.2 \mathrm{~Hz}), 62.96(\mathrm{t}, J=29.3 \mathrm{~Hz}), 29.70 ;{ }^{19} \mathrm{~F}$ NMR $(471 \mathrm{MHz}$, $\left.\mathrm{CDCl}_{3}\right) \delta-124.38(\mathrm{t}, J=11.5 \mathrm{~Hz}),-139.07(\mathrm{~d}, J=53.0 \mathrm{~Hz})$; ATR-FTIR $\left(\mathrm{cm}^{-1}\right): 2923$, 1669, 1624, 1456, 1305, 1194, 1115, 1027; HRMS (ESI+): Calculated for $\mathrm{C}_{12} \mathrm{H}_{9} \mathrm{BrF}_{4} \mathrm{~N}_{2} \mathrm{O}_{2} \mathrm{H}:[\mathrm{M}+\mathrm{H}]^{+}$368.9857, Found 368.9852 .

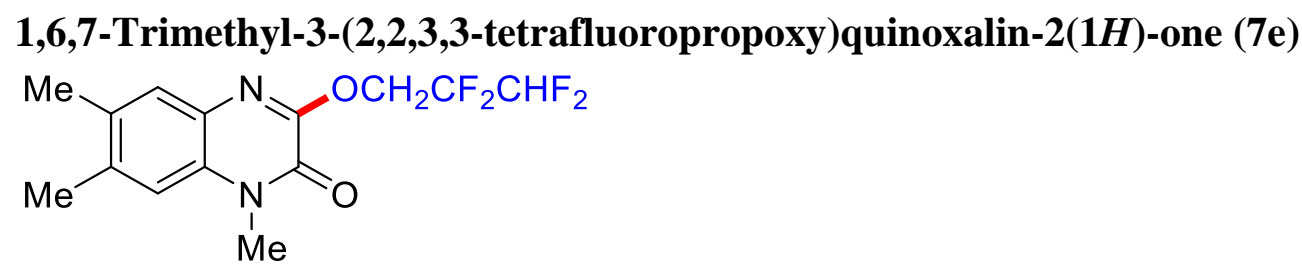

Obtained as a white solid (56 mg, 88\% yield); M.p. $161-162{ }^{\circ} \mathrm{C} .{ }^{1} \mathrm{H}$ NMR (500 MHz, $\left.\mathrm{CDCl}_{3}\right) \delta 7.41(\mathrm{~s}, 1 \mathrm{H}), 7.04(\mathrm{~s}, 1 \mathrm{H}), 6.16(\mathrm{tt}, J=53.0,5.0 \mathrm{~Hz}, 1 \mathrm{H}), 4.81(\mathrm{t}, J=12.2$ $\mathrm{Hz}, 2 \mathrm{H}), 3.70(\mathrm{~s}, 3 \mathrm{H}), 2.39(\mathrm{~s}, 3 \mathrm{H}), 2.34(\mathrm{~s}, 3 \mathrm{H}) ;{ }^{13} \mathrm{C} \mathrm{NMR}\left(126 \mathrm{MHz}, \mathrm{CDCl}_{3}\right) \delta$ 151.64, 150.21, 137.55, 133.15, 129.95, 128.13, 128.11, 115.76 (tt, $J=249.8,27.9$ $\mathrm{Hz}), 114.46,109.04(\mathrm{tt}, J=250.3,36.2 \mathrm{~Hz}), 62.76(\mathrm{t}, J=29.3 \mathrm{~Hz}), 29.48,20.33$, 19.19; ${ }^{19} \mathrm{~F}$ NMR $\left(471 \mathrm{MHz}, \mathrm{CDCl}_{3}\right) \delta-124.70(\mathrm{t}, J=11.5 \mathrm{~Hz}),-139.45(\mathrm{~d}, J=53.0$ $\mathrm{Hz})$; ATR-FTIR $\left(\mathrm{cm}^{-1}\right): 2955,1672,1625,1403,1269,1131,1099,1060$; HRMS (ESI+): Calculated for $\mathrm{C}_{14} \mathrm{H}_{14} \mathrm{~F}_{4} \mathrm{~N}_{2} \mathrm{O}_{2} \mathrm{H}$ : $[\mathrm{M}+\mathrm{H}]^{+} 319.1064$, Found 319.1069 . 
3-(2,2,3,3,4,4,4-Heptafluorobutoxy)-1-methylquinoxalin-2(1H)-one (8a)<smiles>Cn1c(=O)c(OCC(F)(F)F)nc2ccccc21</smiles>

Obtained as a white solid (53 mg, 74\% yield); M.p. $132-133{ }^{\circ} \mathrm{C} .{ }^{1} \mathrm{H}$ NMR $(500 \mathrm{MHz}$, $\left.\mathrm{CDCl}_{3}\right) \delta 7.66(\mathrm{dd}, J=7.9,1.2 \mathrm{~Hz}, 1 \mathrm{H}), 7.51-7.46(\mathrm{~m}, 1 \mathrm{H}), 7.37-7.32(\mathrm{~m}, 1 \mathrm{H})$, $7.30(\mathrm{~d}, J=8.3 \mathrm{~Hz}, 1 \mathrm{H}), 5.01(\mathrm{t}, J=13.5 \mathrm{~Hz}, 2 \mathrm{H}), 3.75(\mathrm{~s}, 3 \mathrm{H}) ;{ }^{13} \mathrm{C}$ NMR $(126 \mathrm{MHz}$, $\left.\mathrm{CDCl}_{3}\right) \delta 152.11,150.13,132.15,129.92,128.21,127.88,124.23,120.45$ (qt, $J=$ $288.6,34.4 \mathrm{~Hz}), 116.67(\mathrm{tt}, J=255.3,30.7 \mathrm{~Hz}), 113.83,109.61(\mathrm{tq}, J=264.7,37.8$ $\mathrm{Hz}), 61.84(\mathrm{t}, J=26.5 \mathrm{~Hz}), 29.60 ;{ }^{19} \mathrm{~F}$ NMR $\left(471 \mathrm{MHz}, \mathrm{CDCl}_{3}\right) \delta-80.74(\mathrm{t}, J=9.7$ $\mathrm{Hz}),-119.92(\mathrm{~m}),-127.54(\mathrm{~m})$; ATR-FTIR $\left(\mathrm{cm}^{-1}\right)$ : 2923, 1678, 1614, 1478, 1337, 1225, 1175, 1066, 1003; HRMS (ESI+): Calculated for $\mathrm{C}_{13} \mathrm{H}_{9} \mathrm{~F}_{7} \mathrm{~N}_{2} \mathrm{O}_{2} \mathrm{H}:[\mathrm{M}+\mathrm{H}]^{+}$ 359.0625, Found 359.0627.

1-Ethyl-3-(2,2,3,3,4,4,4-heptafluorobutoxy)quinoxalin-2(1H)-one $(8 \mathrm{~b})$<smiles>CCn1c(=O)c(OCC(F)(F)F)nc2ccccc21</smiles>

Obtained as a white solid (56 mg, 75\% yield); M.p. $122-123{ }^{\circ} \mathrm{C} .{ }^{1} \mathrm{H}$ NMR $(500 \mathrm{MHz}$, $\left.\mathrm{CDCl}_{3}\right) \delta 7.67(\mathrm{dd}, J=8.3,1.5 \mathrm{~Hz}, 1 \mathrm{H}), 7.50-7.45(\mathrm{~m}, 1 \mathrm{H}), 7.33(\mathrm{dd}, J=7.7,6.7 \mathrm{~Hz}$, $2 \mathrm{H}), 5.00(\mathrm{t}, J=13.5 \mathrm{~Hz}, 2 \mathrm{H}), 4.36(\mathrm{q}, J=7.2 \mathrm{~Hz}, 2 \mathrm{H}), 1.40(\mathrm{t}, J=7.2 \mathrm{~Hz}, 3 \mathrm{H}) ;{ }^{13} \mathrm{C}$ NMR $\left(126 \mathrm{MHz}, \mathrm{CDCl}_{3}\right) \delta 152.11,149.62,131.05,130.27,128.17,127.63,124.02$, 120.44 (qt, $J=288.6,34.4 \mathrm{~Hz}), 117.02(\mathrm{tt}, J=255.3,30.7 \mathrm{~Hz}), 113.67,109.71(\mathrm{tq}, J=$ 264.7, 37.8 Hz), 61.83 (t, $J=26.5 \mathrm{~Hz}), 37.88,12.37 ;{ }^{19} \mathrm{~F}$ NMR (471 MHz, $\left.\mathrm{CDCl}_{3}\right) \delta$ $-80.73(\mathrm{t}, J=9.6 \mathrm{~Hz}),-119.92(\mathrm{~m}),-127.50(\mathrm{~m})$; ATR-FTIR $\left(\mathrm{cm}^{-1}\right): 2922,1672,1651$, 1489, 1338, 1221, 1122, 1022; HRMS (ESI+): Calculated for $\mathrm{C}_{14} \mathrm{H}_{11} \mathrm{~F}_{7} \mathrm{~N}_{2} \mathrm{O}_{2} \mathrm{H}$ : $[\mathrm{M}+\mathrm{H}]^{+}$373.0782, Found 373.0781.

$3-(2,2,3,3,4,4,4-H e p t a f l u o r o b u t o x y) q u i n o x a l i n-2(1 H)-o n e ~(8 c)$<smiles>O=c1[nH]c2ccccc2nc1OCC(F)(F)C(F)(F)F</smiles>

Obtained as a white solid (47 mg, 68\% yield); M.p. $181-182{ }^{\circ} \mathrm{C} .{ }^{1} \mathrm{H}$ NMR $(500 \mathrm{MHz}$, $\left.\mathrm{CDCl}_{3}\right) \delta 11.95(\mathrm{~s}, 1 \mathrm{H}), 7.64(\mathrm{~d}, J=7.8 \mathrm{~Hz}, 1 \mathrm{H}), 7.43(\mathrm{dt}, J=7.0,3.5 \mathrm{~Hz}, 2 \mathrm{H}), 7.33$ (ddd, $J=8.4,6.1,2.5 \mathrm{~Hz}, 1 \mathrm{H}), 5.05(\mathrm{t}, J=13.6 \mathrm{~Hz}, 2 \mathrm{H}) ;{ }^{13} \mathrm{C} \mathrm{NMR}\left(126 \mathrm{MHz}, \mathrm{CDCl}_{3}\right)$ $\delta 152.40,151.43,130.10,129.89,128.35,126.97,124.75,120.78$ (qt, $J=288.6,34.4$ $\mathrm{Hz}), 117.38$ (tt, $J=255.3,30.7 \mathrm{~Hz}), 115.86,109.69$ (tq, $J=264.7,37.8 \mathrm{~Hz}), 61.97$ (t, $J=26.5 \mathrm{~Hz}) ;{ }^{19} \mathrm{~F}$ NMR $\left(471 \mathrm{MHz}, \mathrm{CDCl}_{3}\right) \delta-80.72(\mathrm{t}, J=9.7 \mathrm{~Hz}),-119.96(\mathrm{~m})$, -127.47 (m); ATR-FTIR ( $\left.\mathrm{cm}^{-1}\right)$ : 3198, 1692, 1614, 1420, 1228, 1124, 1022; HRMS (ESI+): Calculated for $\mathrm{C}_{12} \mathrm{H}_{7} \mathrm{~F}_{7} \mathrm{~N}_{2} \mathrm{O}_{2} \mathrm{H}:[\mathrm{M}+\mathrm{H}]^{+}$345.0469, Found 345.0461. 
6-Bromo-3-(2,2,3,3,4,4,4-heptafluorobutoxy)-1-methylquinoxalin-2(1H)-one (8d)<smiles>Cn1c(=O)c(OCC(F)(F)F)nc2cc(Br)ccc21</smiles>

Obtained as a white solid (61 mg, 70\% yield); M.p. 122-123 ${ }^{\circ} \mathrm{C} .{ }^{1} \mathrm{H}$ NMR $(500 \mathrm{MHz}$, $\left.\mathrm{CDCl}_{3}\right) \delta 7.84-7.79(\mathrm{~m}, 1 \mathrm{H}), 7.61-7.55(\mathrm{~m}, 1 \mathrm{H}), 7.17(\mathrm{dd}, J=8.9,1.1 \mathrm{~Hz}, 1 \mathrm{H})$, $5.00(\mathrm{dd}, J=13.4,12.5 \mathrm{~Hz}, 2 \mathrm{H}), 3.72(\mathrm{~s}, 3 \mathrm{H}) ;{ }^{13} \mathrm{C} \mathrm{NMR}\left(126 \mathrm{MHz}, \mathrm{CDCl}_{3}\right) \delta 152.81$, $149.76,131.29,130.98,130.91,130.36,120.26$ (qt, $J=288.6,34.4 \mathrm{~Hz}), 117.19$ (tt, $J$ $=255.3,30.7 \mathrm{~Hz}), 116.84,115.18,109.42(\mathrm{tq}, J=264.7,37.8 \mathrm{~Hz}), 61.99$ (t, $J=26.5$ $\mathrm{Hz}), 29.76 ;{ }^{19} \mathrm{~F}$ NMR $\left(471 \mathrm{MHz}, \mathrm{CDCl}_{3}\right) \delta-80.73(\mathrm{t}, J=10.1 \mathrm{~Hz}),-119.91(\mathrm{~m})$, -127.50 (m); ATR-FTIR $\left(\mathrm{cm}^{-1}\right)$ : 2923, 1668, 1613, 1462, 1298, 1228, 1120, 1017; HRMS (ESI+): Calculated for $\mathrm{C}_{13} \mathrm{H}_{8} \mathrm{BrF}_{7} \mathrm{~N}_{2} \mathrm{O}_{2} \mathrm{H}$ : $[\mathrm{M}+\mathrm{H}]^{+} 436.9730$, Found 436.9732.

3-(2,2,3,3,4,4,4-Heptafluorobutoxy)-1,6,7-trimethylquinoxalin-2(1H)-one (8e)<smiles>Cc1cc2nc(OCC(F)C(F)(F)F)c(=O)n(C)c2cc1C</smiles>

Obtained as a white solid (46 mg, 60\% yield); M.p. $136-137{ }^{\circ} \mathrm{C} .{ }^{1} \mathrm{H}$ NMR $(500 \mathrm{MHz}$, $\left.\mathrm{CDCl}_{3}\right) \delta 7.41(\mathrm{~s}, 1 \mathrm{H}), 7.05(\mathrm{~s}, 1 \mathrm{H}), 4.98(\mathrm{t}, J=13.6 \mathrm{~Hz}, 2 \mathrm{H}), 3.71(\mathrm{~s}, 3 \mathrm{H}), 2.40(\mathrm{~s}$, $3 \mathrm{H}), 2.34(\mathrm{~s}, 3 \mathrm{H}) ;{ }^{13} \mathrm{C}$ NMR $\left(126 \mathrm{MHz}, \mathrm{CDCl}_{3}\right) \delta 151.62,150.15,137.58,133.07$, 130.07, 128.11, 127.97, 120.48 (qt, $J=288.6,34.4 \mathrm{~Hz}$ ), 117.25 (tt, $J=255.3,30.7$ $\mathrm{Hz}), 114.46,109.48(\mathrm{tq}, J=264.7,37.8 \mathrm{~Hz}), 61.68(\mathrm{t}, J=26.5 \mathrm{~Hz}), 29.49,20.31$, 19.16; ${ }^{19} \mathrm{~F}$ NMR $\left(471 \mathrm{MHz}, \mathrm{CDCl}_{3}\right) \delta-80.75(\mathrm{t}, J=9.7 \mathrm{~Hz}, 3 \mathrm{~F}),-119.93(\mathrm{~m}),-127.49$ (m); ATR-FTIR $\left(\mathrm{cm}^{-1}\right): 2925,1668,1623,1470,1306,1269,1117,1026$; HRMS (ESI+): Calculated for $\mathrm{C}_{15} \mathrm{H}_{13} \mathrm{~F}_{7} \mathrm{~N}_{2} \mathrm{O}_{2} \mathrm{H}:[\mathrm{M}+\mathrm{H}]^{+}$387.0938, Found 387.0932.

1-Methyl-3-(4,4,4-trifluorobutoxy)quinoxalin-2(1H)-one (9a)<smiles>Cn1c(=O)c(OCCCC(F)F)nc2ccccc21</smiles>

Obtained as a white solid (46 mg, 81\% yield); M.p. 103-104 ${ }^{\circ} \mathrm{C} .{ }^{1} \mathrm{H}$ NMR $(500 \mathrm{MHz}$, $\left.\mathrm{CDCl}_{3}\right) \delta 7.62(\mathrm{dd}, J=7.9,1.4 \mathrm{~Hz}, 1 \mathrm{H}), 7.45-7.40(\mathrm{~m}, 1 \mathrm{H}), 7.31(\mathrm{td}, J=7.8,1.2 \mathrm{~Hz}$, $1 \mathrm{H}), 7.27(\mathrm{dd}, J=8.3,1.0 \mathrm{~Hz}, 1 \mathrm{H}), 4.54(\mathrm{t}, J=6.3 \mathrm{~Hz}, 2 \mathrm{H}), 3.73(\mathrm{~s}, 3 \mathrm{H}), 2.39-2.30$ (m, 2H), $2.17(\mathrm{ddd}, J=16.1,10.8,6.3 \mathrm{~Hz}, 2 \mathrm{H}) ;{ }^{13} \mathrm{C} \mathrm{NMR}\left(126 \mathrm{MHz}, \mathrm{CDCl}_{3}\right) \delta$ 153.59, 150.94, 131.67, 130.94, 127.63, 127.28, 127.04 (q, $J=277.2 \mathrm{~Hz}$ ), 124.05, $113.68,65.63,30.77$ (q, $J=31.5 \mathrm{~Hz}), 29.49,21.50(\mathrm{q}, J=3.8 \mathrm{~Hz}) ;{ }^{19} \mathrm{~F}$ NMR $(471$ $\left.\mathrm{MHz}, \mathrm{CDCl}_{3}\right) \delta$-66.30; ATR-FTIR $\left(\mathrm{cm}^{-1}\right):$ 2958, 1676, 1608, 1478, 1339, 1244, 1154, 1080, 1001; HRMS (ESI+): Calculated for $\mathrm{C}_{13} \mathrm{H}_{13} \mathrm{~F}_{3} \mathrm{~N}_{2} \mathrm{O}_{2} \mathrm{H}:[\mathrm{M}+\mathrm{H}]^{+}$287.1002, Found 287.1002 . 
1-Ethyl-3-(4,4,4-trifluorobutoxy)quinoxalin-2(1H)-one (9b)<smiles>CCn1c(=O)c(OCCCC(F)F)nc2ccccc21</smiles>

Obtained as a white solid (50 mg, 83\% yield); M.p. $134-135{ }^{\circ} \mathrm{C} .{ }^{1} \mathrm{H}$ NMR $(500 \mathrm{MHz}$, $\left.\mathrm{CDCl}_{3}\right) \delta 7.64(\mathrm{dd}, J=8.3,1.5 \mathrm{~Hz}, 1 \mathrm{H}), 7.45-7.40(\mathrm{~m}, 1 \mathrm{H}), 7.30(\mathrm{dd}, J=7.7,6.6 \mathrm{~Hz}$, $2 \mathrm{H}), 4.54(\mathrm{t}, J=6.3 \mathrm{~Hz}, 2 \mathrm{H}), 4.35(\mathrm{q}, J=7.2 \mathrm{~Hz}, 2 \mathrm{H}), 2.41-2.31(\mathrm{~m}, 2 \mathrm{H}), 2.17(\mathrm{dt}, J$ $=13.5,6.3 \mathrm{~Hz}, 2 \mathrm{H}), 1.39(\mathrm{t}, J=7.2 \mathrm{~Hz}, 3 \mathrm{H}) ;{ }^{13} \mathrm{C} \mathrm{NMR}\left(126 \mathrm{MHz}, \mathrm{CDCl}_{3}\right) \delta 153.60$, $150.41,131.28,130.52,127.92,127.26,127.06$ (q, $J=277.2 \mathrm{~Hz}), 123.83,113.53$, 65.60, 37.69, 30.79 (q, $J=31.5 \mathrm{~Hz}), 21.51(\mathrm{q}, J=3.8 \mathrm{~Hz}), 12.36 ;{ }^{19} \mathrm{~F}$ NMR $(471 \mathrm{MHz}$, $\left.\mathrm{CDCl}_{3}\right) \delta$-66.30; ATR-FTIR $\left(\mathrm{cm}^{-1}\right): 2948,1664,1609,1452,1394,1220,1147,1009$; HRMS (ESI+): Calculated for $\mathrm{C}_{14} \mathrm{H}_{15} \mathrm{~F}_{3} \mathrm{~N}_{2} \mathrm{O}_{2} \mathrm{H}:[\mathrm{M}+\mathrm{H}]^{+}$301.1158, Found 301.1164.

6-Bromo-1-methyl-3-(4,4,4-trifluorobutoxy)quinoxalin-2(1H)-one $(9 \mathrm{c})$<smiles>Cn1c(=O)c(OCCCC(F)F)nc2cc(Br)ccc21</smiles>

Obtained as a white solid (52 mg, 72\% yield); M.p. 127-128 ${ }^{\circ} \mathrm{C} .{ }^{1} \mathrm{H}$ NMR $(500 \mathrm{MHz}$, $\left.\mathrm{CDCl}_{3}\right) \delta 7.77(\mathrm{~d}, J=2.2 \mathrm{~Hz}, 1 \mathrm{H}), 7.51(\mathrm{dd}, J=8.8,2.2 \mathrm{~Hz}, 1 \mathrm{H}), 7.13(\mathrm{~d}, J=8.8 \mathrm{~Hz}$, $1 \mathrm{H}), 4.52$ (t, $J=6.3 \mathrm{~Hz}, 2 \mathrm{H}), 3.70(\mathrm{~s}, 3 \mathrm{H}), 2.38-2.30(\mathrm{~m}, 2 \mathrm{H}), 2.19-2.14(\mathrm{~m}, 2 \mathrm{H})$; ${ }^{13} \mathrm{C}$ NMR $\left(126 \mathrm{MHz}, \mathrm{CDCl}_{3}\right) \delta 154.24,150.57,131.99,130.82,130.10,129.99$, $126.98(\mathrm{q}, J=277.2 \mathrm{~Hz}), 116.63,115.01,65.95,30.72(\mathrm{q}, J=31.5 \mathrm{~Hz}), 29.64,21.43$ $(\mathrm{q}, J=3.8 \mathrm{~Hz}) ;{ }^{19} \mathrm{~F}$ NMR $\left(471 \mathrm{MHz}, \mathrm{CDCl}_{3}\right) \delta-66.28$; ATR-FTIR $\left(\mathrm{cm}^{-1}\right): 2947,1679$, 1608, 1463, 1315, 1239, 1131, 1027; HRMS (ESI+): Calculated for $\mathrm{C}_{13} \mathrm{H}_{12} \mathrm{BrF}_{3} \mathrm{~N}_{2} \mathrm{O}_{2} \mathrm{H}:[\mathrm{M}+\mathrm{H}]^{+}$365.0107, Found 365.0116.

1-Benzyl-3-(4,4,4-trifluorobutoxy)quinoxalin-2(1H)-one (9d)<smiles>O=c1c(OCCCC(F)F)nc2ccccc2n1Cc1ccccc1</smiles>

Obtained as a white solid (53 mg, 73\% yield); M.p. $121-122{ }^{\circ} \mathrm{C} .{ }^{1} \mathrm{H}$ NMR (500 MHz, $\left.\mathrm{CDCl}_{3}\right) \delta 7.62(\mathrm{dd}, J=7.7,1.7 \mathrm{~Hz}, 1 \mathrm{H}), 7.31(\mathrm{t}, J=4.3 \mathrm{~Hz}, 1 \mathrm{H}), 7.30-7.20(\mathrm{~m}, 7 \mathrm{H})$, $5.51(\mathrm{~s}, 2 \mathrm{H}), 4.56(\mathrm{t}, J=6.3 \mathrm{~Hz}, 2 \mathrm{H}), 2.42-2.33(\mathrm{~m}, 2 \mathrm{H}), 2.19(\mathrm{dt}, J=13.4,6.3 \mathrm{~Hz}$, $2 \mathrm{H}) ;{ }^{13} \mathrm{C}$ NMR $\left(126 \mathrm{MHz}, \mathrm{CDCl}_{3}\right) \delta 153.68,151.15,135.13,135.10,131.19,130.99$, 128.93, 127.76, 127.27, 127.08 (q, $J=277.2 \mathrm{~Hz}$ ), 126.98, 124.07, 114.54, 65.79, 46.31, $30.82(\mathrm{q}, J=31.5 \mathrm{~Hz}), 21.54(\mathrm{q}, J=3.8 \mathrm{~Hz}) ;{ }^{19} \mathrm{~F}$ NMR $\left(471 \mathrm{MHz}, \mathrm{CDCl}_{3}\right) \delta$ -66.26; ATR-FTIR ( $\left.\mathrm{cm}^{-1}\right)$ : 2965, 1671, 1611, 1452, 1344, 1245, 1150, 1015, 754, 696; HRMS (ESI+): Calculated for $\mathrm{C}_{19} \mathrm{H}_{17} \mathrm{~F}_{3} \mathrm{~N}_{2} \mathrm{O}_{2} \mathrm{H}$ : $[\mathrm{M}+\mathrm{H}]^{+}$363.1315, Found 363.1319. 


\section{1-(4-Chlorobenzyl)-3-(4,4,4-trifluorobutoxy)quinoxalin-2(1H)-one (9e)}<smiles>O=c1c(OCCCC(F)(F)F)nc2ccccc2n1Cc1ccc(Cl)cc1</smiles>

Obtained as a white solid (55 mg, 69\% yield); M.p. 138-139 ${ }^{\circ} \mathrm{C} .{ }^{1} \mathrm{H}$ NMR $(500 \mathrm{MHz}$, $\left.\mathrm{CDCl}_{3}\right) \delta 7.64(\mathrm{dd}, J=7.7,1.7 \mathrm{~Hz}, 1 \mathrm{H}), 7.33-7.26(\mathrm{~m}, 4 \mathrm{H}), 7.21(\mathrm{~d}, J=8.5 \mathrm{~Hz}, 2 \mathrm{H})$, $7.17(\mathrm{dd}, J=8.1,1.3 \mathrm{~Hz}, 1 \mathrm{H}), 5.47(\mathrm{~s}, 2 \mathrm{H}), 4.56(\mathrm{t}, J=6.3 \mathrm{~Hz}, 2 \mathrm{H}), 2.42-2.32(\mathrm{~m}$, $2 \mathrm{H}), 2.19(\mathrm{dt}, J=13.5,6.3 \mathrm{~Hz}, 2 \mathrm{H}) ;{ }^{13} \mathrm{C} \mathrm{NMR}\left(126 \mathrm{MHz}, \mathrm{CDCl}_{3}\right) \delta 153.60,151.09$, $133.70,133.65,131.20,130.76,129.12,128.47,127.91,127.35,127.05$ (q, $J=277.2$ $\mathrm{Hz}), 124.25,114.26,65.85,45.68,30.80(\mathrm{q}, J=31.5 \mathrm{~Hz}), 21.52(\mathrm{q}, J=3.8 \mathrm{~Hz}) ;{ }^{19} \mathrm{~F}$ NMR (471 MHz, $\left.\mathrm{CDCl}_{3}\right) \delta$-66.25; ATR-FTIR ( $\left.\mathrm{cm}^{-1}\right)$ : 2972, 1679, 1636, 1491, 1340 , 1248, 1128, 1073, 800; HRMS (ESI+): Calculated for $\mathrm{C}_{19} \mathrm{H}_{16} \mathrm{ClF}_{3} \mathrm{~N}_{2} \mathrm{O}_{2} \mathrm{H}:[\mathrm{M}+\mathrm{H}]^{+}$ 397.0925, Found 397.0919.

\section{2-Chloro-3-(2,2,2-trifluoroethoxy)quinoxaline (10)}<smiles>FC(F)(F)COc1nc2ccccc2nc1Cl</smiles>

Obtained as a white solid (1.17 g, 89\% yield); M.p. $112-113{ }^{\circ} \mathrm{C} .{ }^{1} \mathrm{H}$ NMR $(500 \mathrm{MHz}$, $\left.\mathrm{CDCl}_{3}\right) \delta 7.98(\mathrm{dd}, J=8.3,1.2 \mathrm{~Hz}, 1 \mathrm{H}), 7.86(\mathrm{dd}, J=8.3,1.1 \mathrm{~Hz}, 1 \mathrm{H}), 7.75-7.70(\mathrm{~m}$, $1 \mathrm{H}), 7.68-7.63(\mathrm{~m}, 1 \mathrm{H}), 4.97(\mathrm{q}, J=8.2 \mathrm{~Hz}, 2 \mathrm{H}) ;{ }^{13} \mathrm{C} \mathrm{NMR}\left(126 \mathrm{MHz}, \mathrm{CDCl}_{3}\right) \delta$ 151.16, 139.10, 138.70, 138.64, 130.73, 128.46, 128.14, 126.89, 123.08 (q, $J=277.2$ $\mathrm{Hz}), 63.28(\mathrm{q}, J=37.8 \mathrm{~Hz}) ;{ }^{19} \mathrm{~F}$ NMR $\left(471 \mathrm{MHz}, \mathrm{CDCl}_{3}\right) \delta-73.43$; ATR-FTIR $\left(\mathrm{cm}^{-1}\right)$ : 2977, 2574, 1429, 1334, 1277, 1155, 1088, 973, 768; HRMS (ESI+): Calculated for $\mathrm{C}_{10} \mathrm{H}_{6} \mathrm{ClF}_{3} \mathrm{~N}_{2} \mathrm{OH}:[\mathrm{M}+\mathrm{H}]^{+}$263.0194, Found 263.0199.

\section{2-(p-Tolyl)-3-(2,2,2-trifluoroethoxy)quinoxaline (11a)}<smiles>CC(F)(F)COc1nc2ccccc2nc1O[Na]</smiles>

Obtained as a white solid (59 mg, 93\% yield); M.p. $132-133{ }^{\circ} \mathrm{C} .{ }^{1} \mathrm{H}$ NMR $(500 \mathrm{MHz}$, $\left.\mathrm{CDCl}_{3}\right) \delta 8.07(\mathrm{dd}, J=8.1,1.3 \mathrm{~Hz}, 1 \mathrm{H}), 8.05(\mathrm{~d}, J=8.2 \mathrm{~Hz}, 2 \mathrm{H}), 7.80(\mathrm{dd}, J=8.2$, $1.2 \mathrm{~Hz}, 1 \mathrm{H}), 7.65-7.61(\mathrm{~m}, 1 \mathrm{H}), 7.60-7.56(\mathrm{~m}, 1 \mathrm{H}), 7.31(\mathrm{~d}, J=8.0 \mathrm{~Hz}, 2 \mathrm{H}), 4.96$ $(\mathrm{q}, J=8.4 \mathrm{~Hz}, 2 \mathrm{H}), 2.42(\mathrm{~s}, 3 \mathrm{H}) ;{ }^{13} \mathrm{C} \mathrm{NMR}\left(126 \mathrm{MHz}, \mathrm{CDCl}_{3}\right) \delta 153.31,145.85$, $140.31,139.70,138.82,132.46,129.87,129.60,129.14,129.04,127.63,126.68$, $123.63(\mathrm{q}, J=277.2 \mathrm{~Hz}), 62.54(\mathrm{q}, J=37.8 \mathrm{~Hz}), 21.44 ;{ }^{19} \mathrm{~F} \mathrm{NMR}\left(471 \mathrm{MHz}, \mathrm{CDCl}_{3}\right)$ $\delta$-73.16; ATR-FTIR $\left(\mathrm{cm}^{-1}\right): 2960,1609,1576,1422,1255,1165,1065,828 ;$ HRMS (ESI+): Calculated for $\mathrm{C}_{17} \mathrm{H}_{13} \mathrm{~F}_{3} \mathrm{~N}_{2} \mathrm{OH}:[\mathrm{M}+\mathrm{H}]^{+} 319.1053$, Found 319.1048. 
2-(p-Tolylethynyl)-3-(2,2,2-trifluoroethoxy)quinoxaline (11b)<smiles>[O+]C#Cc1nc2ccccc2nc1OCC(F)(F)F</smiles>

Obtained as a white solid (58 mg, 85\% yield); M.p. $122-123{ }^{\circ} \mathrm{C} .{ }^{1} \mathrm{H}$ NMR $(500 \mathrm{MHz}$, $\left.\mathrm{CDCl}_{3}\right) \delta 8.02(\mathrm{dd}, J=8.5,0.8 \mathrm{~Hz}, 1 \mathrm{H}), 7.82-7.77(\mathrm{~m}, 1 \mathrm{H}), 7.67-7.63(\mathrm{~m}, 1 \mathrm{H})$, $7.62-7.58(\mathrm{~m}, 1 \mathrm{H}), 7.57(\mathrm{dd}, J=9.9,4.5 \mathrm{~Hz}, 2 \mathrm{H}), 7.19(\mathrm{~d}, J=8.0 \mathrm{~Hz}, 2 \mathrm{H}), 4.95(\mathrm{q}, J$ $=8.3 \mathrm{~Hz}, 2 \mathrm{H}), 2.38(\mathrm{~s}, 3 \mathrm{H}) ;{ }^{13} \mathrm{C} \mathrm{NMR}\left(126 \mathrm{MHz}, \mathrm{CDCl}_{3}\right) \delta 154.75,140.37,139.38$, $138.39,132.45,130.71,129.33,128.74,128.41,127.94,126.92,123.43$ (q, $J=277.2$ $\mathrm{Hz}), 118.53,97.73,83.74,62.87$ (q, $J=37.8 \mathrm{~Hz}), 21.67 ;{ }^{19} \mathrm{~F} \mathrm{NMR}\left(471 \mathrm{MHz}, \mathrm{CDCl}_{3}\right.$ ) $\delta$-73.50; ATR-FTIR $\left(\mathrm{cm}^{-1}\right): 3055,2209,1614,1589,1514,1429,1267,1177,1130$, 1060, 965, 766; HRMS (ESI+): Calculated for $\mathrm{C}_{19} \mathrm{H}_{13} \mathrm{~F}_{3} \mathrm{~N}_{2} \mathrm{OH}:[\mathrm{M}+\mathrm{H}]^{+} 343.1053$, Found 343.1050.

4-(3-(2,2,2-Trifluoroethoxy)quinoxalin-2-yl)morpholine (11c)<smiles>FC(F)(F)COc1nc2ccccc2nc1N1CCOCC1</smiles>

Obtained as a white solid (58 mg, 92\% yield); M.p. $101-102{ }^{\circ} \mathrm{C} .{ }^{1} \mathrm{H}$ NMR (500 MHz, $\left.\mathrm{CDCl}_{3}\right) \delta 7.77(\mathrm{dd}, J=8.2,1.0 \mathrm{~Hz}, 1 \mathrm{H}), 7.69(\mathrm{dd}, J=8.1,1.2 \mathrm{~Hz}, 1 \mathrm{H}), 7.53-7.47(\mathrm{~m}$, $1 \mathrm{H}), 7.47-7.37(\mathrm{~m}, 1 \mathrm{H}), 4.92(\mathrm{q}, J=8.4 \mathrm{~Hz}, 2 \mathrm{H}), 3.92-3.83(\mathrm{~m}, 4 \mathrm{H}), 3.78-3.68$ $(\mathrm{m}, 4 \mathrm{H}) ;{ }^{13} \mathrm{C}$ NMR $\left(126 \mathrm{MHz}, \mathrm{CDCl}_{3}\right) \delta 148.16,146.12,138.71,135.52,127.61$, 126.25, 126.20, 123.54 (q, $J=277.2 \mathrm{~Hz}), 66.78,62.56$ (q, $J=37.8 \mathrm{~Hz}), 48.39 ;{ }^{19} \mathrm{~F}$ NMR $\left(471 \mathrm{MHz}, \mathrm{CDCl}_{3}\right) \delta$-73.33; ATR-FTIR $\left(\mathrm{cm}^{-1}\right): 2893,1635,1618,1502,1436$, 1250, 1165, 1058; HRMS (ESI+): Calculated for $\mathrm{C}_{14} \mathrm{H}_{14} \mathrm{~F}_{3} \mathrm{~N}_{3} \mathrm{O}_{2} \mathrm{H}:[\mathrm{M}+\mathrm{H}]^{+}$314.1111, Found 314.1118.

\section{2-Methoxy-3-(2,2,2-trifluoroethoxy)quinoxaline (11d)}<smiles>COc1nc2ccccc2nc1OCC(F)(F)F</smiles>

Obtained as a white solid (48 mg, 93\% yield); M.p. 96-97 ${ }^{\circ} \mathrm{C} .{ }^{1} \mathrm{H}$ NMR (500 MHz, $\left.\mathrm{CDCl}_{3}\right) \delta 7.79(\mathrm{dd}, J=7.9,1.5 \mathrm{~Hz}, 1 \mathrm{H}), 7.76-7.71(\mathrm{~m}, 1 \mathrm{H}), 7.55-7.48(\mathrm{~m}, 2 \mathrm{H})$, $4.96(\mathrm{q}, J=8.4 \mathrm{~Hz}, 2 \mathrm{H}), 4.16(\mathrm{~s}, 3 \mathrm{H}) ;{ }^{13} \mathrm{C} \mathrm{NMR}\left(126 \mathrm{MHz}, \mathrm{CDCl}_{3}\right) \delta 149.54,147.55$, $137.90,136.26,127.55,126.95,126.54,126.52,123.35$ (q, $J=277.2 \mathrm{~Hz}), 62.51$ (q, $J$ $=37.8 \mathrm{~Hz}), 54.34 ;{ }^{19} \mathrm{~F}$ NMR $\left(471 \mathrm{MHz}, \mathrm{CDCl}_{3}\right) \delta-73.27$; ATR-FTIR $\left(\mathrm{cm}^{-1}\right): 2995$, 1617, 1582, 1482, 1316, 1253, 1155, 1005; HRMS (ESI+): Calculated for $\mathrm{C}_{11} \mathrm{H}_{9} \mathrm{~F}_{3} \mathrm{~N}_{2} \mathrm{O}_{2} \mathrm{H}:[\mathrm{M}+\mathrm{H}]^{+}$259.0689, Found 259.0695. 
Diphenyl(3-(2,2,2-trifluoroethoxy)quinoxalin-2-yl)phosphine oxide (11e)<smiles>O=P(c1ccccc1)(c1ccccc1)c1nc2ccccc2nc1OCC(F)(F)F</smiles>

Obtained as a white solid (77 mg, 90\% yield); M.p. 198-199 ${ }^{\circ} \mathrm{C} .{ }^{31} \mathrm{P}$ NMR (202 MHz, $\left.\mathrm{CDCl}_{3}\right) \delta 23.68 ;{ }^{1} \mathrm{H} \mathrm{NMR}\left(500 \mathrm{MHz}, \mathrm{CDCl}_{3}\right) \delta 8.08(\mathrm{~d}, J=8.3 \mathrm{~Hz}, 1 \mathrm{H}), 7.85(\mathrm{dd}, J=$ 10.4, $9.2 \mathrm{~Hz}, 5 \mathrm{H}), 7.78$ (t, $J=7.6 \mathrm{~Hz}, 1 \mathrm{H}), 7.64$ (t, $J=7.5 \mathrm{~Hz}, 1 \mathrm{H}), 7.57$ (t, $J=7.4 \mathrm{~Hz}$, $2 \mathrm{H}), 7.49(\mathrm{dd}, J=7.2,5.6 \mathrm{~Hz}, 4 \mathrm{H}), 4.84(\mathrm{q}, J=8.3 \mathrm{~Hz}, 2 \mathrm{H}) ;{ }^{13} \mathrm{C}$ NMR $(126 \mathrm{MHz}$, $\left.\mathrm{CDCl}_{3}\right) \delta 155.70,155.56,145.31,144.34,140.19,139.45,139.32,132.62,132.18$, $132.16,132.04,131.96,130.98,130.36,130.12,128.44,128.34,128.05,126.99$, 123.88, 121.67, 62.47, 62.18, 61.89, 61.59; ${ }^{19} \mathrm{~F}$ NMR (471 $\left.\mathrm{MHz}, \mathrm{CDCl}_{3}\right) \delta$-73.35; ATR-FTIR ( $\left.\mathrm{cm}^{-1}\right)$ : 2925, 1621, 1576, 1442, 1337, 1272, 1152, 1115, 771, 706; HRMS (ESI+): Calculated for $\mathrm{C}_{22} \mathrm{H}_{16} \mathrm{~F}_{3} \mathrm{~N}_{2} \mathrm{O}_{2} \mathrm{PH}:[\mathrm{M}+\mathrm{H}]^{+}$429.0974, Found 429.0977.

2-(p-Tolylthio)-3-(2,2,2-trifluoroethoxy)quinoxaline (11f)<smiles>FC(F)(F)Oc1nc2ccccc2nc1SOc1ccccc1</smiles>

Obtained as a white solid (62 mg, 89\% yield); M.p. $118-119{ }^{\circ} \mathrm{C} .{ }^{1} \mathrm{H}$ NMR $(500 \mathrm{MHz}$, $\left.\mathrm{CDCl}_{3}\right) \delta 7.66(\mathrm{~d}, J=8.2 \mathrm{~Hz}, 1 \mathrm{H}), 7.60(\mathrm{~d}, J=8.2 \mathrm{~Hz}, 1 \mathrm{H}), 7.44(\mathrm{t}, J=9.2 \mathrm{~Hz}, 3 \mathrm{H})$, $7.38(\mathrm{t}, J=7.6 \mathrm{~Hz}, 1 \mathrm{H}), 7.19$ (d, $J=7.9 \mathrm{~Hz}, 2 \mathrm{H}), 4.89$ (q, $J=8.3 \mathrm{~Hz}, 2 \mathrm{H}), 2.35$ (s, $3 \mathrm{H}) ;{ }^{13} \mathrm{C}$ NMR $\left(126 \mathrm{MHz}, \mathrm{CDCl}_{3}\right) \delta 150.38,147.95,138.84,138.50,136.53,134.38$, 128.97, 127.51, 126.79, 126.30, 125.63, 122.88, 122.24 (q, $J=277.2 \mathrm{~Hz}), 61.64$ (q, $J$ $=37.8 \mathrm{~Hz}), 20.37 ;{ }^{19} \mathrm{~F}$ NMR $\left(471 \mathrm{MHz}, \mathrm{CDCl}_{3}\right) \delta-73.35$; ATR-FTIR $\left(\mathrm{cm}^{-1}\right): 2957$, 1616, 1556, 1422, 1327, 1285, 1157, 1085, 811; HRMS (ESI+): Calculated for $\mathrm{C}_{17} \mathrm{H}_{13} \mathrm{~F}_{3} \mathrm{~N}_{2} \mathrm{OSH}:[\mathrm{M}+\mathrm{H}]^{+}$351.0774, Found 351.0773.

\section{2-Tosyl-3-(2,2,2-trifluoroethoxy)quinoxaline (11g)}

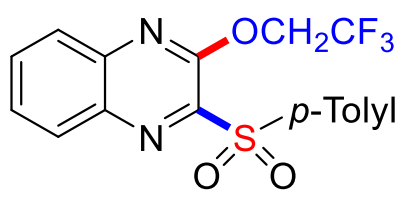

Obtained as a white solid (66 mg, 87\% yield); M.p. $178-179{ }^{\circ} \mathrm{C} .{ }^{1} \mathrm{H}$ NMR $(500 \mathrm{MHz}$, $\left.\mathrm{CDCl}_{3}\right) \delta 8.27-8.22(\mathrm{~m}, 1 \mathrm{H}), 8.00(\mathrm{~d}, J=8.3 \mathrm{~Hz}, 2 \mathrm{H}), 7.85(\mathrm{dtd}, J=9.6,8.3,1.2 \mathrm{~Hz}$, 2H), $7.73(\mathrm{ddd}, J=8.4,6.6,1.7 \mathrm{~Hz}, 1 \mathrm{H}), 7.35(\mathrm{~d}, J=8.1 \mathrm{~Hz}, 2 \mathrm{H}), 4.94(\mathrm{q}, J=8.3 \mathrm{~Hz}$, $2 \mathrm{H}), 2.44(\mathrm{~s}, 3 \mathrm{H}) ;{ }^{13} \mathrm{C} \mathrm{NMR}\left(126 \mathrm{MHz}, \mathrm{CDCl}_{3}\right) \delta 151.37,145.59,144.07,141.30$, $138.09,134.96,133.48,130.39,129.74,129.72,128.95,126.87,122.97$ (q, $J=277.2$ $\mathrm{Hz}), 62.82(\mathrm{q}, J=37.8 \mathrm{~Hz}), 21.71 ;{ }^{19} \mathrm{~F}$ NMR $\left(471 \mathrm{MHz}, \mathrm{CDCl}_{3}\right) \delta-72.81$; ATR-FTIR $\left(\mathrm{cm}^{-1}\right): 2958,1596,1559,1445,1306,1278,1166,1105,1074,792$; HRMS (ESI+): Calculated for $\mathrm{C}_{17} \mathrm{H}_{13} \mathrm{~F}_{3} \mathrm{~N}_{2} \mathrm{O}_{3} \mathrm{SH}:[\mathrm{M}+\mathrm{H}]^{+}$383.0672, Found 383.0672. 


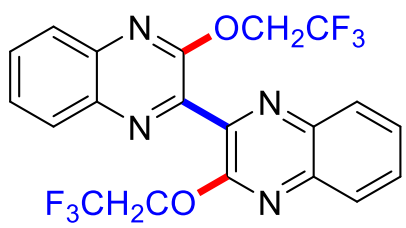

Obtained as a pale yellow solid (56 mg, 62\% yield); M.p. $217-218{ }^{\circ} \mathrm{C} .{ }^{1} \mathrm{H}$ NMR (500 $\left.\mathrm{MHz} \mathrm{CDCl}_{3}\right) \delta 8.22(\mathrm{dd}, J=8.3,1.1 \mathrm{~Hz}, 1 \mathrm{H}), 7.97(\mathrm{dd}, J=8.4,1.0 \mathrm{~Hz}, 1 \mathrm{H}), 7.81$ (ddd, $J=8.4,7.1,1.4 \mathrm{~Hz}, 1 \mathrm{H}), 7.71$ (ddd, $J=8.3,7.1,1.4 \mathrm{~Hz}, 1 \mathrm{H}), 4.93$ (q, $J=8.3$ $\mathrm{Hz}, 2 \mathrm{H}) ;{ }^{13} \mathrm{C}$ NMR $\left(126 \mathrm{MHz}, \mathrm{CDCl}_{3}\right) \delta 153.67,142.48,140.16,139.51,131.47$, $129.72,128.07,127.19,123.16(\mathrm{q}, J=277.2 \mathrm{~Hz}), 62.62(\mathrm{q}, J=37.8 \mathrm{~Hz}) ;{ }^{19} \mathrm{~F}$ NMR $\left(471 \mathrm{MHz}, \mathrm{CDCl}_{3}\right) \delta$-73.62; ATR-FTIR $\left(\mathrm{cm}^{-1}\right)$ : 2922, 1624, 1579, 1442, 1344, 1270, 1170, 1070, 965, 768; HRMS (ESI+): Calculated for $\mathrm{C}_{20} \mathrm{H}_{12} \mathrm{~F}_{6} \mathrm{~N}_{4} \mathrm{O}_{2} \mathrm{H}:[\mathrm{M}+\mathrm{H}]^{+}$ 455.0937, Found 455.0929.

\section{1-Methyl-3-morpholinoquinoxalin-2(1H)-one (12a)}<smiles>Cn1c(=O)c(N2CCOCC2)nc2ccccc21</smiles>

Obtained as a white solid (38 mg, 77\% yield); M.p. $160-161{ }^{\circ} \mathrm{C} .{ }^{1} \mathrm{H}$ NMR $(500 \mathrm{MHz}$, $\left.\mathrm{CDCl}_{3}\right) \delta 7.65-7.52(\mathrm{~m}, 1 \mathrm{H}), 7.32-7.27(\mathrm{~m}, 1 \mathrm{H}), 7.26-7.23(\mathrm{~m}, 1 \mathrm{H}), 7.21(\mathrm{dd}, J=$ 8.0, $1.4 \mathrm{~Hz}, 1 \mathrm{H}), 4.01-3.94(\mathrm{~m}, 4 \mathrm{H}), 3.89-3.82(\mathrm{~m}, 4 \mathrm{H}), 3.68(\mathrm{~s}, 3 \mathrm{H}) ;{ }^{13} \mathrm{C} \mathrm{NMR}$ $\left(126 \mathrm{MHz}, \mathrm{CDCl}_{3}\right) \delta 152.23,150.62,132.84,130.79,126.74,125.43,123.89,113.31$, 67.00, 47.65, 29.36; ATR-FTIR $\left(\mathrm{cm}^{-1}\right)$ : 2988, 1660, 1543, 1252, 1122, 1037; HRMS (ESI+): Calculated for $\mathrm{C}_{13} \mathrm{H}_{15} \mathrm{~N}_{3} \mathrm{O}_{2} \mathrm{H}:[\mathrm{M}+\mathrm{H}]^{+}$246.1237, Found 246.1236.

3-Morpholinoquinoxalin-2(1H)-one (12b)<smiles>O=c1[nH]c2ccccc2nc1N1CCOCC1</smiles>

Obtained as a white solid (33 mg, 72\% yield); M.p. 192-193 ${ }^{\circ} \mathrm{C} .{ }^{1} \mathrm{H}$ NMR $(500 \mathrm{MHz}$, $\left.\mathrm{CDCl}_{3}\right) \delta 10.83(\mathrm{~s}, 1 \mathrm{H}), 7.63-7.52(\mathrm{~m}, 1 \mathrm{H}), 7.24(\mathrm{dd}, J=6.0,3.3 \mathrm{~Hz}, 2 \mathrm{H}), 7.12(\mathrm{dd}$, $J=5.9,3.4 \mathrm{~Hz}, 1 \mathrm{H}), 4.13-3.97(\mathrm{~m}, 4 \mathrm{H}), 3.94-3.81(\mathrm{~m}, 4 \mathrm{H}) ;{ }^{13} \mathrm{C} \mathrm{NMR}(126 \mathrm{MHz}$, $\left.\mathrm{CDCl}_{3}\right) \delta 153.37,150.66,132.83,128.40,125.92,125.42,124.36,114.40,66.97$, 47.45; ATR-FTIR $\left(\mathrm{cm}^{-1}\right)$ : 2943, 1667, 1544, 1370, 1225, 1120, 1049; HRMS (ESI+): Calculated for $\mathrm{C}_{12} \mathrm{H}_{13} \mathrm{~N}_{3} \mathrm{O}_{2} \mathrm{H}$ : $[\mathrm{M}+\mathrm{H}]^{+} 232.1081$, Found 232.1089 .

6,7-Dichloro-3-(4-methylpiperazin-1-yl)quinoxalin-2(1H)-one (13)<smiles>CN1CCN(c2nc3cc(Cl)c(Cl)cc3[nH]c2=O)CC1</smiles> 
Obtained as a pale yellow solid (106 mg, 68\% yield); M.p. $234-235{ }^{\circ} \mathrm{C} .{ }^{1} \mathrm{H}$ NMR (500 MHz, DMSO) $\delta 12.43$ (s, 1H), $7.46(\mathrm{~s}, 1 \mathrm{H}), 7.37$ (s, 1H), $3.96(\mathrm{~s}, 4 \mathrm{H}), 2.59$ (s, 4H), 2.29 (s, 3H); ${ }^{13} \mathrm{C}$ NMR (126 MHz, DMSO) $\delta 152.18,151.67,132.96,129.74,125.89$, 125.80, 125.03, 115.79, 55.02, 46.38, 46.08; ATR-FTIR $\left(\mathrm{cm}^{-1}\right): 2965,1683,1624$, 1462, 1287, 1177, 1069; HRMS (ESI+): Calculated for $\mathrm{C}_{13} \mathrm{H}_{14} \mathrm{Cl}_{2} \mathrm{~N}_{4} \mathrm{OH}:[\mathrm{M}+\mathrm{H}]^{+}$ 312.0618, Found 312.0612. 


\section{X-ray Crystal Data for 31 and 7d}

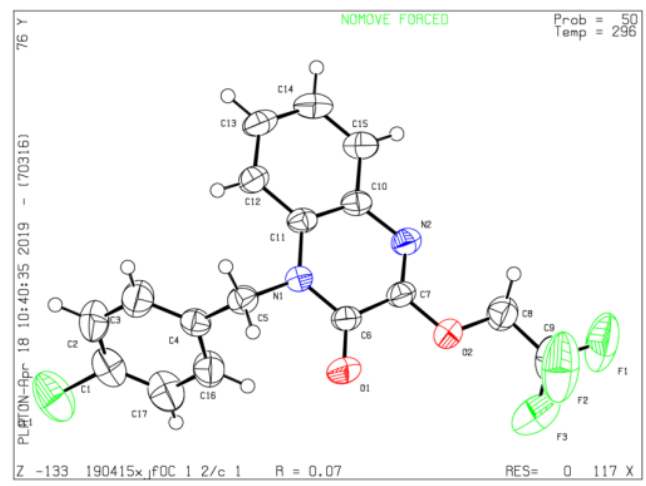

Figure S1 Single-crystal X-ray structure of 31. Ellipsoids are represented at $30 \%$ probability.

Table S2. Crystallographic data and structure refinement for $\mathbf{3 1}$

\begin{tabular}{|c|c|}
\hline $\mathrm{CCDC}$ & 1911863 \\
\hline Empirical formula & $\mathrm{C}_{17} \mathrm{H}_{12} \mathrm{ClF}_{3} \mathrm{~N}_{2} \mathrm{O}_{2}$ \\
\hline Formula weight & 368.74 \\
\hline Temperature, $\mathrm{K}$ & 296.15 \\
\hline Wavelength, $\AA$ & 0.71073 \\
\hline Crystal system & Monoclinic \\
\hline Space group & $\mathrm{C} 12 / \mathrm{c} 1$ \\
\hline$a, b, c, \AA$ & $15.921(7), 9.096(4), 23.502(9)$ \\
\hline$\alpha, \beta, \gamma,^{\mathrm{o}}$ & $90,104.100(14), 90$ \\
\hline Volume, $\AA^{3}$ & $3301(2)$ \\
\hline$Z$ & 8 \\
\hline Calculated density, $\mathrm{Mg} / \mathrm{m}^{\wedge} 3$ & 1.484 \\
\hline Absorption coefficient, $\mathrm{mm}^{-1}$ & 0.276 \\
\hline$F(000)$ & 1504 \\
\hline Theta range for data collection, ${ }^{\circ}$ & 2.599 to 25.026 \\
\hline Limiting indices & $-18<=\mathrm{h}<=18,-10<=\mathrm{k}<=8,-27<=\mathrm{l}<=27$ \\
\hline Reflections collected / unique & $8027 / 2900[\mathrm{R}(\mathrm{int})=0.0928]$ \\
\hline Absorption correction & Semi-empirical from equivalents \\
\hline Refinement method & Full-matrix least-squares on $\mathrm{F}^{2}$ \\
\hline Data / restraints / parameters & $2900 / 0 / 226$ \\
\hline Goodness of fit on $F^{2}$ & 1.043 \\
\hline Final $\mathrm{R}$ indices $[\mathrm{I}>2 \operatorname{sigma}(\mathrm{I})]$ & $\mathrm{R} 1=0.0674, \mathrm{wR} 2=0.1652$ \\
\hline $\mathrm{R}$ indices (all data) & $\mathrm{R} 1=0.1063, \mathrm{wR} 2=0.1894$ \\
\hline
\end{tabular}




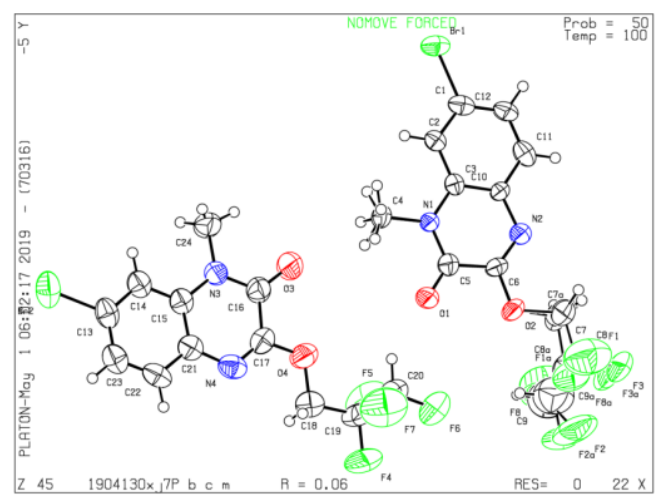

Figure S2 Single-crystal X-ray structure of 7d. Ellipsoids are represented at $30 \%$ probability.

Table S3. Crystallographic data and structure refinement for 7d

\begin{tabular}{|c|c|}
\hline $\mathrm{CCDC}$ & 1913960 \\
\hline Empirical formula & $\mathrm{C}_{12} \mathrm{H}_{9} \mathrm{BrF}_{4} \mathrm{~N}_{2} \mathrm{O}_{2}$ \\
\hline Formula weight & 369.12 \\
\hline Temperature, $\mathrm{K}$ & 100.15 \\
\hline Wavelength, $\AA$ & 0.71073 \\
\hline Crystal system & Orthorhombic \\
\hline Space group & $\mathrm{Pbcm}$ \\
\hline$a, b, c, \AA$ & $11.665(2), 17.410(4), 20.688(4)$ \\
\hline$\alpha, \beta, \gamma,{ }^{\circ}$ & $90,90,90$ \\
\hline Volume, $\AA^{3}$ & $4201.5(15)$ \\
\hline$Z$ & 12 \\
\hline Calculated density, $\mathrm{Mg} / \mathrm{m}^{\wedge} 3$ & 1.751 \\
\hline Absorption coefficient, $\mathrm{mm}^{-1}$ & 2.986 \\
\hline$F(000)$ & 2184 \\
\hline Theta range for data collection, ${ }^{\circ}$ & 2.321 to 25.681 \\
\hline Limiting indices & $-11<=\mathrm{h}<=14,-21<=\mathrm{k}<=18,-24<=1<=24$ \\
\hline Reflections collected / unique & $17875 / 4086[\mathrm{R}(\mathrm{int})=0.0816]$ \\
\hline Absorption correction & Semi-empirical from equivalents \\
\hline Refinement method & Full-matrix least-squares on $\mathrm{F}^{2}$ \\
\hline Data / restraints / parameters & 4086 / 94 / 339 \\
\hline Goodness of fit on $F^{2}$ & 1.015 \\
\hline Final R indices [I $>2 \operatorname{sigma}(\mathrm{I})]$ & $\mathrm{R} 1=0.0572, \mathrm{wR} 2=0.1359$ \\
\hline $\mathrm{R}$ indices (all data) & $\mathrm{R} 1=0.1318, \mathrm{wR} 2=0.1661$ \\
\hline
\end{tabular}




\section{References}

(1) Liu, S.; Huang, Y.; Qing, F.-L.; Xu, X.-H. Org. Lett. 2018, 20, 5497.

(2) Č́žková, M.; Kolivoška, V.; Císařová, I.; Šaman, D.; Pospíšil, L.; Teplý, F. Org. Biomol. Chem. 2011, 9, 450.

(3) Abbas, H.-A. S.; Al-Marhabi, A. R.; Eissa, S. I.; Ammar, Y. A. Bioorg. Med. Chem. 2015, 23, 6560.

(4) Zhang, H.-Y.; Sun, M.; Ma, Y.-N.; Tian, Q.-P.; Yang, S.-D. Org. Biomol. Chem. 2012, 10, 9627.

(5) Takahashi, F.; Nogi, K.; Yorimitsu, H. Org. Lett. 2018, 20, 6601.

(6) Bao, P.; Wang, L.; Liu, Q.; Yang, D.; Wang, H.; Zhao, X.; Yue, H.; Wei, W. Tetrahedron Lett. 2019, 60, 214.

(7) Liao, L.-Y.; Kong, X.-R.; Duan, X.-F. J. Org. Chem. 2014, 79, 777.

(8) Fisher, E. L.; am Ende, C. W.; Humphrey, J. M. J. Org. Chem. 2019, 84, 4904. 


\section{Copies of ${ }^{1} \mathrm{H},{ }^{13} \mathrm{C}$ and ${ }^{19} \mathrm{~F}$ NMR Spectra}

\section{3a ${ }^{1}$ H NMR}

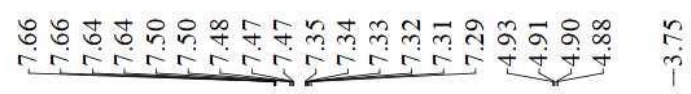

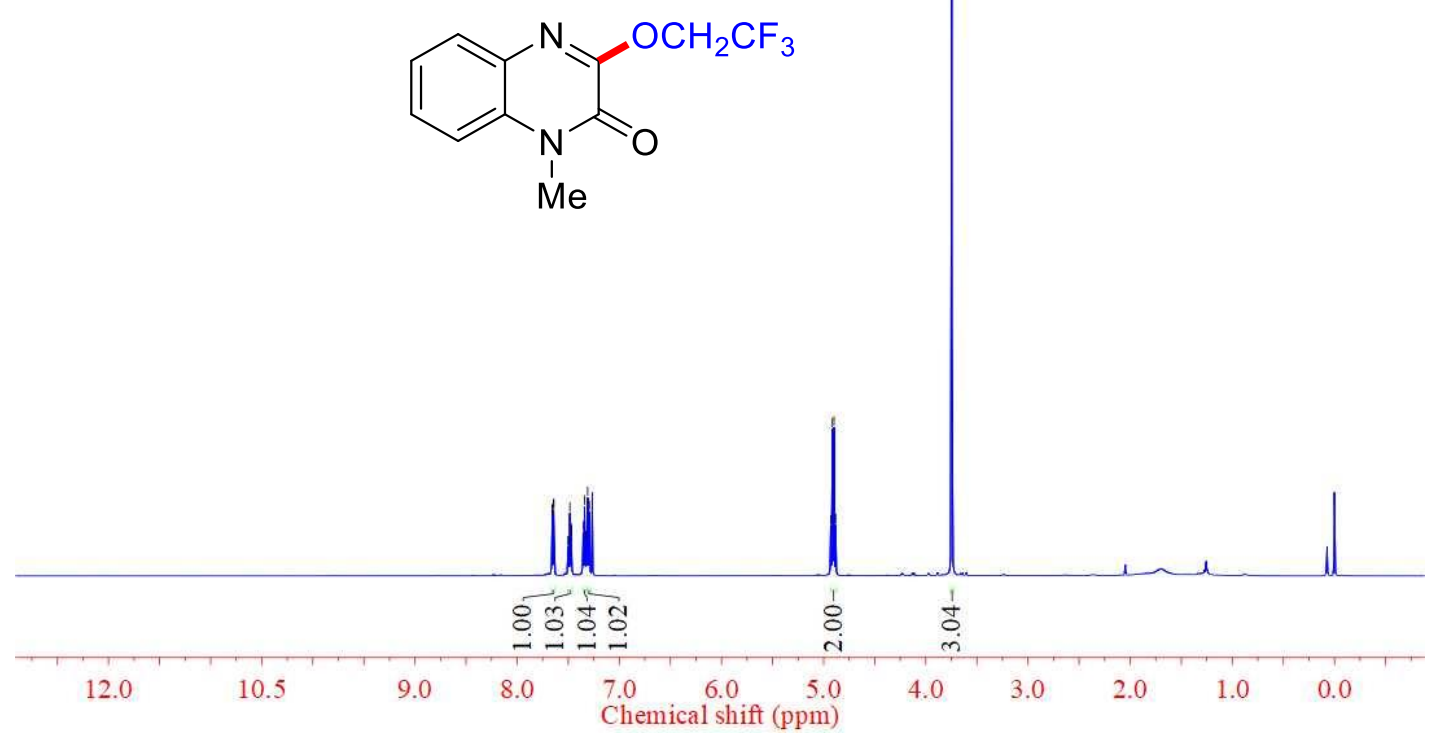

$3 a^{13}$ C NMR

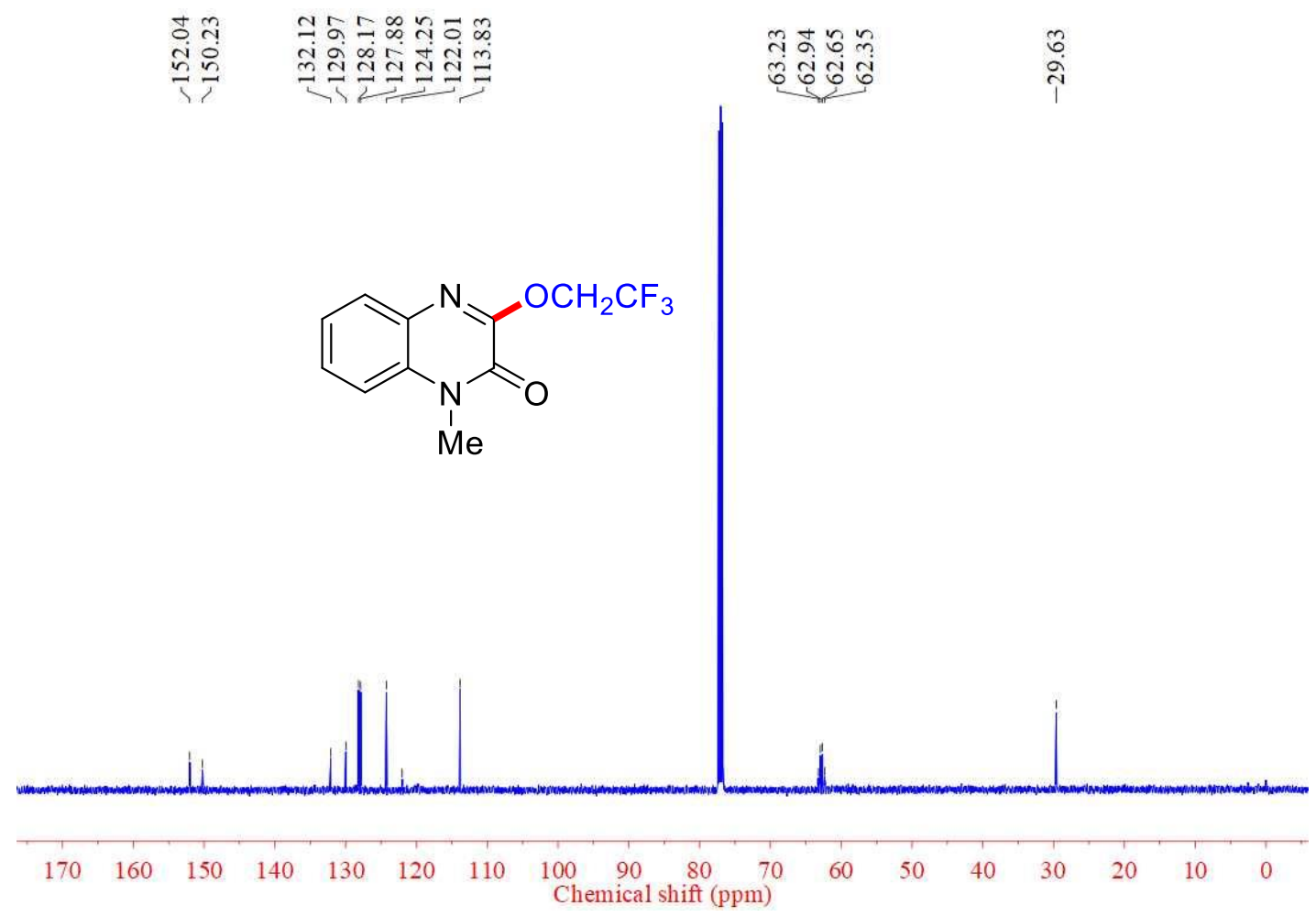




\section{$3 a^{19}$ F NMR}
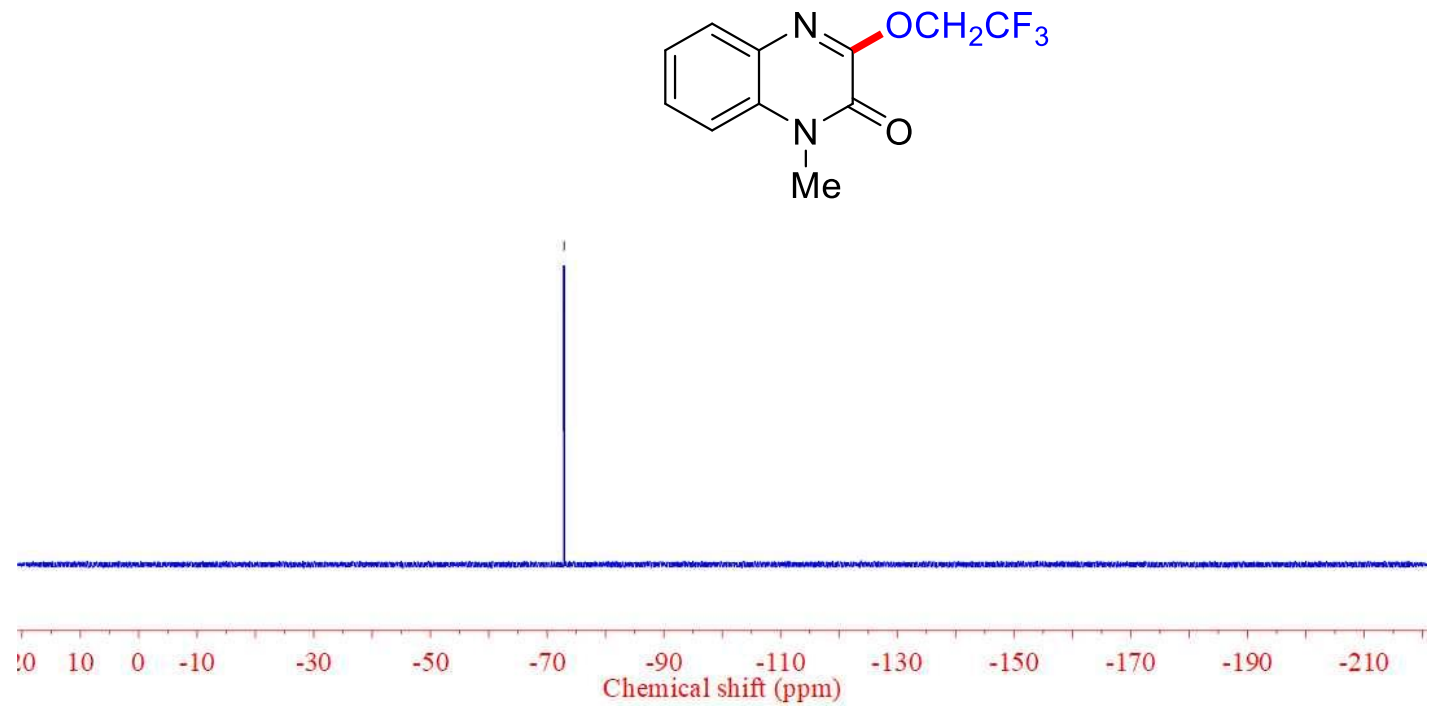

\section{3b ${ }^{1}$ H NMR}

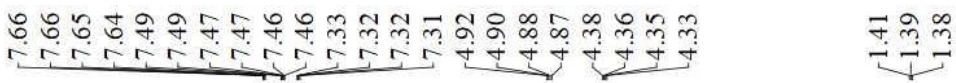<smiles>CCn1c(=O)c(OCC(F)(F)F)nc2ccccc21</smiles>

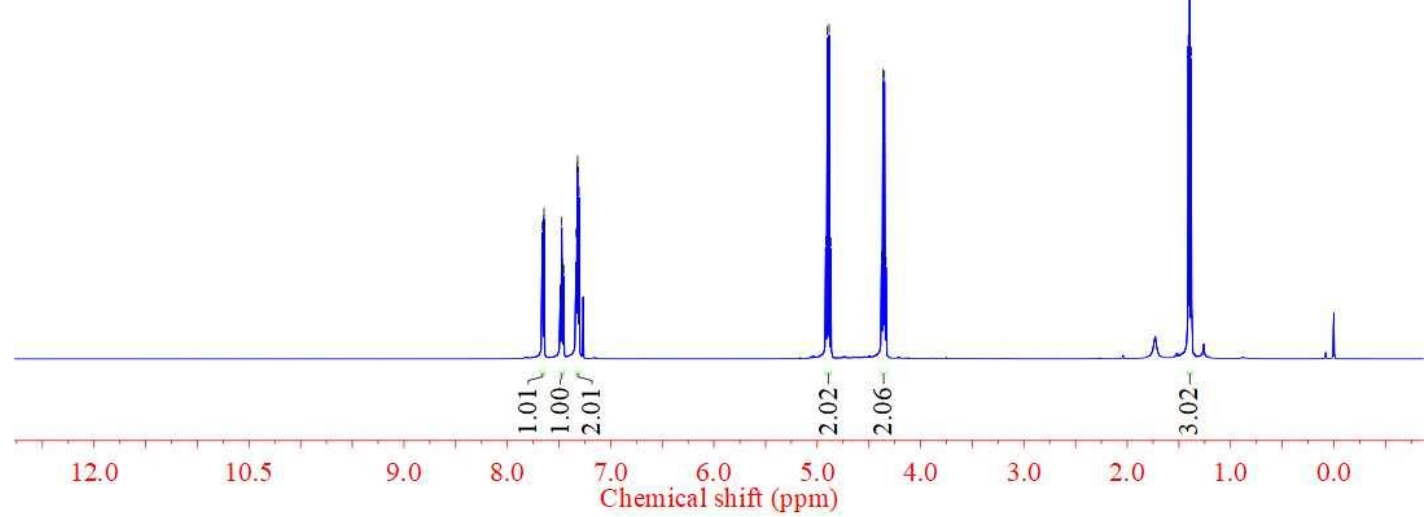


$3 b^{13}$ C NMR

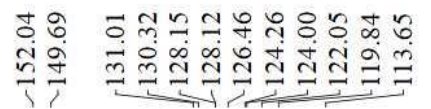

ำ ํํำ

กิ สู่

+
$\infty$
$\infty$
1

$\stackrel{+}{\stackrel{m}{i}}$<smiles>CCn1c(=O)c(OCC(F)(F)F)nc2ccccc21</smiles>

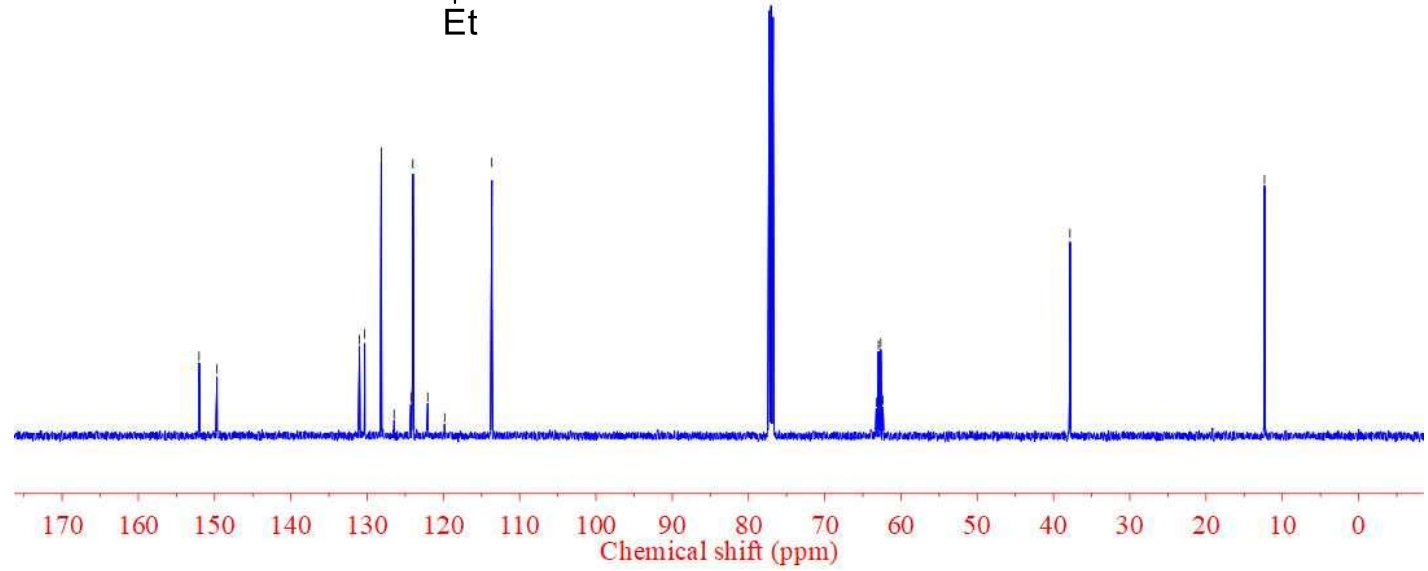

\section{$3 b{ }^{19}$ F NMR}

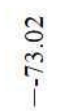<smiles>CCn1c(=O)c(OCC(F)(F)F)nc2ccccc21</smiles>

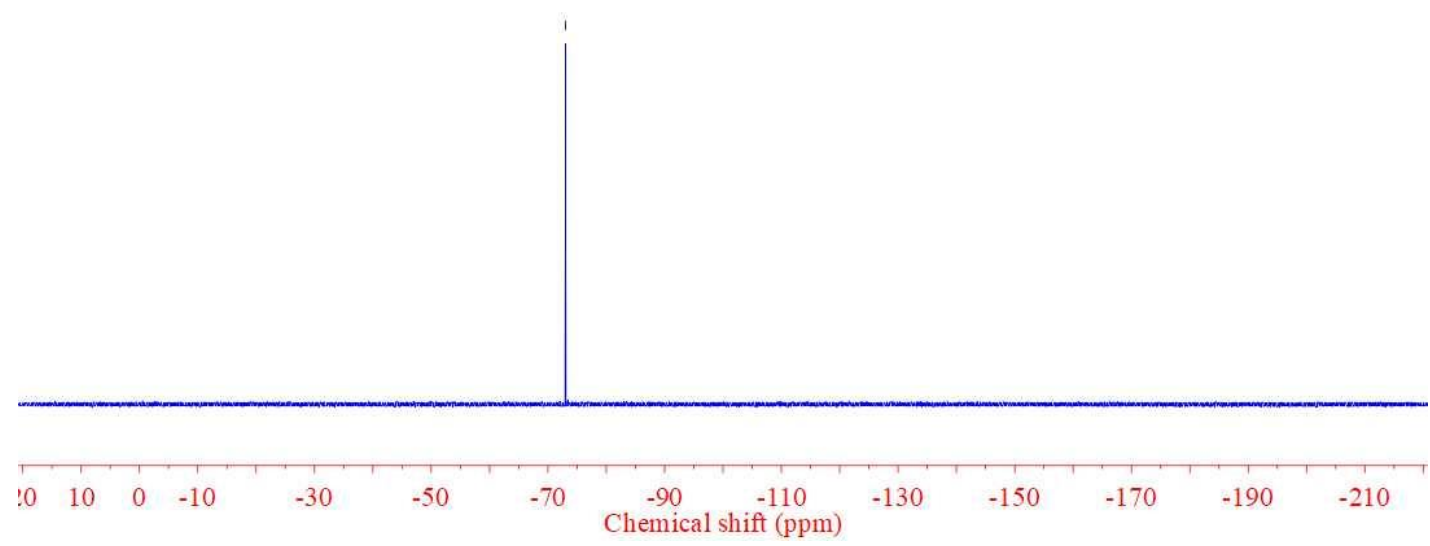




\section{$3 c^{1}$ H NMR}

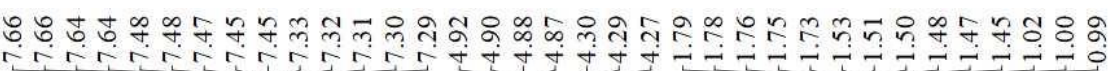<smiles>Cn1c(=O)c(OCC(F)(F)F)nc2ccccc21</smiles>

${ }^{n} \mathrm{Bu}$

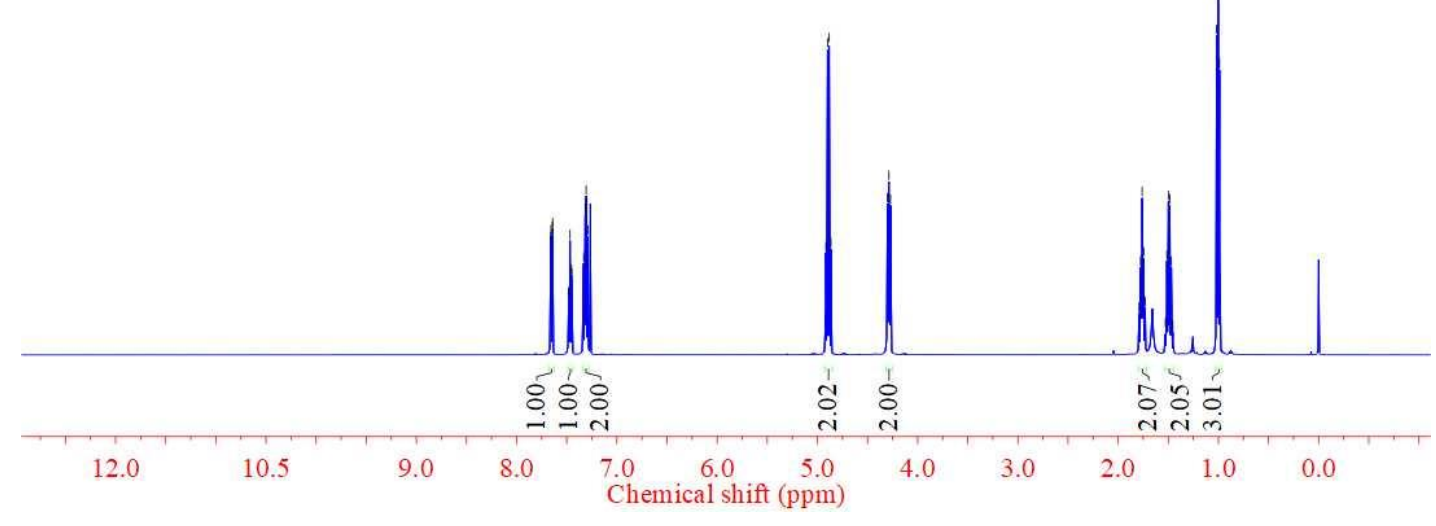

\section{3c ${ }^{13}$ C NMR}

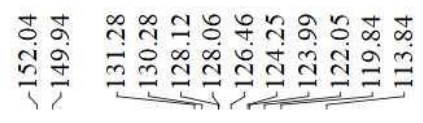

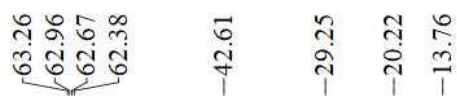<smiles>CC(C)(C)n1c(=O)c(OCC(F)F)nc2ccccc21</smiles>

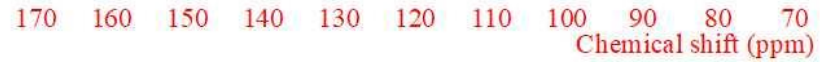




\section{$3 c^{19}$ F NMR}

$$
\text { 함 }
$$<smiles>CC(C)(C)n1c(=O)c(OCC(F)(F)F)nc2ccccc21</smiles>

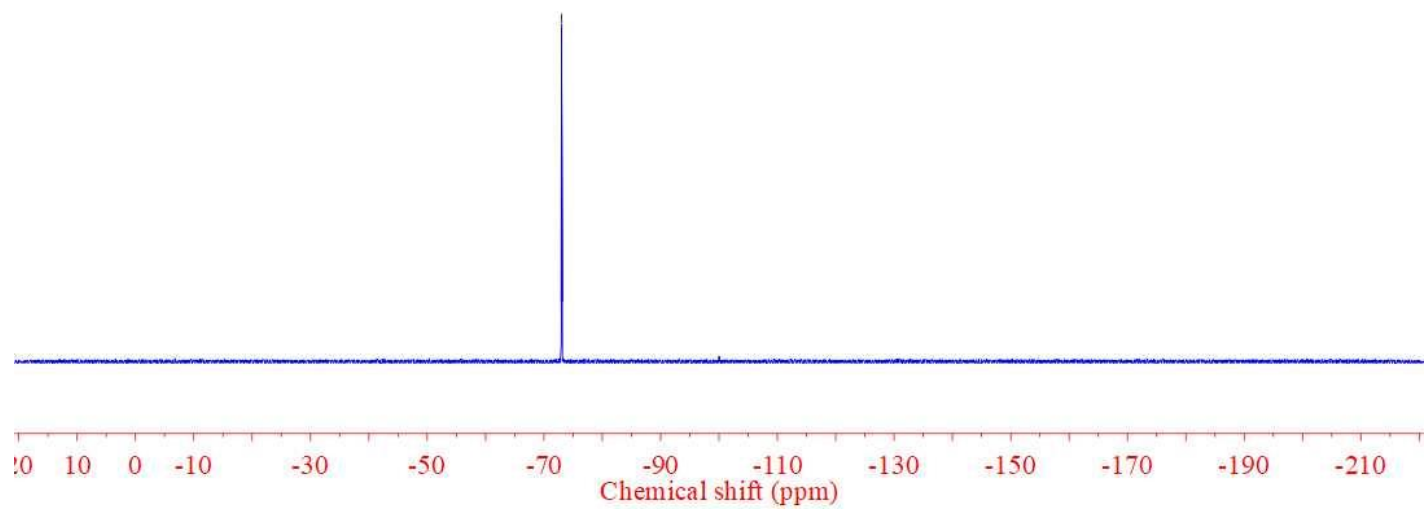

\section{3d ${ }^{1}$ H NMR}

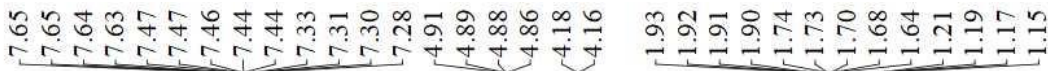<smiles>Cn1c(=O)c(OCC(F)(F)F)nc2ccccc21</smiles>

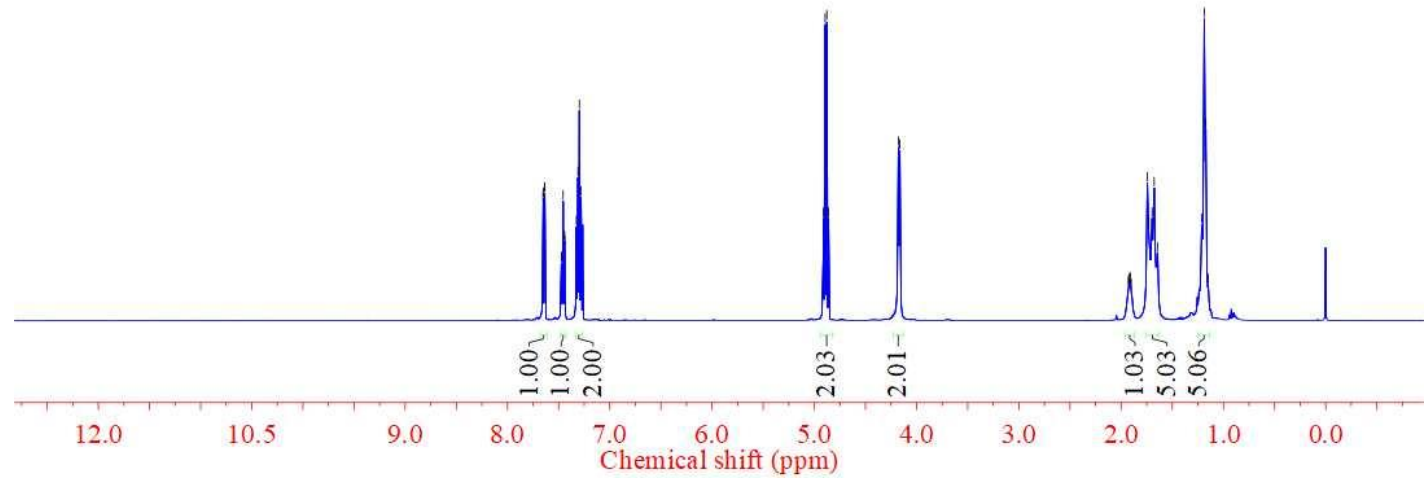




\section{$3 d{ }^{13}$ C NMR}

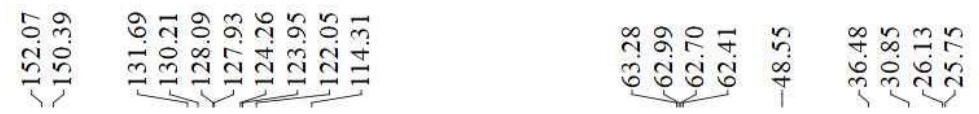<smiles>Cn1c(=O)c(OCC(F)F)nc2ccccc21</smiles>

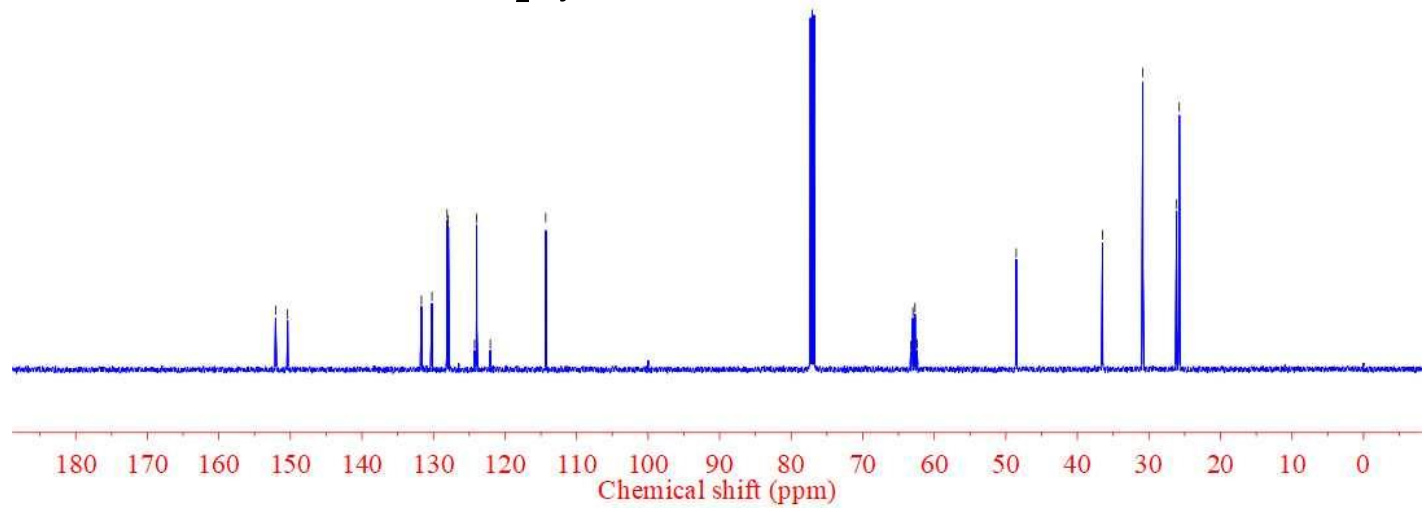

\section{$3{ }^{19}$ F NMR}

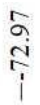

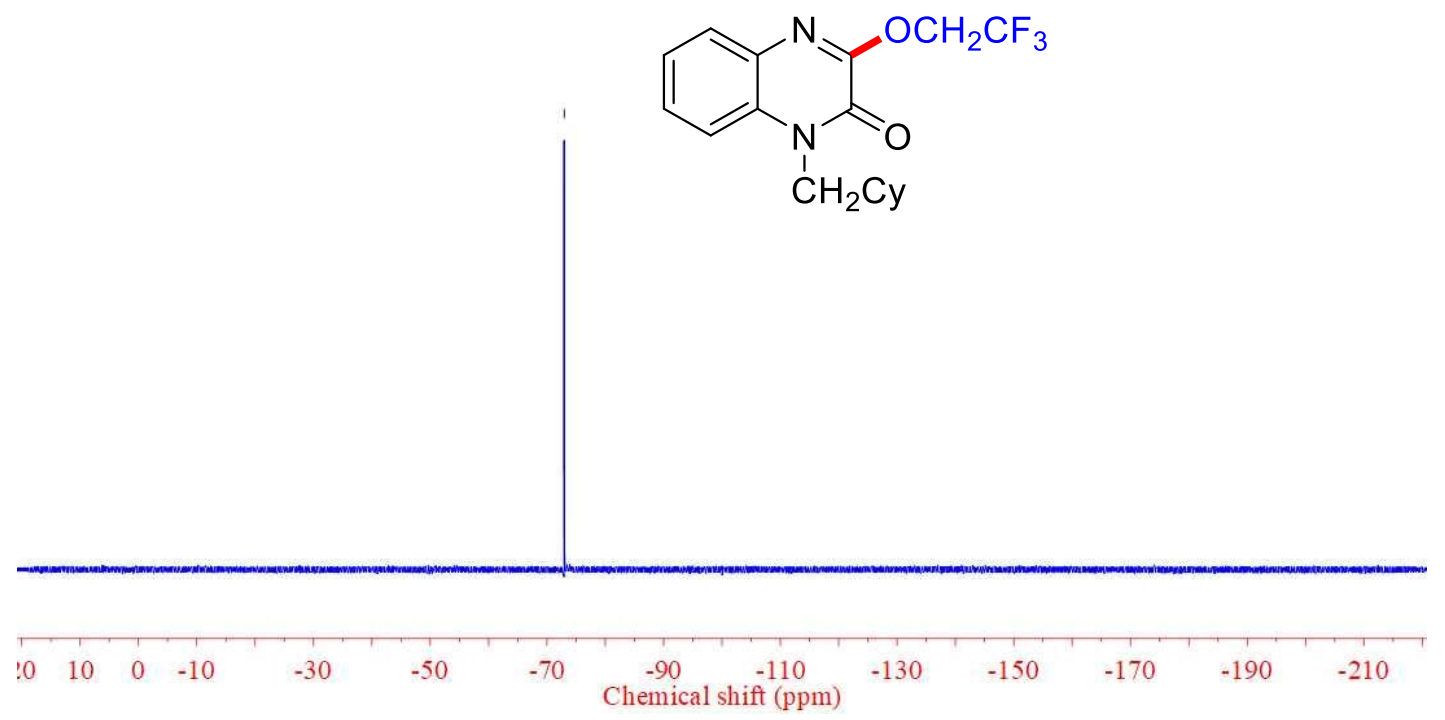




\section{$3 e^{1}$ H NMR}

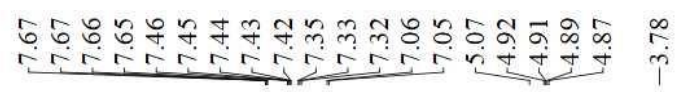

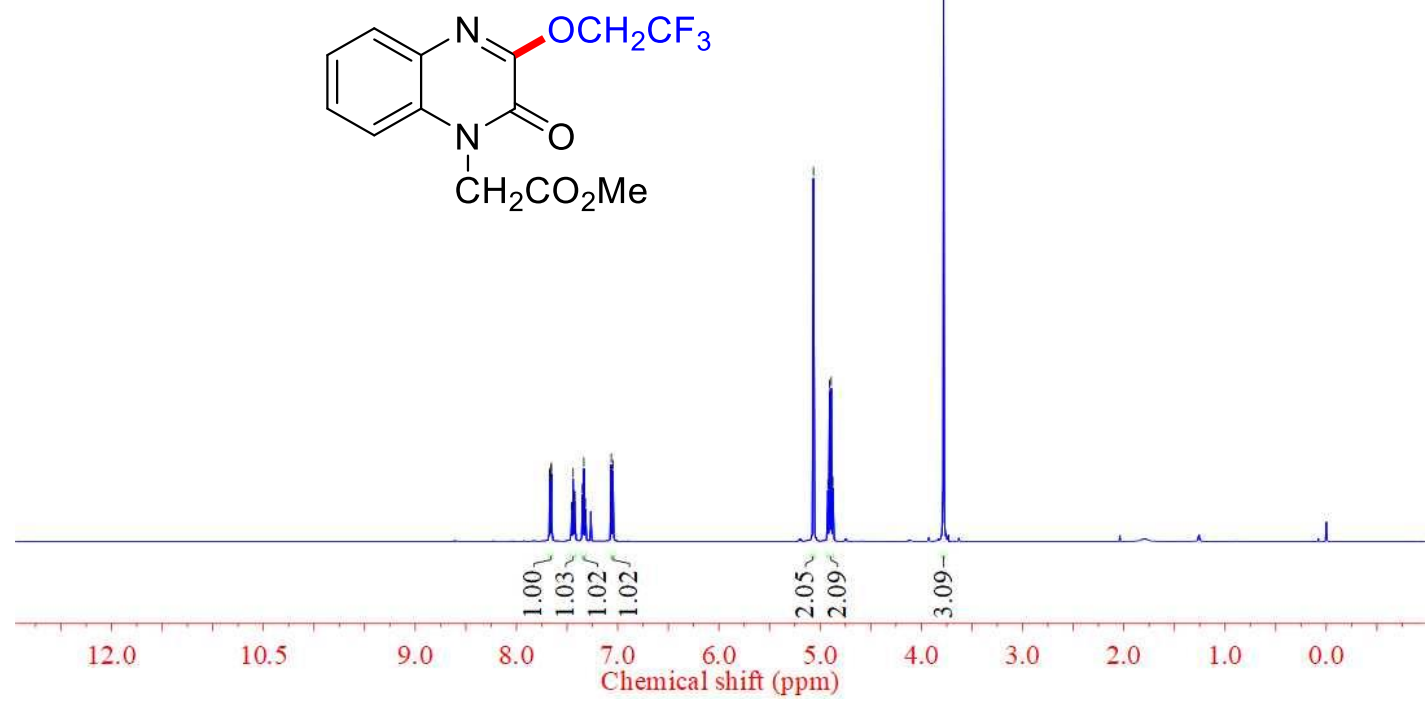

\section{$3 e^{13} \mathrm{C}$ NMR}
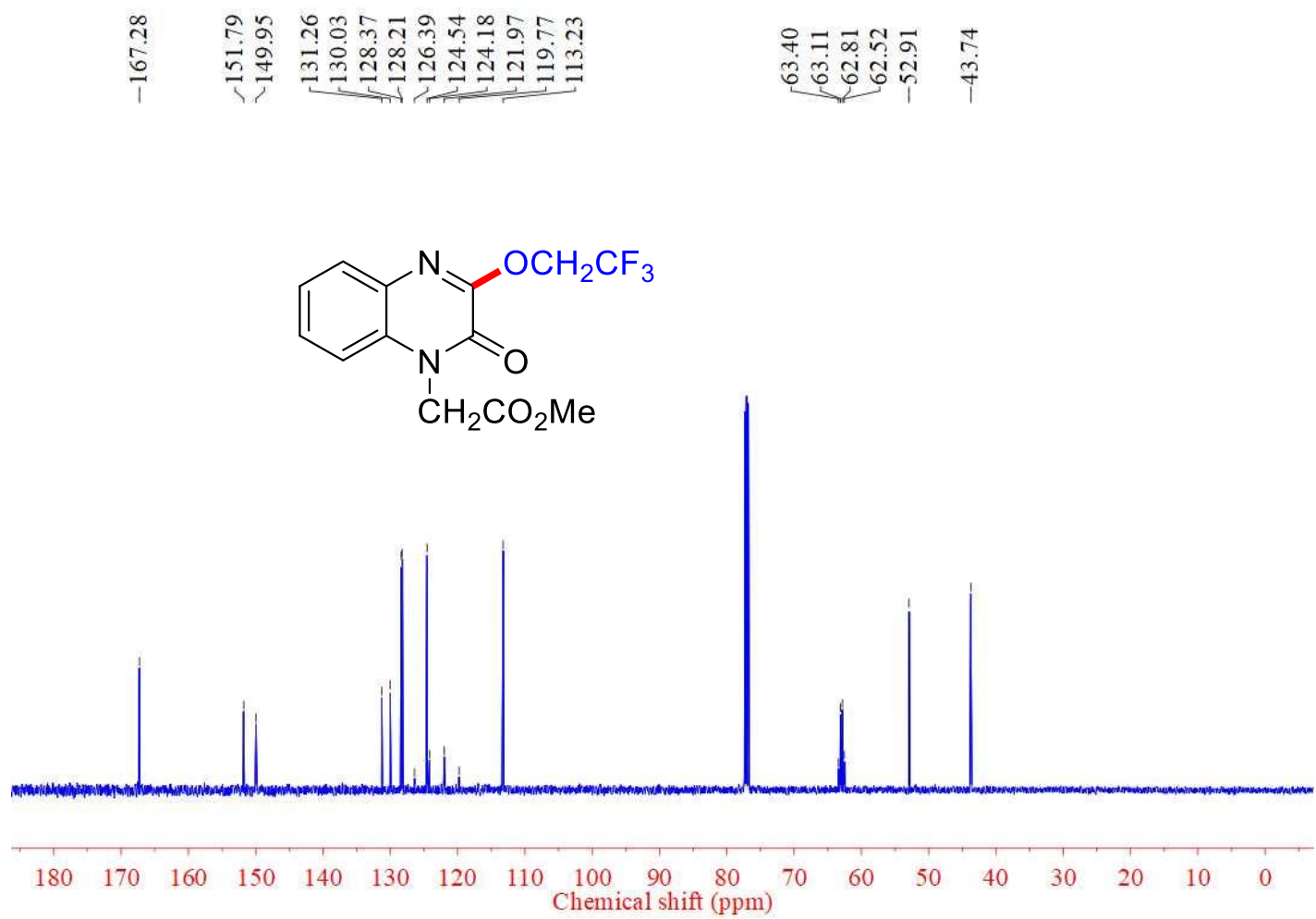


\section{$3 e^{19}$ F NMR}

i্
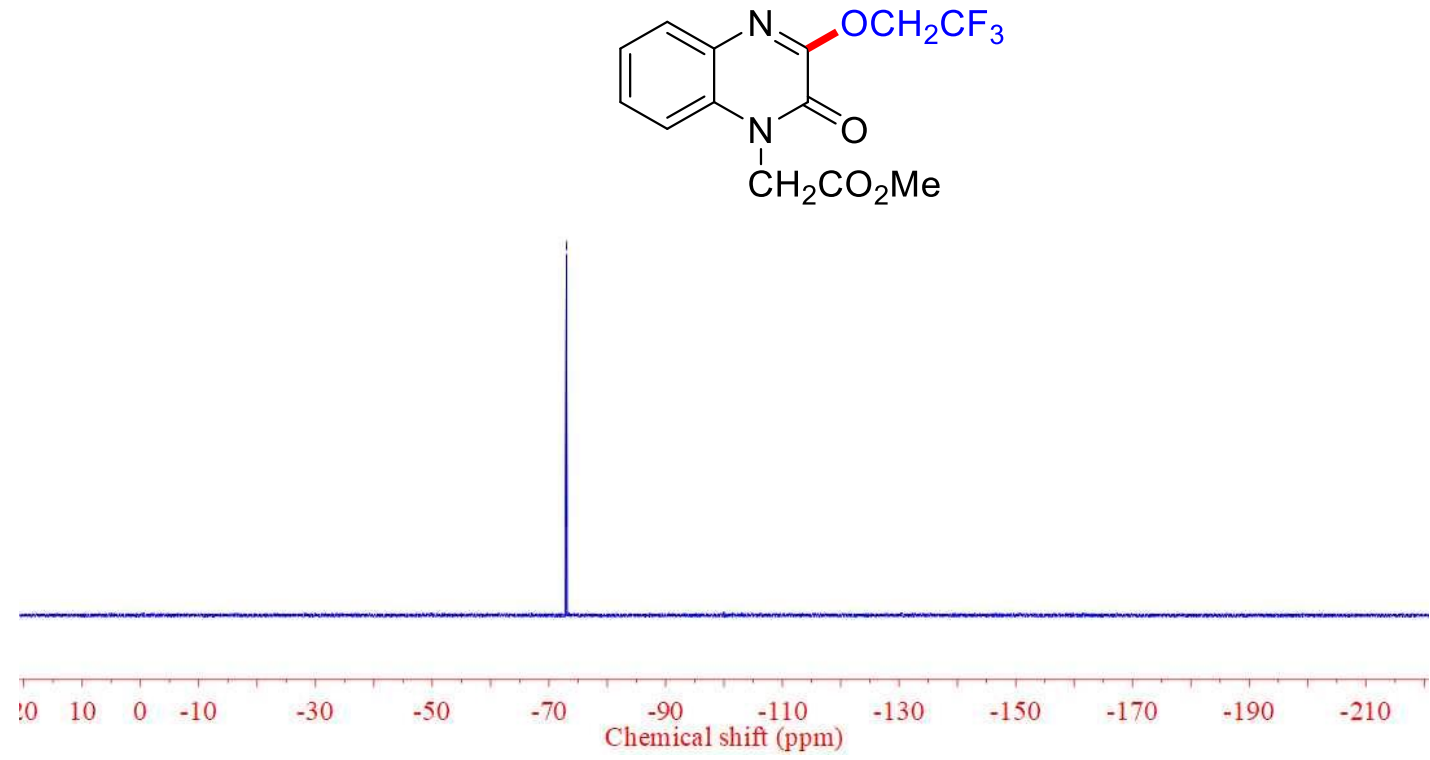

\section{$3 f^{1}$ H NMR}

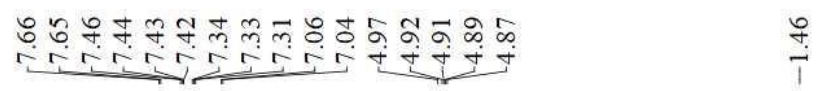<smiles>Cn1c(=O)c(OCC(F)(F)F)nc2ccccc21</smiles>

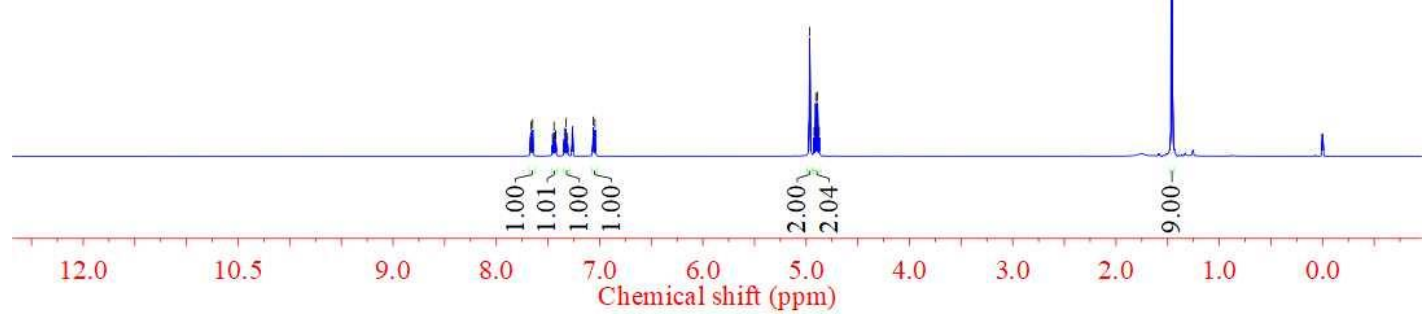




\section{$3 f{ }^{13}$ C NMR}

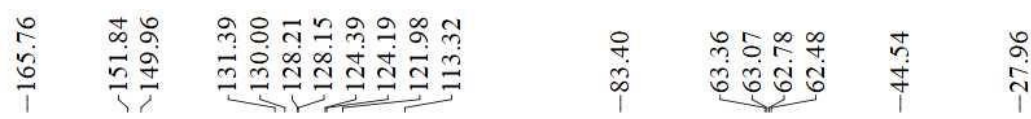

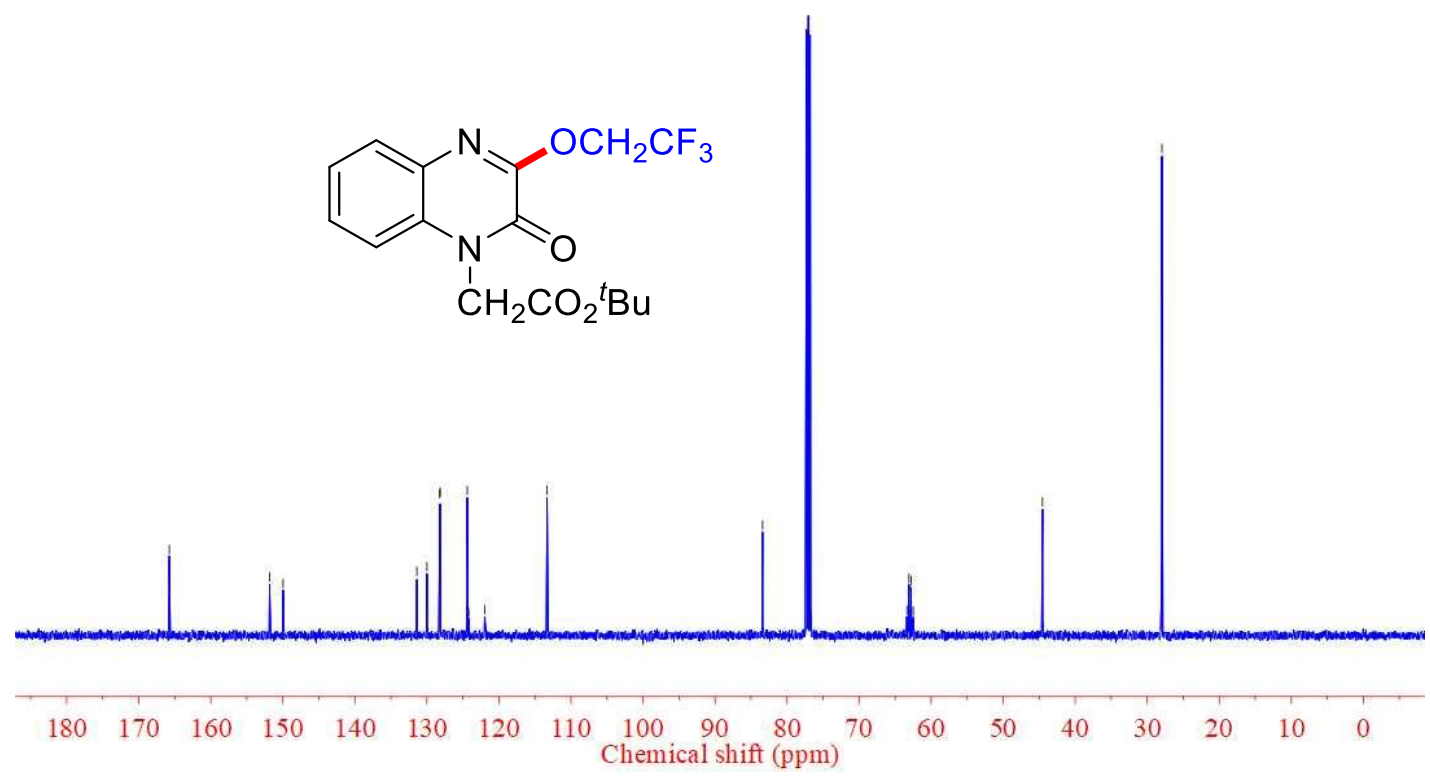

$3 f{ }^{19}$ F NMR

$\frac{8}{i}$<smiles>CCOC(=O)Cn1c(=O)c(OCC(F)(F)F)nc2ccccc21</smiles>

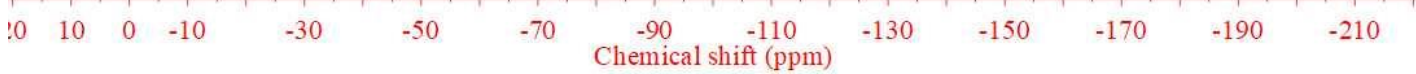




\section{$3 g{ }^{1} H$ NMR}

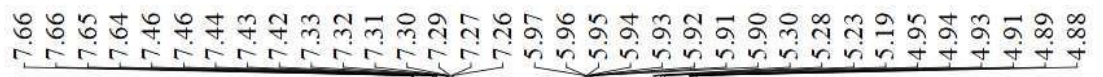<smiles>C=CCn1c(=O)c(OCC(F)(F)F)nc2ccccc21</smiles>

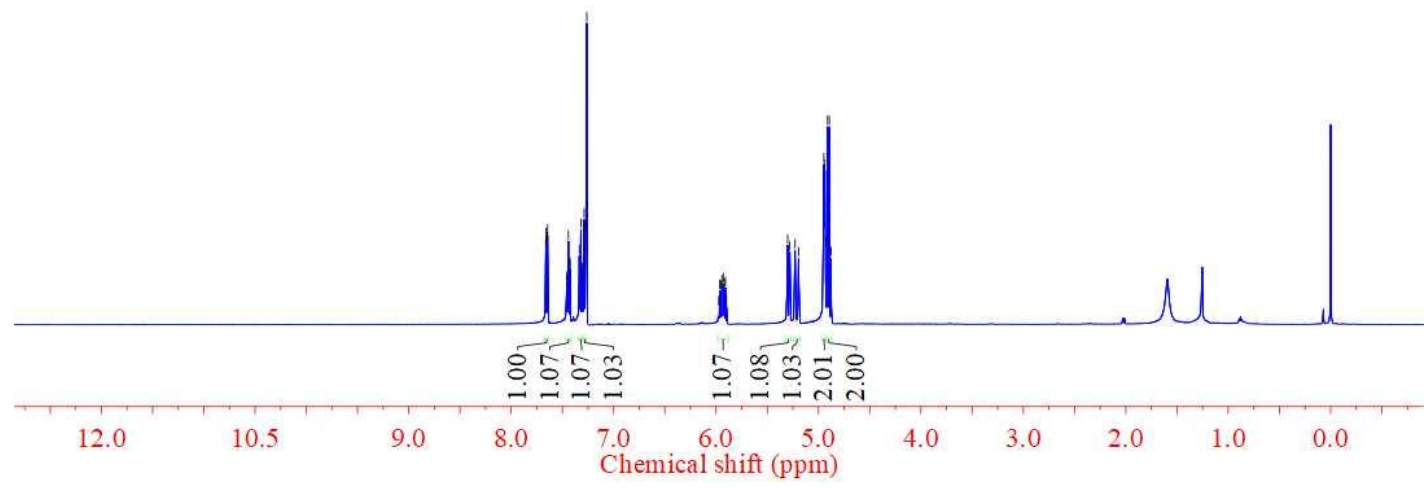

$3 g{ }^{13}$ C NMR

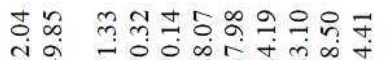

约

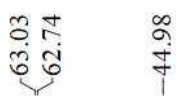<smiles>C=CCn1c(=O)c(OCC(F)(F)F)nc2ccccc21</smiles>

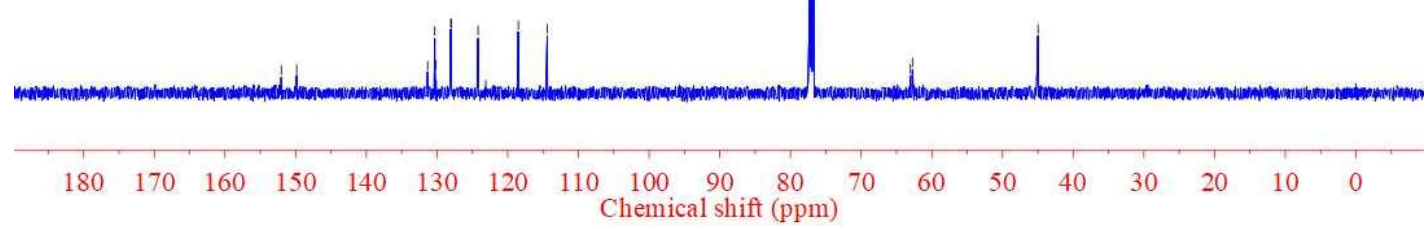




\section{$3 g^{19}$ F NMR}
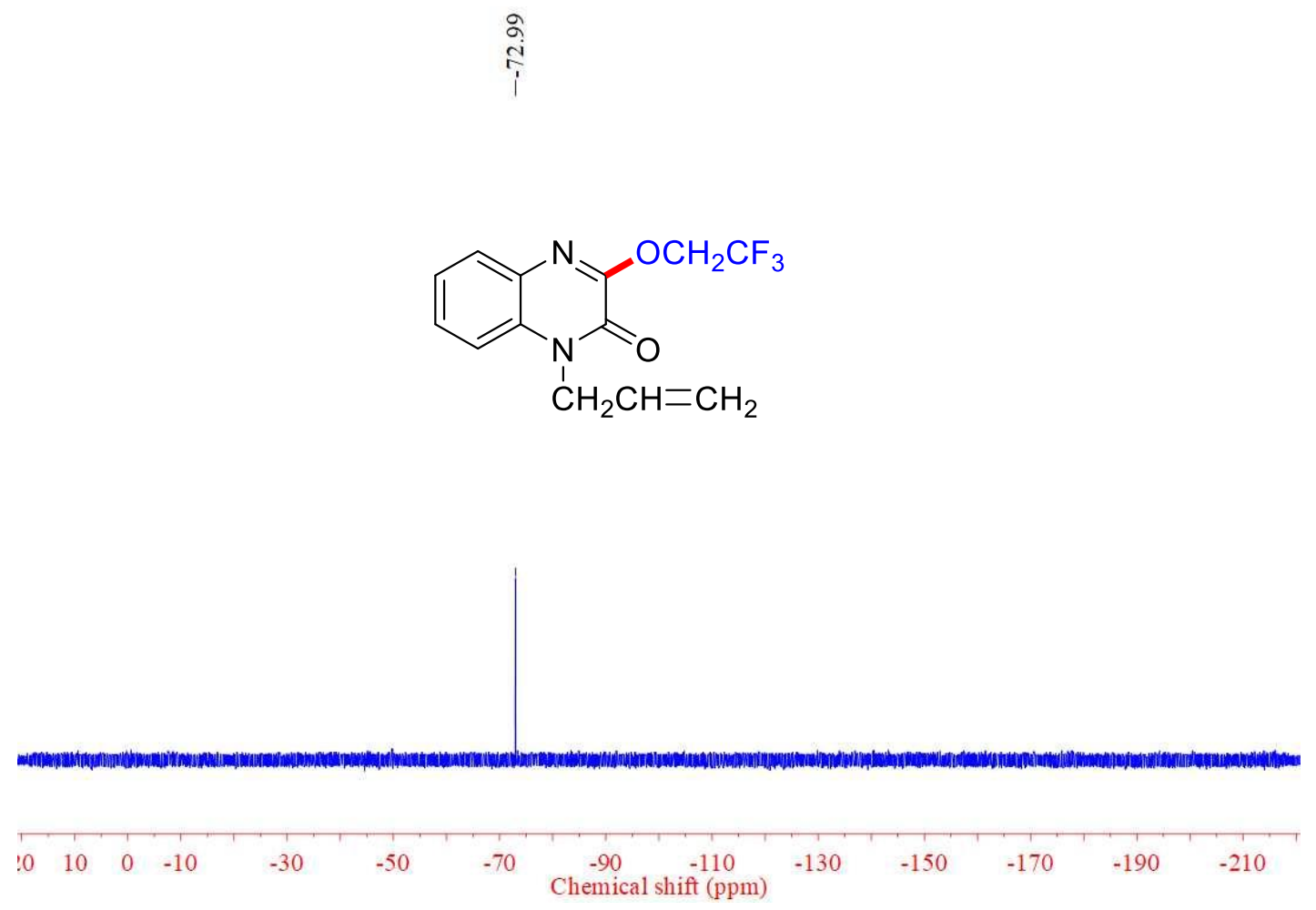

$3 h{ }^{1}$ H NMR

1
11

$\overbrace{\mathrm{H}}^{\mathrm{OCH}_{2} \mathrm{CF}_{3}}$

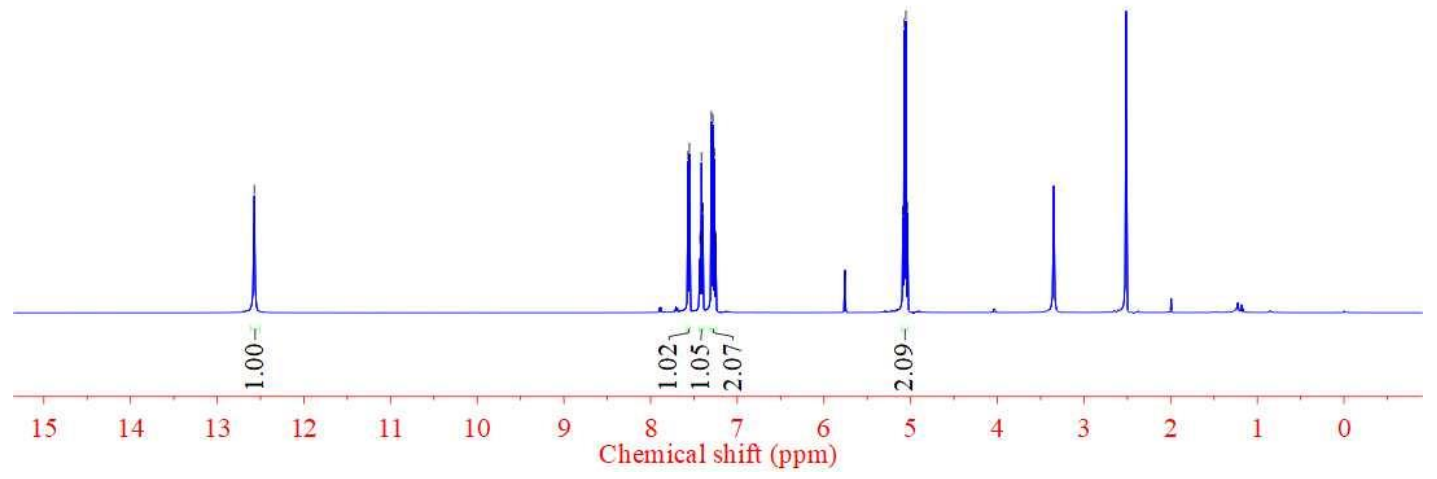




\section{$3 h{ }^{13}$ C NMR}

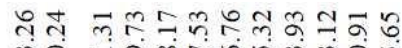

ถึ่

$7 \underset{1}{0}+\infty$

ถู่ ปู่<smiles>O=c1[nH]c2ccccc2nc1OCC(F)(F)F</smiles>
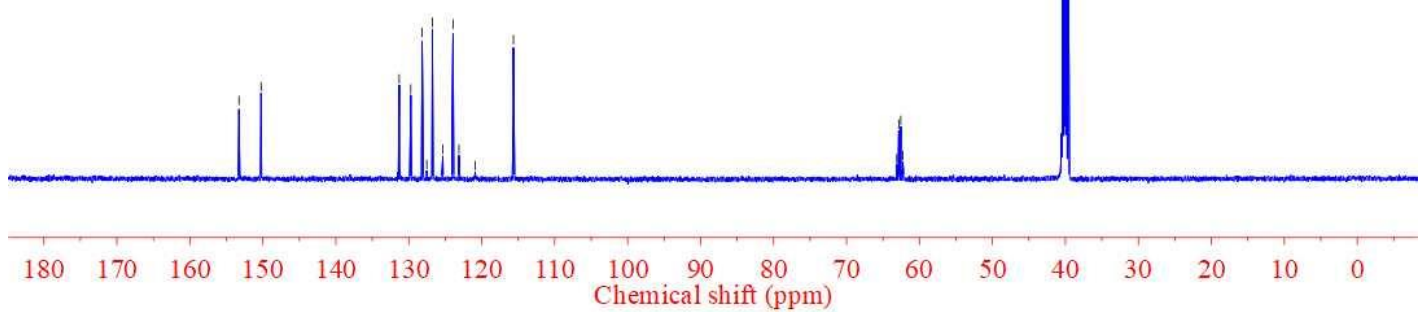

\section{$3 h{ }^{19}$ F NMR}

$\stackrel{+}{\stackrel{5}{i}}$<smiles>O=c1[nH]c2ccccc2nc1OCC(F)(F)F</smiles>

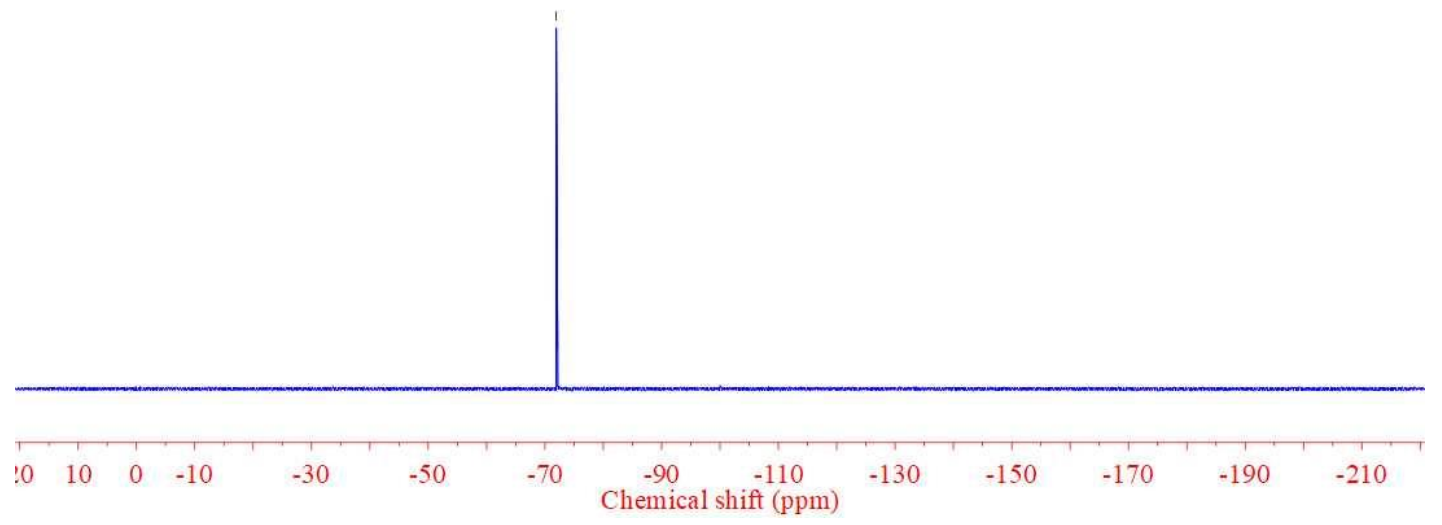




\section{$3 i^{1}$ H NMR}

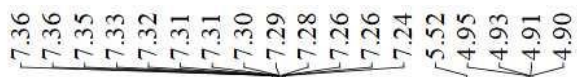
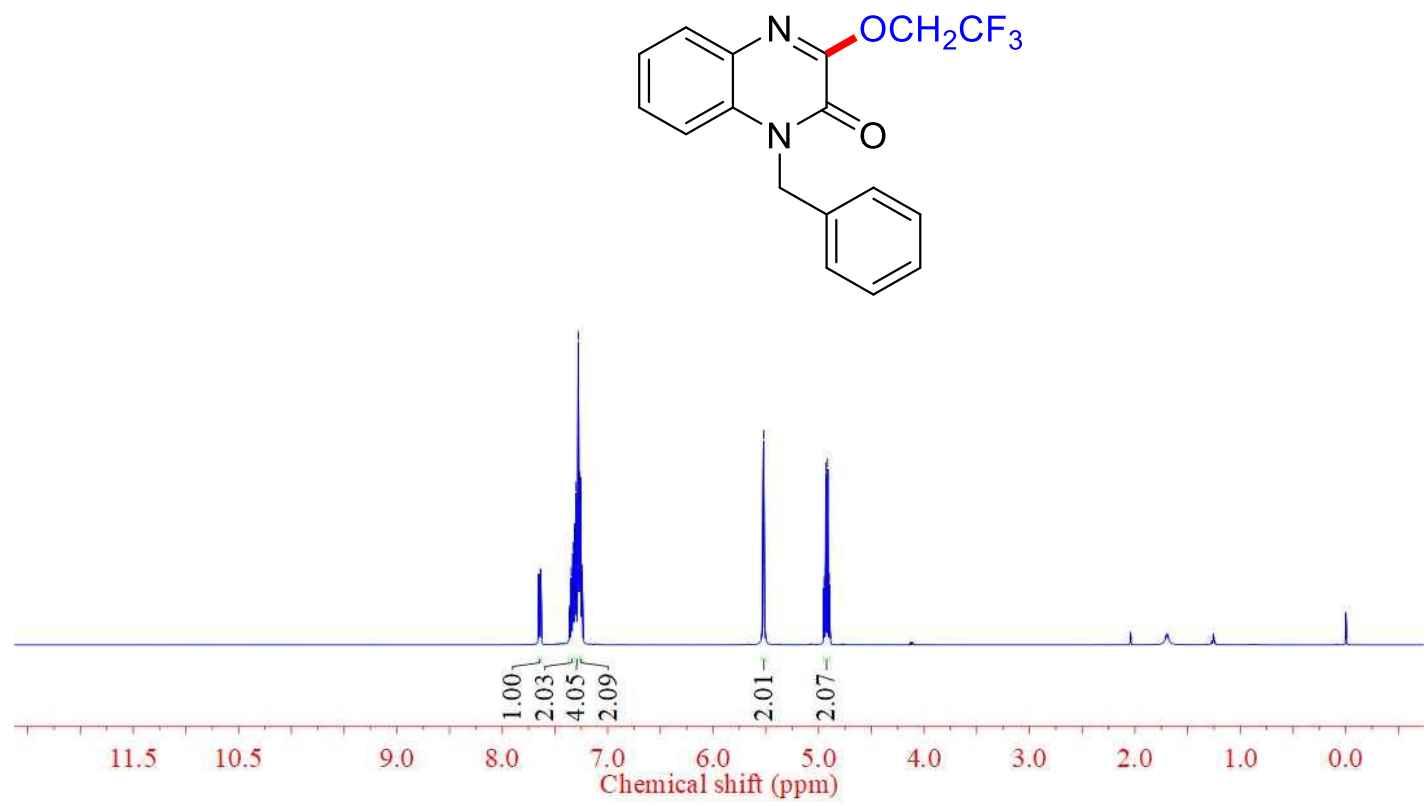

\section{$3 i{ }^{13} \mathrm{C}$ NMR}

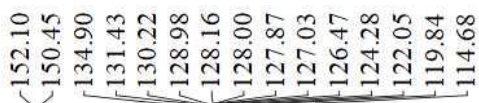

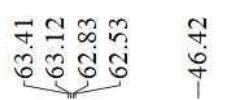

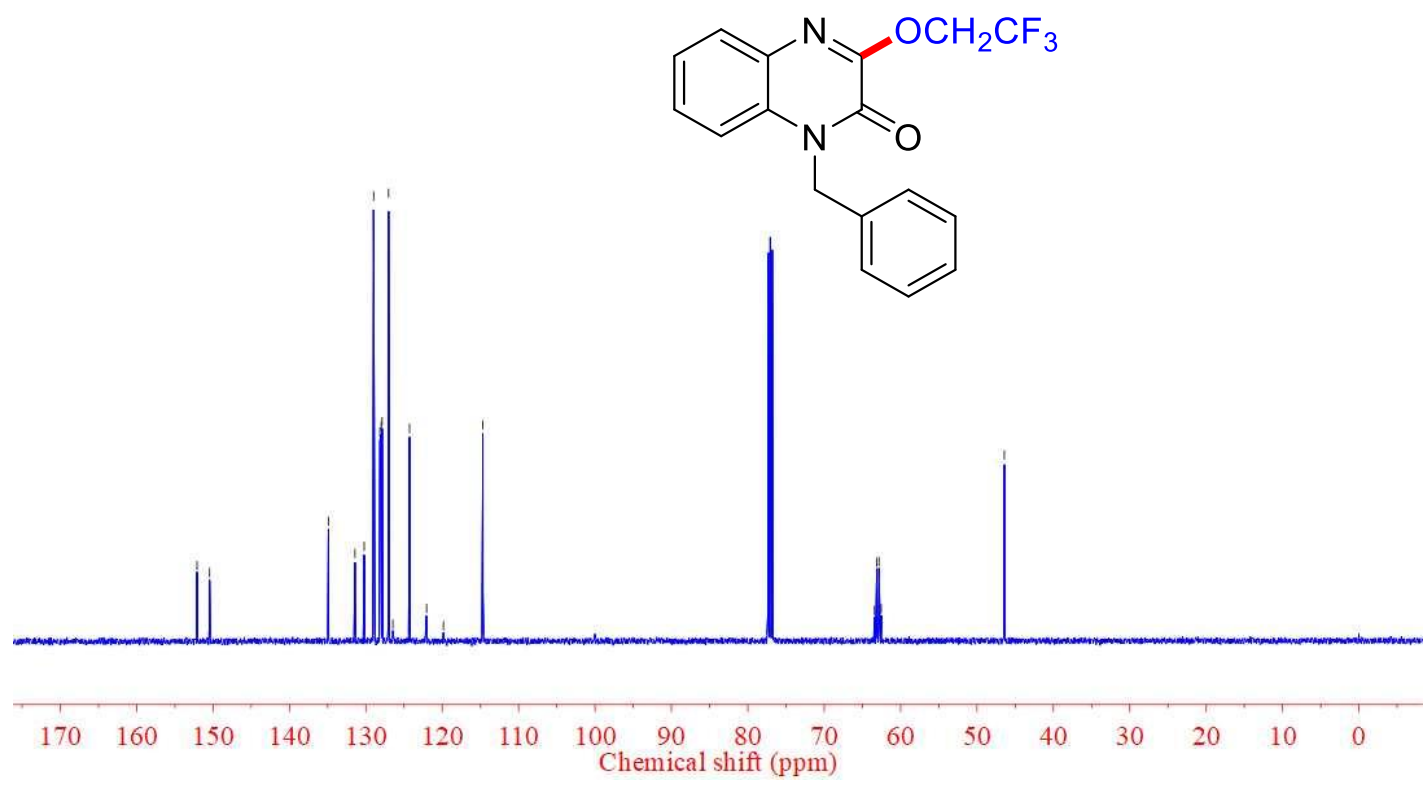




\section{$3 i^{19}$ F NMR}

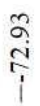

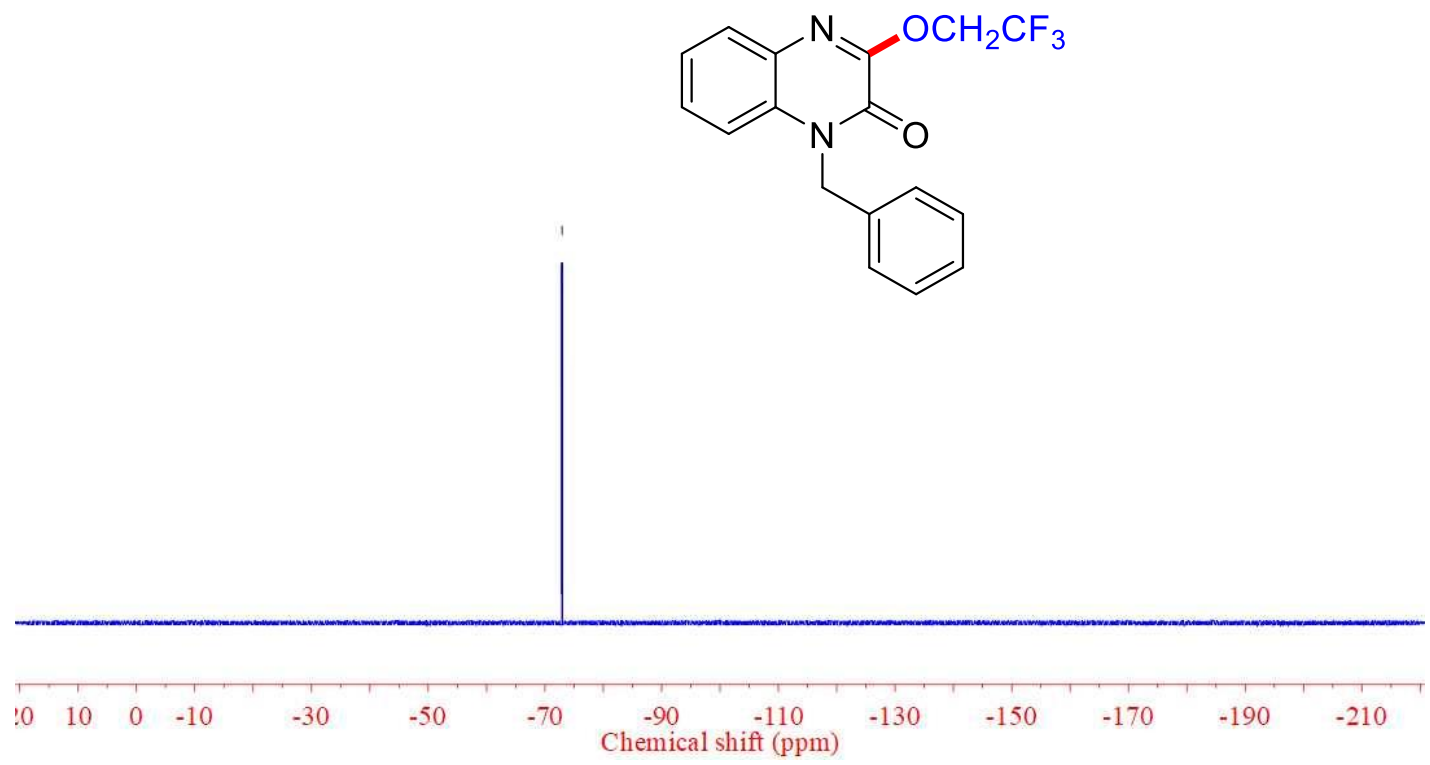

\section{3j ${ }^{1}$ H NMR}

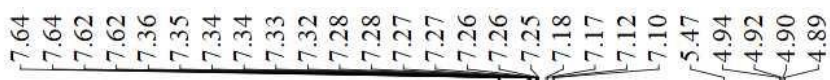
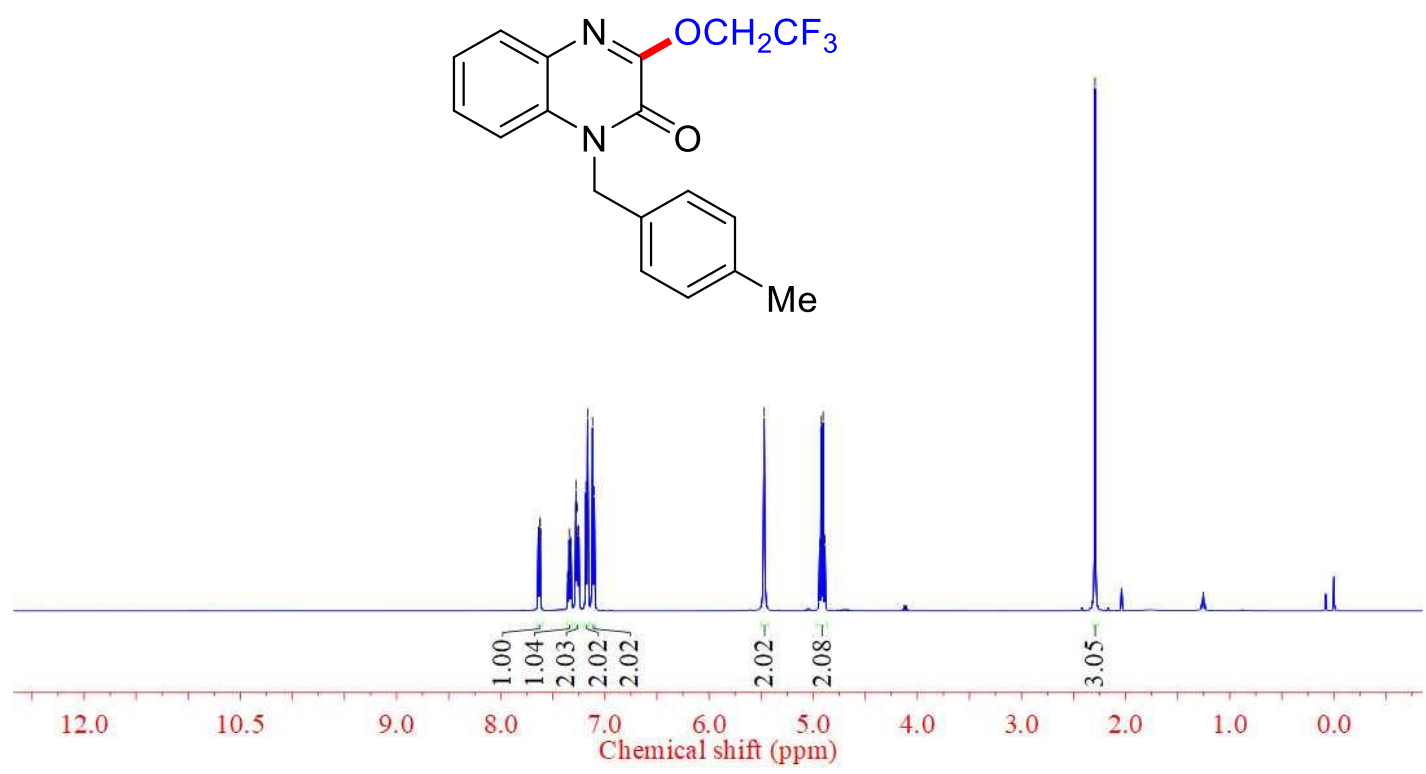


\section{$3 j^{13}$ C NMR}

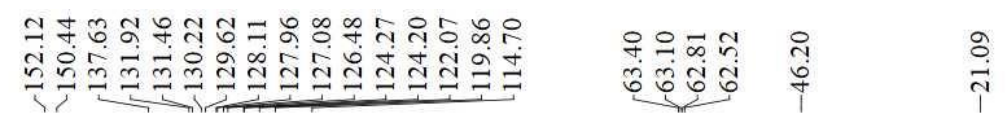

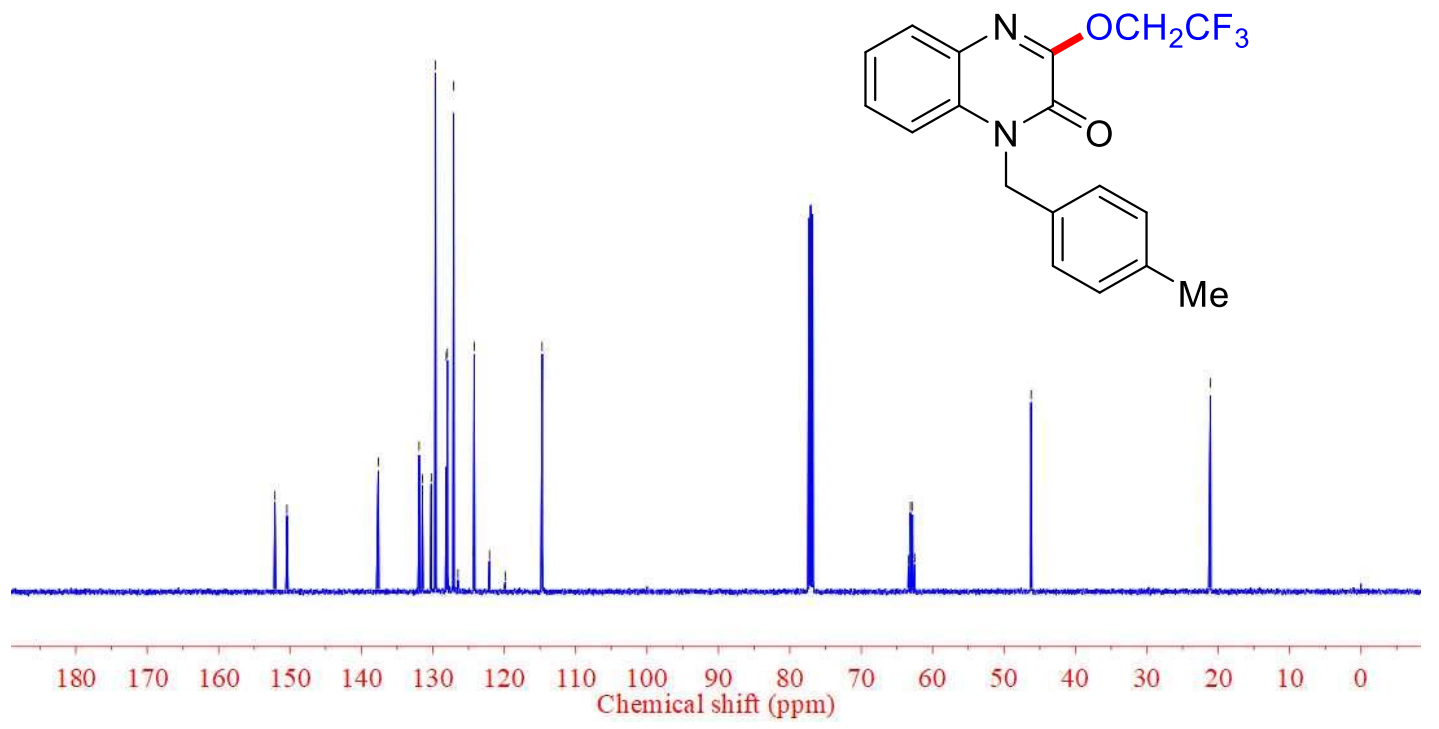

$3 \mathbf{j}^{19}$ F NMR

$\stackrel{m}{i}$

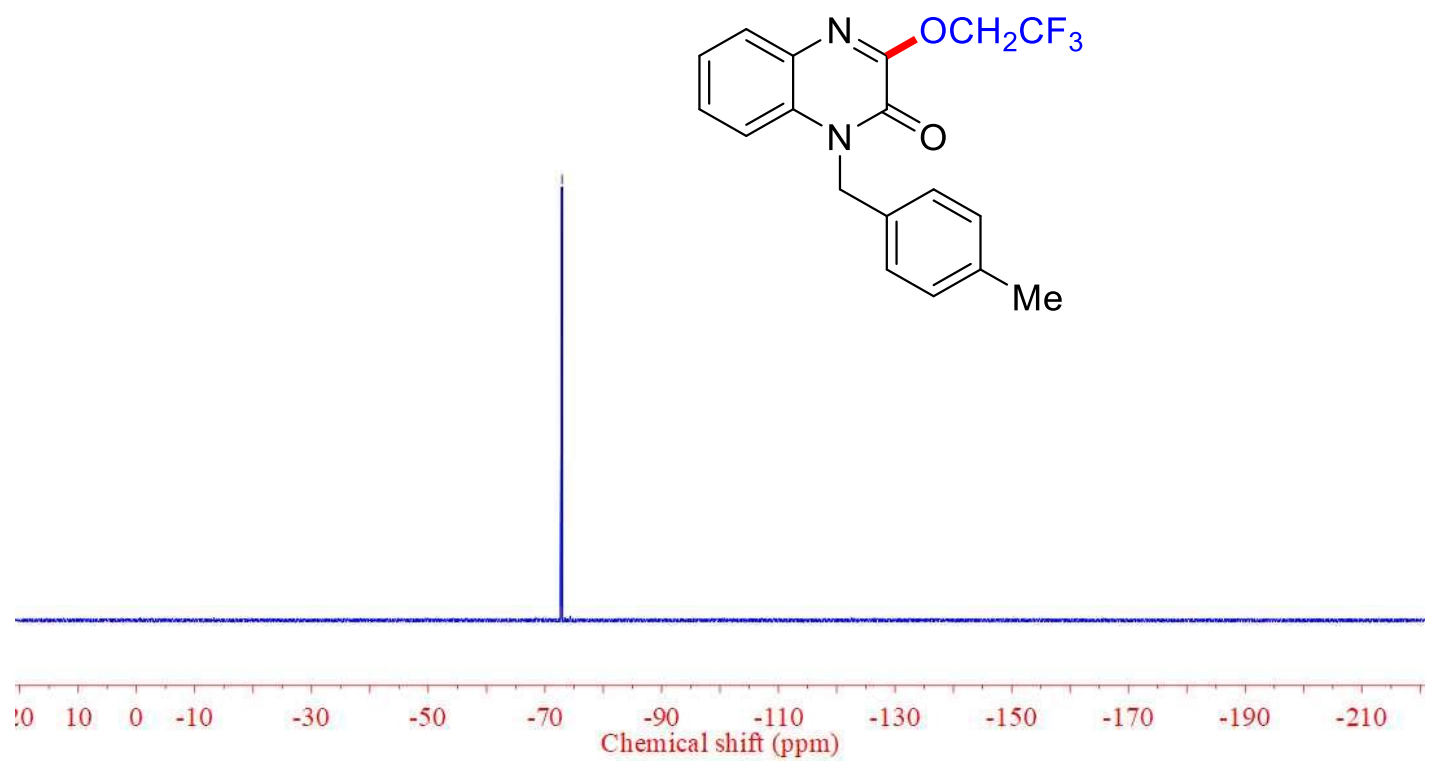




\section{$3 \mathrm{k}^{1} \mathrm{H}$ NMR}

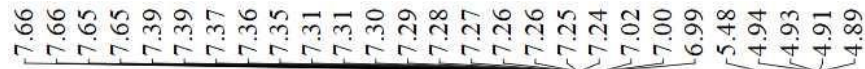
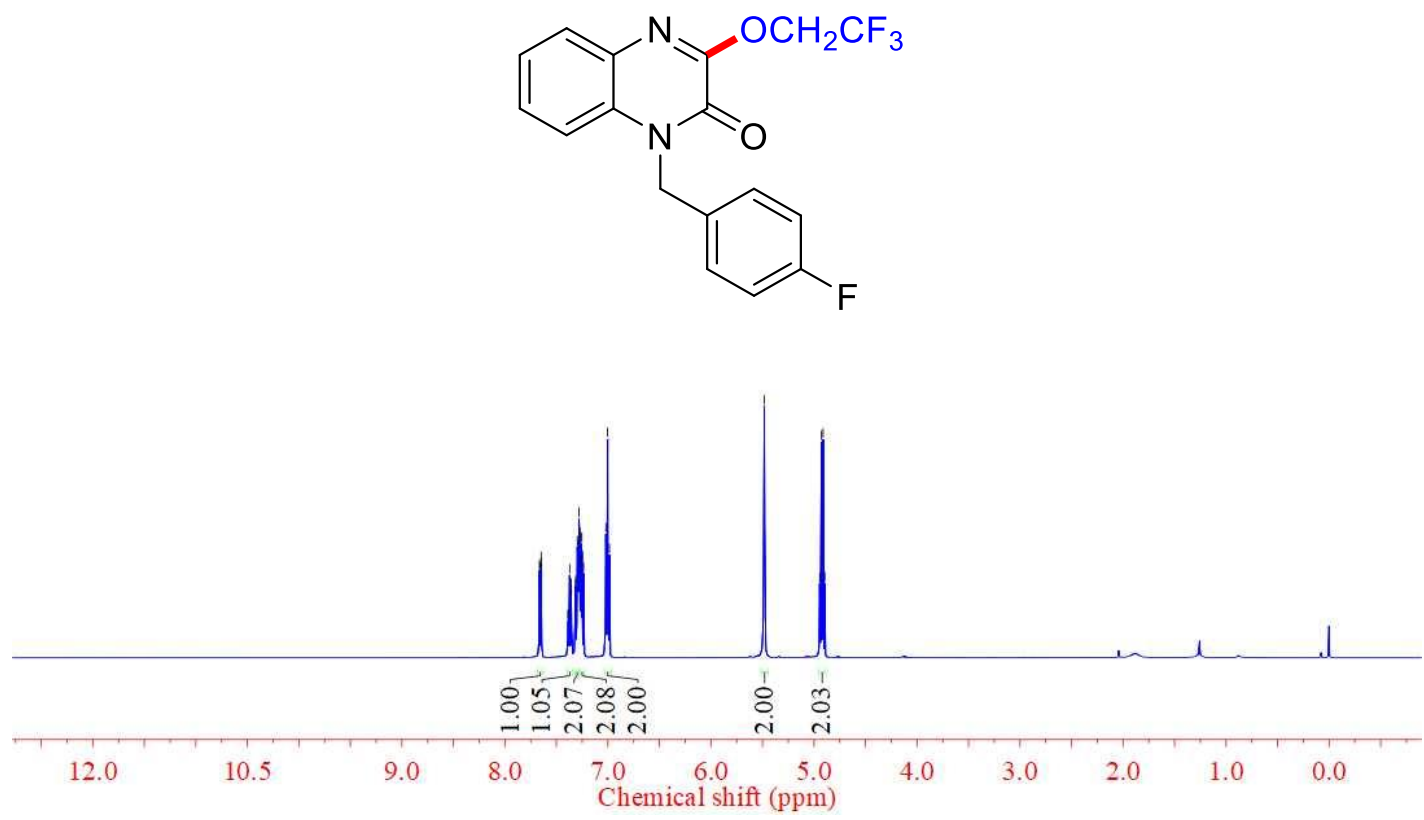

\section{$3{ }^{13} \mathrm{C}$ NMR}

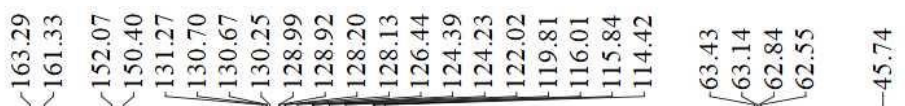<smiles>O=c1c(OCC(F)(F)F)nc2ccccc2n1Cc1ccc(F)cc1</smiles>

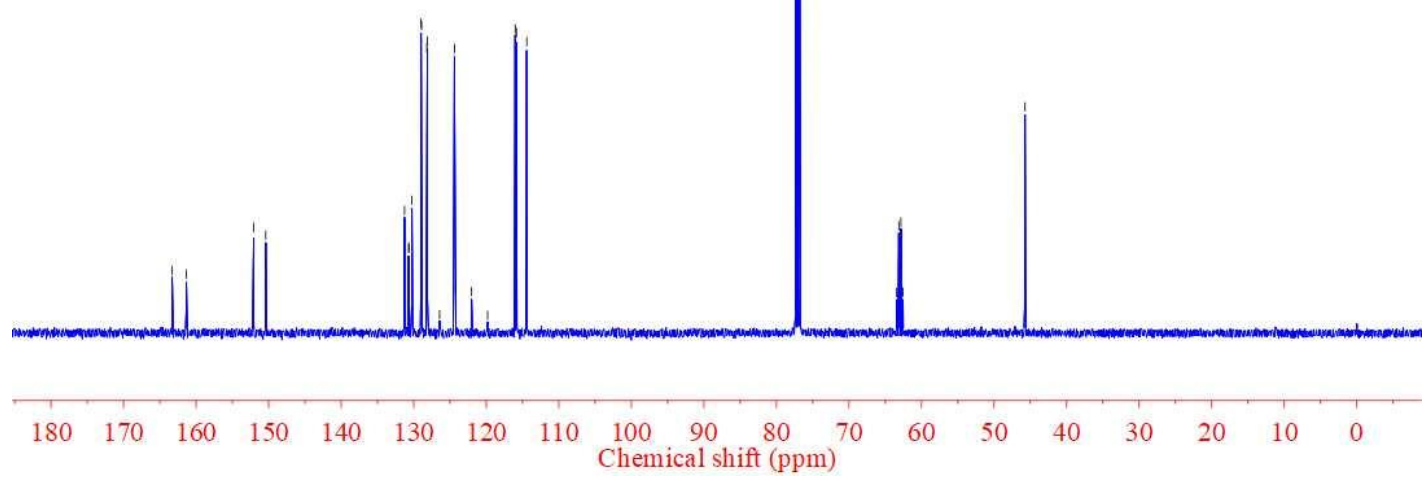




\section{$3 \mathrm{k}^{19}$ F NMR}

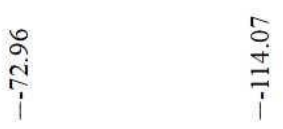

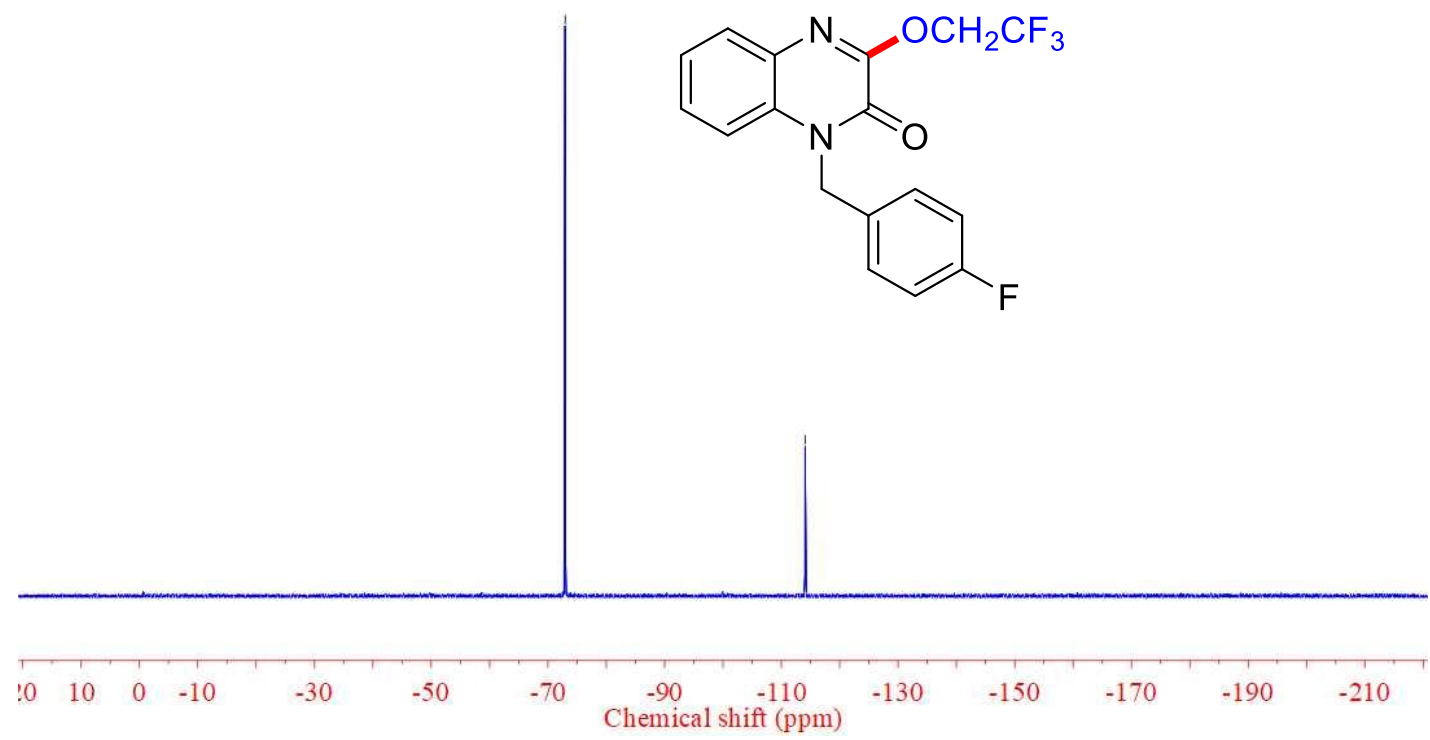

\section{I ${ }^{1}$ H NMR}

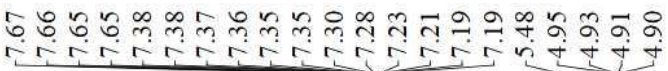
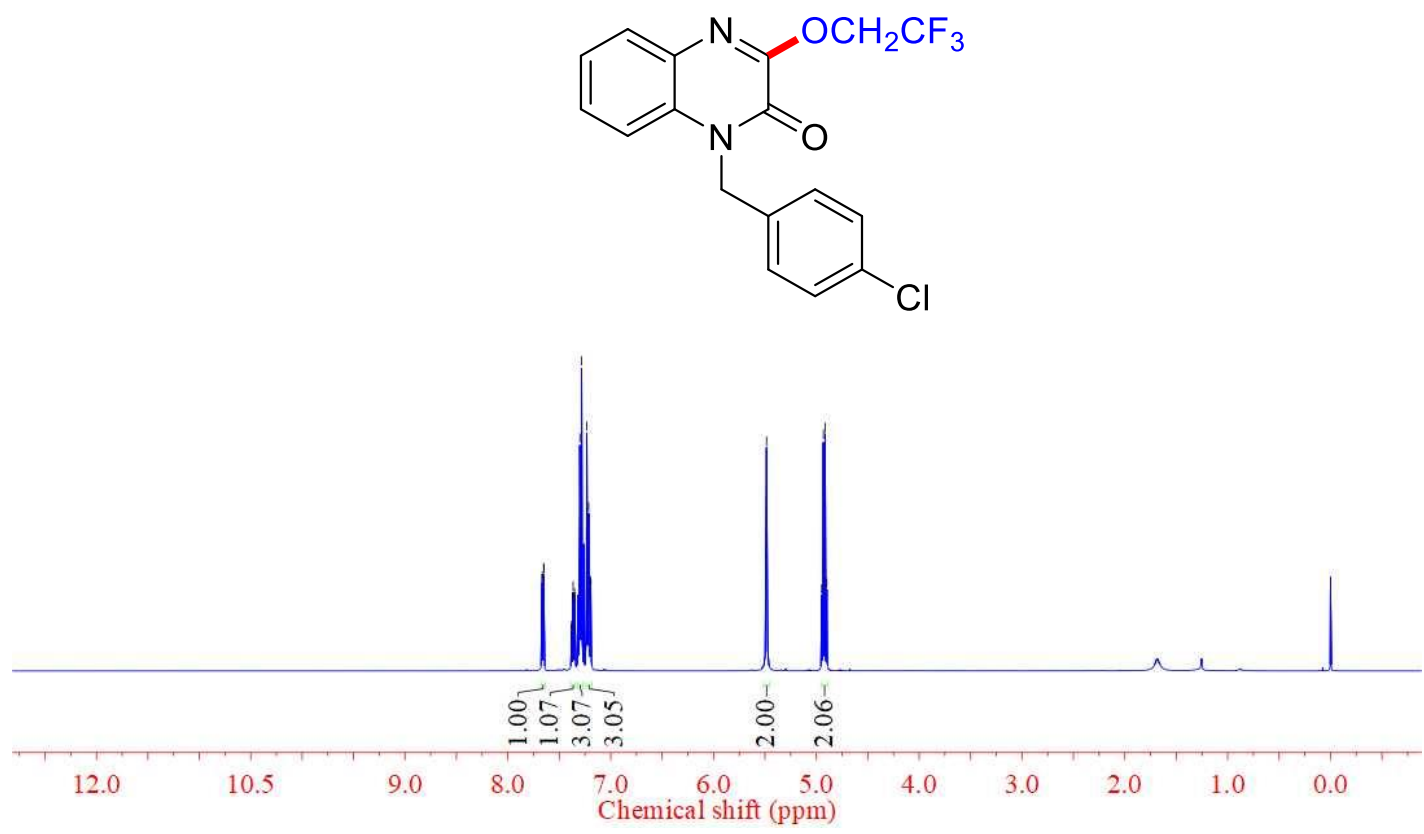


\section{$31{ }^{13} \mathrm{C}$ NMR}

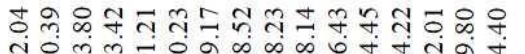

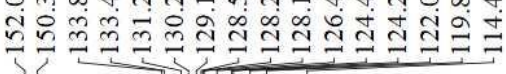

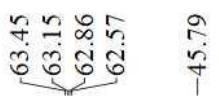

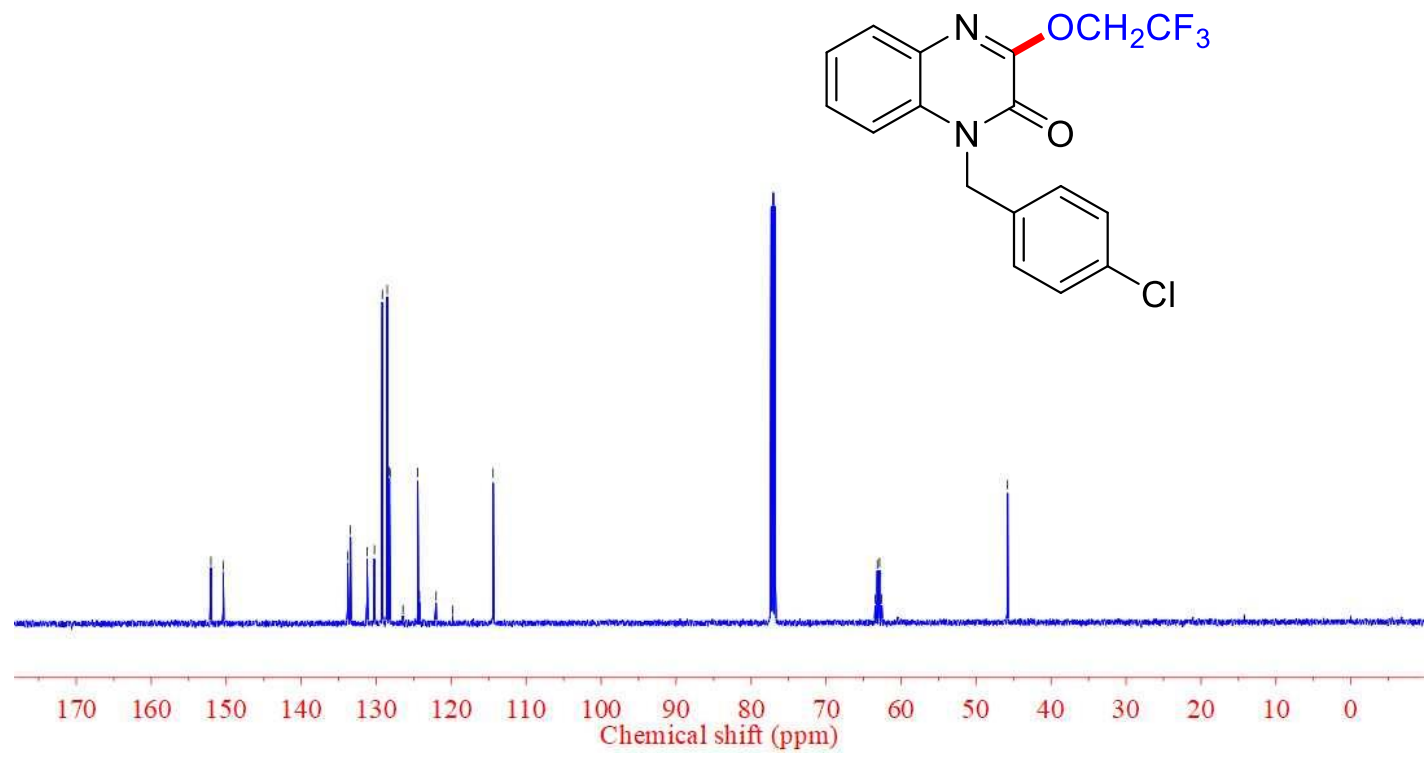

\section{$31{ }^{19}$ F NMR}

ลั

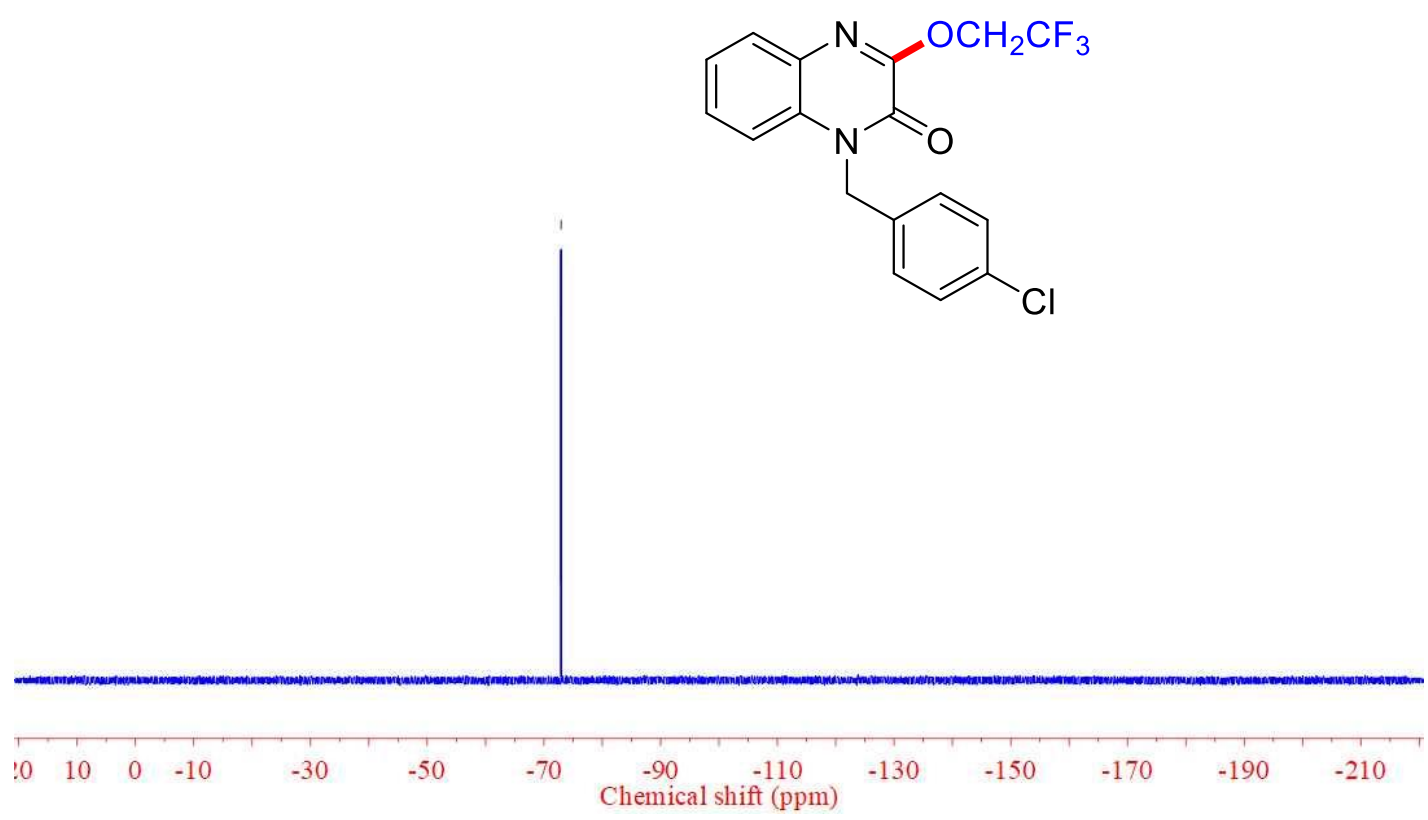




\section{3m ${ }^{1}$ H NMR}

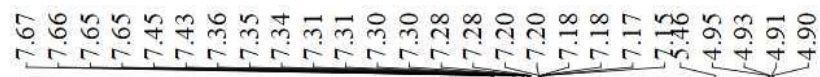

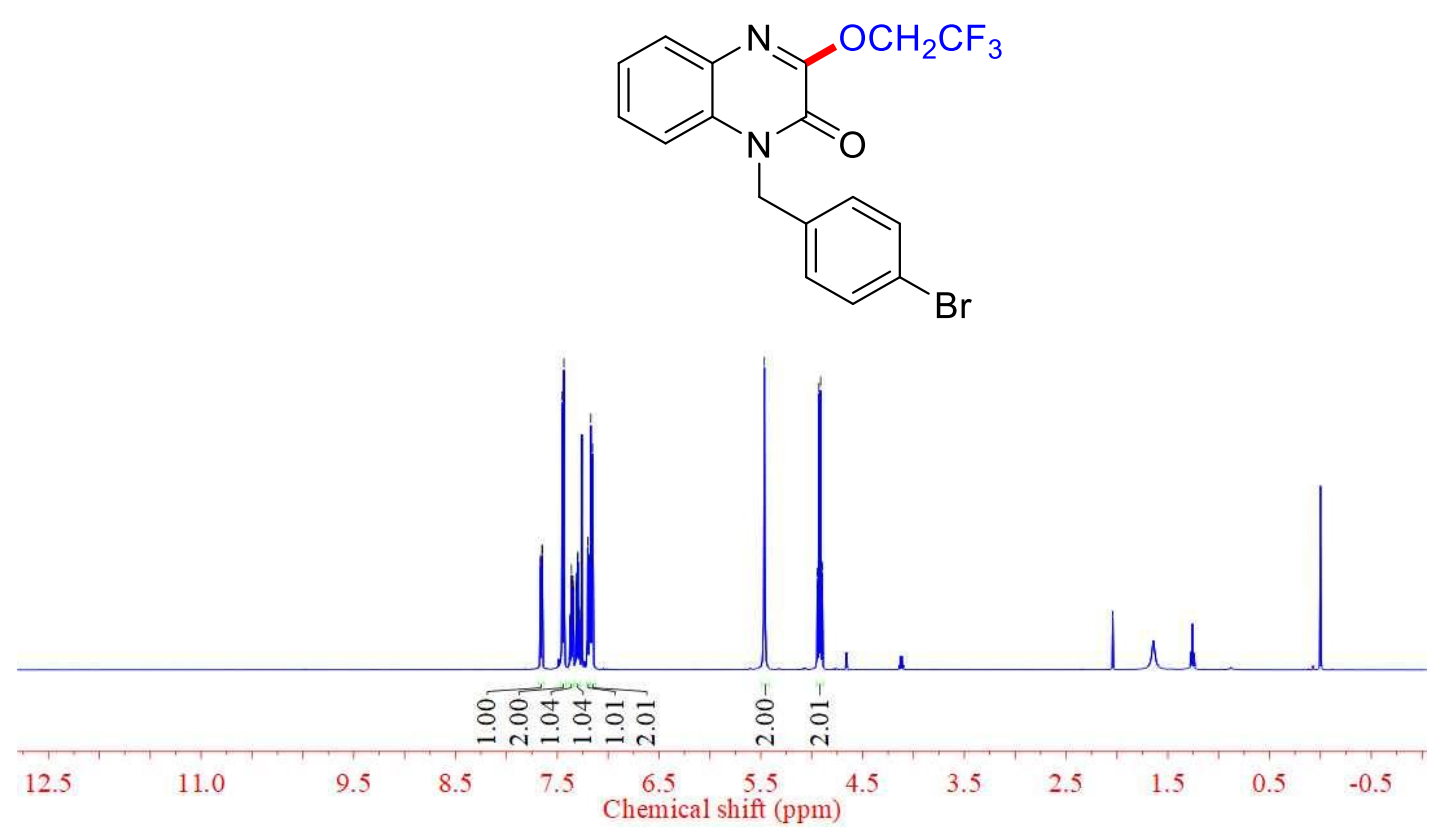

\section{$3 m^{13} \mathrm{C}$ NMR}

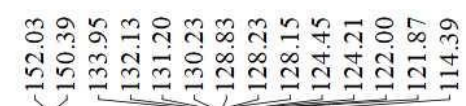

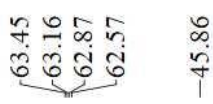<smiles>O=c1c(OCC(F)(F)F)nc2ccccc2n1Cc1ccc(Br)cc1</smiles>

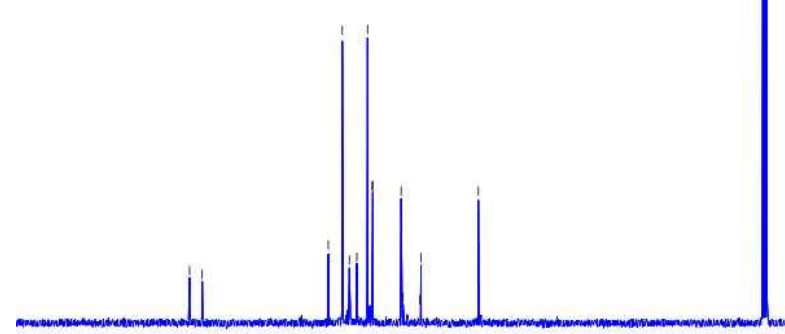

$\begin{array}{lllllllllllllllll}170 & 160 & 150 & 140 & 130 & 120 & 110 & 100 \begin{array}{c}90 \\ \text { Chemical shift }\end{array}\left(\begin{array}{c}70 \\ (\mathrm{ppm})\end{array}\right. & 60 & 50 & 40 & 30 & 20 & 10 & 0\end{array}$ 


\section{$3 m^{19}$ F NMR}

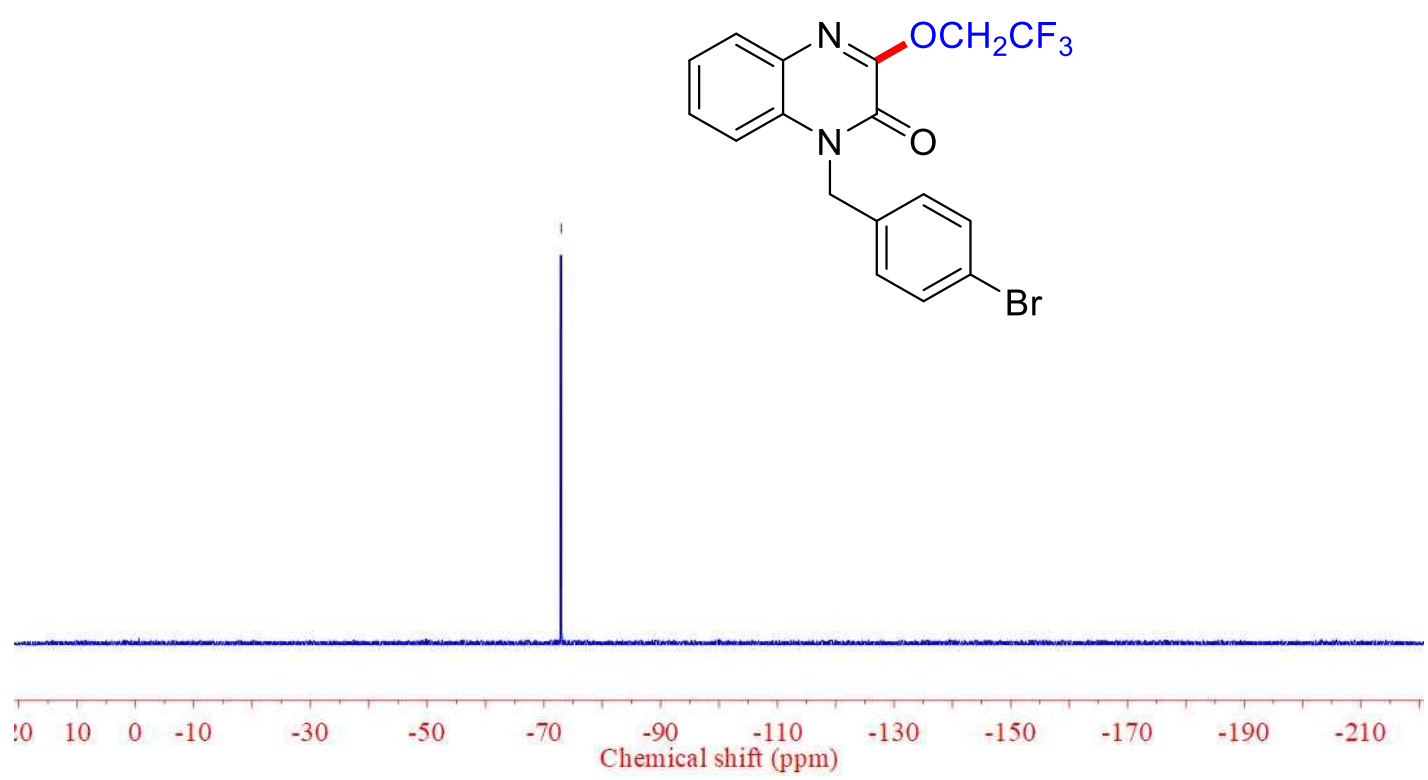

\section{3n ${ }^{1}$ H NMR}

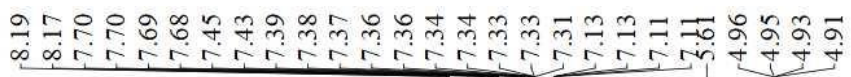

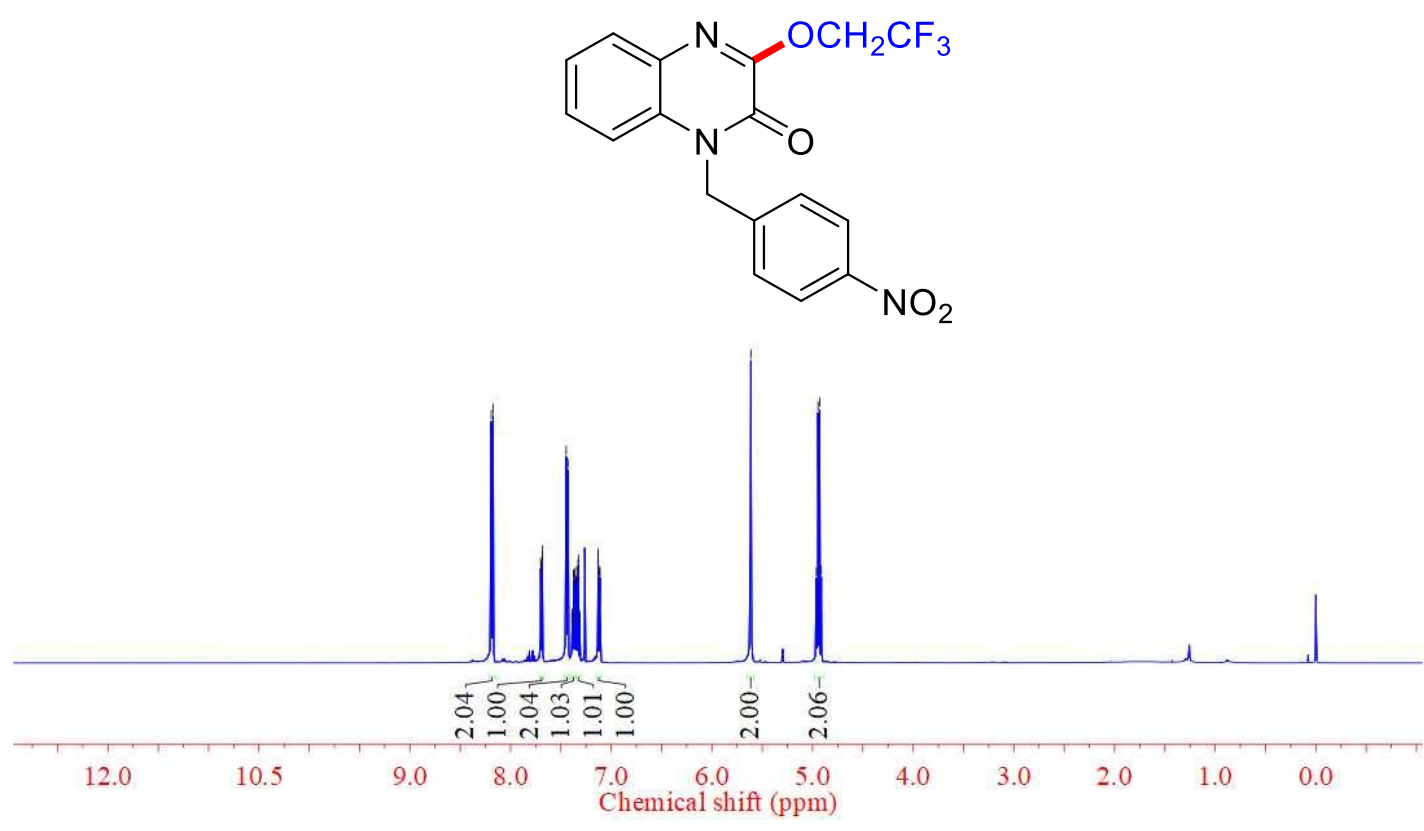




\section{$3 n{ }^{13} \mathrm{C}$ NMR}

ณㅇํำ

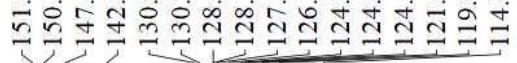

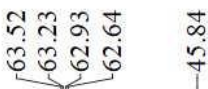

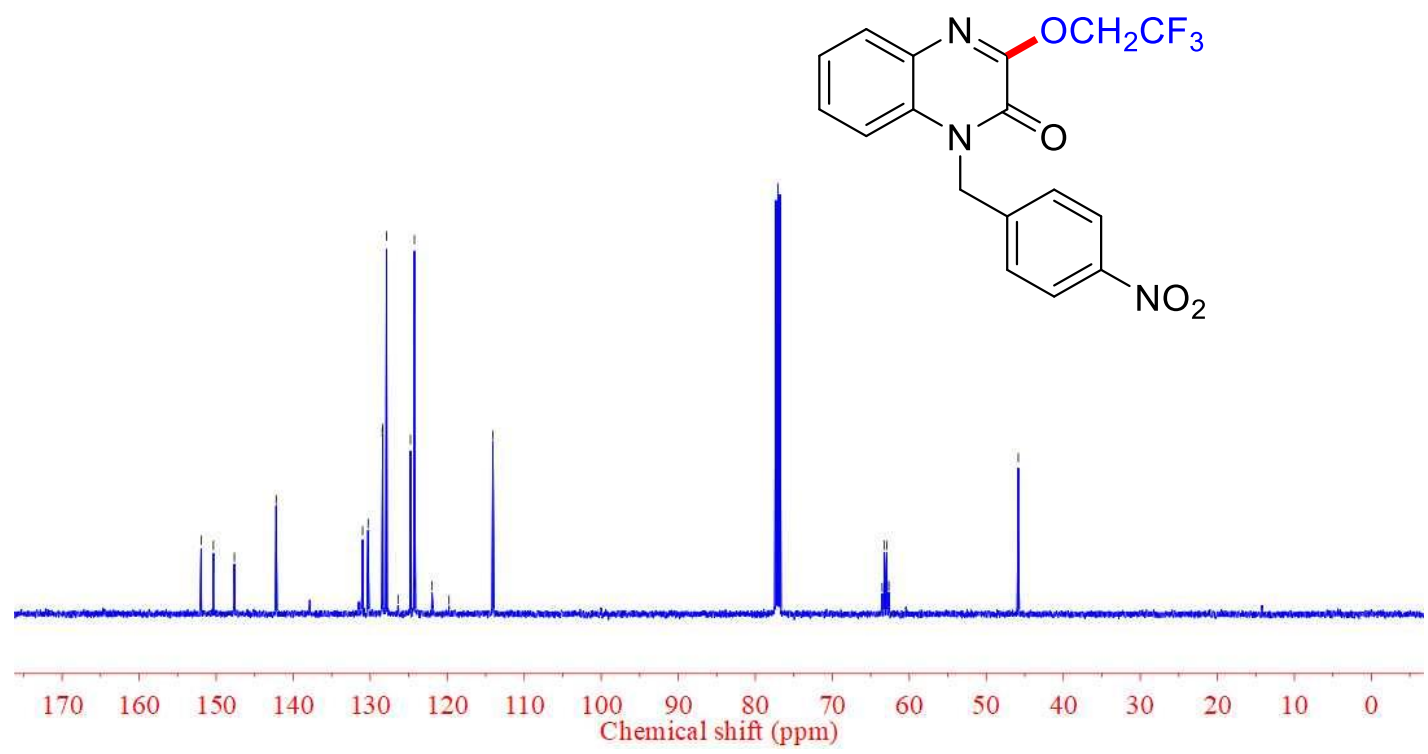

\section{$3 n{ }^{19}$ F NMR}

ลุำ

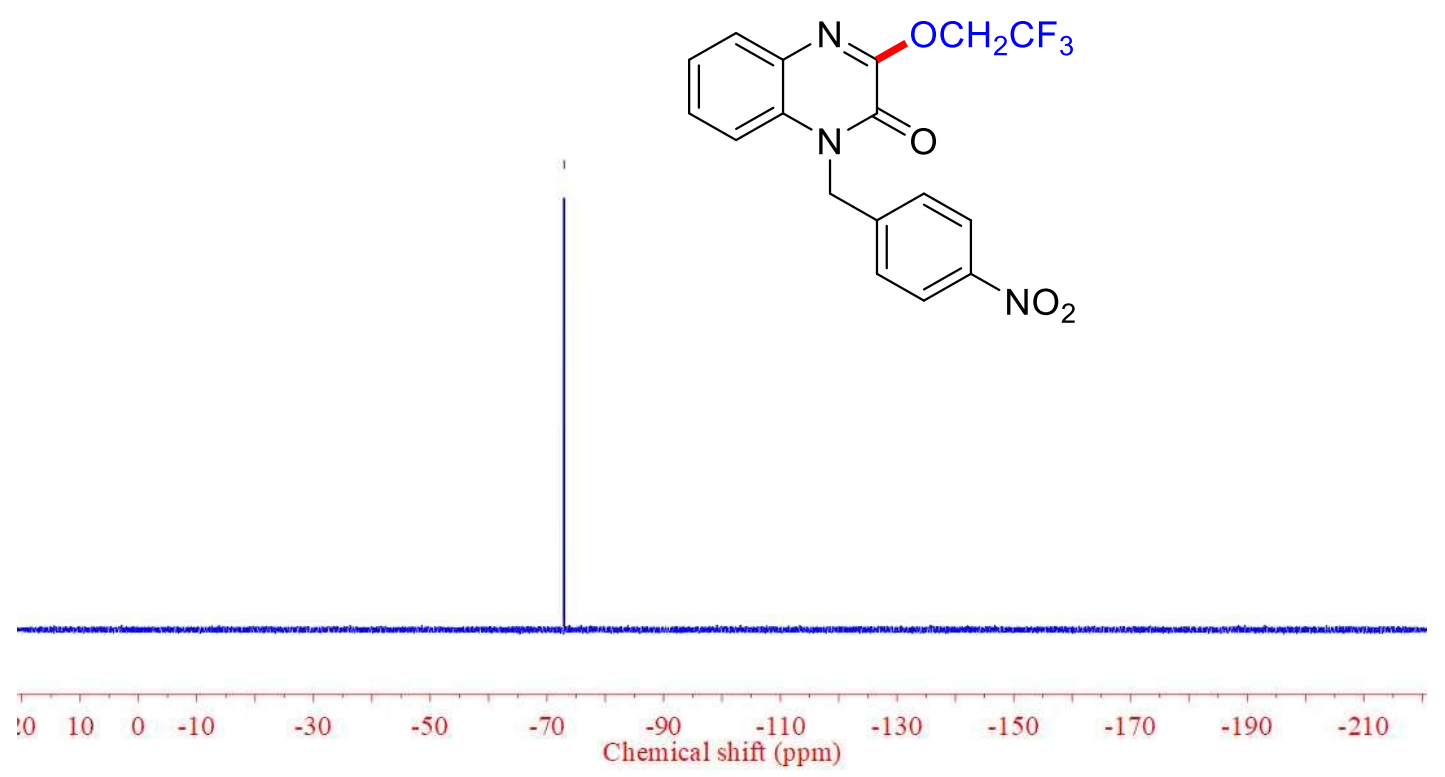




\section{3o ${ }^{1}$ H NMR}

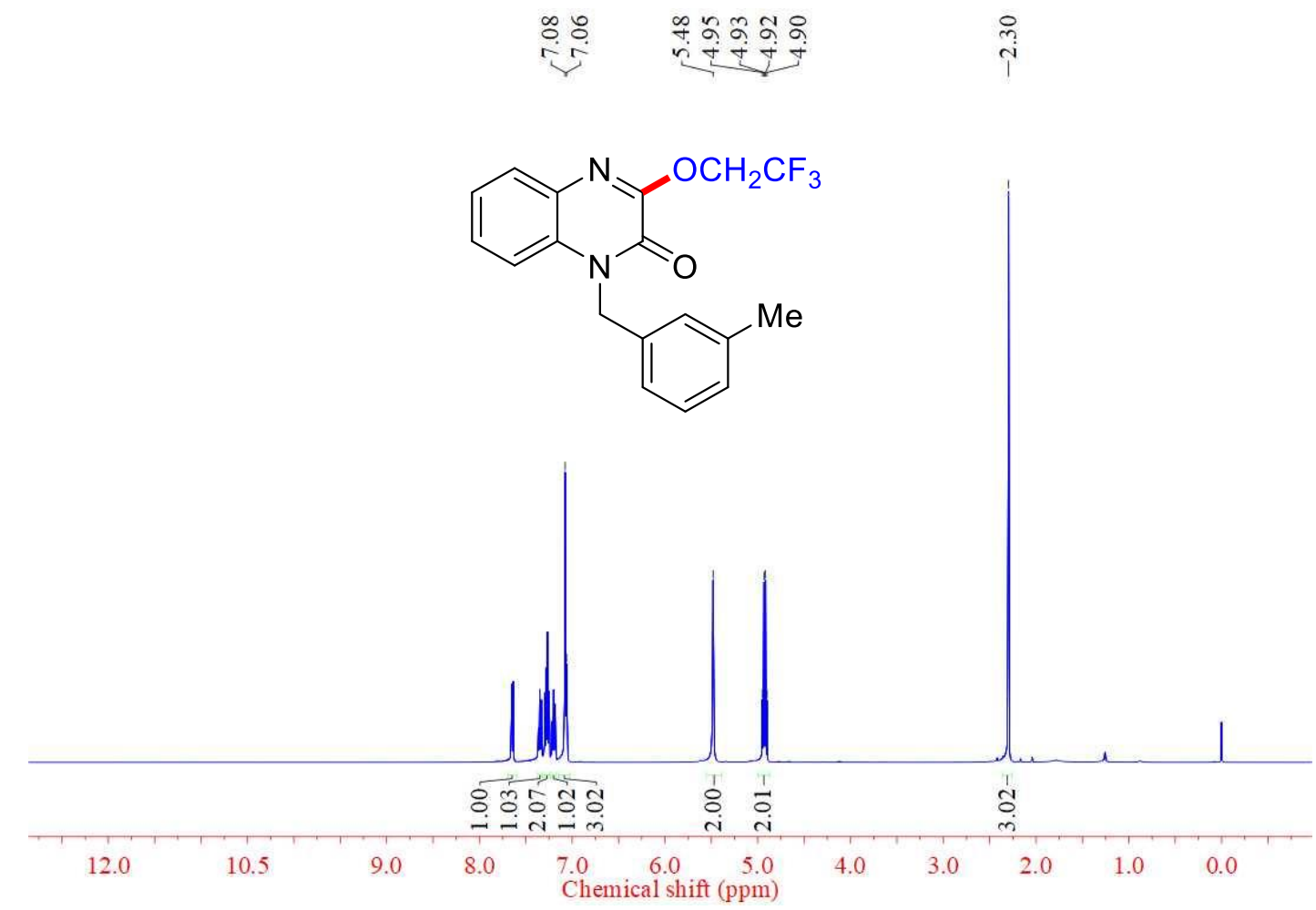

\section{$30{ }^{13} \mathrm{C}$ NMR}

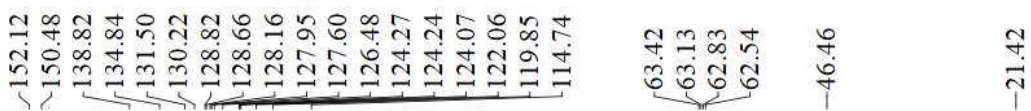

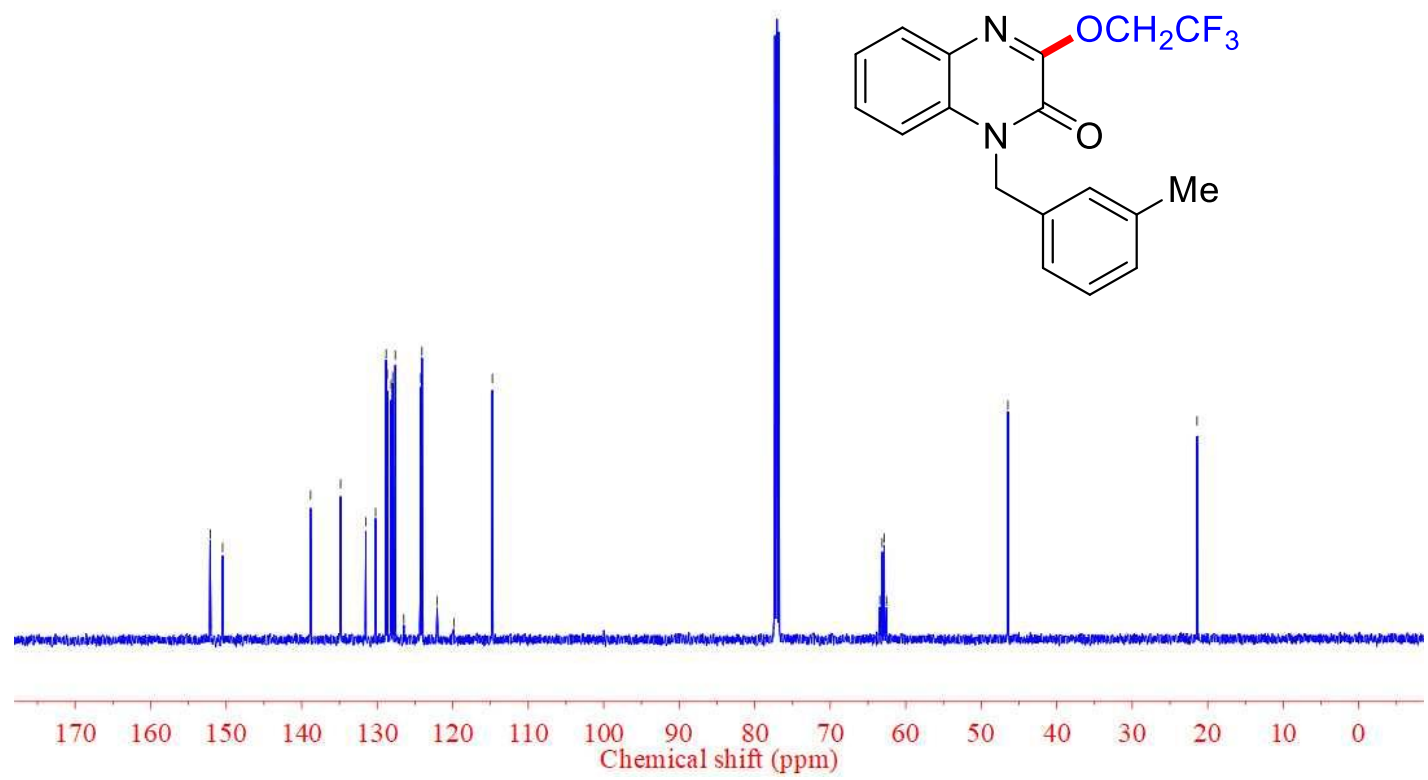




\section{$30{ }^{19}$ F NMR}

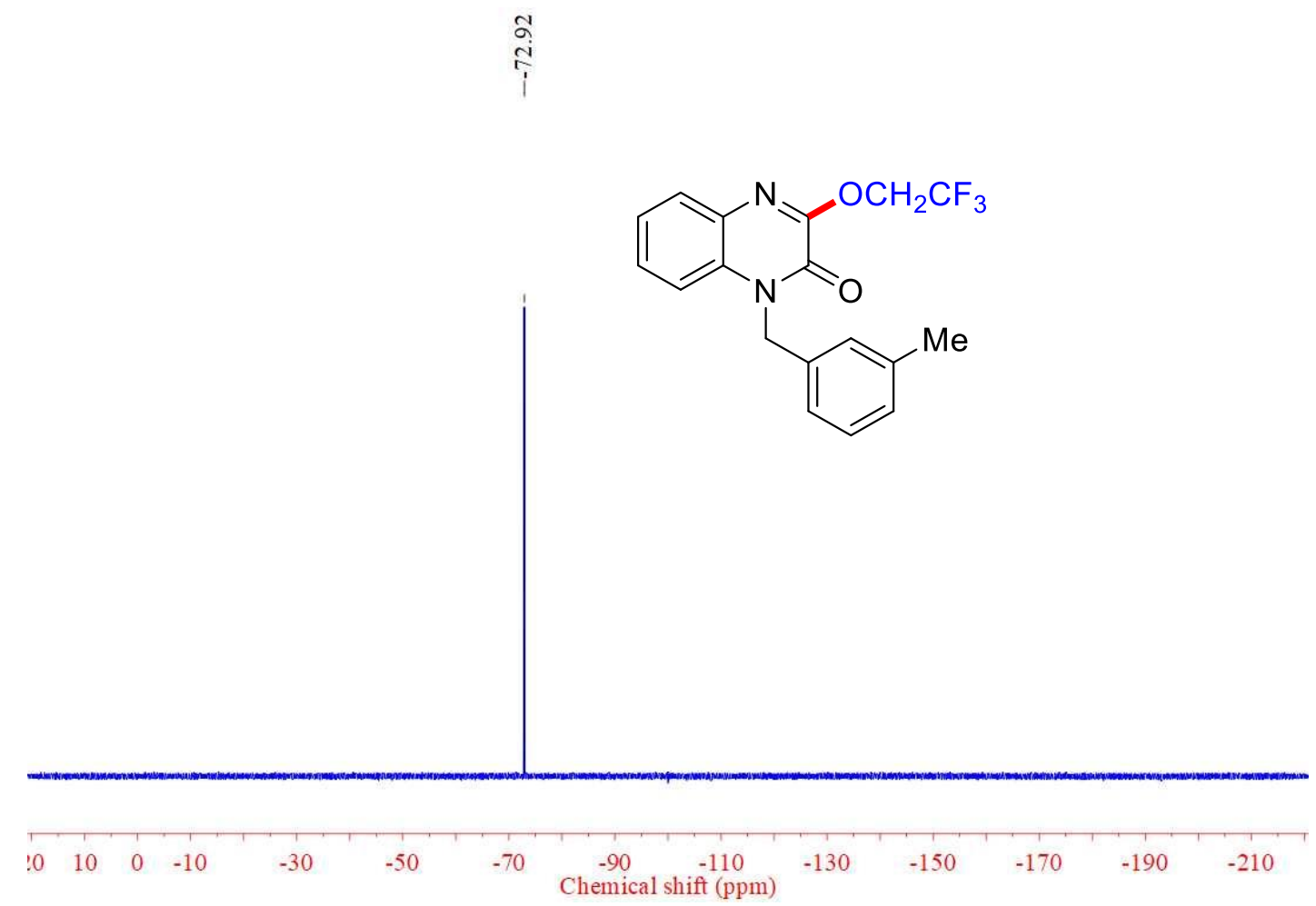

\section{3p ${ }^{1}$ H NMR}

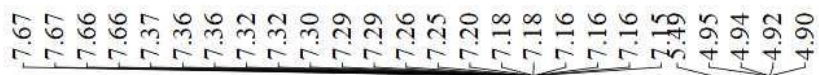

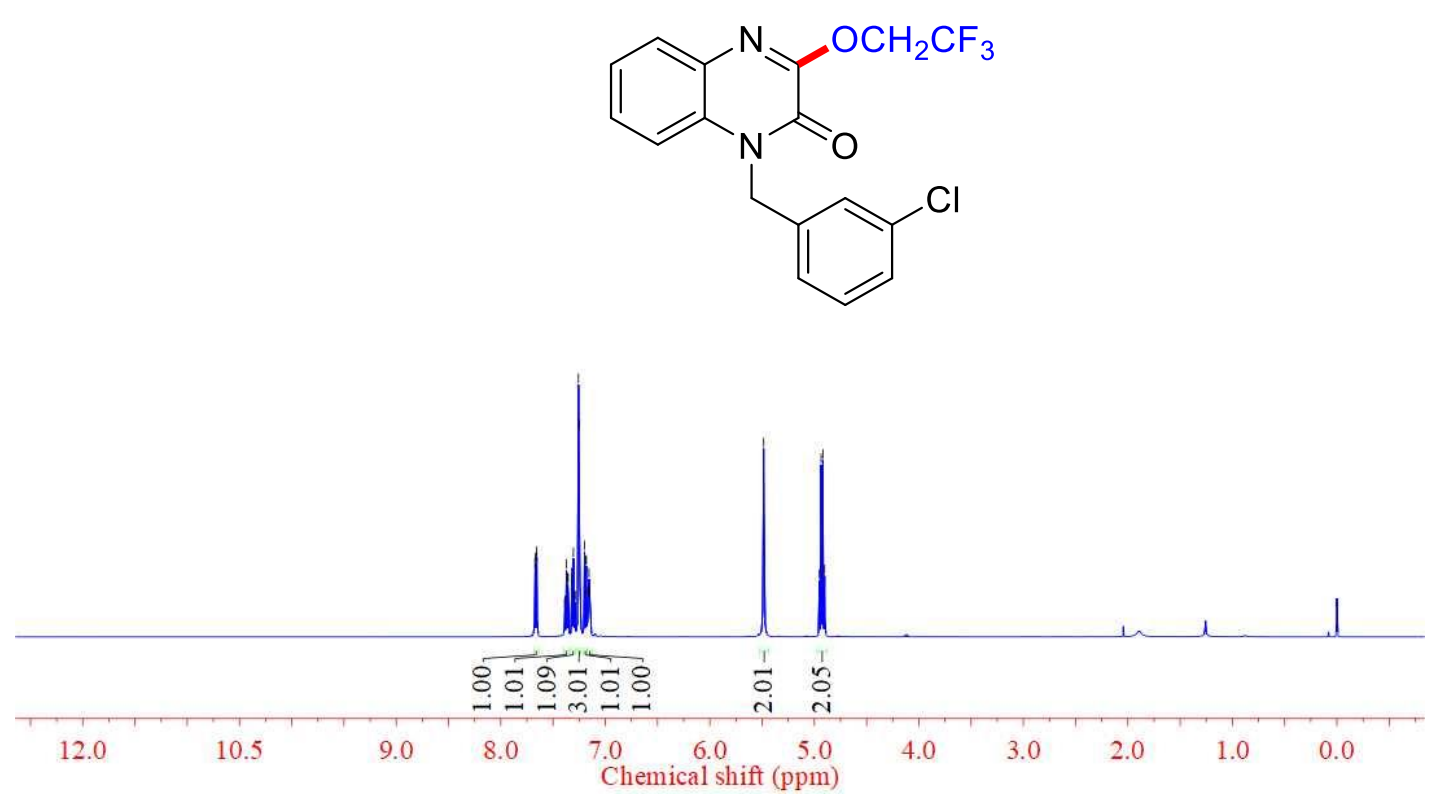




\section{$3 p{ }^{13}$ C NMR}

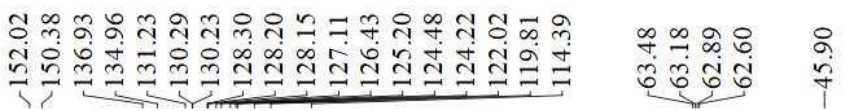

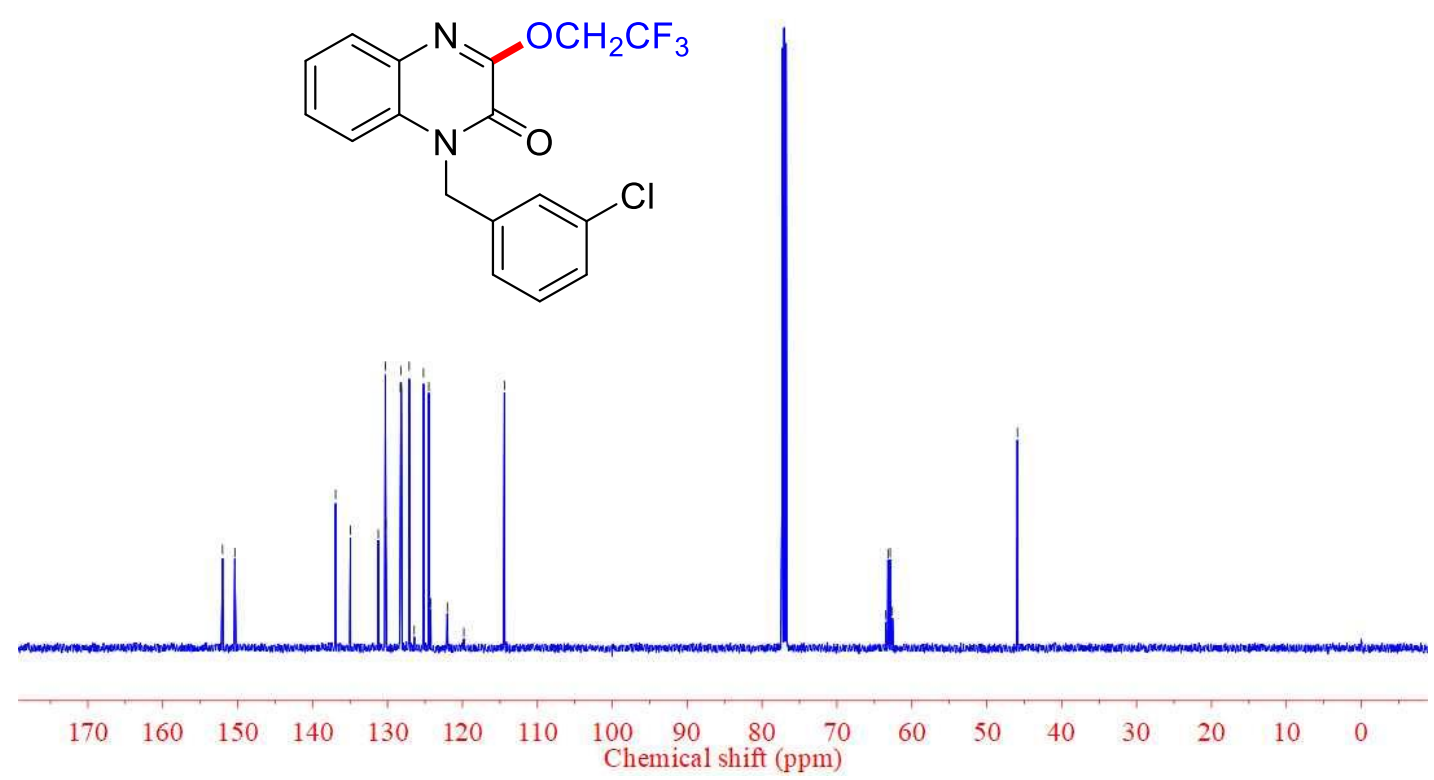

\section{$3 p{ }^{19}$ F NMR}

ลั

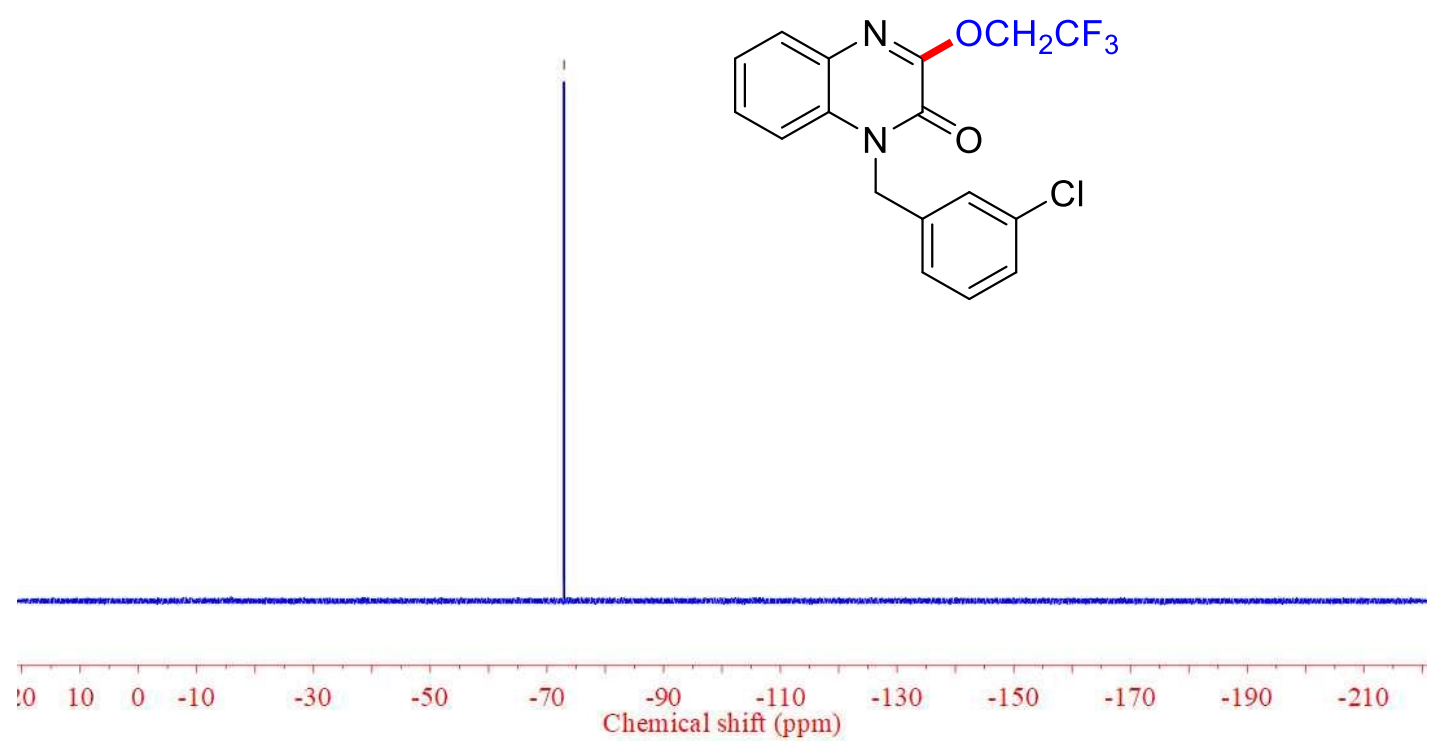




\section{$3 q^{1}$ H NMR}

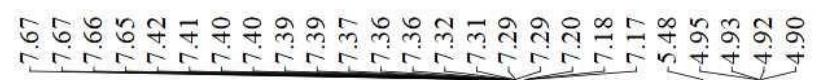

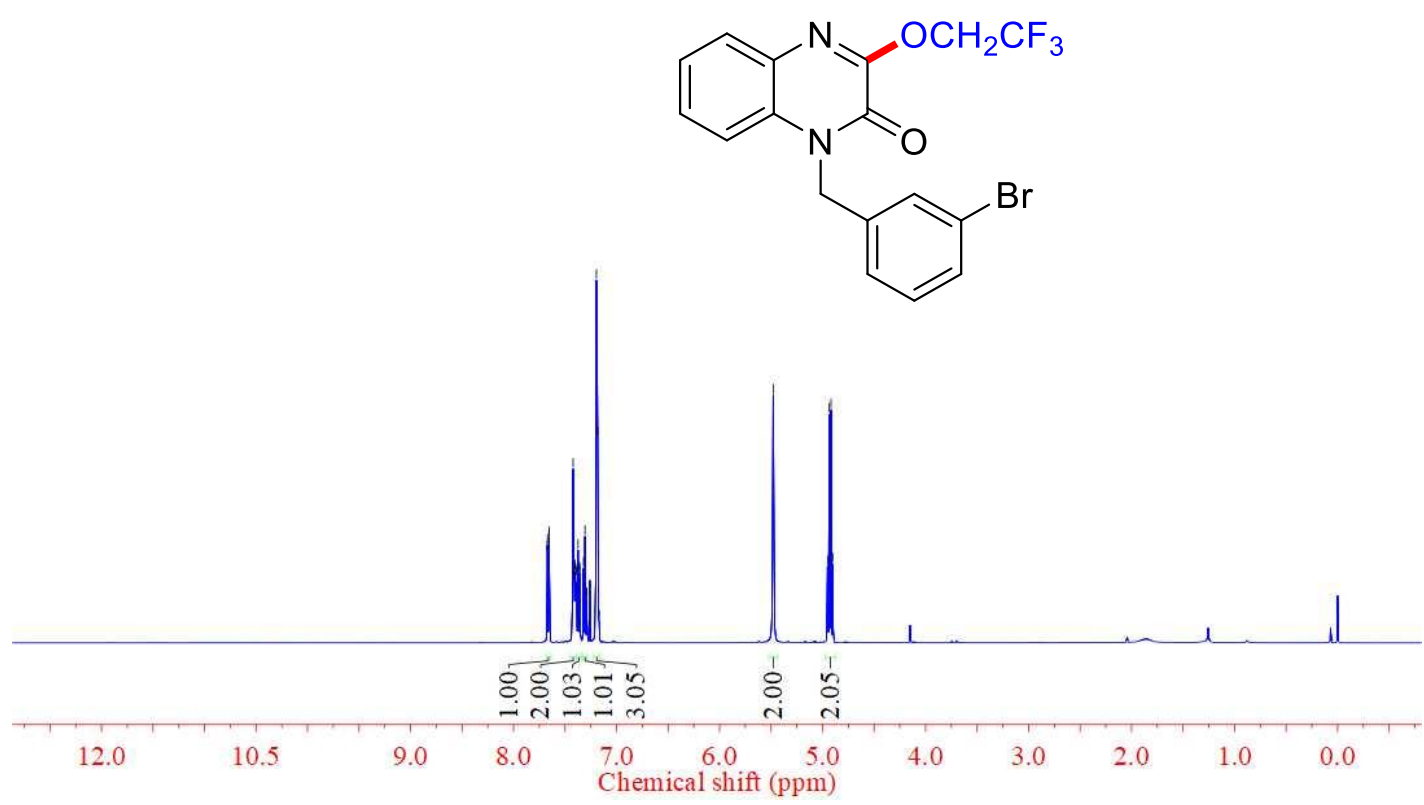

\section{$3 q{ }^{13} \mathrm{C}$ NMR}

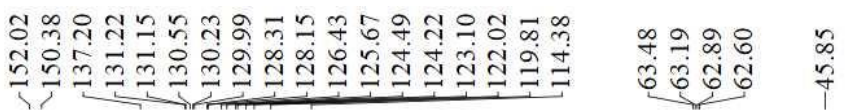<smiles>O=c1c(OCC(F)(F)F)nc2ccccc2n1Cc1cccc(Br)c1</smiles>

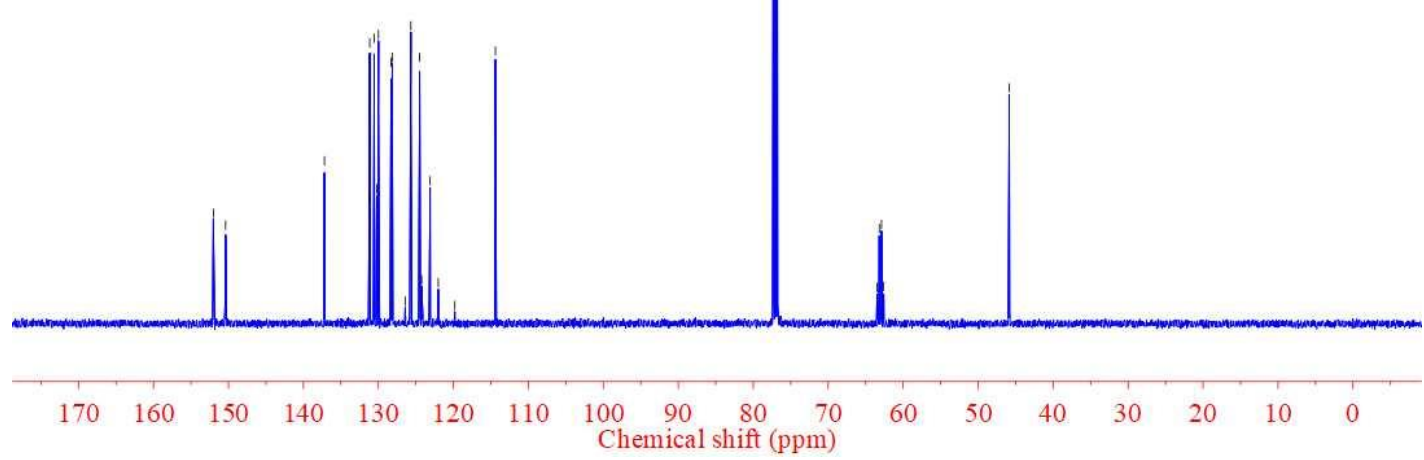




\section{$3 q{ }^{19}$ F NMR}

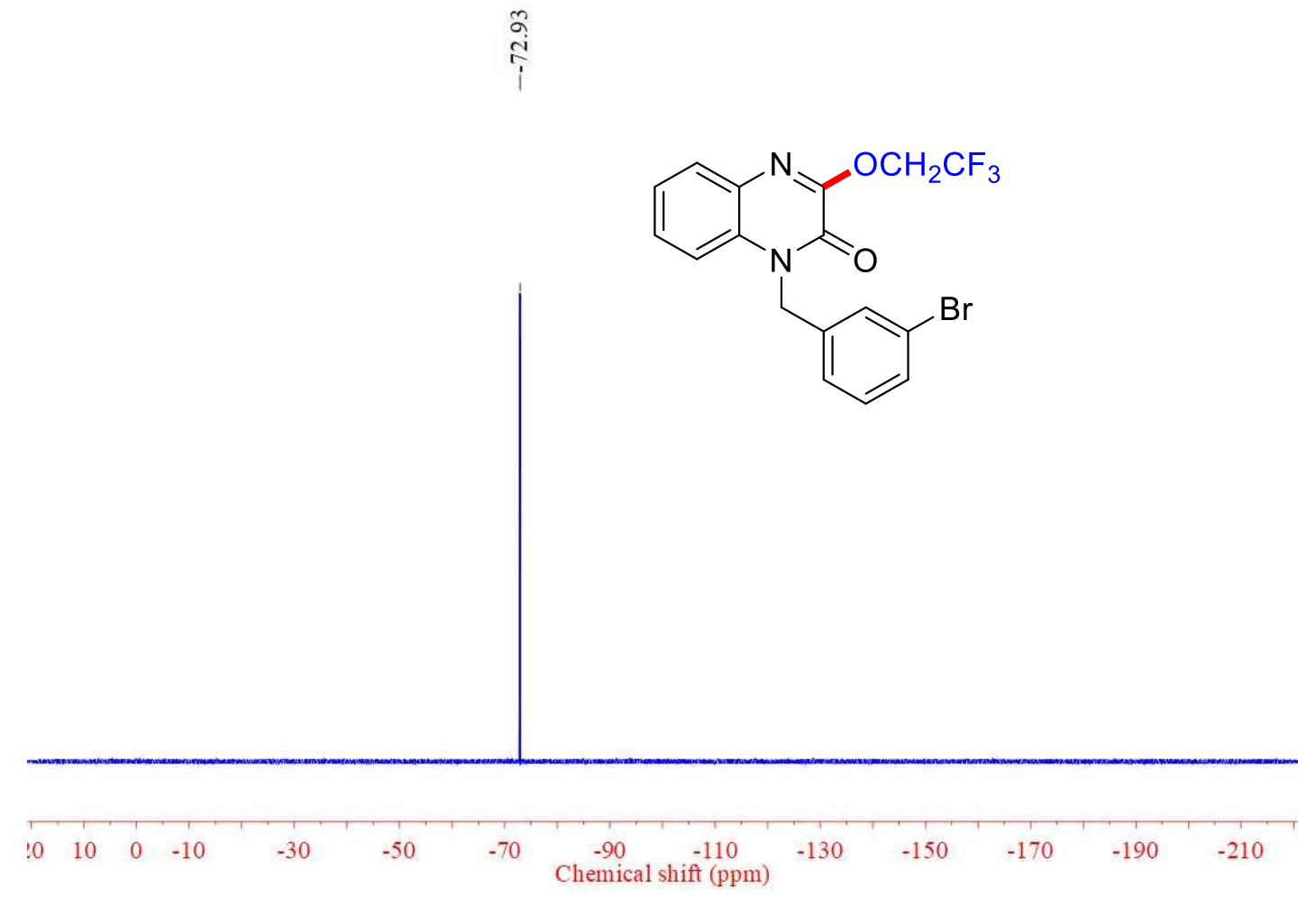

\section{$3 r^{1} H$ NMR}

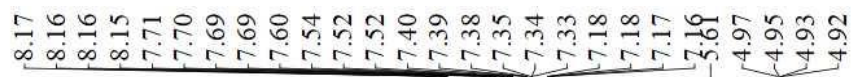

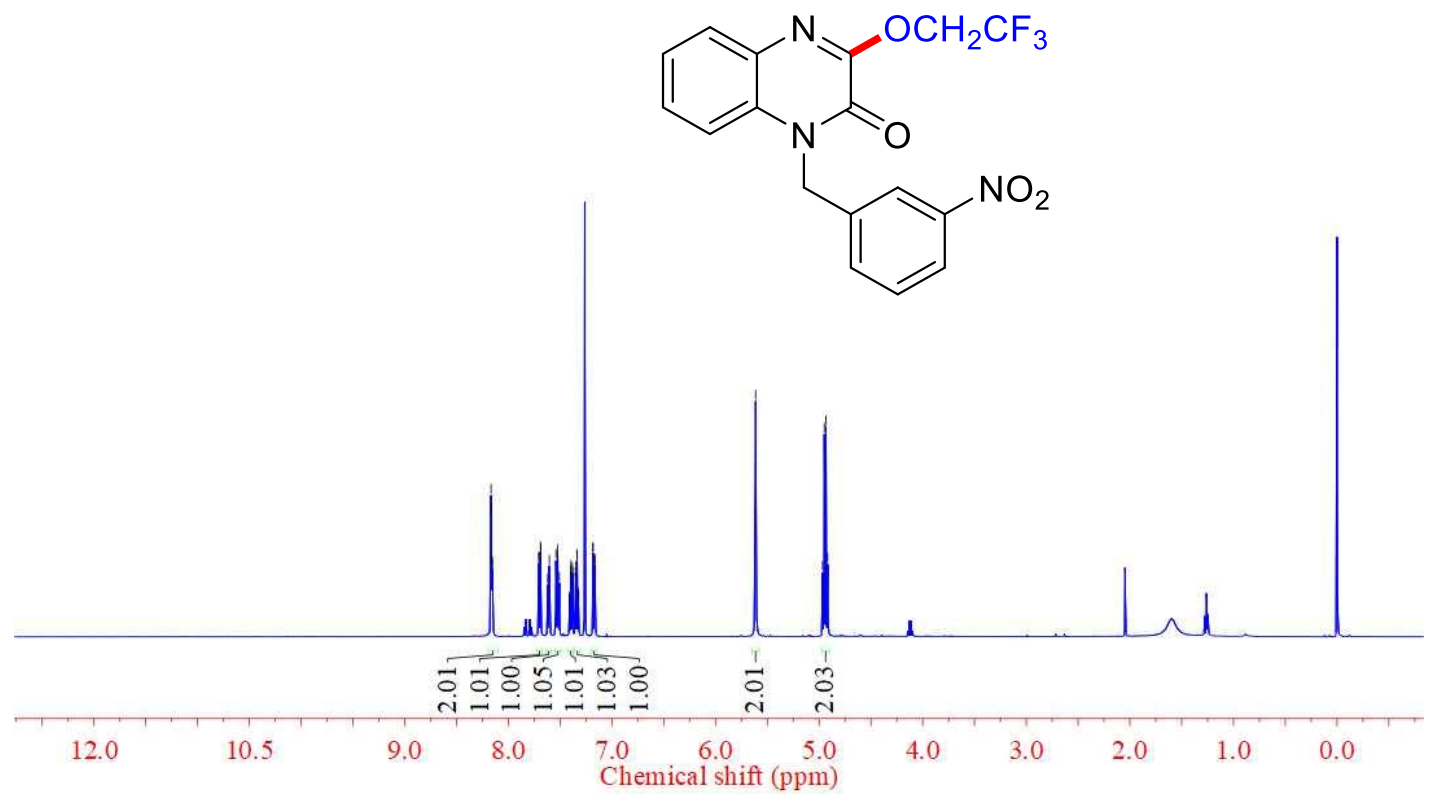




\section{$3 r{ }^{13} \mathrm{C}$ NMR}

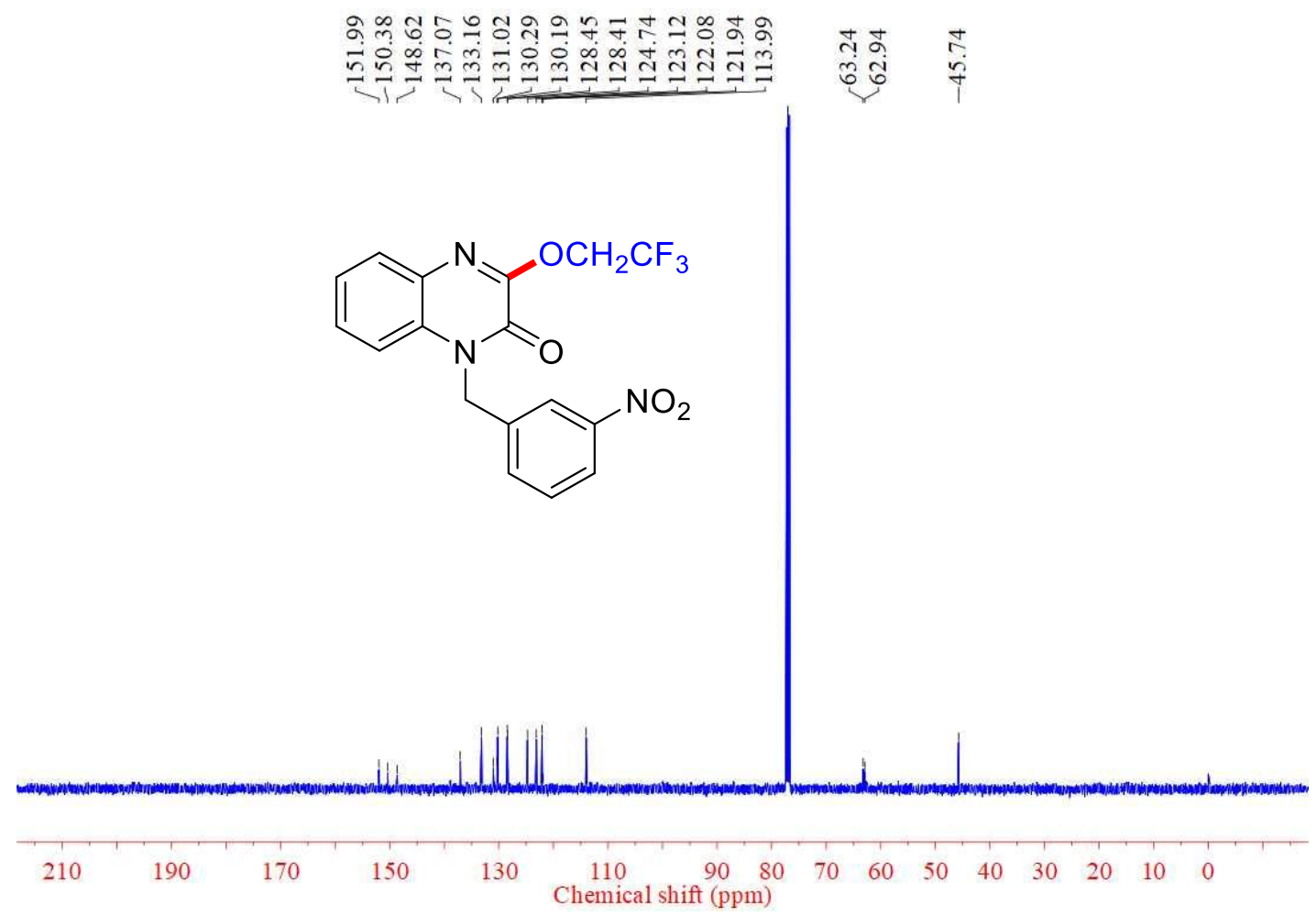

$3 r^{19}$ F NMR<smiles>O=c1c(OCC(F)(F)F)nc2ccccc2n1Cc1cccc([N+](=O)[O-])c1</smiles>

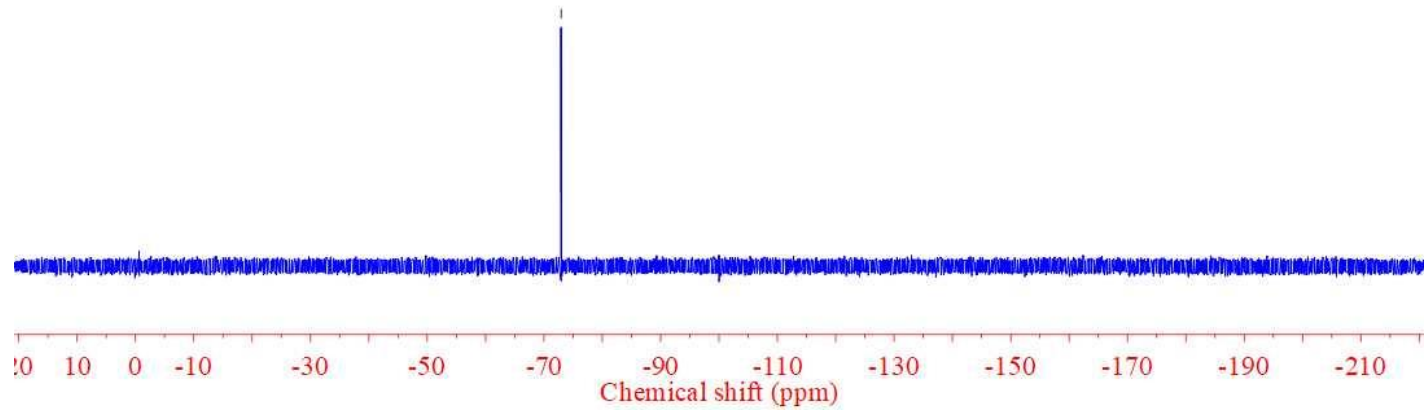




\section{3s ${ }^{1}$ H NMR}

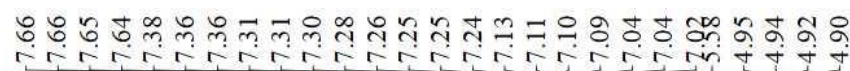

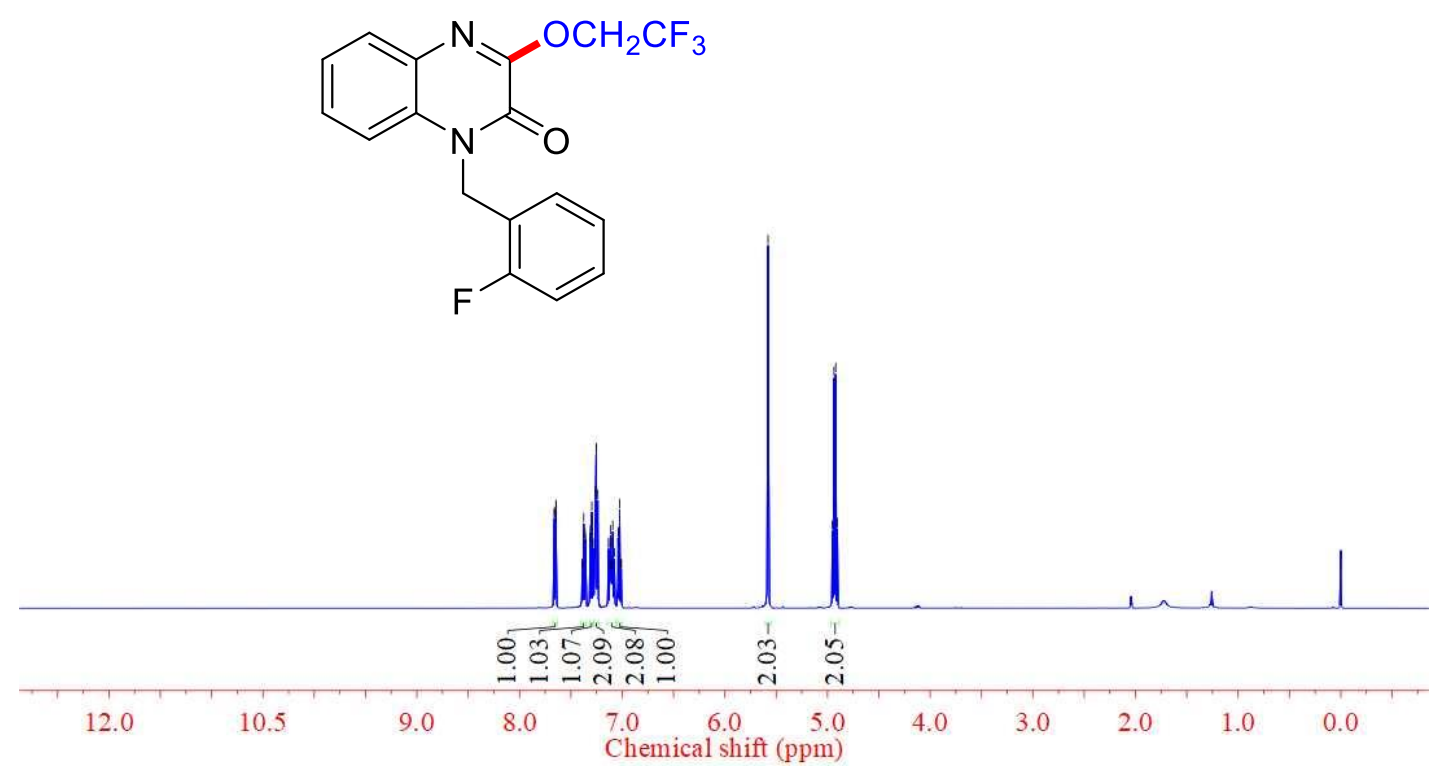

\section{3s ${ }^{13}$ C NMR}

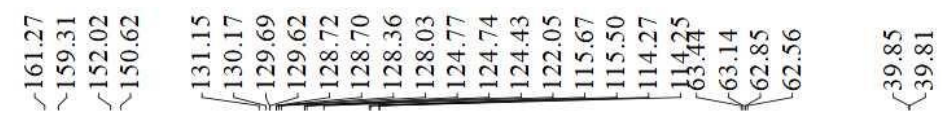

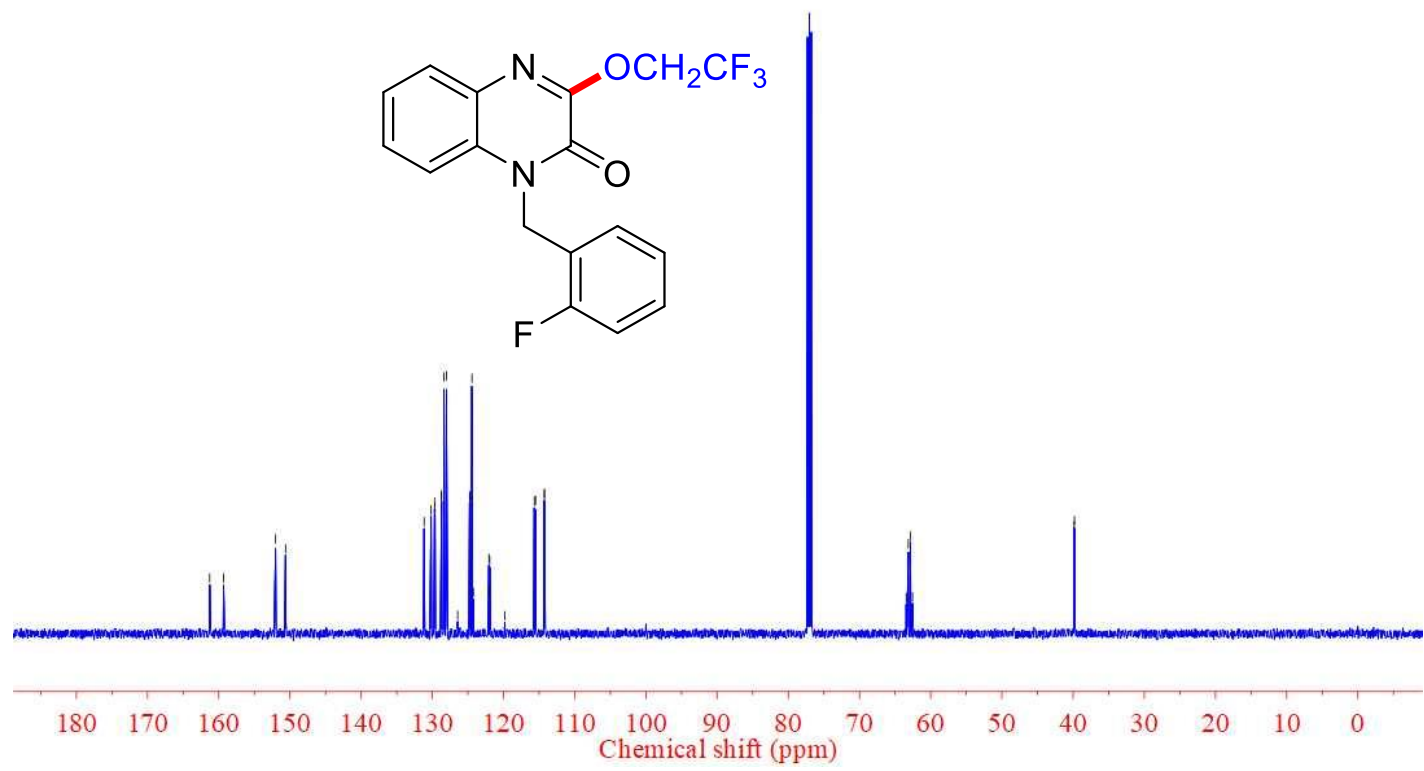




\section{$3 \mathrm{~s}{ }^{19} \mathrm{~F}$ NMR}

$\stackrel{\substack{i \\ i}}{\stackrel{\sim}{\infty}}$

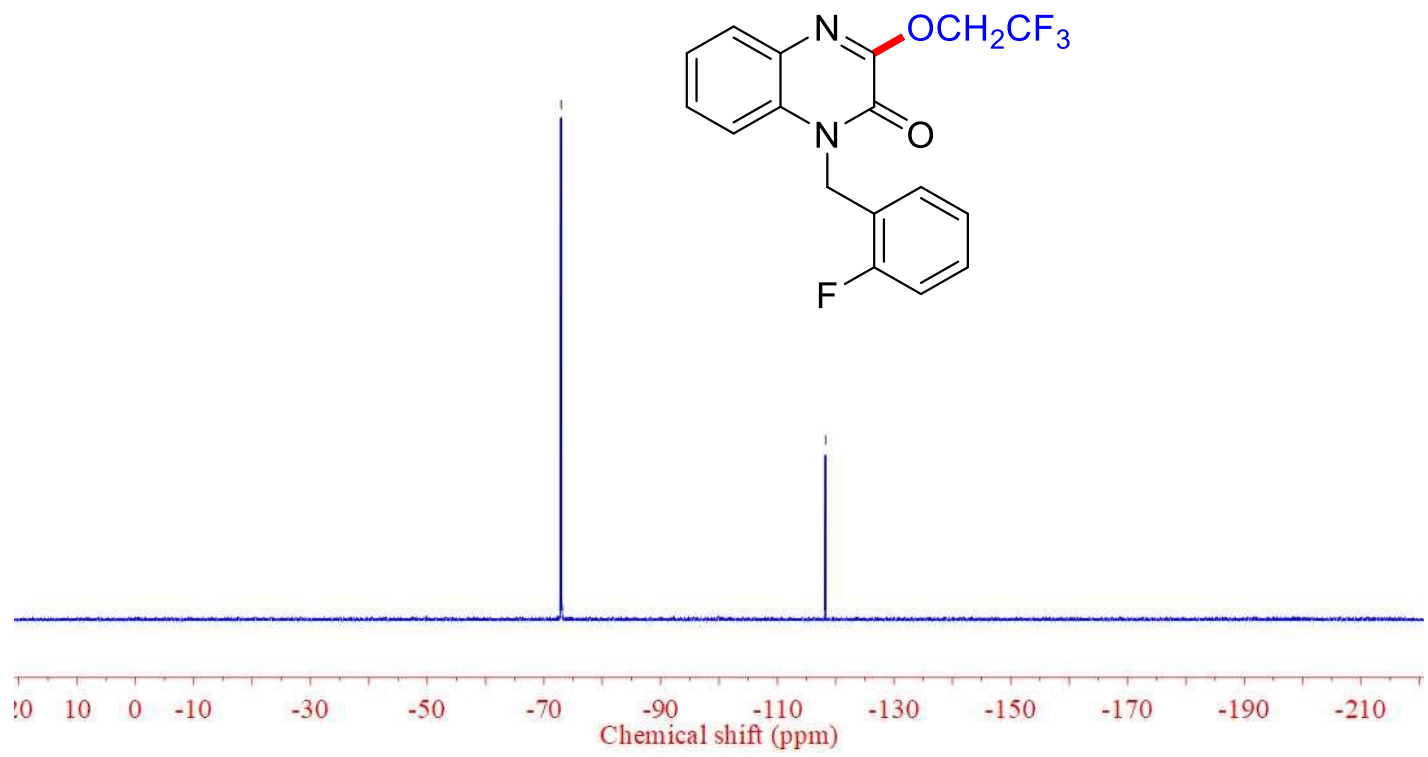

\section{$3 \mathrm{t}^{1} \mathrm{H}$ NMR}

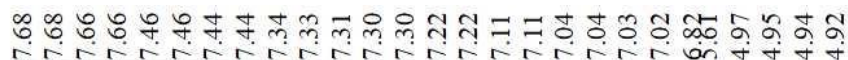

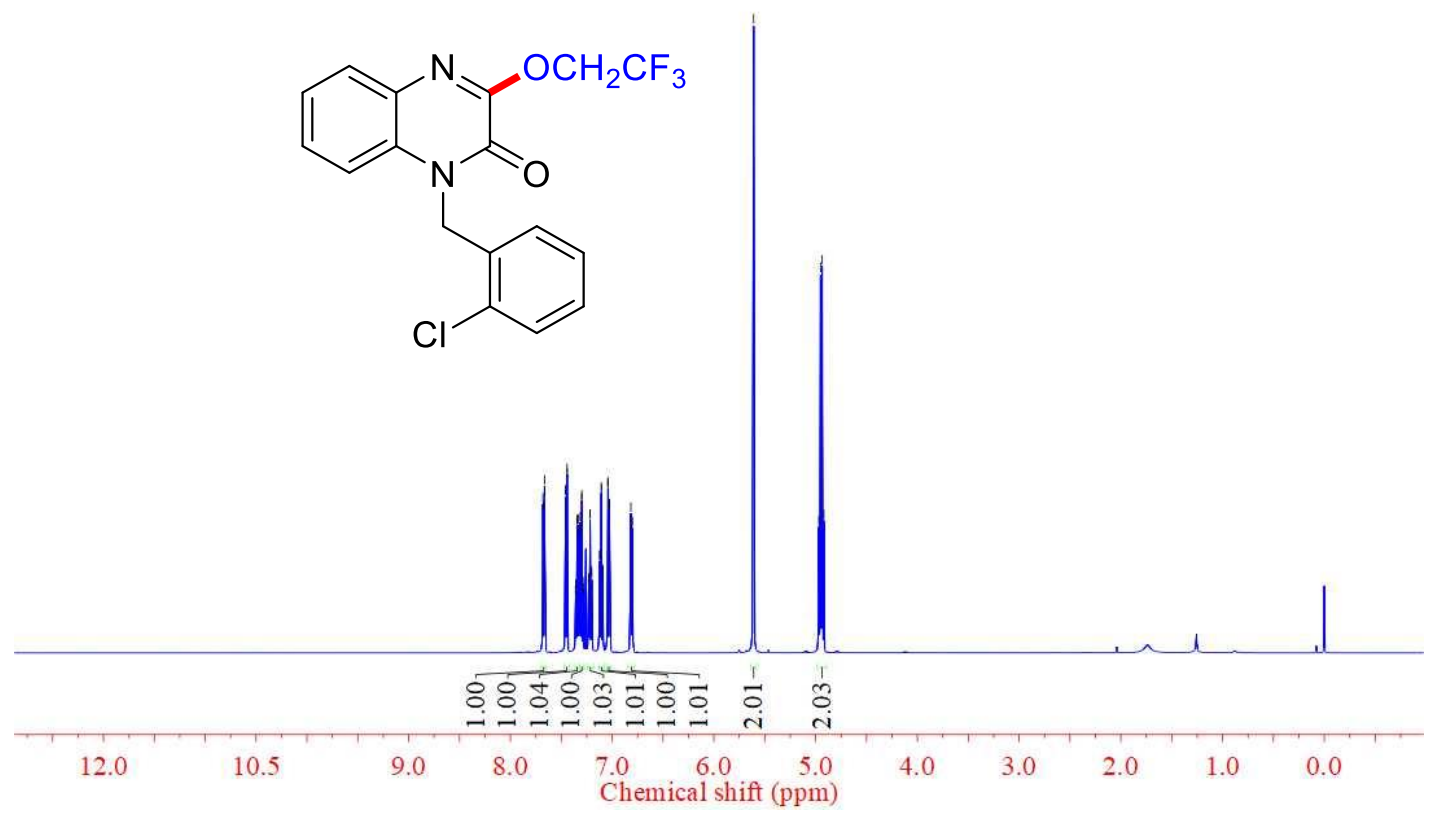




\section{$3 \mathrm{t}^{13} \mathrm{C}$ NMR}

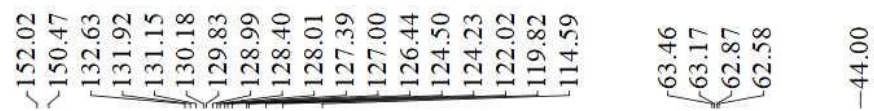

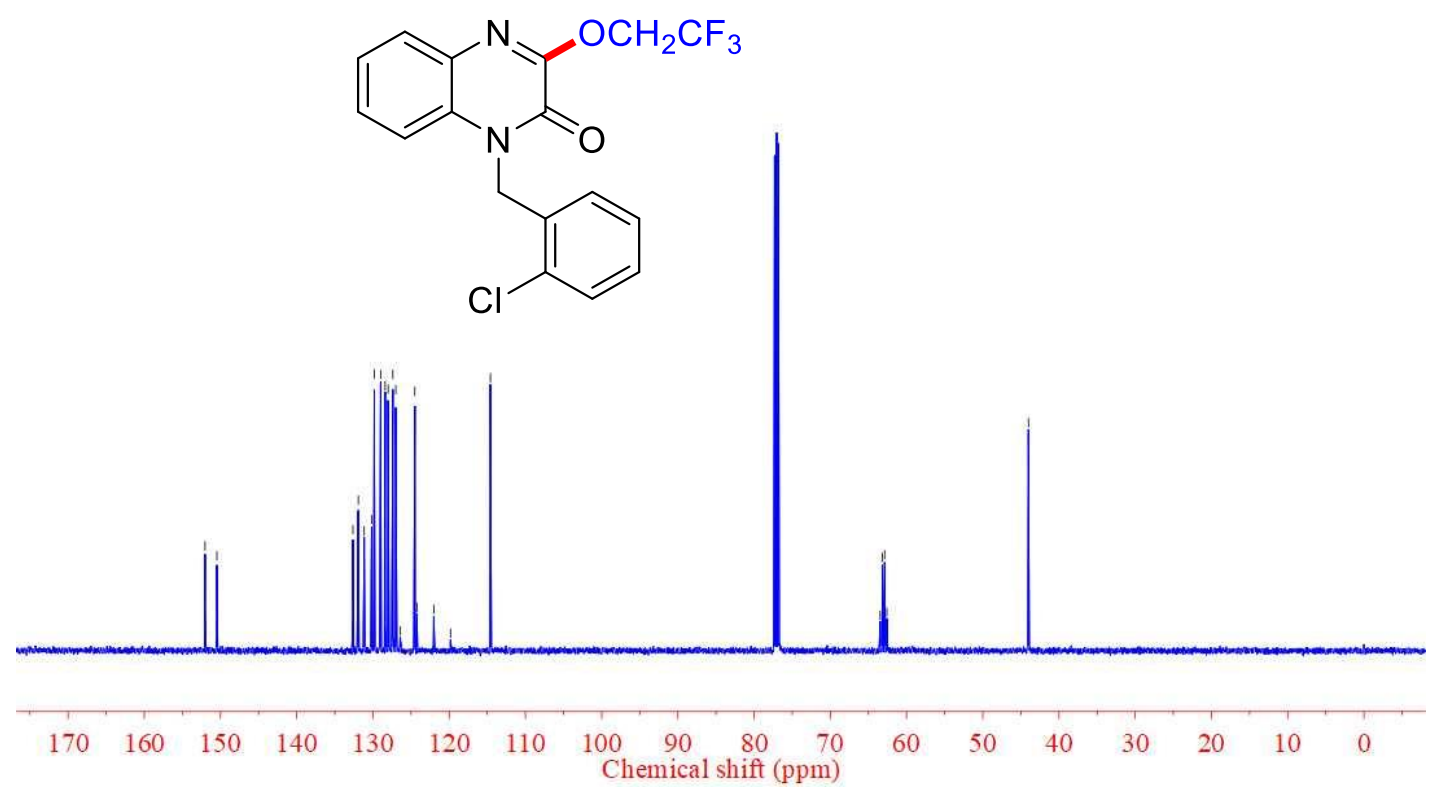

$3 t^{19}$ F NMR

$\stackrel{a}{i}$<smiles>O=c1c(OCC(F)(F)F)nc2ccccc2n1Cc1ccccc1Cl</smiles>

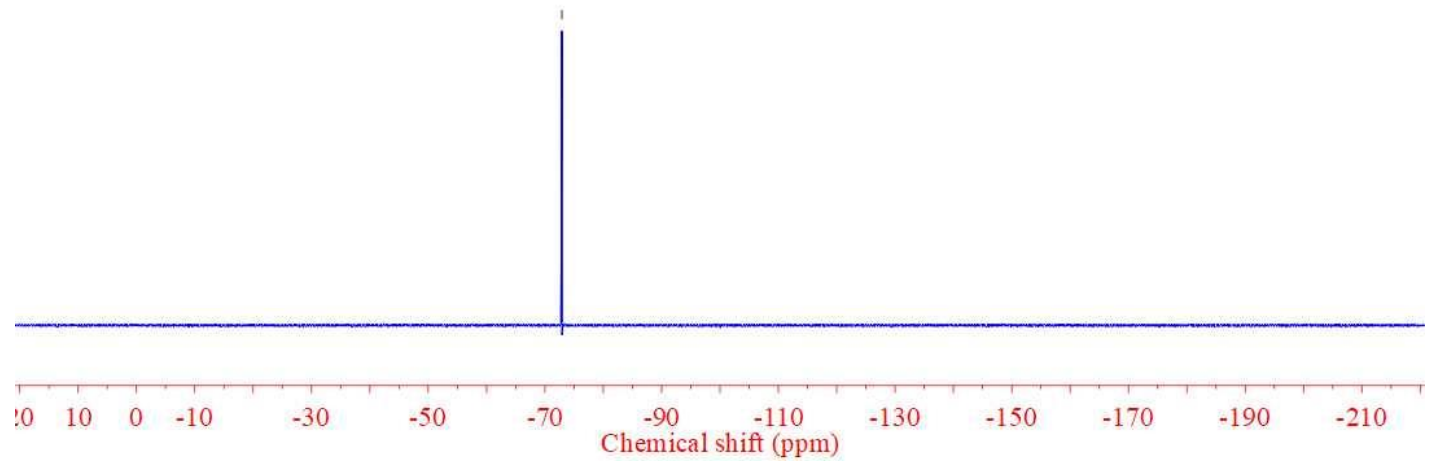




\section{$3 \mathbf{u}^{1}$ H NMR}

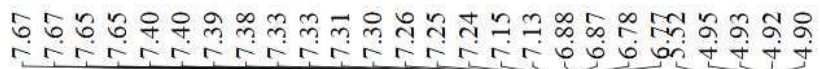

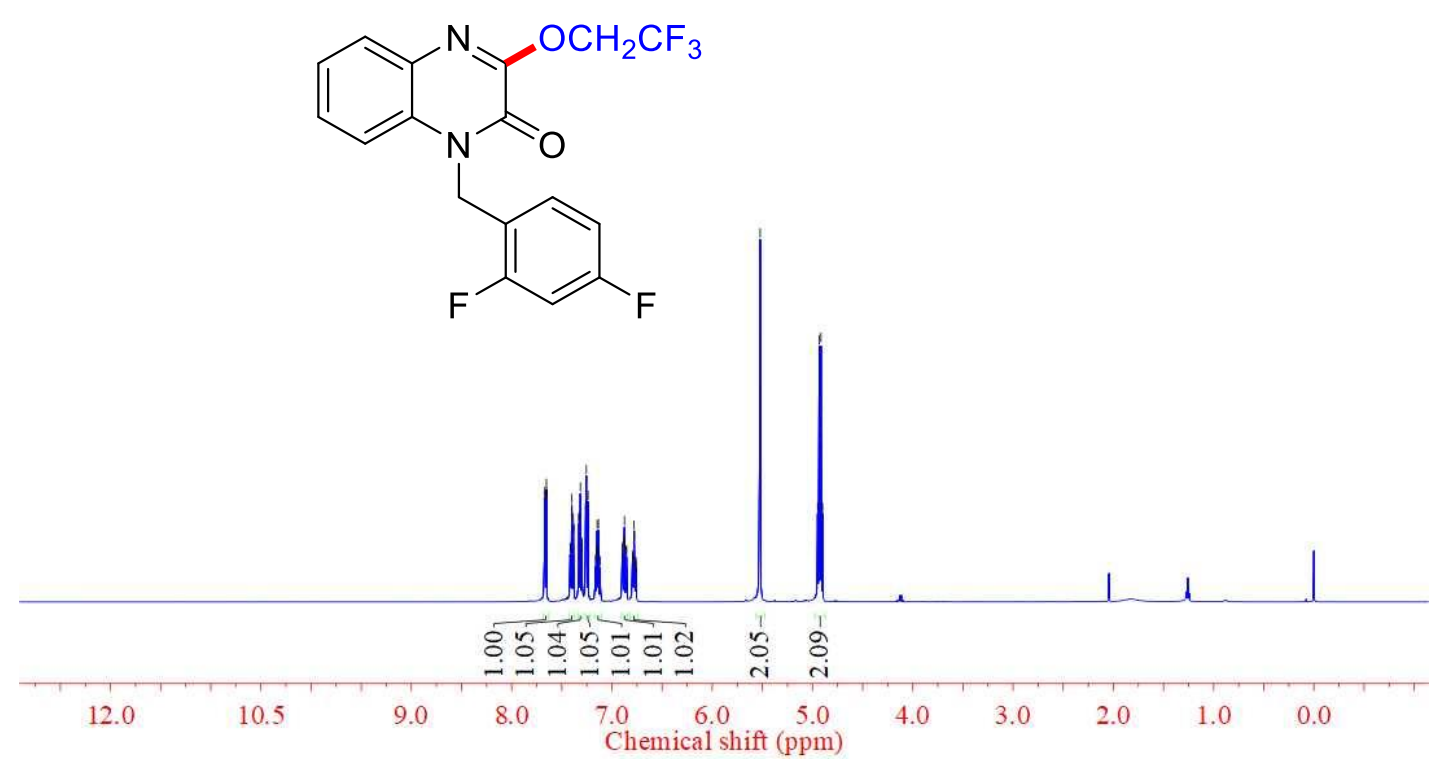

\section{$3{ }^{13}$ C NMR}

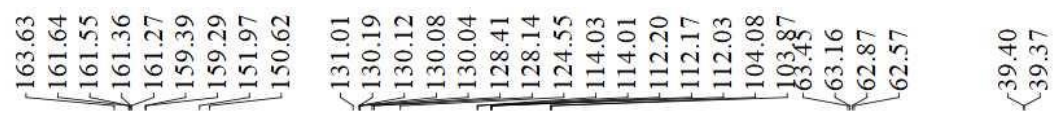

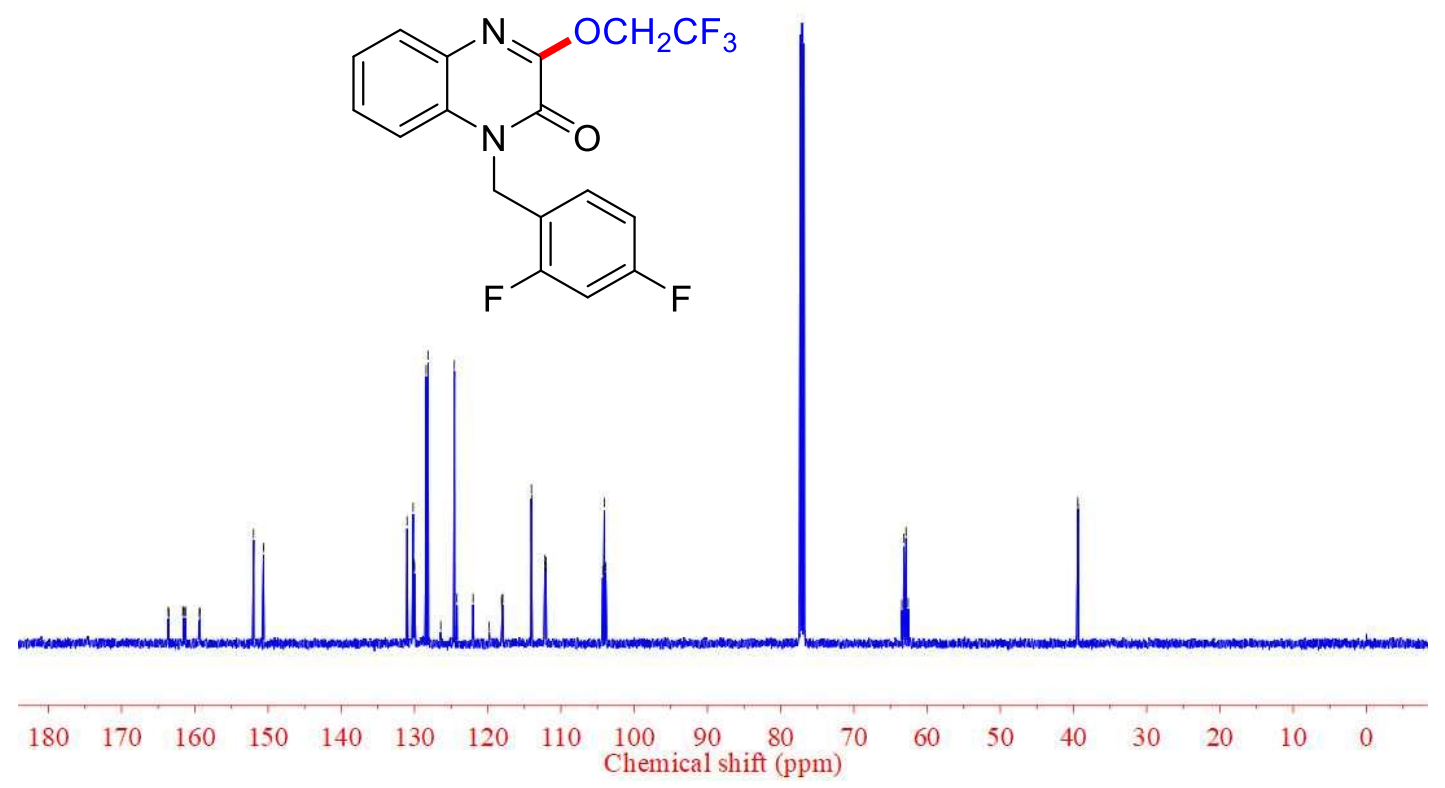




\section{$3 u{ }^{19}$ F NMR}

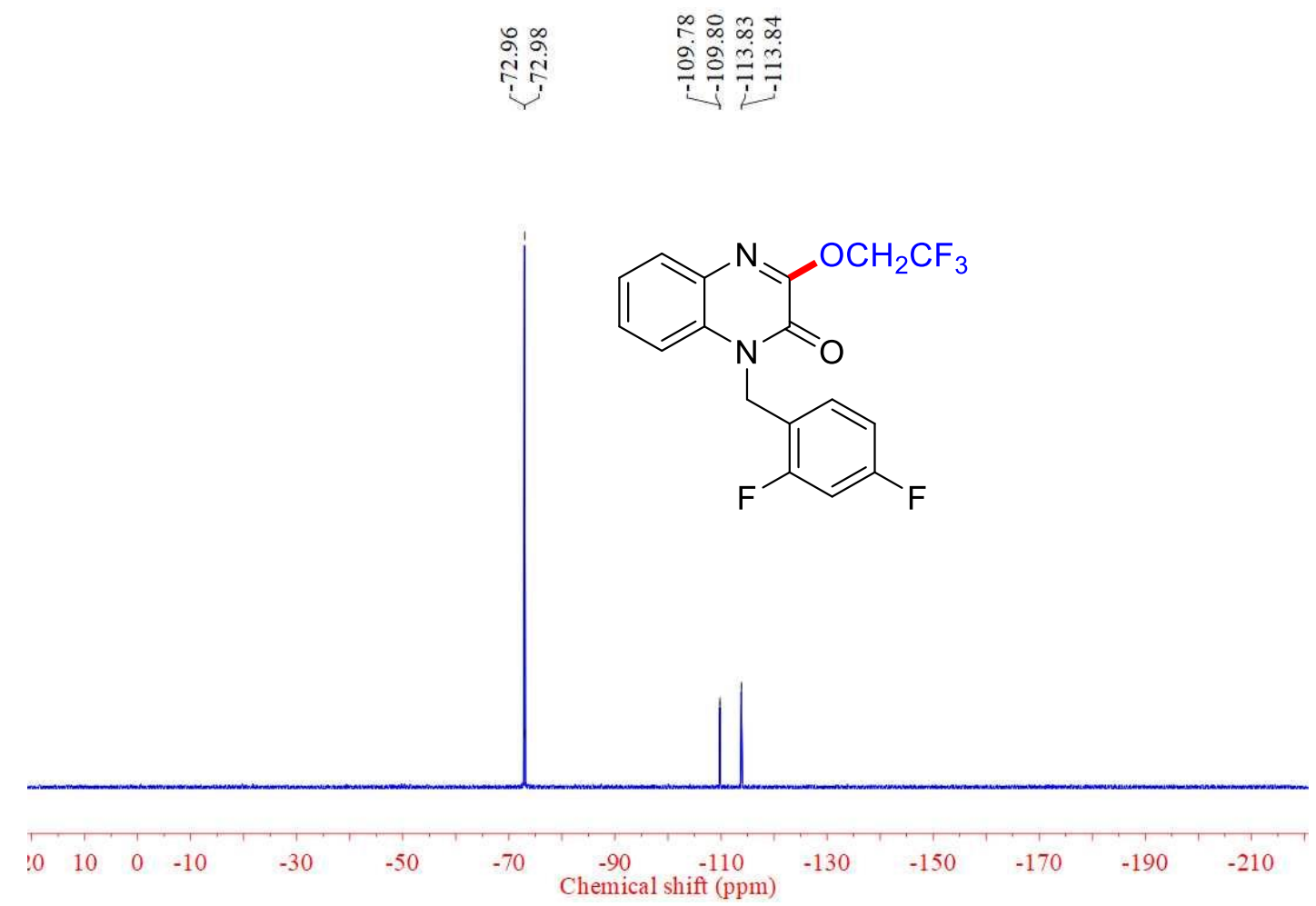

\section{$3 v^{1}$ H NMR}

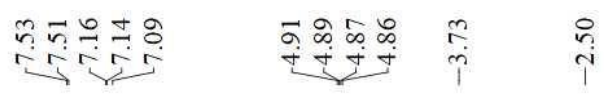

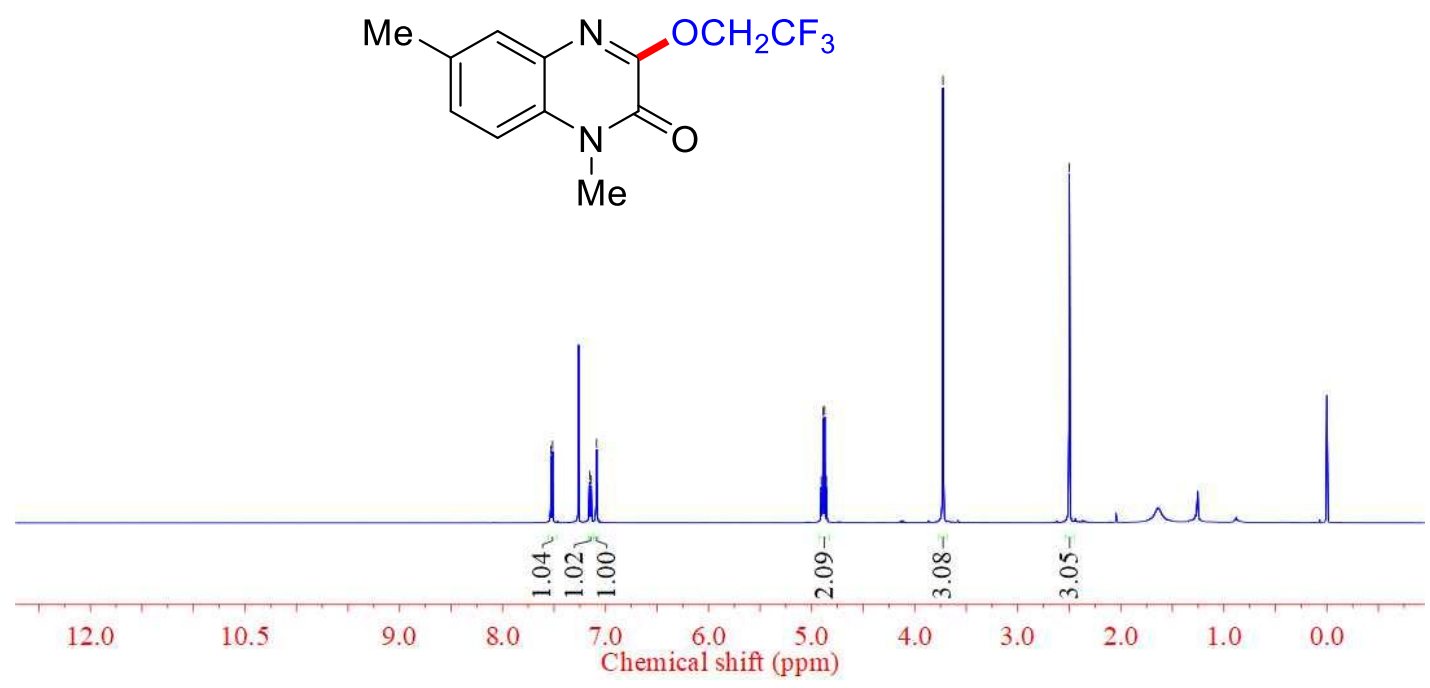




\section{$3 v{ }^{13}$ C NMR}

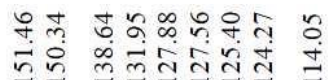

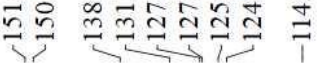

$\infty$
în
î̉

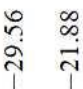
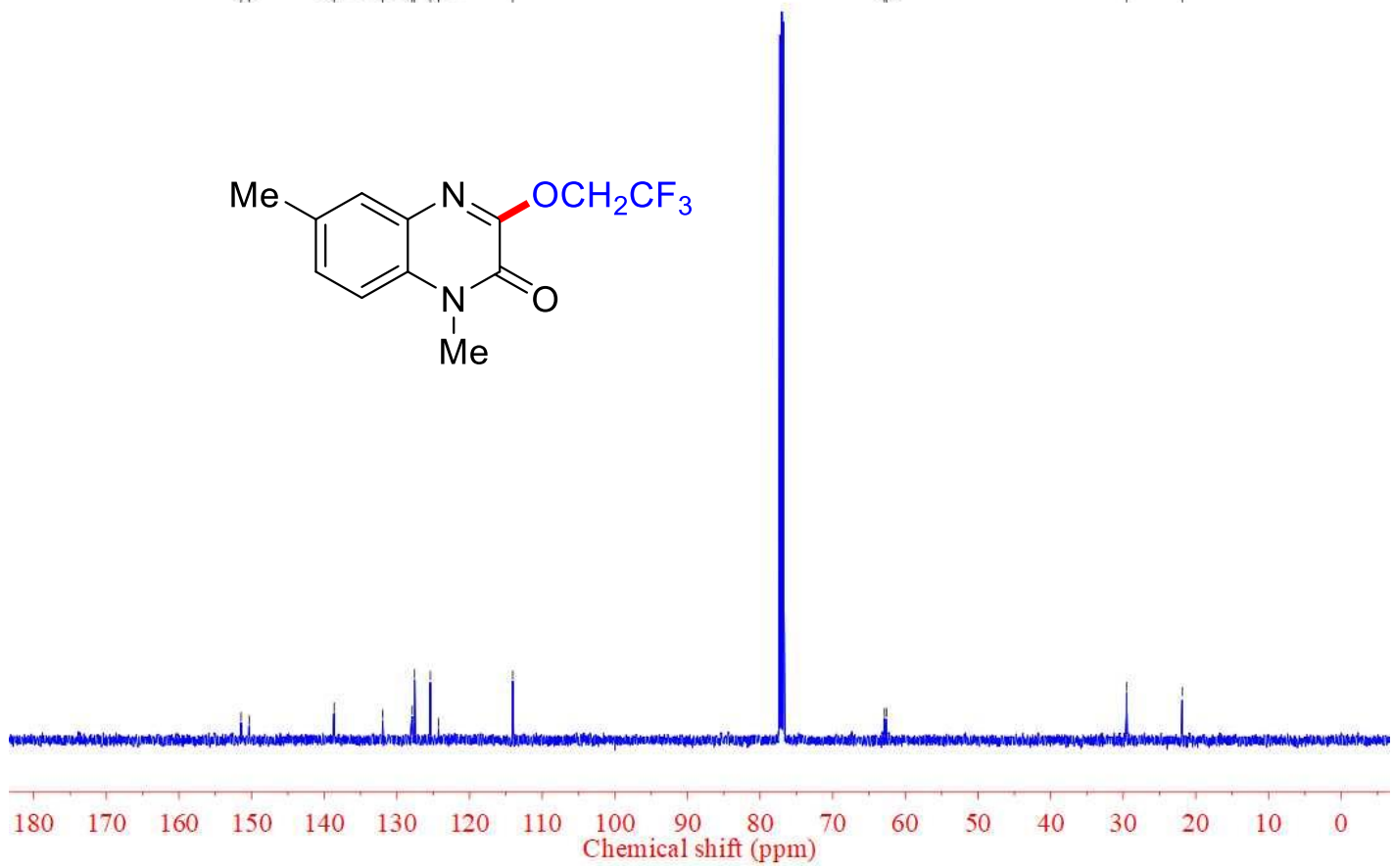

$3 v{ }^{19}$ F NMR

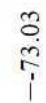<smiles>Cc1ccc2c(c1)nc(OCC(F)(F)F)c(=O)n2C</smiles>

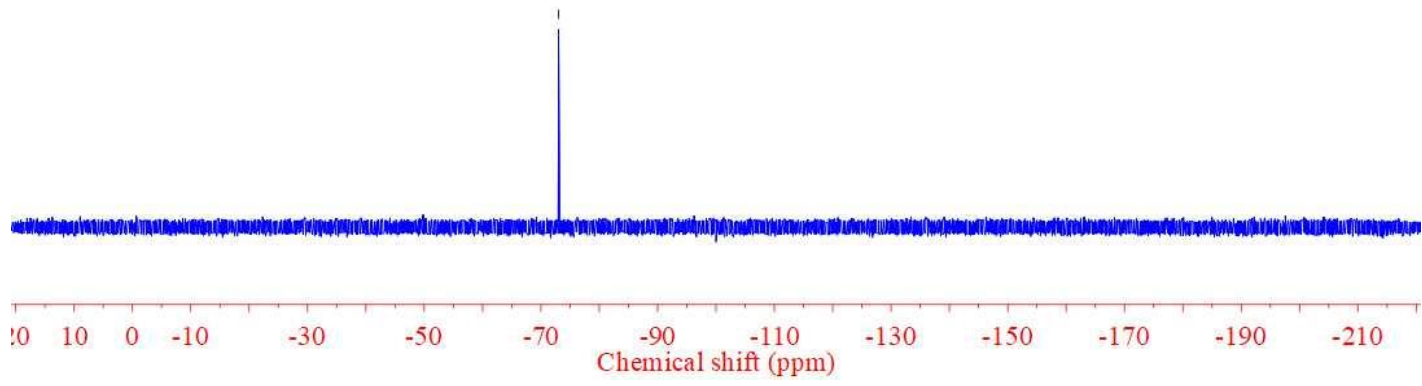




\section{3w ${ }^{1} \mathrm{H}$ NMR}

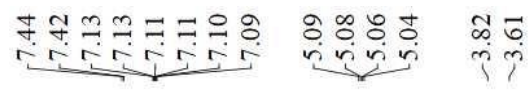

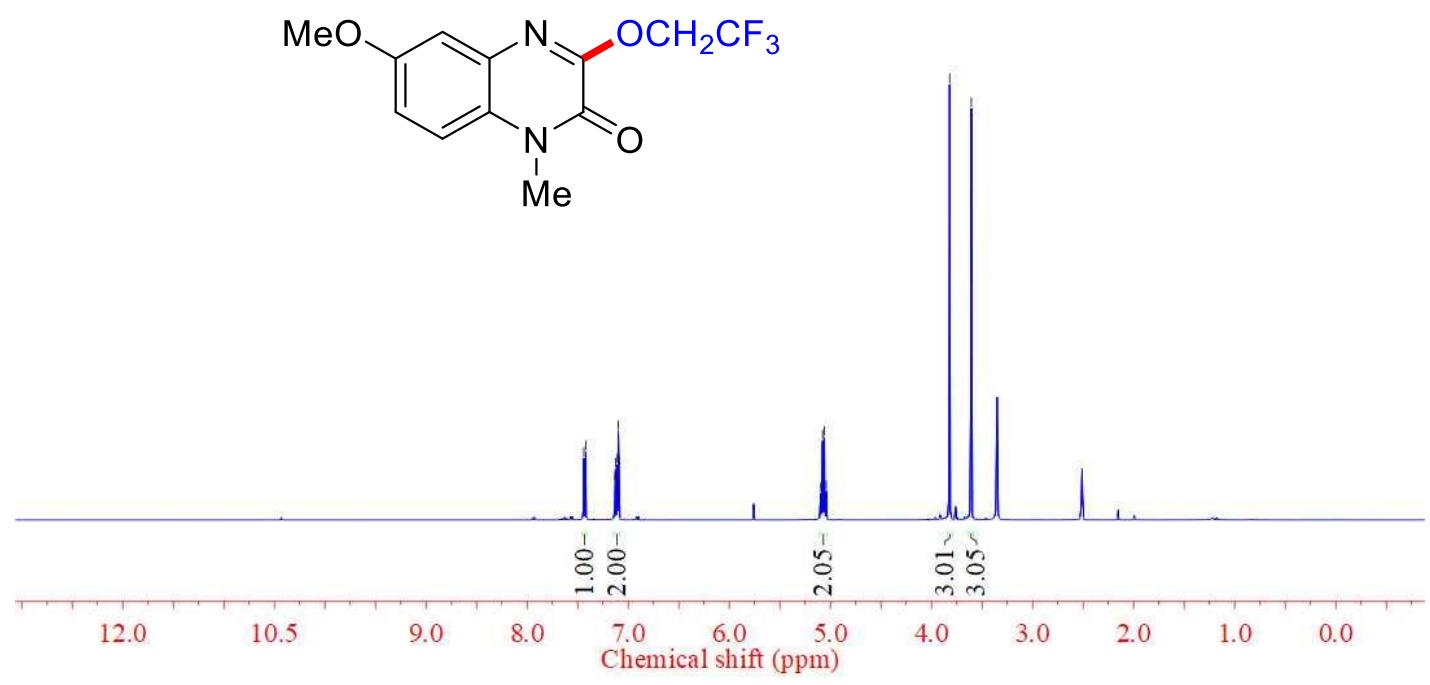

3w ${ }^{13}$ C NMR

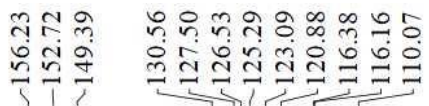

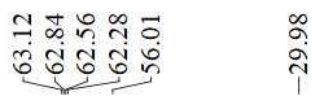

$\underbrace{\mathrm{MeO}}_{\substack{1 \\ \mathrm{Me}}} \mathrm{OCH}_{2} \mathrm{CF}_{3}$

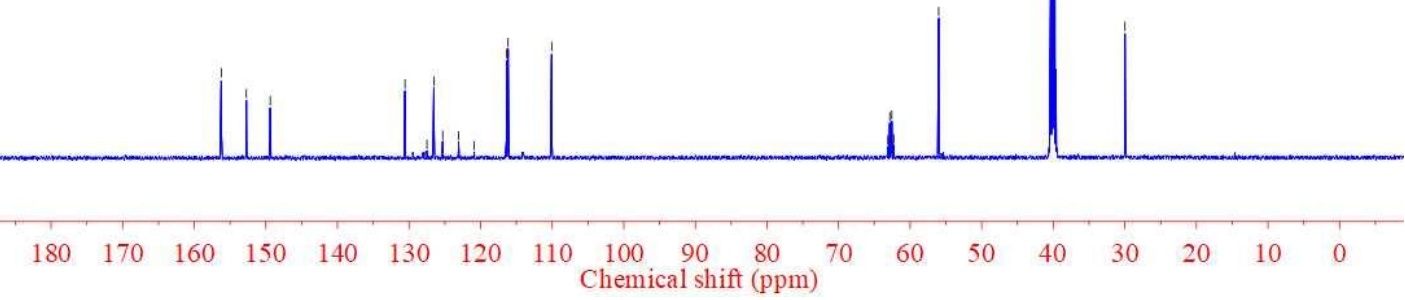




\section{$3 \mathrm{w}^{19}$ F NMR}

$$
\frac{5}{i}
$$

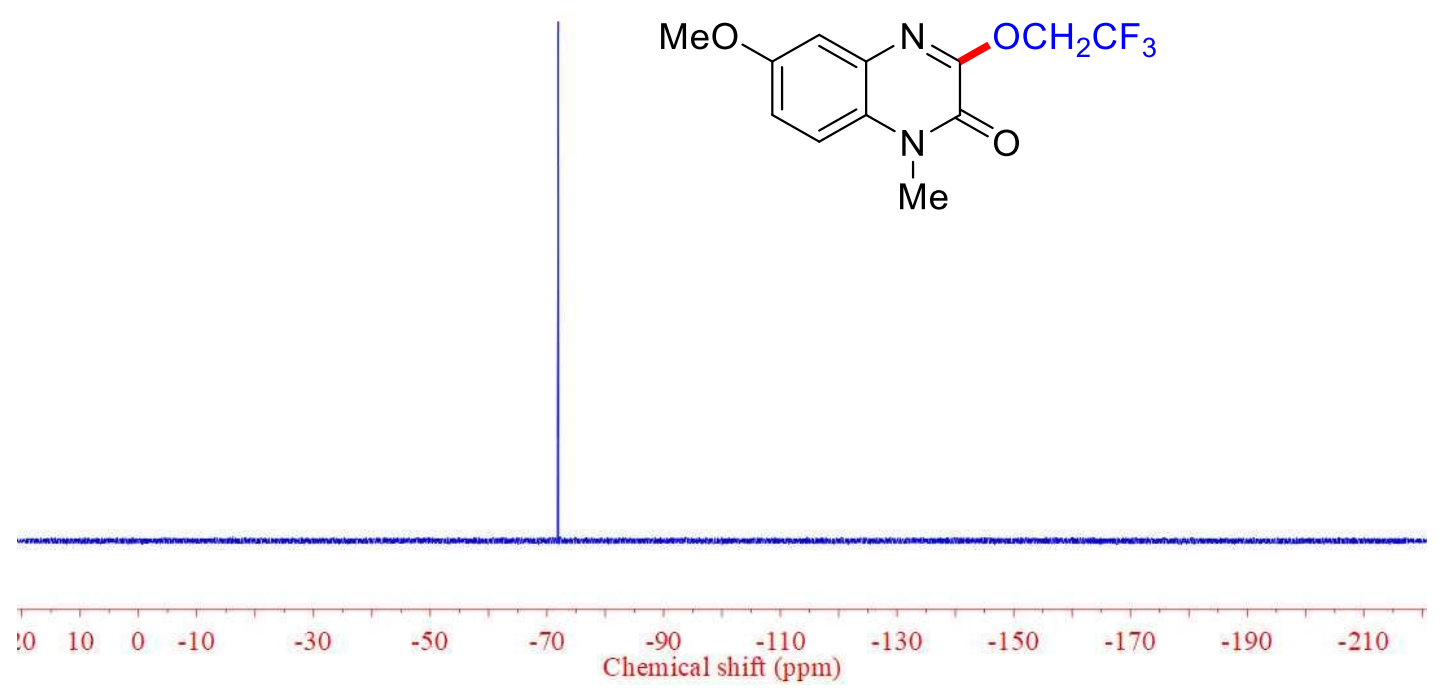

\section{$3 \mathbf{x}^{1}$ H NMR}

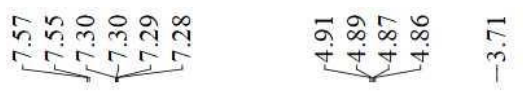

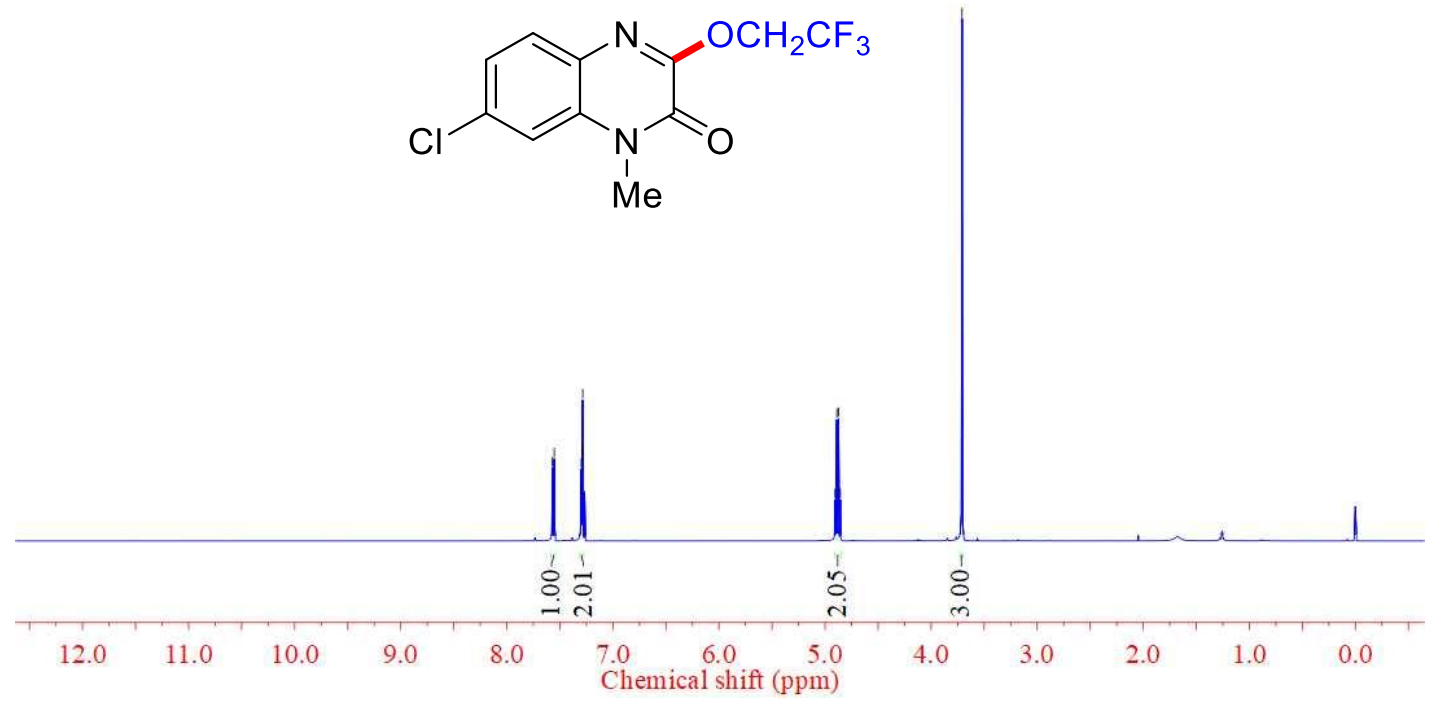




\section{$3 \mathrm{X}^{13} \mathrm{C}$ NMR}

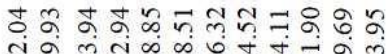

तु

ํํㅇํำ

की

$\stackrel{2}{2}$

iิ<smiles>Cn1c(=O)c(OCC(F)F)nc2ccc(Cl)cc21</smiles>

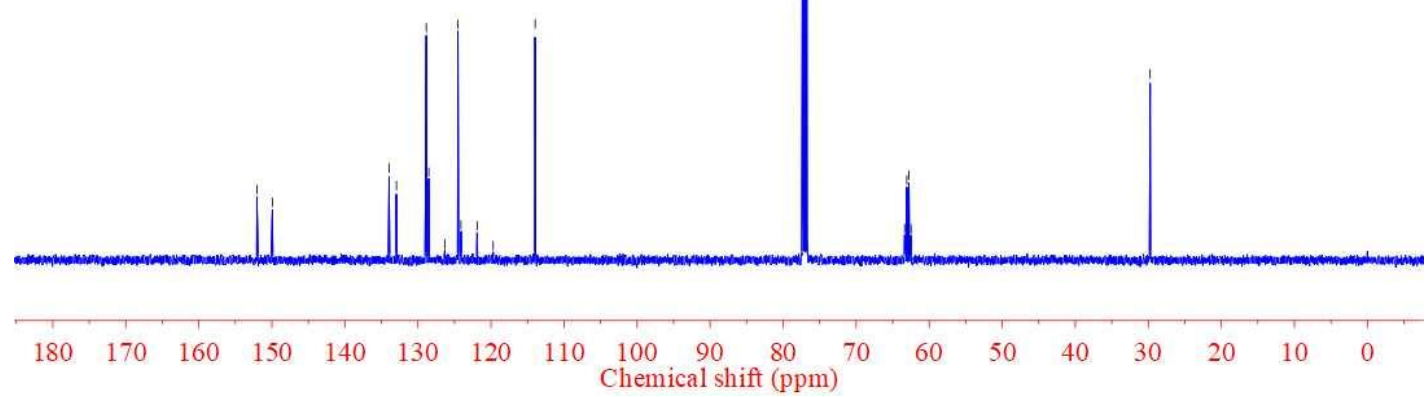

$3 x^{19}$ F NMR

๙ั<smiles>Cn1c(=O)c(OCC(F)(F)F)nc2ccc(Cl)cc21</smiles>

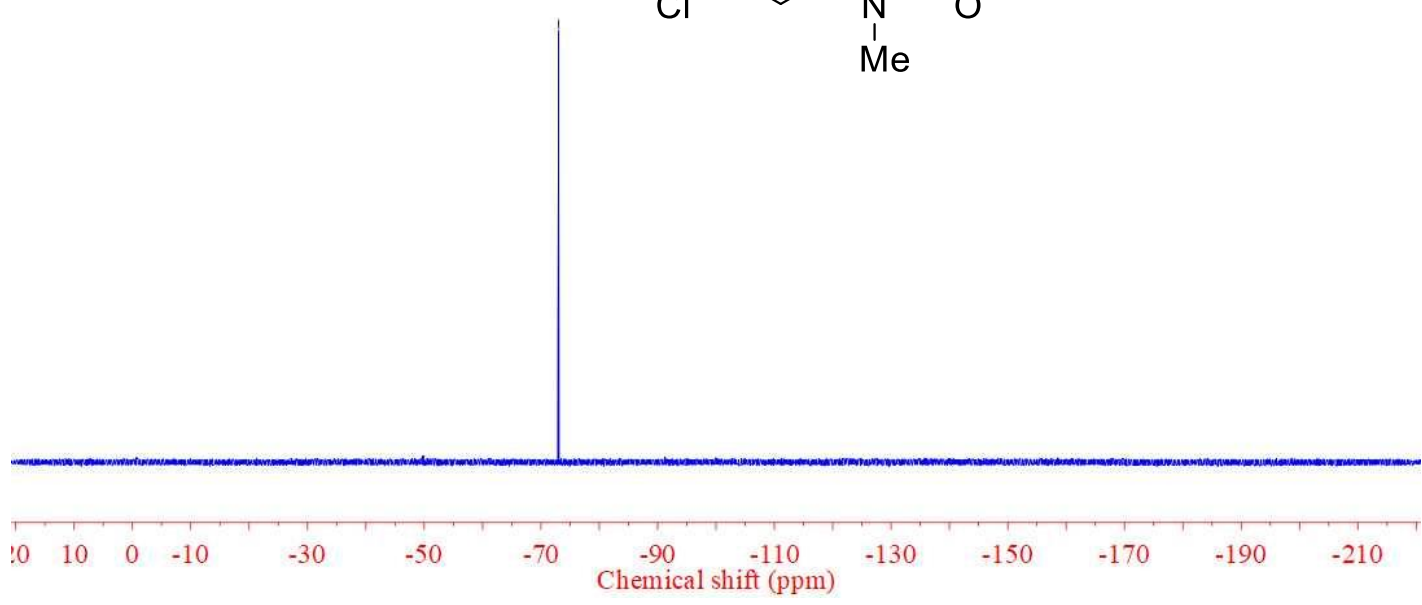




\section{$3{ }^{1}$ H NMR}

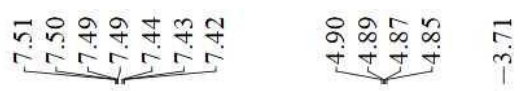

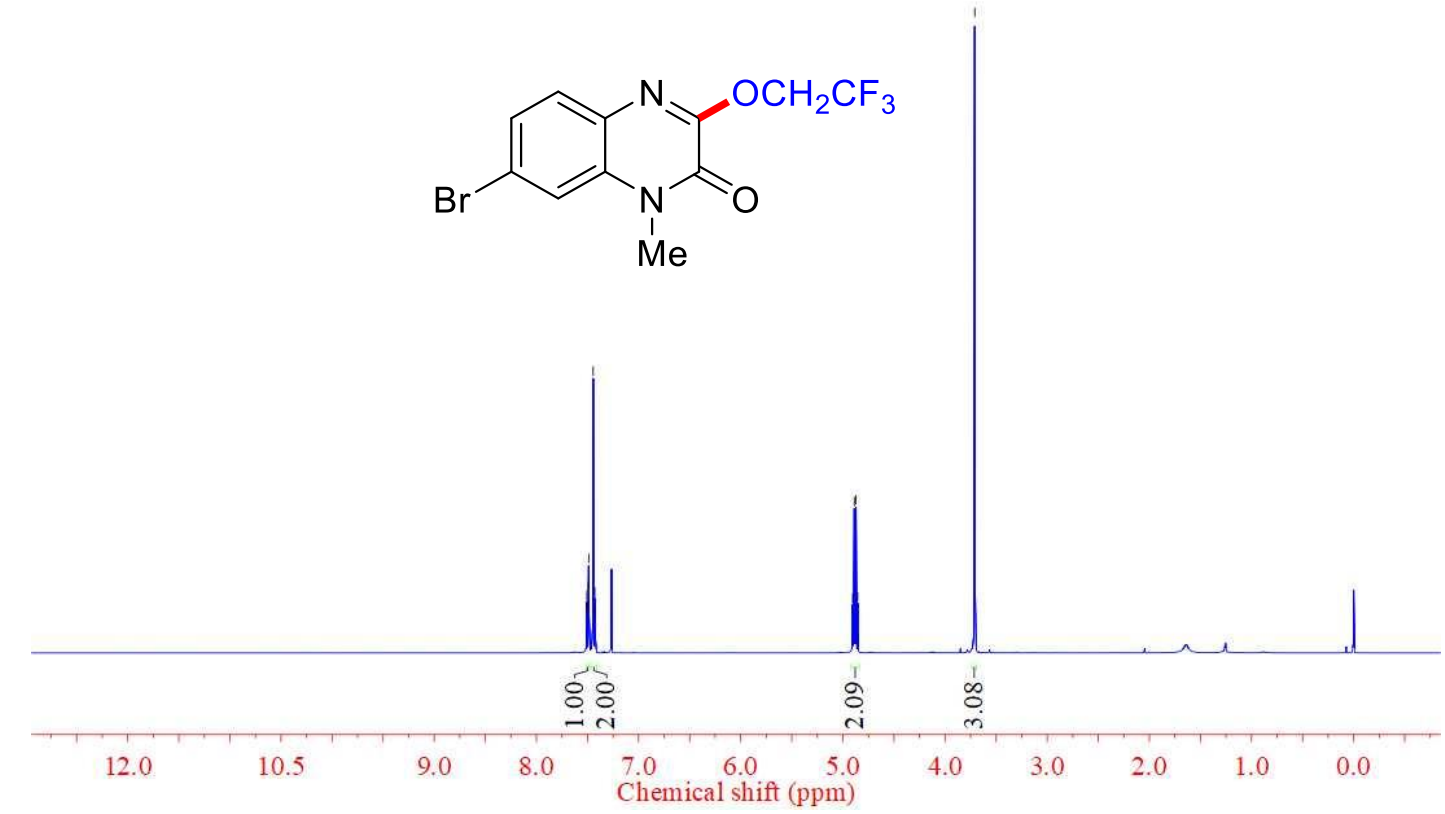

$3 y{ }^{13}$ C NMR

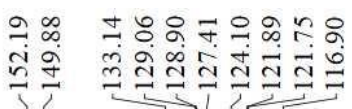

m

ชู่

$\stackrel{2}{2}$<smiles>Cn1c(=O)c(OCC(F)(F)F)nc2ccc(Br)cc21</smiles>

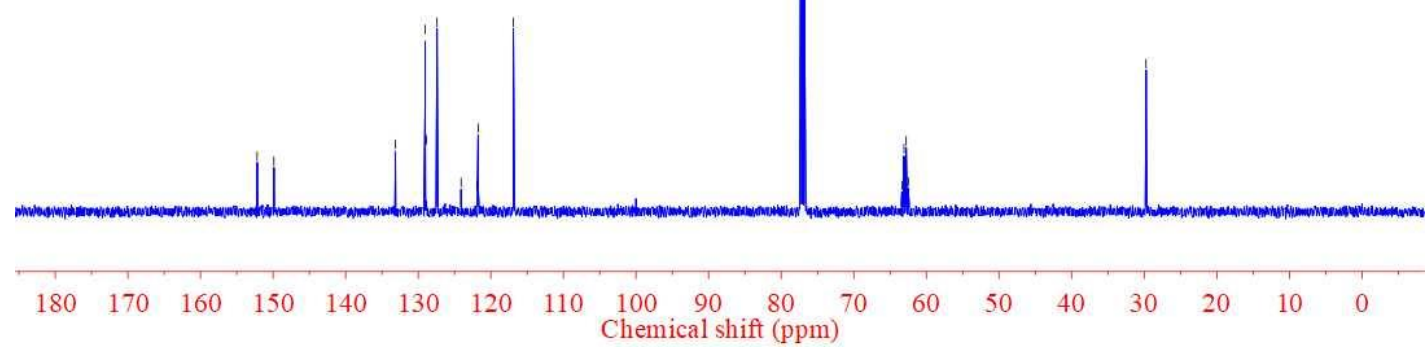




\section{$3 y{ }^{19}$ F NMR}

$\frac{\tilde{c}}{\mathfrak{r}}$<smiles>Cn1c(=O)c(OCC(F)(F)F)nc2ccc(Br)cc21</smiles>

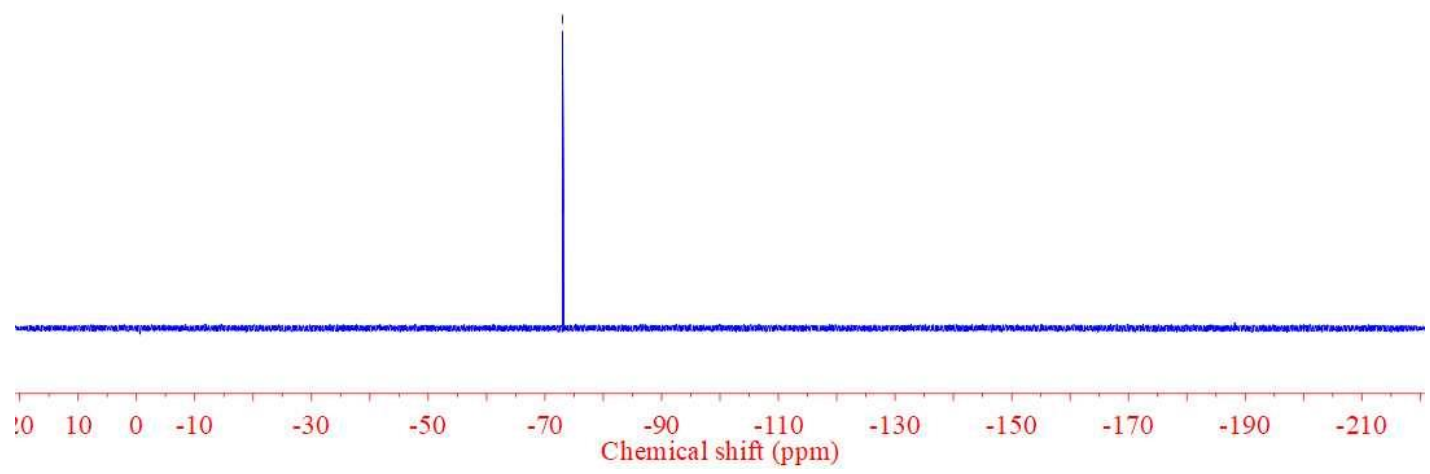

\section{$3 z^{1}$ H NMR}

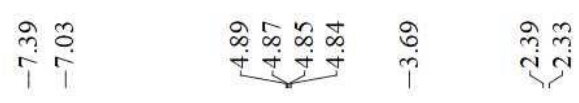

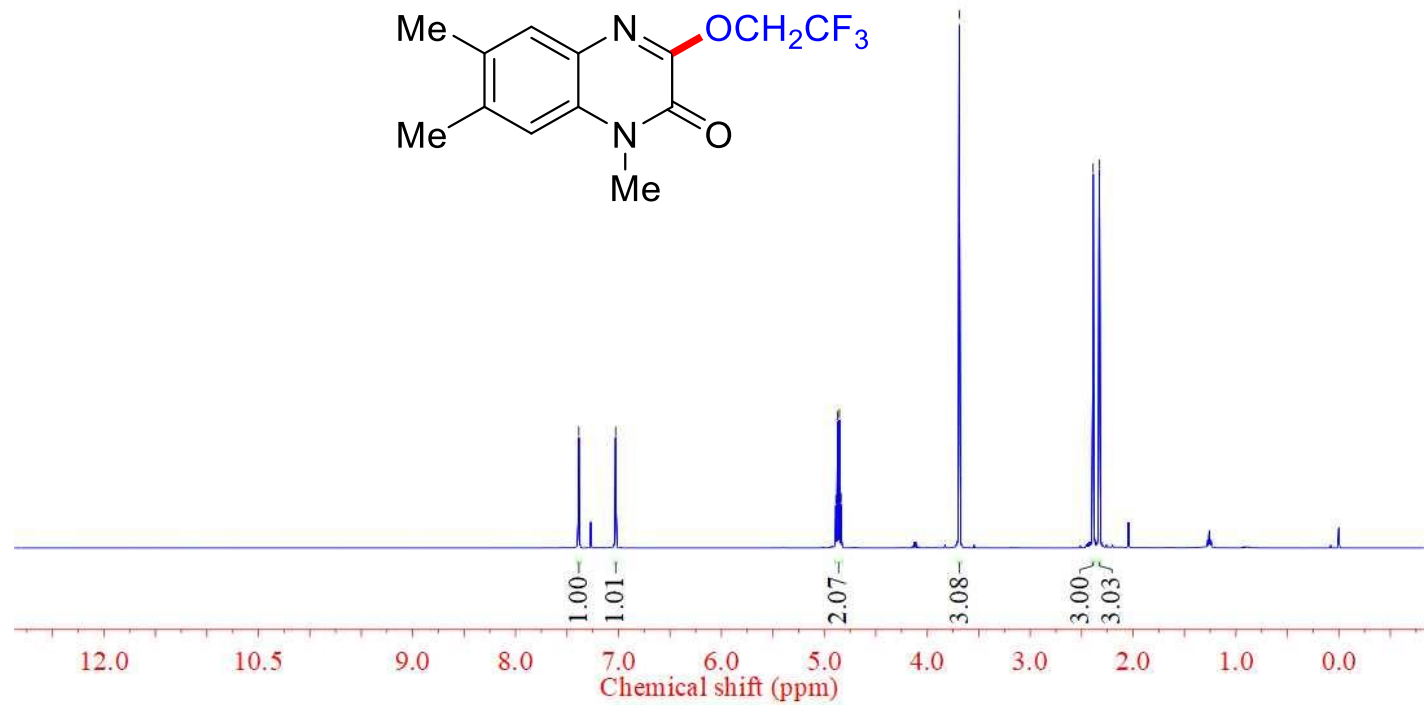




\section{$3 z^{13}$ C NMR}

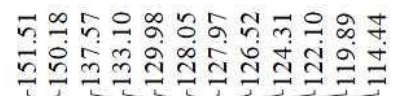

웅 원워

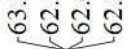

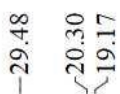<smiles>Cc1cc2nc(OCC(F)(F)F)c(=O)n(C)c2cc1C</smiles>

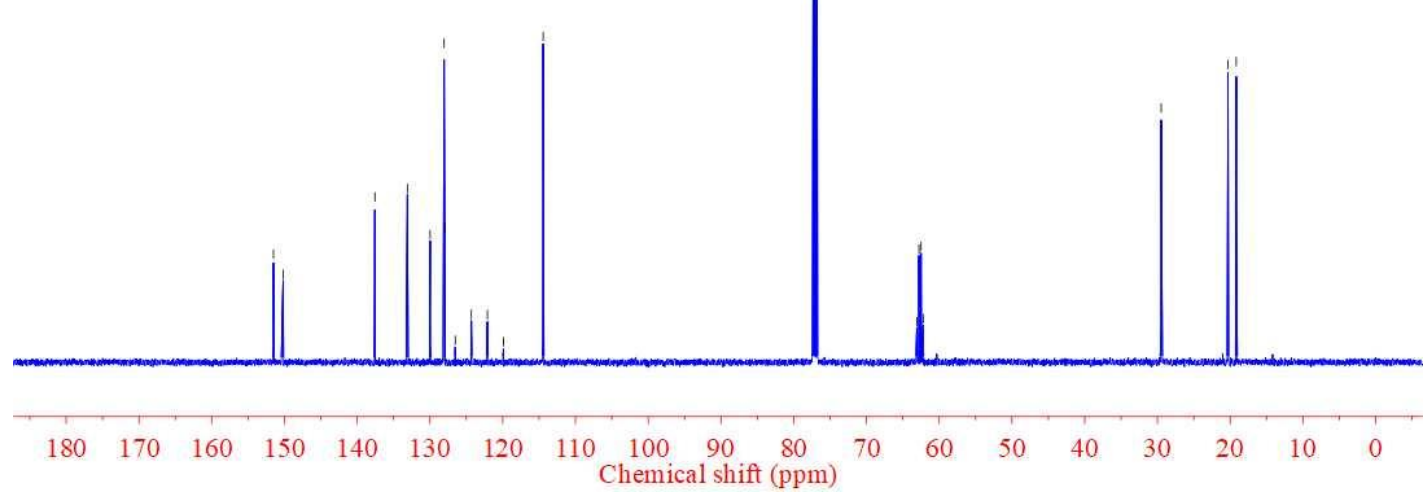

\section{$3 z^{19}$ F NMR}

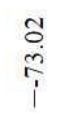<smiles>Cc1cc2nc(OCC(F)(F)F)c(=O)n(C)c2cc1C</smiles>

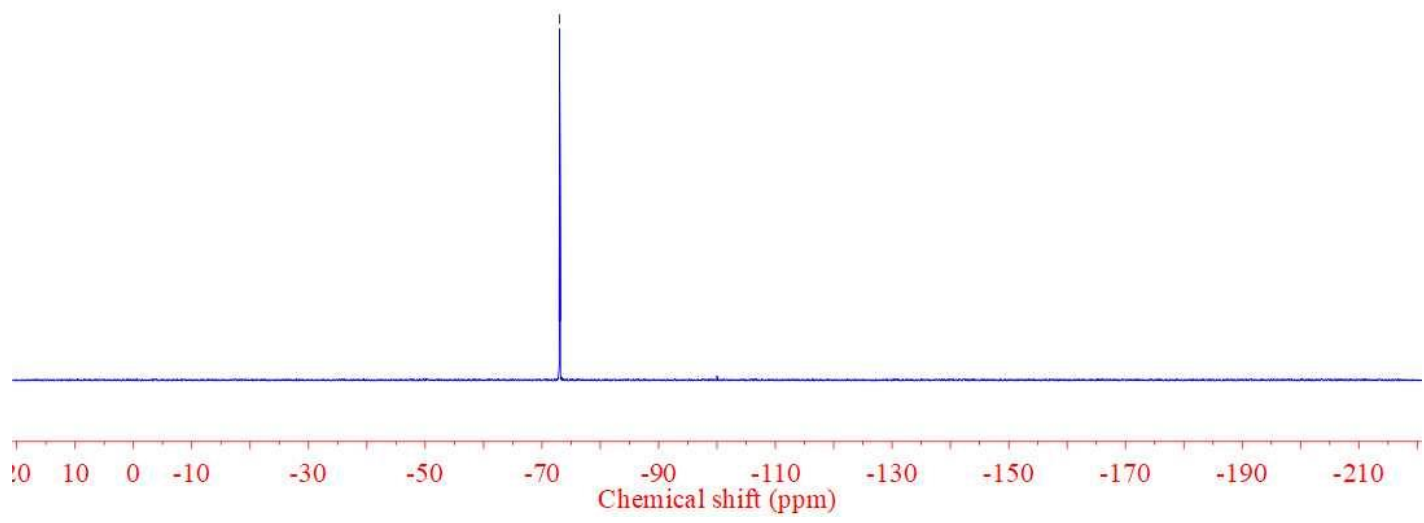




\section{3aa ${ }^{1}$ H NMR}

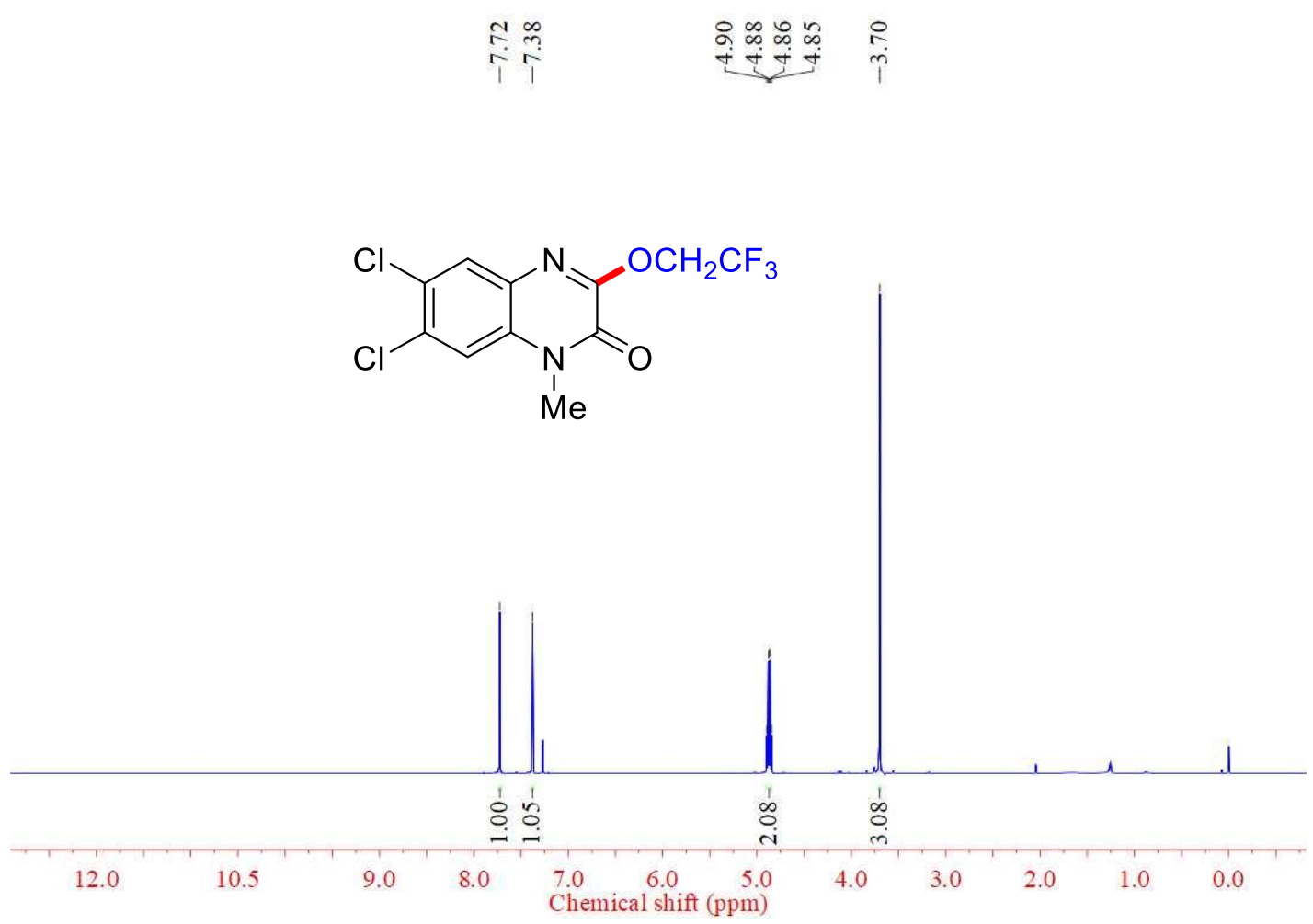

\section{3aa ${ }^{13}$ C NMR}

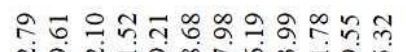

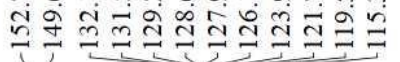

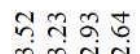

กิ่ กิ่

ลั่<smiles>Cn1c(=O)c(OCC(F)(F)F)nc2cc(Cl)c(Cl)cc21</smiles>

Me

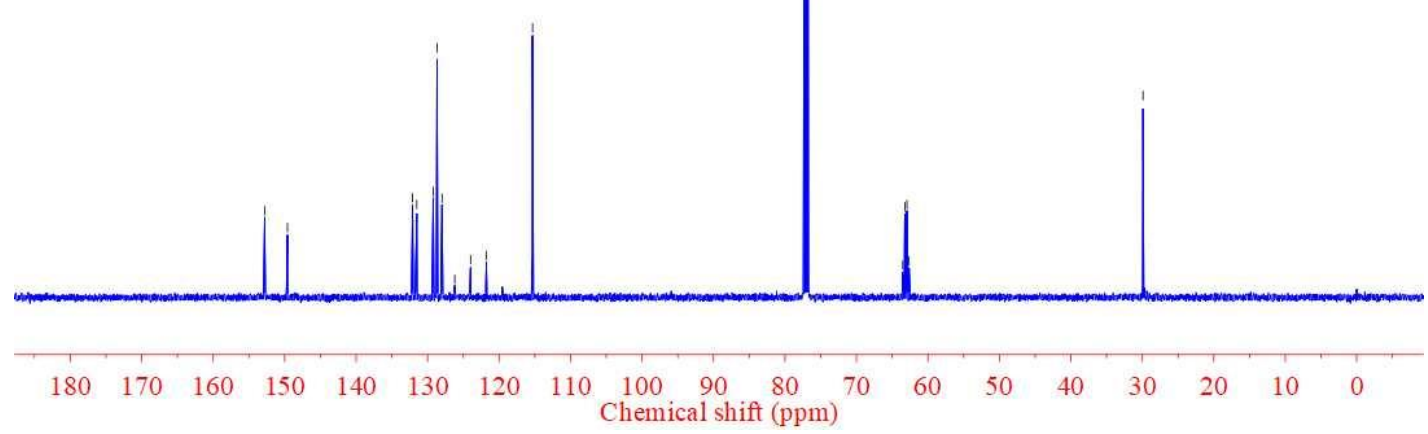




\section{3aa ${ }^{19}$ F NMR}

$$
\text { ja }
$$

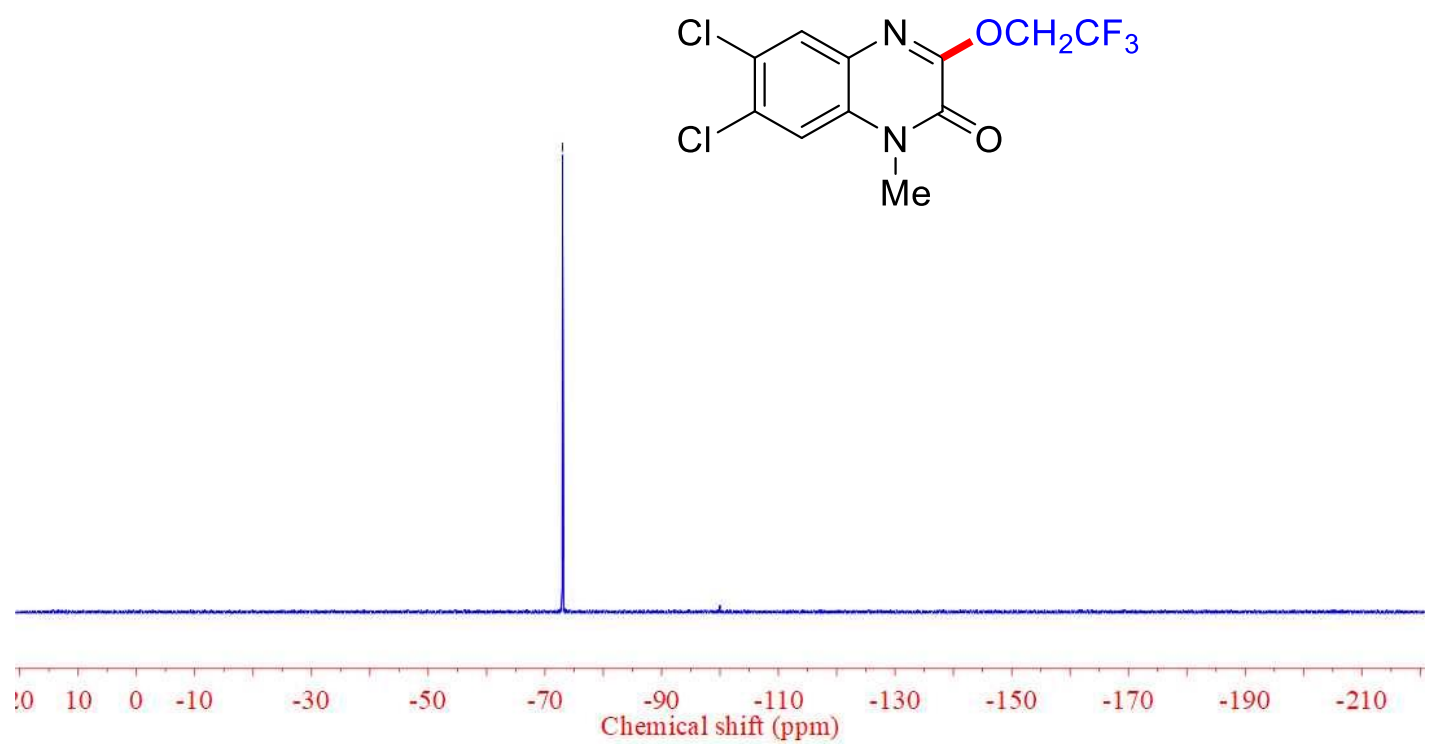

\section{4a ${ }^{1} H$ NMR}

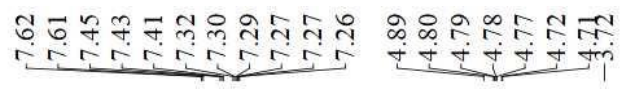

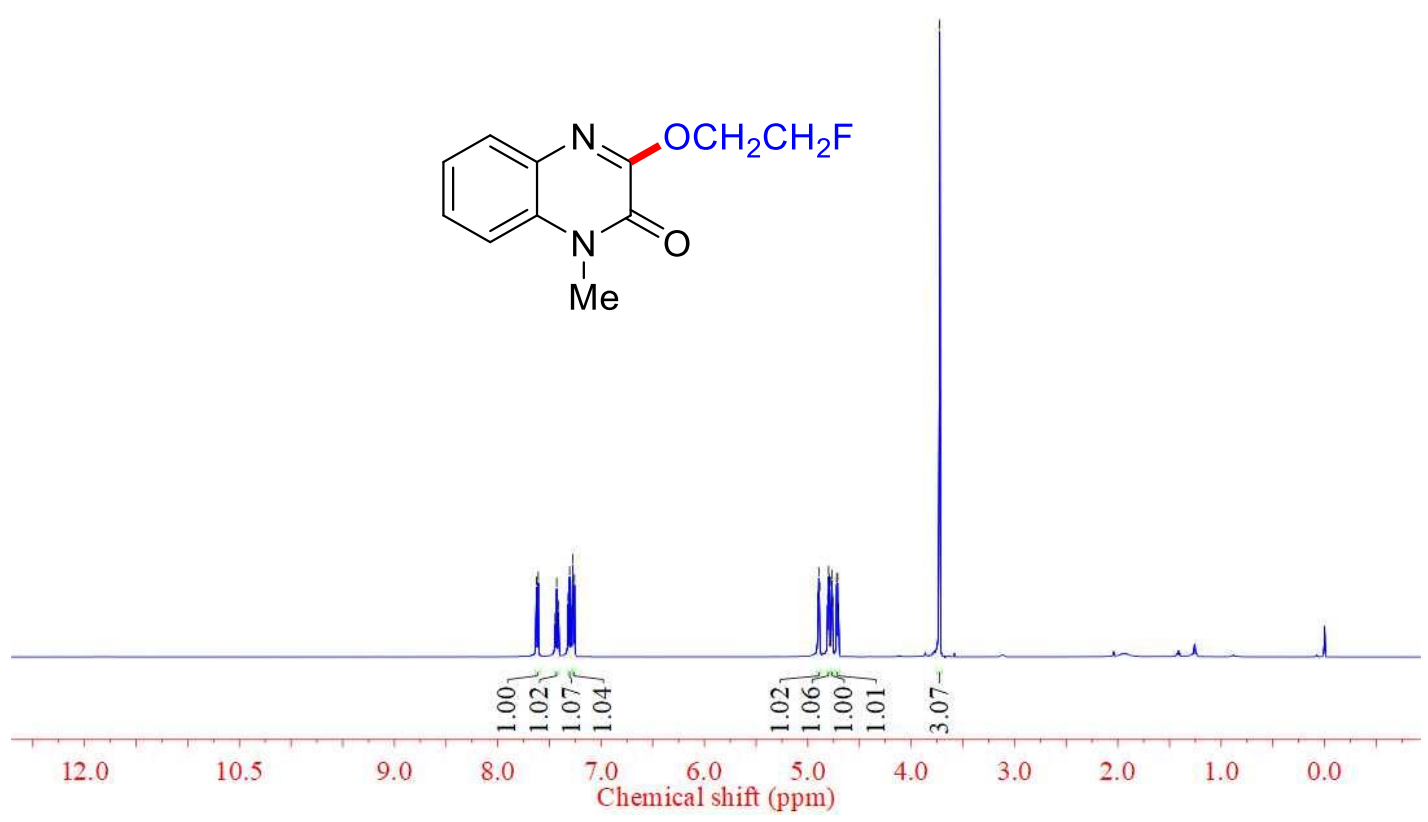




\section{$4 a^{13}$ C NMR}

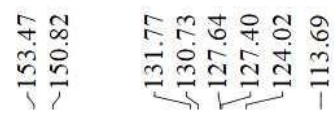

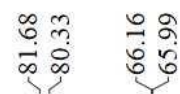

กั่<smiles>Cn1c(=O)c(OCCF)nc2ccccc21</smiles>

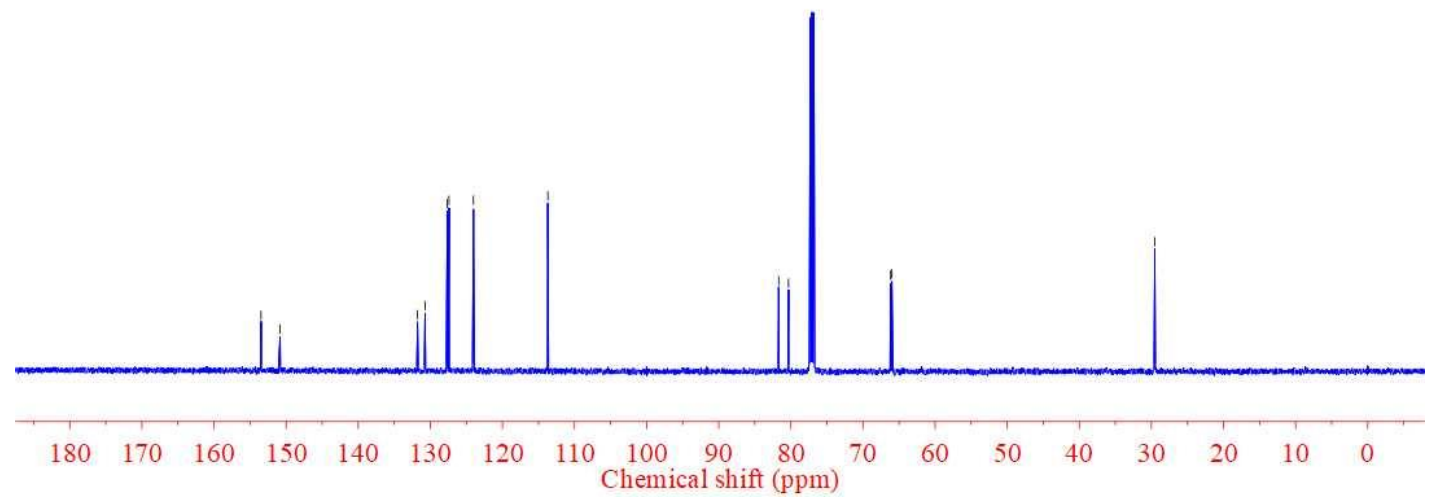

\section{$4 a^{19}$ F NMR}<smiles>Cn1c(=O)c(OCCF)nc2ccccc21</smiles>

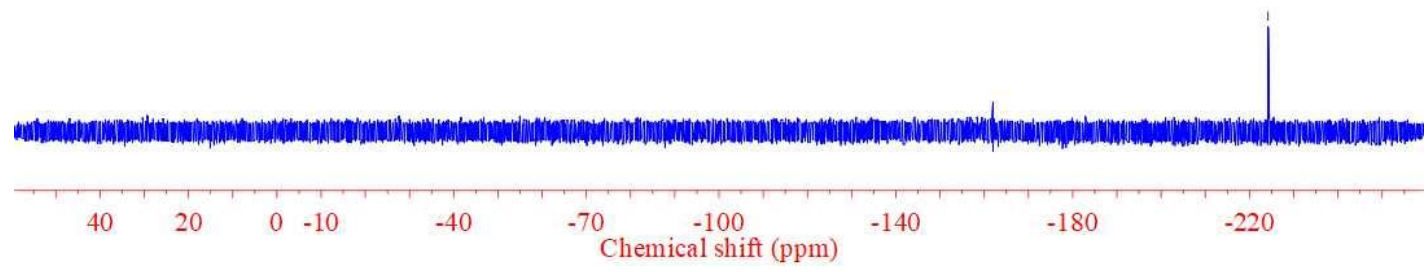




\section{$4 b^{1}$ H NMR}

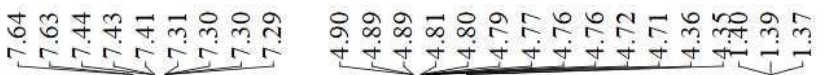

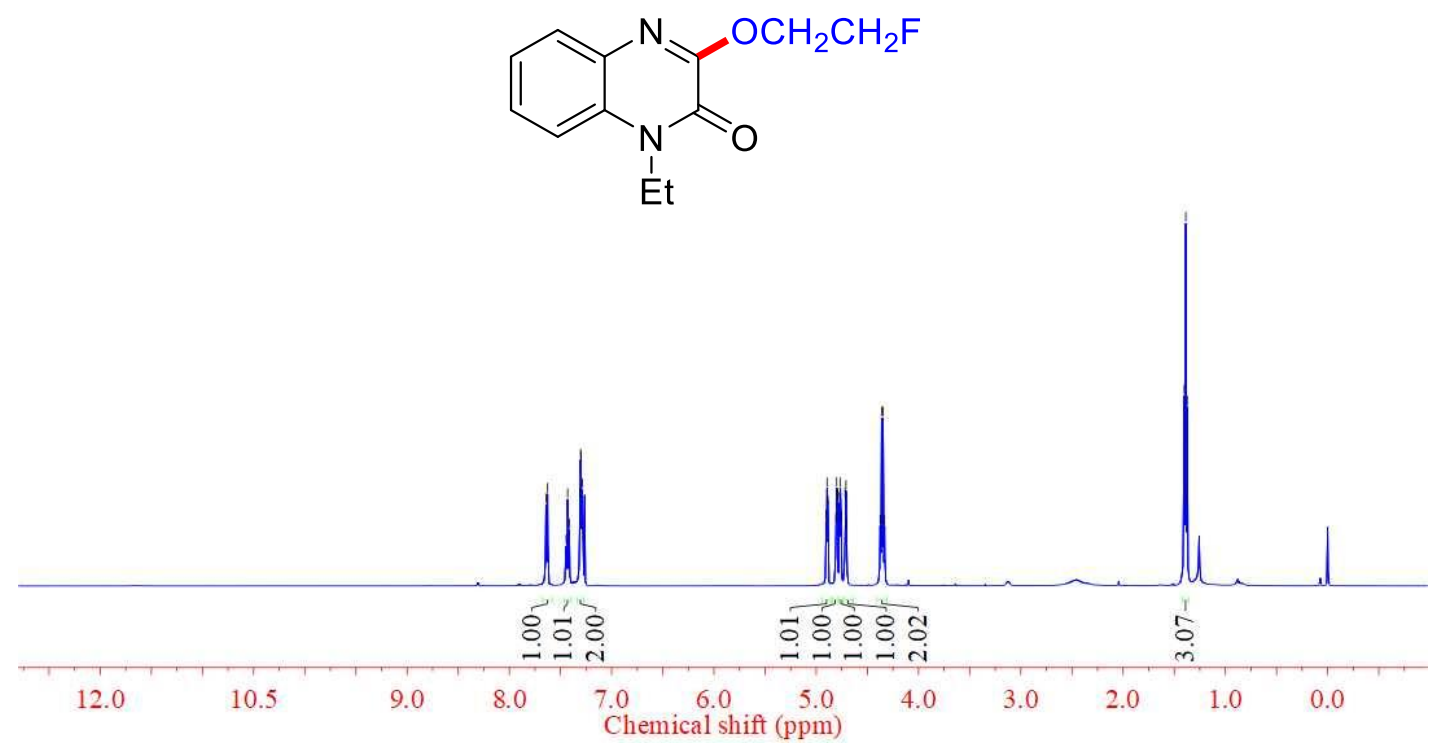

$4 b{ }^{13} \mathrm{C}$ NMR

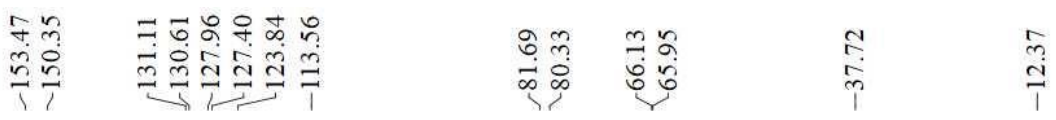<smiles>CCn1c(=O)c(OCCF)nc2ccccc21</smiles>

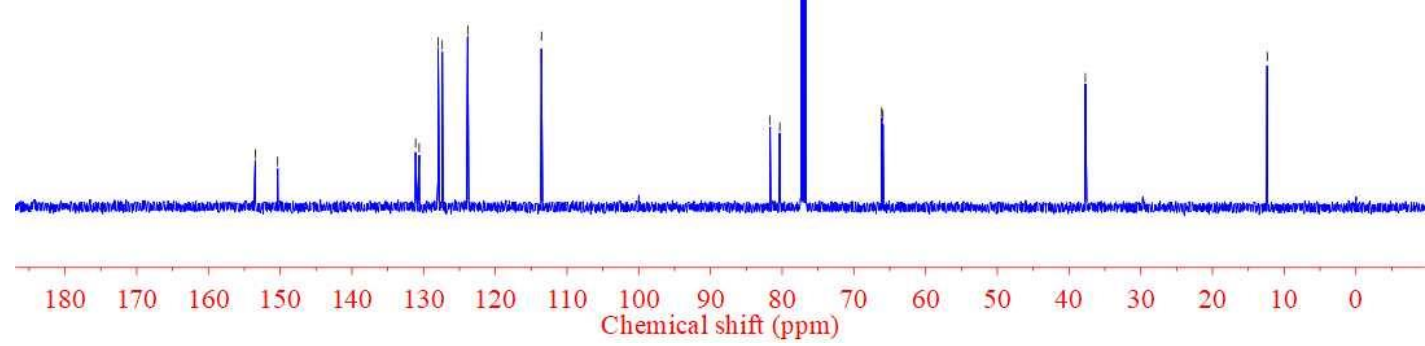




\section{$4 b{ }^{19}$ F NMR}
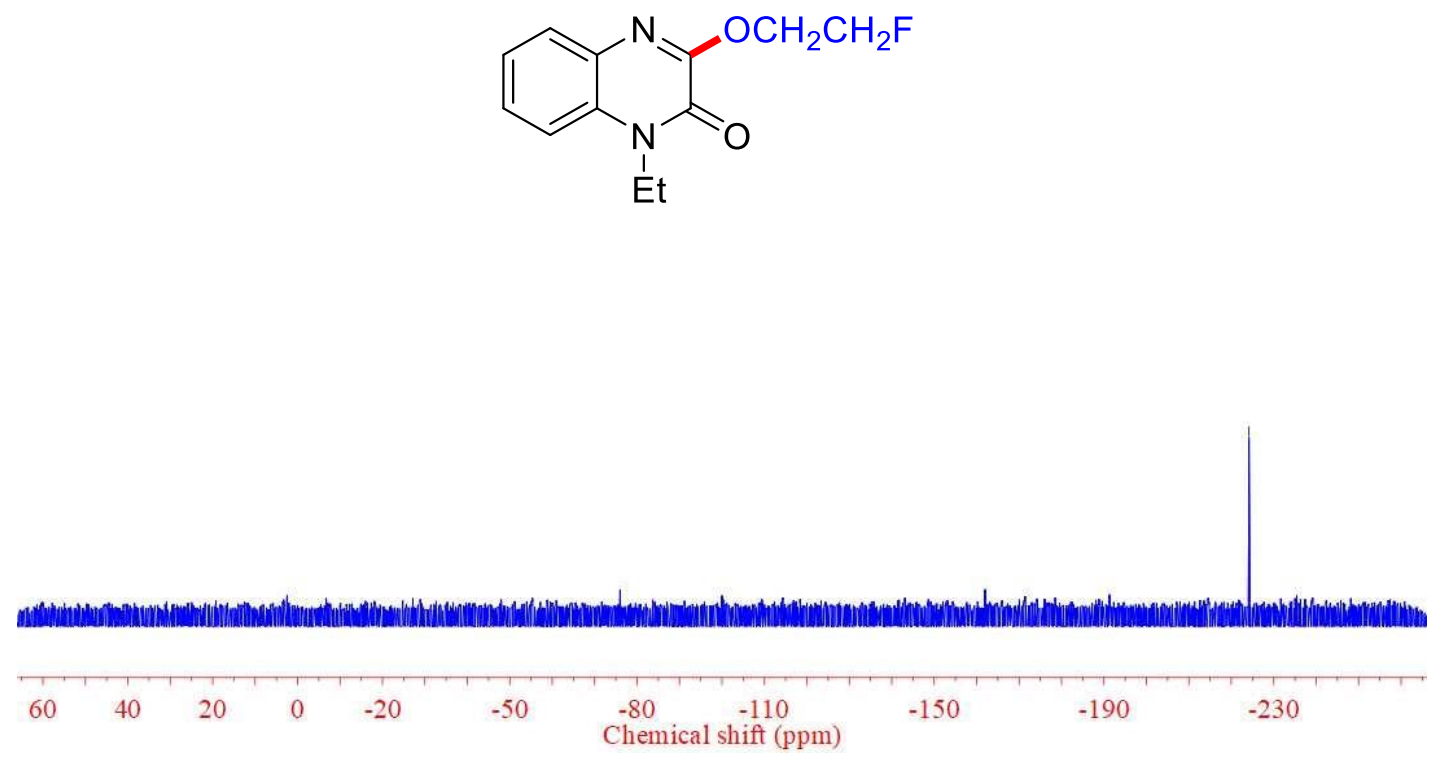

\section{$4 c^{1} H$ NMR}

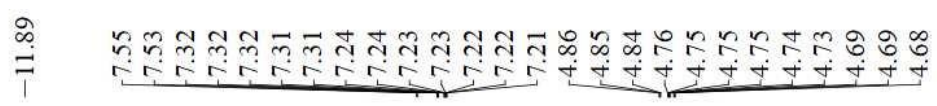<smiles>O=c1[nH]c2ccccc2nc1OCCF</smiles>

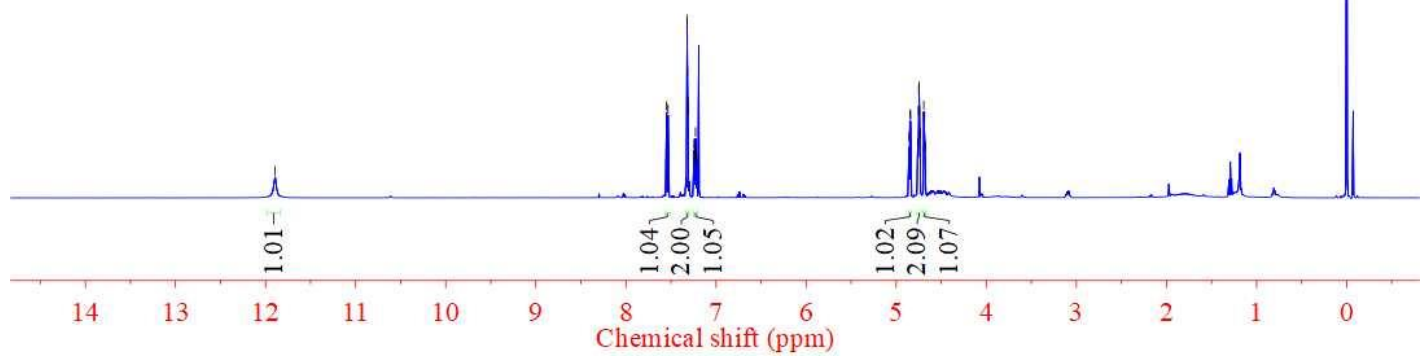


$4 c^{13} \mathrm{C}$ NMR

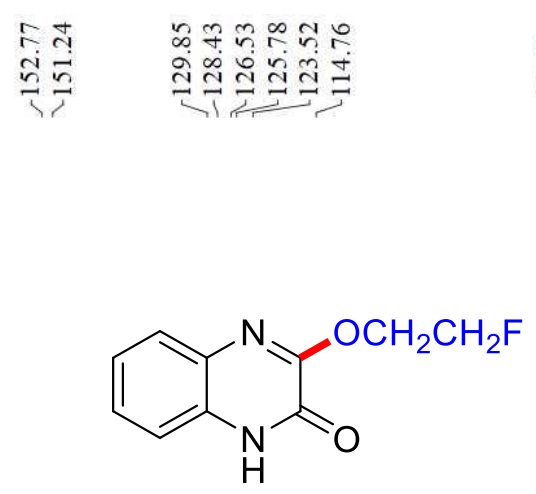

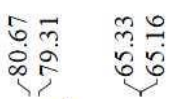

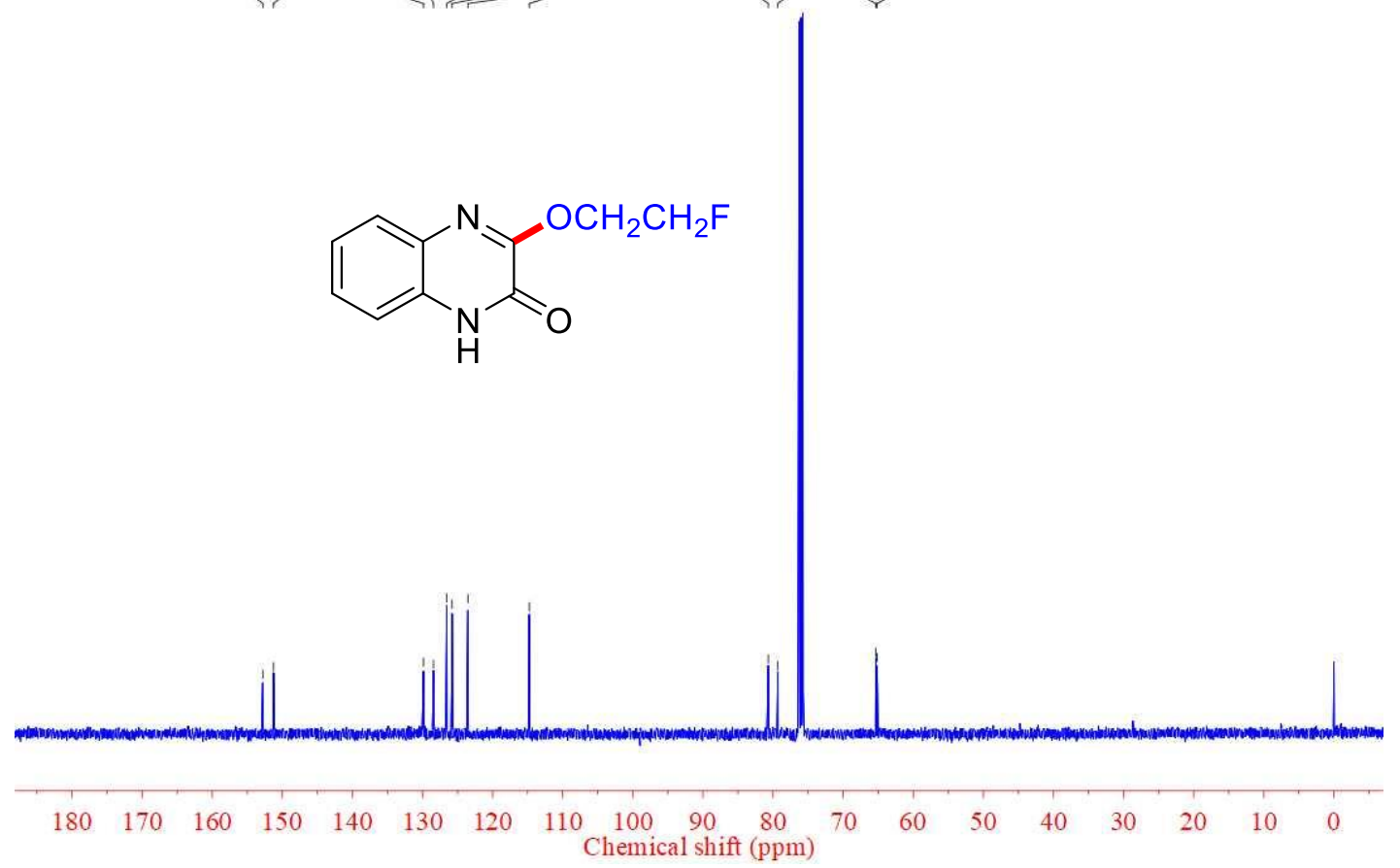

$4 c^{19}$ F NMR

ָับ<smiles>O=c1[nH]c2ccccc2nc1OCCF</smiles>

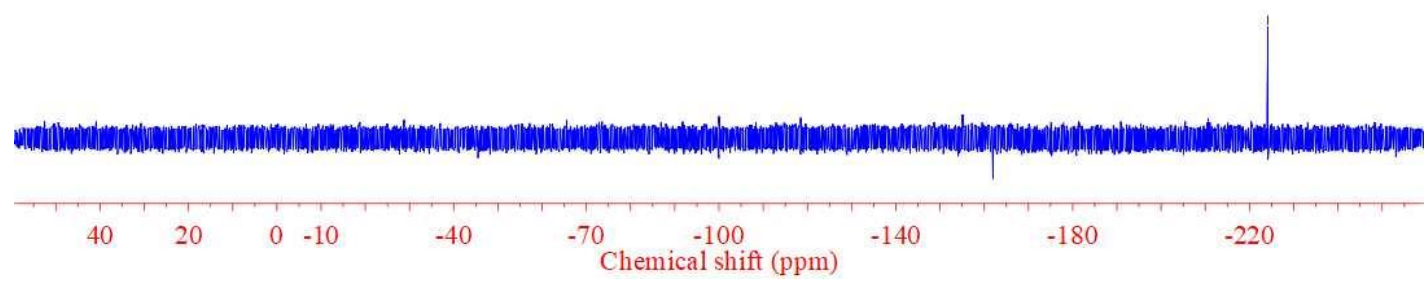




\section{4d ${ }^{1}$ H NMR}

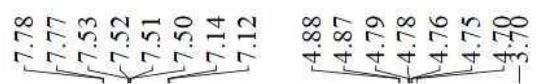

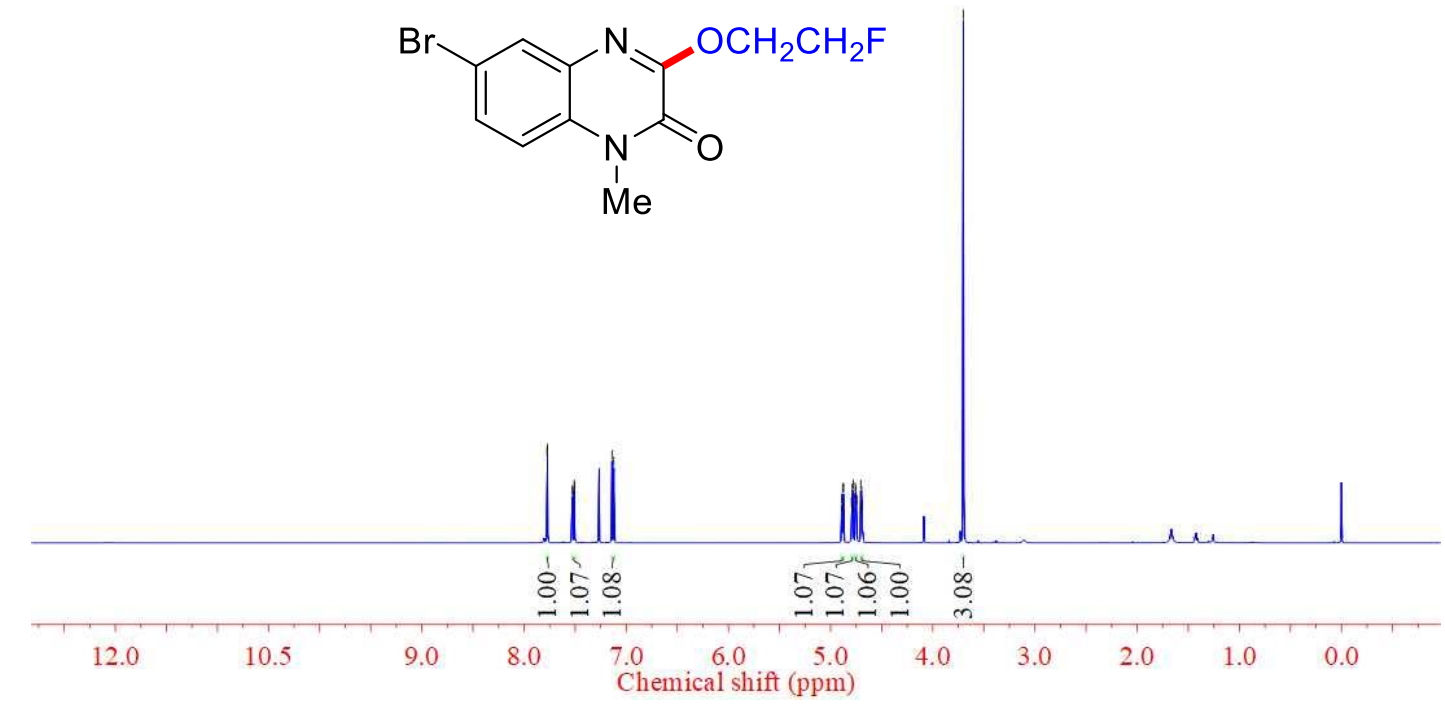

4d ${ }^{13}$ C NMR

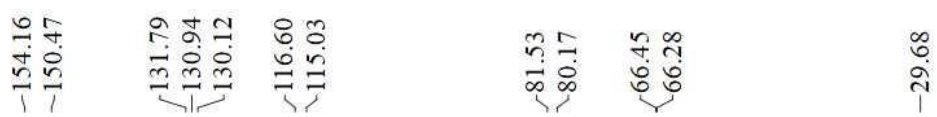<smiles>Cn1c(=O)c(OCCF)nc2cc(Br)ccc21</smiles>

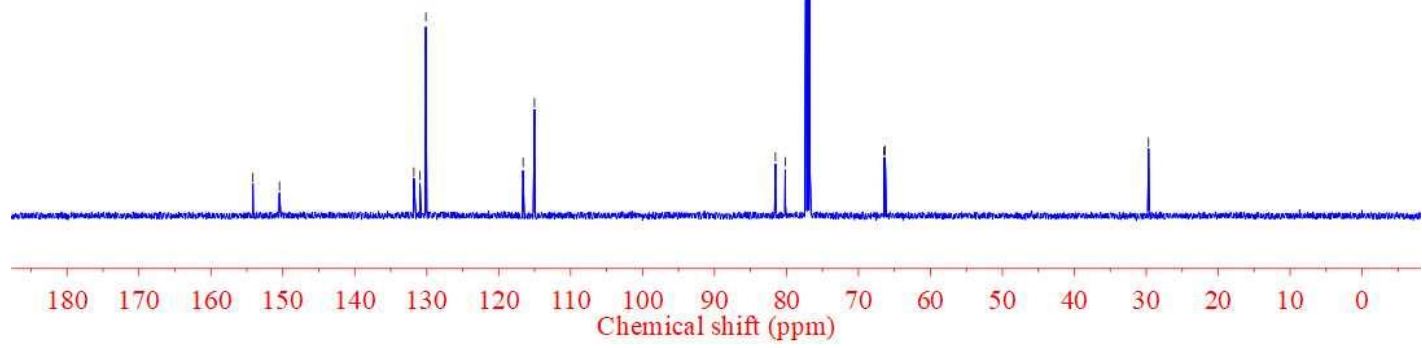




\section{4d ${ }^{19}$ F NMR}<smiles>O=c1c(OCCF)nc2cc(Br)ccc2n1[N+](=O)[O-]</smiles>

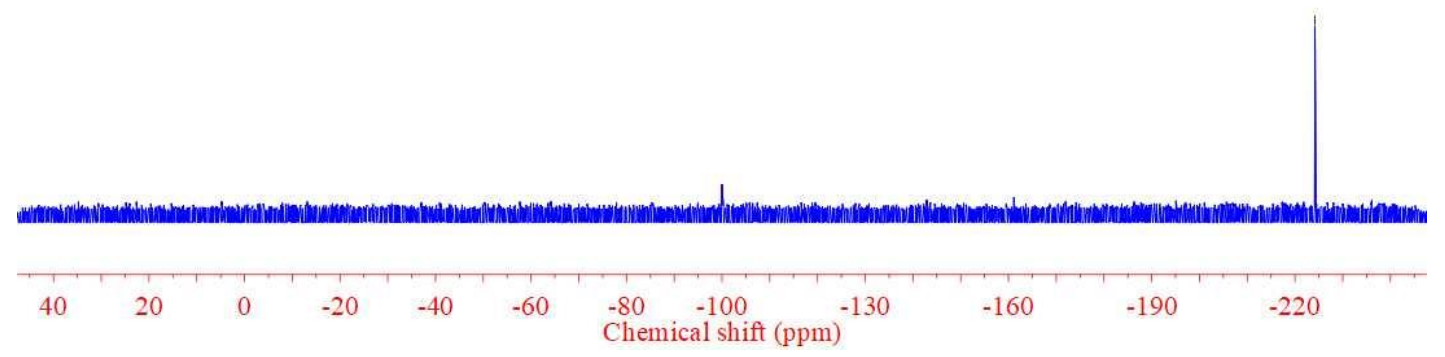

\section{$4 \mathrm{e}^{1} \mathrm{H}$ NMR}

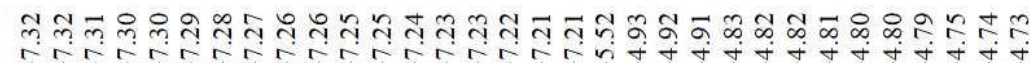

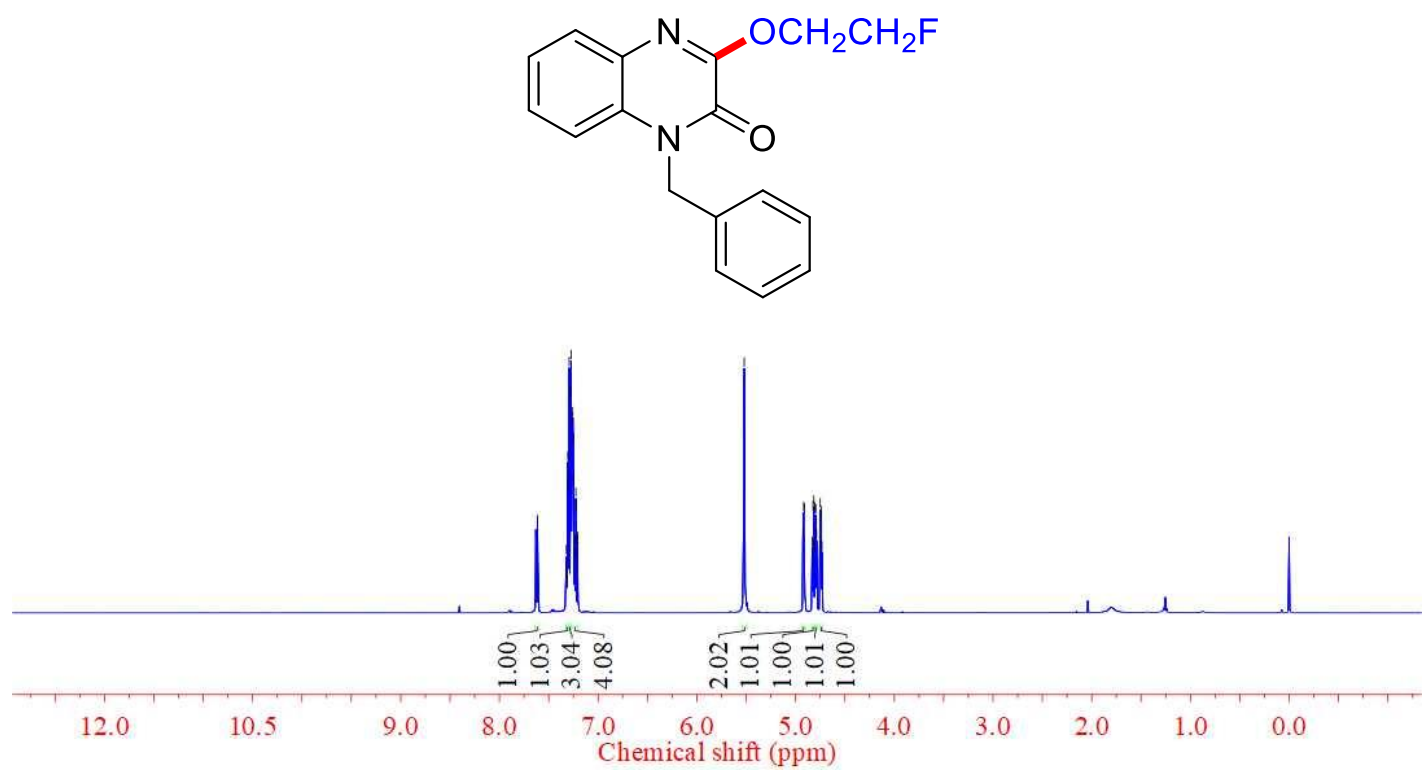




\section{$4 e^{13} C$ NMR}

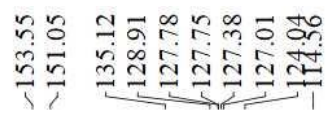

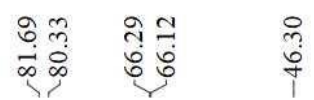

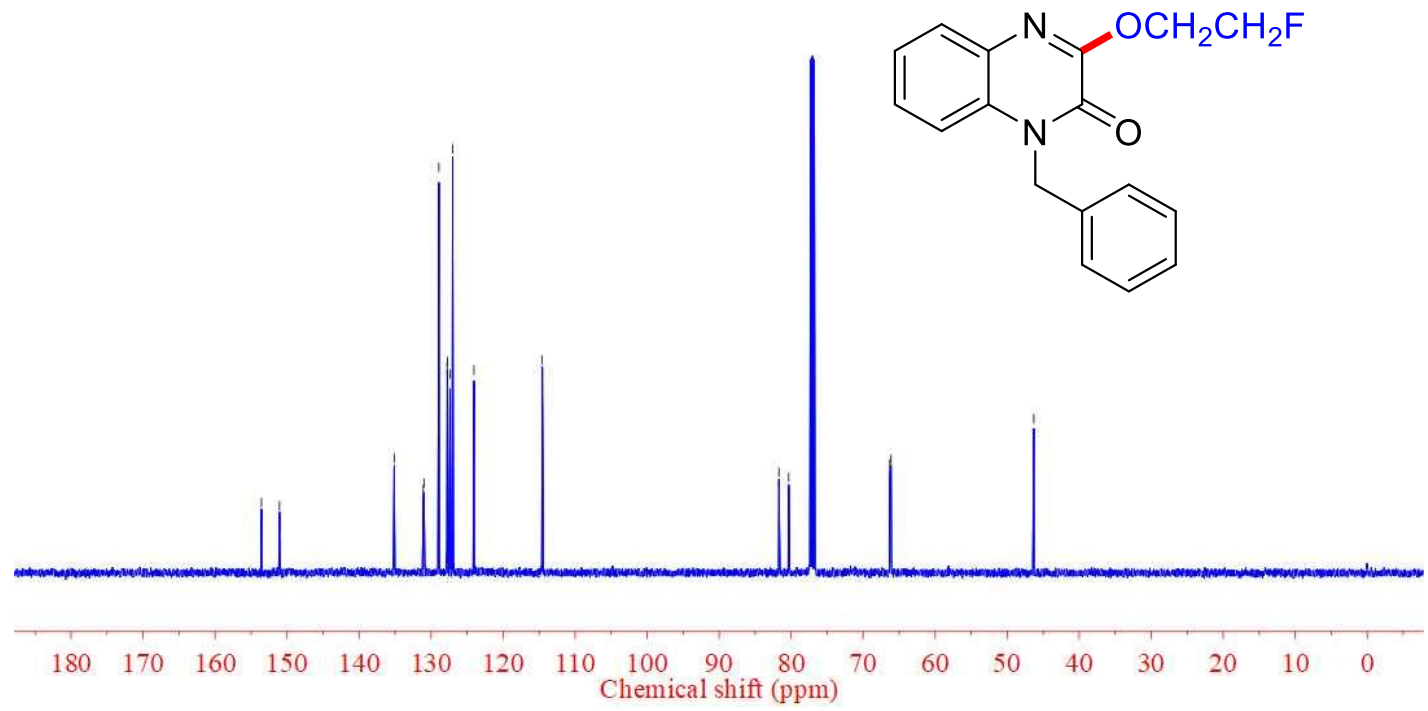

$4 e^{19}$ F NMR

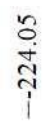<smiles>O=c1c(OCCF)nc2ccccc2n1Cc1ccccc1</smiles>

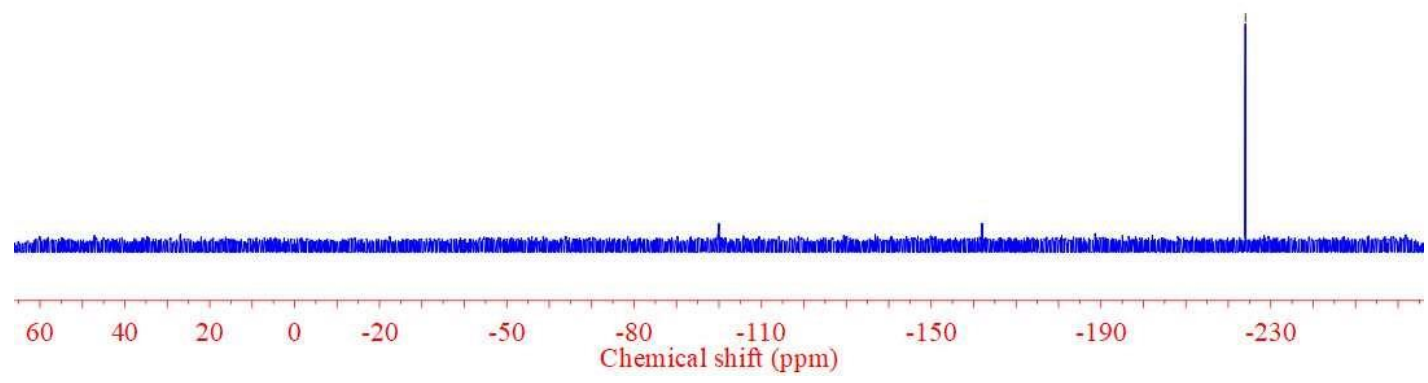




\section{$4 f^{1} H$ NMR}

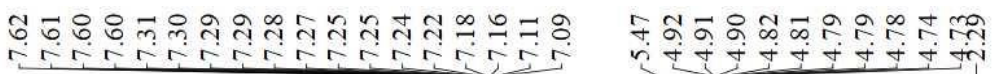

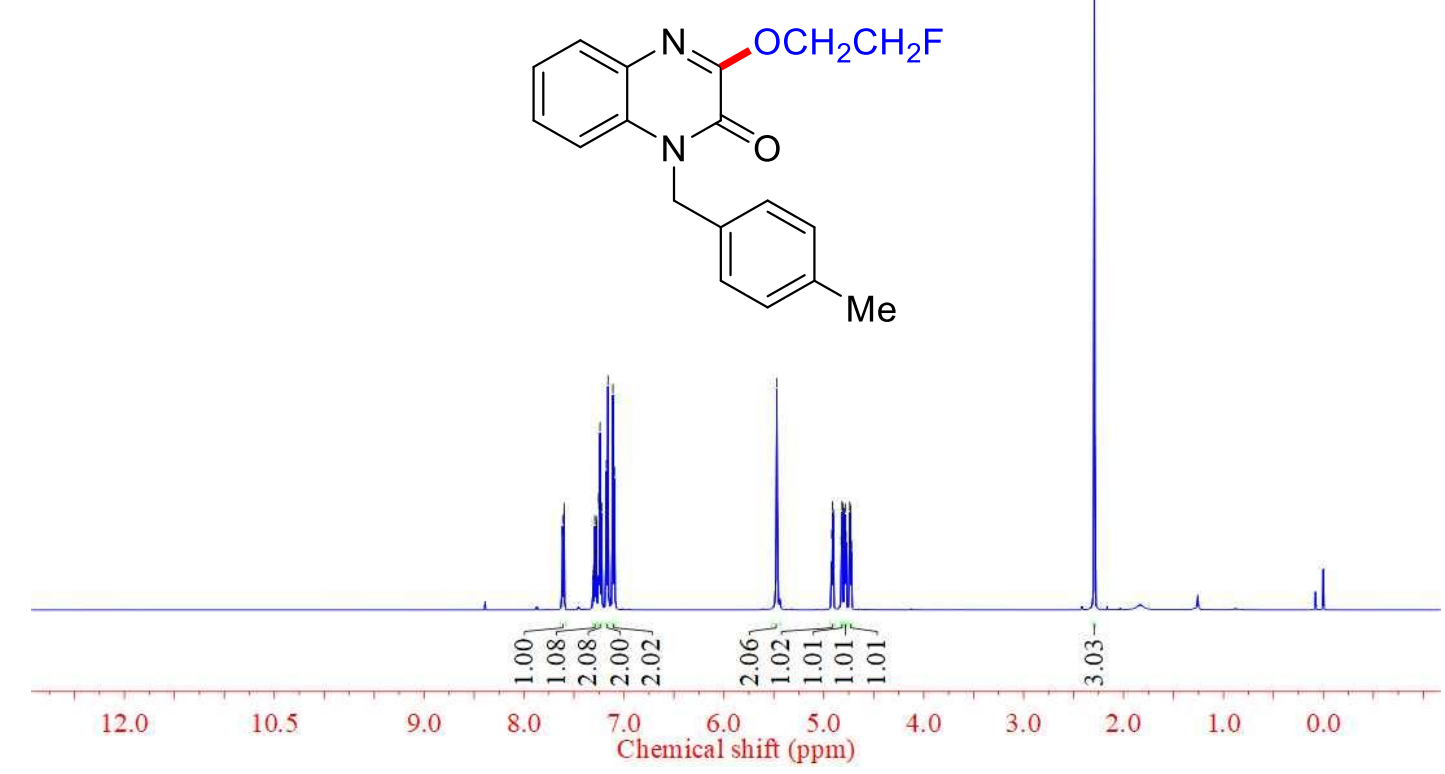

$4 f^{13} \mathrm{C}$ NMR

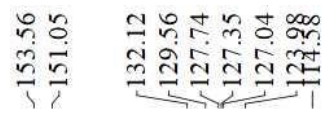

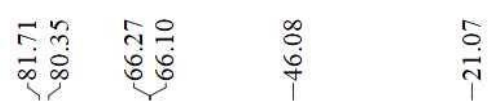

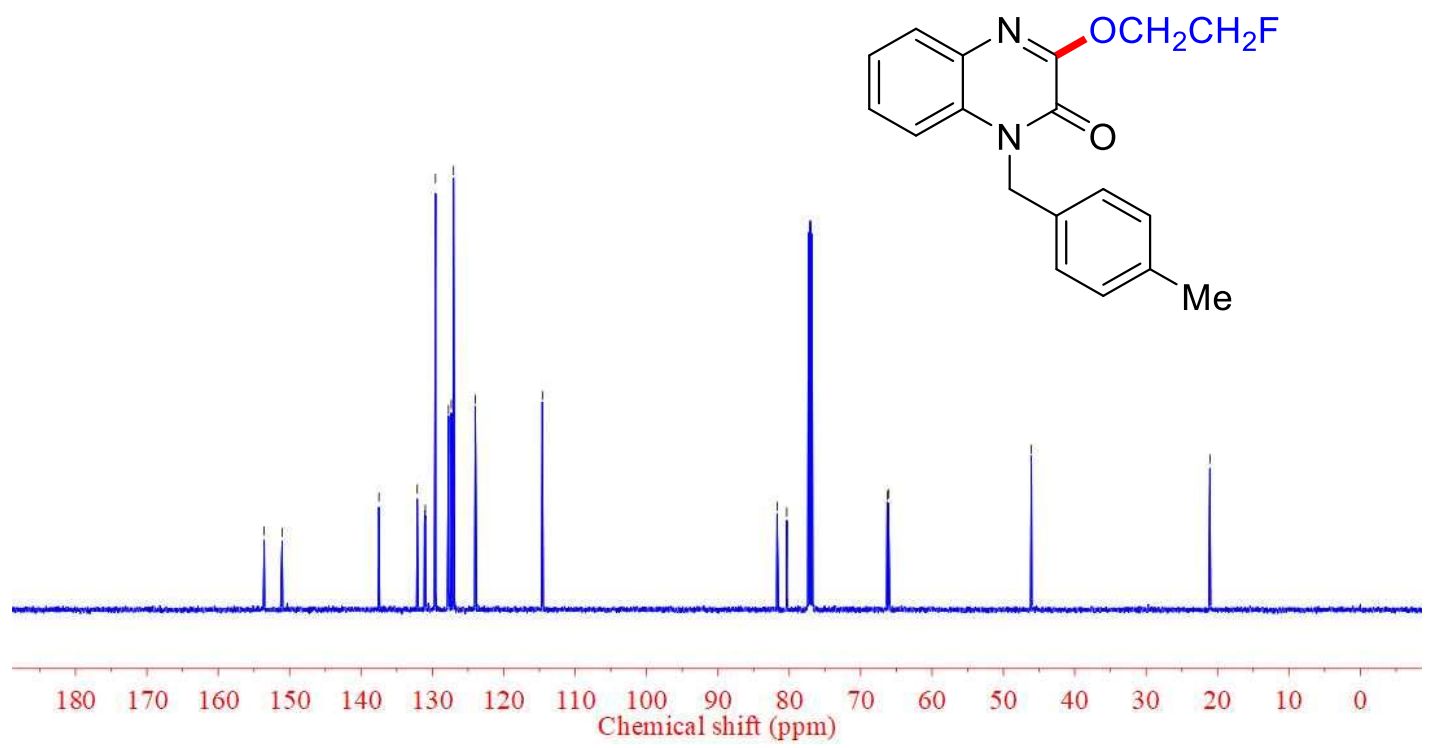


4f ${ }^{19}$ F NMR

8
$\stackrel{+}{c}$
$i$<smiles>Cc1ccc(Cn2c(=O)c(OCCF)nc3ccccc32)cc1</smiles>

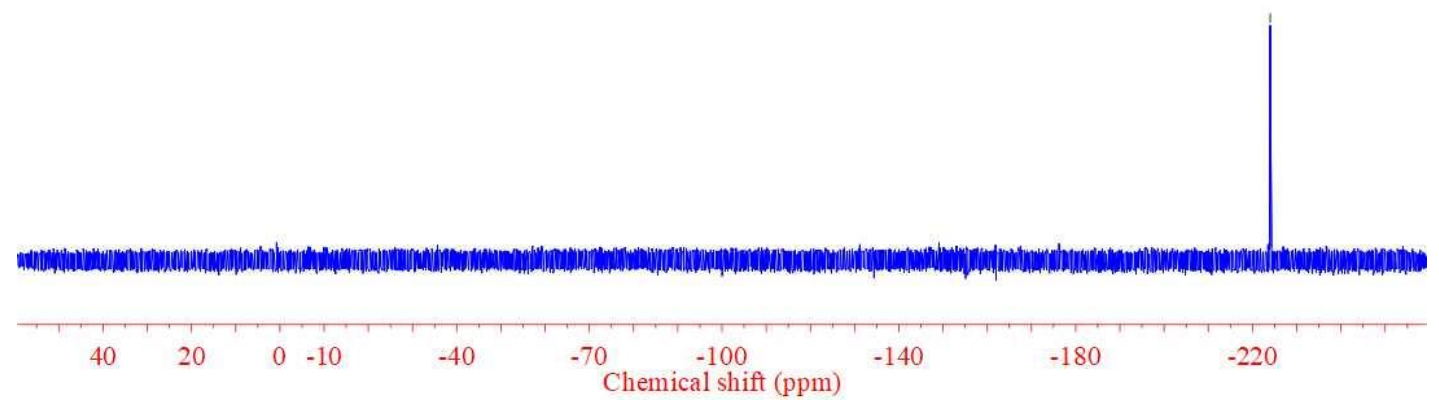

\section{5a ${ }^{1}$ H NMR}

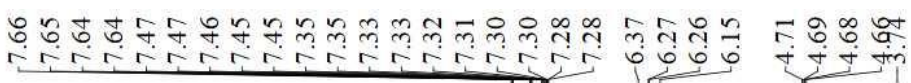

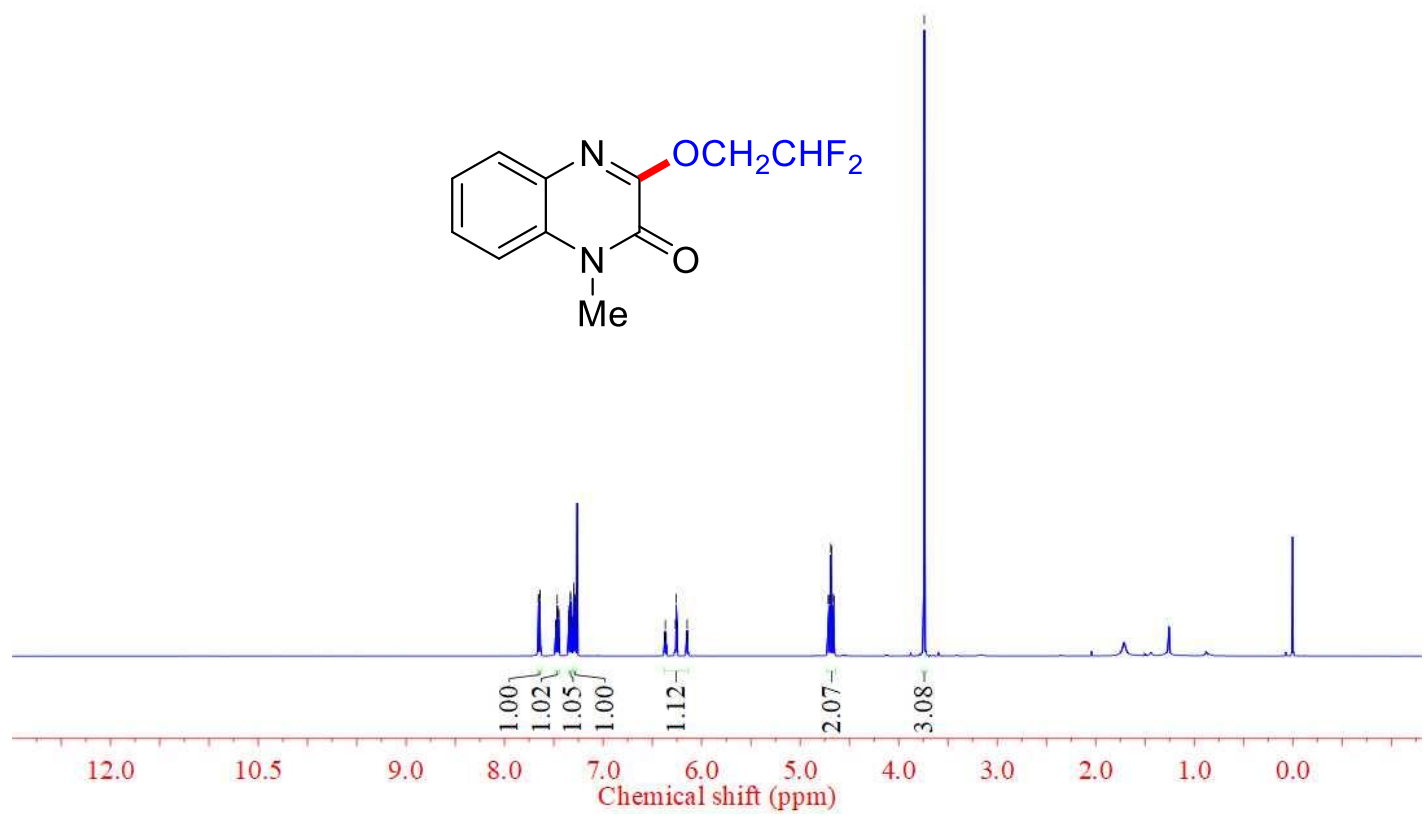




\section{$5 a^{13}$ C NMR}

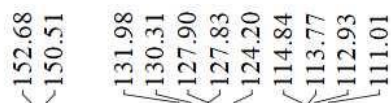

우을

630

iั<smiles>Cn1c(=O)c(OCC(F)F)nc2ccccc21</smiles>

$\mathrm{Me}$

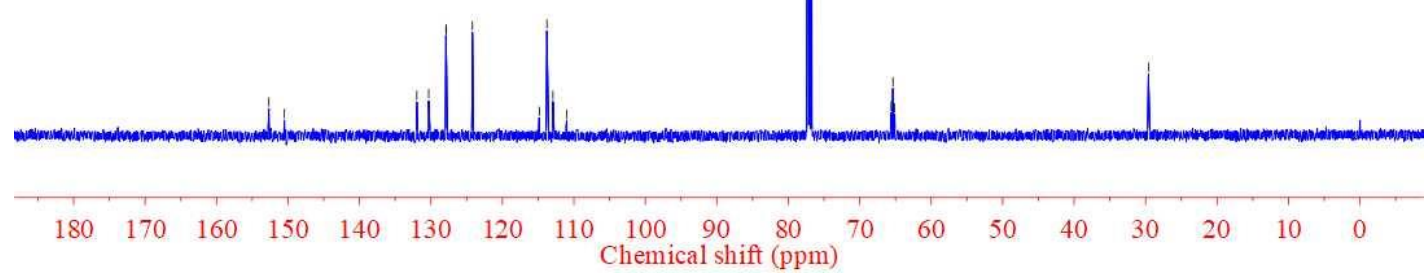

\section{$5 a^{19}$ F NMR}<smiles>Cn1c(=O)c(OCC(F)F)nc2ccccc21</smiles>

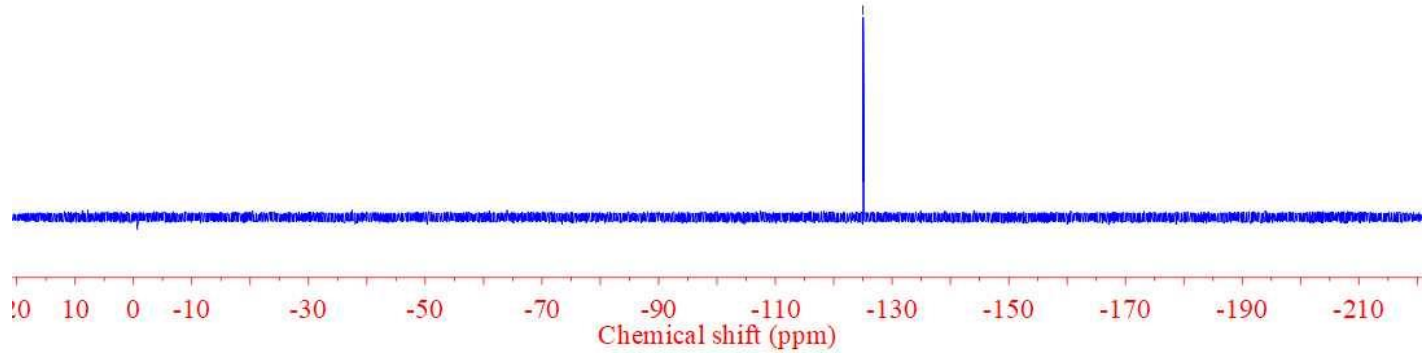




\section{$5 b{ }^{1} \mathrm{H}$ NMR}

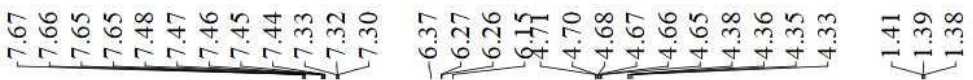<smiles>CCn1c(=O)c(OCC(F)F)nc2ccccc21</smiles>

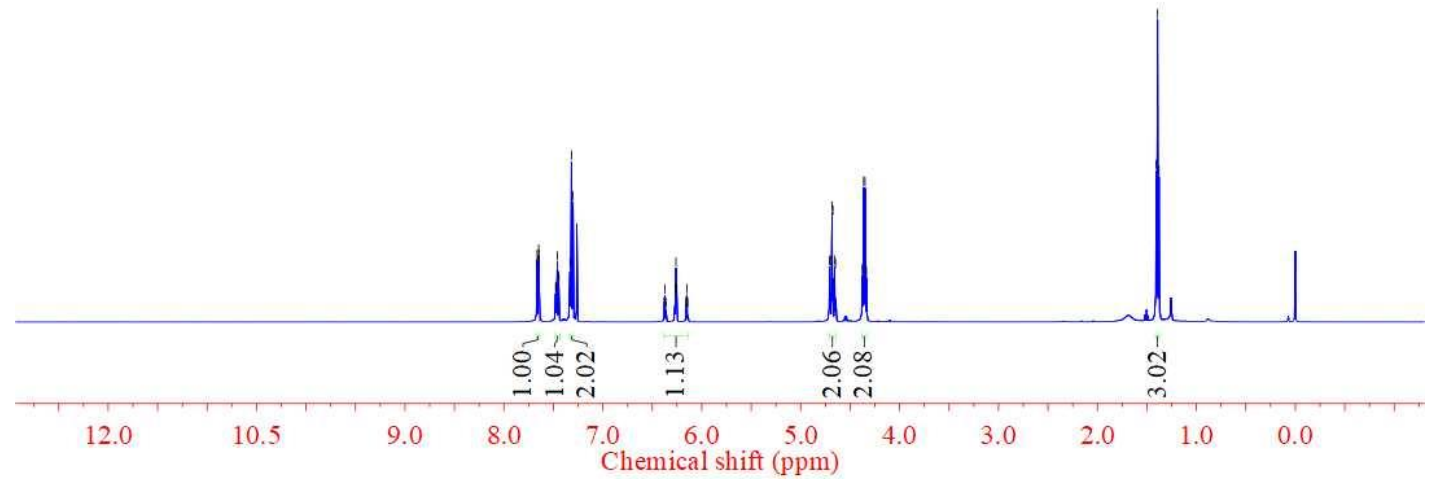

$5 b{ }^{13} \mathrm{C}$ NMR

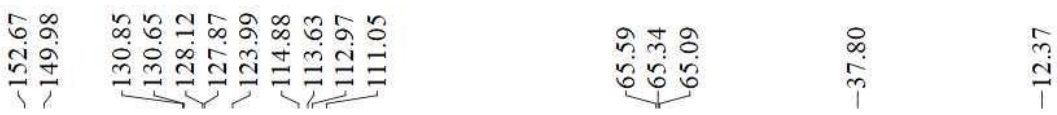<smiles>CCn1c(=O)c(OCC(F)F)nc2ccccc21</smiles>

Et

$\begin{array}{llllllllllllllllll}180 & 170 & 160 & 150 & 140 & 130 & 120 & 110 & \begin{array}{l}100 \\ \text { Chemical shift }(\mathrm{ppm})\end{array}\end{array}$ 


\section{$5 b{ }^{19}$ F NMR}

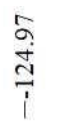<smiles>CCn1c(=O)c(OCC(F)F)nc2ccccc21</smiles>

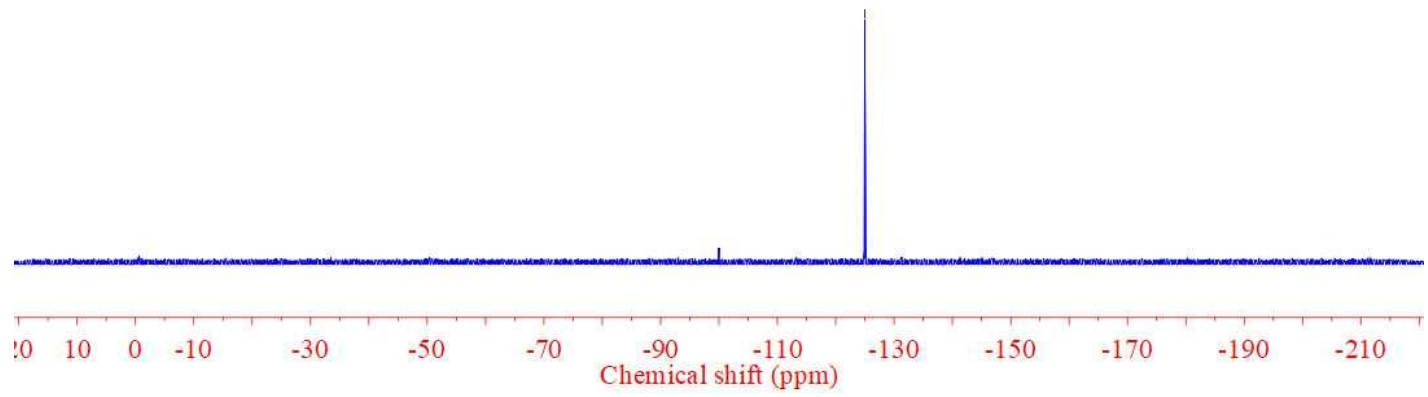

\section{$5 c^{1} H$ NMR}

彳亍
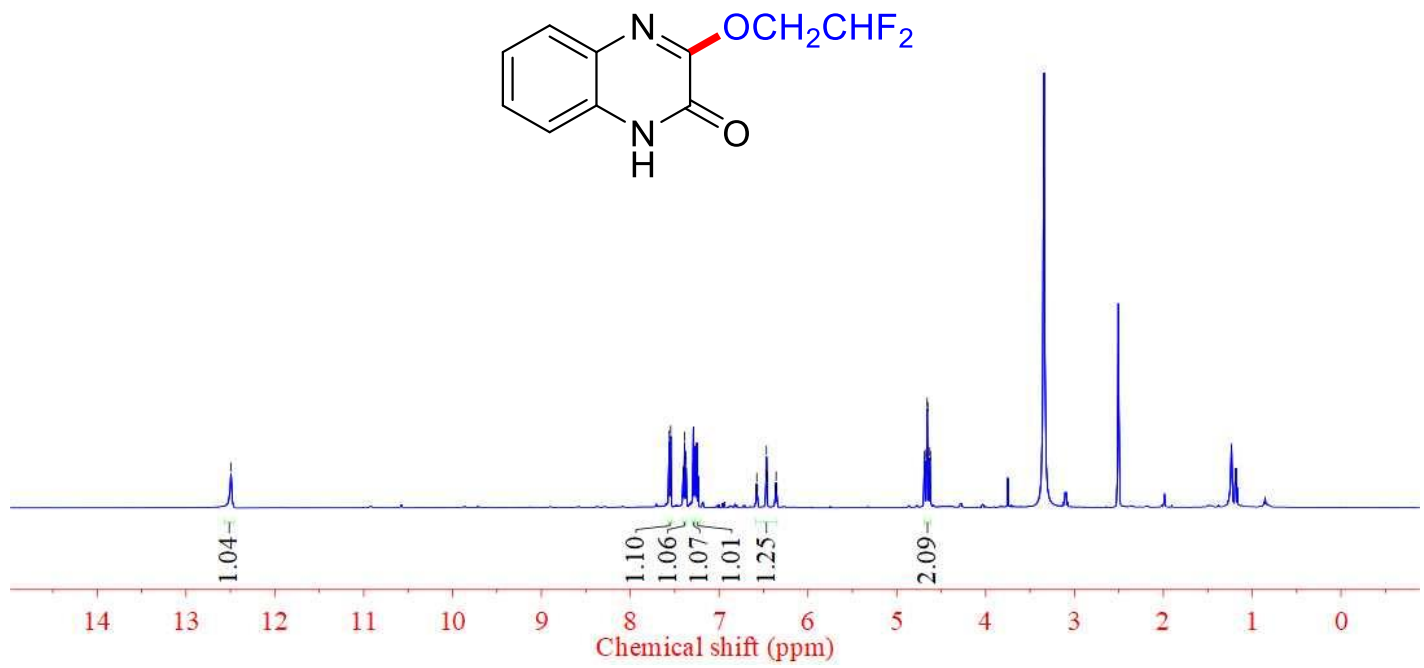


\section{$5 c^{13} \mathrm{C}$ NMR}

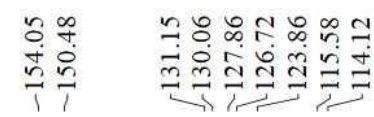

ㅇํㅇ

ठิ<smiles>O=c1[nH]c2ccccc2nc1OCC(F)F</smiles>

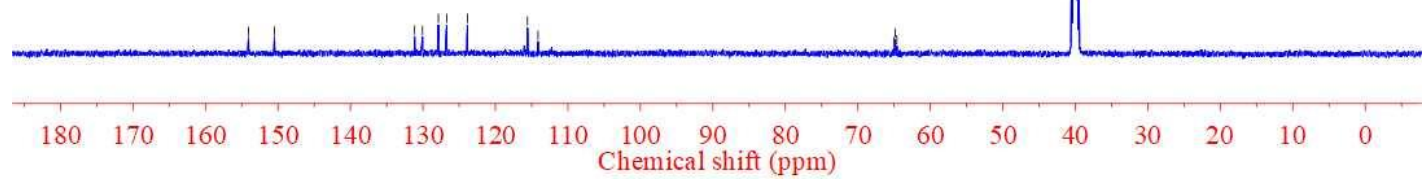

\section{$5 c^{19}$ F NMR}

$$
\text { ลี }
$$<smiles>O=c1[nH]c2ccccc2nc1OCC(F)F</smiles>

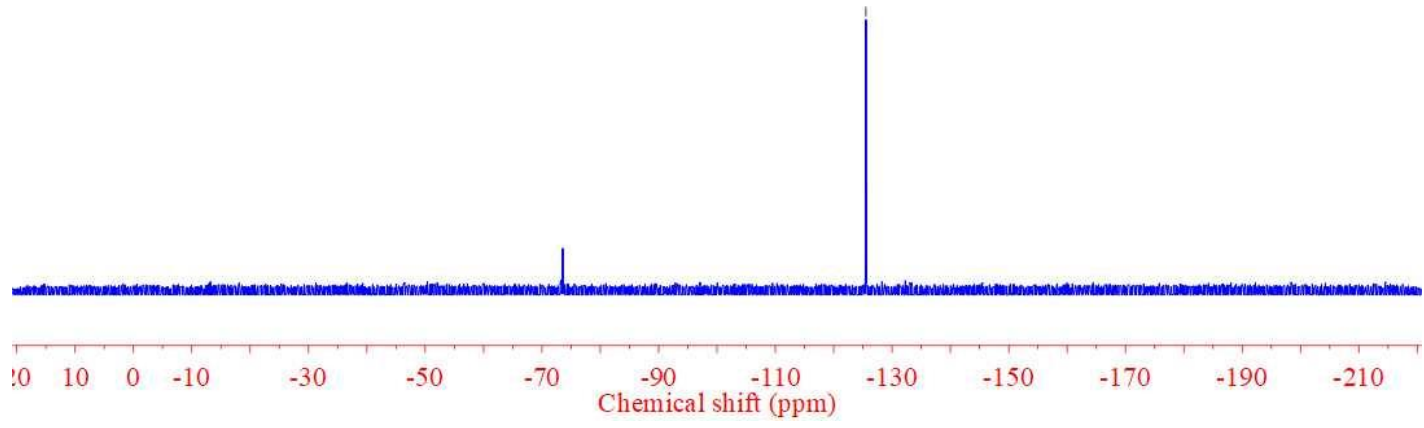




\section{5d ${ }^{1}$ H NMR}

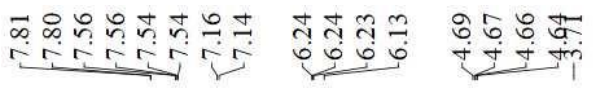

$\underbrace{\mathrm{OCH}_{2} \mathrm{CHF}_{2}}_{\substack{1 \\ \mathrm{Me}}}$

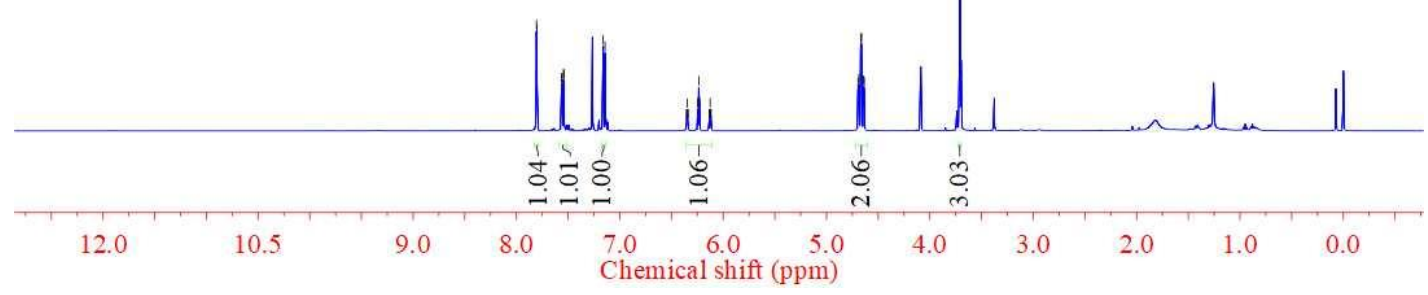

5d ${ }^{13} \mathrm{C}$ NMR

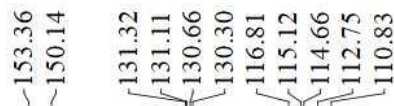

ำกำ

ถู่อ

$\stackrel{n}{\stackrel{2}{1}}$<smiles>Cn1c(=O)c(OCC(F)F)nc2cc(Br)ccc21</smiles>

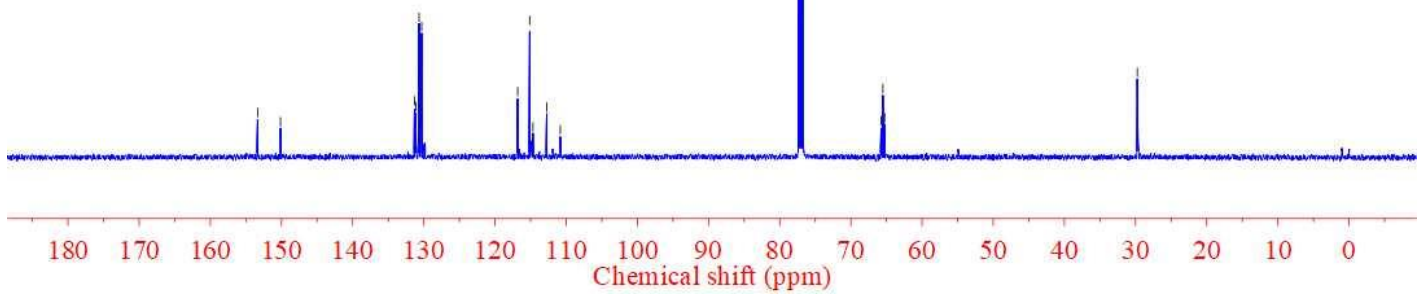




\section{5d ${ }^{19}$ F NMR}

$$
\begin{aligned}
& 8 \\
& \multirow{2}{1}{} \\
& \frac{1}{1}
\end{aligned}
$$<smiles>Cn1c(=O)c(OCC(F)F)nc2cc(Br)ccc21</smiles>

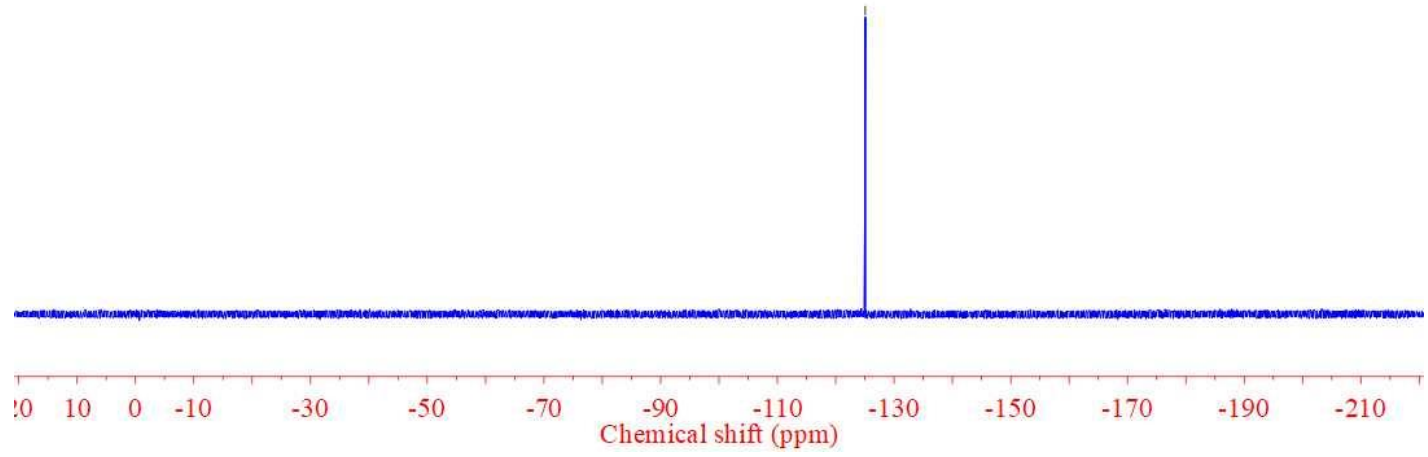

\section{$6 a^{1} H$ NMR}

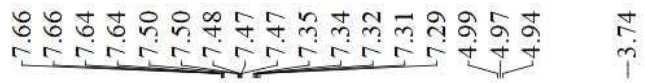

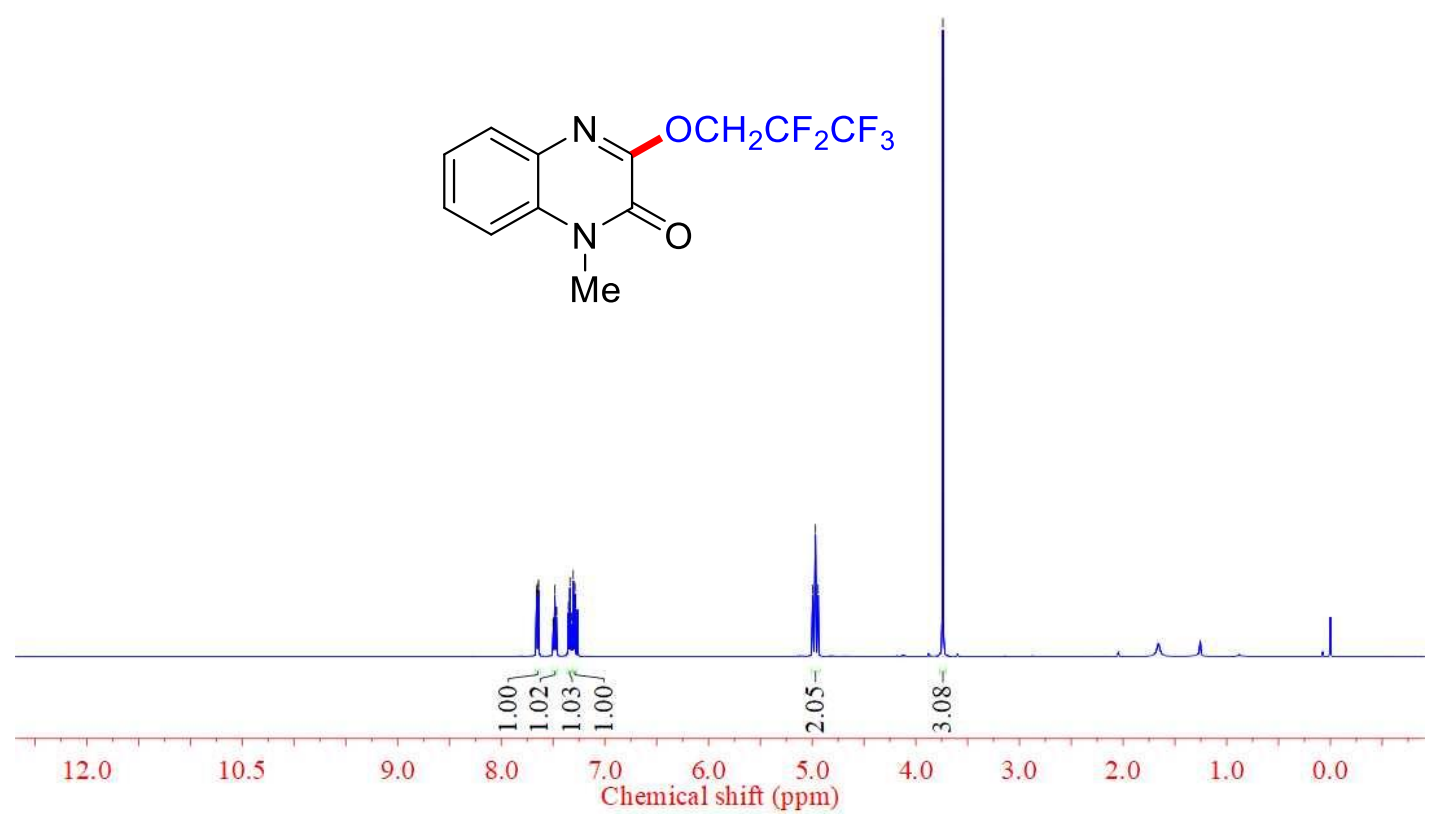




\section{$6 a^{13} \mathrm{C}$ NMR}

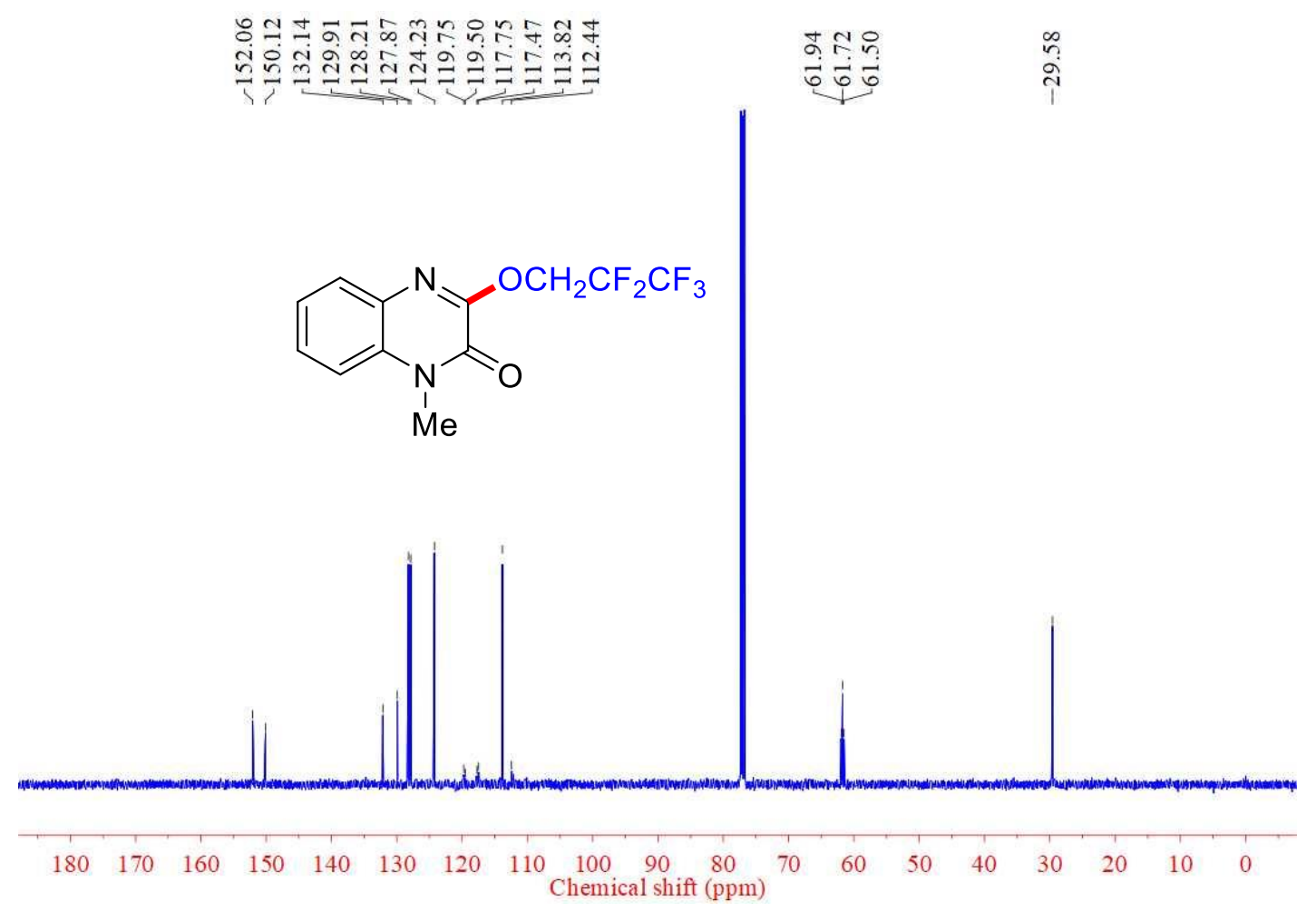

\section{$6 a^{19}$ F NMR}

$\begin{array}{ll}\infty & \vec{a} \\ \infty & \text { ते } \\ \infty & 1\end{array}$
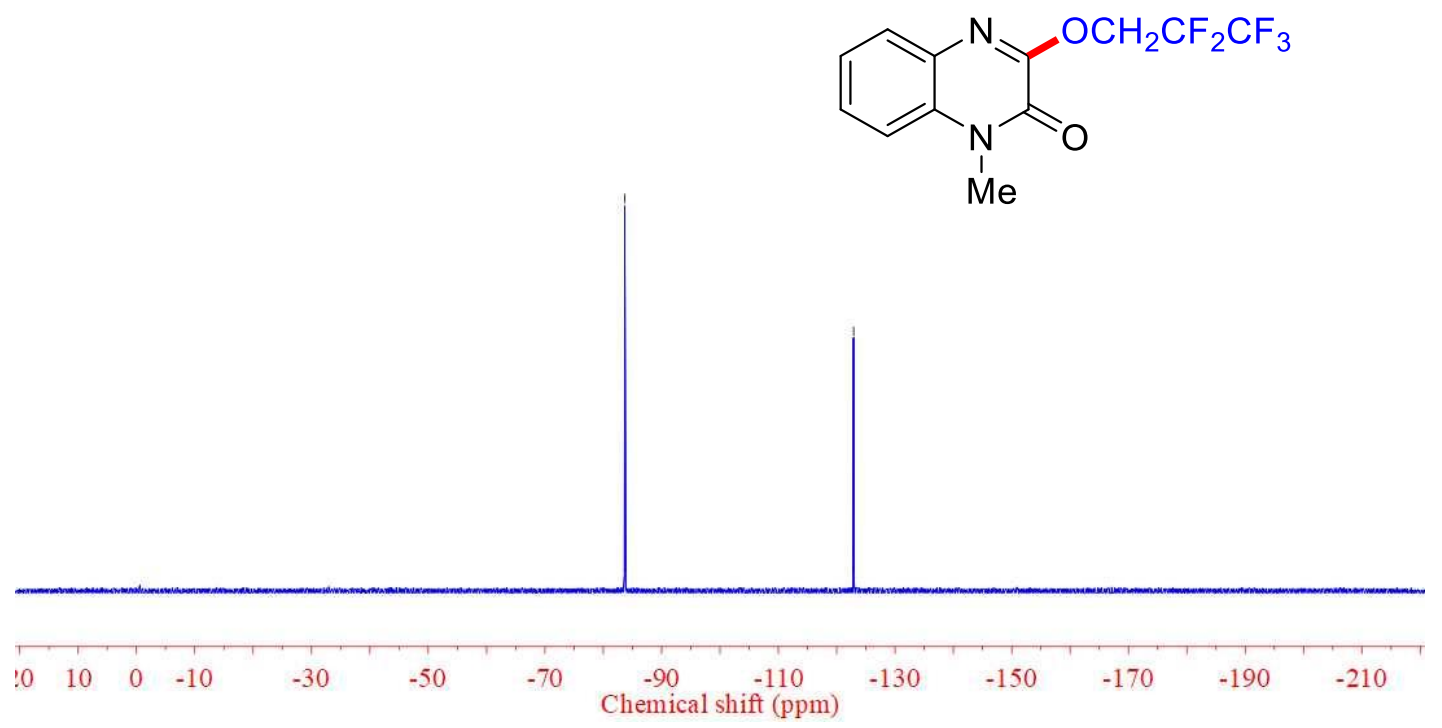


\section{6b ${ }^{1}$ H NMR}

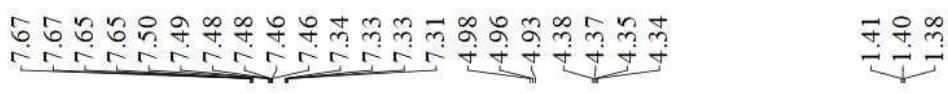<smiles>CCn1c(=O)c(OCC(F)(F)C(F)(F)F)nc2ccccc21</smiles>

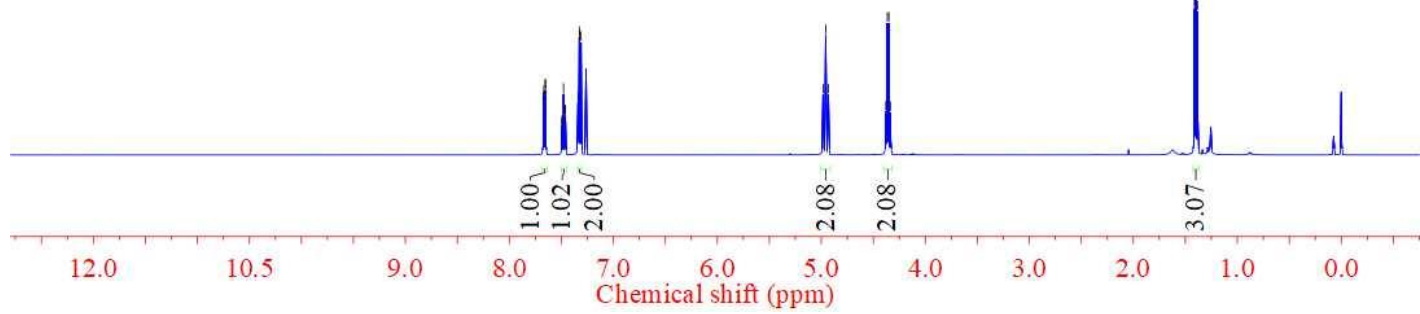

$6 \mathrm{~b}{ }^{13} \mathrm{C}$ NMR

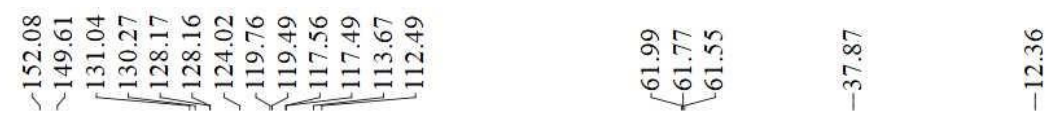<smiles>CCn1c(=O)c(OCC(F)(F)F)nc2ccccc21</smiles>

$\begin{array}{lllllllllllllllllll}180 & 170 & 160 & 150 & 140 & 130 & 120 & 110 & \begin{array}{c}100 \\ \text { Chemical shift }\end{array}(\mathrm{ppm}) & 70 & 60 & 50 & 40 & 30 & 20 & 10 & 0\end{array}$ 


\section{6b ${ }^{19}$ F NMR}

$\begin{array}{ll}0 & 8 \\ 0 & \text { ป } \\ 0 & 1\end{array}$

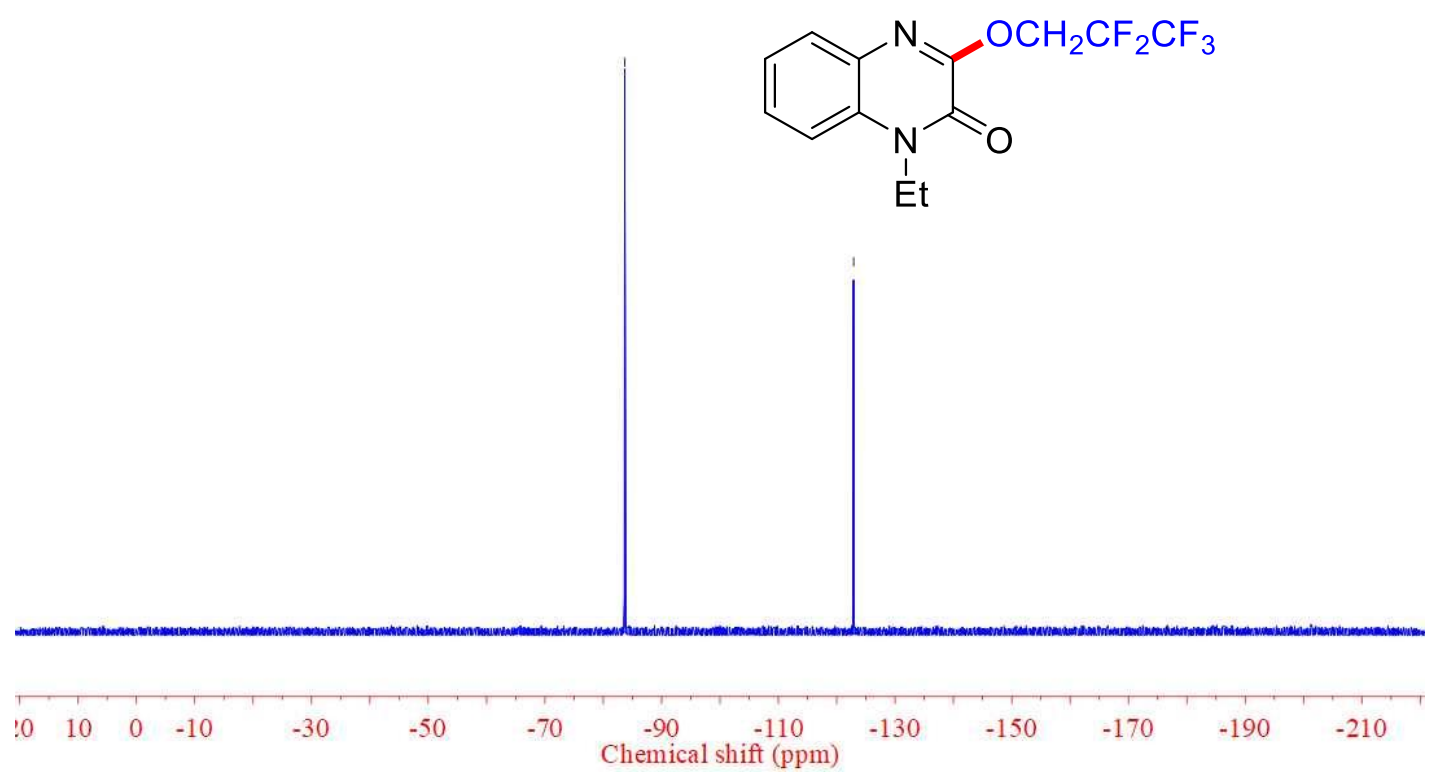

\section{$6 c^{1} \mathrm{H}$ NMR}

彳े
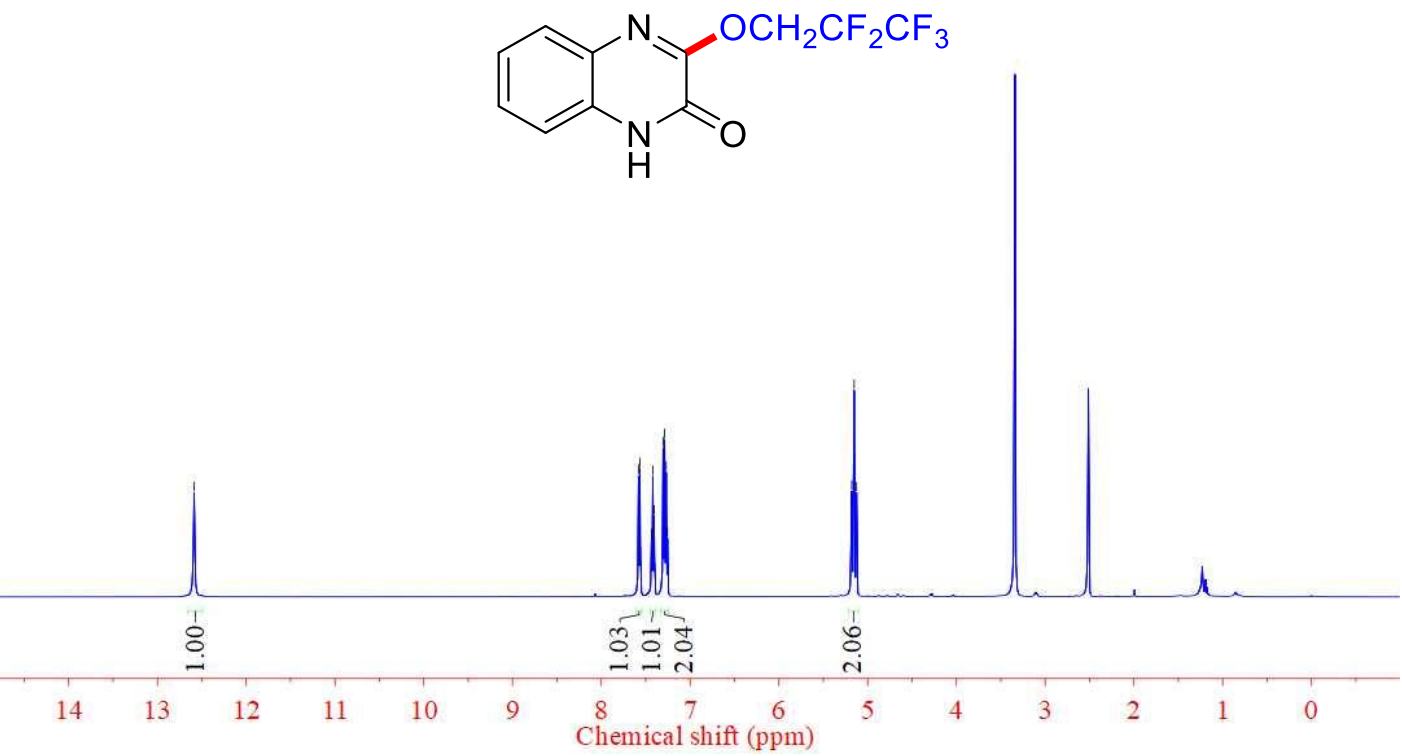


\section{$6 c^{13} \mathrm{C}$ NMR}

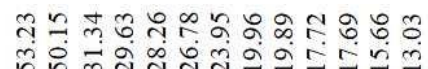

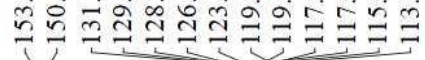

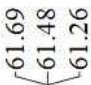

$\overbrace{\mathrm{H}}^{\mathrm{OCH}_{2} \mathrm{CF}_{2} \mathrm{CF}_{3}}$

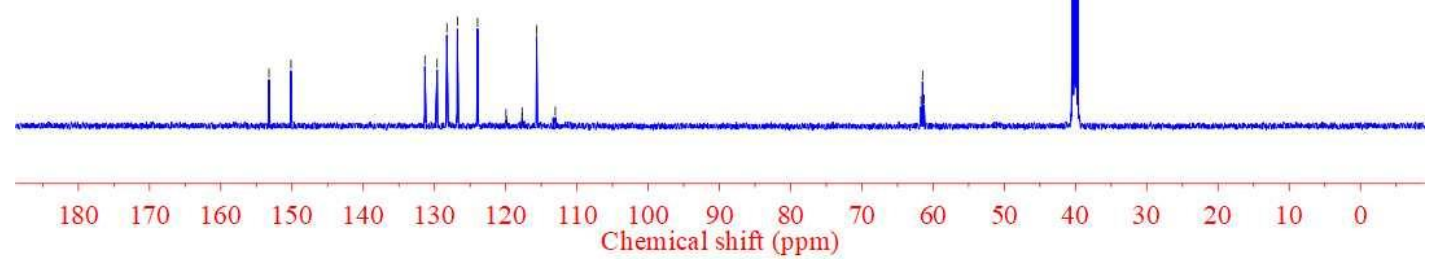

$6 c^{19}$ F NMR

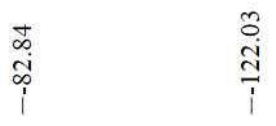<smiles>O=c1[nH]c2ccccc2nc1OCC(F)(F)F</smiles>

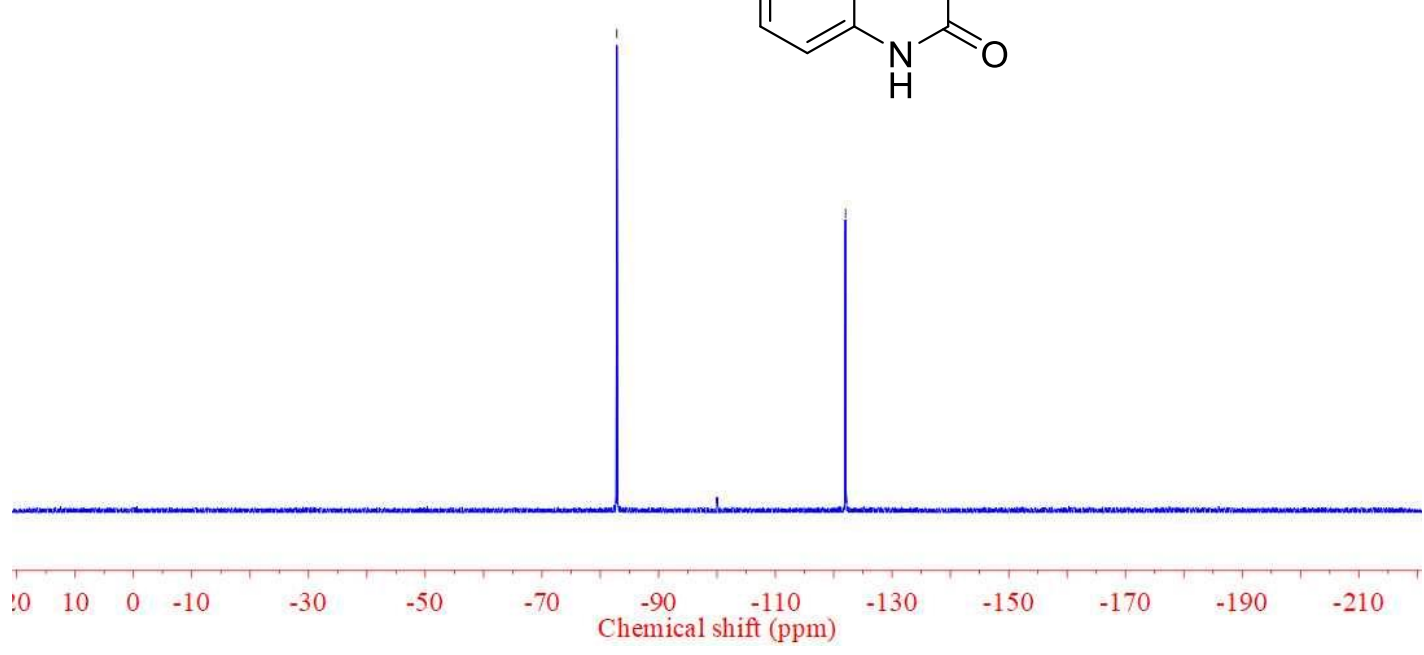




\section{6d ${ }^{1}$ H NMR}

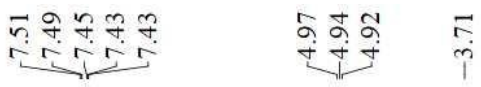

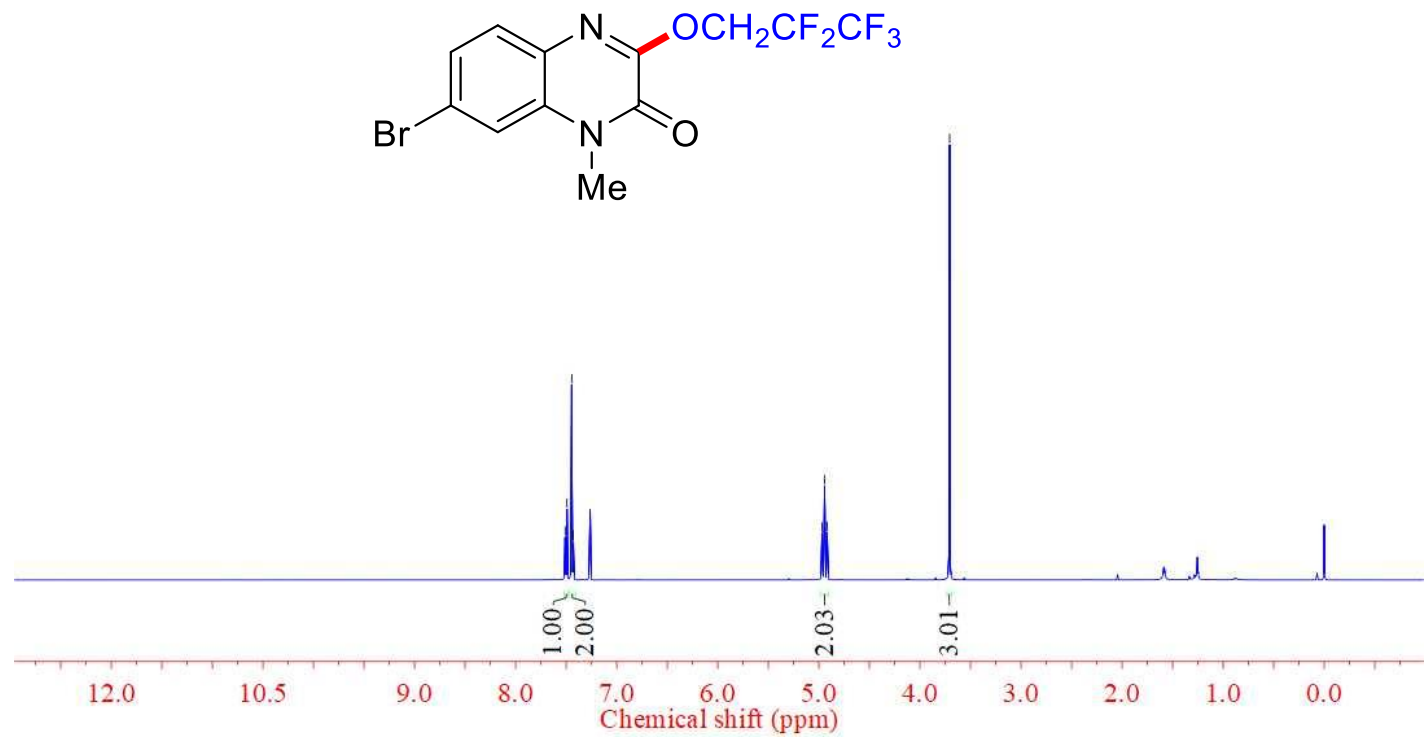

\section{6d ${ }^{13}$ C NMR}

สุณ

ง่ำ

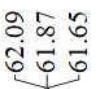

交<smiles>Cn1c(=O)c(OCC(F)(F)C(F)(F)C(F)(F)F)nc2ccc(Br)cc21</smiles>

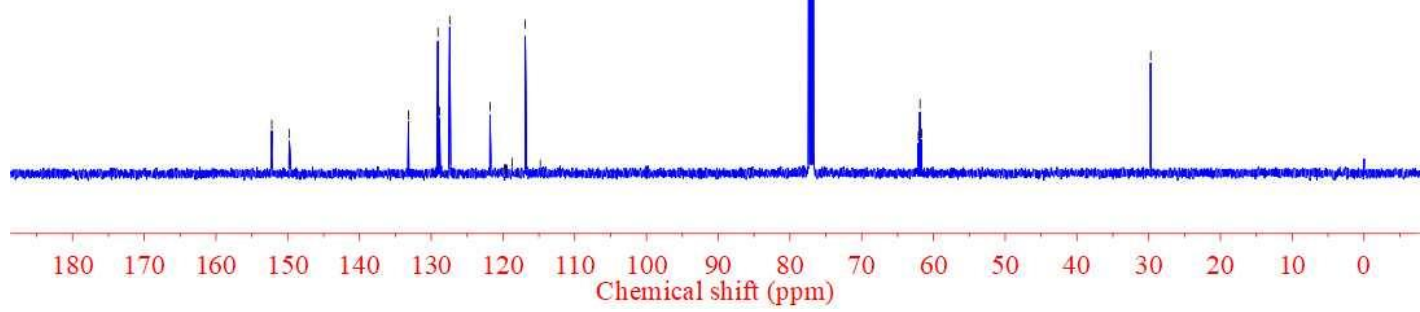




\section{6d ${ }^{19}$ F NMR}

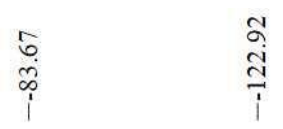<smiles>Cn1c(=O)c(OCC(F)(F)F)nc2ccc(Br)cc21</smiles>

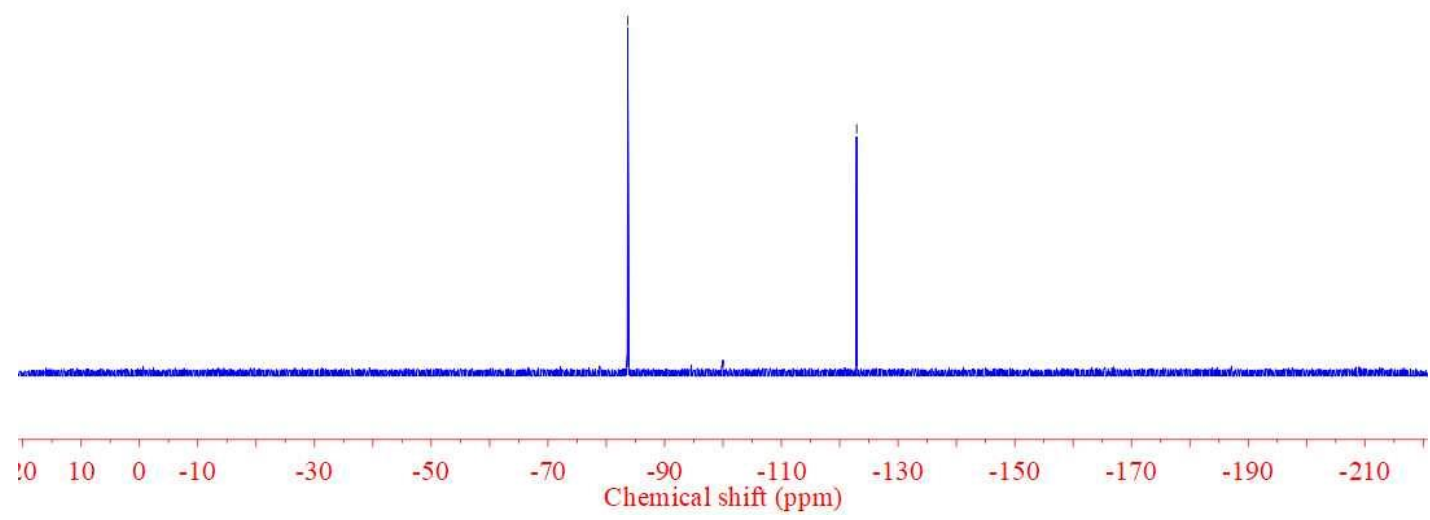

\section{$6 \mathrm{e}^{1} \mathrm{H}$ NMR}

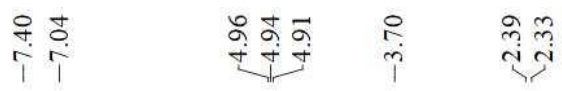

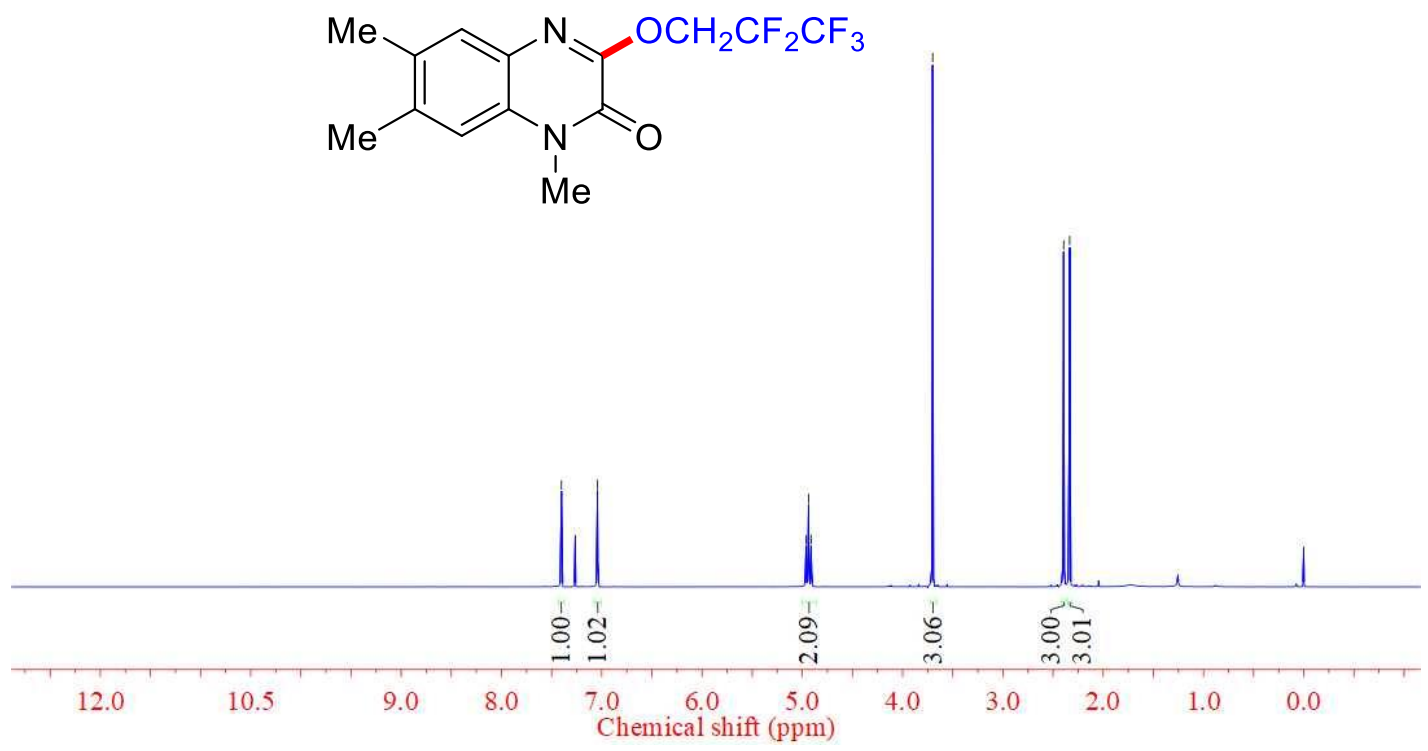




\section{$6 e^{13}$ C NMR}

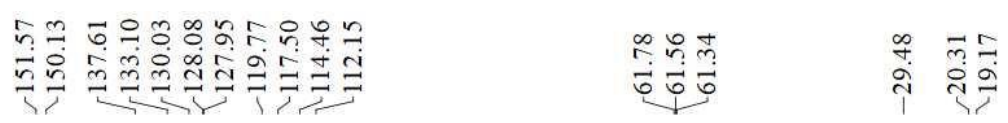

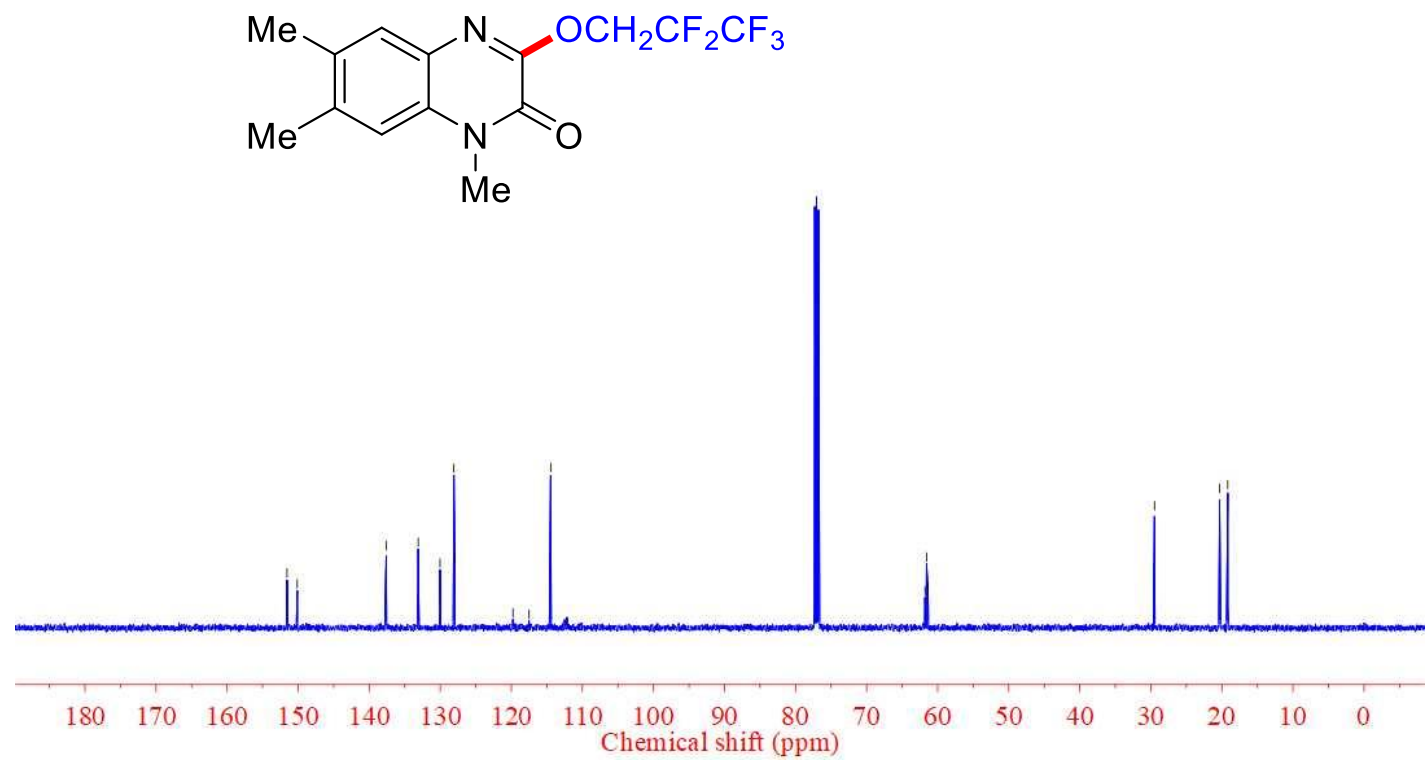

$6 e^{19}$ F NMR

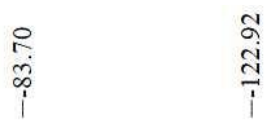
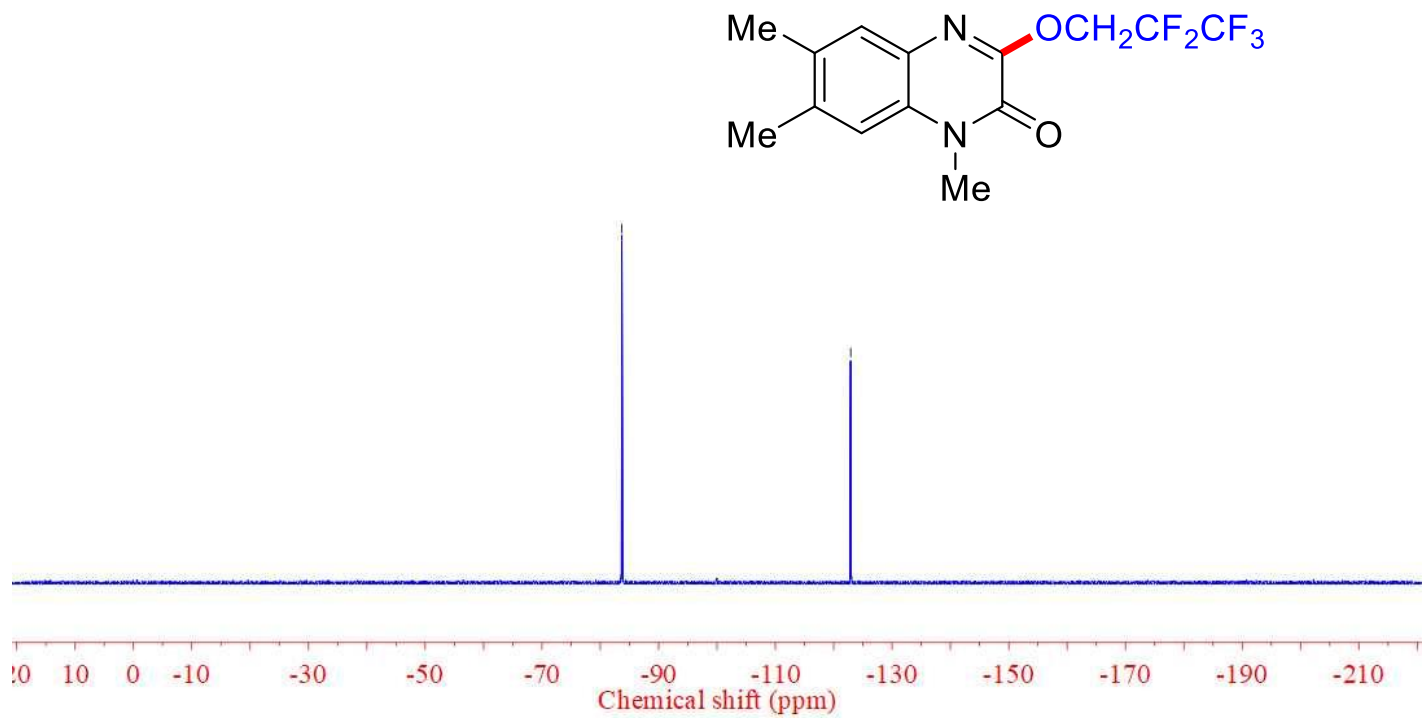


\section{6f ${ }^{1} \mathrm{H}$ NMR}

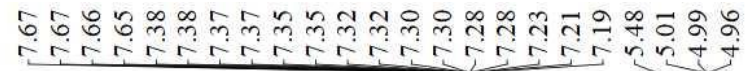

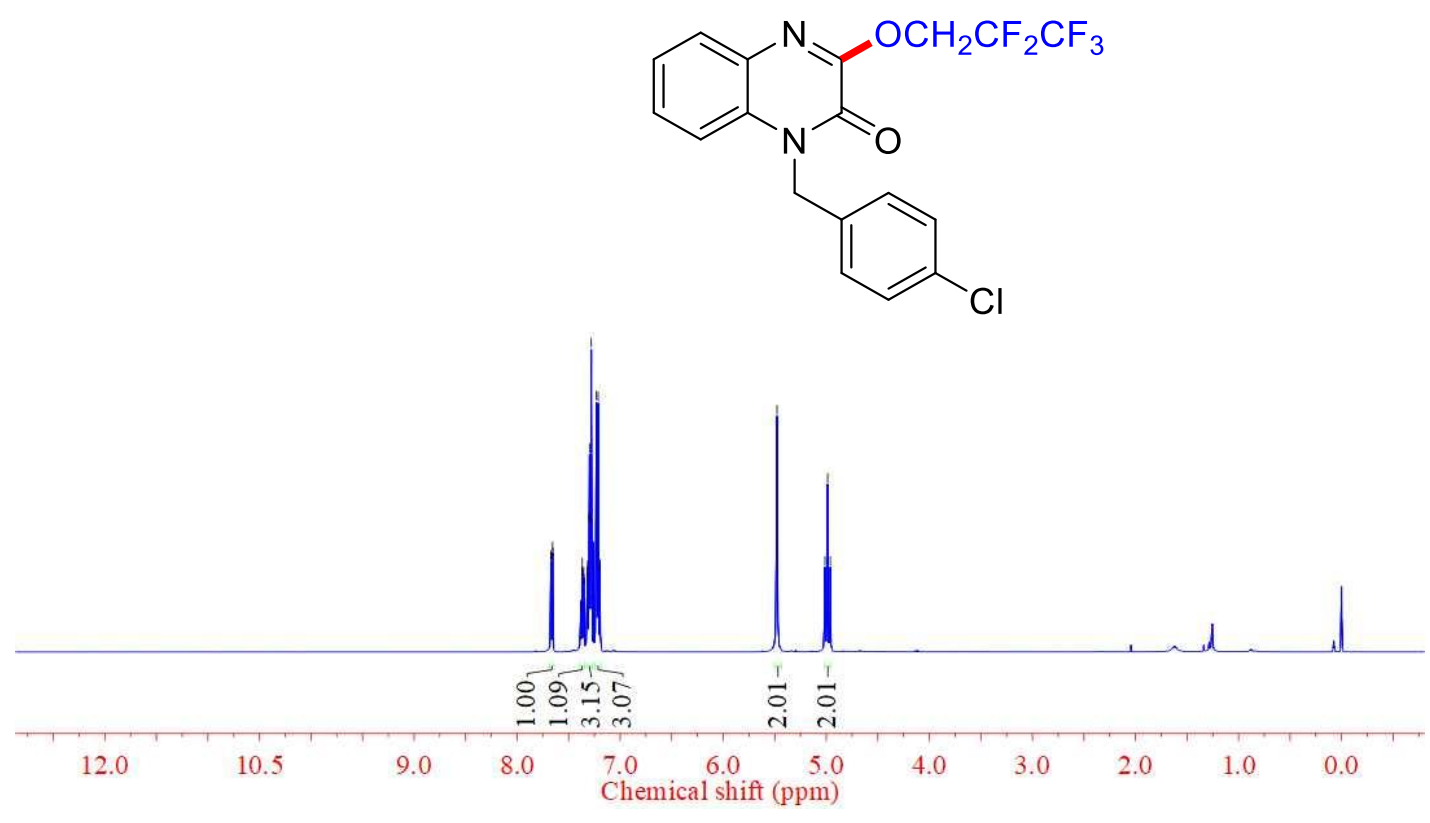

\section{6f ${ }^{13} \mathrm{C}$ NMR}

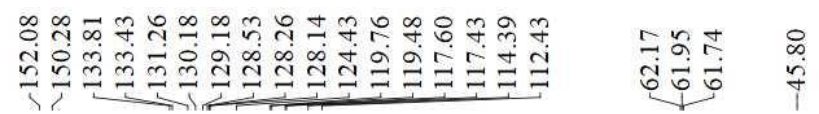

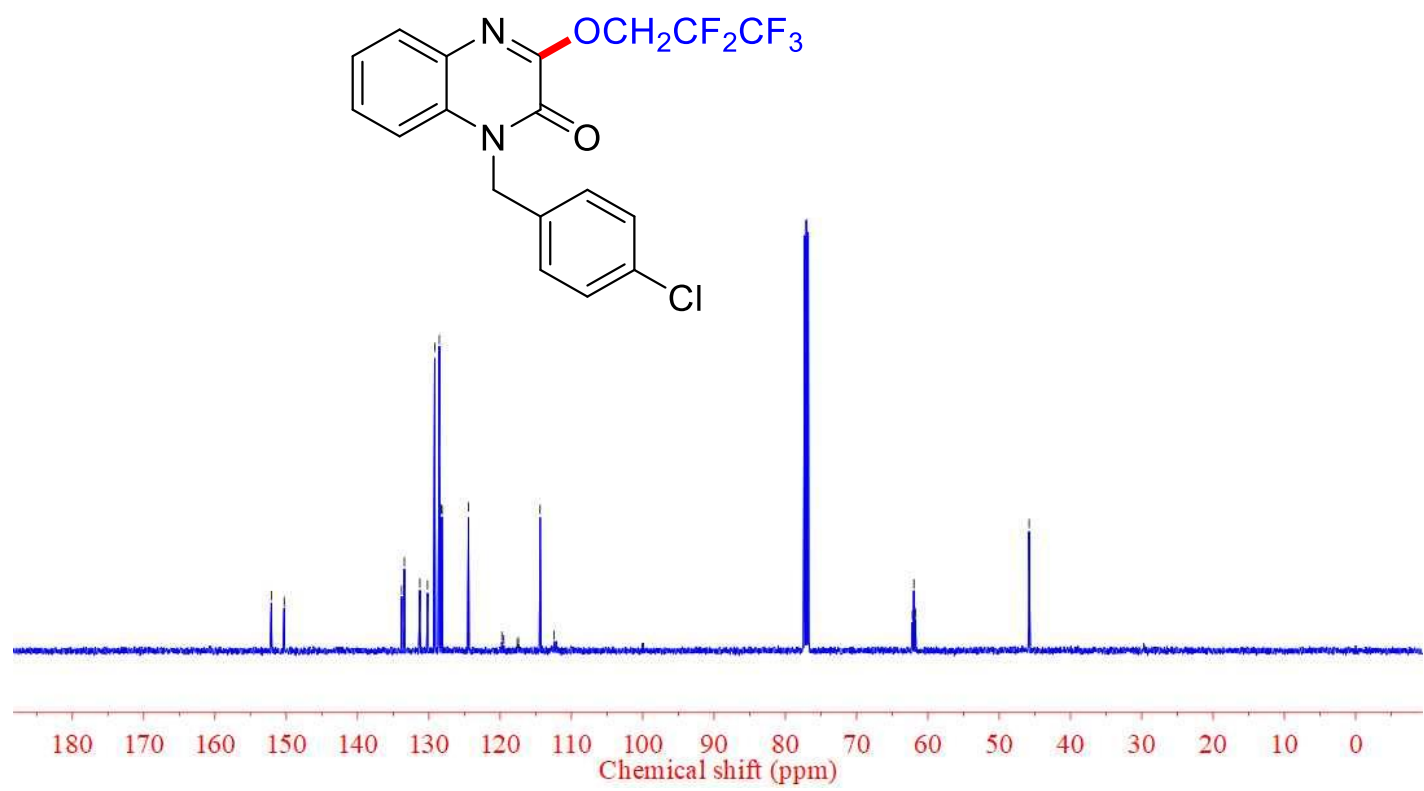




\section{6f ${ }^{19}$ F NMR}

$\begin{array}{ll}3 & \infty \\ \infty & \infty \\ 0 & \text { i } \\ 0 & \frac{1}{1}\end{array}$

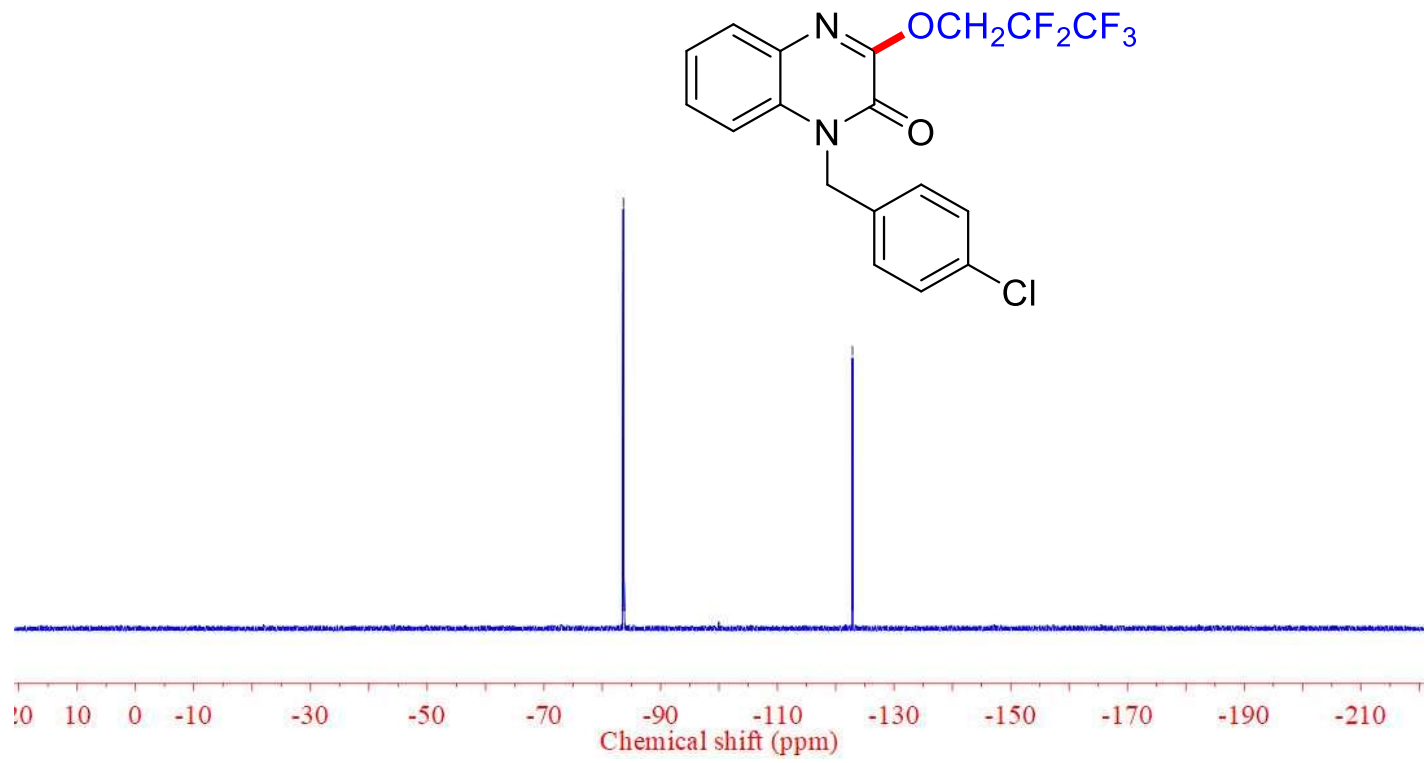

\section{$6 \mathrm{~g}{ }^{1} \mathrm{H}$ NMR}

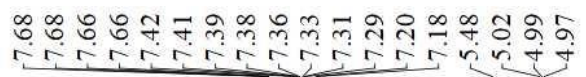

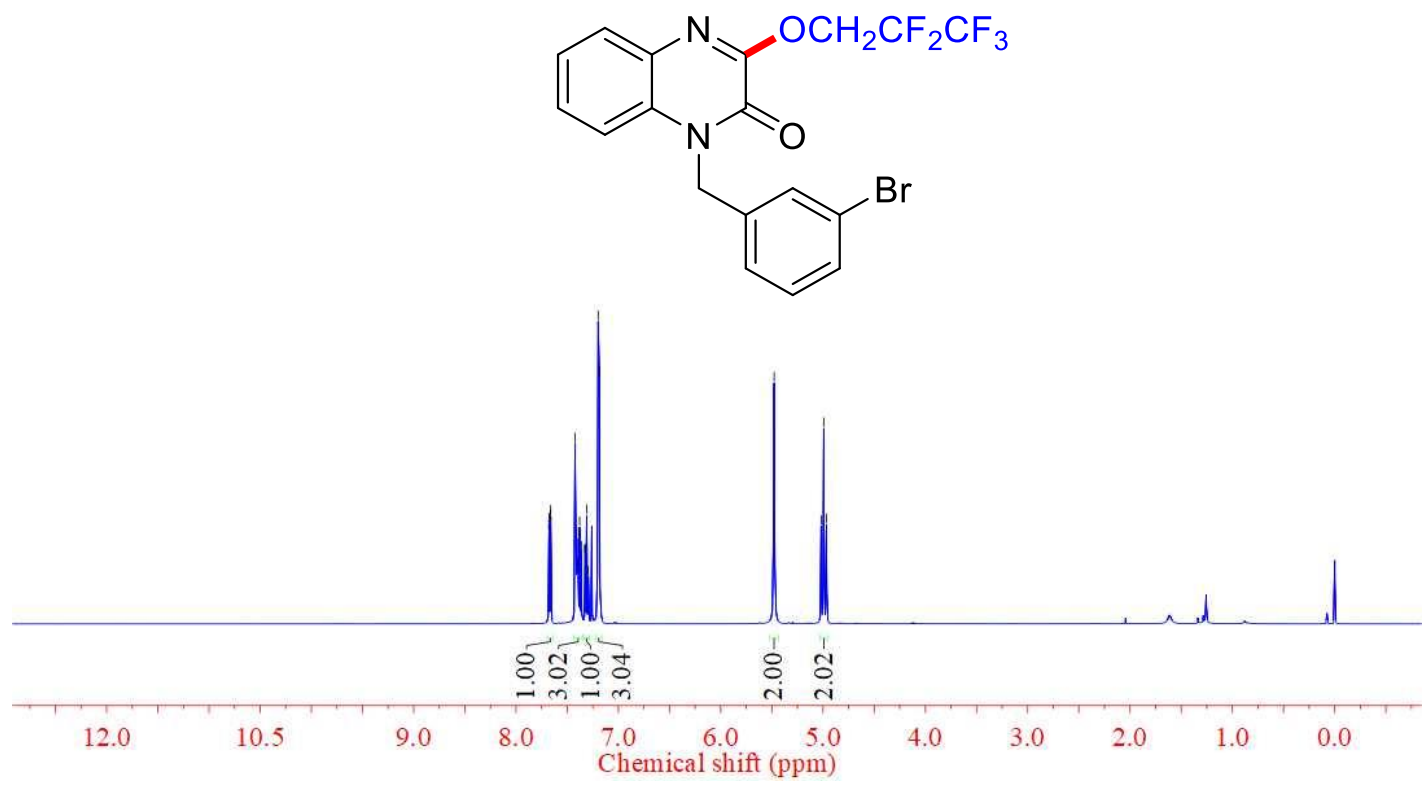




\section{$6 \mathrm{~g}{ }^{13} \mathrm{C}$ NMR}

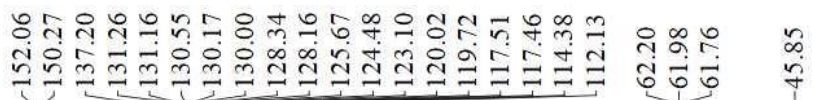

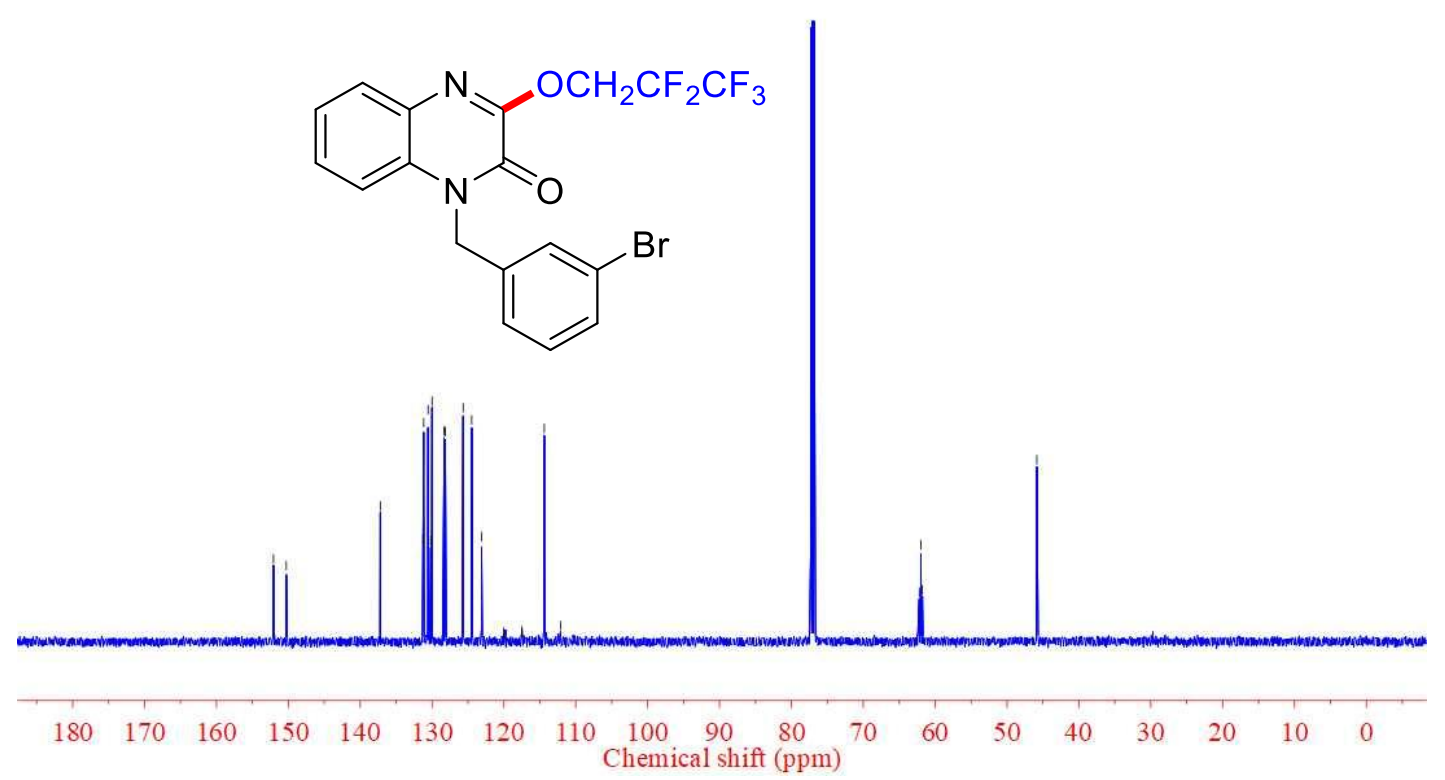

\section{$6{ }^{19}$ F NMR}

$\begin{array}{ll}1 & \frac{1}{1} \\ \infty & \frac{1}{1}\end{array}$

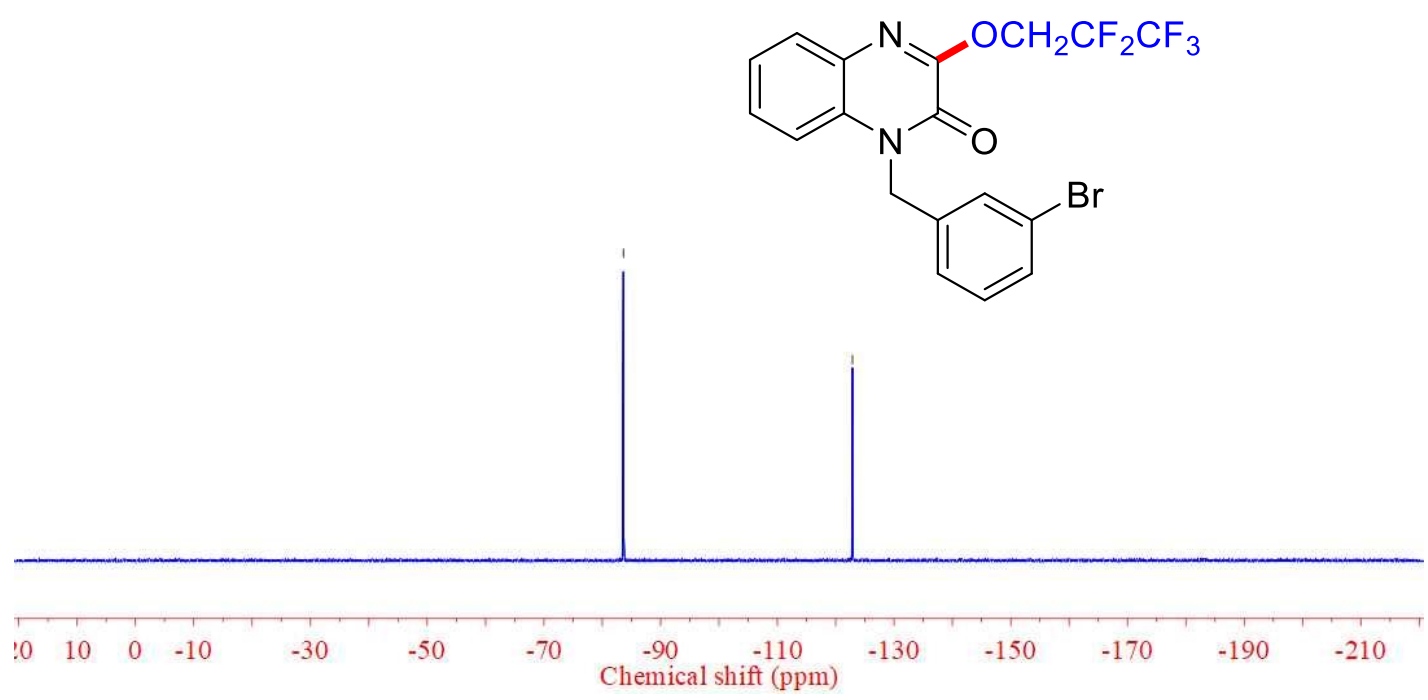




\section{7a ${ }^{1}$ H NMR}

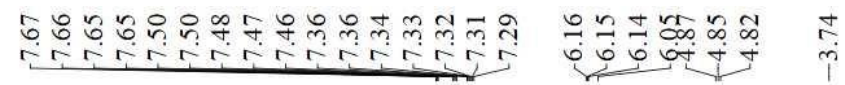

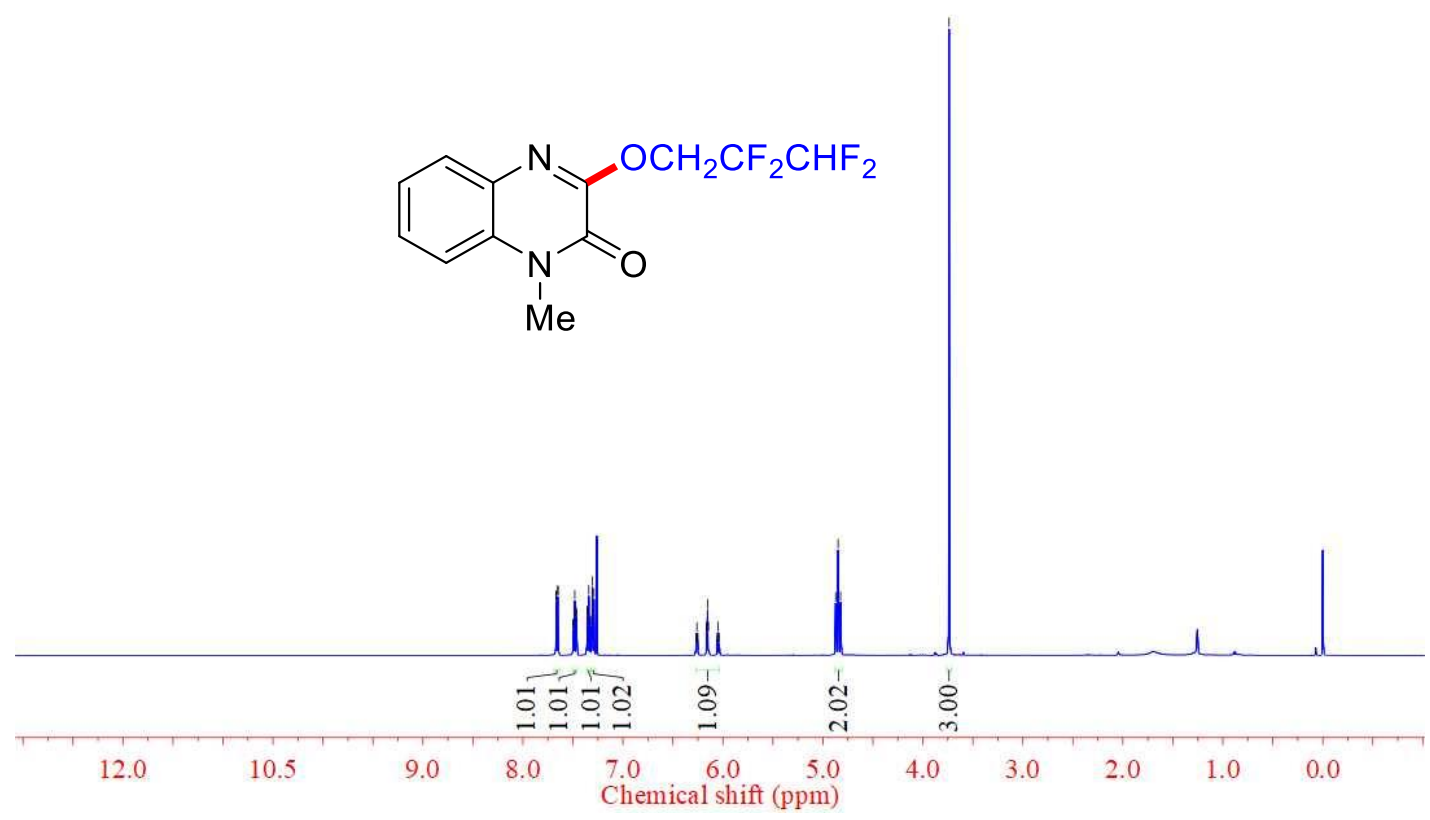

$7 \mathbf{a}^{13} \mathrm{C}$ NMR

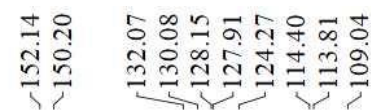

$\rightarrow \infty$

तु

$\stackrel{\infty}{\dddot{\imath}}$
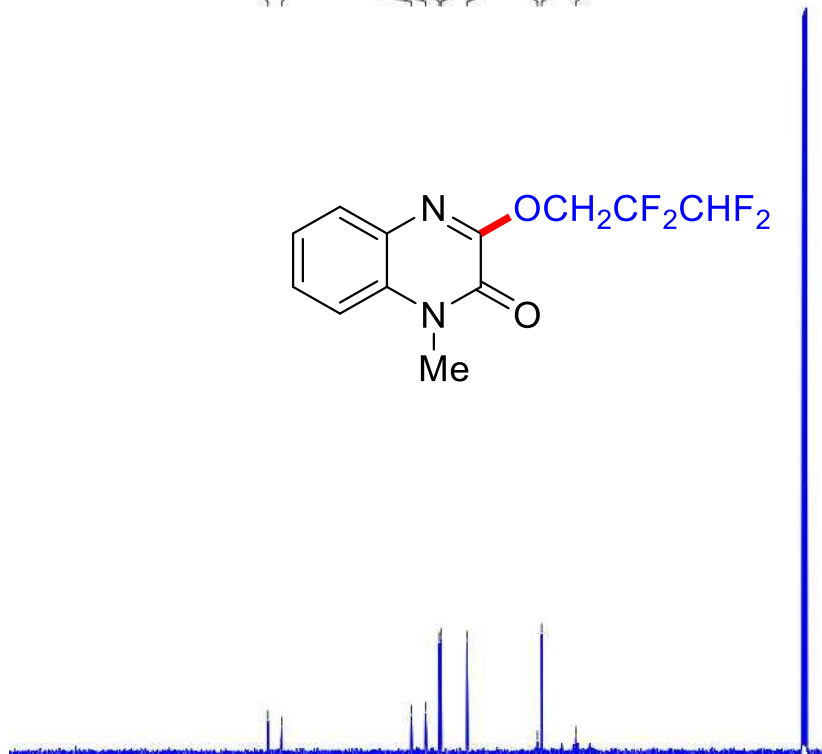

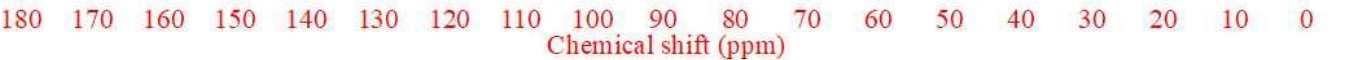




\section{$7 a^{19}$ F NMR}

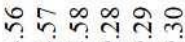

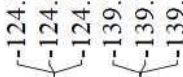
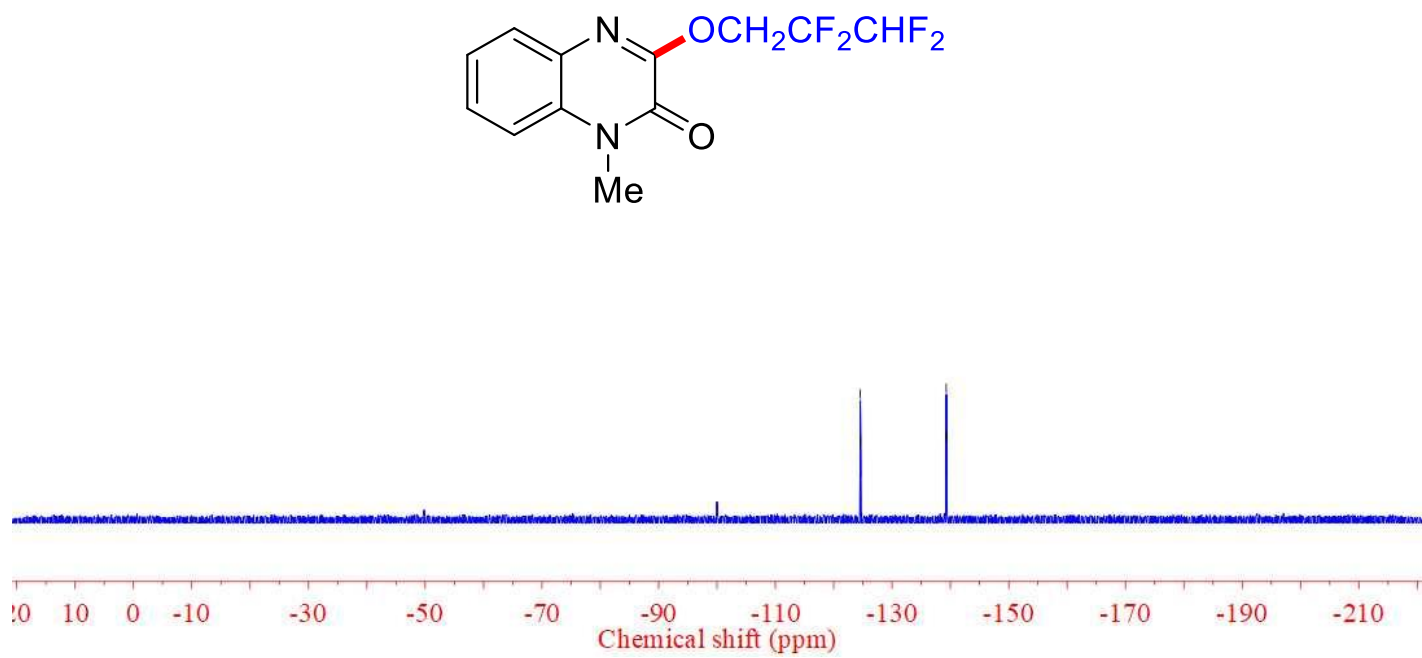

\section{$7 b^{1} H$ NMR}

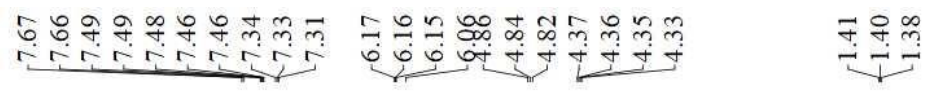<smiles>CCn1c(=O)c(OCC(F)C(F)F)nc2ccccc21</smiles>

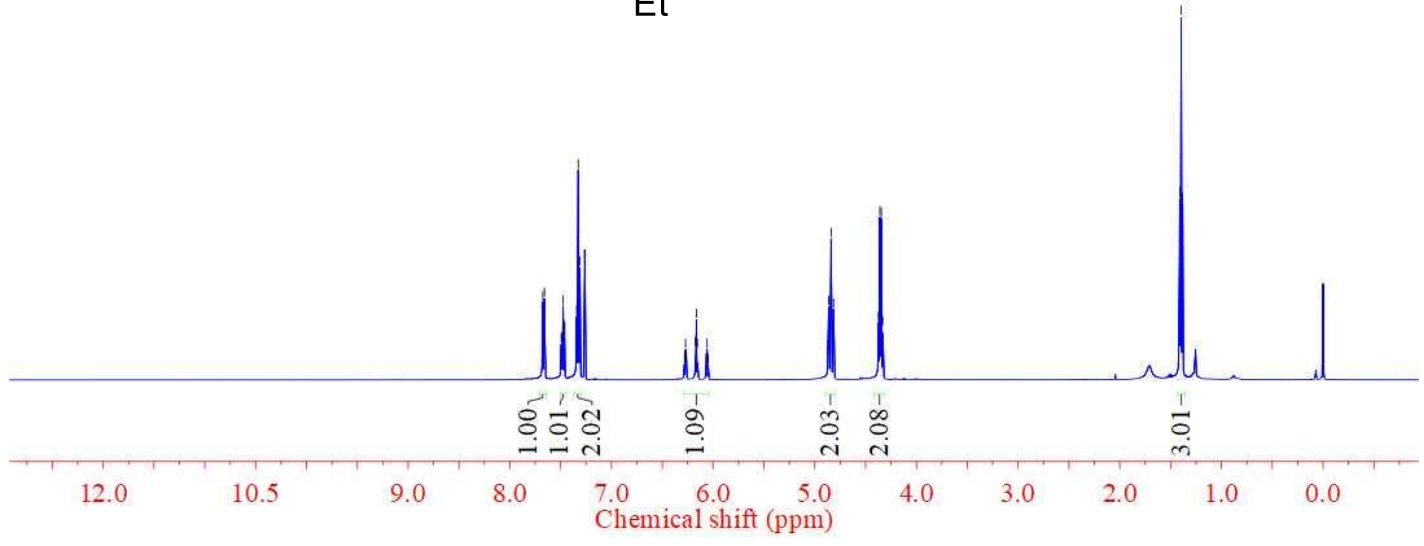




\section{$7 b{ }^{13}$ C NMR}

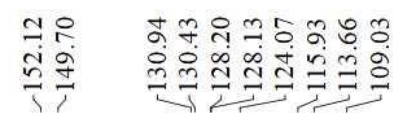

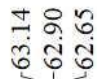

$\underset{\infty}{\infty}$

$\stackrel{\substack{i \\ i}}{i}$

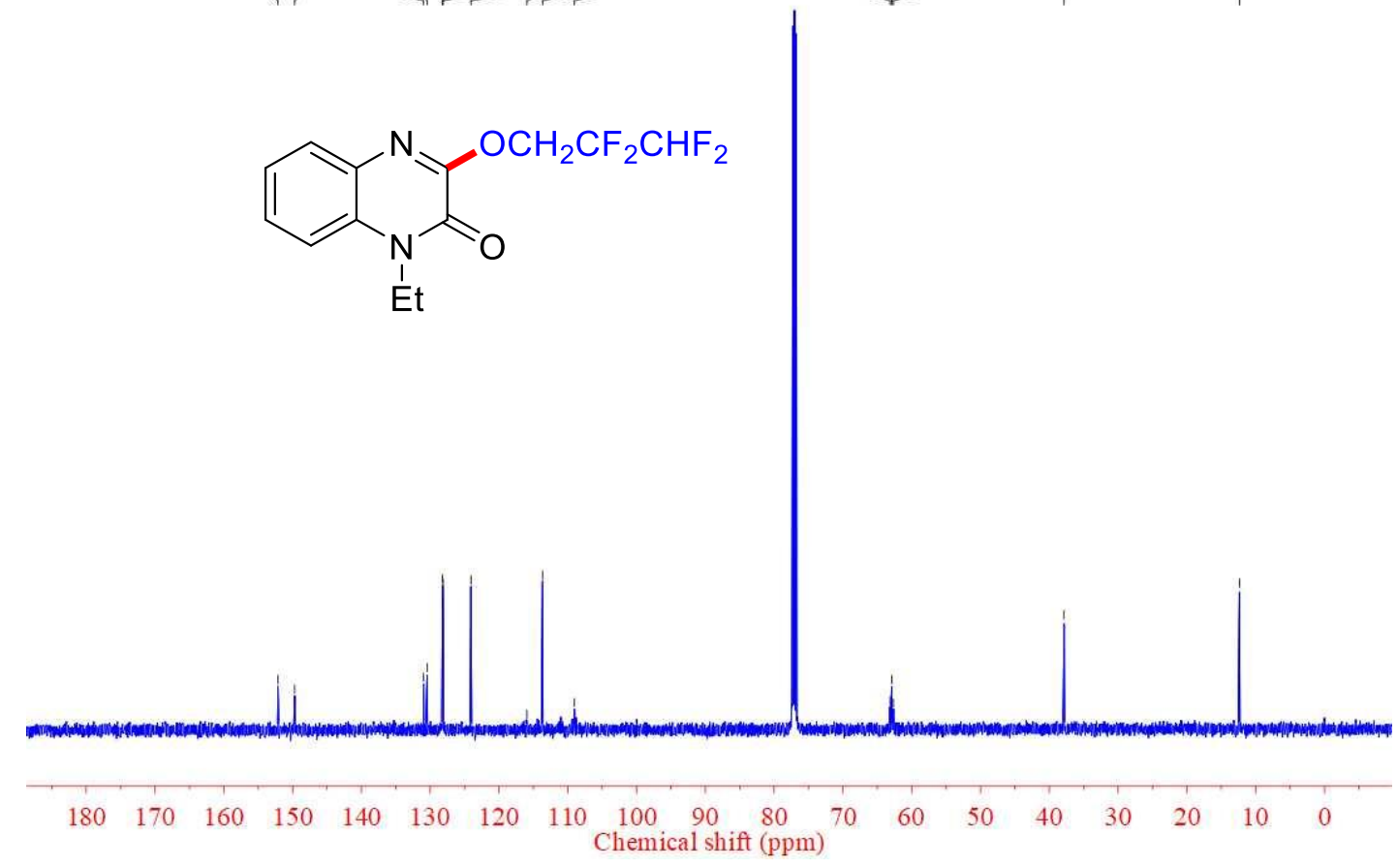

7b ${ }^{19}$ F NMR

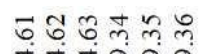

整焉

$\underbrace{\mathrm{N}}_{\mathrm{O}} \mathrm{NCH}_{\mathrm{E}} \mathrm{CF}_{2} \mathrm{CHF}_{2}$

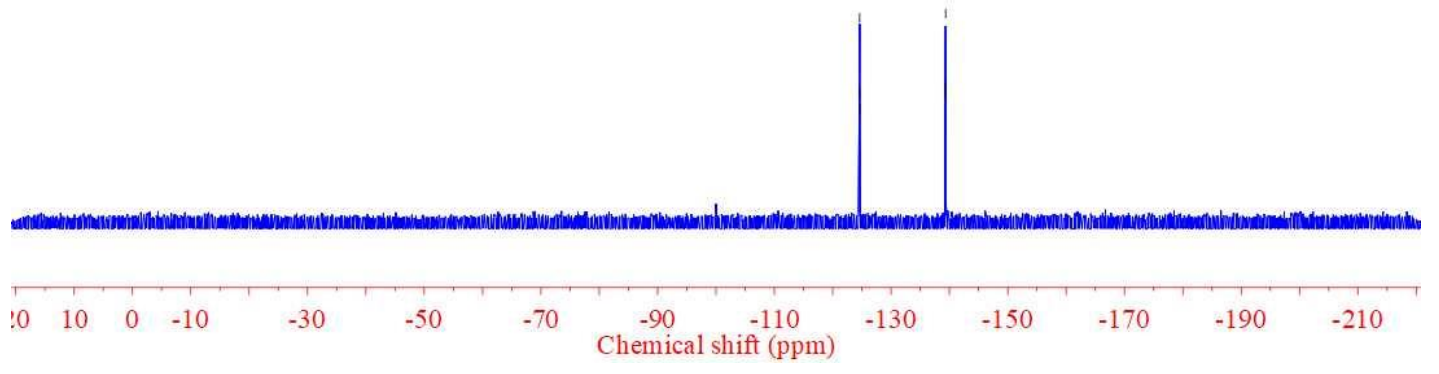




\section{$7 c^{1}$ H NMR}

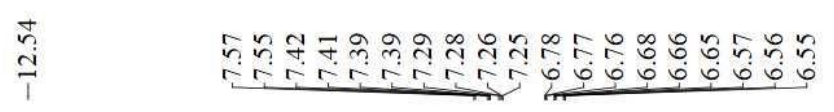<smiles>O=c1[nH]c2ccccc2nc1OCC(F)F</smiles>

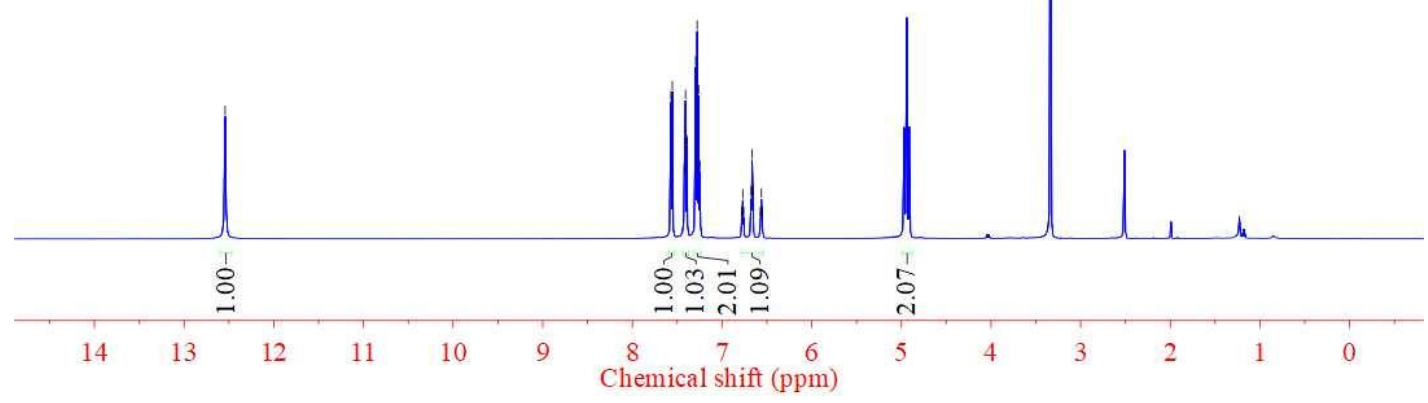

\section{$7 c^{13} \mathrm{C}$ NMR}

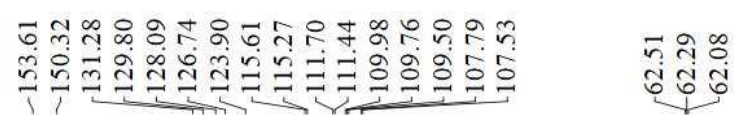
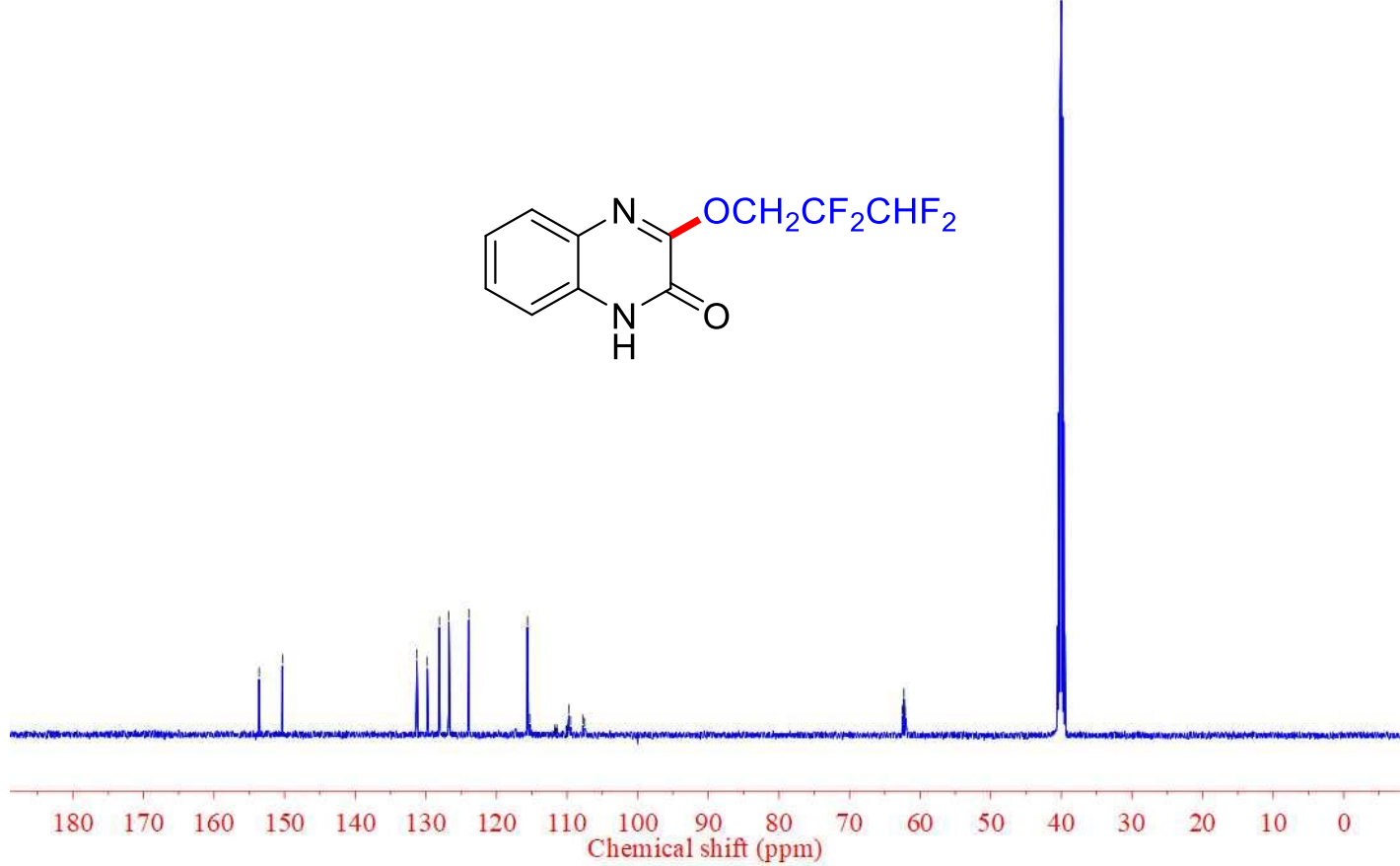


\section{$7 c^{19}$ F NMR}

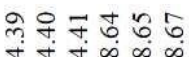

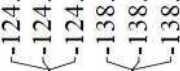<smiles>O=c1[nH]c2ccccc2nc1OCC(F)C(F)F</smiles>

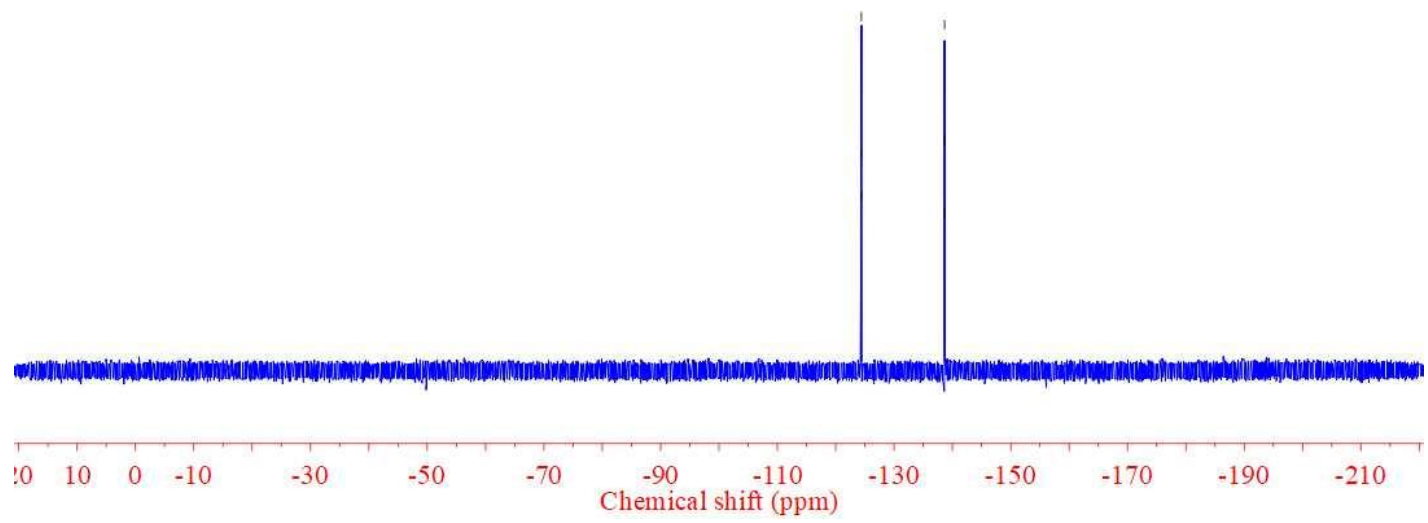

\section{7d ${ }^{1} \mathrm{H}$ NMR}

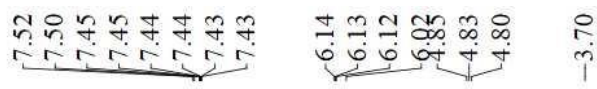<smiles>Cn1c(=O)c(OCC(F)C(F)F)nc2ccc(Br)cc21</smiles>

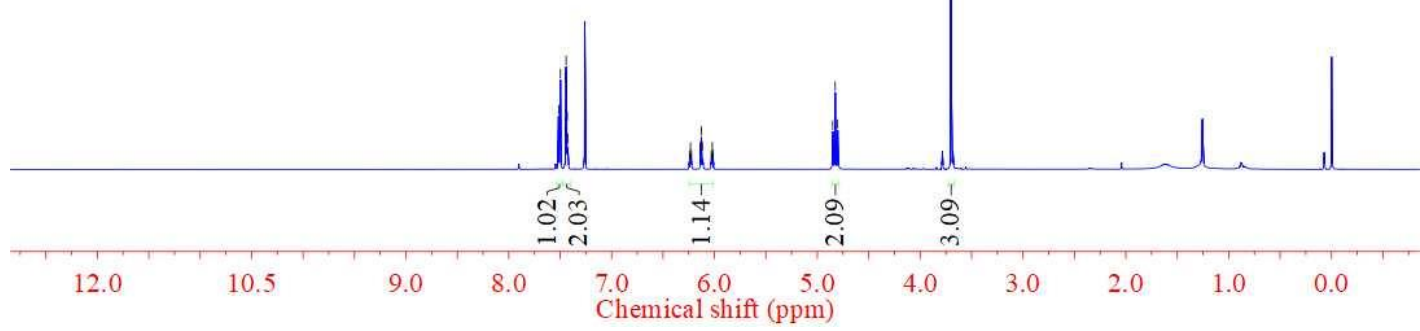




\section{7d ${ }^{13}$ C NMR}

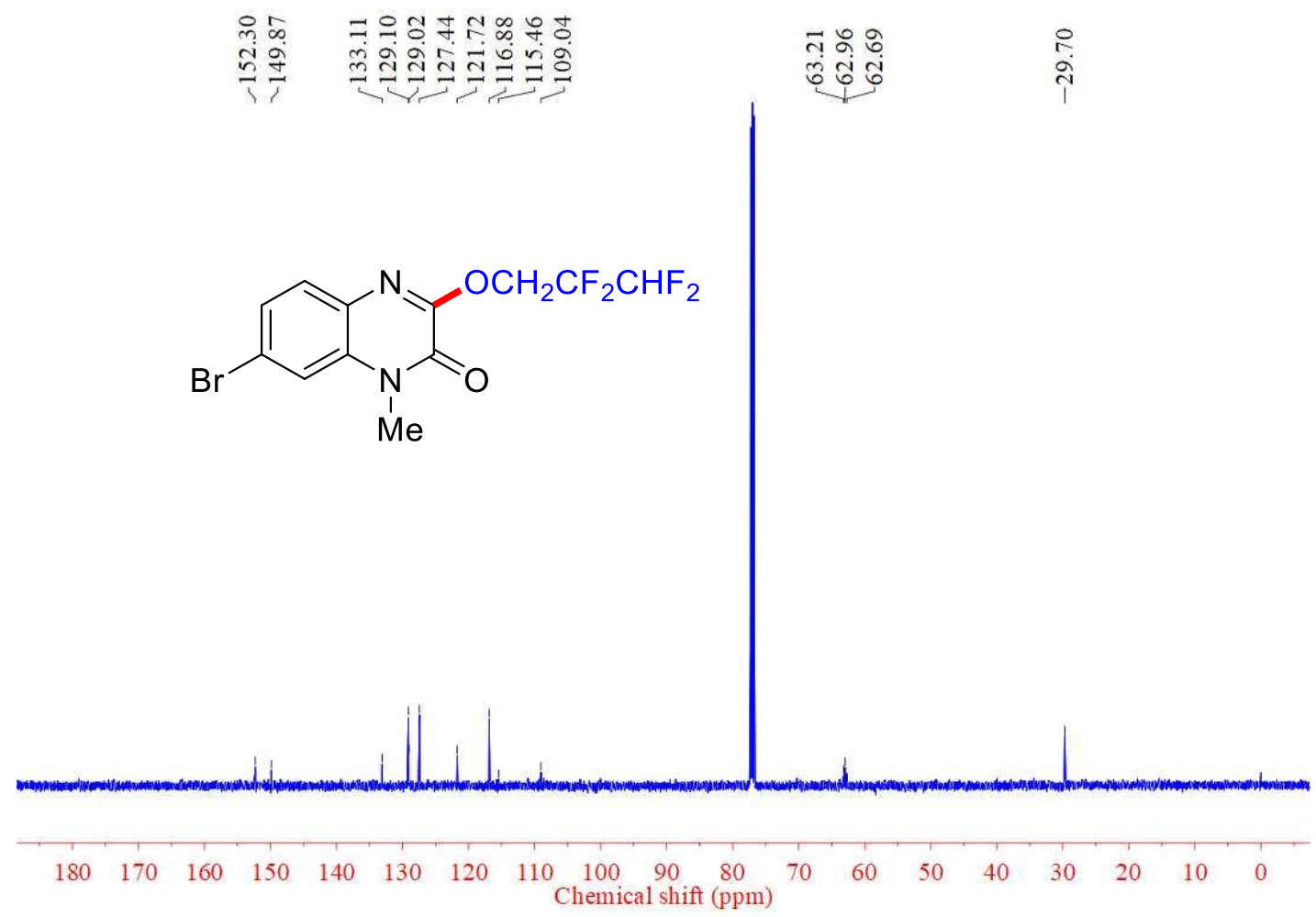

\section{7d ${ }^{19}$ F NMR}

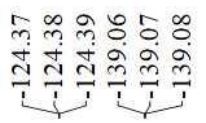<smiles>Cn1c(=O)c(OCC(F)C(F)F)nc2ccc(Br)cc21</smiles>

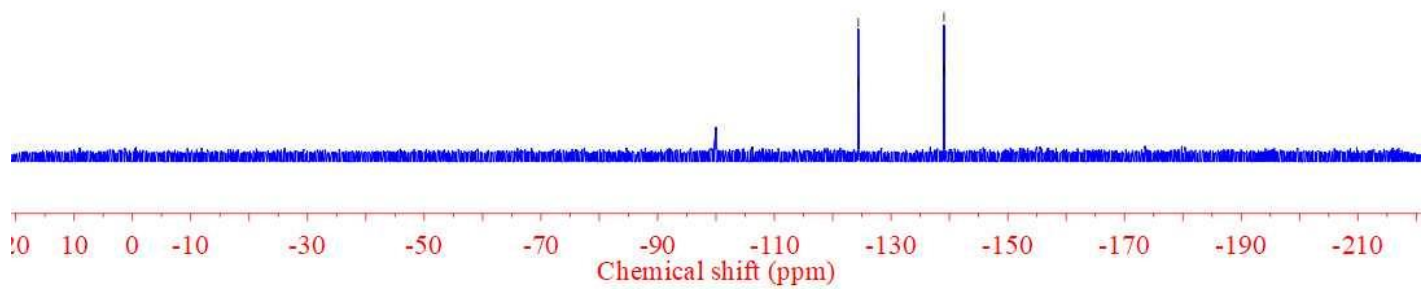




\section{$7 e^{1}$ H NMR}

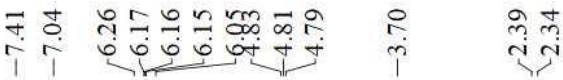

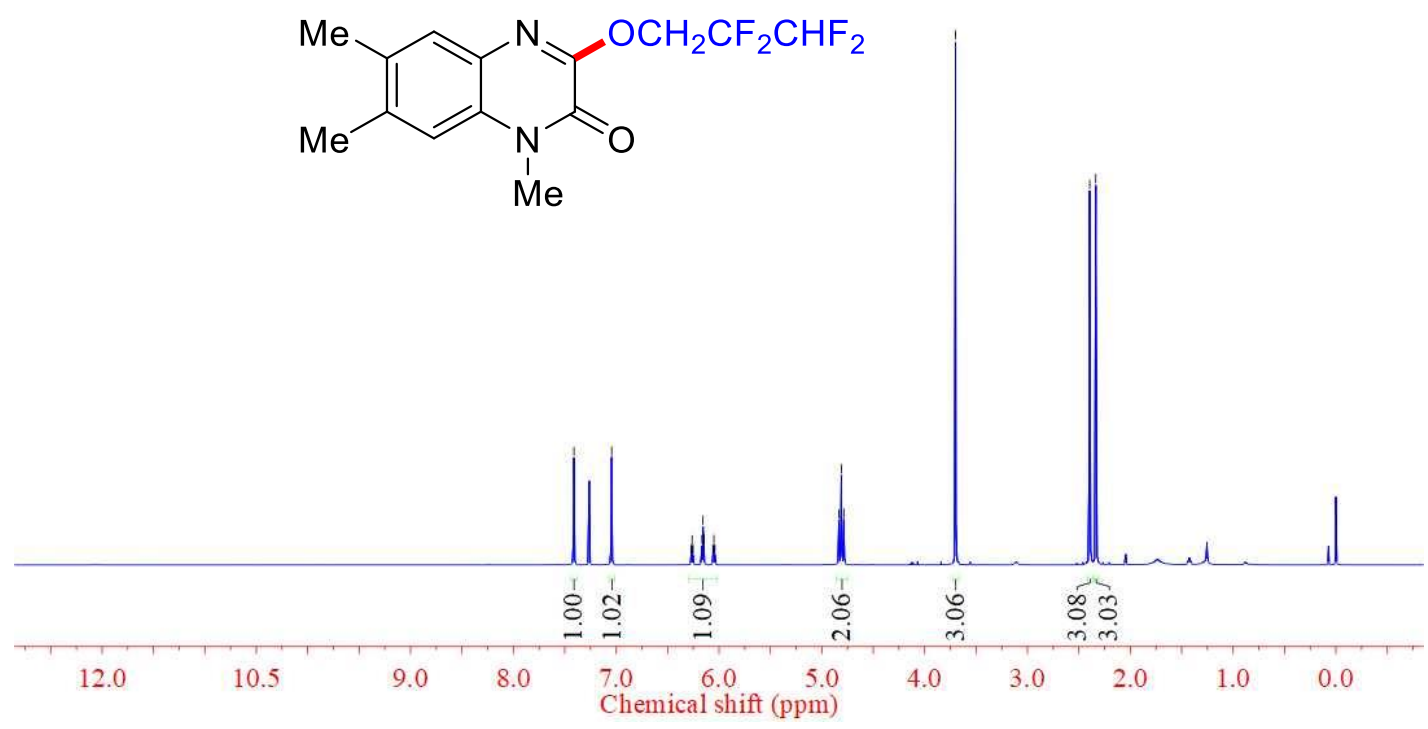

\section{$7 e^{13}$ C NMR}

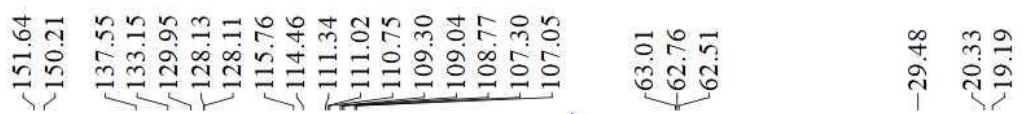<smiles>Cc1cc2nc(OCC(F)C(F)F)c(=O)n(C)c2cc1C</smiles>

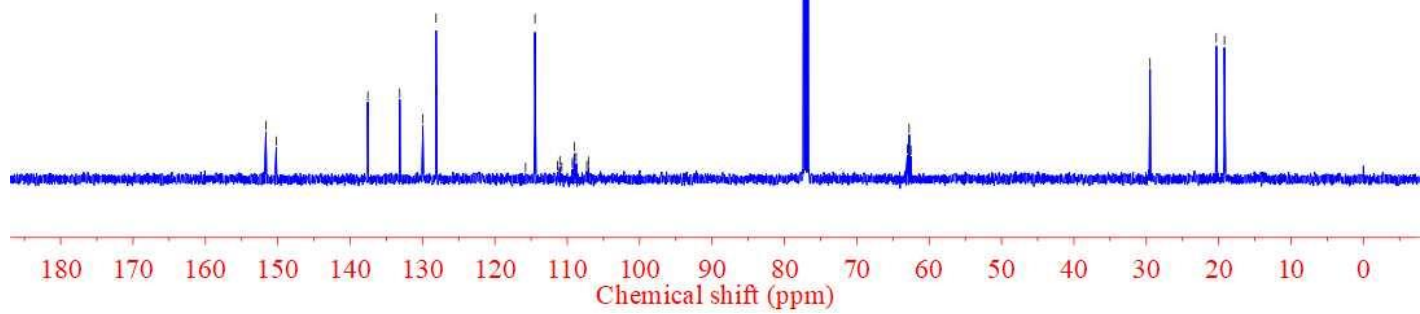


$7 e^{19}$ F NMR

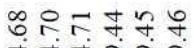

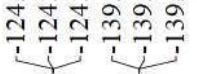

$\overbrace{\substack{1 \\ \mathrm{Me}}}^{\mathrm{OCH} \mathrm{CF}_{2} \mathrm{CHF}_{2}}$

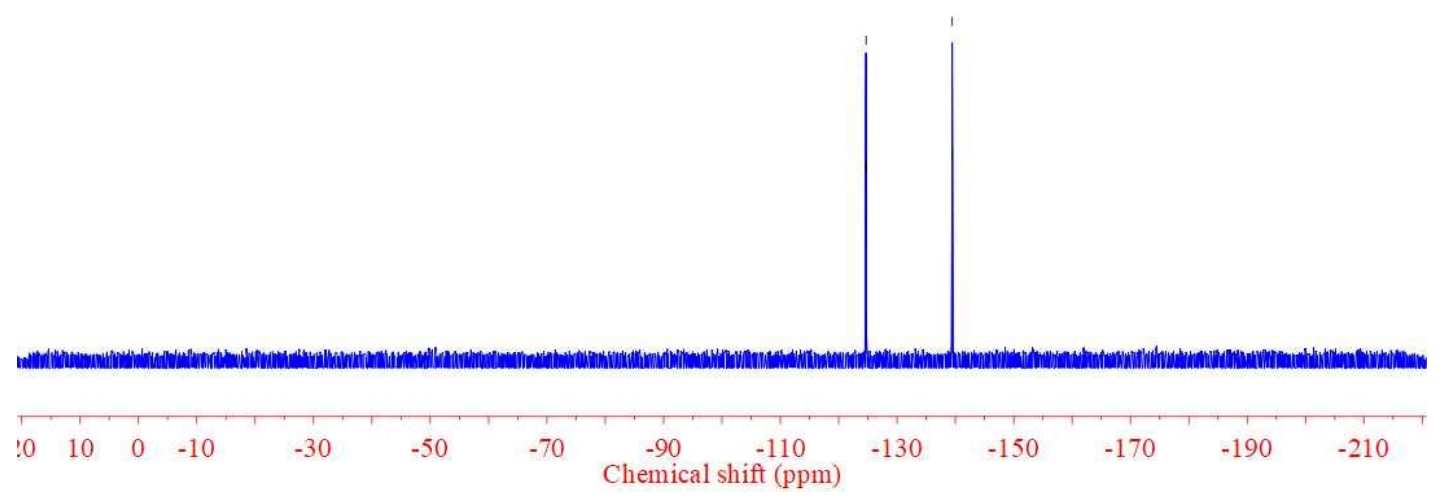

\section{8a ${ }^{1}$ H NMR}

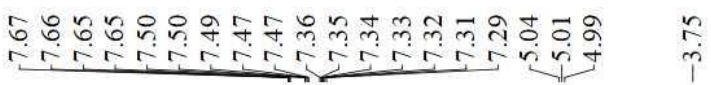

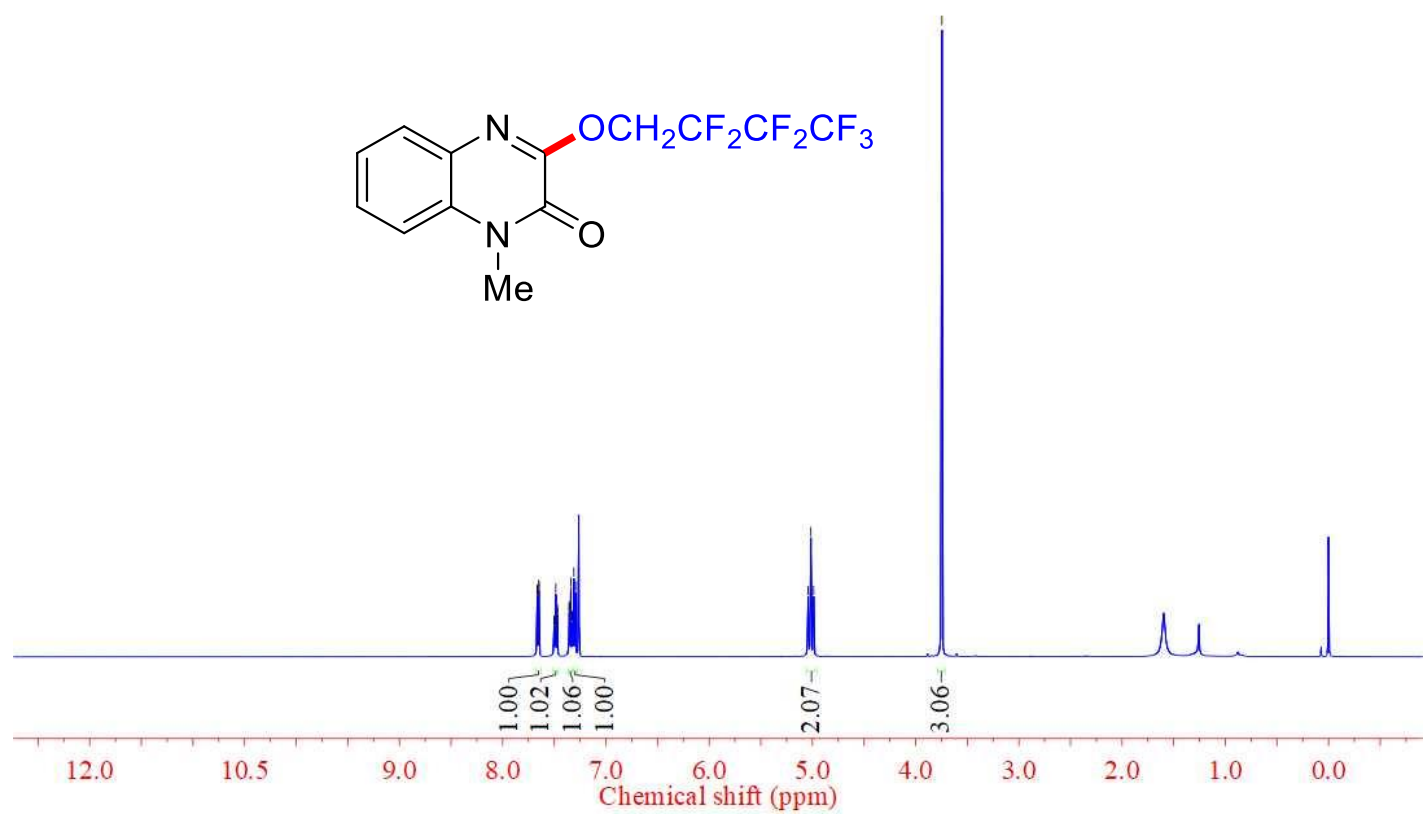




\section{$8 a^{13}$ C NMR}

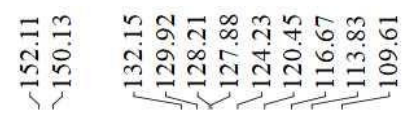

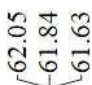

ํํํ<smiles>[Y6]n1c(=O)c(OCC(F)(F)C(F)(F)C(F)(F)C(C)(F)F)nc2ccccc21</smiles>

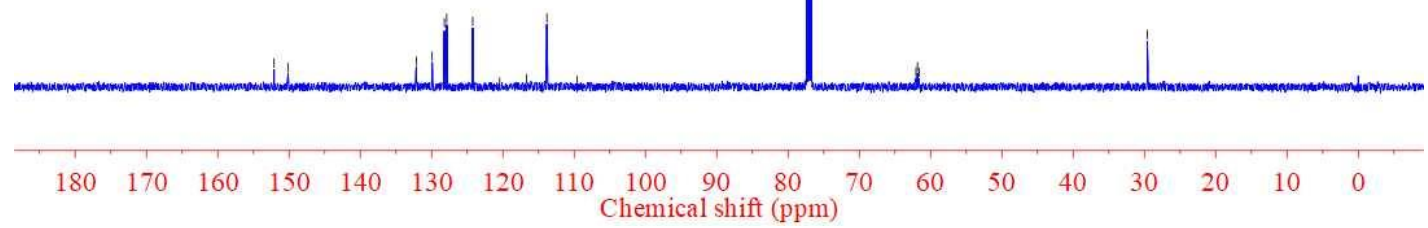

\section{$8 a^{19}$ F NMR}

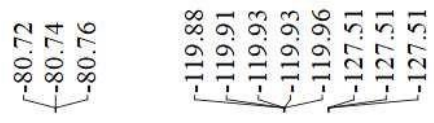<smiles>Cn1c(=O)c(OCC(F)C(F)(F)F)nc2ccccc21</smiles>

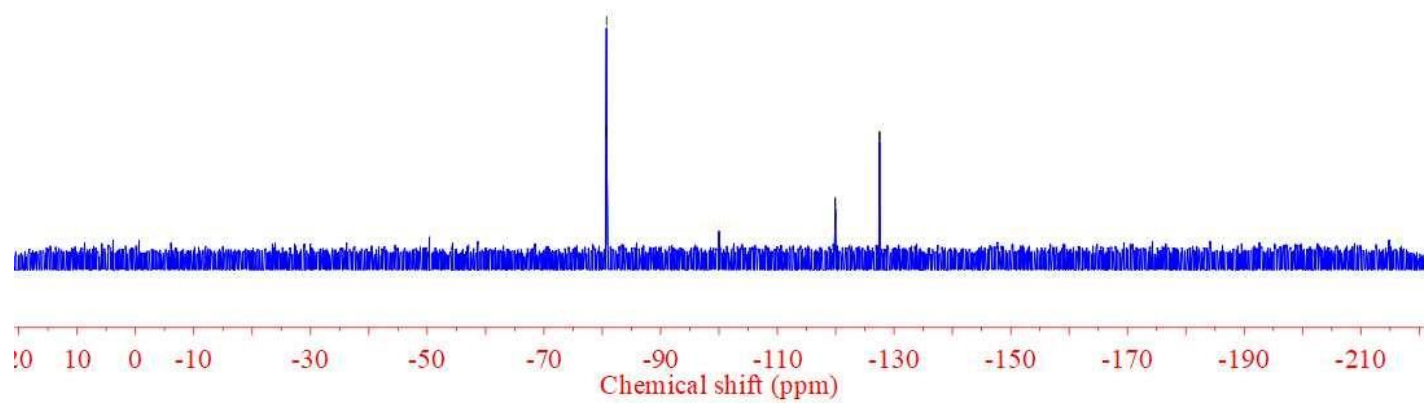




\section{8b ${ }^{1}$ H NMR}

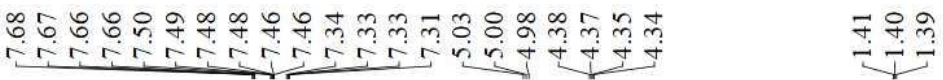<smiles>CCn1c(=O)c(OCC(F)F)nc2ccccc21</smiles>

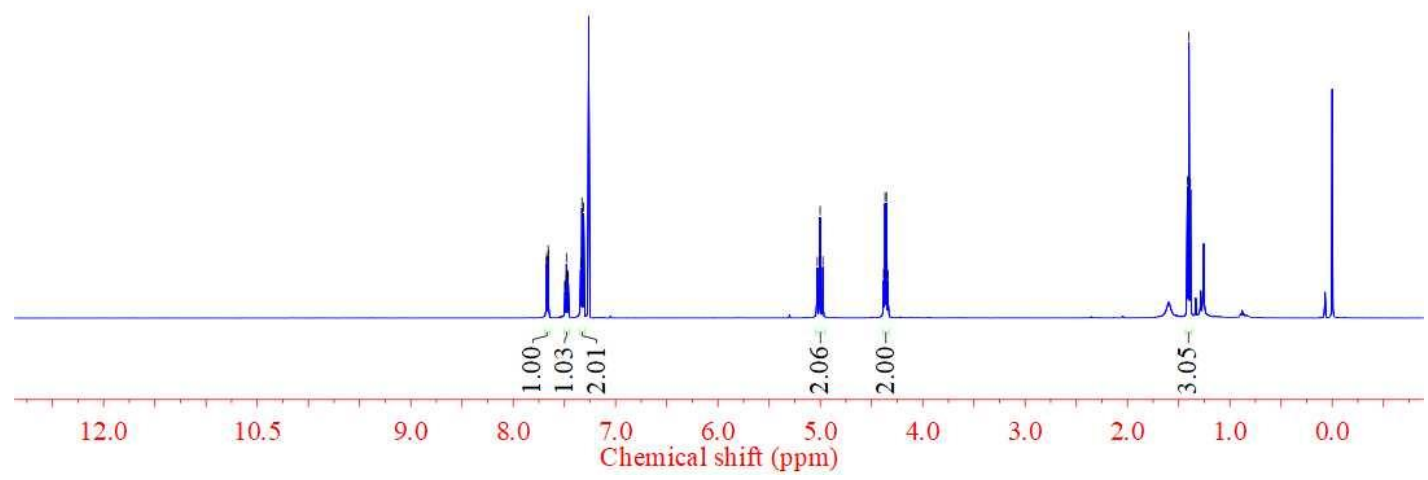

$8 b{ }^{13} \mathrm{C}$ NMR

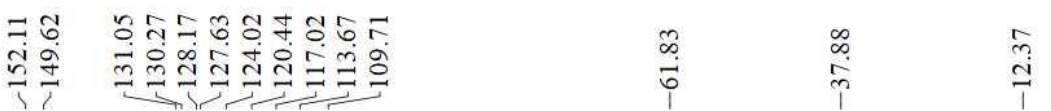
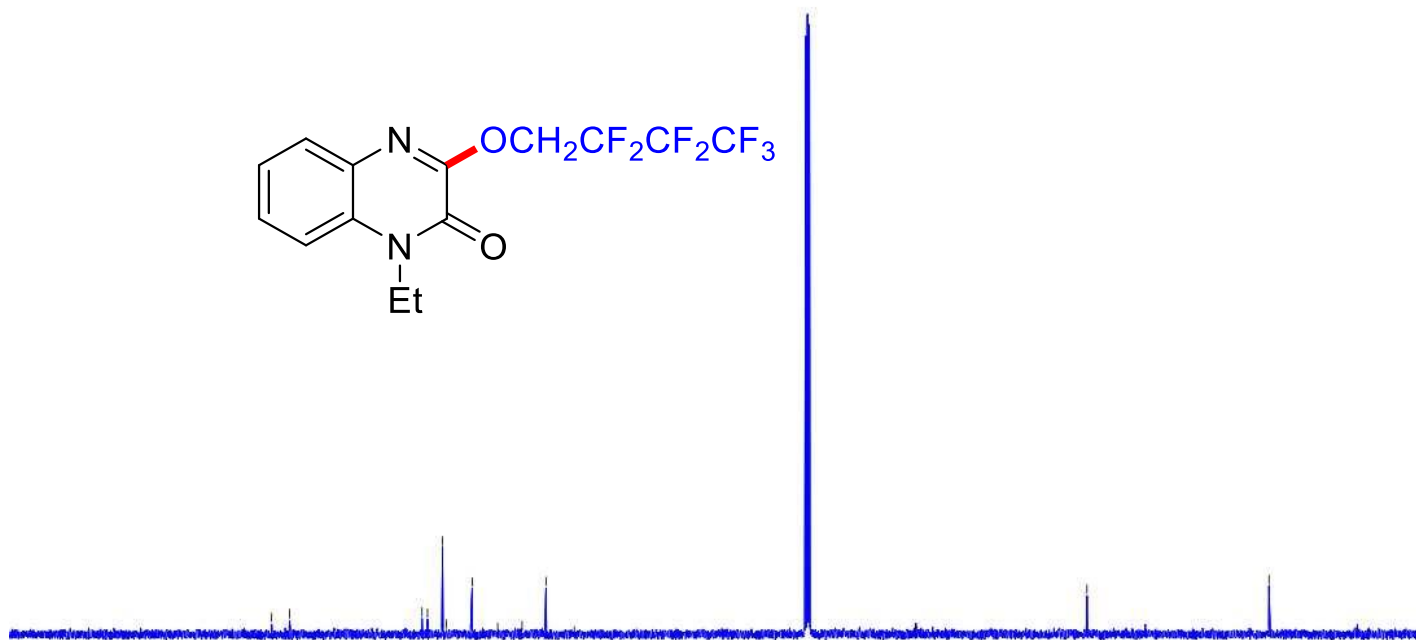

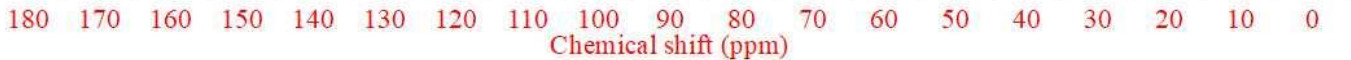




\section{$8 b{ }^{19}$ F NMR}

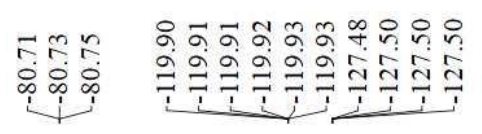

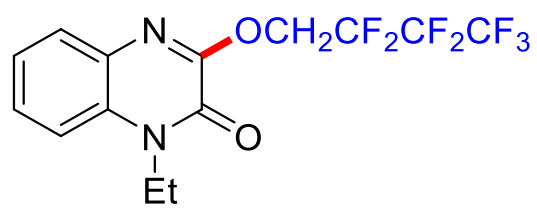

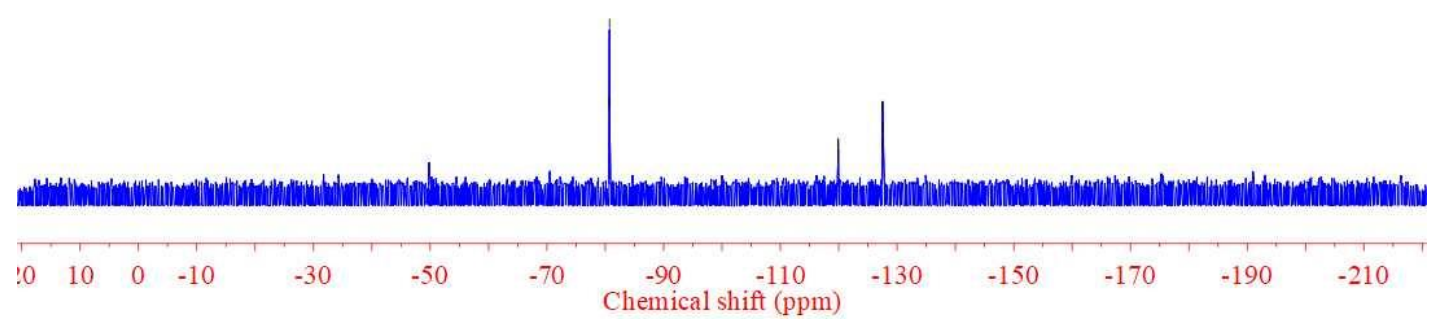

\section{8c ${ }^{1}$ H NMR}

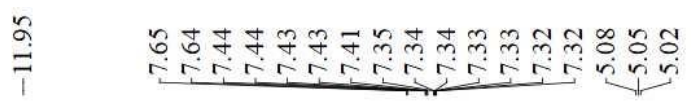<smiles>O=c1[nH]c2ccccc2nc1OCC1CCCCC1(F)F</smiles>

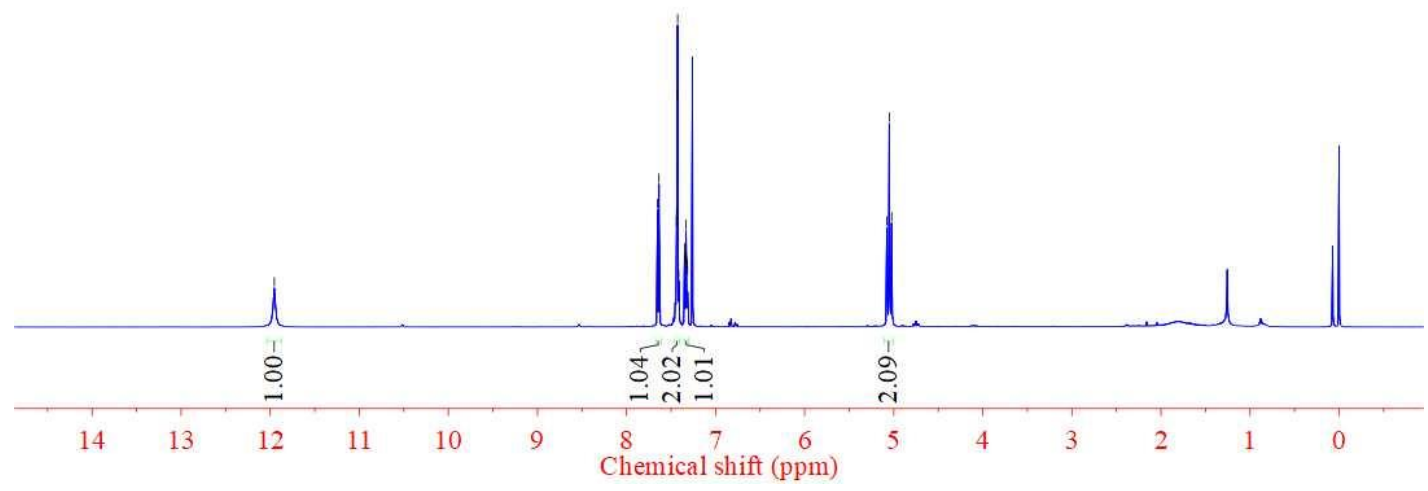


8c ${ }^{13} \mathrm{C}$ NMR

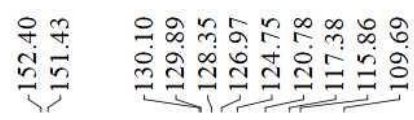

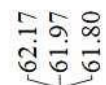

(c)

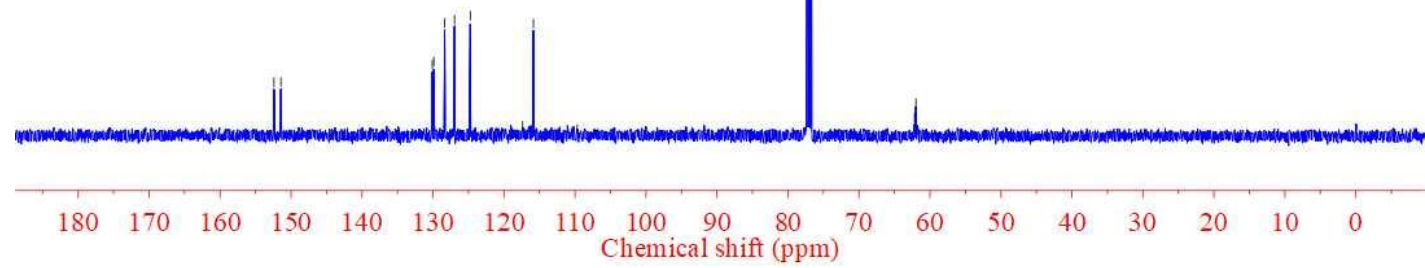

\section{$8 c^{19}$ F NMR}

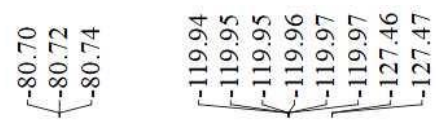

$\mathrm{N}_{\mathrm{H}}^{\mathrm{OCH}_{2} \mathrm{CF}_{2} \mathrm{CF}_{2} \mathrm{CF}_{3}}$

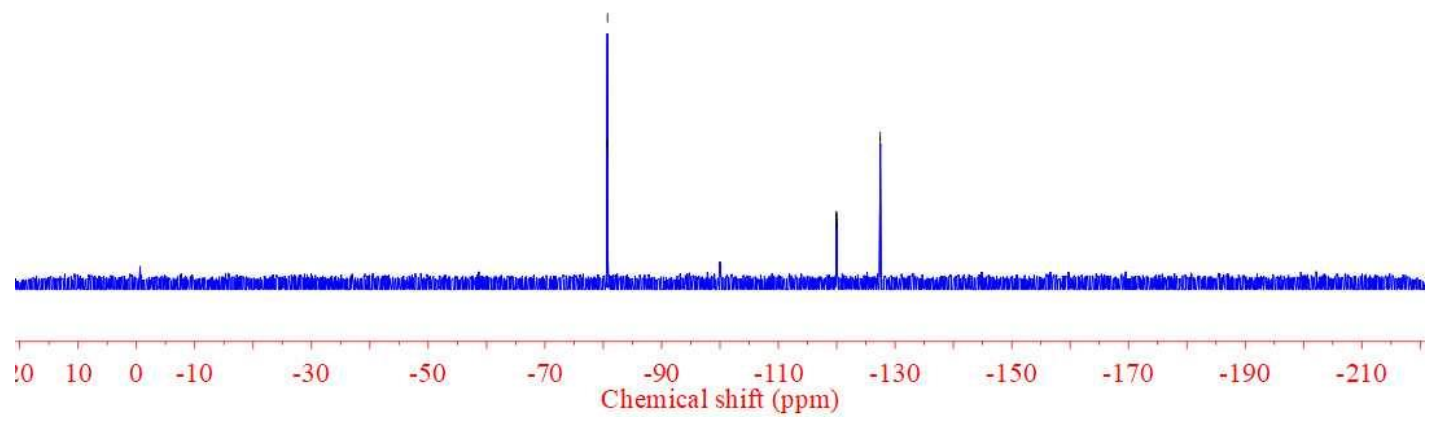




\section{8d ${ }^{1}$ H NMR}

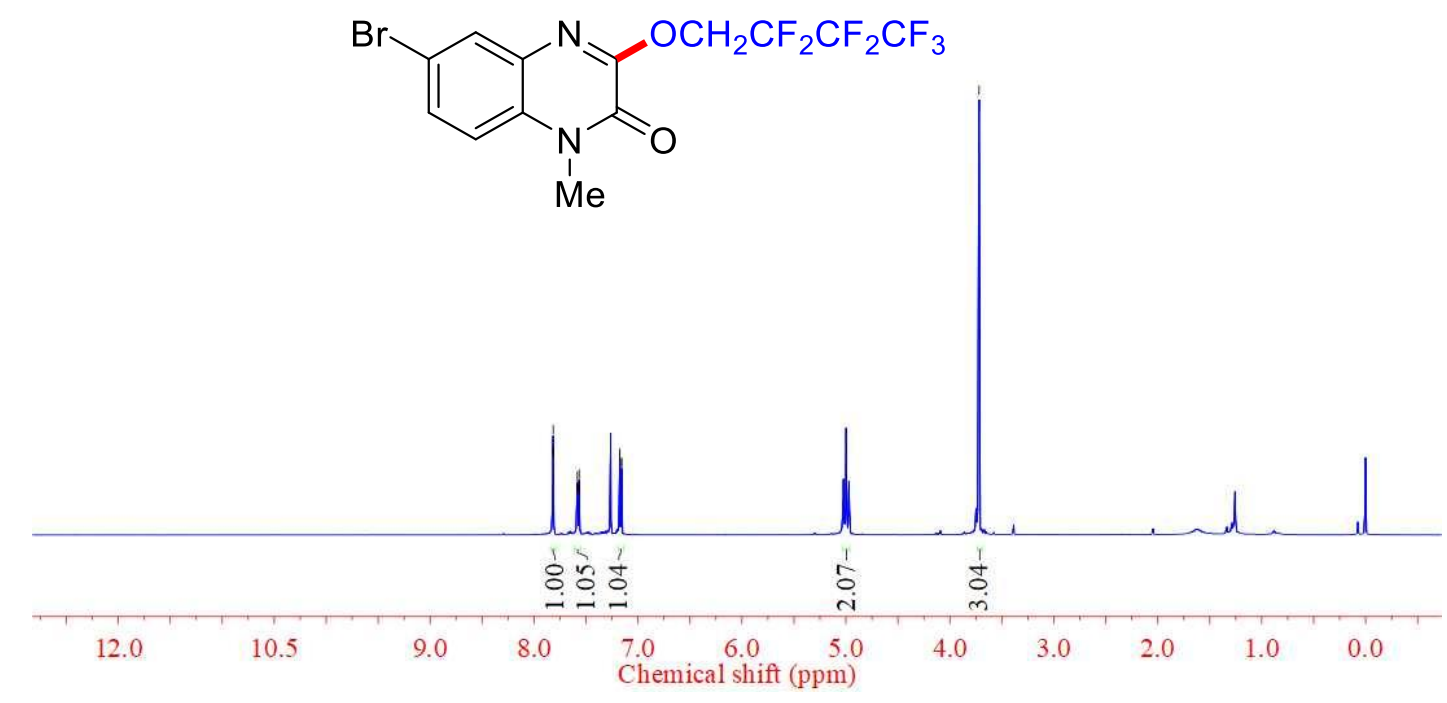

8d ${ }^{13} \mathrm{C}$ NMR

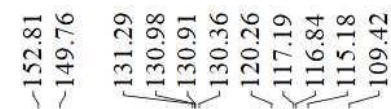

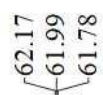

$\stackrel{\circ}{\stackrel{2}{i}}$

$\mathrm{Br}$<smiles></smiles>

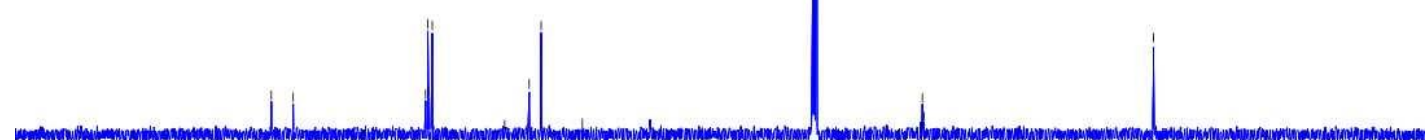

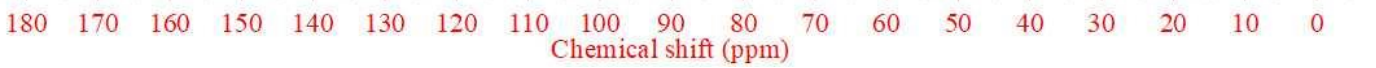




\section{8d ${ }^{19}$ F NMR}

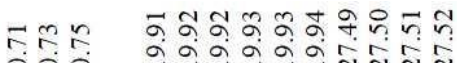 \\ 舟金}
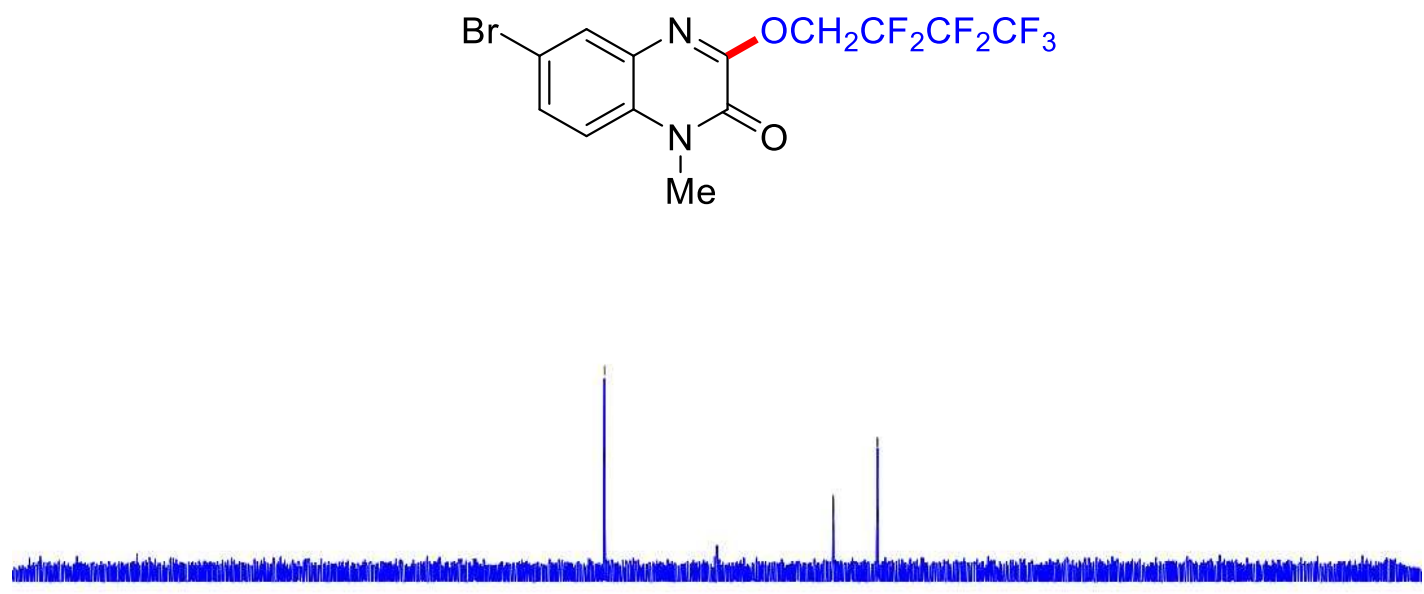

\begin{tabular}{lllllllllllll}
\hline 0 & 10 & 0 & -10 & -30 & -50 & -70 & $\begin{array}{c}-90 \\
\text { Chemical shift }(\mathrm{ppm})\end{array}$ & -130 & -150 & -170 & -190 & -210
\end{tabular}

\section{$8 e^{1}$ H NMR}

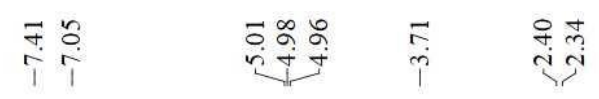<smiles>Cc1cc2nc(OCC(F)C(F)(F)F)c(=O)n(C)c2cc1C</smiles>

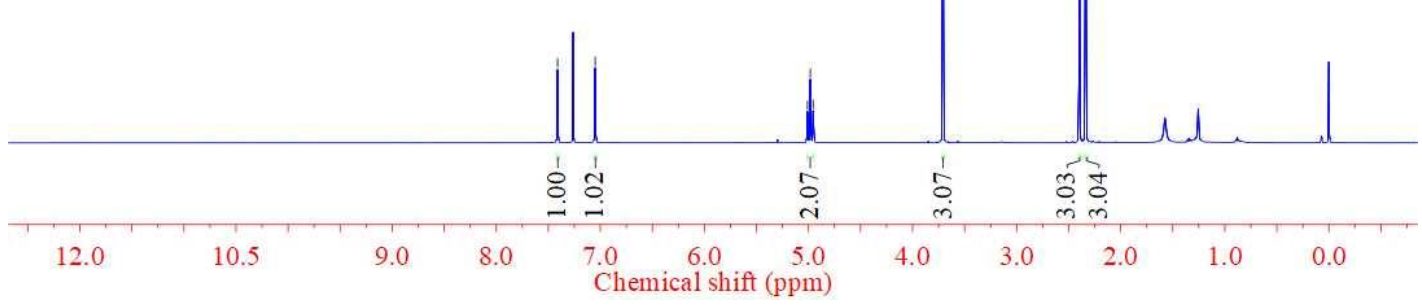




\section{$8 e^{13}$ C NMR}

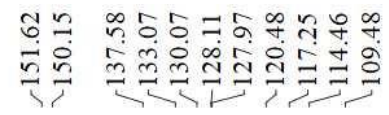

通

ๆे लำ

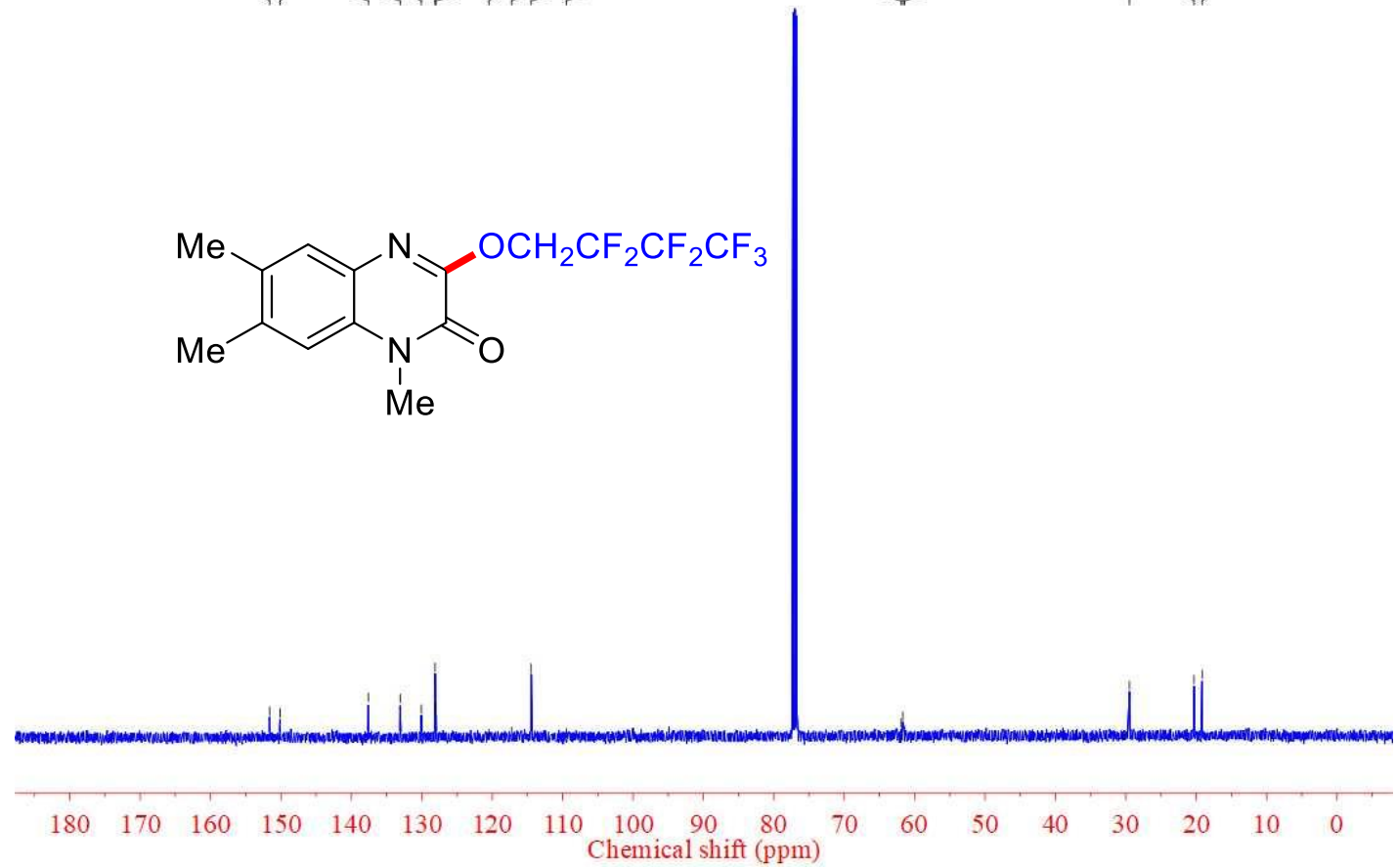

\section{$8 e^{19}$ F NMR}

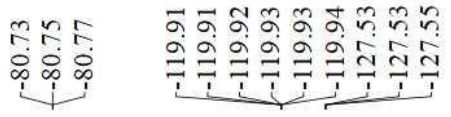<smiles>Cc1cc2nc(OCC(F)C(F)(F)C(F)(F)F)c(=O)n(C)c2cc1C</smiles>

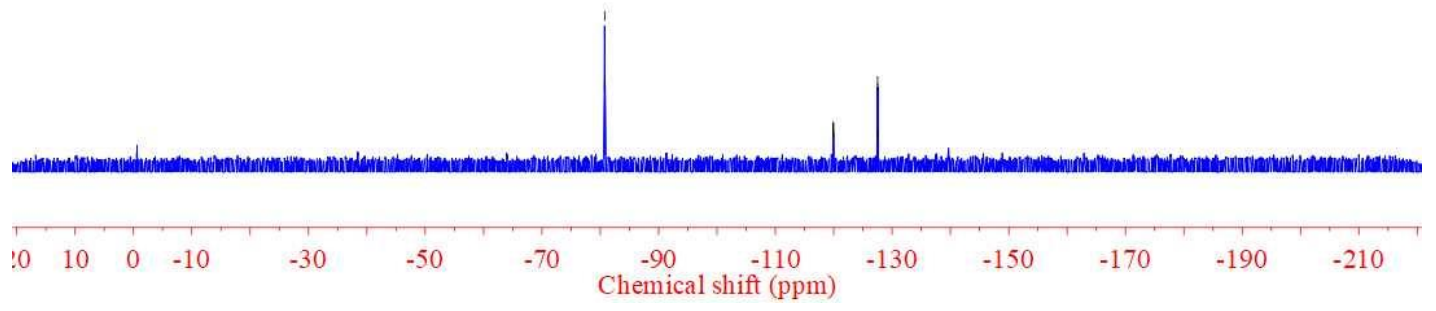




\section{9a ${ }^{1}$ H NMR}

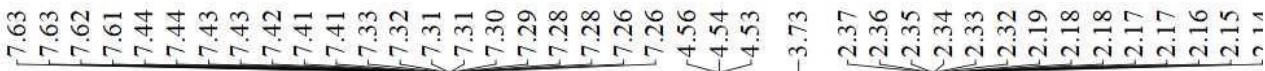

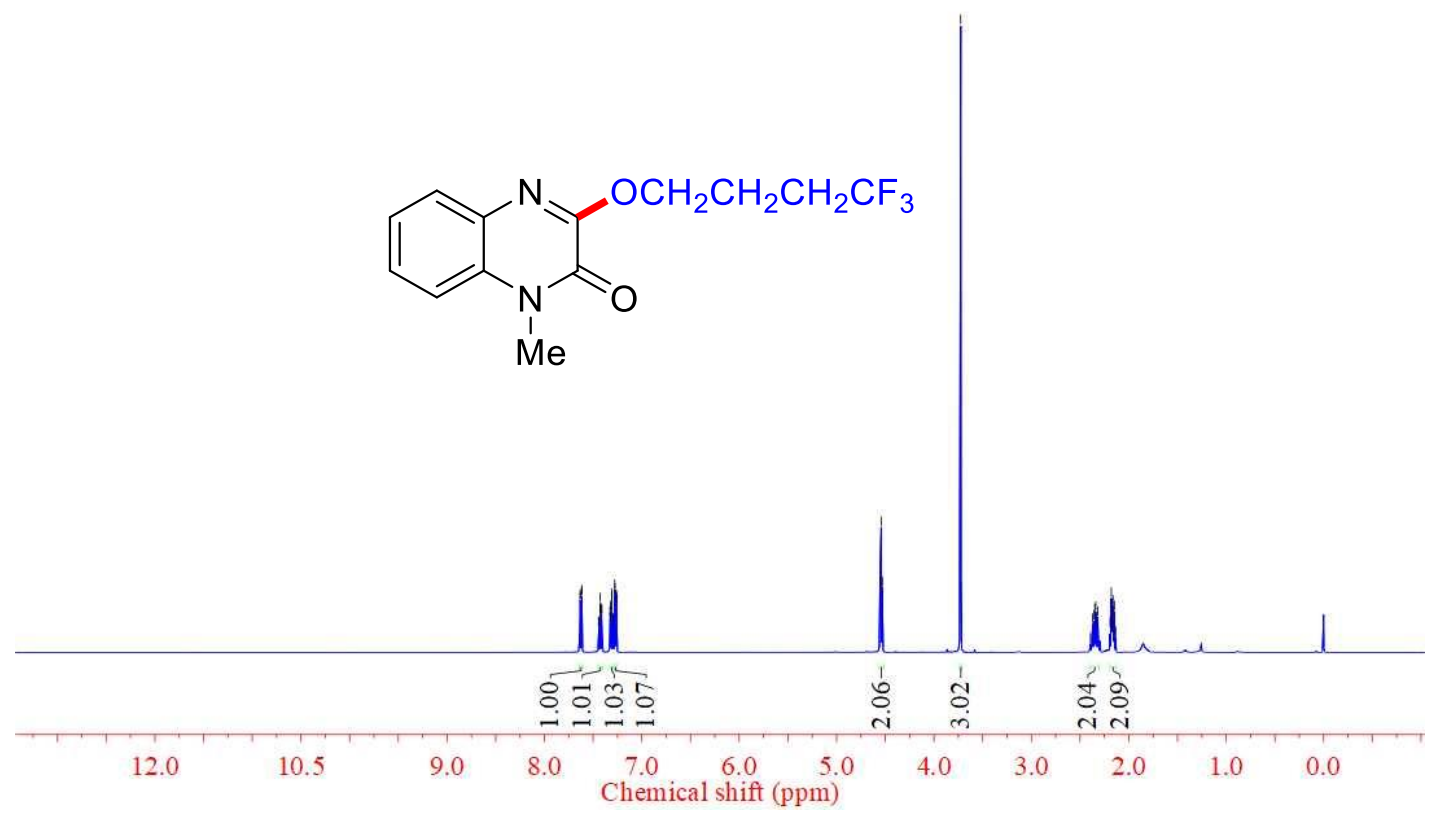

9a ${ }^{13} \mathrm{C}$ NMR

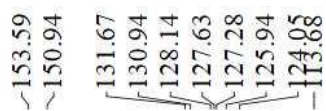

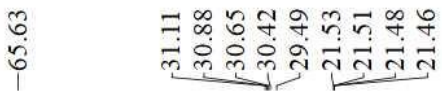
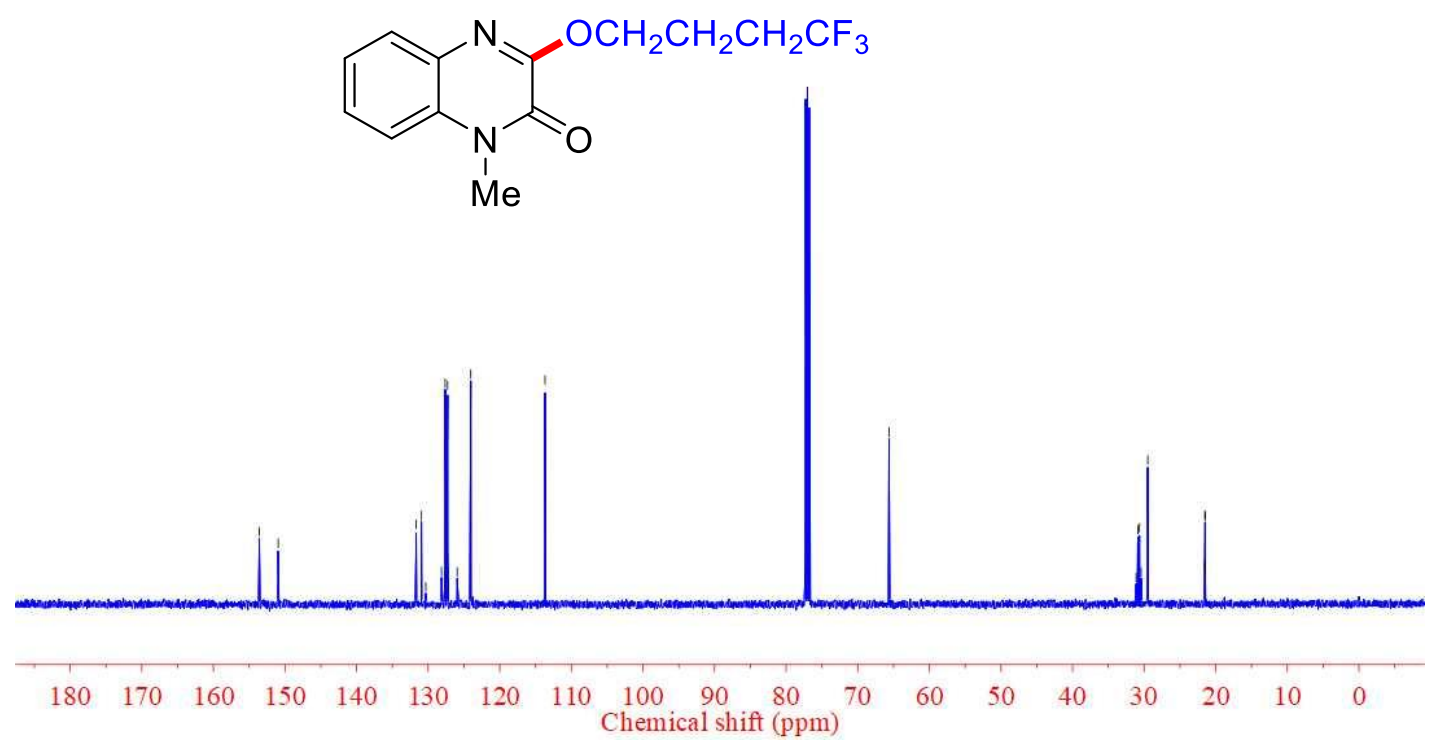


\section{$9 a^{19}$ F NMR}

0
m.
$\vdots$
$\vdots$
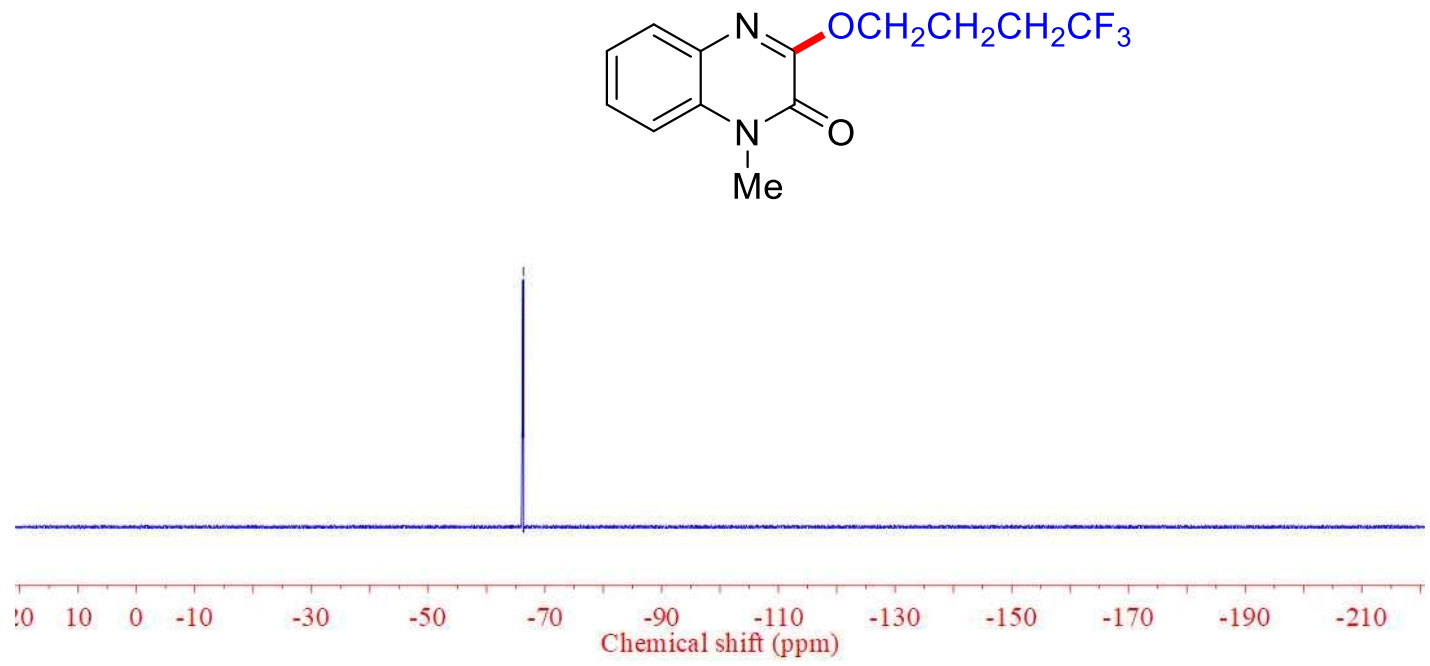

\section{9b ${ }^{1} \mathrm{H}$ NMR}

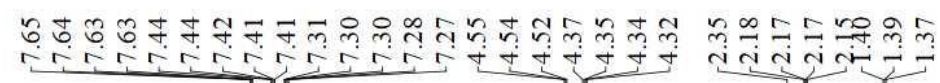<smiles>CCn1c(=O)c(OCCCC(F)(F)F)nc2ccccc21</smiles>

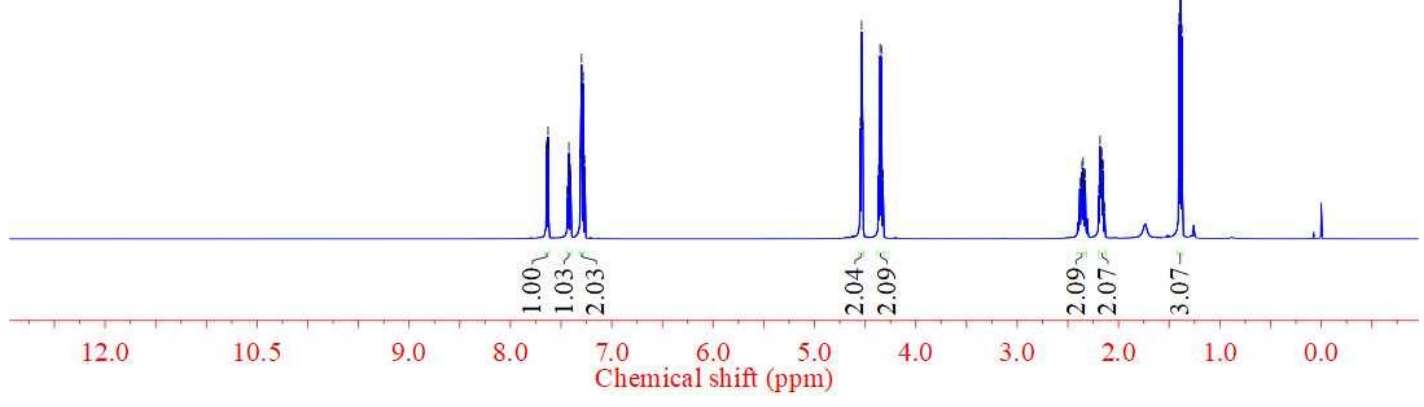


$9 b{ }^{13} \mathrm{C}$ NMR

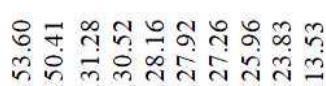

ñ.

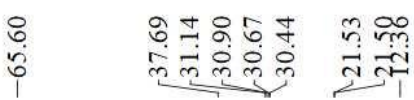
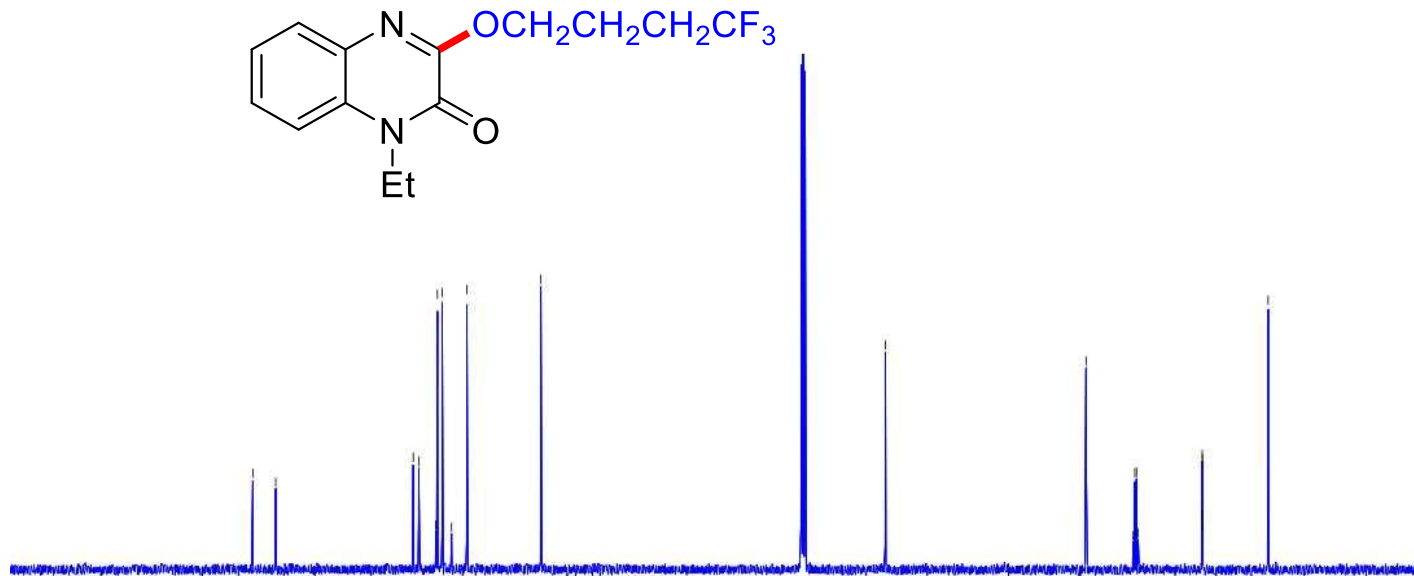

$\begin{array}{llllllllllllllllll}180 & 170 & 160 & 150 & 140 & 130 & 120 & 110 & \begin{array}{c}100 \\ \text { Chemical shift }(\mathrm{ppm})\end{array} & \begin{array}{c}80 \\ \text { Che }\end{array} & 60 & 50 & 40 & 30 & 20 & 10 & 0\end{array}$

\section{9b ${ }^{19}$ F NMR}

0
ñ
0
$i$
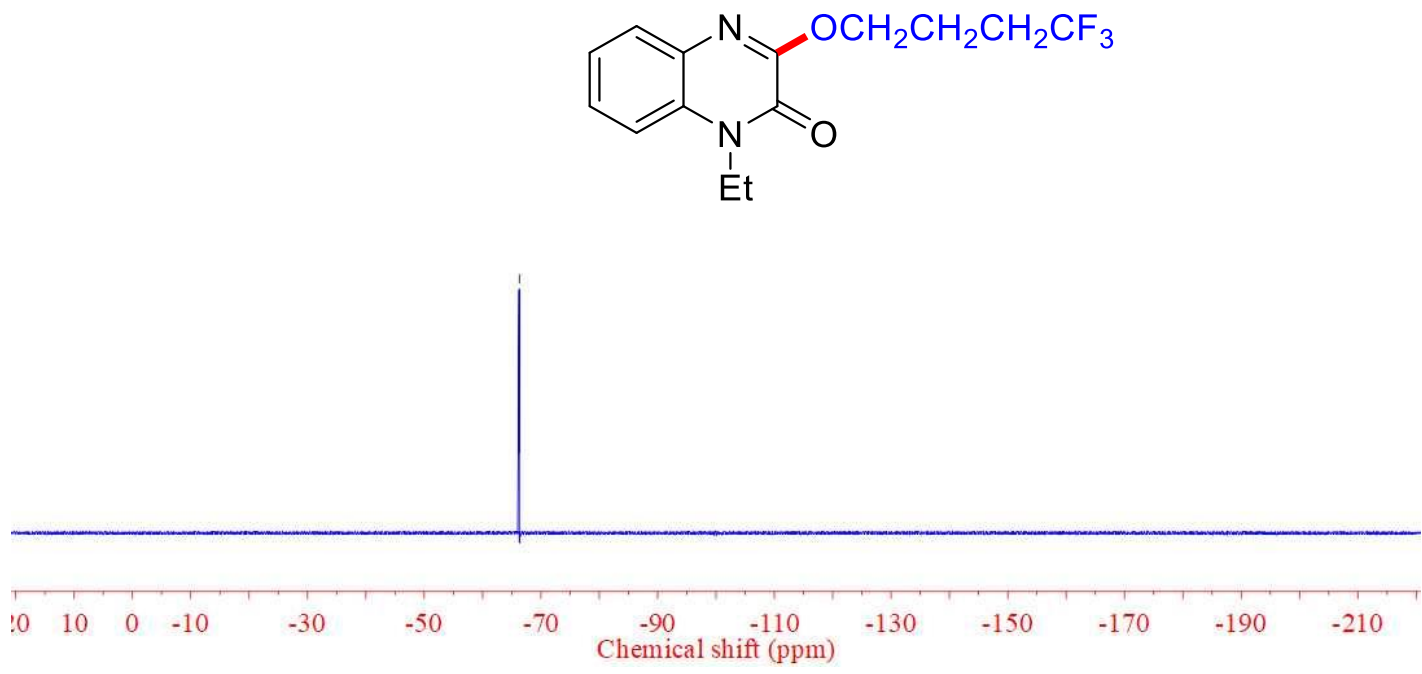


\section{$9 c^{1} \mathrm{H}$ NMR}

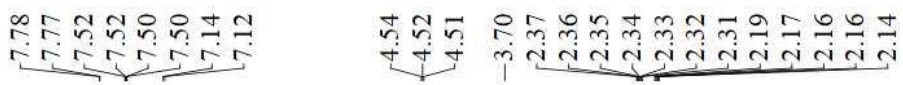

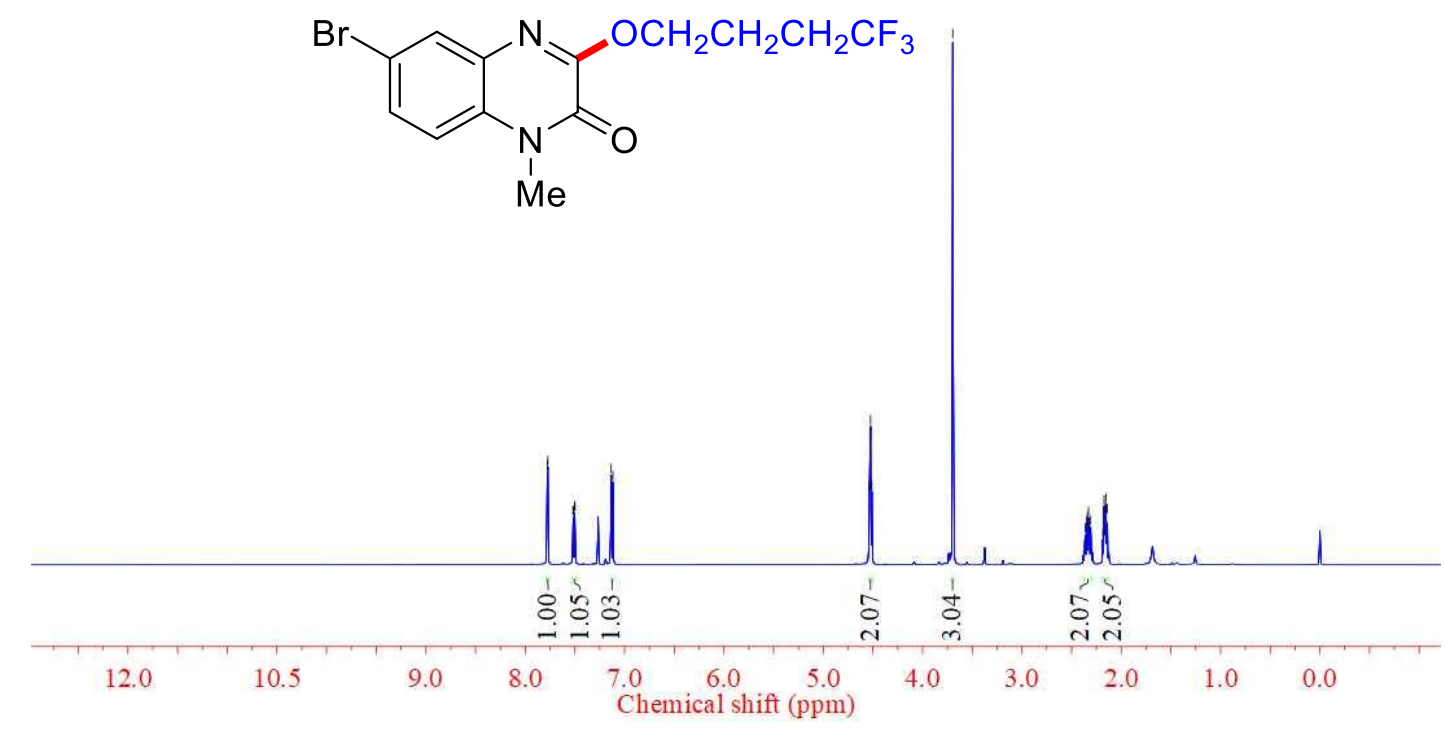

9c ${ }^{13} \mathrm{C}$ NMR

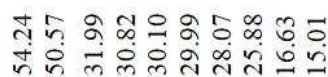

药

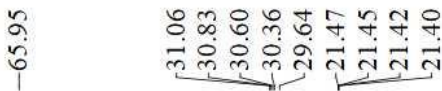<smiles>Cn1c(=O)c(OCCCC(F)(F)F)nc2cc(Br)ccc21</smiles>

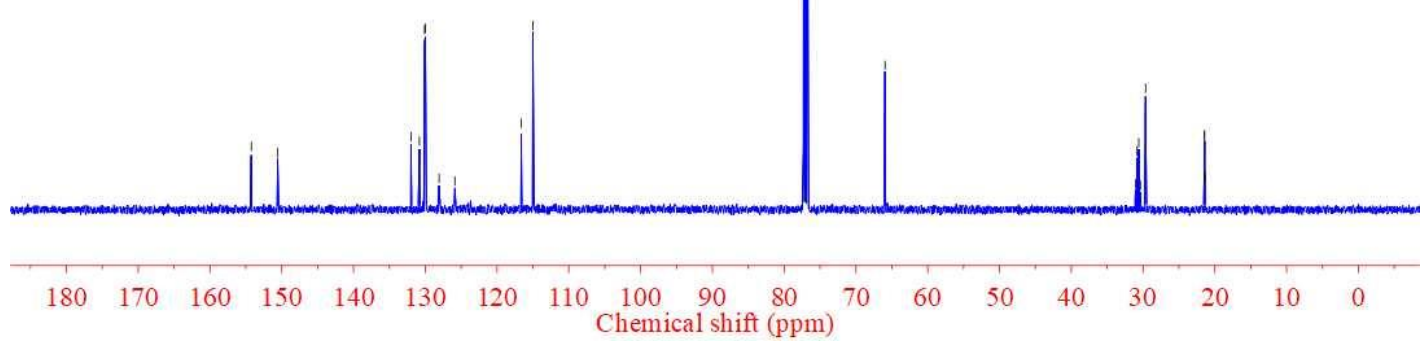




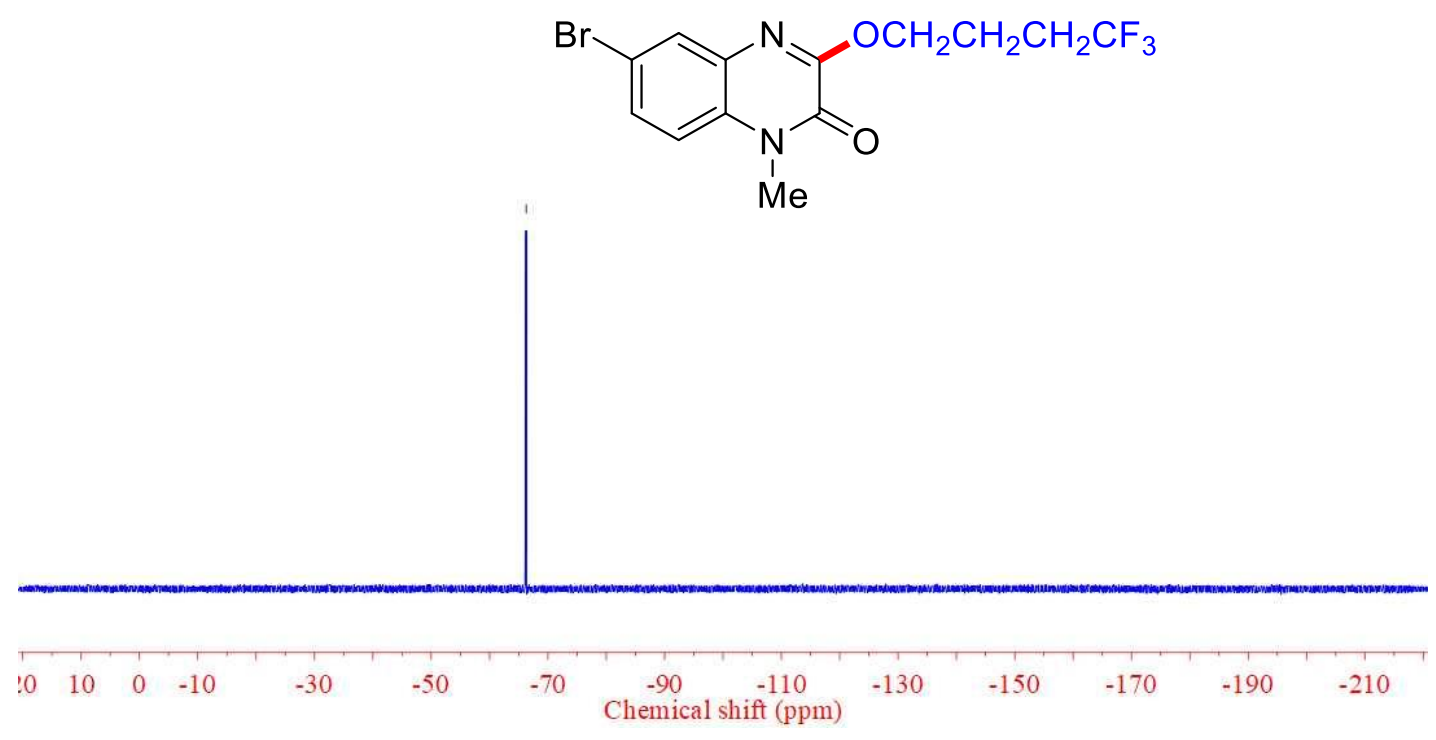

\section{9d ${ }^{1}$ H NMR}

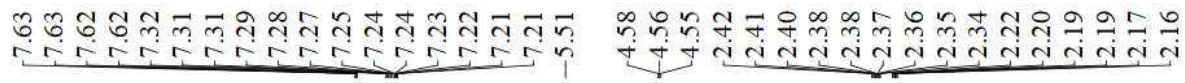

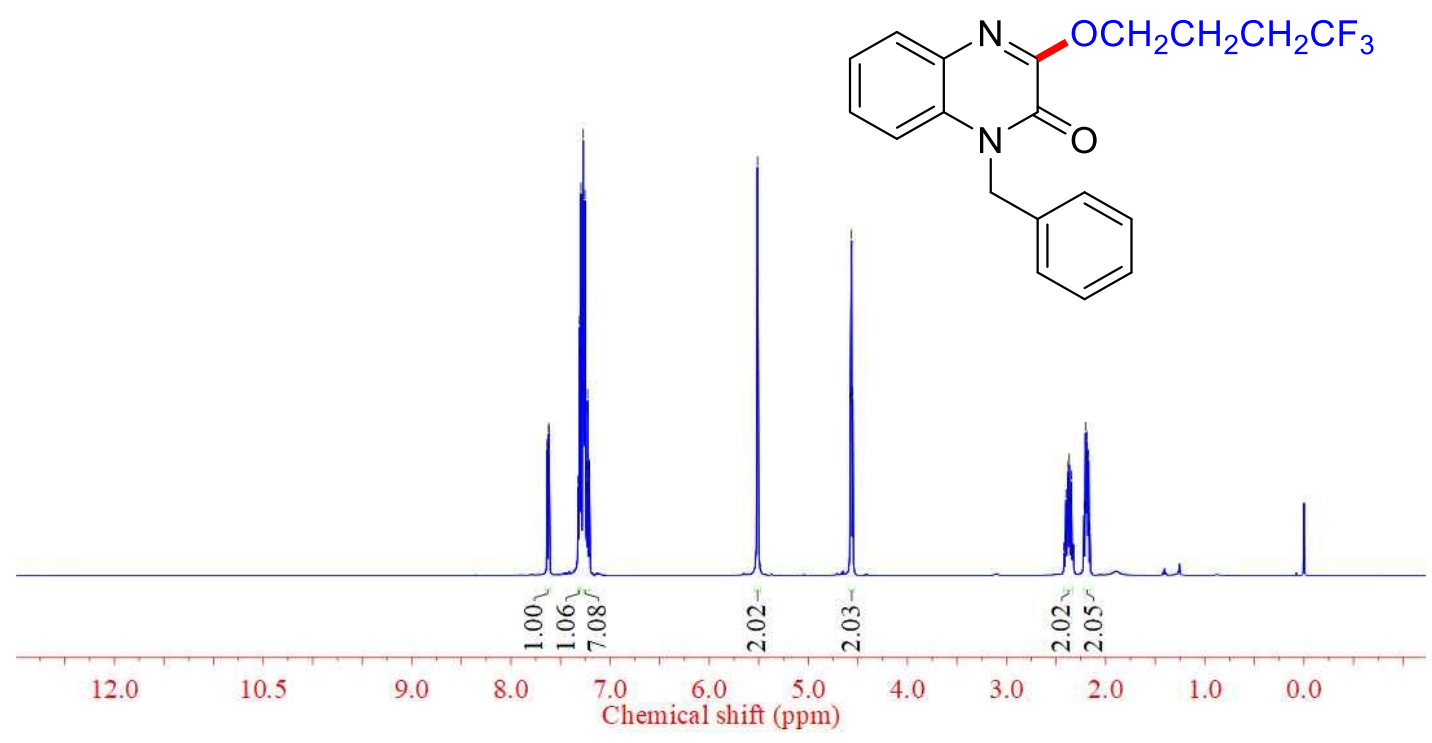




\section{9d ${ }^{13}$ C NMR}

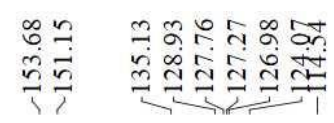

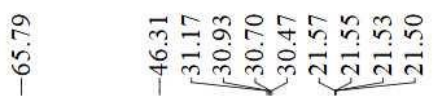

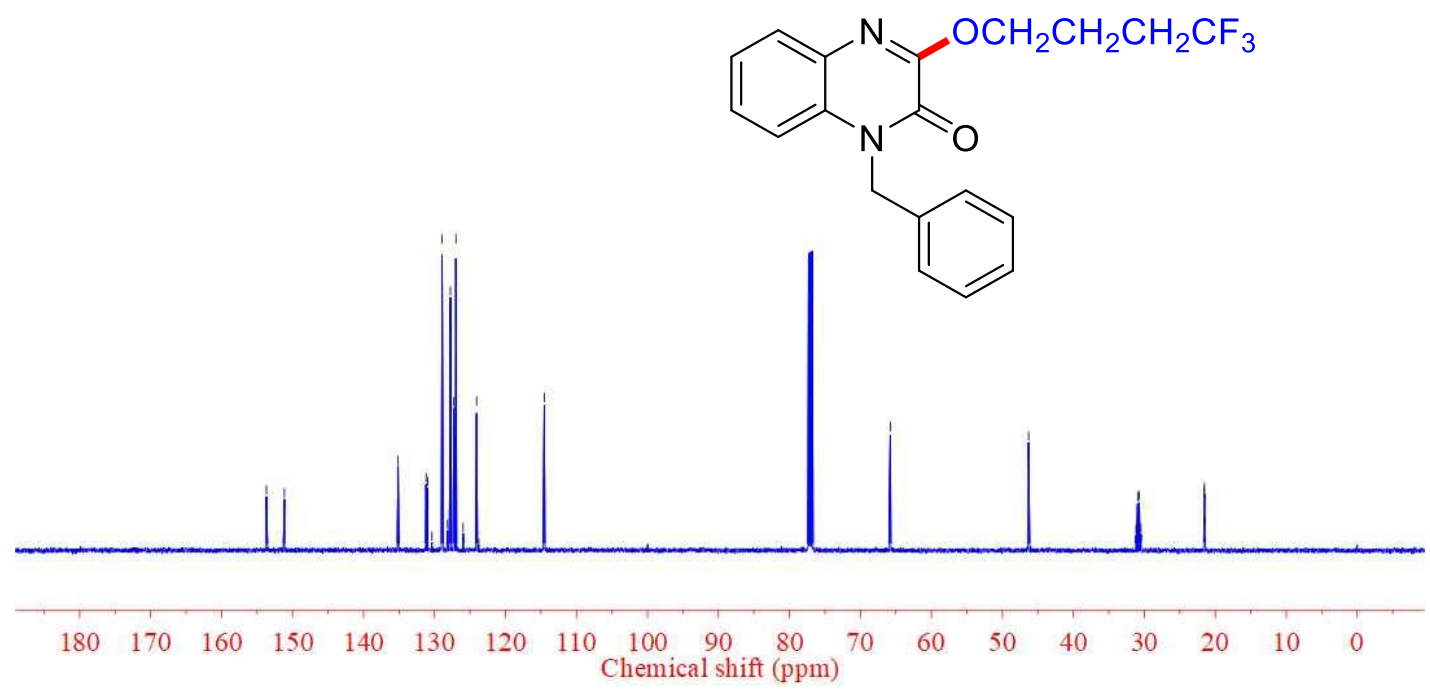

\section{9d ${ }^{19}$ F NMR}

กุ
ర్
$i$
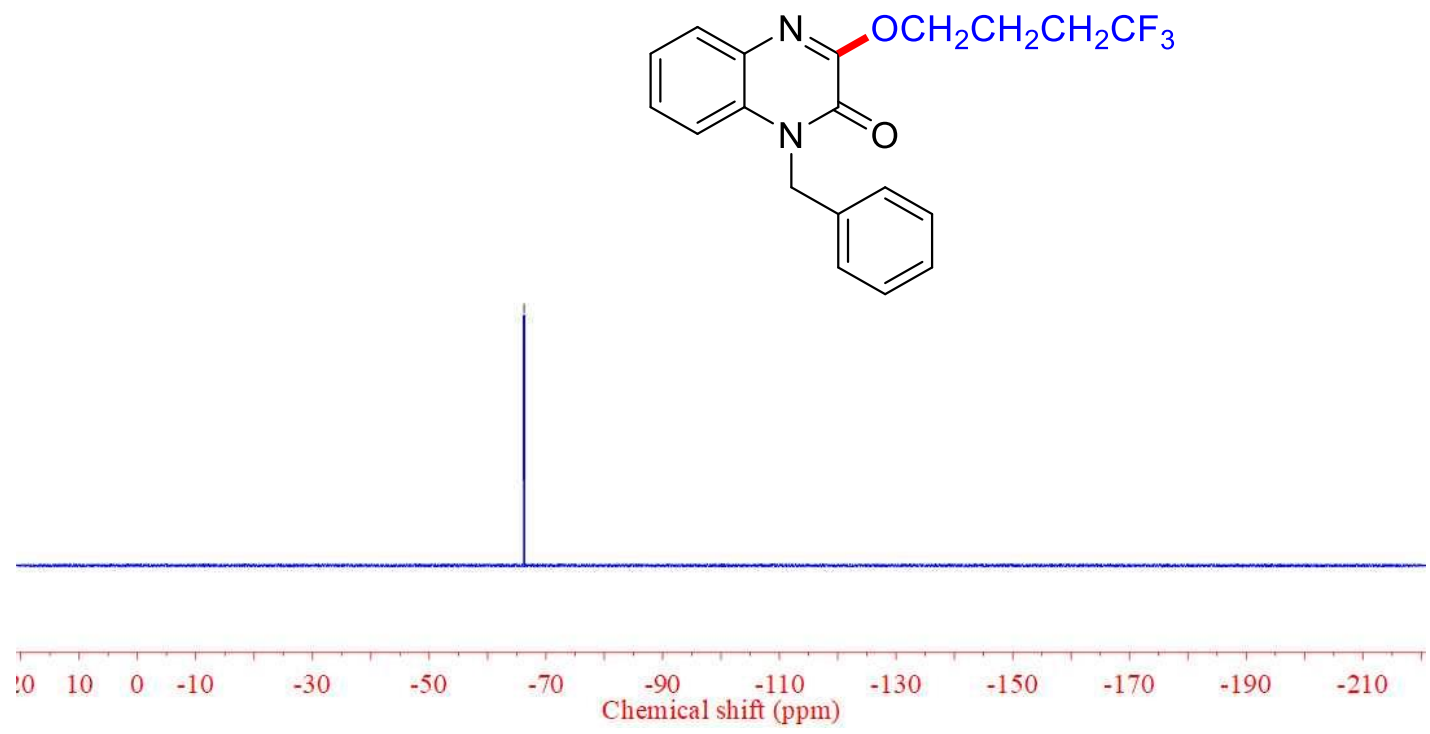


\section{$9 e^{1} \mathrm{H}$ NMR}

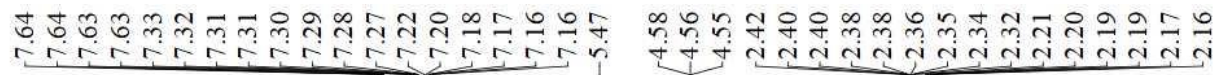

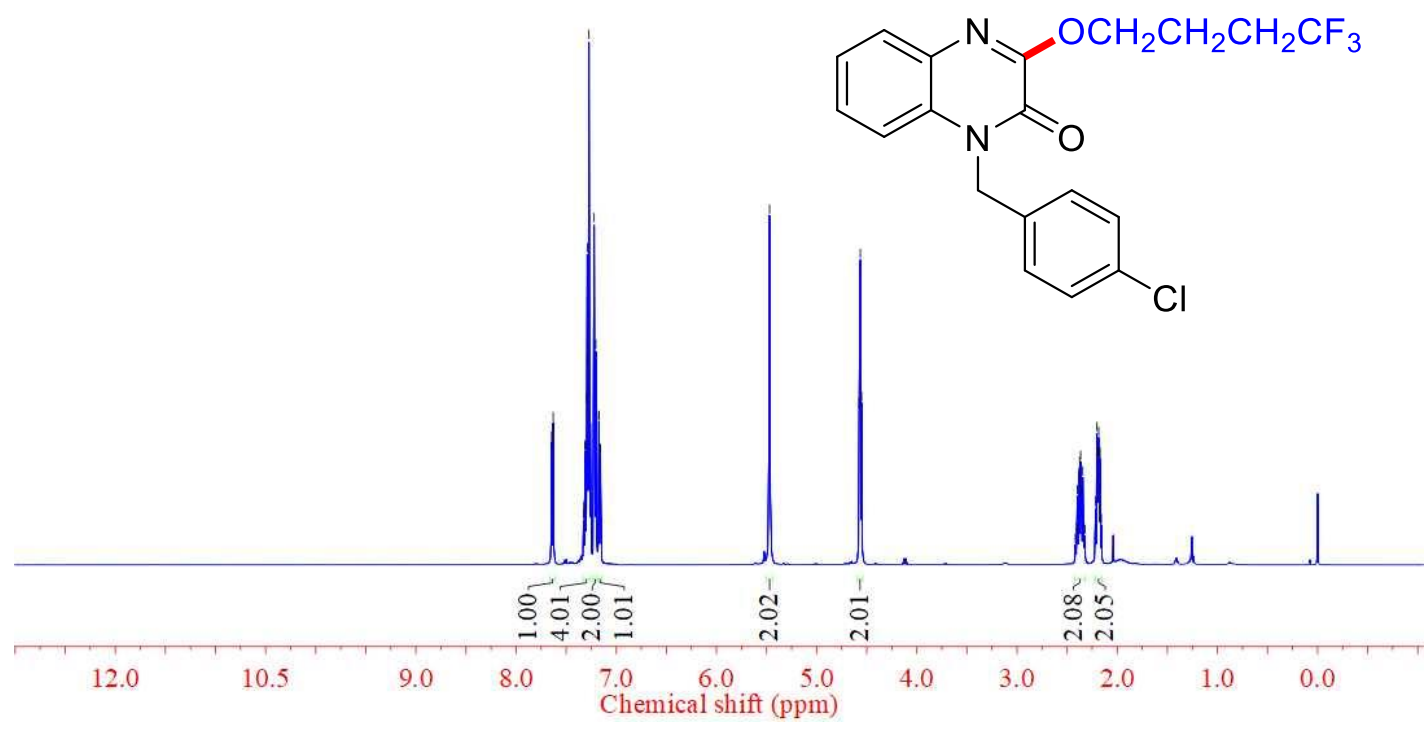

\section{9e ${ }^{13} \mathrm{C}$ NMR}

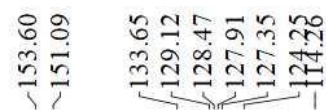

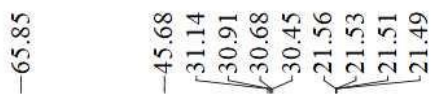

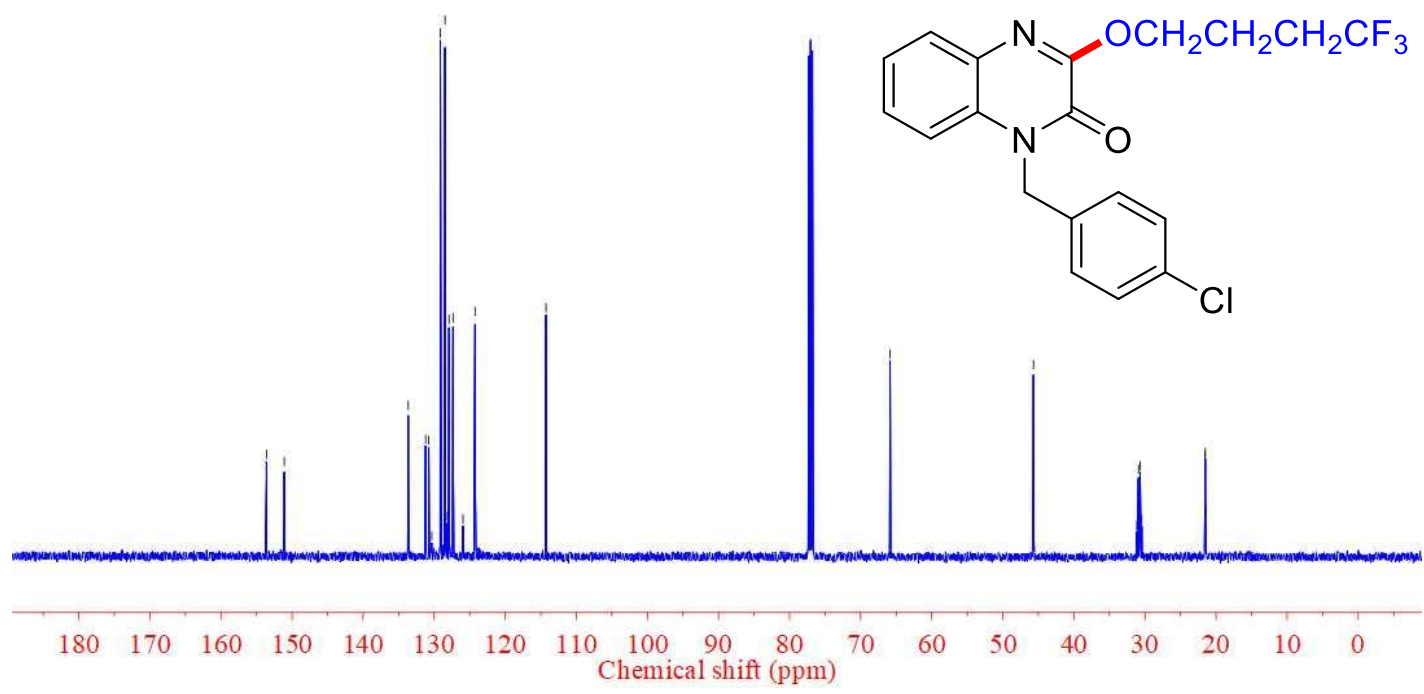


$9 e^{19}$ F NMR

กิ
ò
1

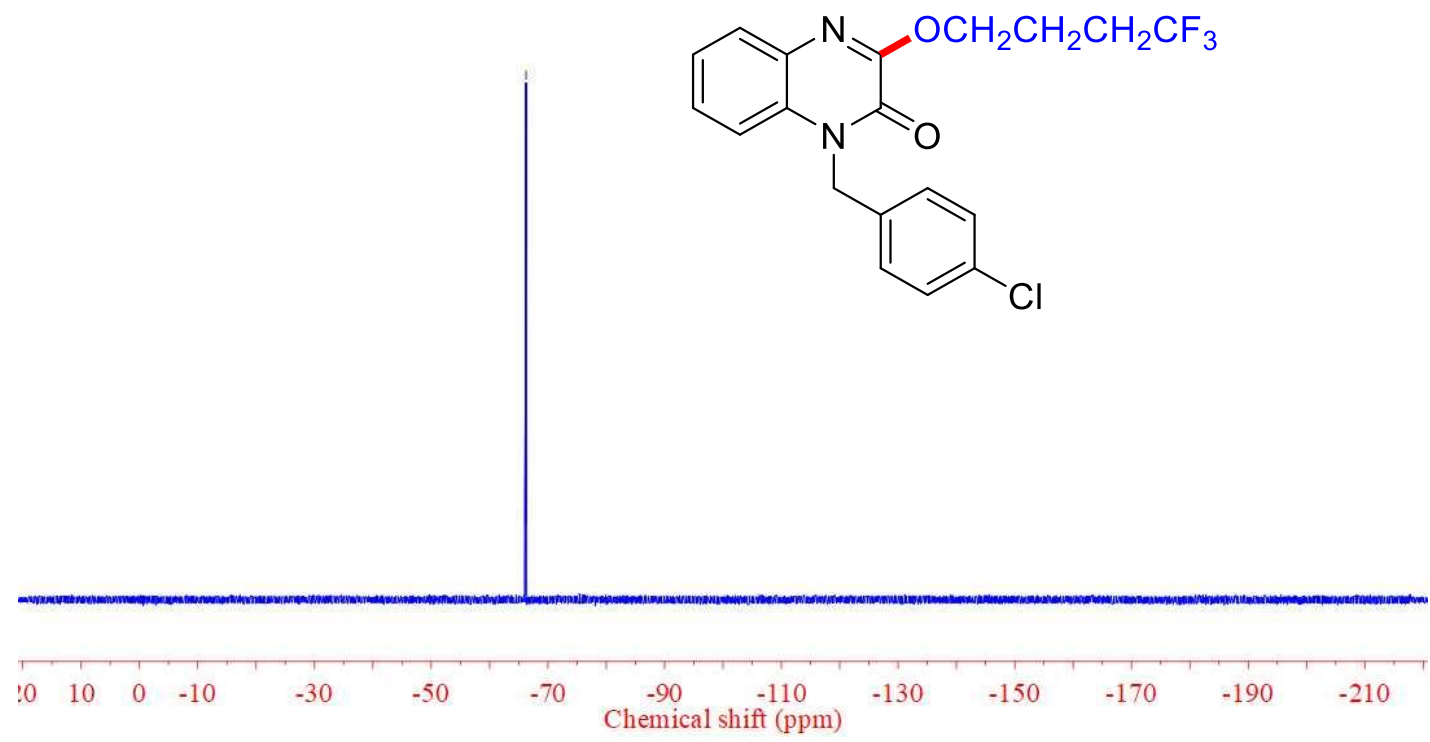

\section{$10{ }^{1} \mathrm{H}$ NMR}

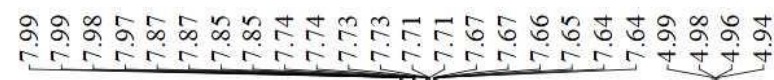

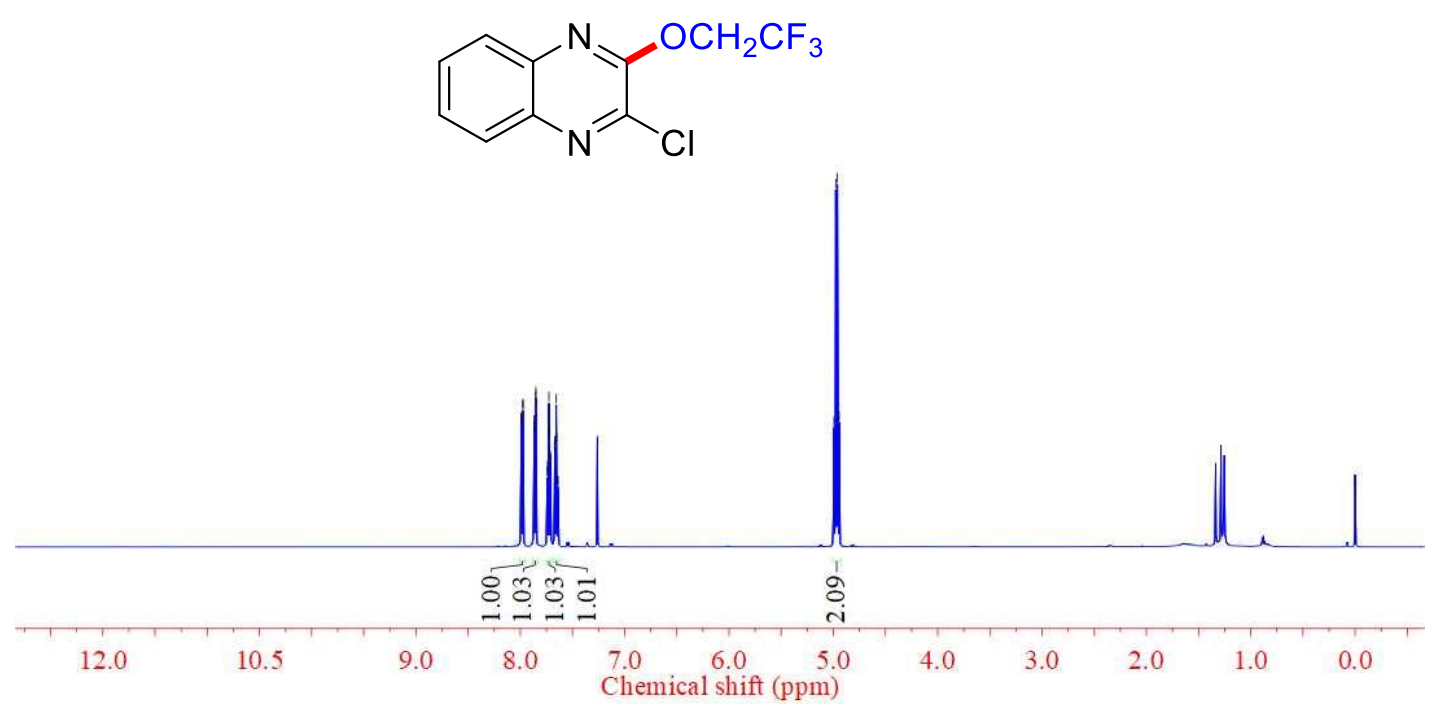




\section{$10{ }^{13} \mathrm{C}$ NMR}

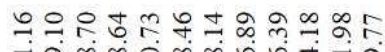

ที่

ำ 9

हुํㅇㅇㅇ
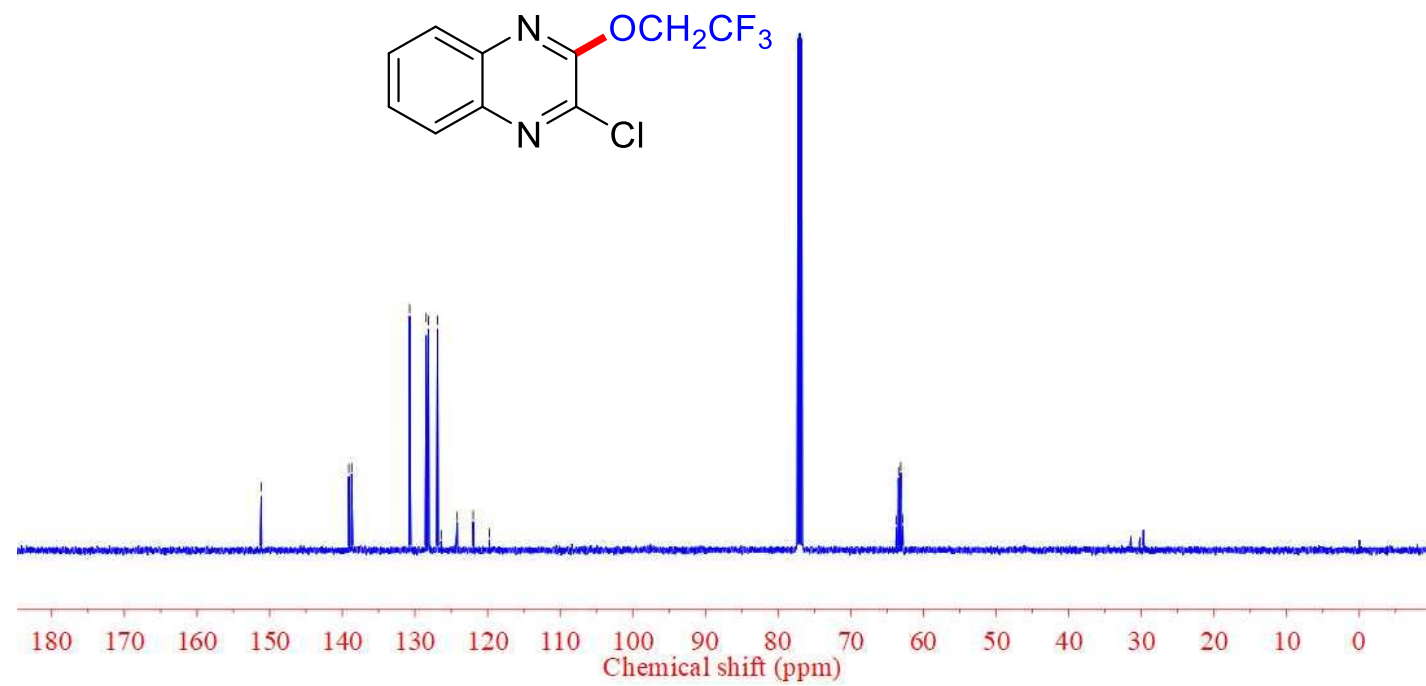

\section{$10{ }^{19}$ F NMR}

$\stackrel{m}{\stackrel{m}{i}}$

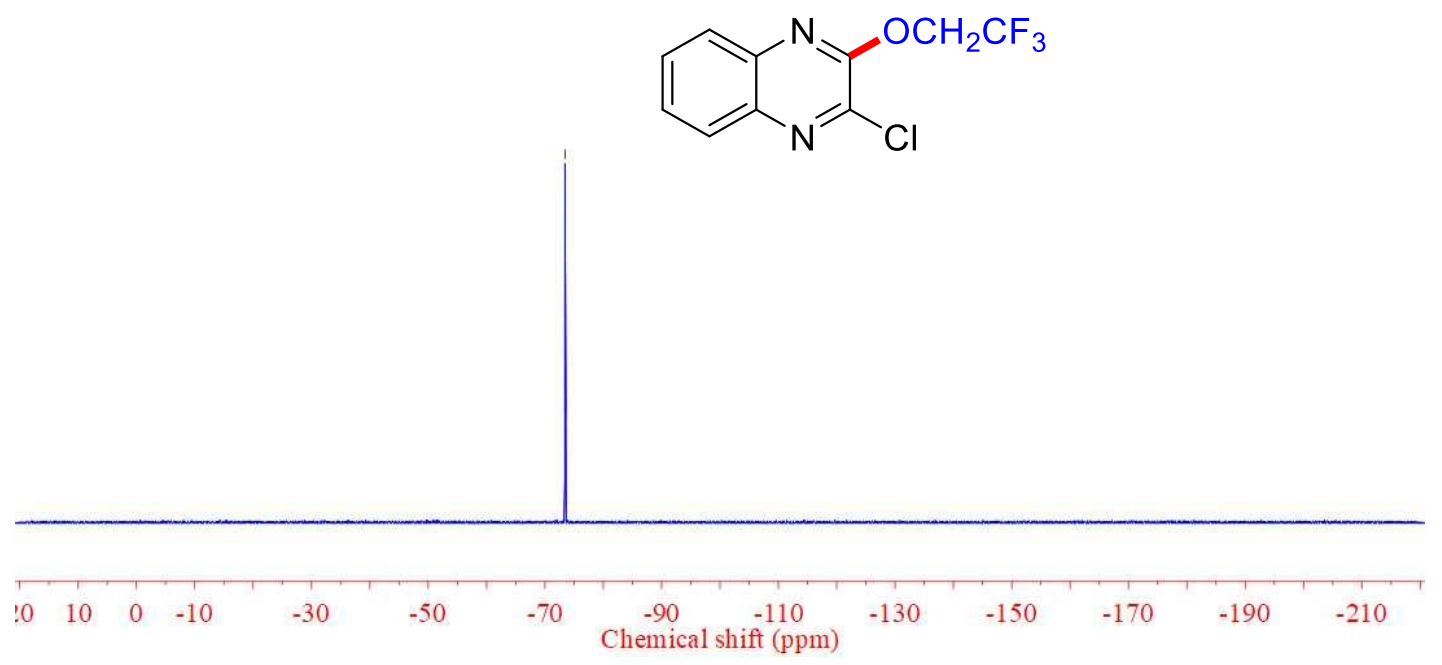




\section{1a ${ }^{1} \mathrm{H}$ NMR}

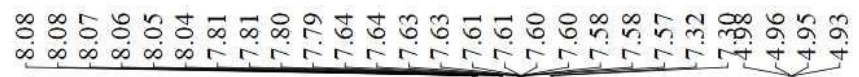

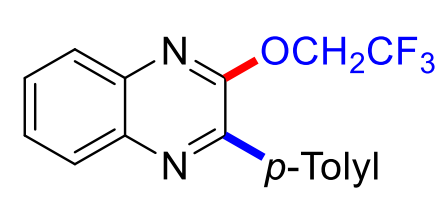

$\mathrm{N} p$-Tolyl

\section{$11 a^{13} \mathrm{C}$ NMR}

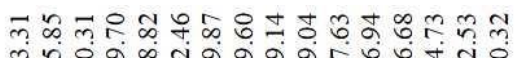

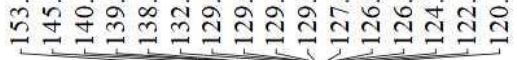

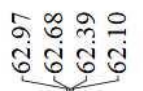

$\frac{ \pm}{i}$

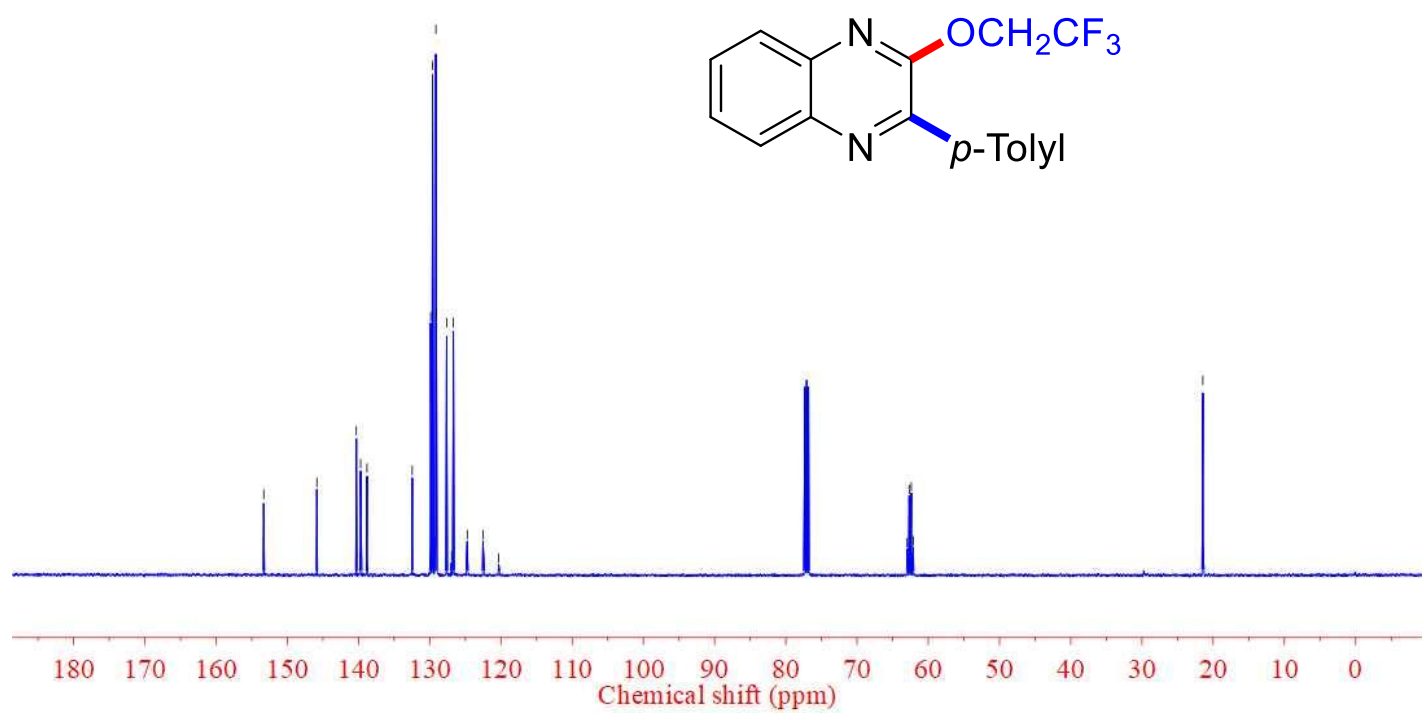




\section{$11 \mathrm{a}{ }^{19}$ F NMR}

$$
\frac{0}{i}
$$<smiles>[O-][O+]c1nc2ccccc2nc1OCC(F)(F)F</smiles>

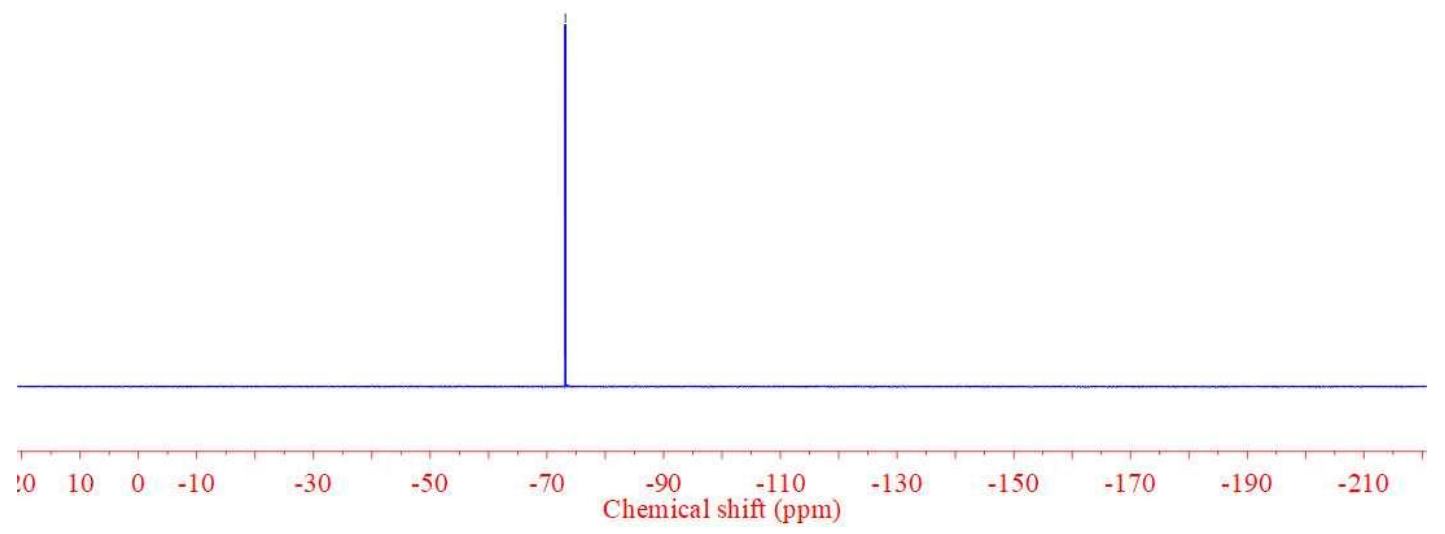

\section{1b ${ }^{1} \mathrm{H}$ NMR}

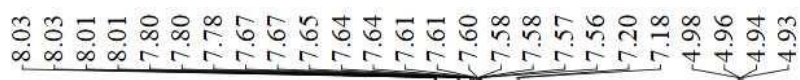
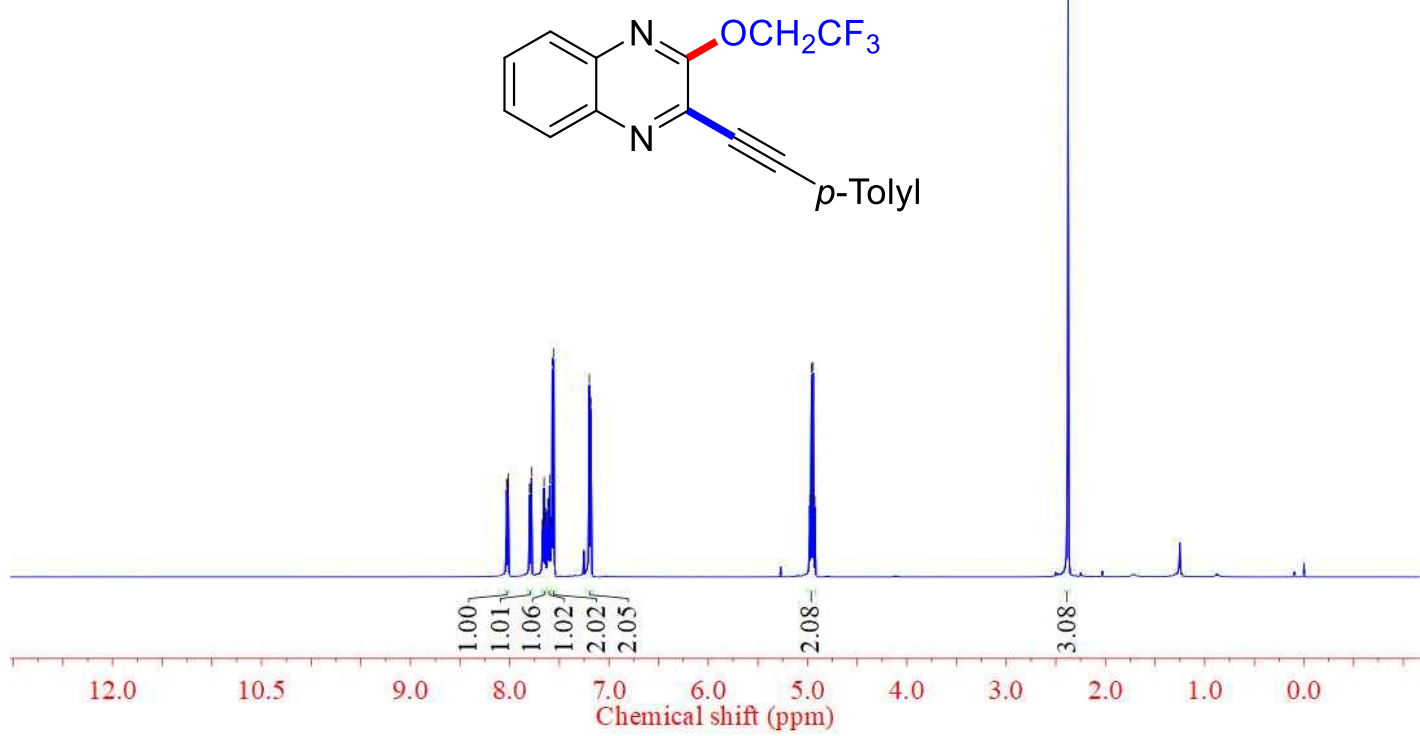


\section{1b ${ }^{13} \mathrm{C}$ NMR}

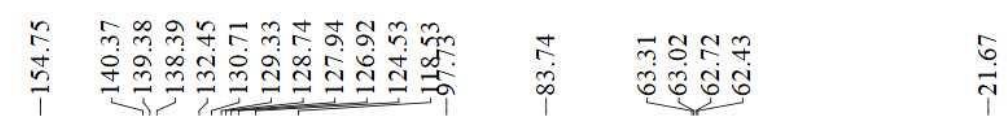

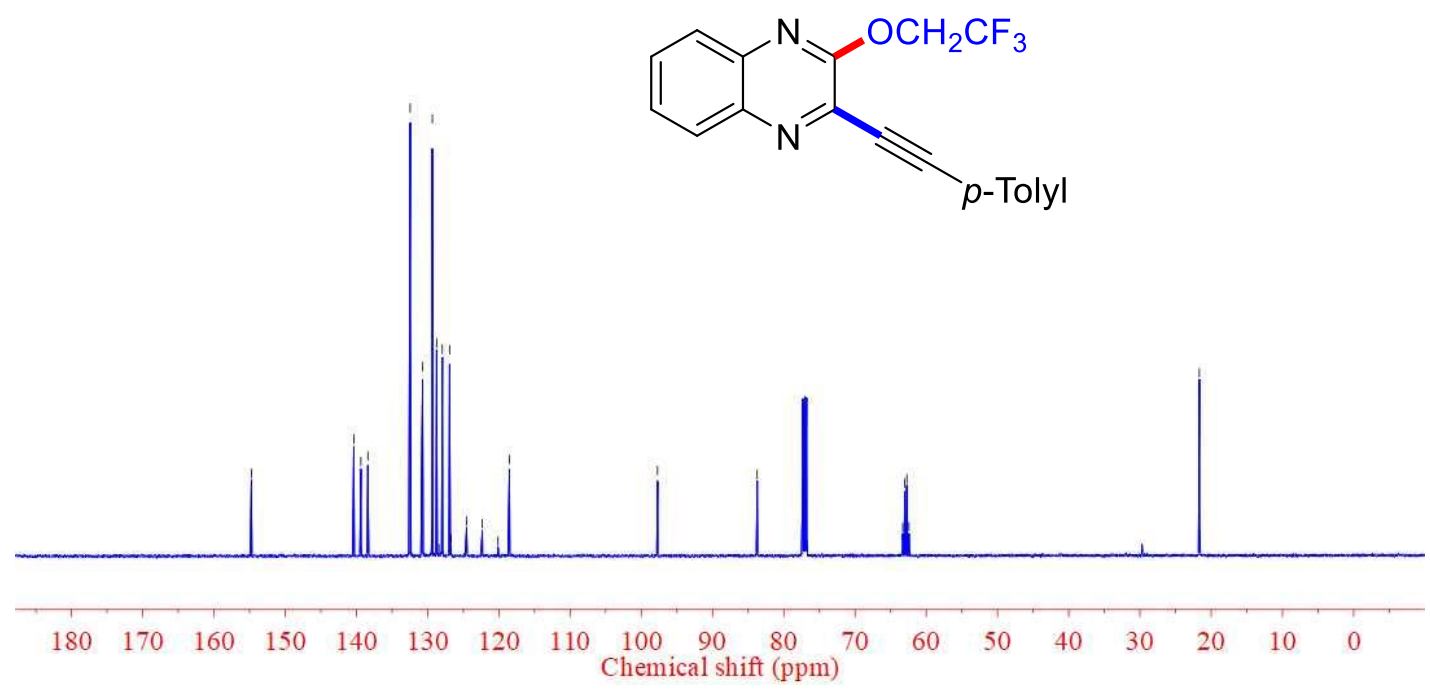

\section{1b ${ }^{19}$ F NMR}

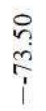

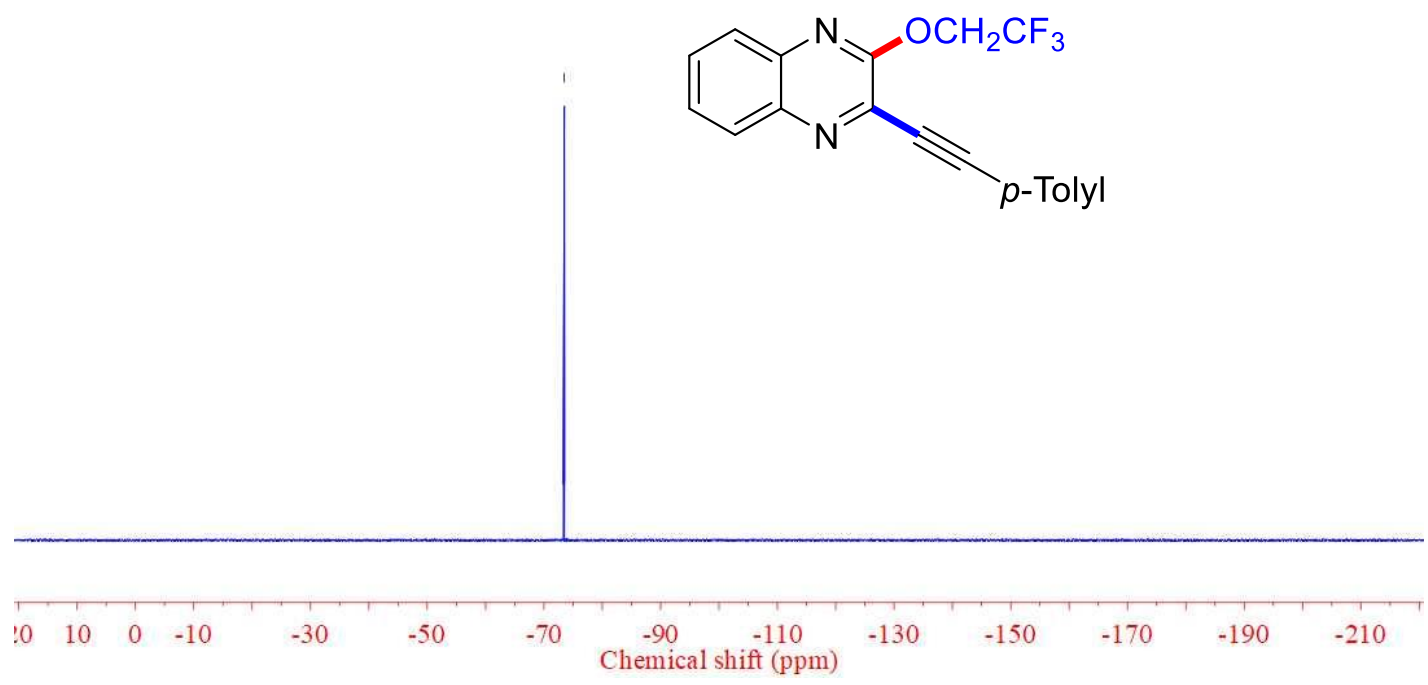




\section{1c ${ }^{1} \mathrm{H}$ NMR}

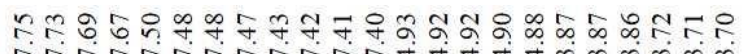

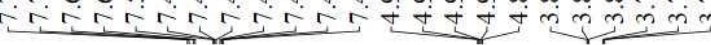<smiles>FC(F)(F)COc1nc2ccccc2nc1N1CCOCC1</smiles>

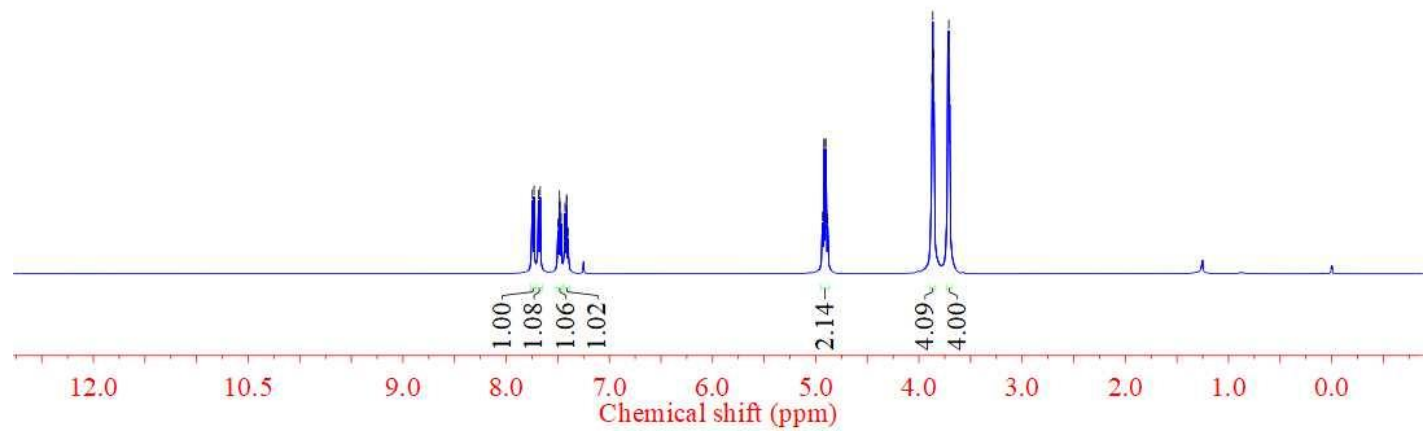

\section{1c ${ }^{13} \mathrm{C}$ NMR}

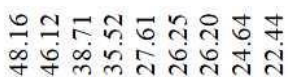

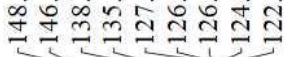

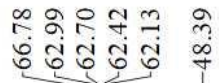<smiles>FC(F)(F)COc1nc2ccccc2nc1N1CCOCC1</smiles>

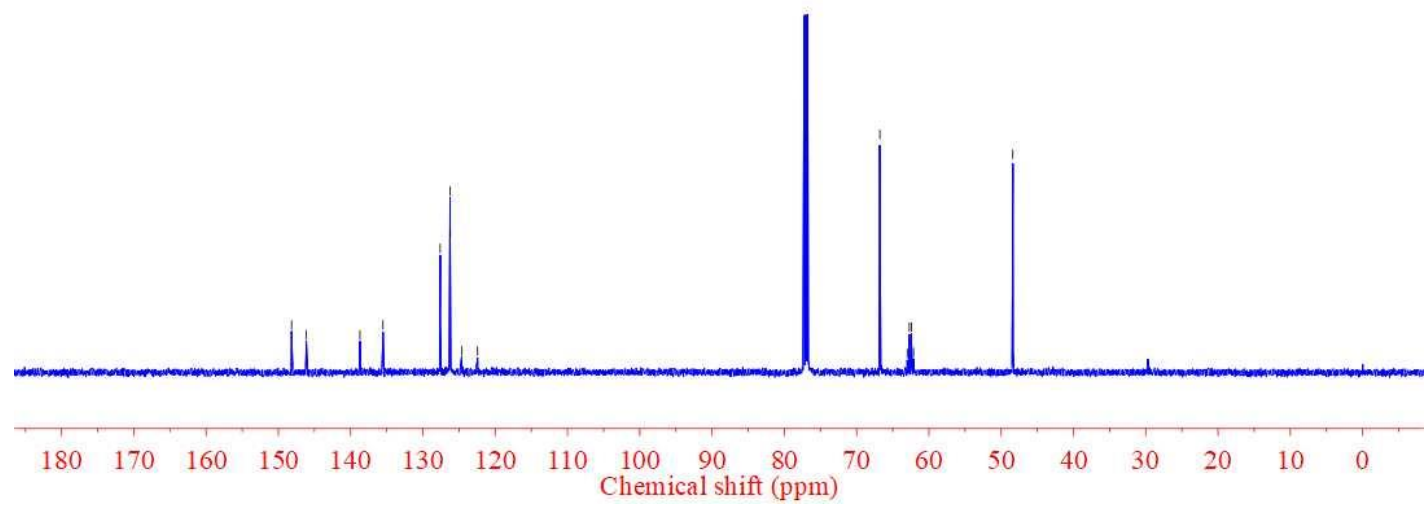




\section{1c ${ }^{19}$ F NMR}

$\frac{m}{m}$<smiles>FC(F)(F)COc1nc2ccccc2nc1N1CCOCC1</smiles>

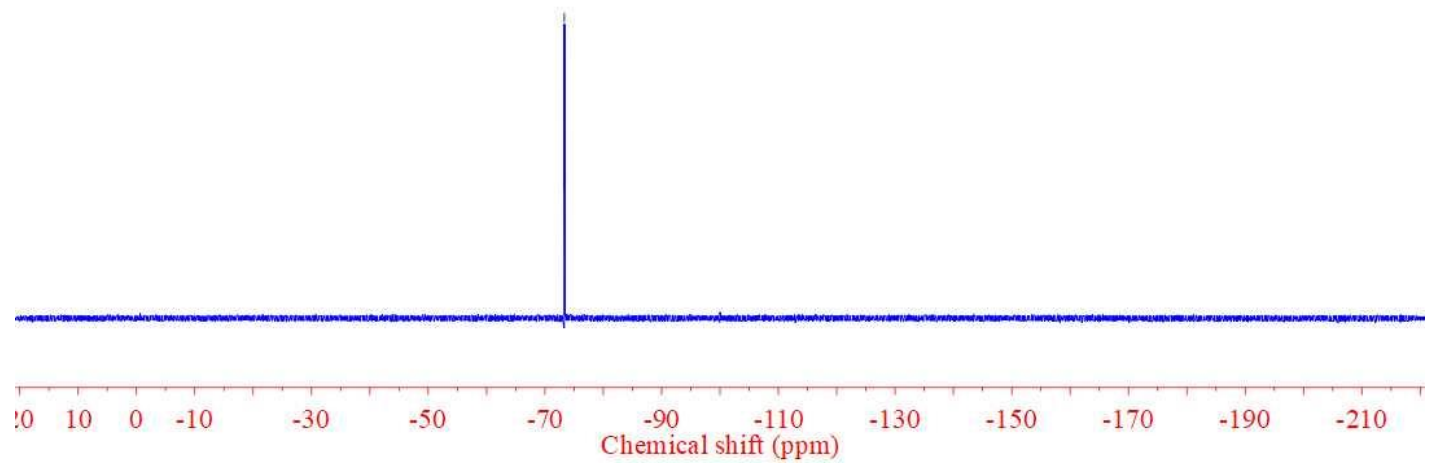

\section{1d ${ }^{1}$ H NMR}

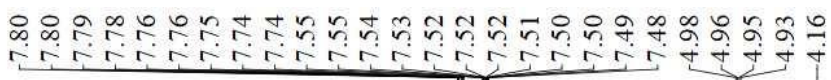

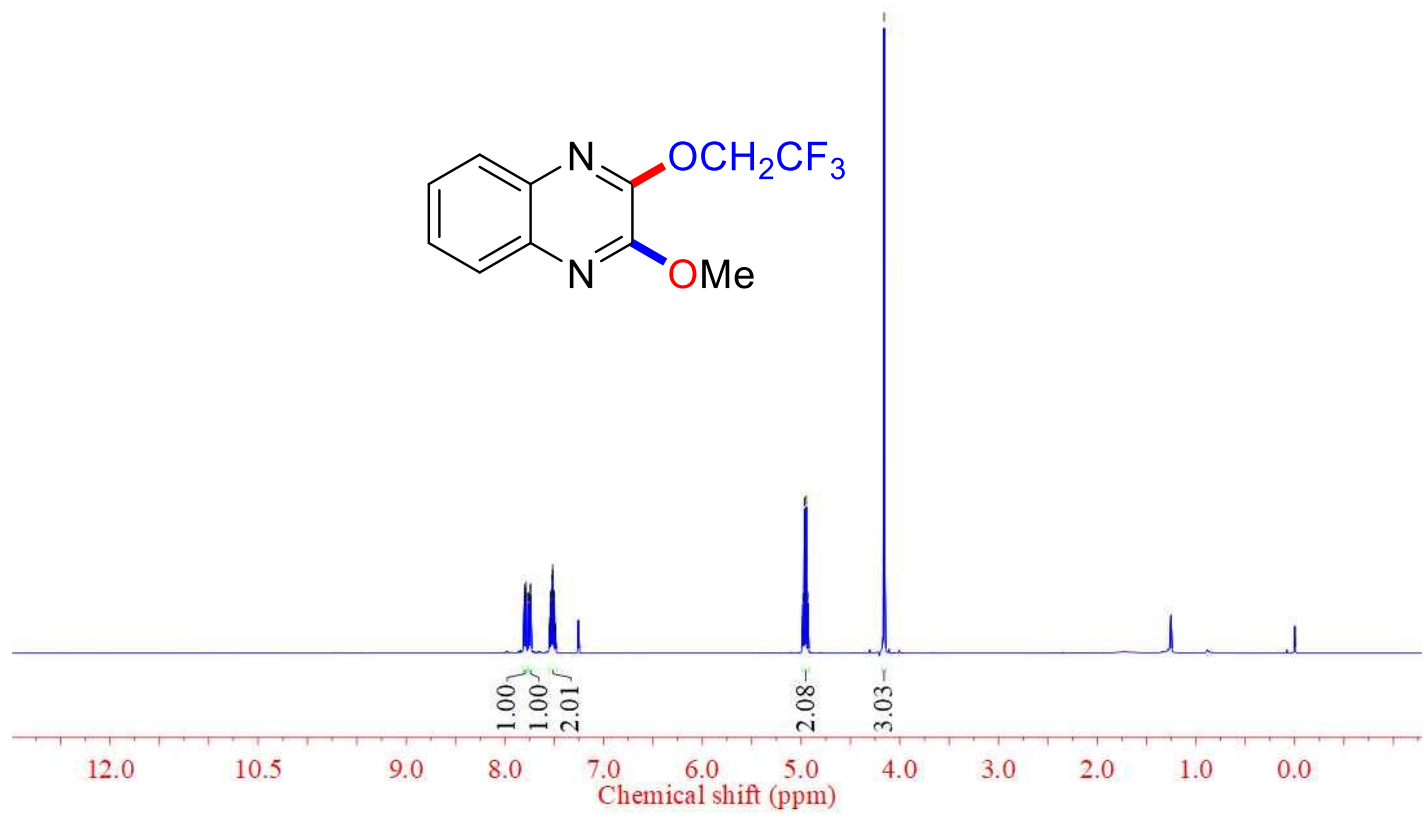




\section{1d ${ }^{13}$ C NMR}

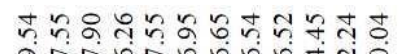

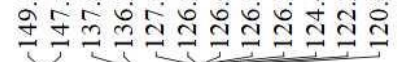

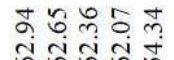

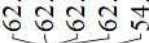

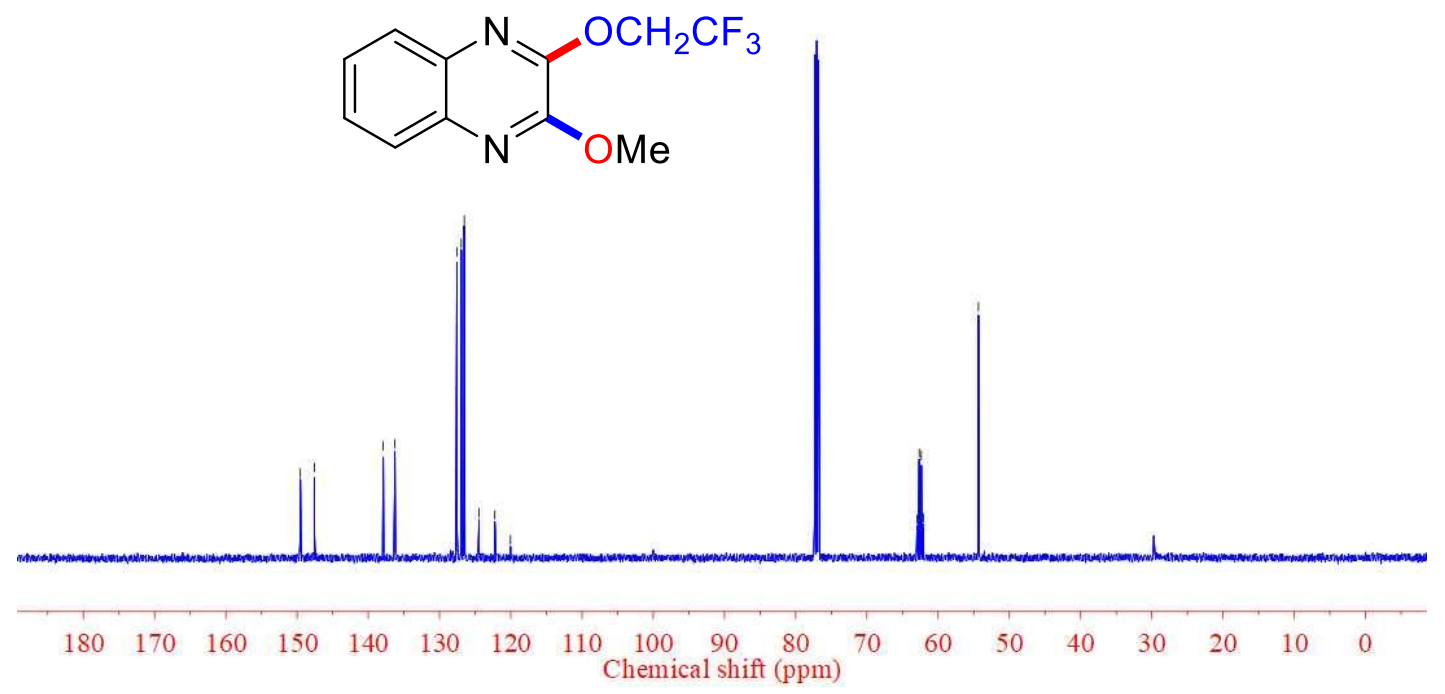

\section{1d ${ }^{19}$ F NMR}

ஸุ

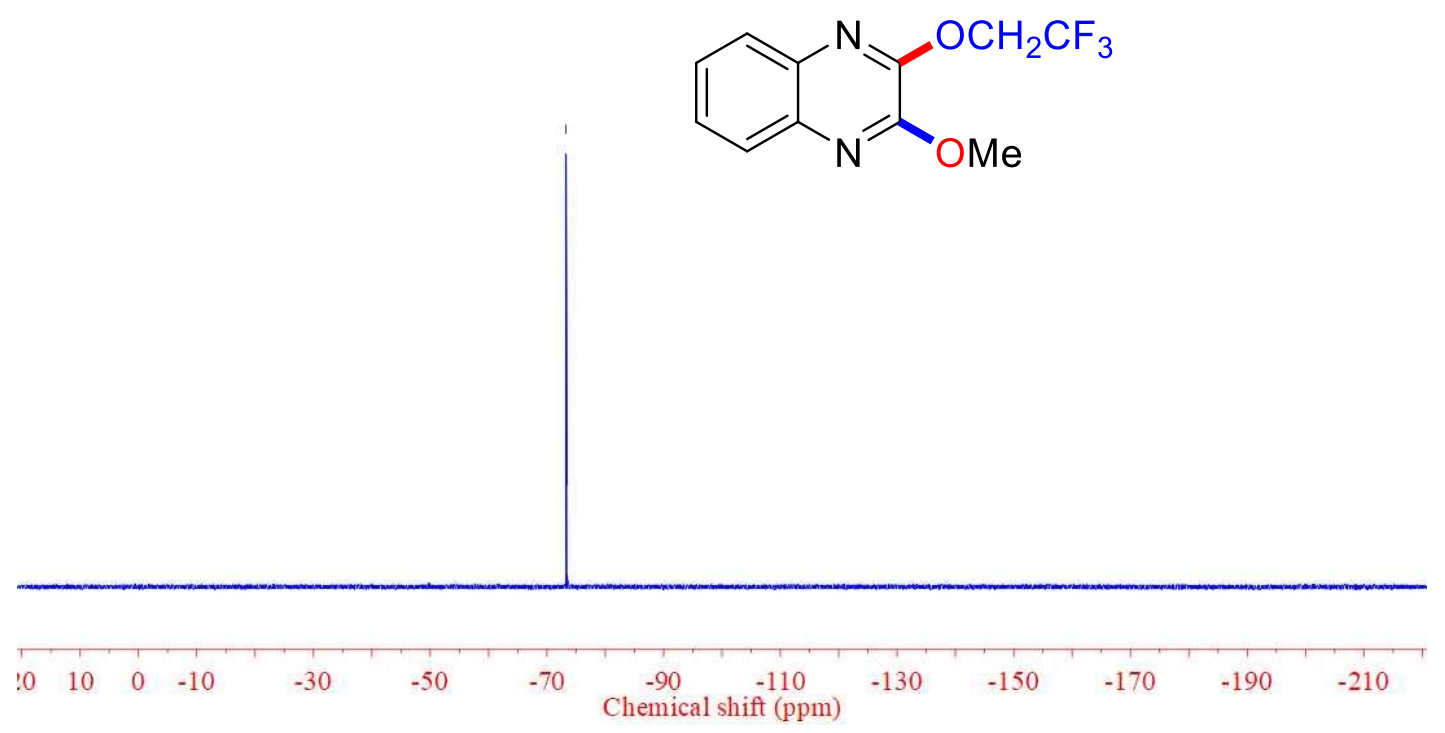




\section{$11 e^{31}$ P NMR}

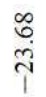

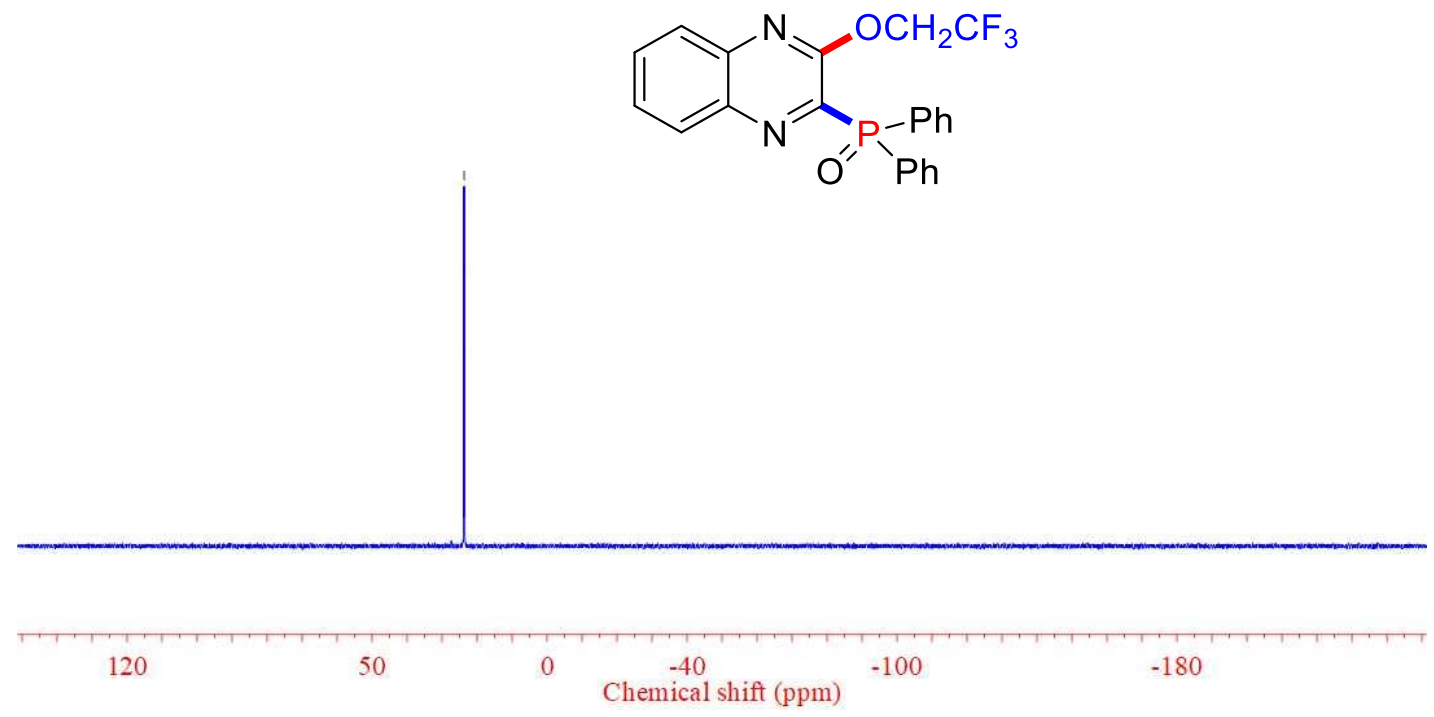

\section{1e ${ }^{1} \mathrm{H}$ NMR}

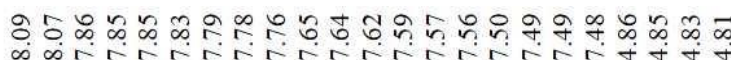

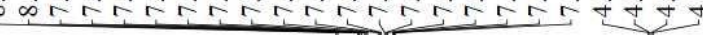
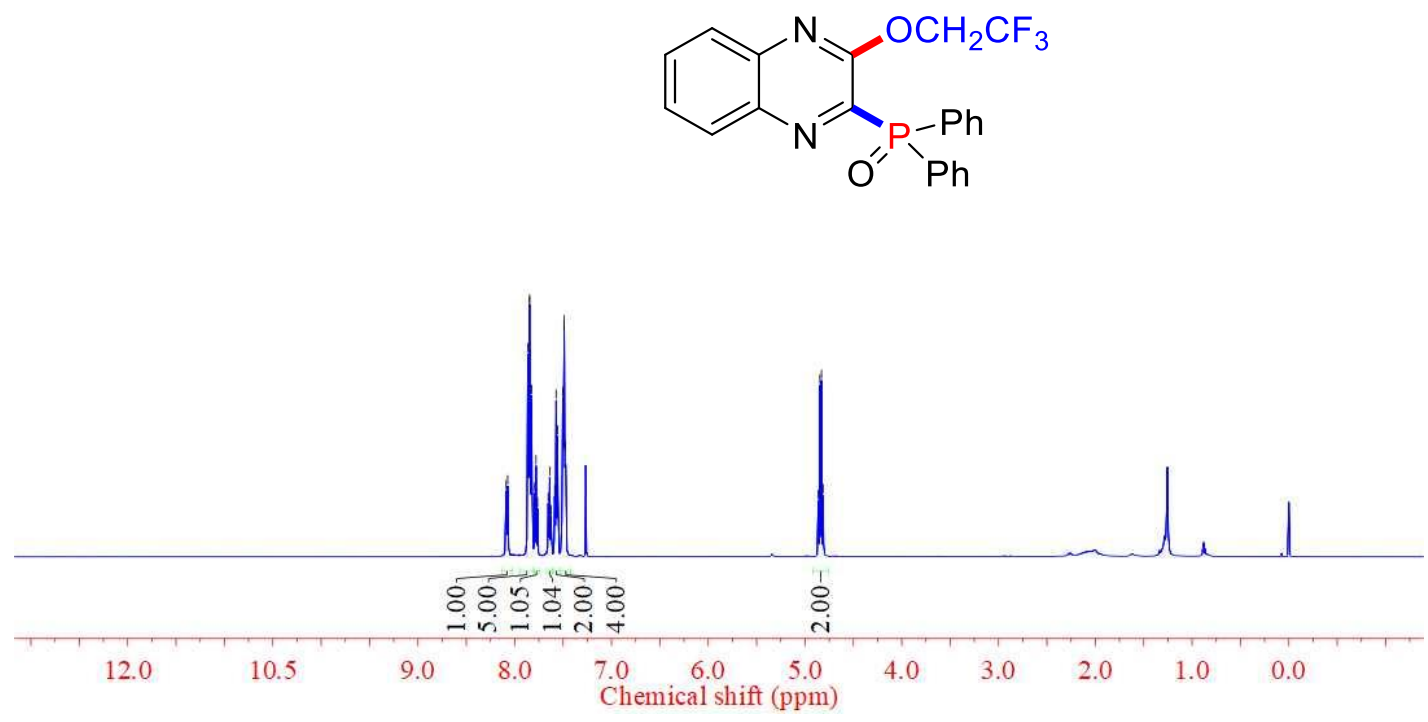


\section{$11 e^{13}$ C NMR}

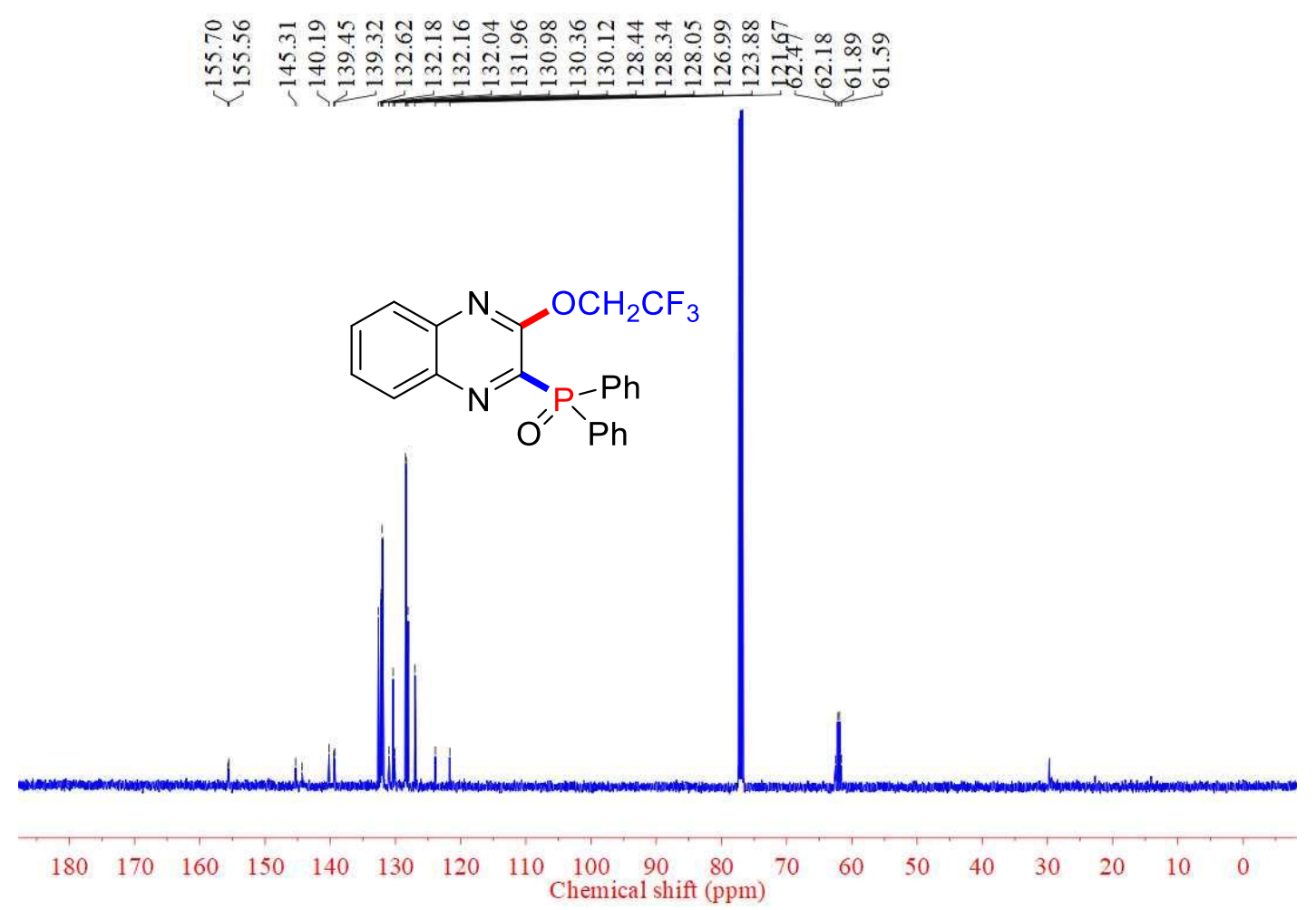

\section{$11 e^{19}$ F NMR}

$\frac{m}{m}$

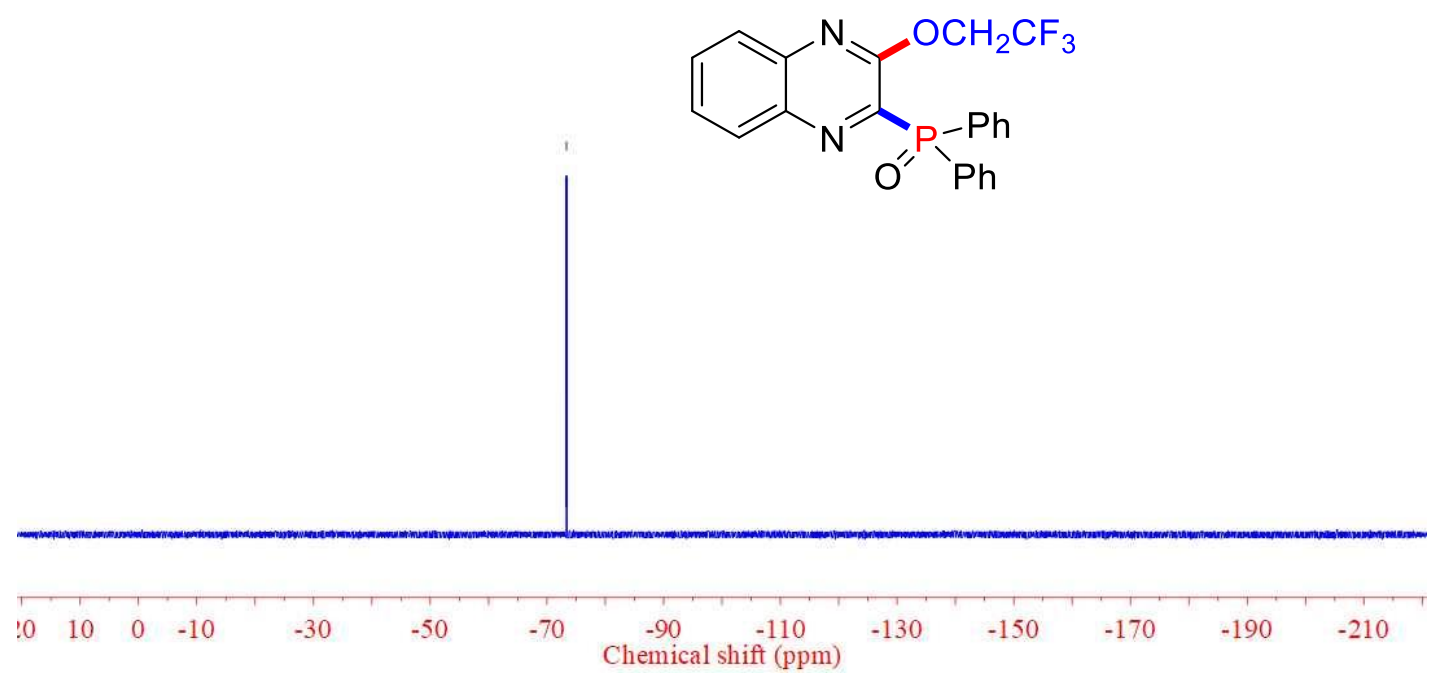




\section{1f $^{1} \mathrm{H}$ NMR}<smiles>[O+]OSc1nc2ccccc2nc1OCC(F)(F)F</smiles>

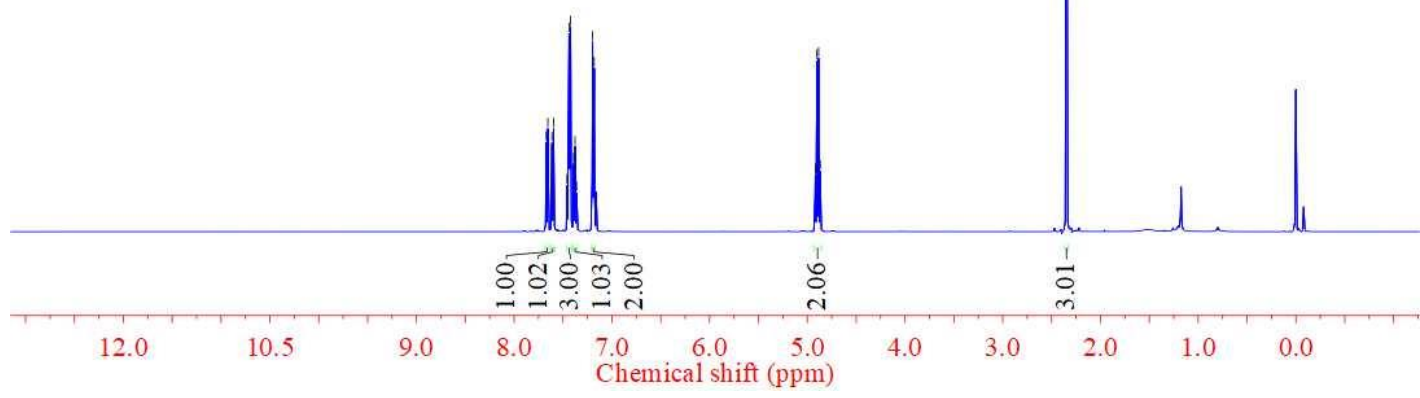

\section{$11 f^{13} \mathrm{C}$ NMR}

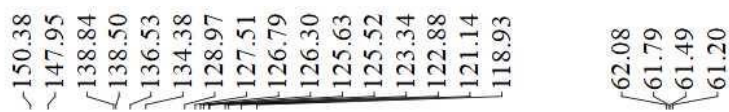

$\stackrel{n}{\tilde{n}}$<smiles>[O+]OSc1nc2ccccc2nc1OCC(F)(F)F</smiles>

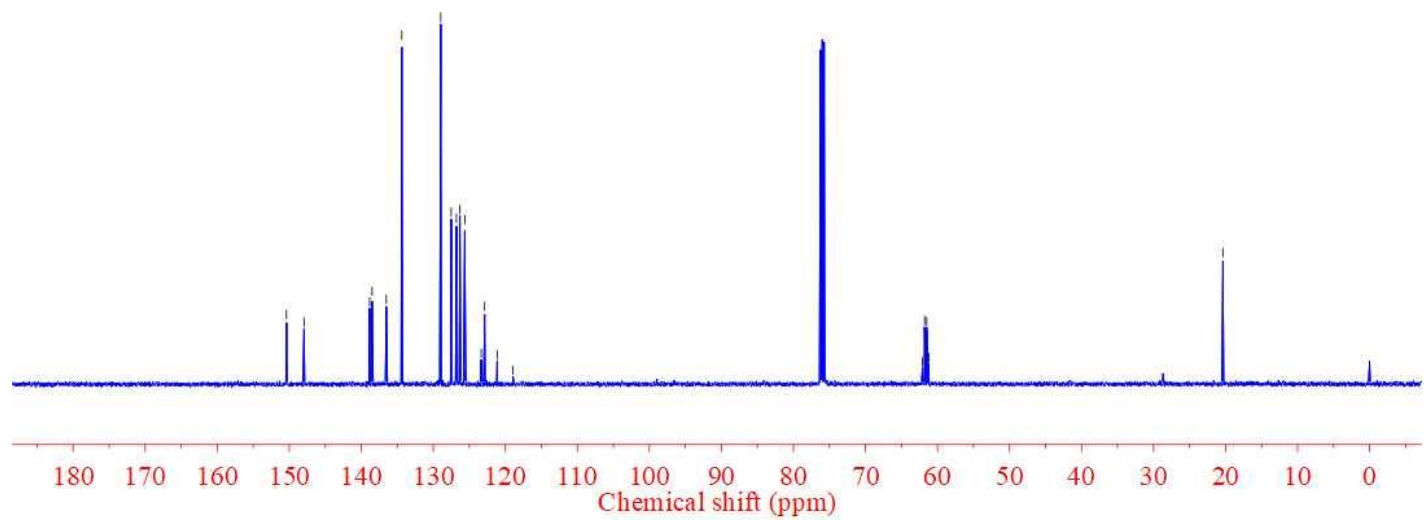




\section{$11 f^{19}$ F NMR}

$\stackrel{n}{m}$

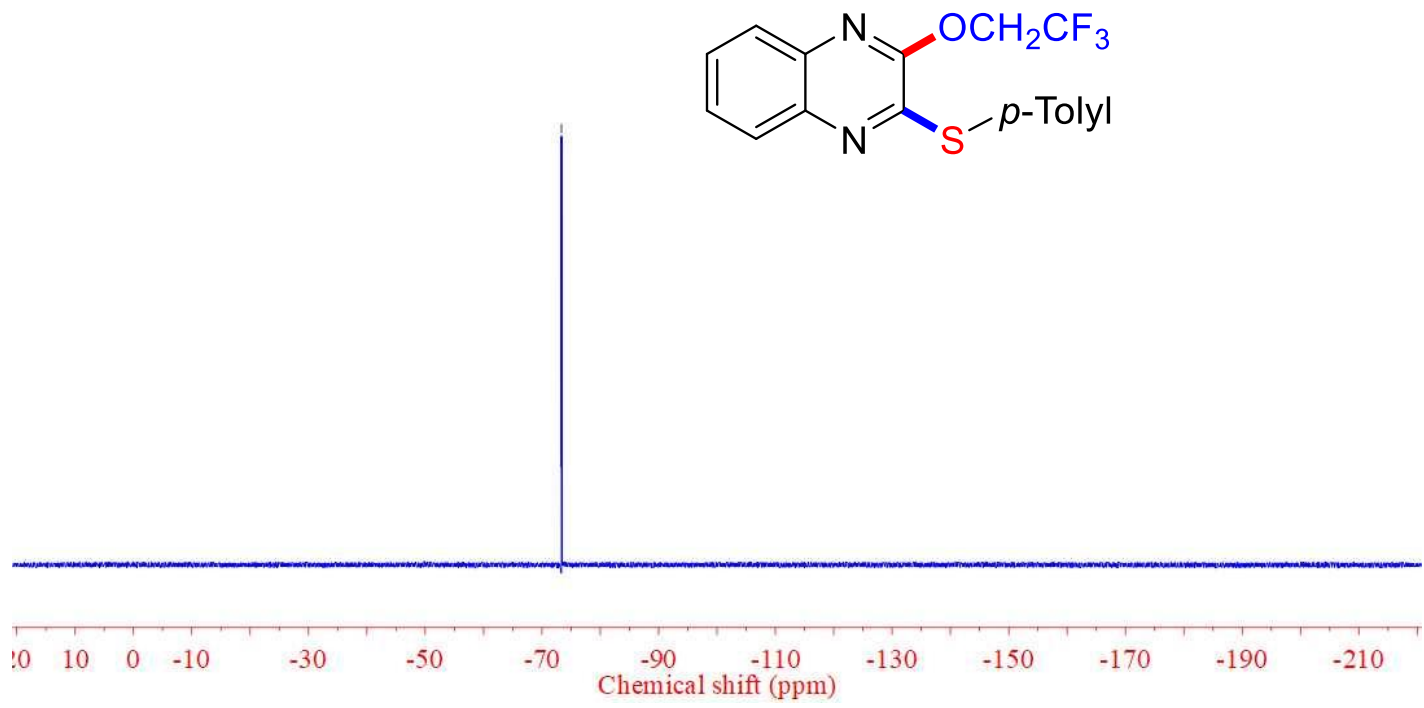

\section{$11{ }^{1}$ H NMR}

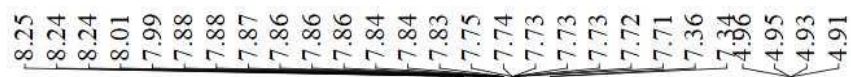

$\stackrel{i}{i}$

$\overbrace{\mathrm{N}}^{\mathrm{N}_{\mathrm{O}}^{\prime \prime} \mathrm{O}-\mathrm{O} \text {-Tolyl }}$

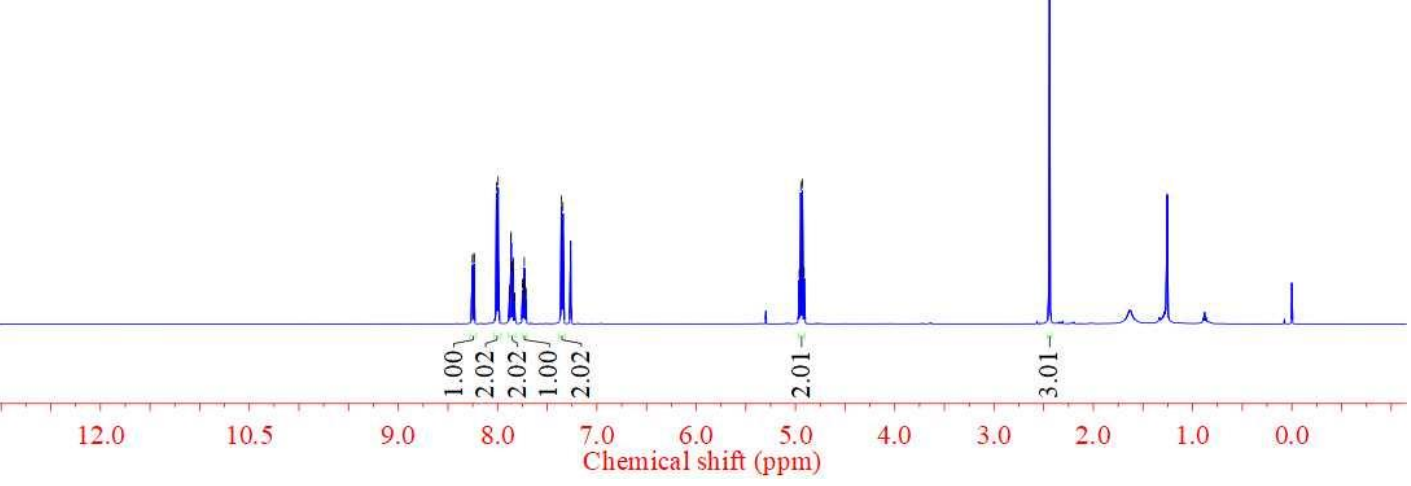




\section{$11 \mathrm{~g}{ }^{13} \mathrm{C}$ NMR}

ตำ

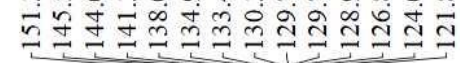

ํํㅇํำ

ชं

$\stackrel{\overrightarrow{1}}{\overrightarrow{1}}$

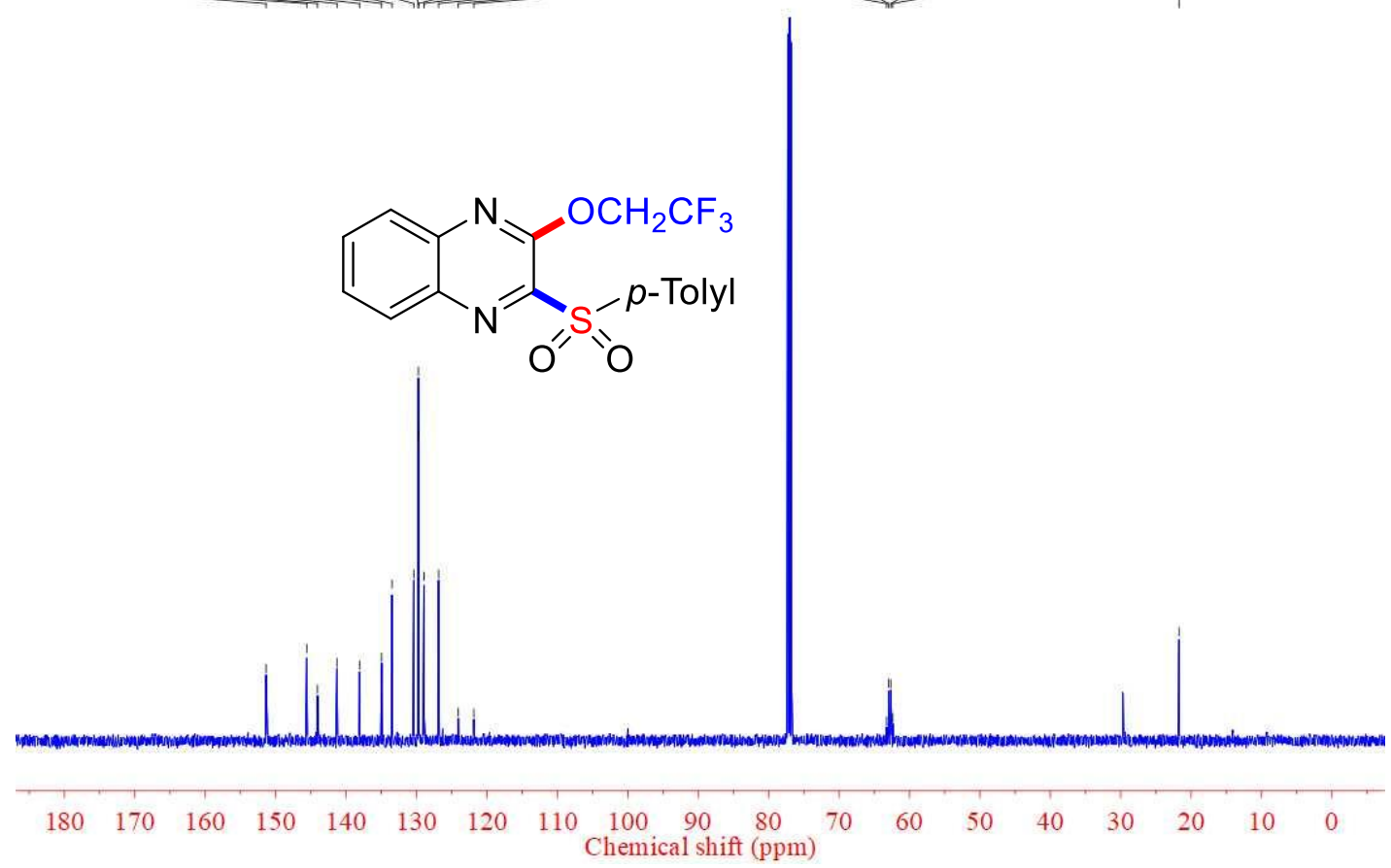

\section{19 ${ }^{19}$ NMR}

$$
\begin{aligned}
& \infty \\
& i \\
& i
\end{aligned}
$$<smiles>O=S(=O)(O[Ge])c1nc2ccccc2nc1OCC(F)(F)F</smiles>

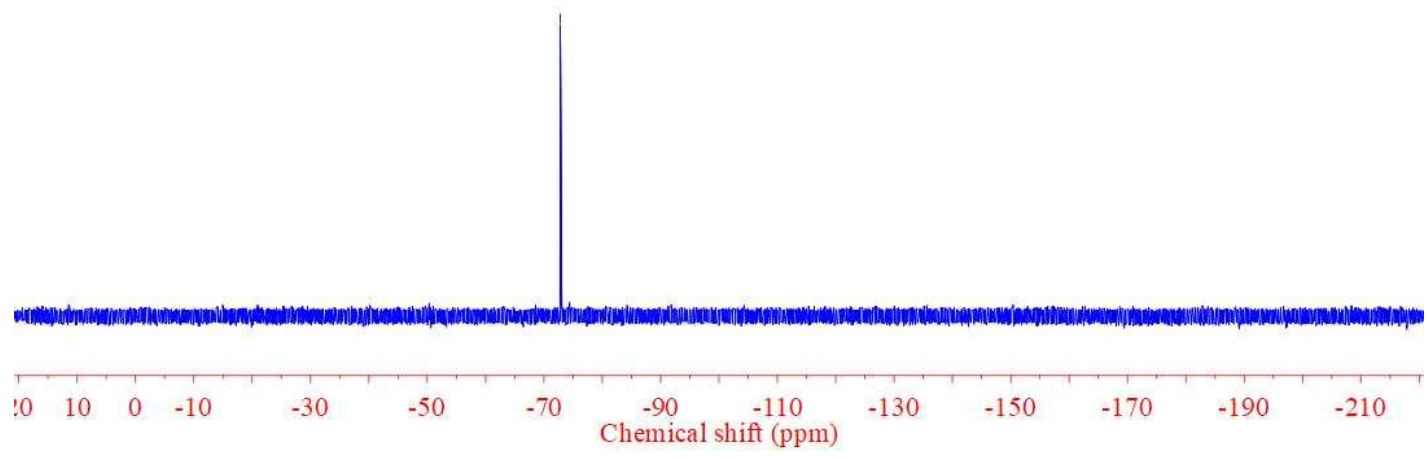




\section{$11 \mathrm{~h}{ }^{1} \mathrm{H}$ NMR}

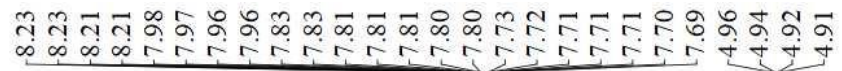
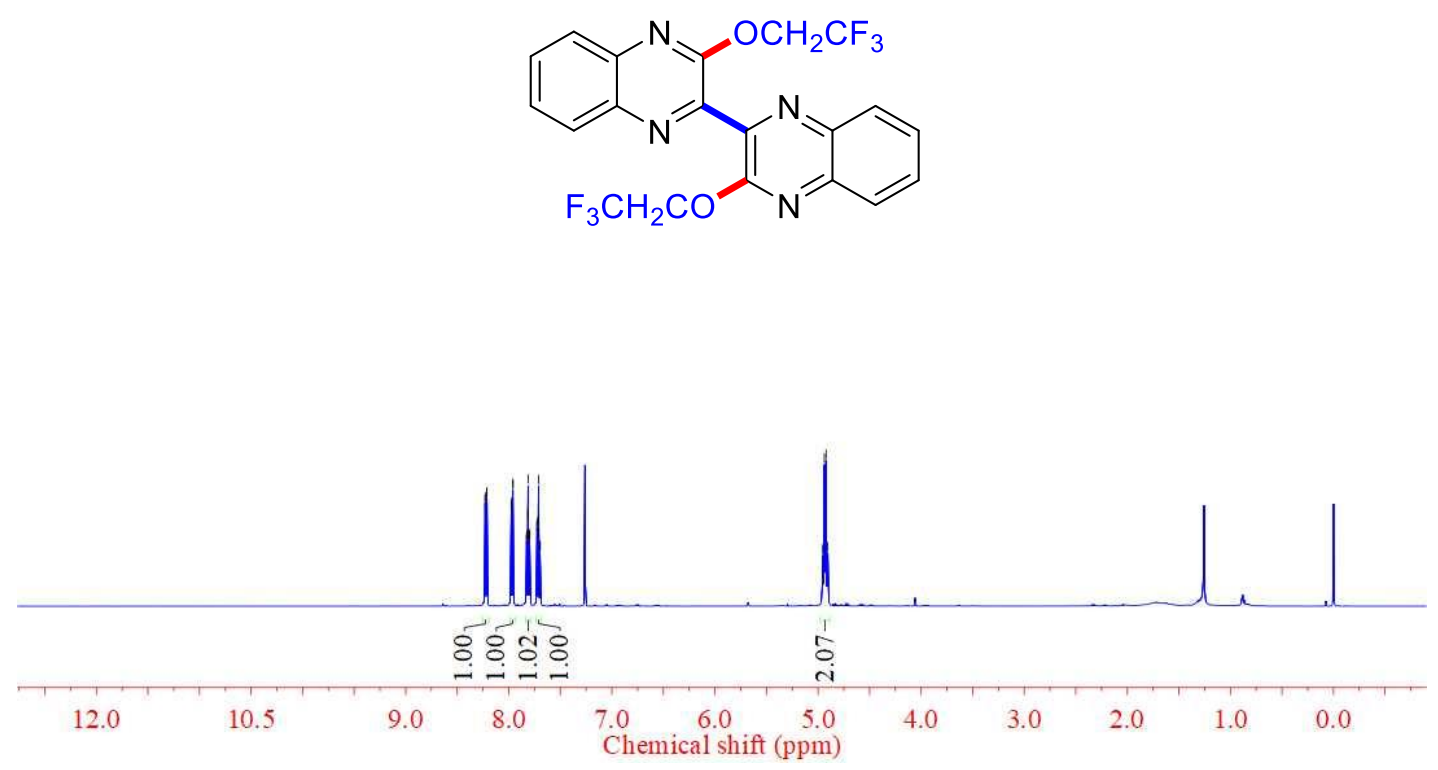

\section{$11 \mathrm{~h}{ }^{13} \mathrm{C}$ NMR}

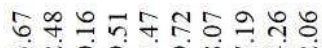

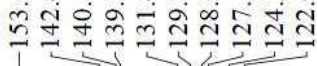

우워

ถู่
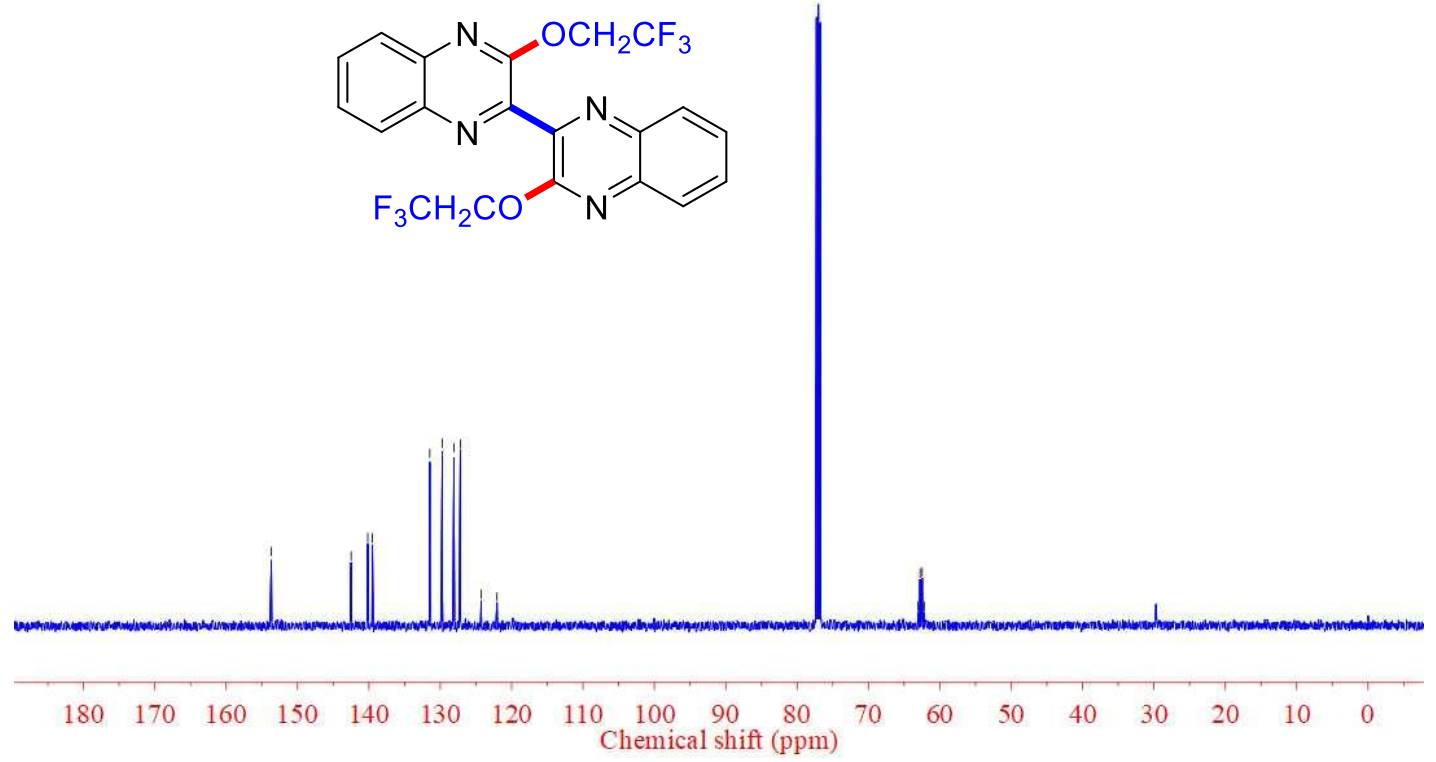


\section{1h ${ }^{19}$ F NMR}

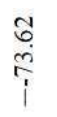

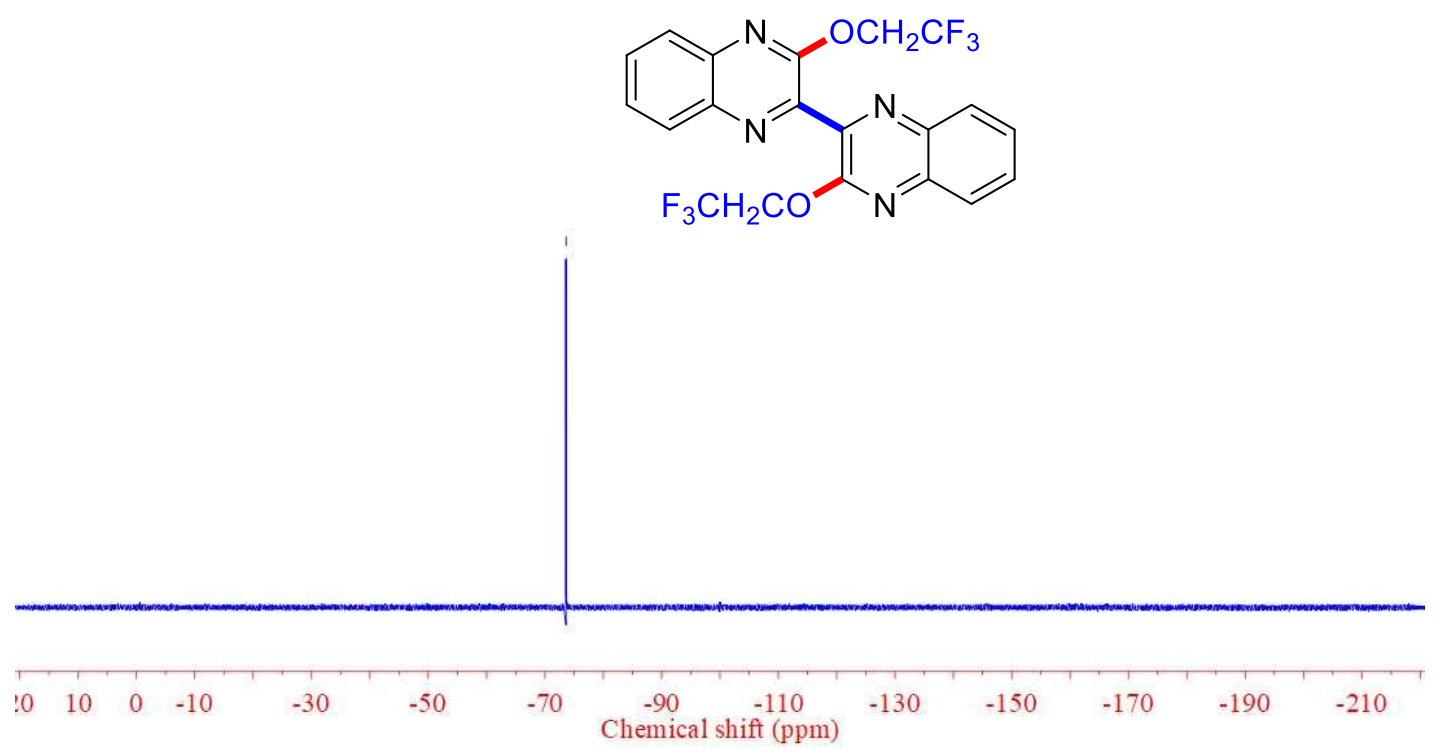

\section{2a ${ }^{1} \mathrm{H}$ NMR}

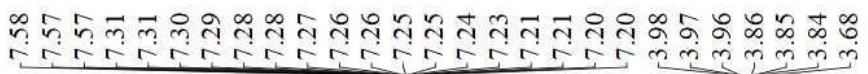

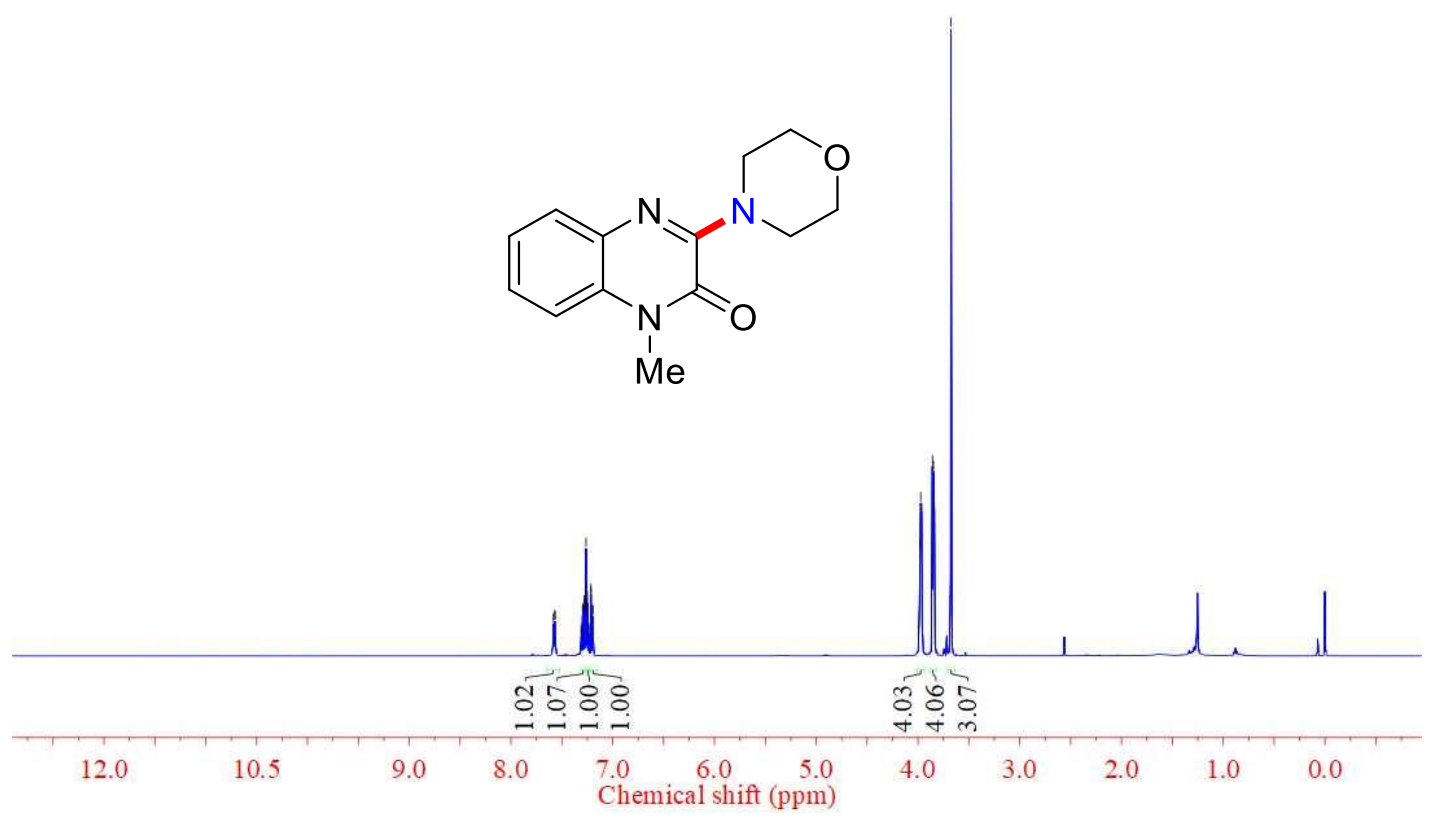




\section{2a ${ }^{13} \mathrm{C}$ NMR}

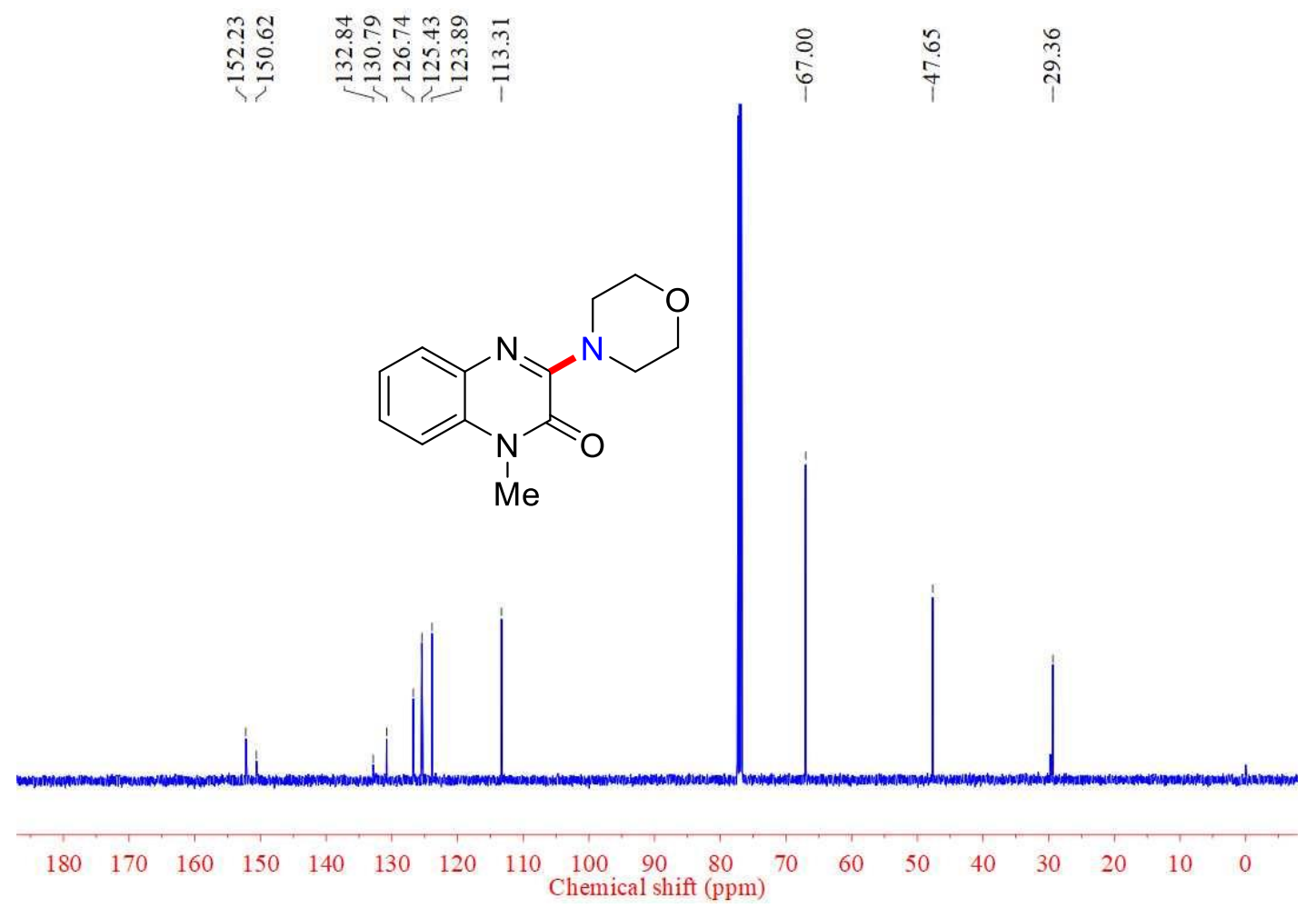

\section{2b ${ }^{1} \mathrm{H}$ NMR}

$\begin{array}{ll}1 & 0 \\ 0 & 0\end{array}$<smiles>O=c1[nH]c2ccccc2nc1N1CCOCC1</smiles>

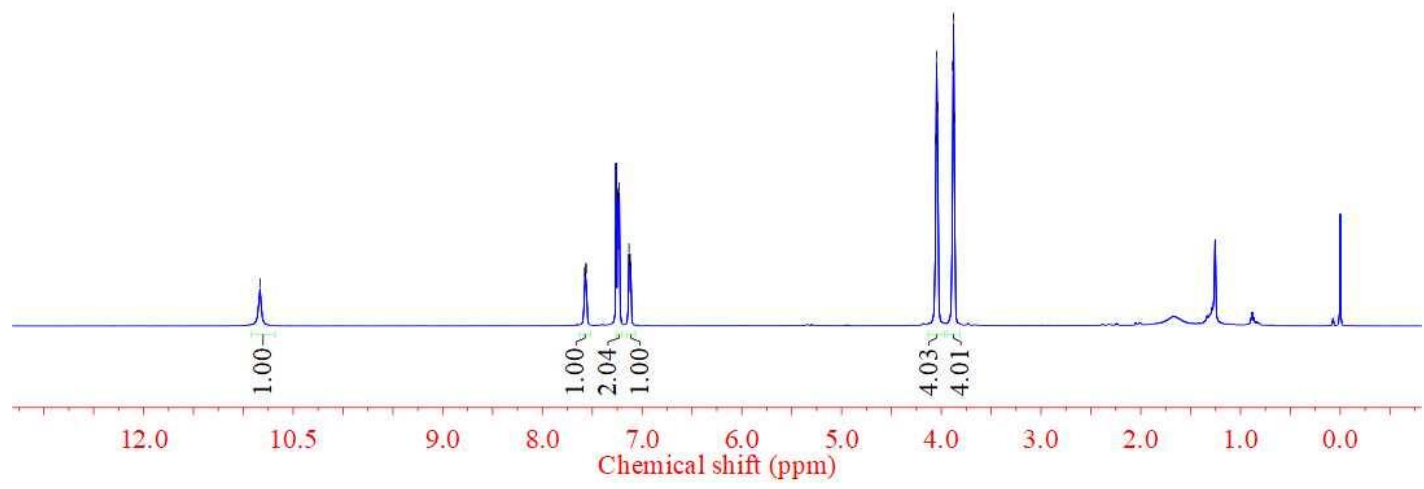




\section{2b ${ }^{13} \mathrm{C}$ NMR}

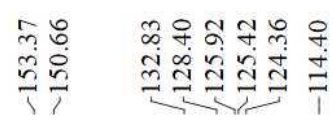

ลิ<smiles>O=c1[nH]c2ccccc2nc1N1CCOCC1</smiles> 


\section{$13{ }^{13} \mathrm{C}$ NMR}

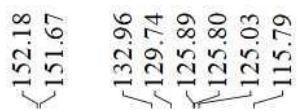

(c)

तٓ요

访泋

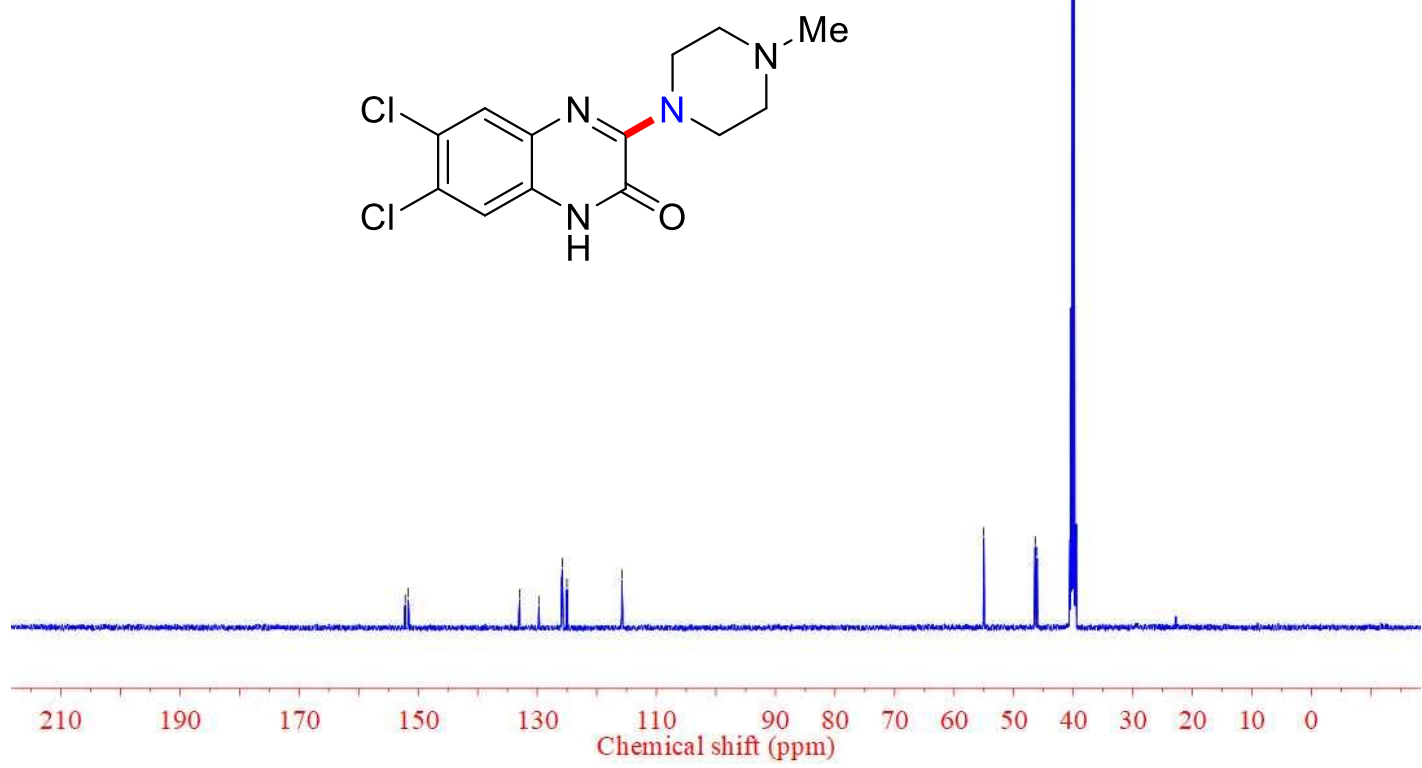




\section{Copies of IR spectra}

\section{$3 \mathbf{a}$}

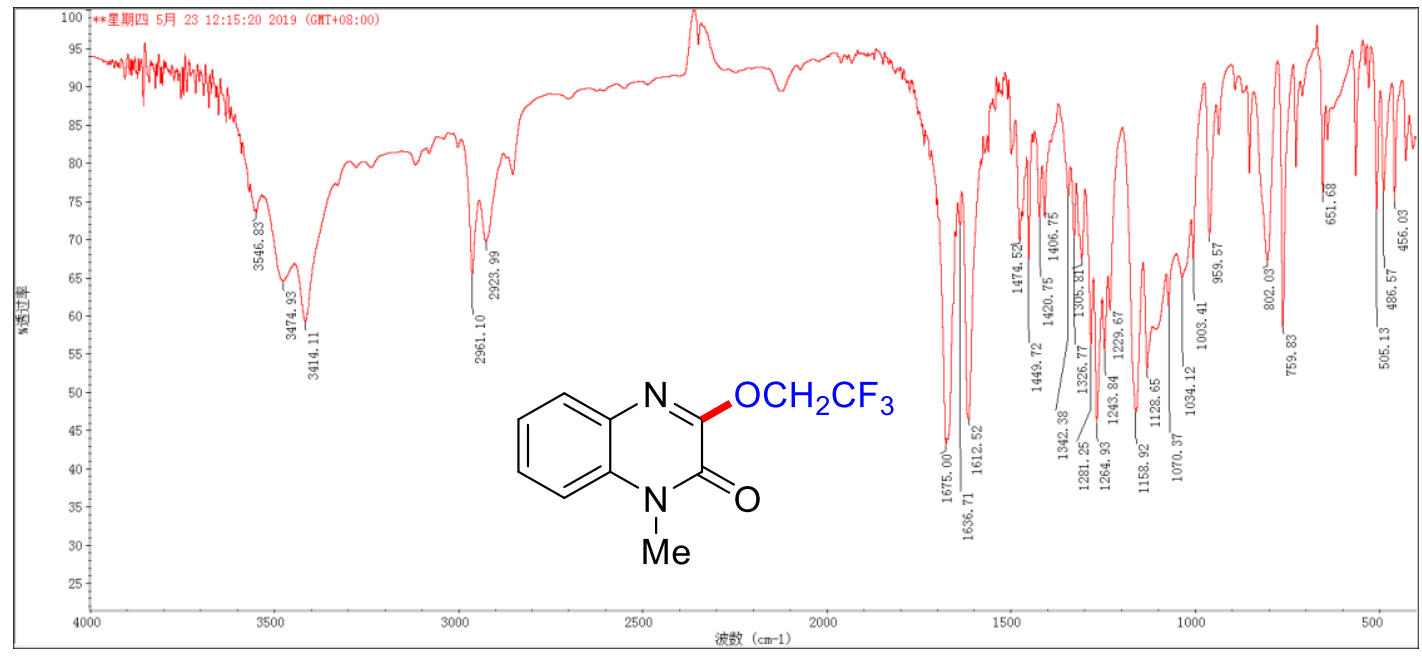

3b

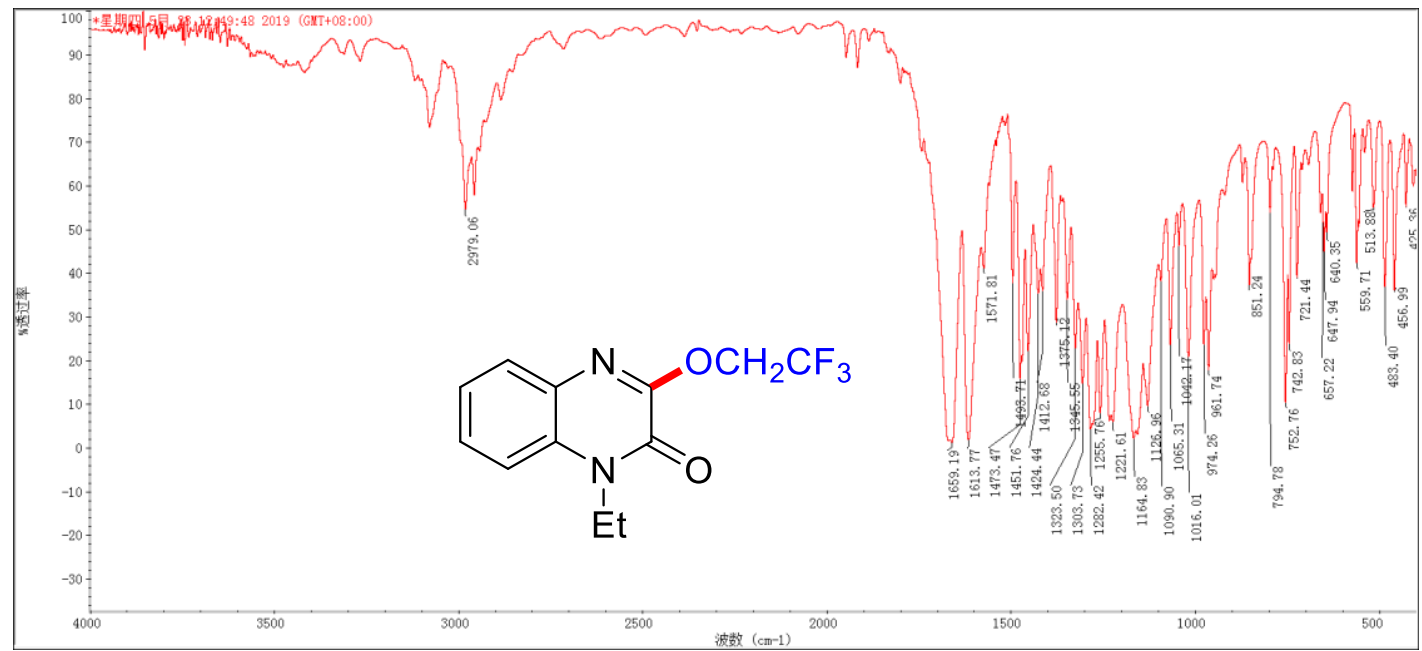

3c

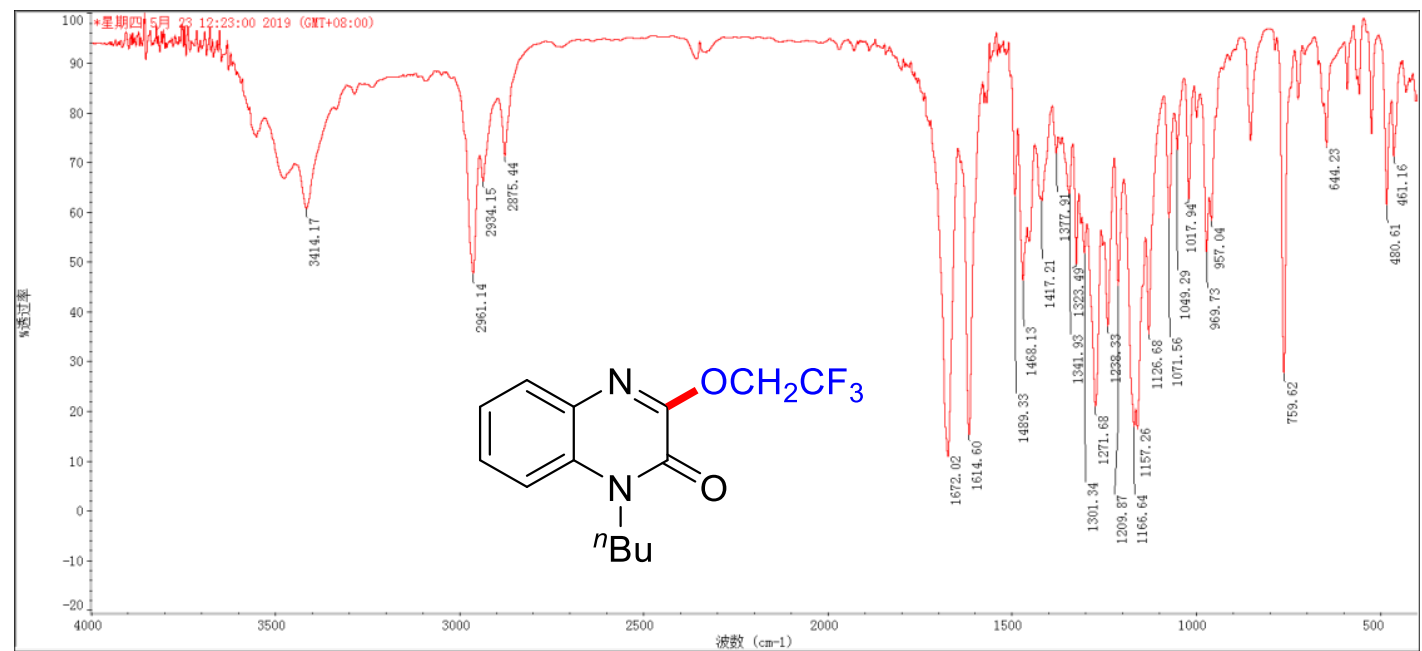


3d

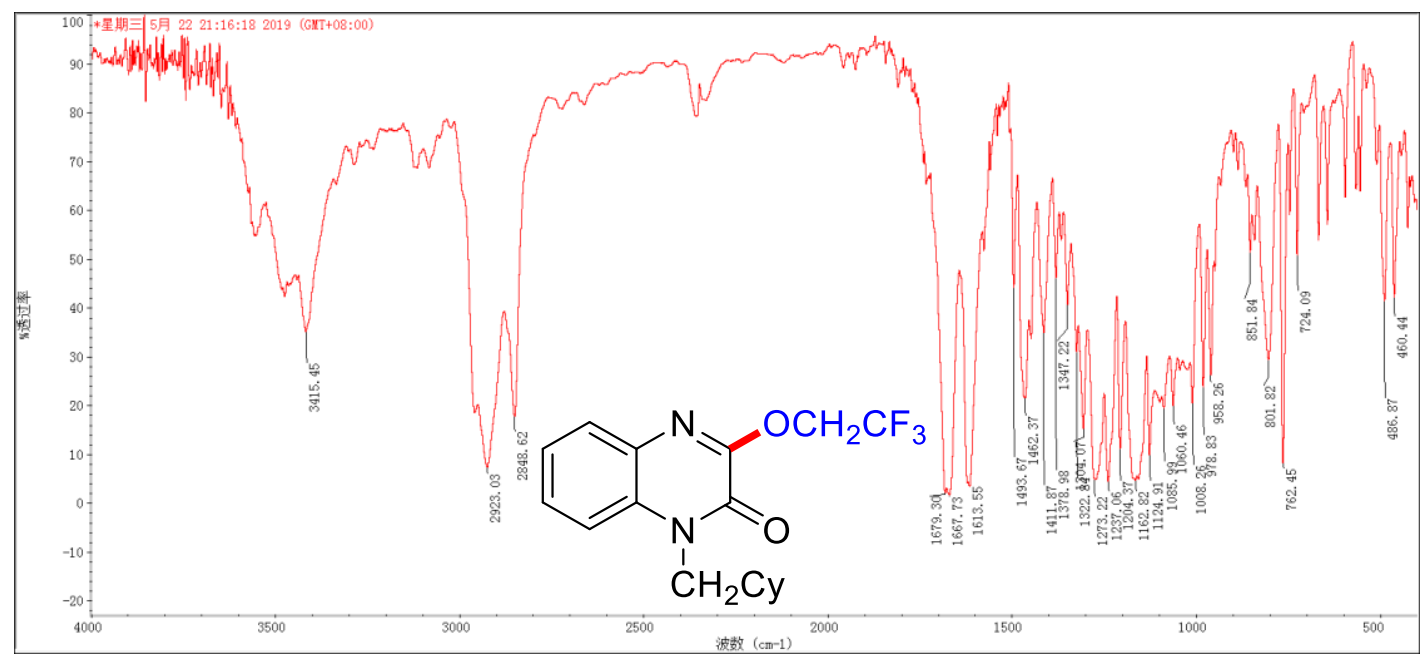

3e

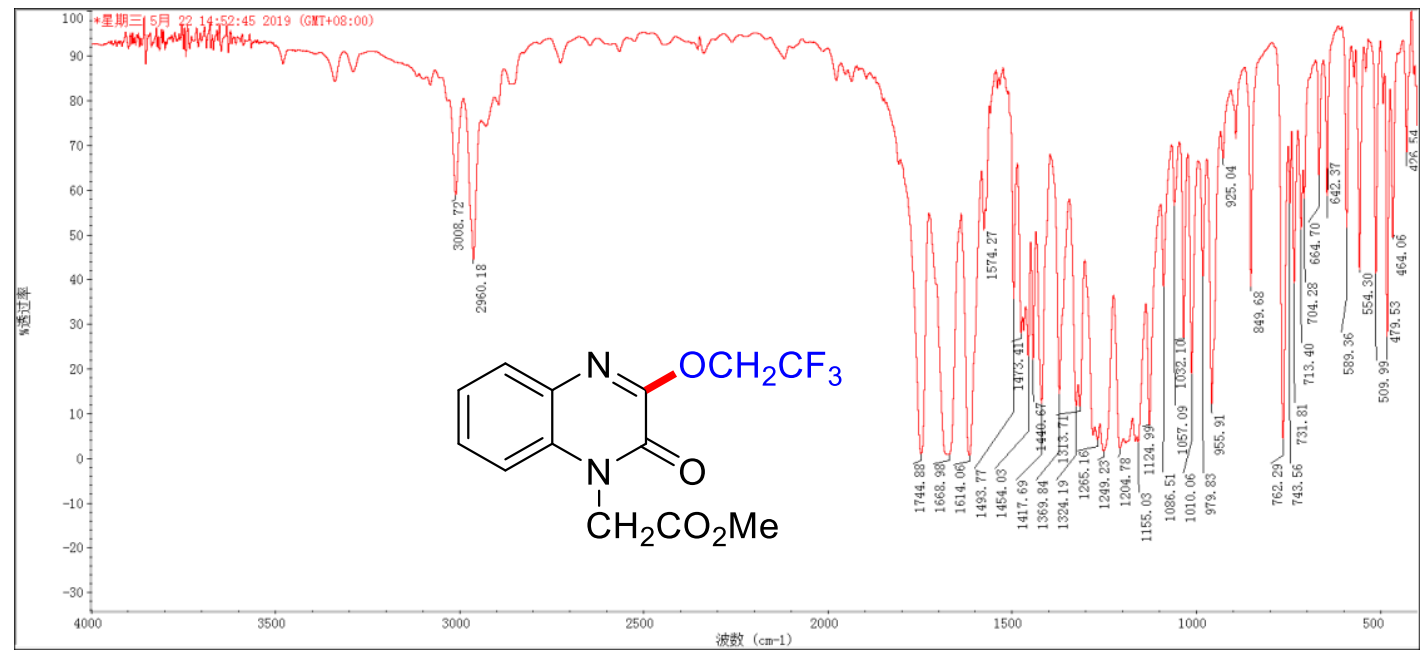

3f

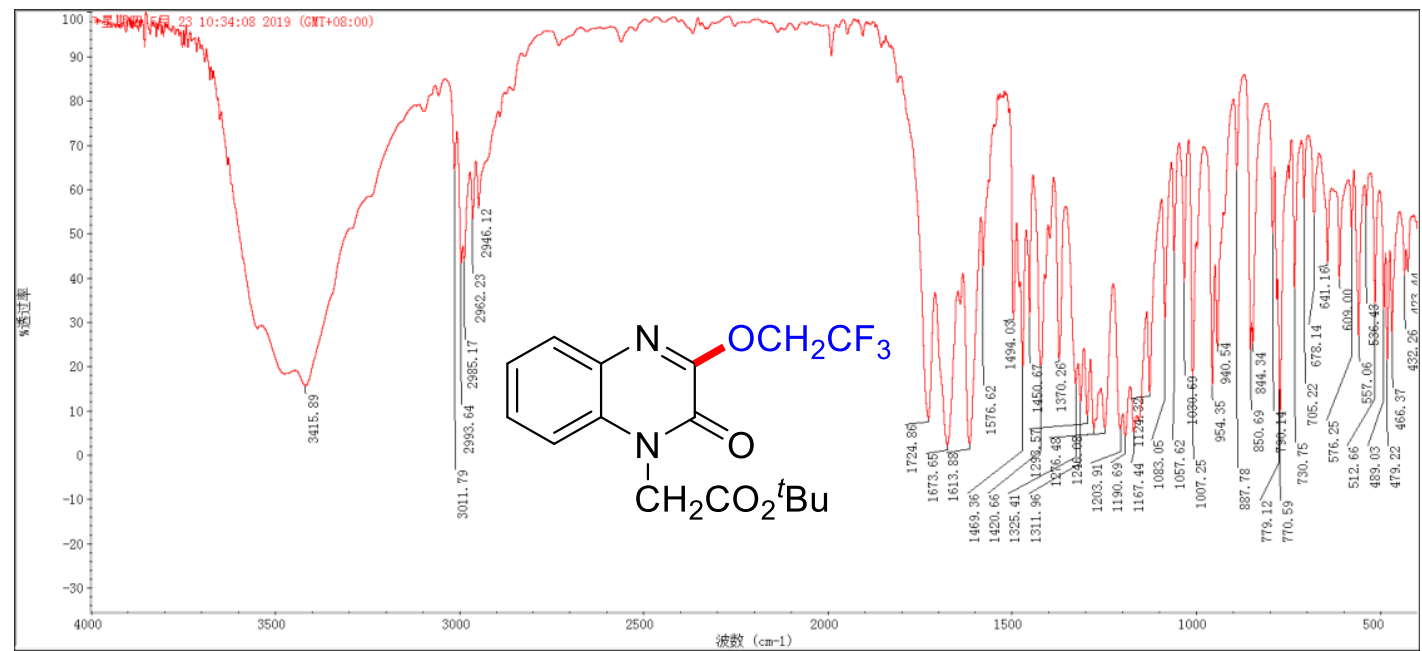




\section{$3 g$}

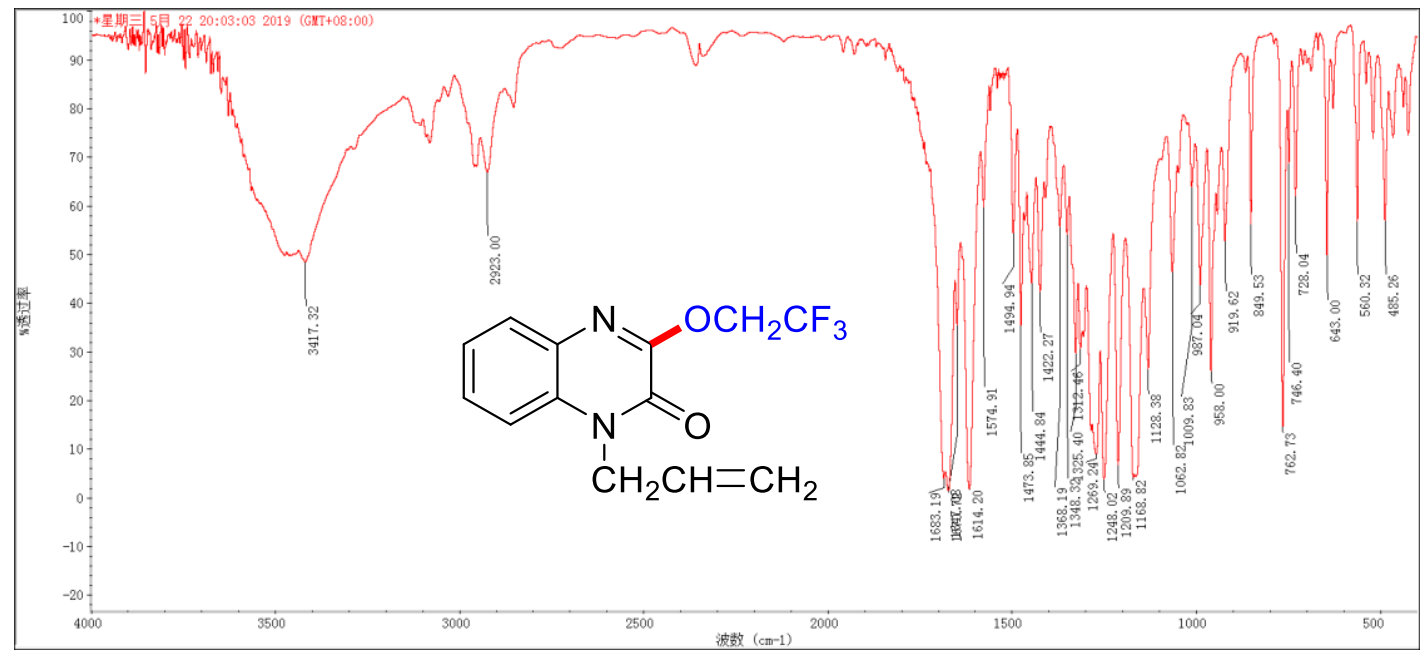

\section{3h}

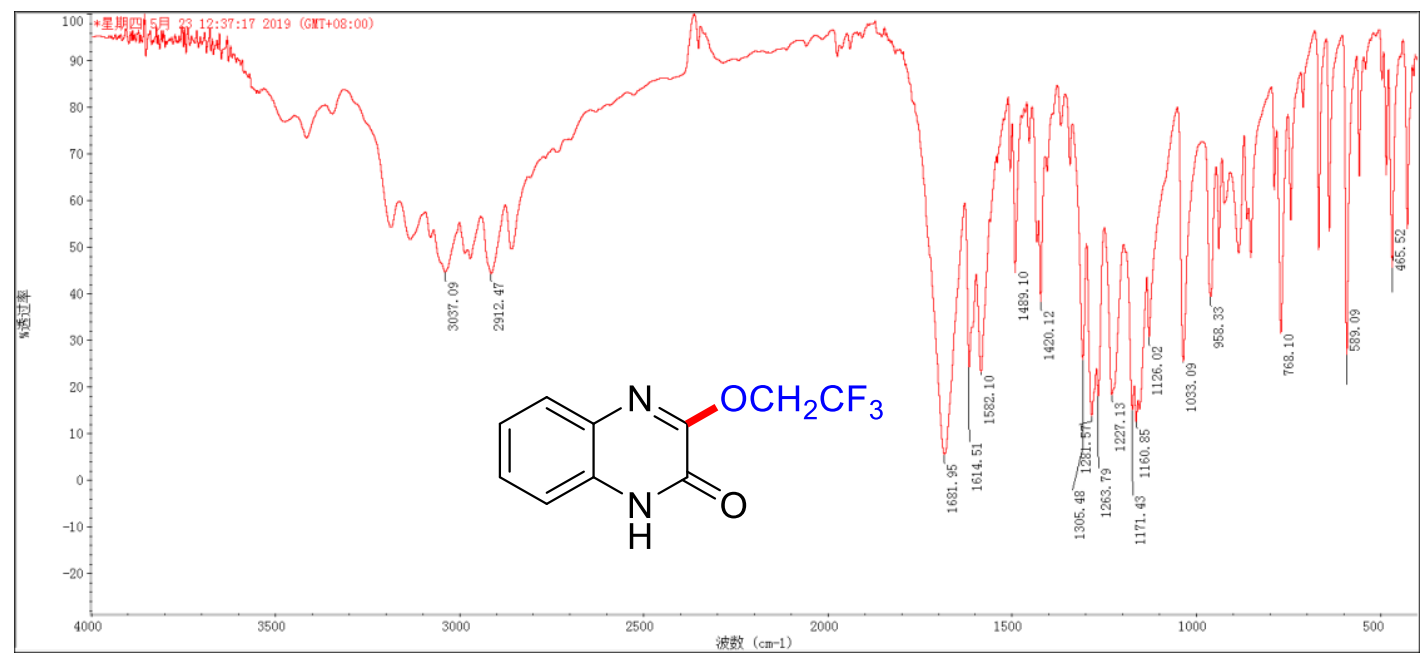

3i

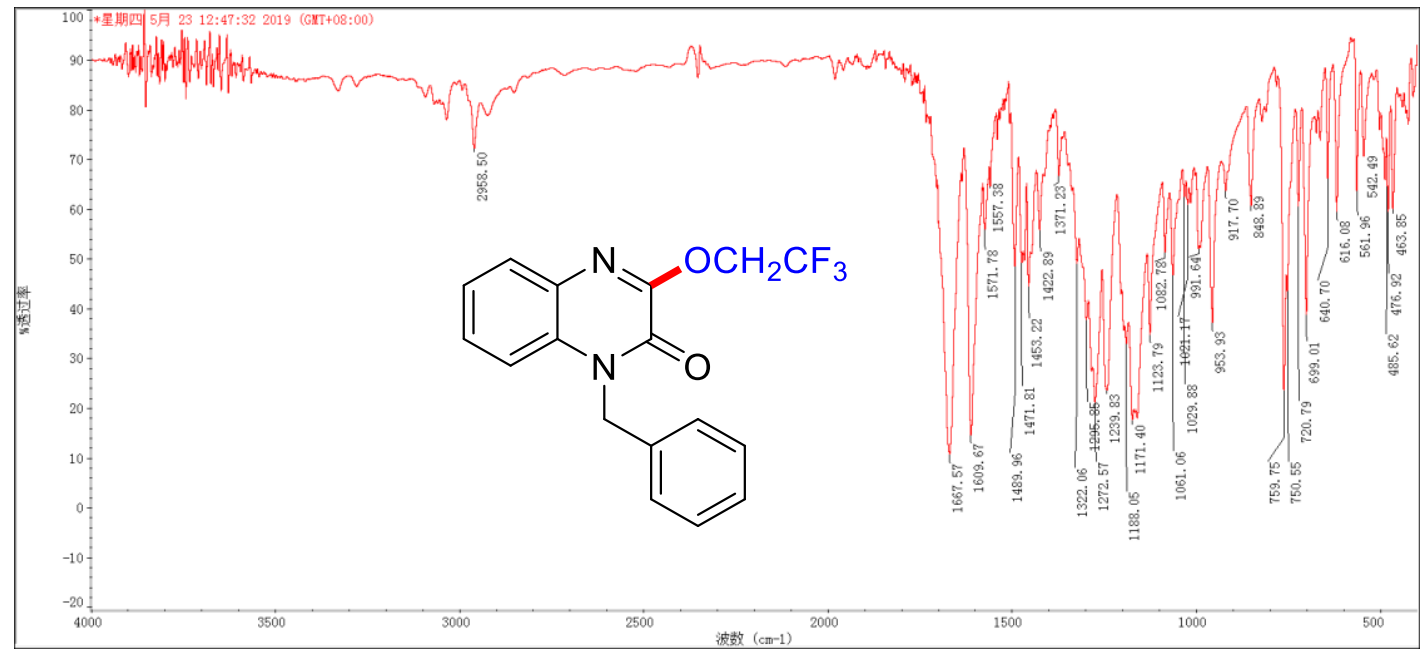


3j

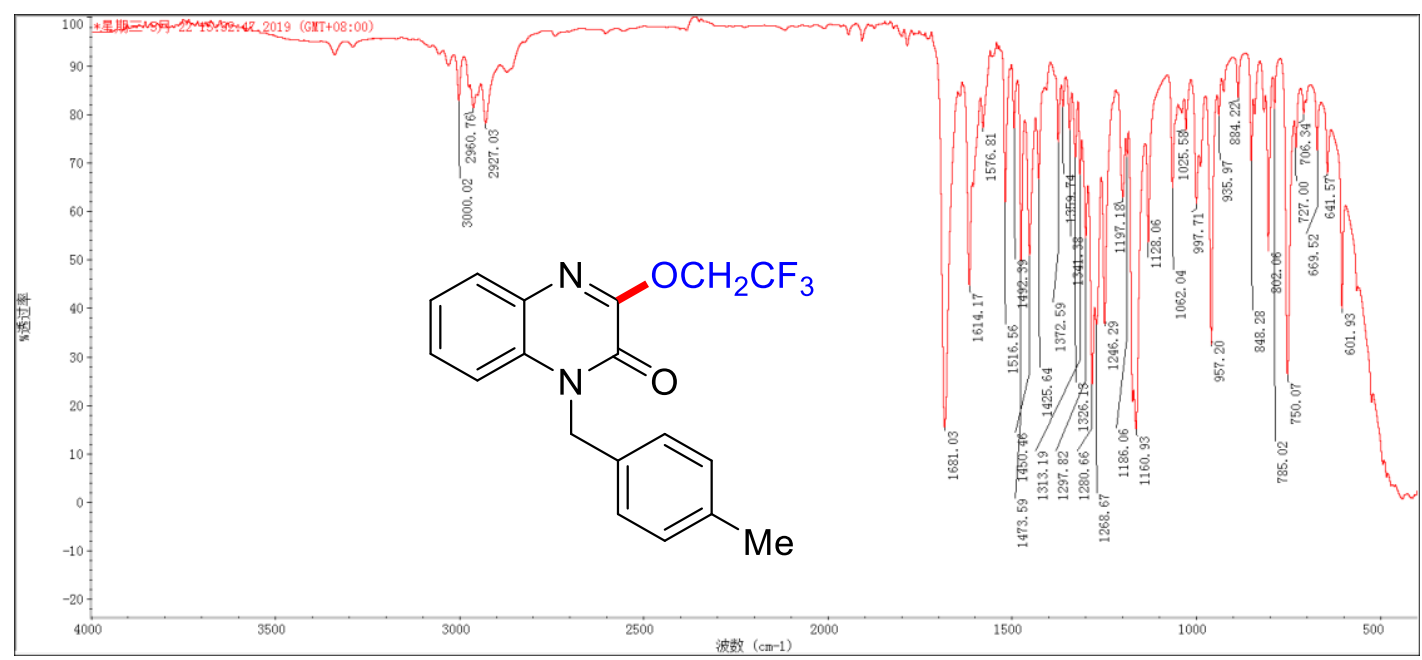

3k

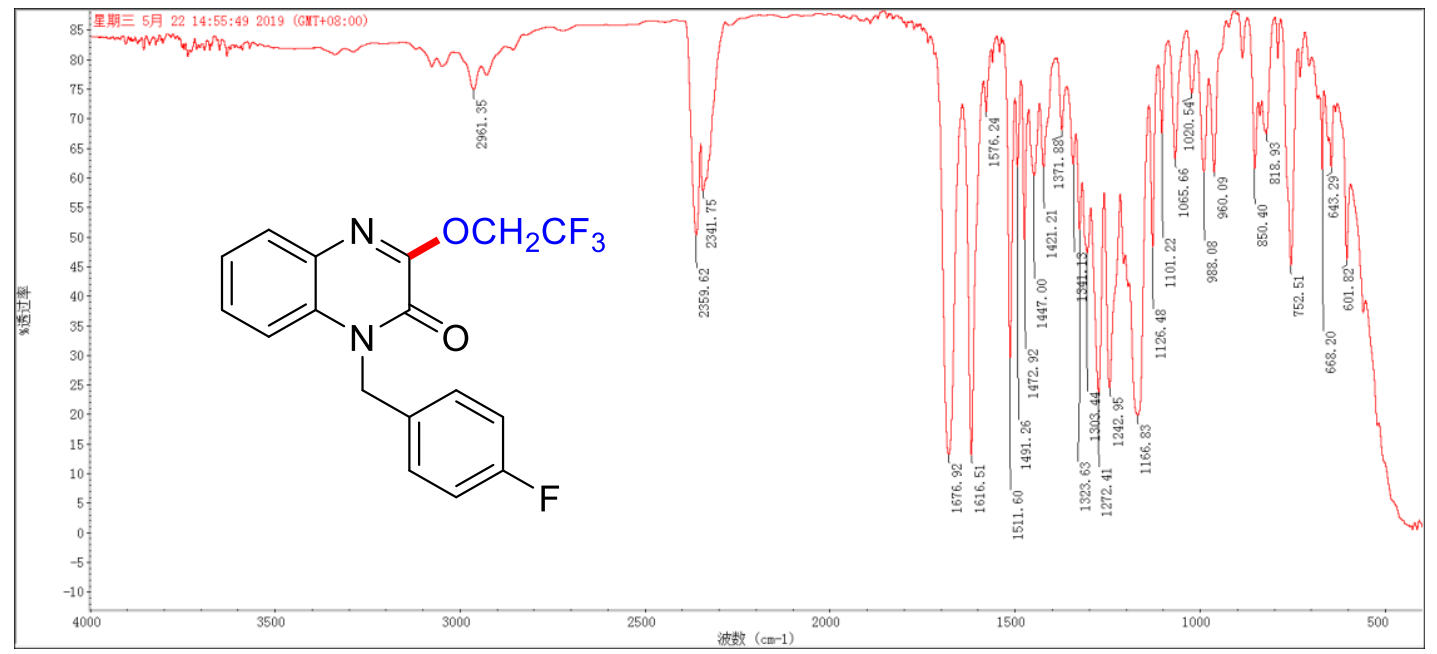

31

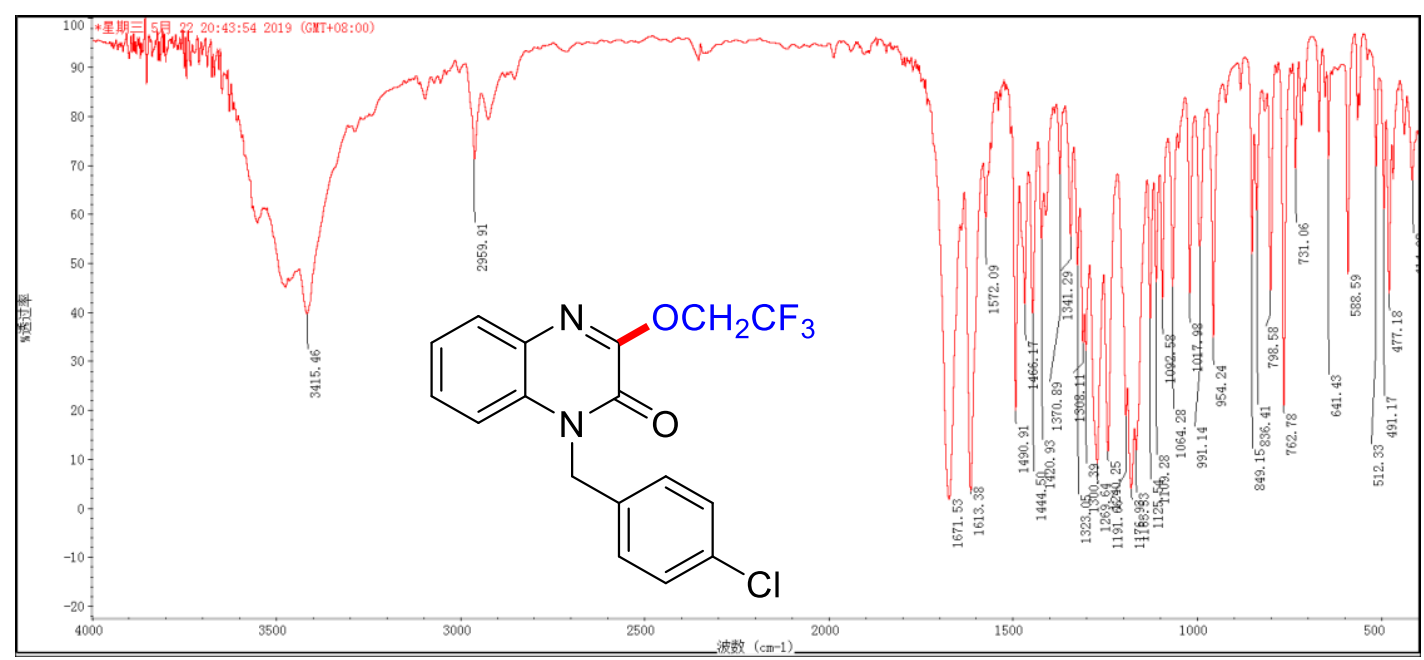


$3 m$

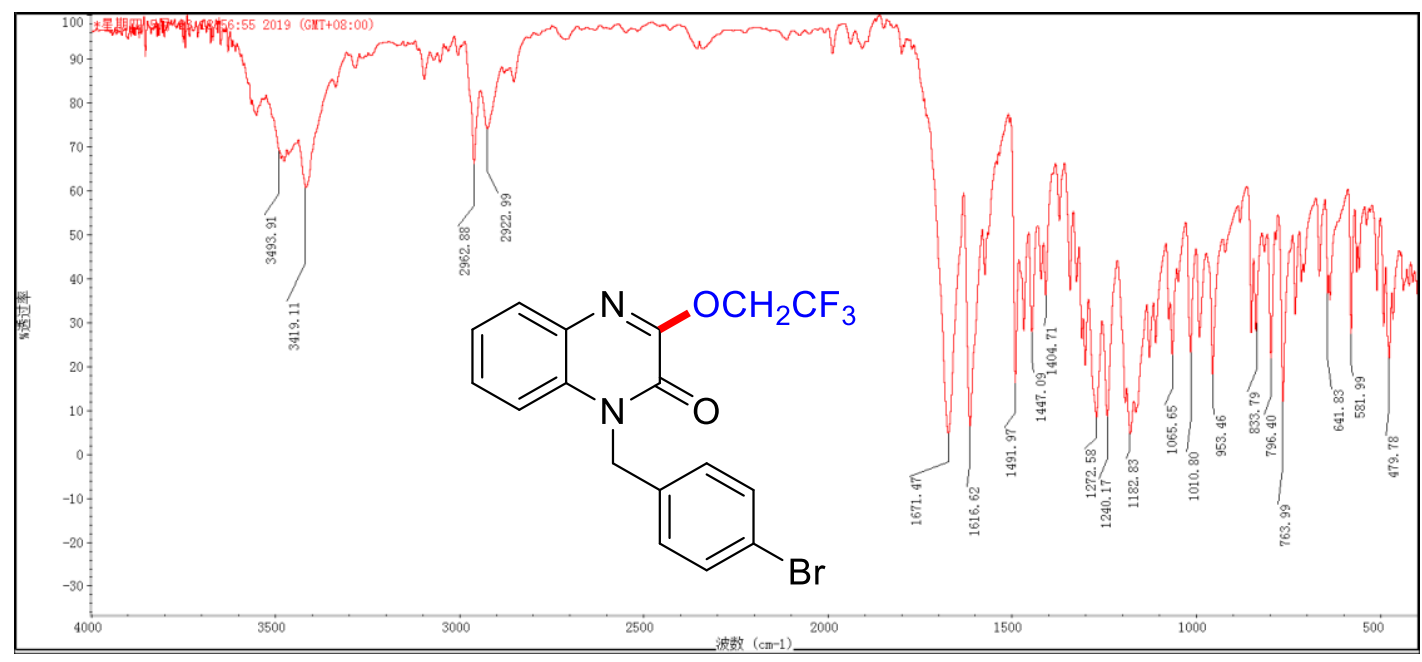

3n

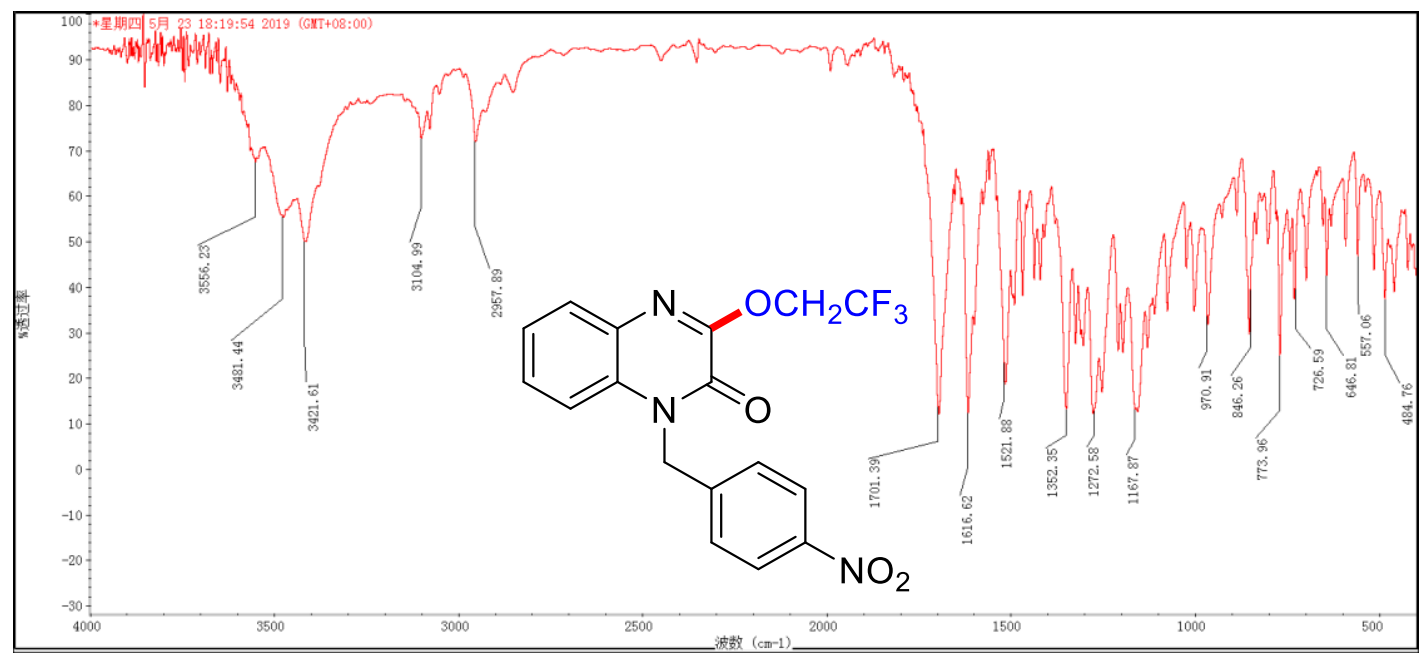

30

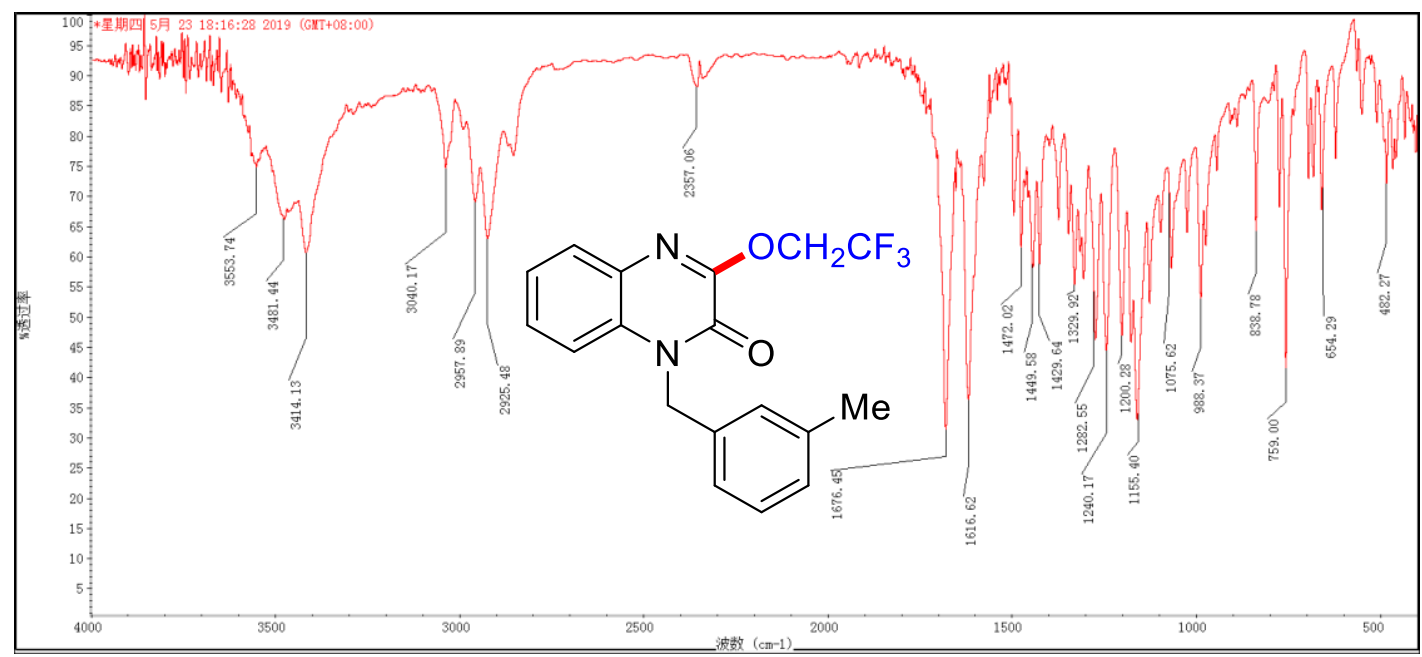


3p

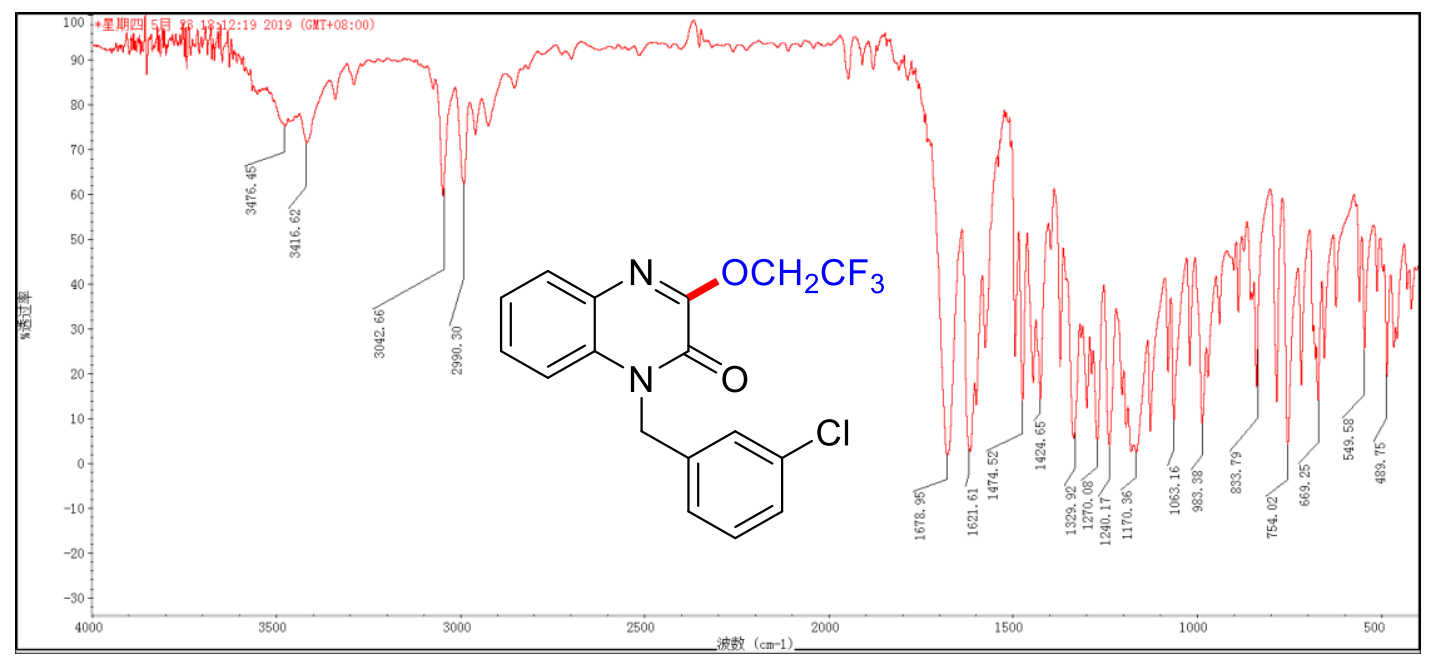

$3 q$

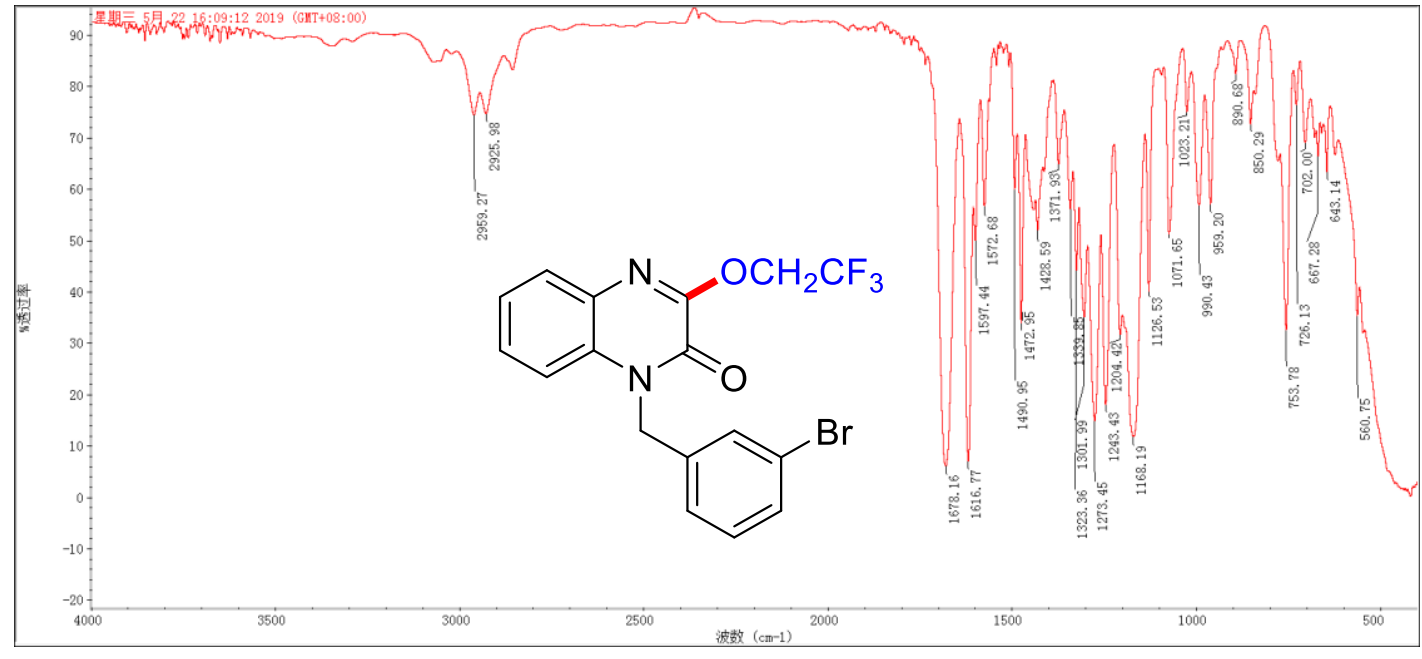

3r

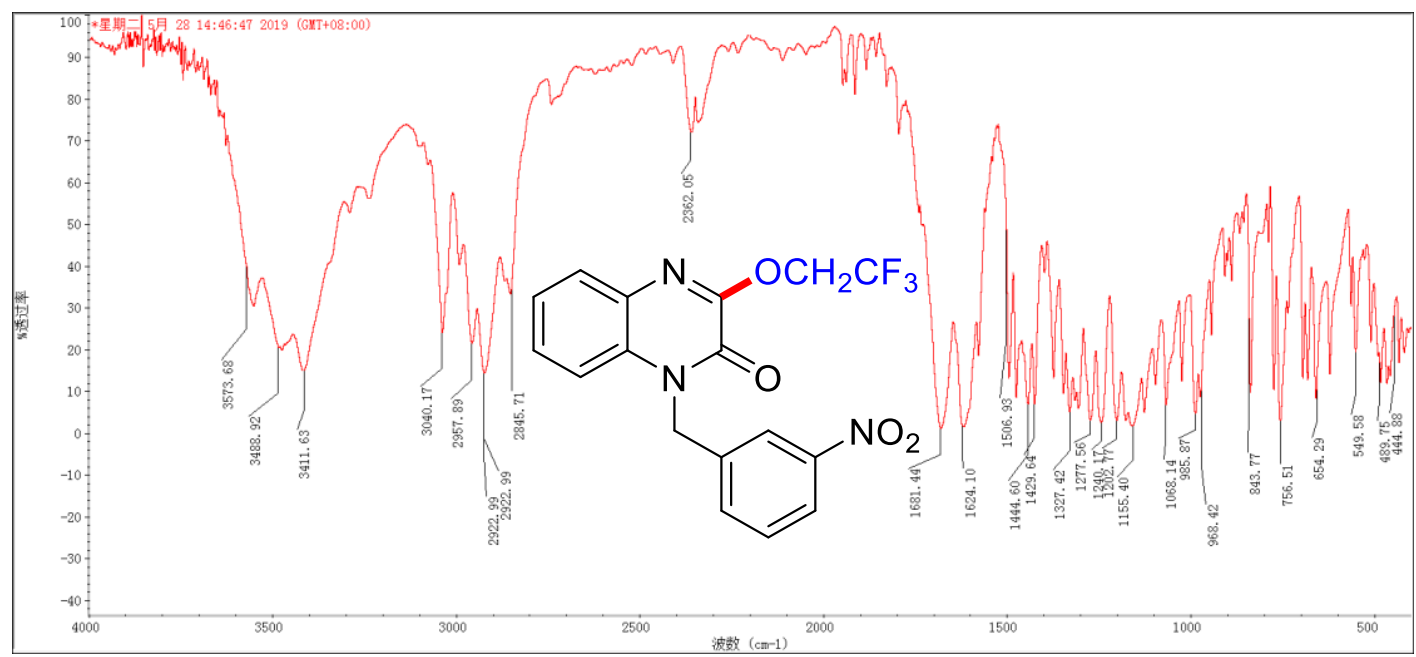


3s

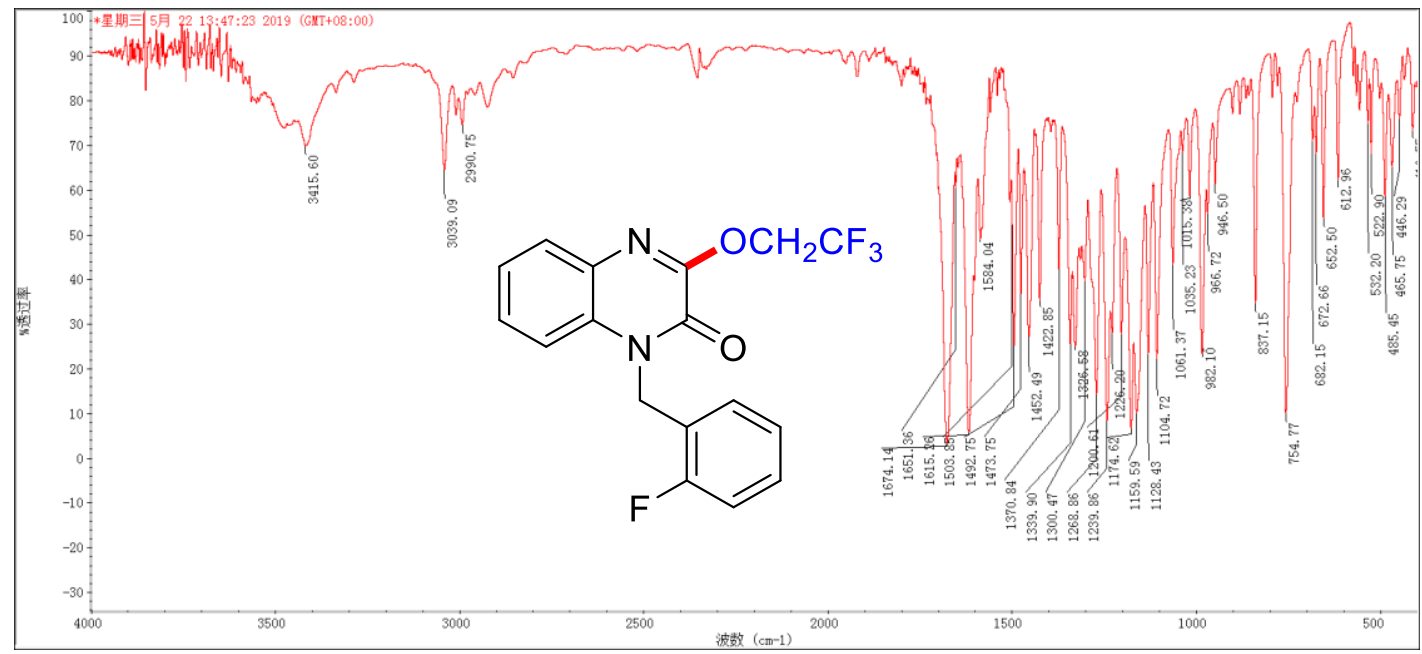

3t

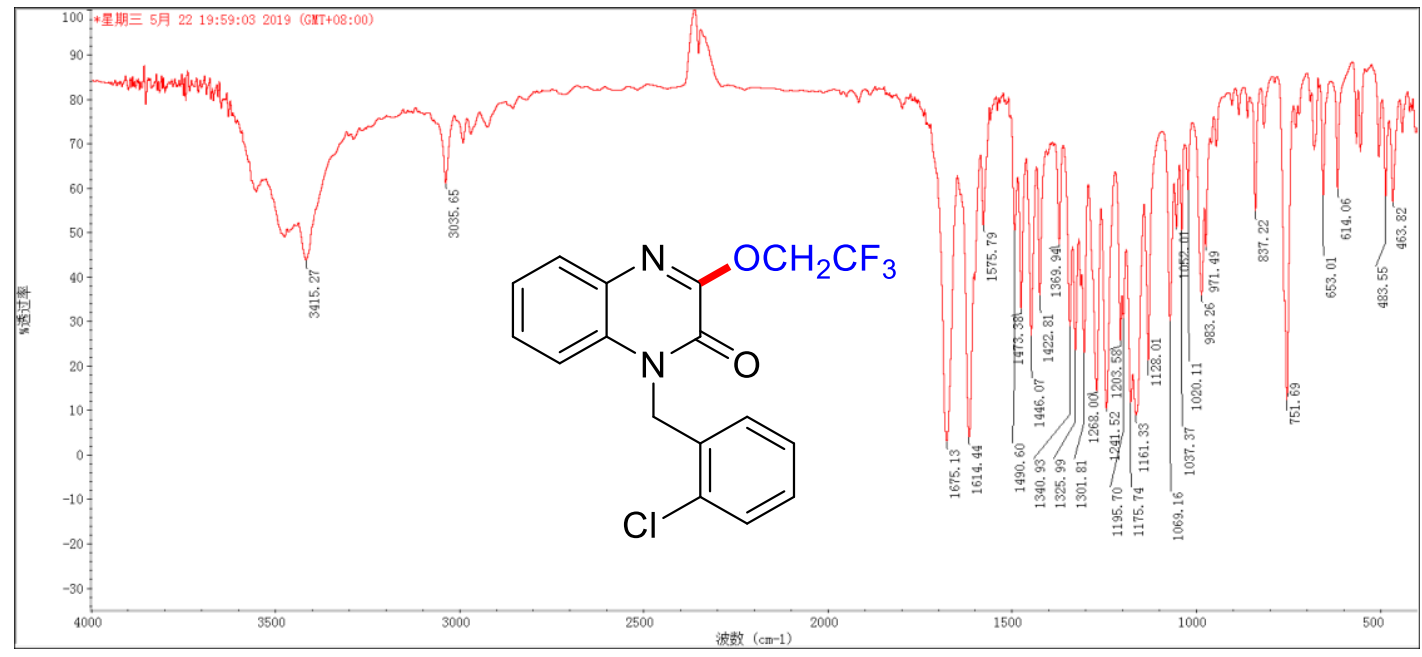

3u

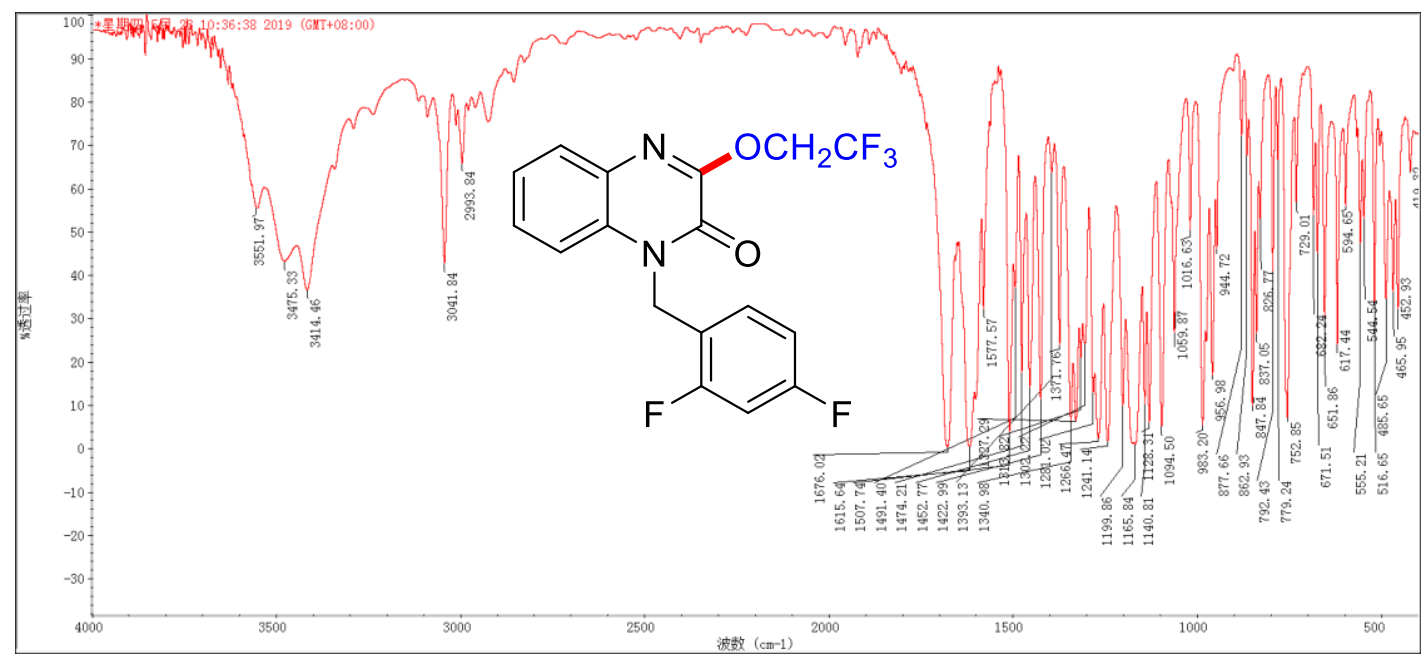


3v

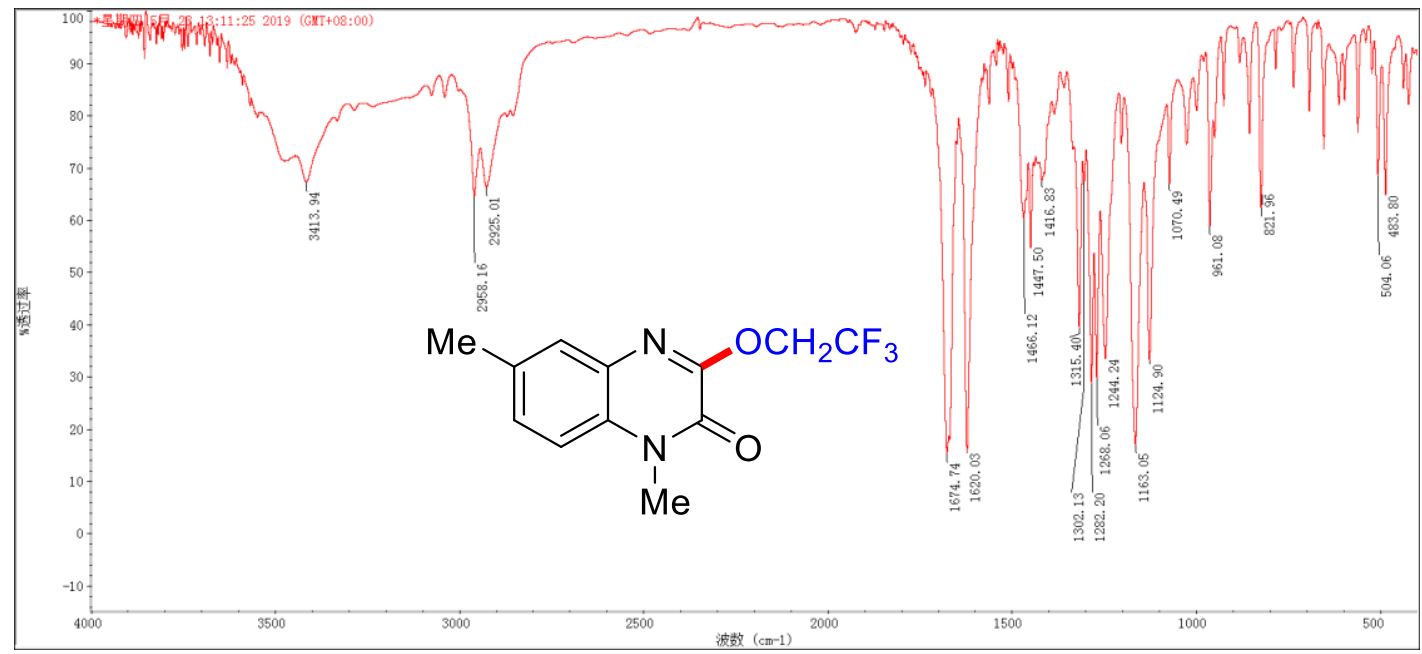

\section{3w}

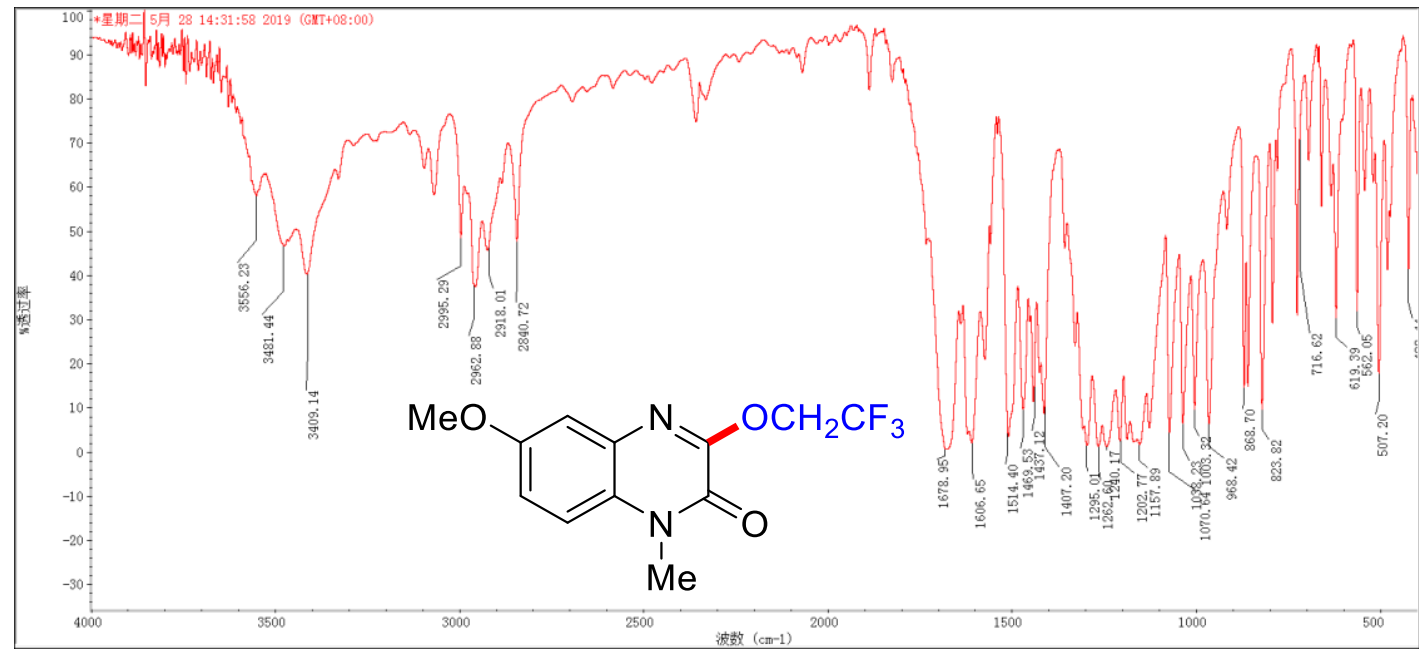

3x

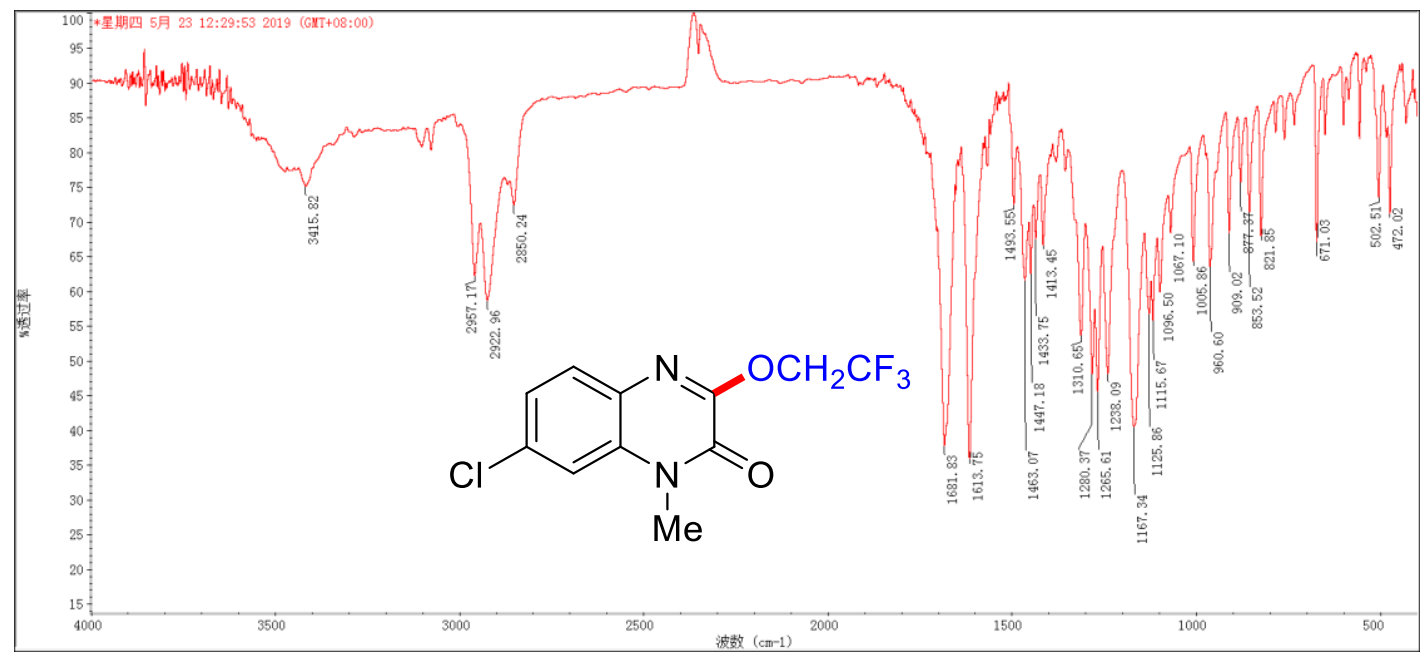




\section{3y}

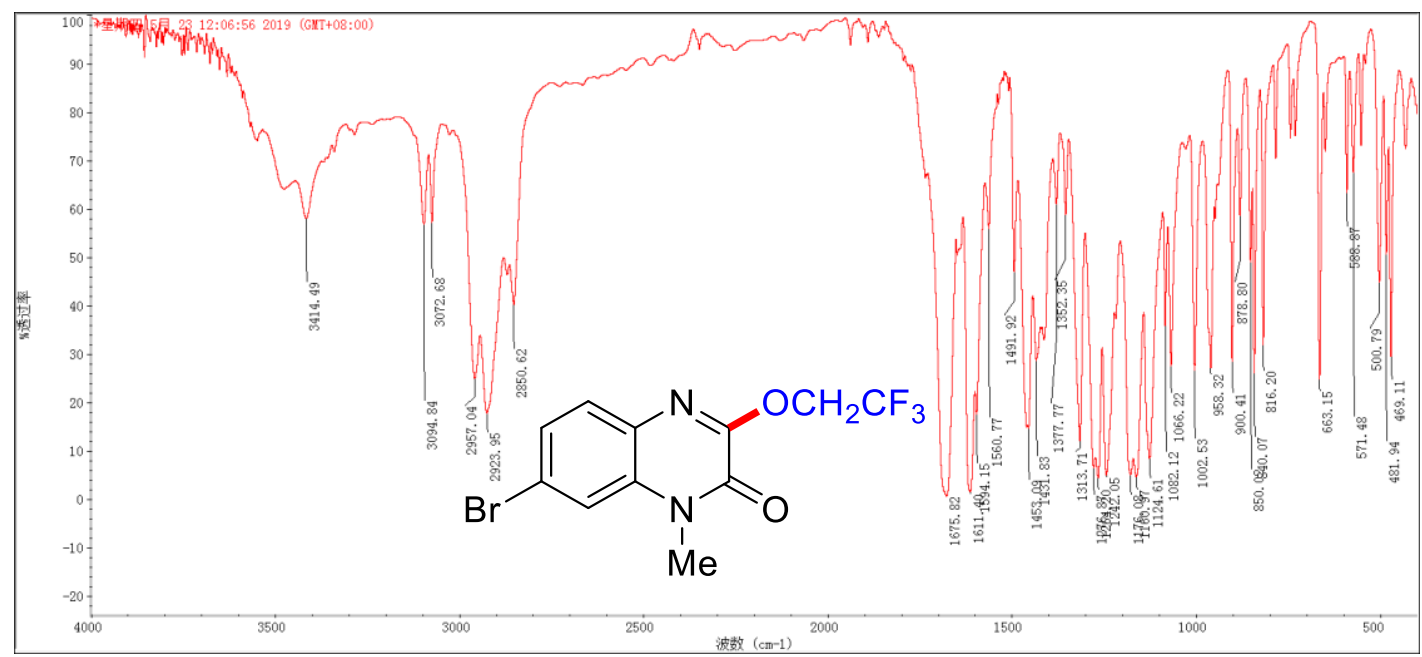

3z

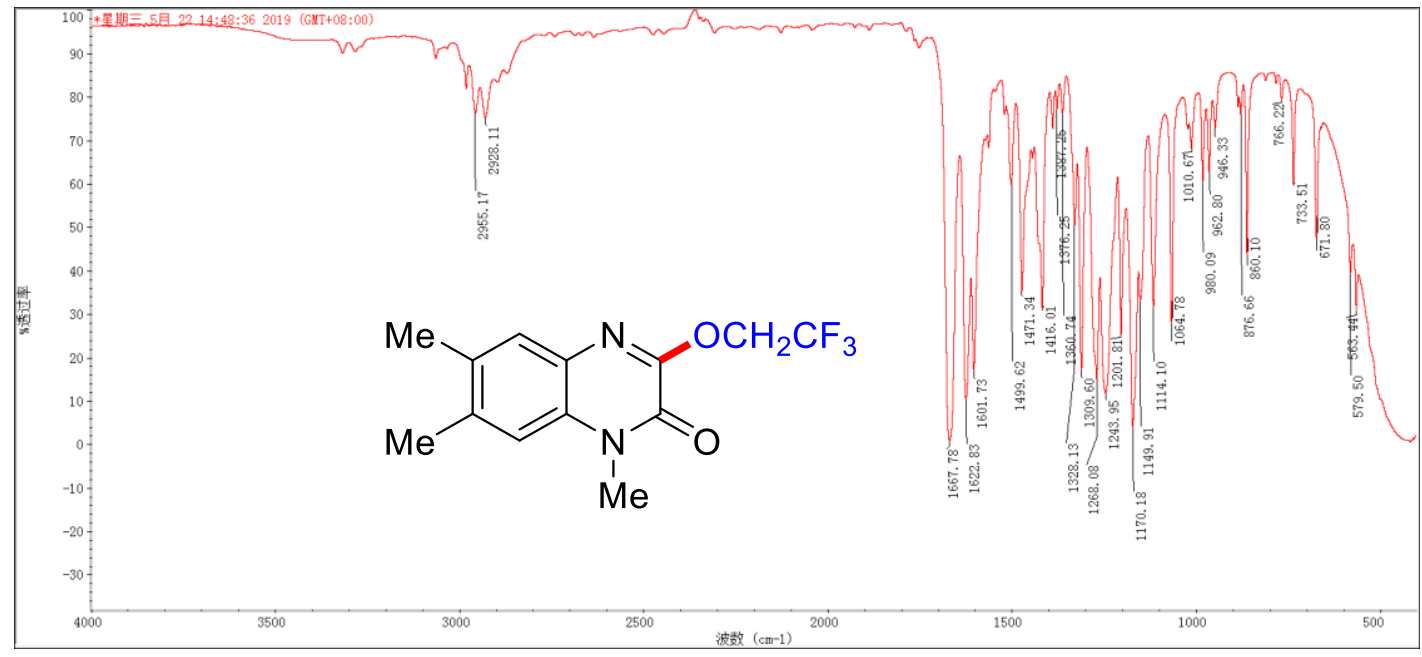

\section{3aa}

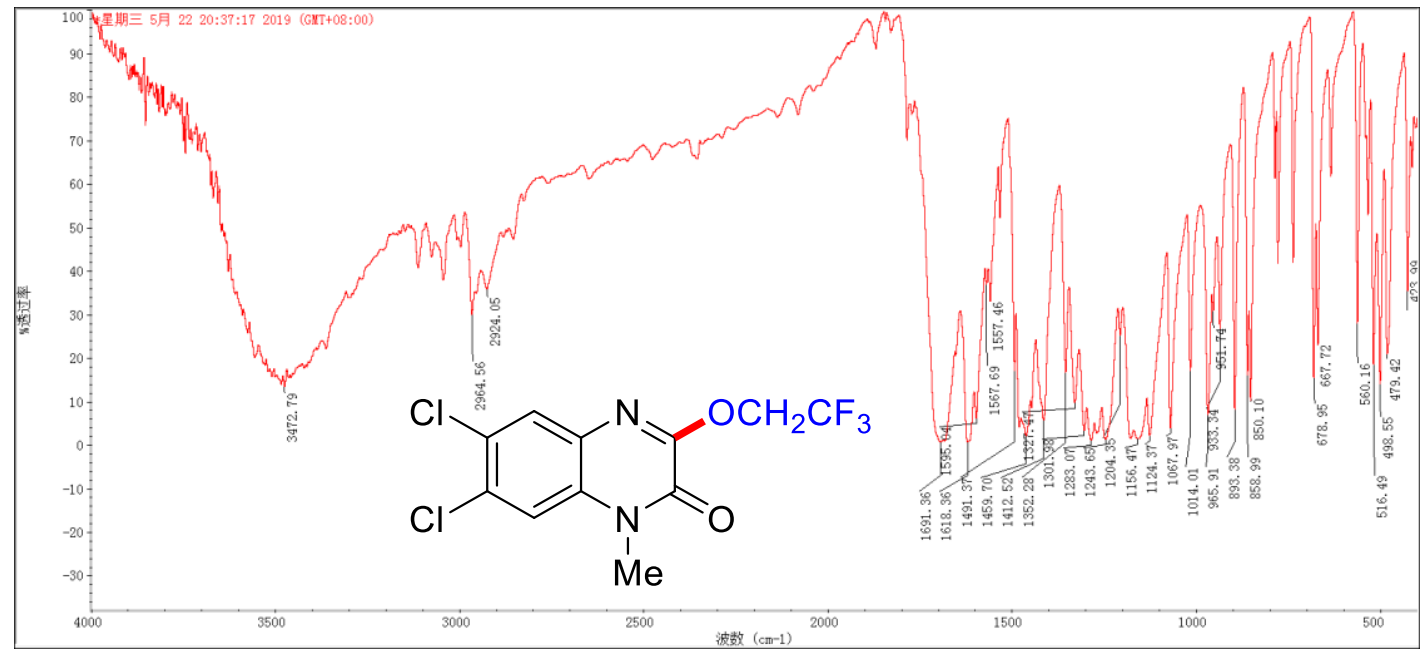


$4 a$

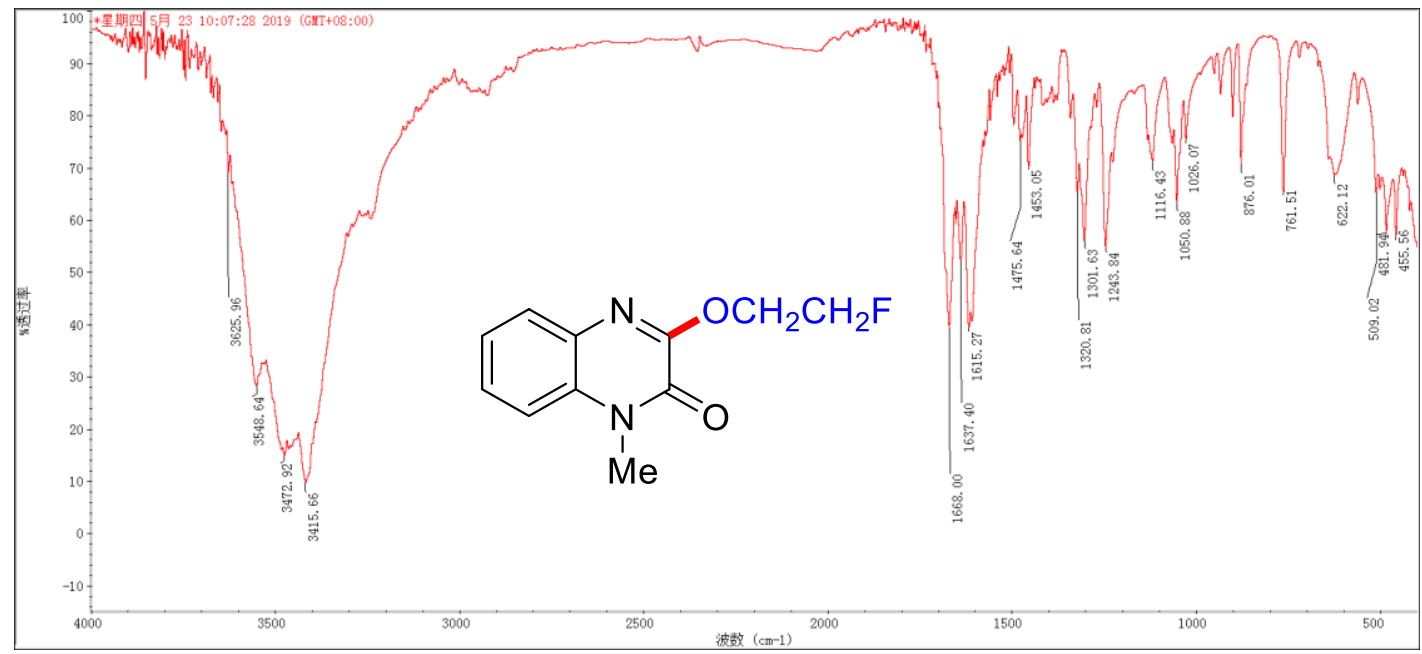

$4 b$

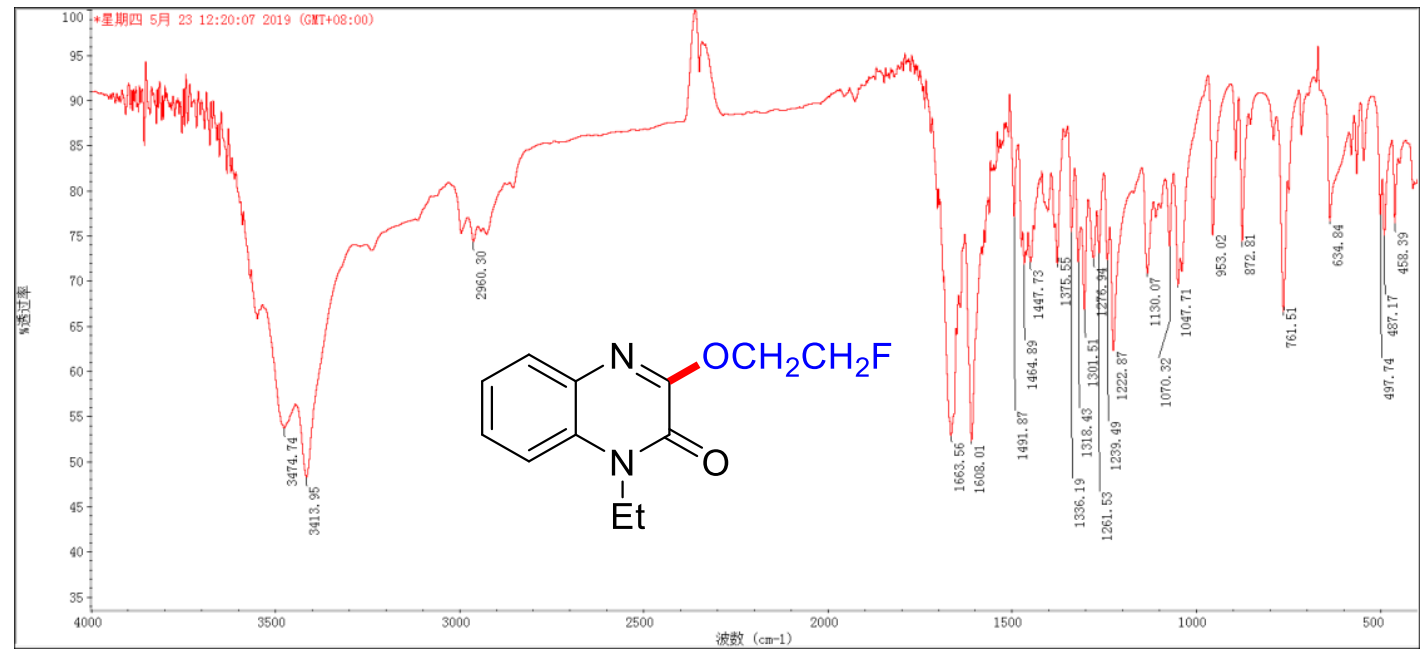

$4 c$

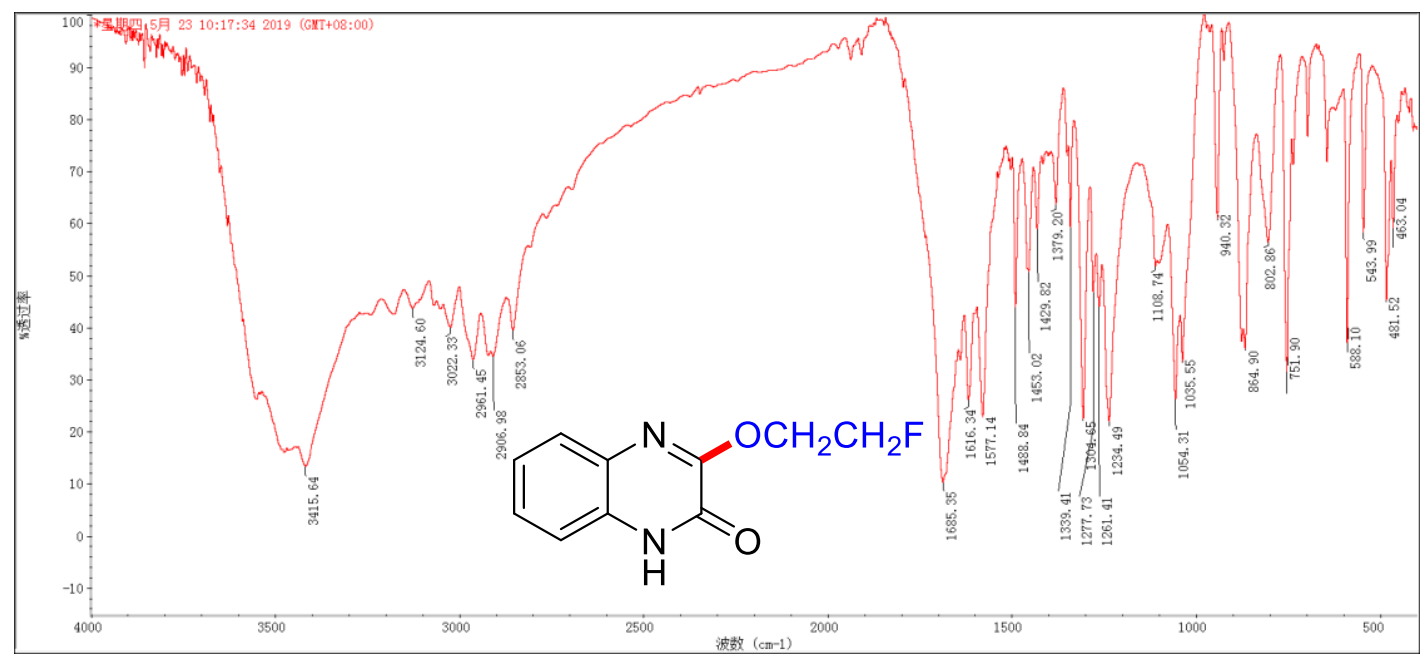


4d

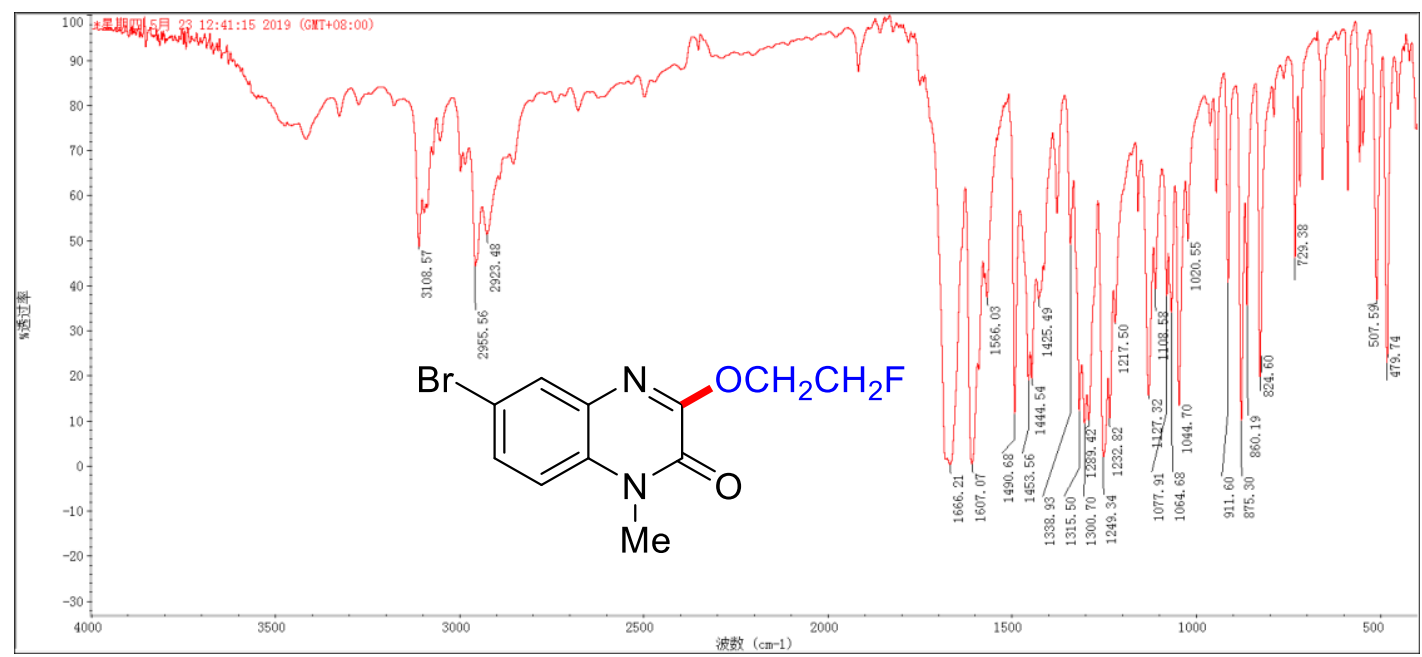

$4 e$

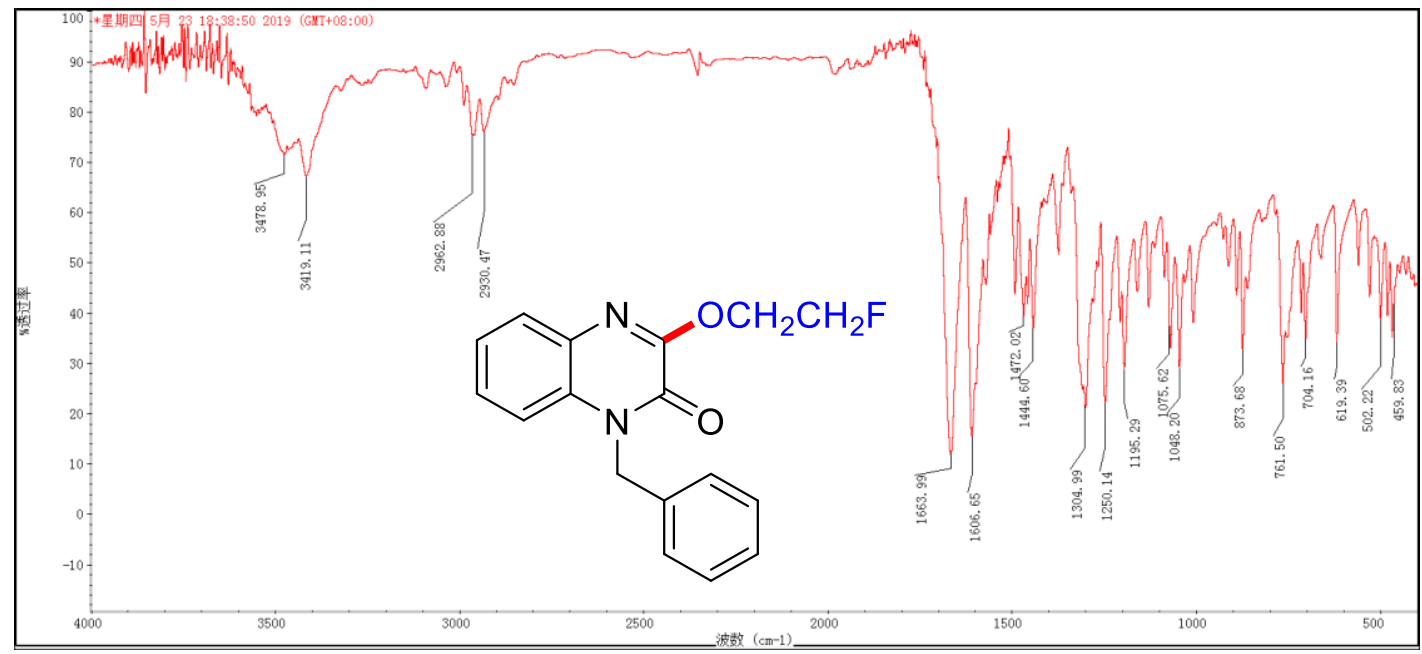

4f

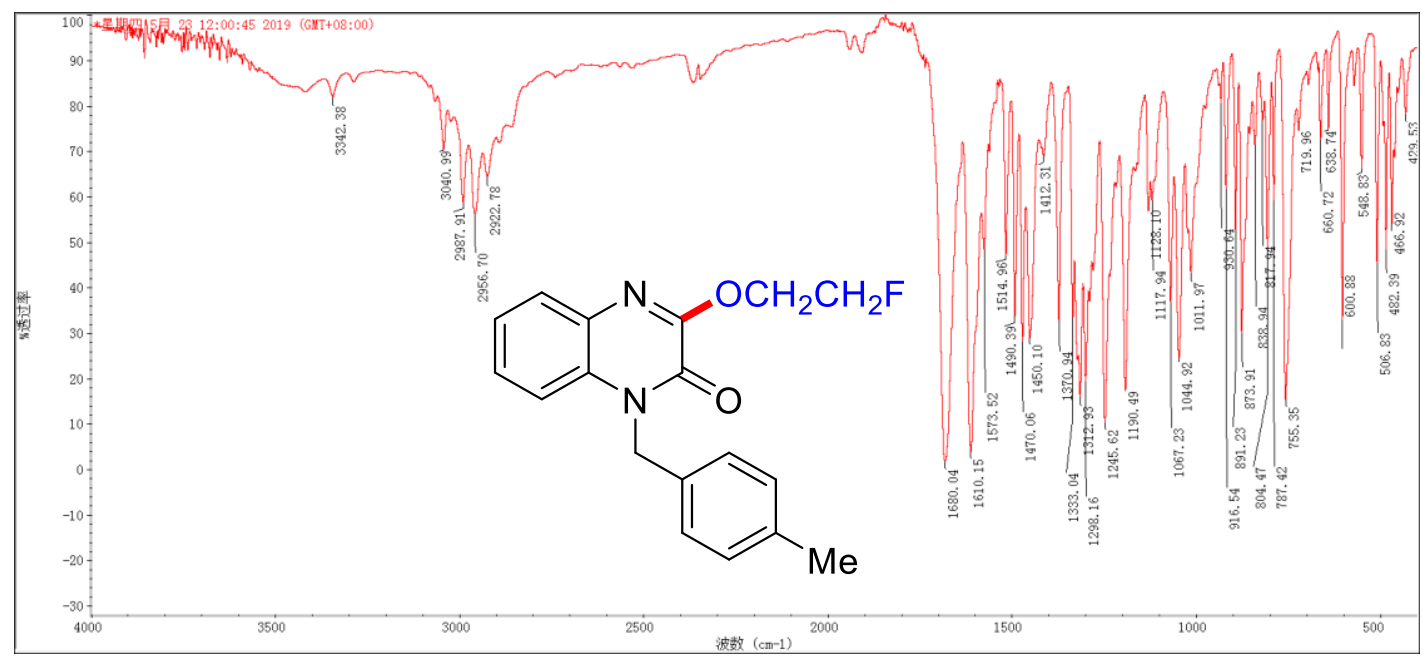


$5 a$

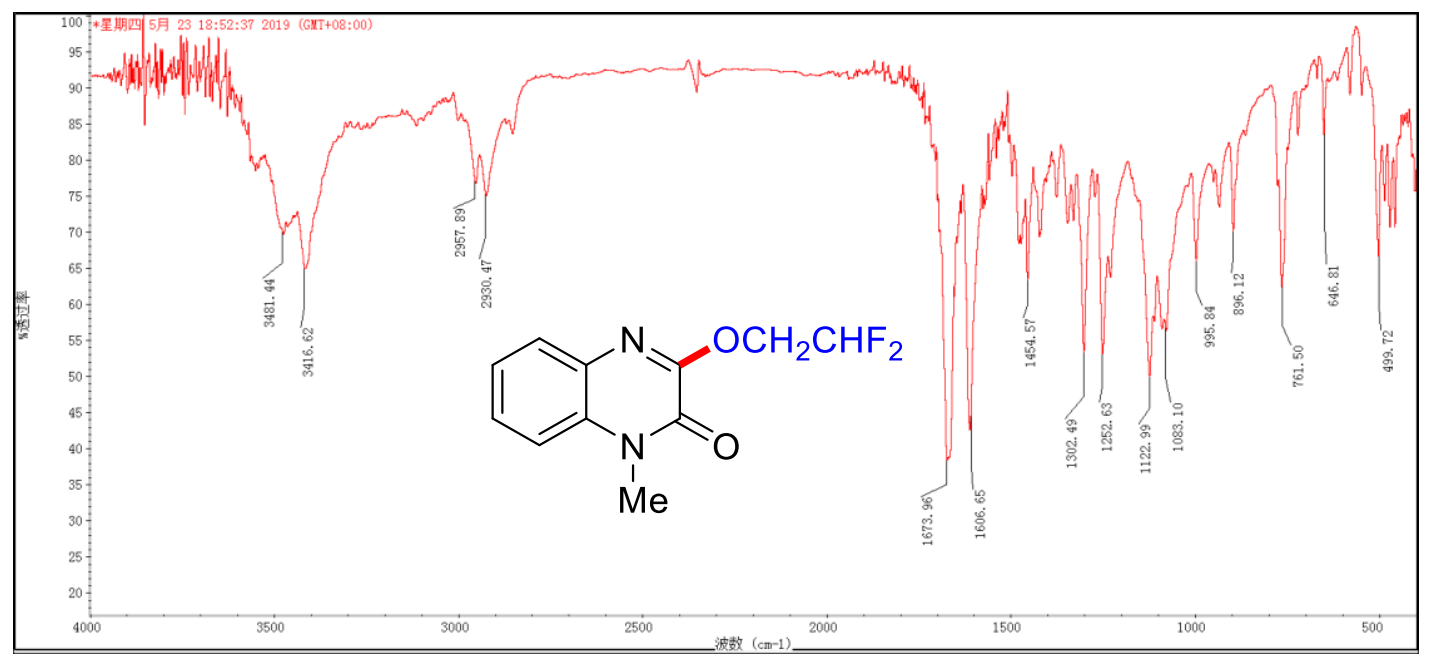

5b

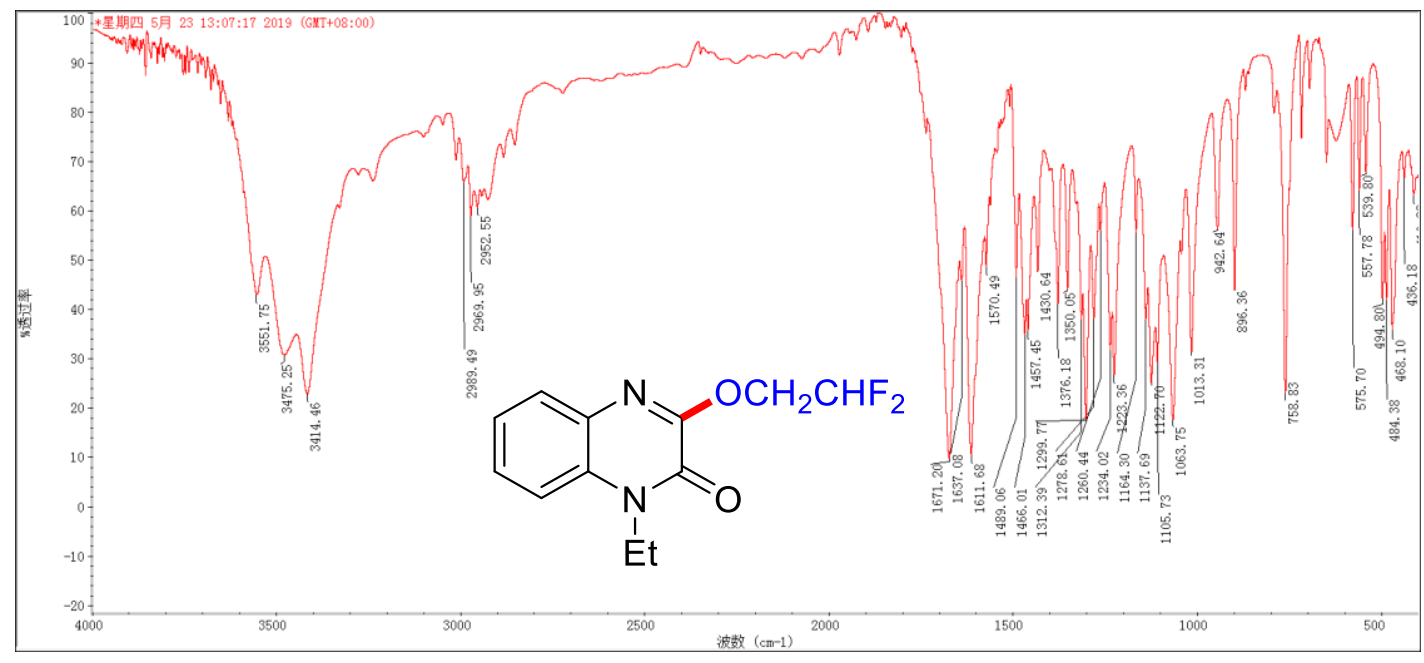

5c

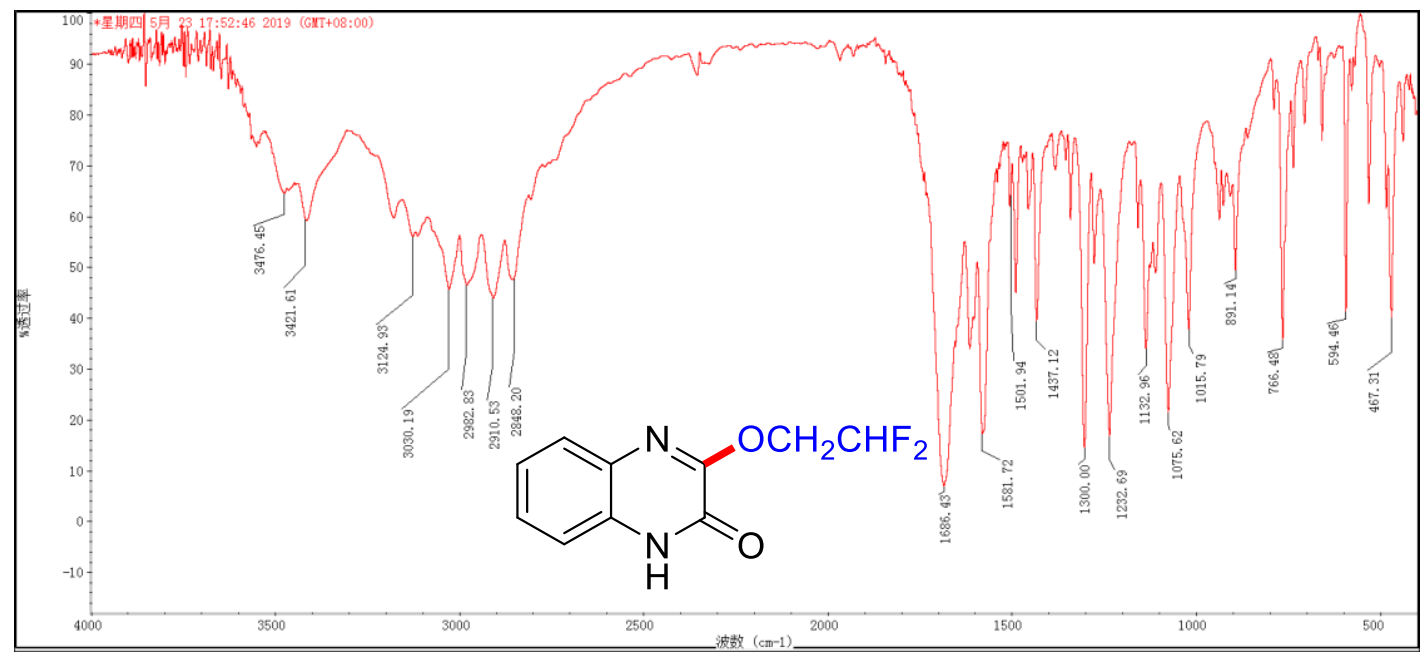


5d

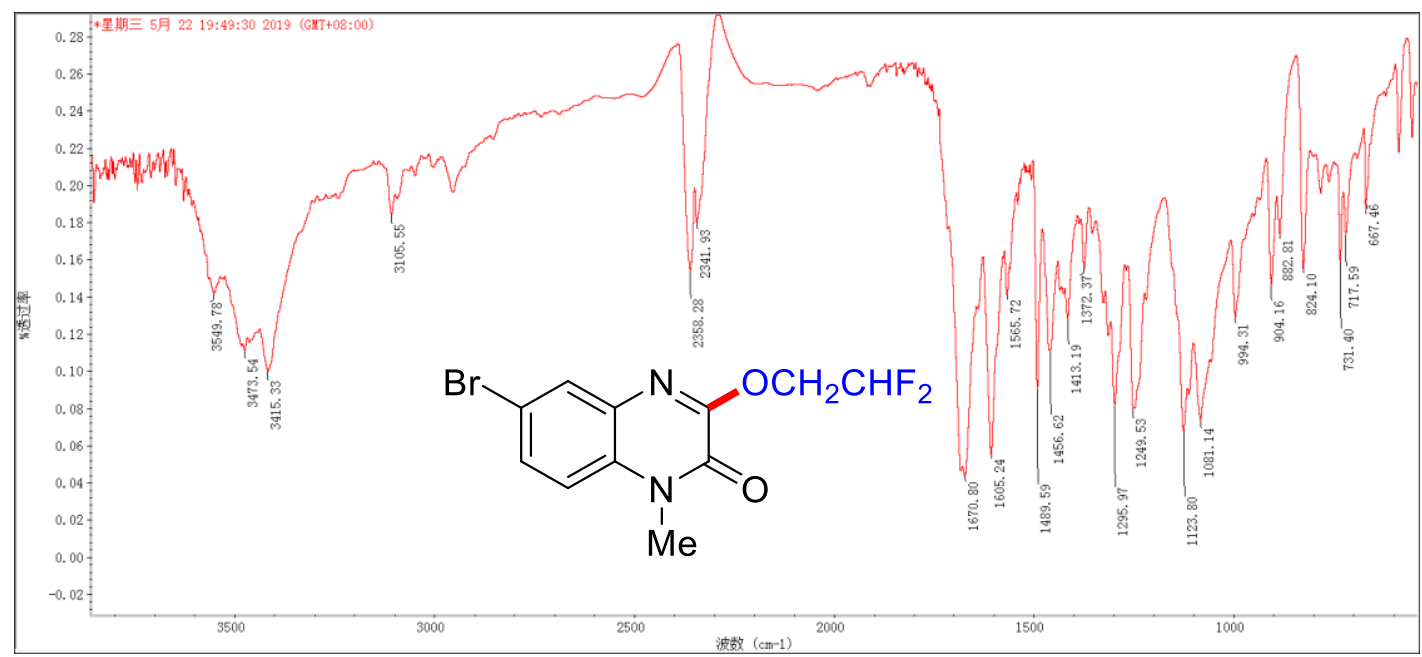

$6 a$

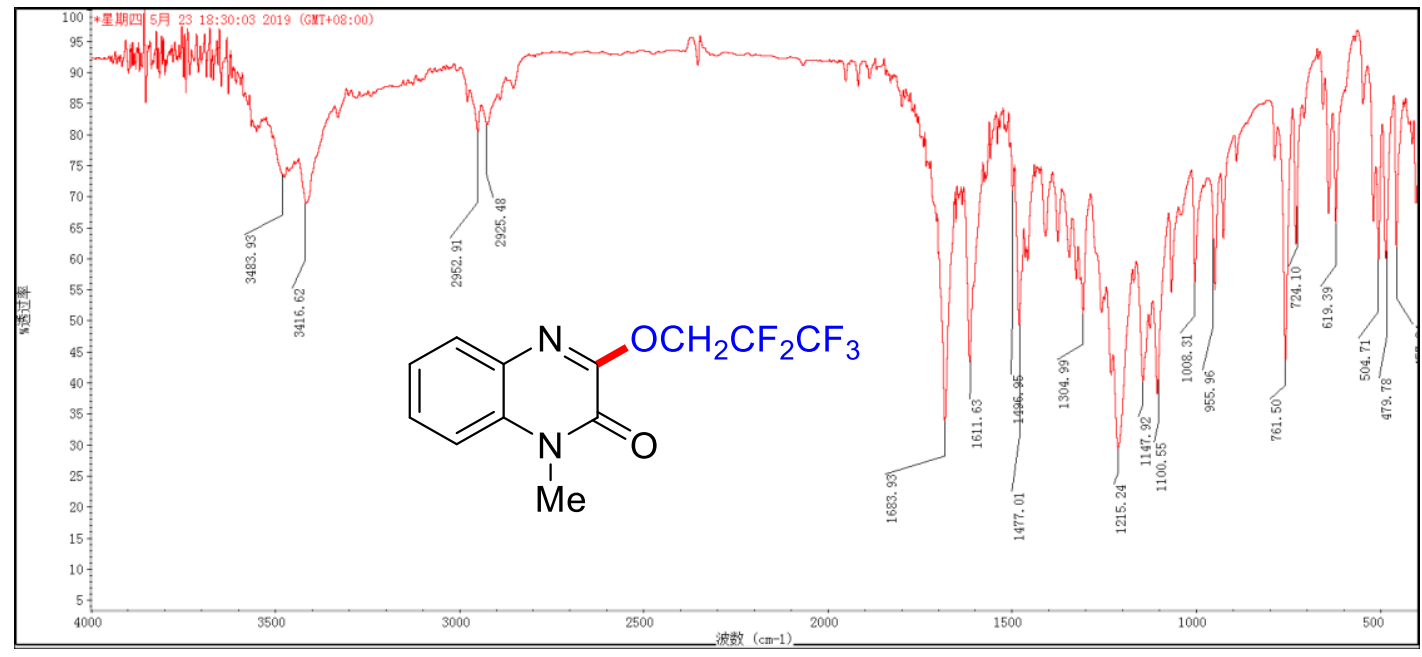

6b

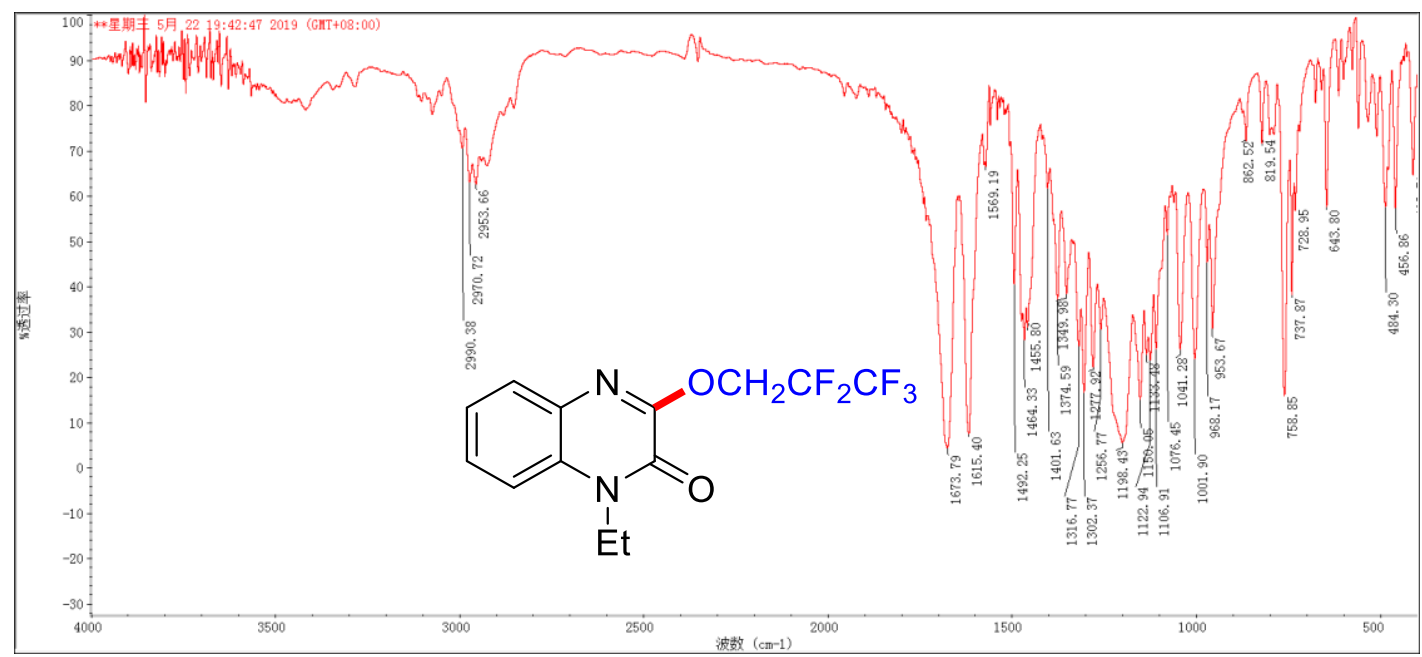


6c

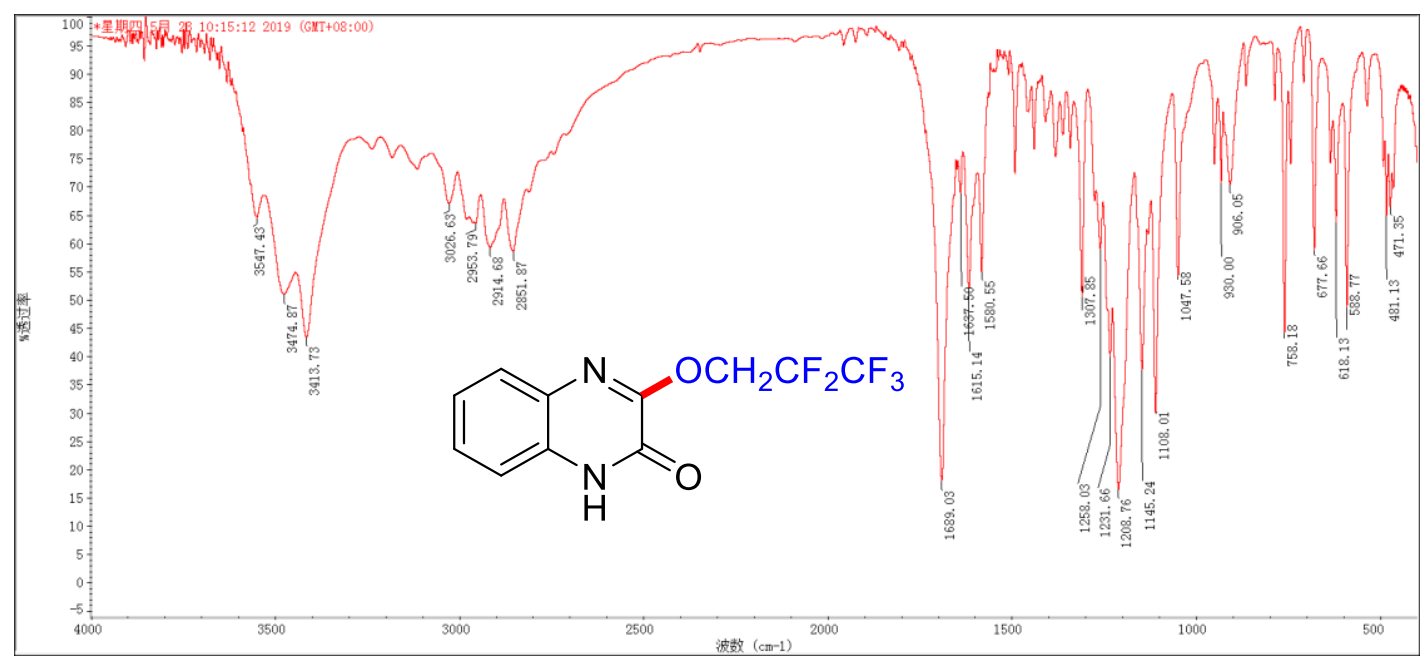

6d

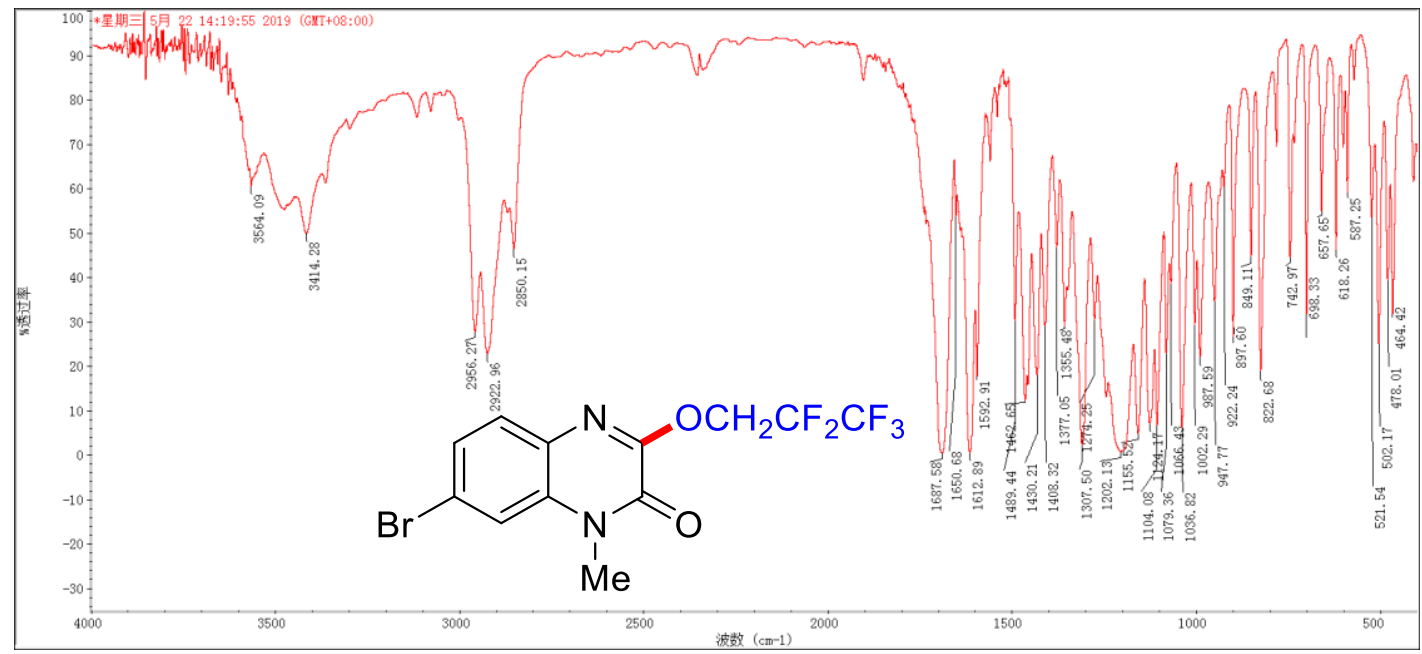

$6 e$

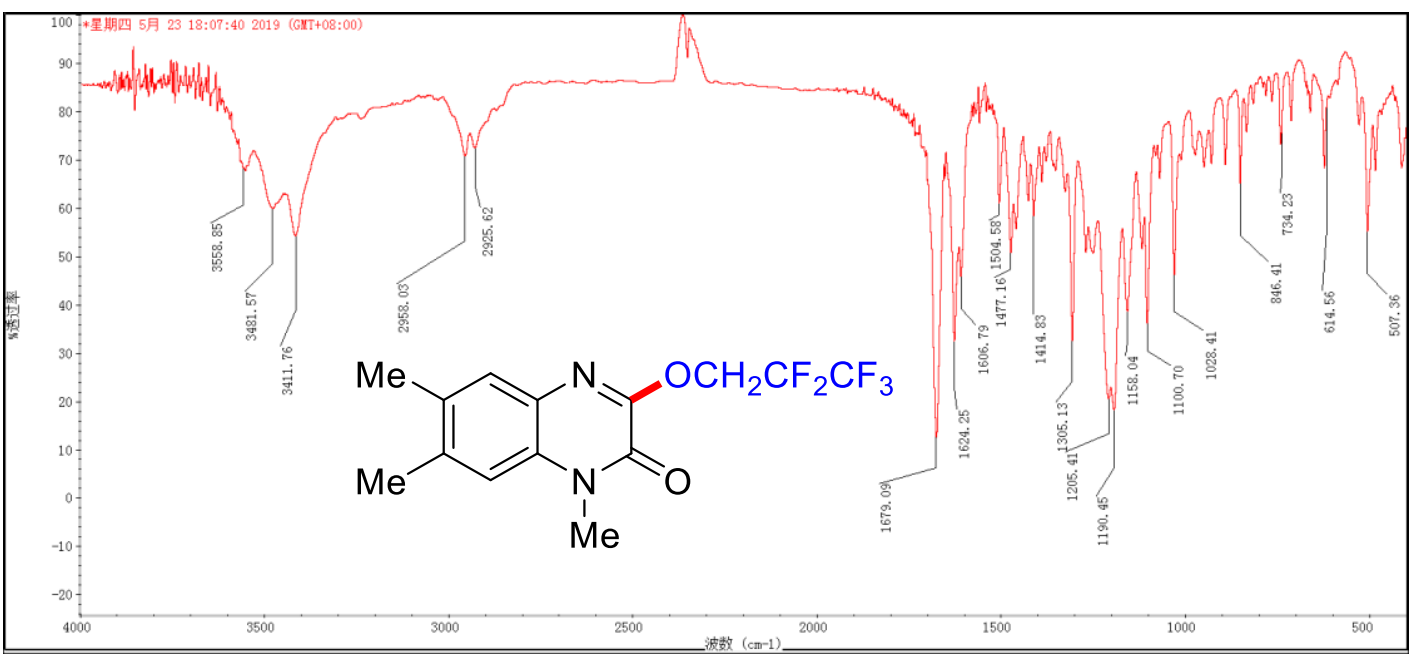


6f

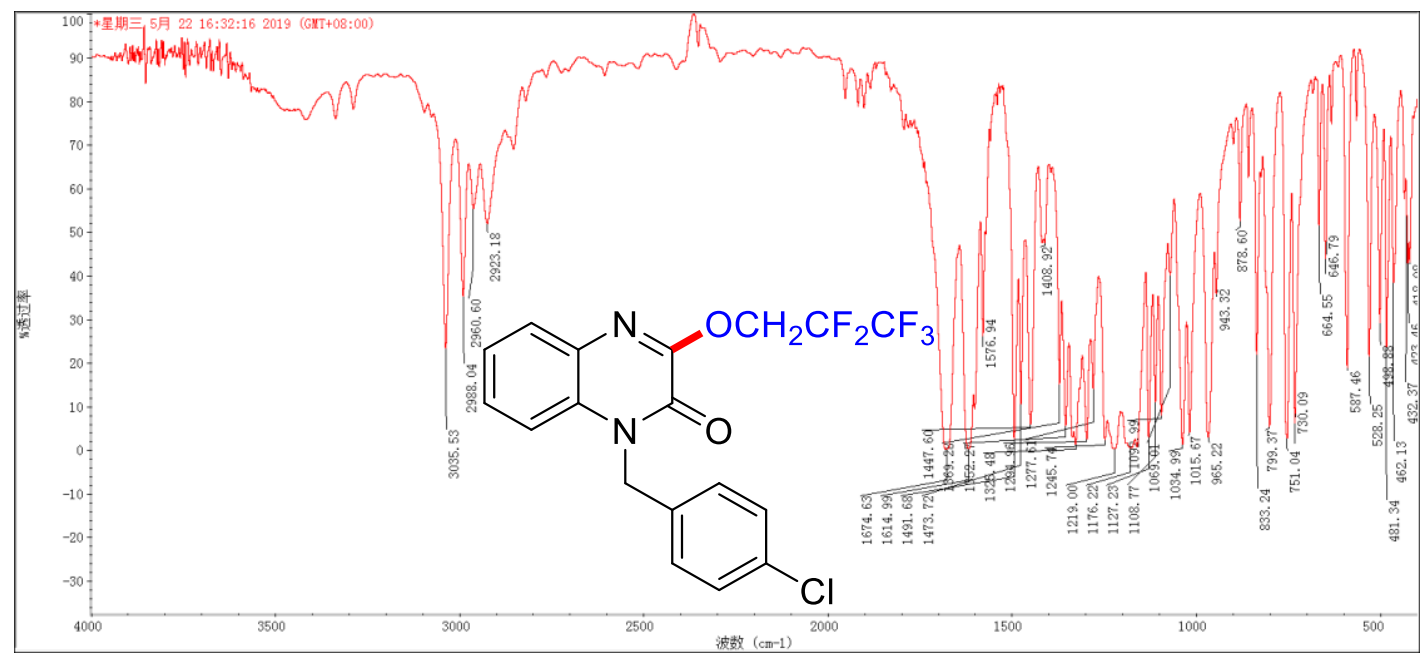

$6 \mathrm{~g}$

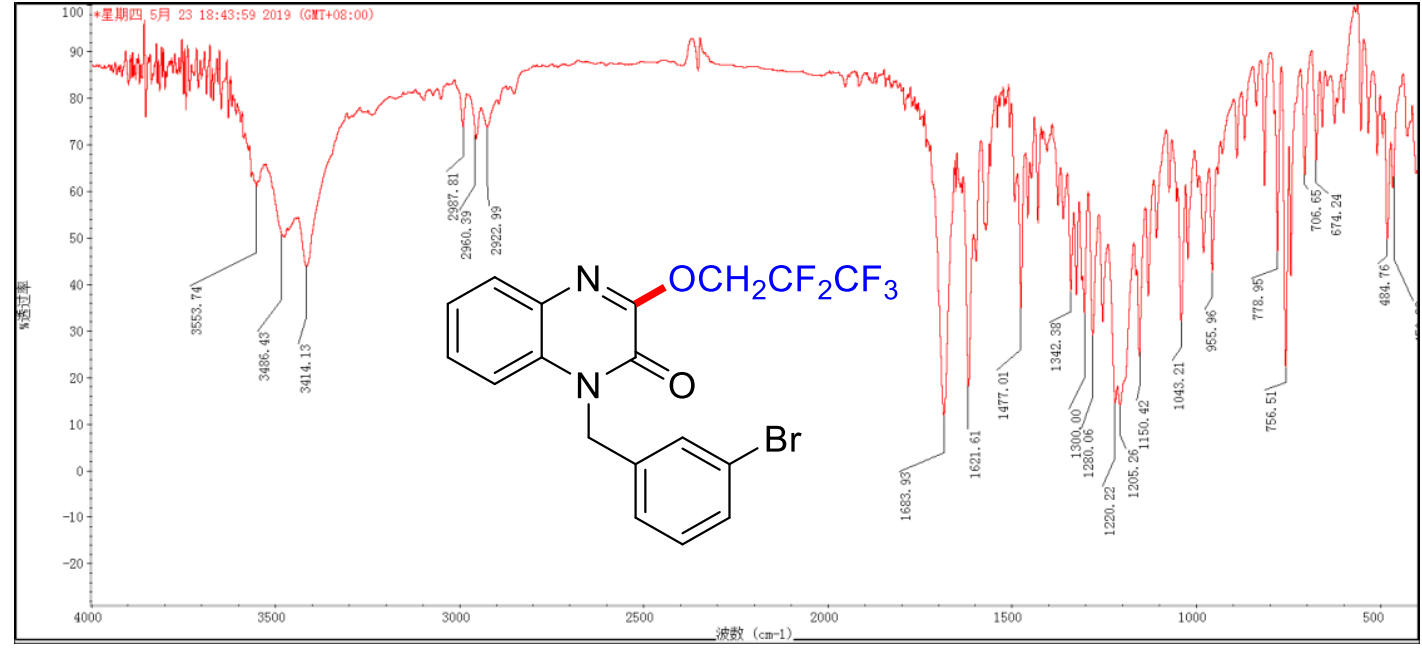

$7 a$

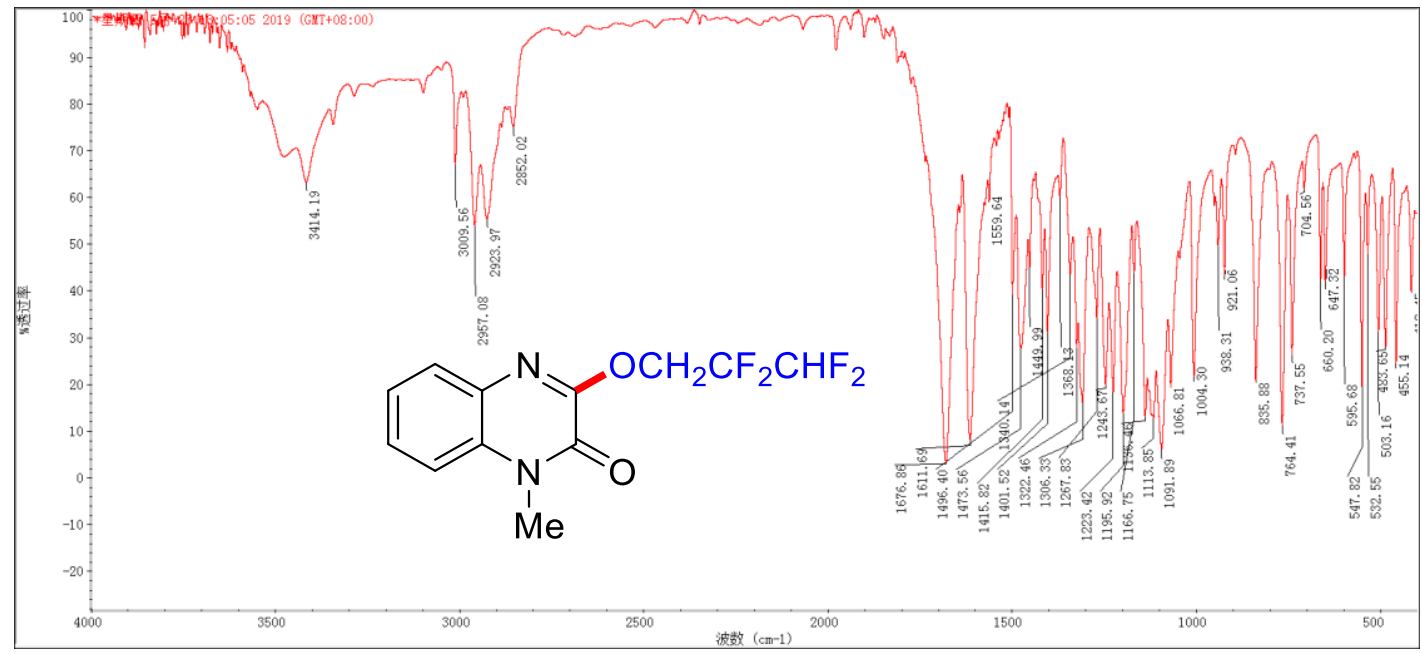


7b

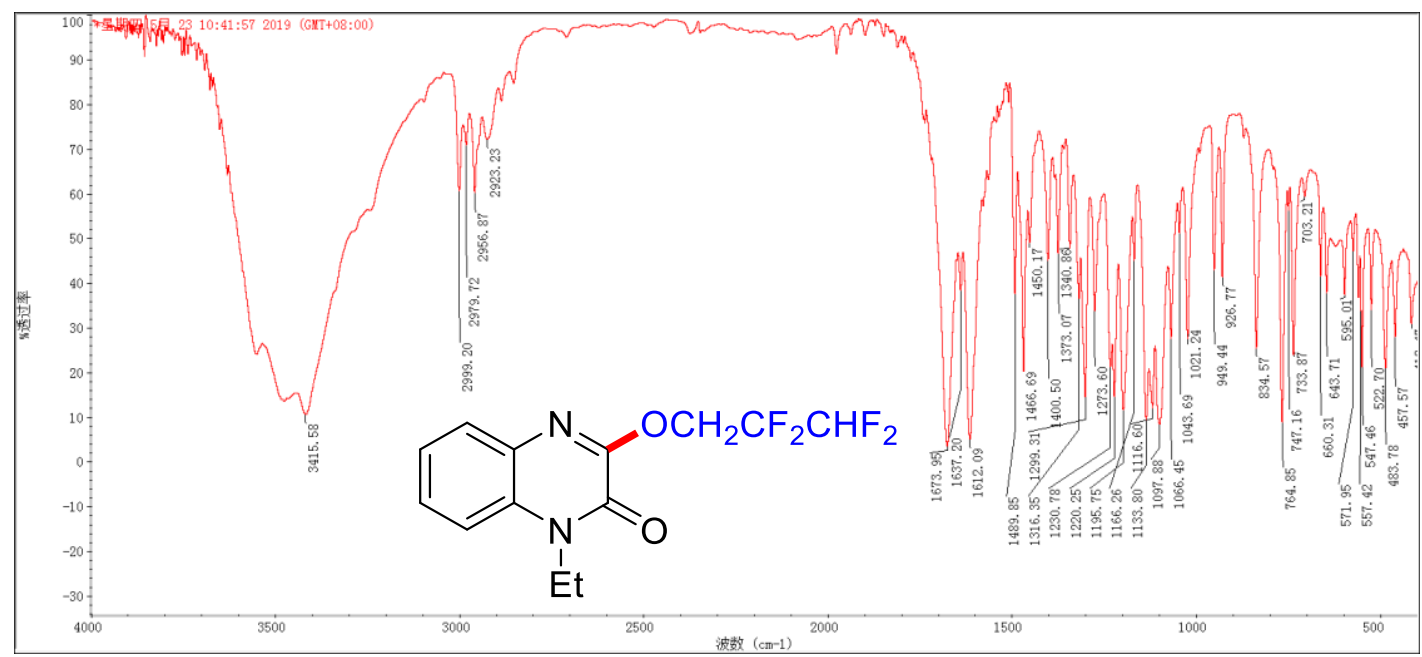

7c

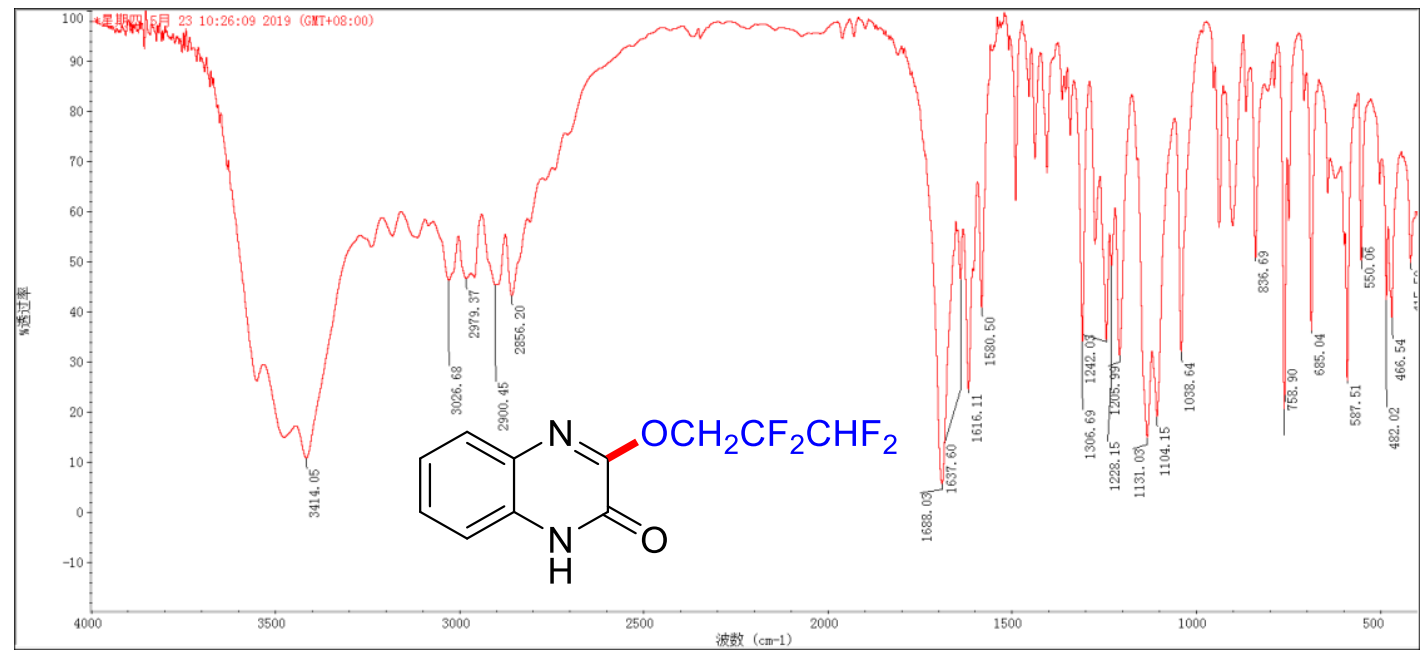

7d

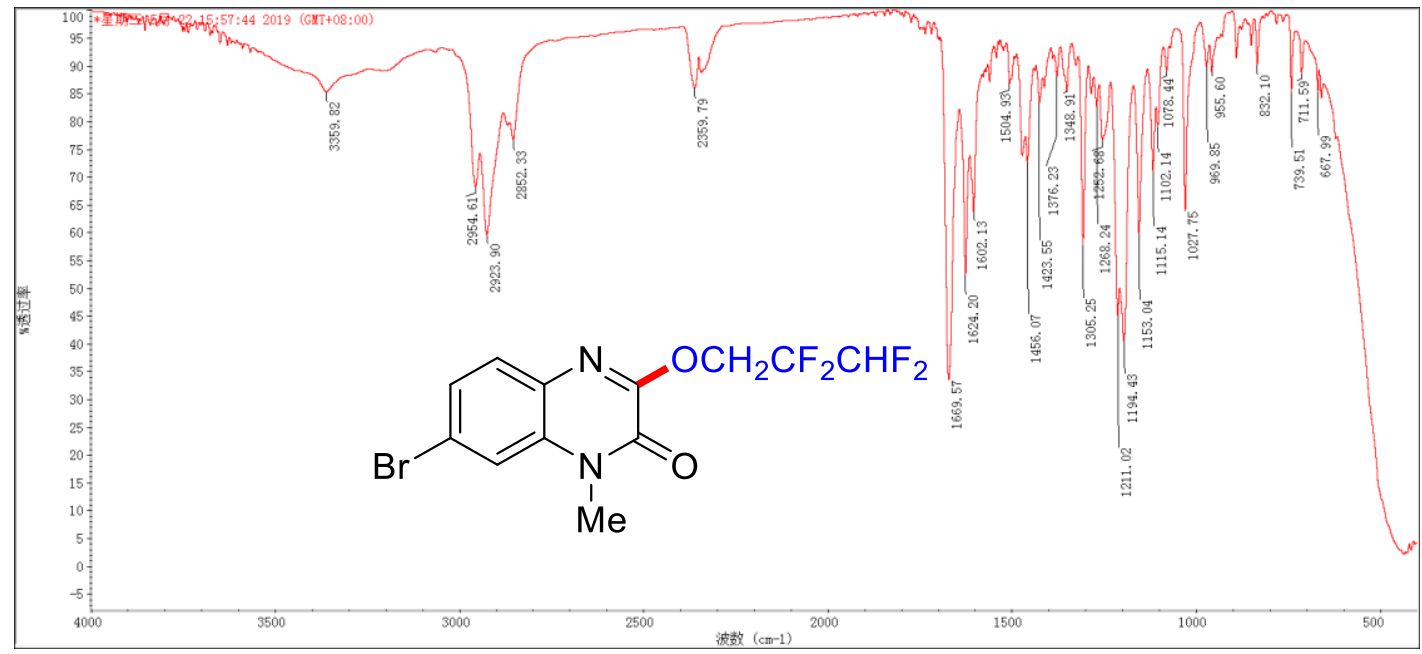


$7 e$

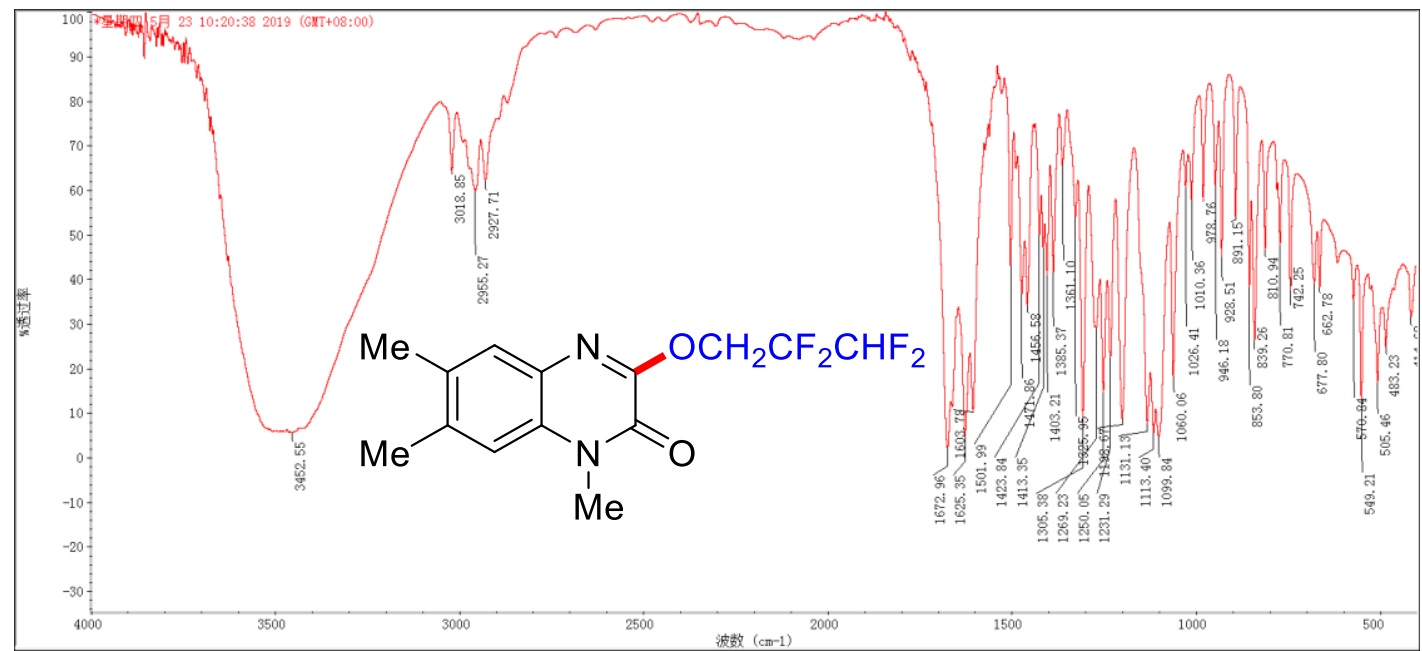

\section{$8 a$}

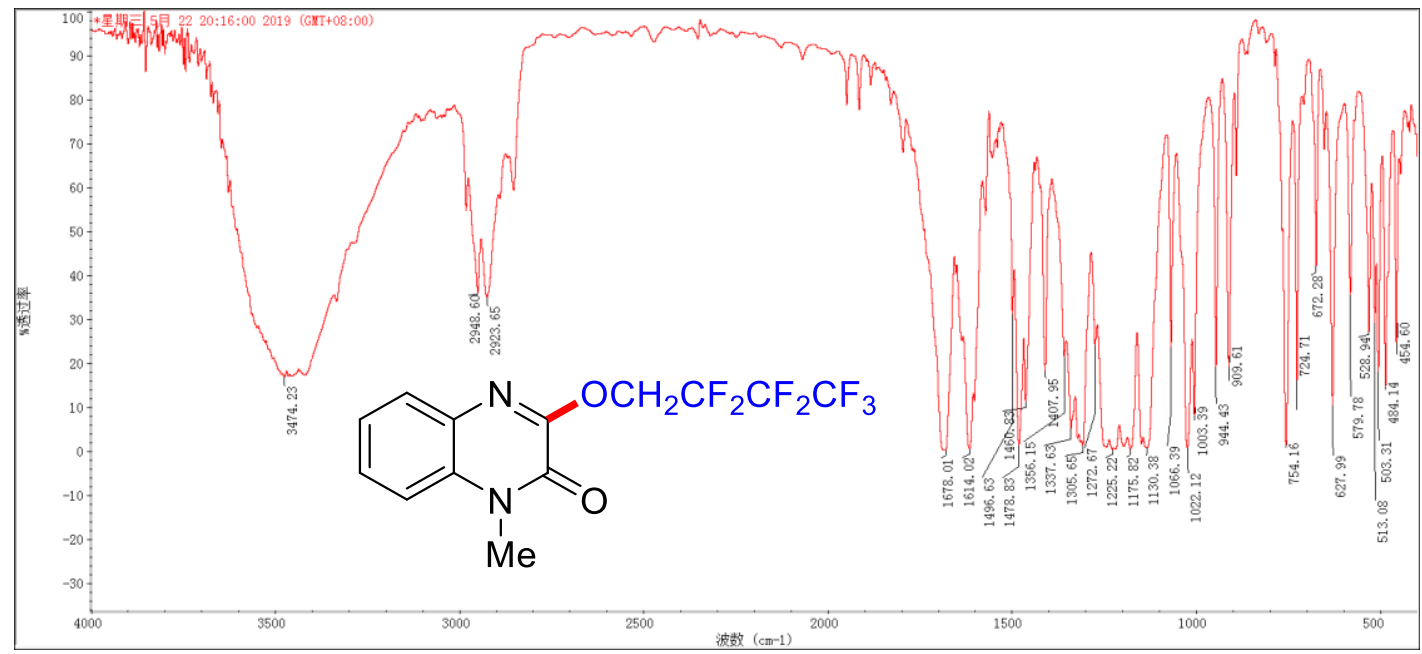

$8 b$

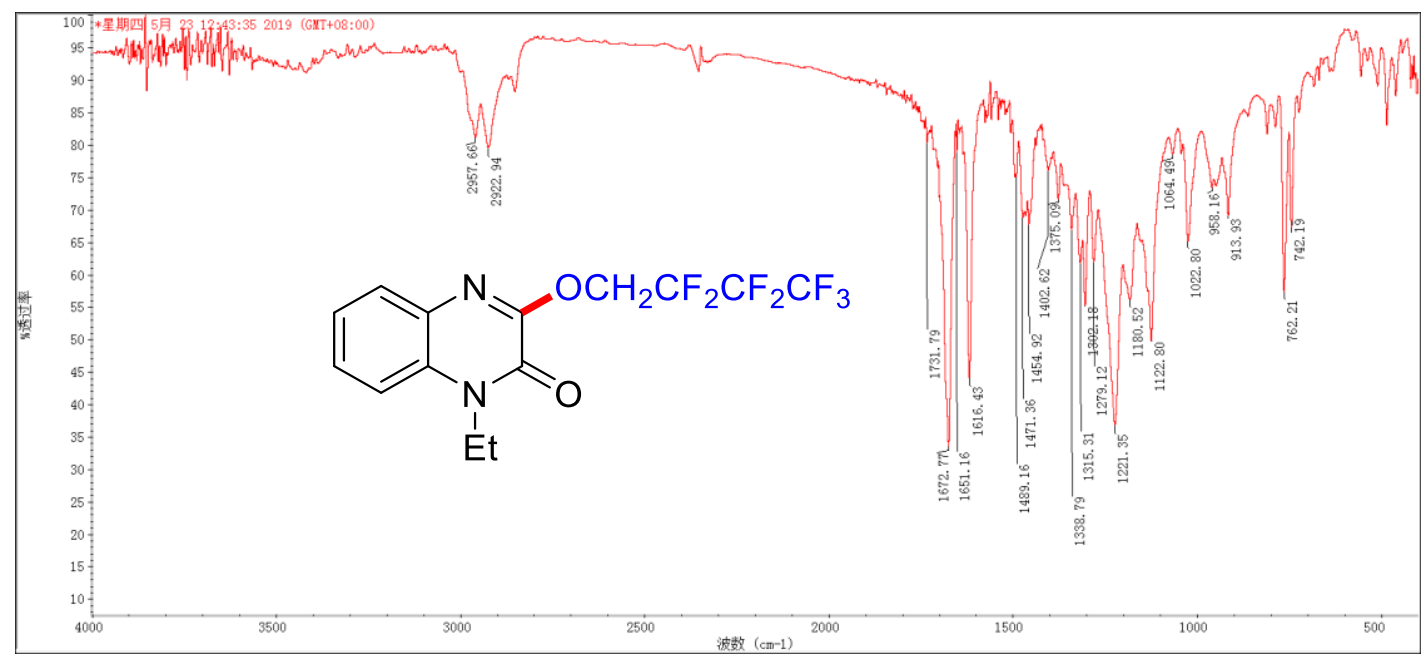


$8 c$

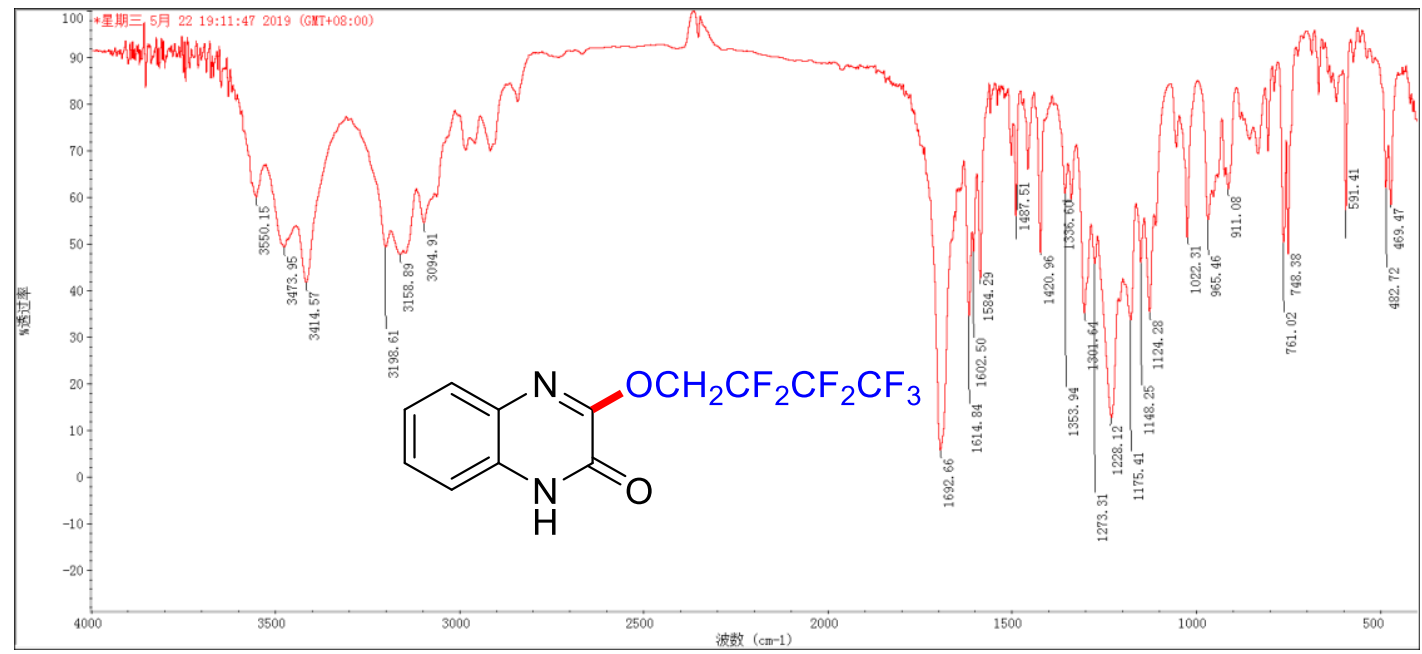

\section{8d}

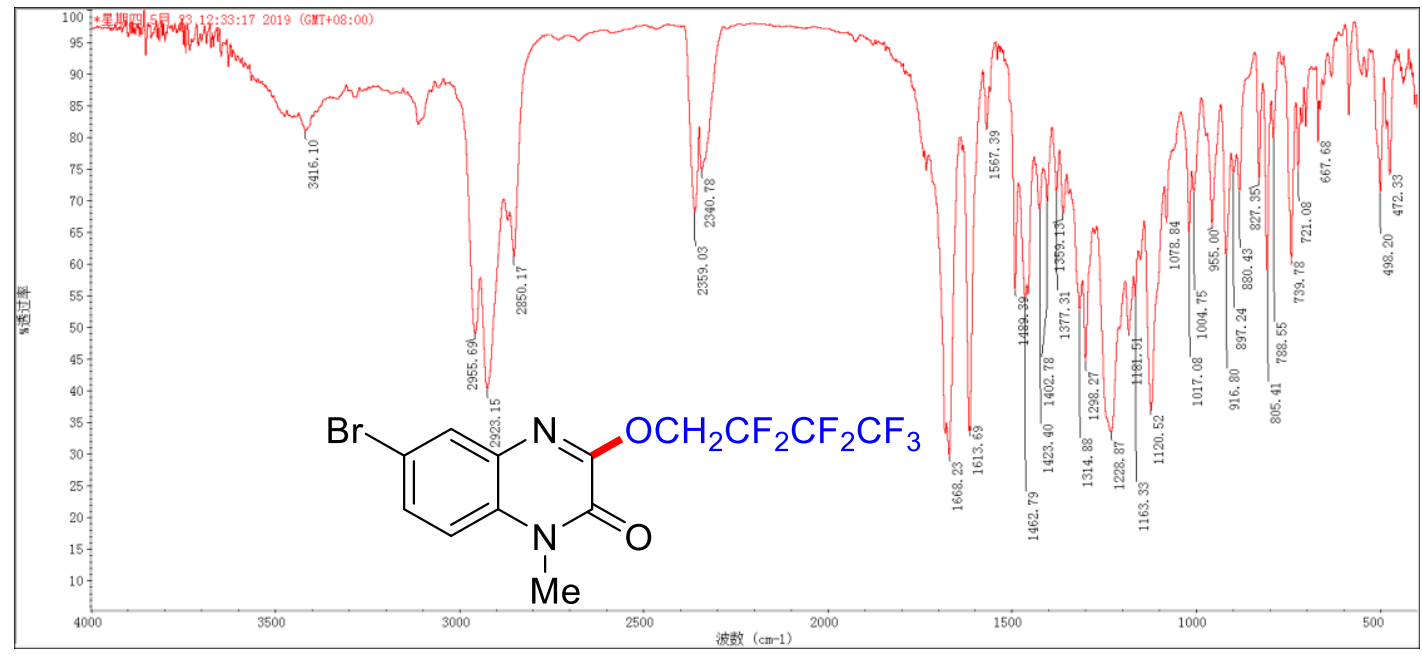

\section{$8 e$}

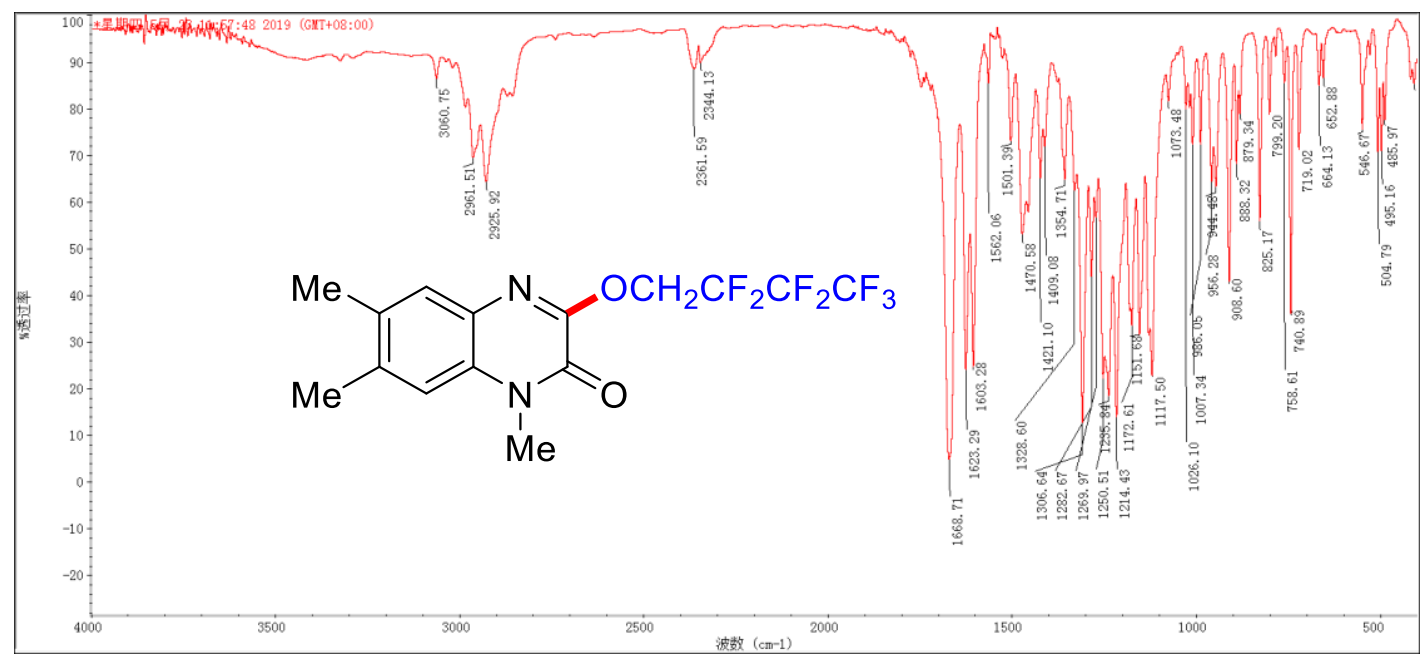


9a

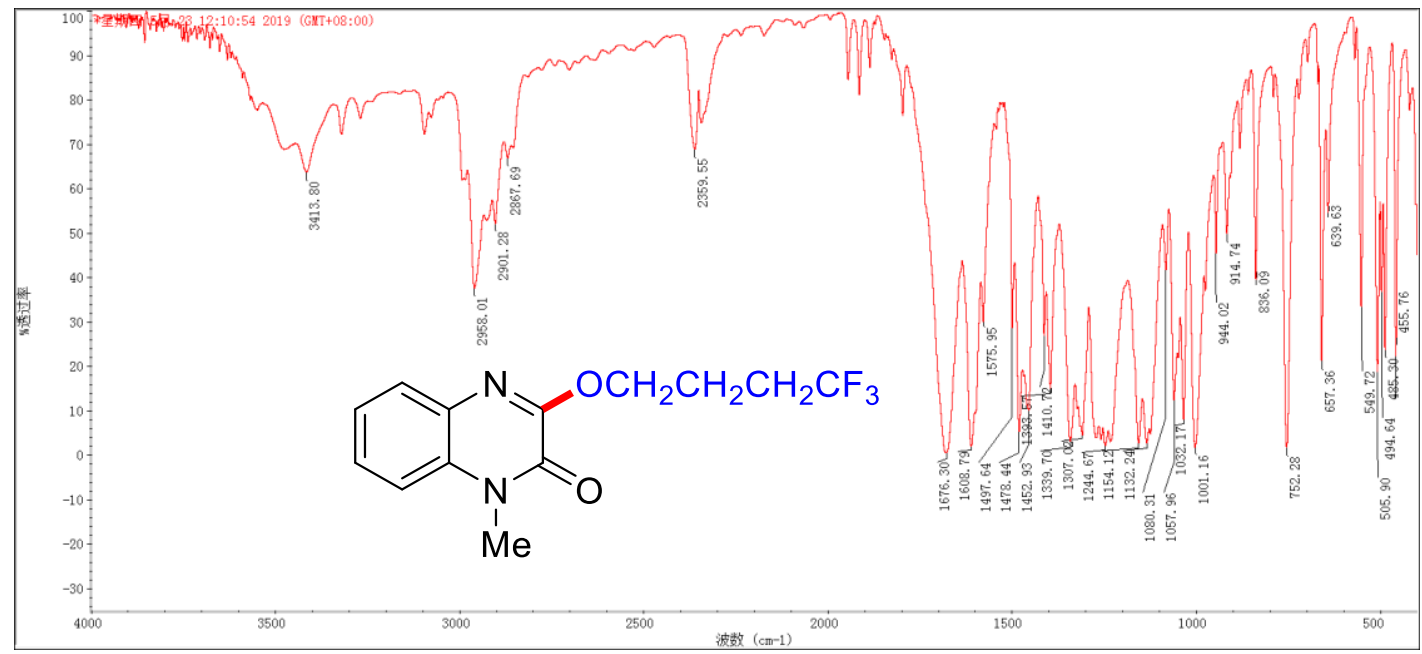

9b

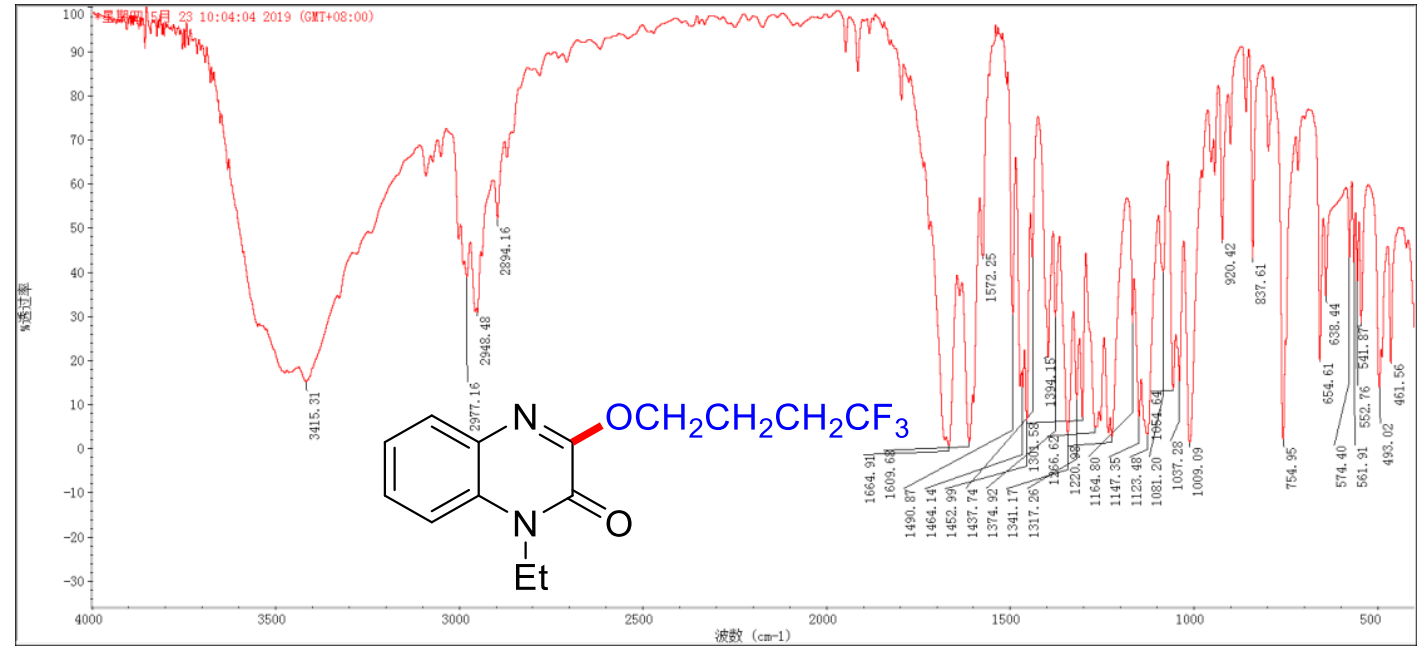

$9 c$

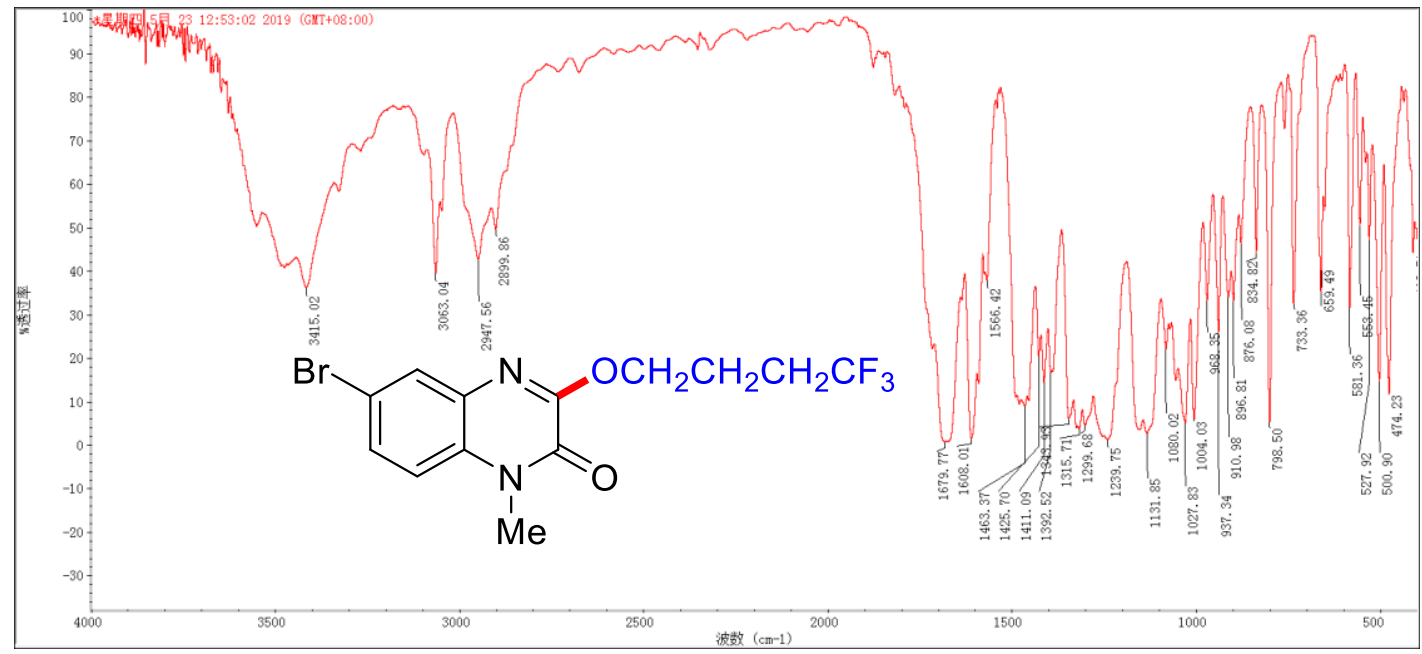




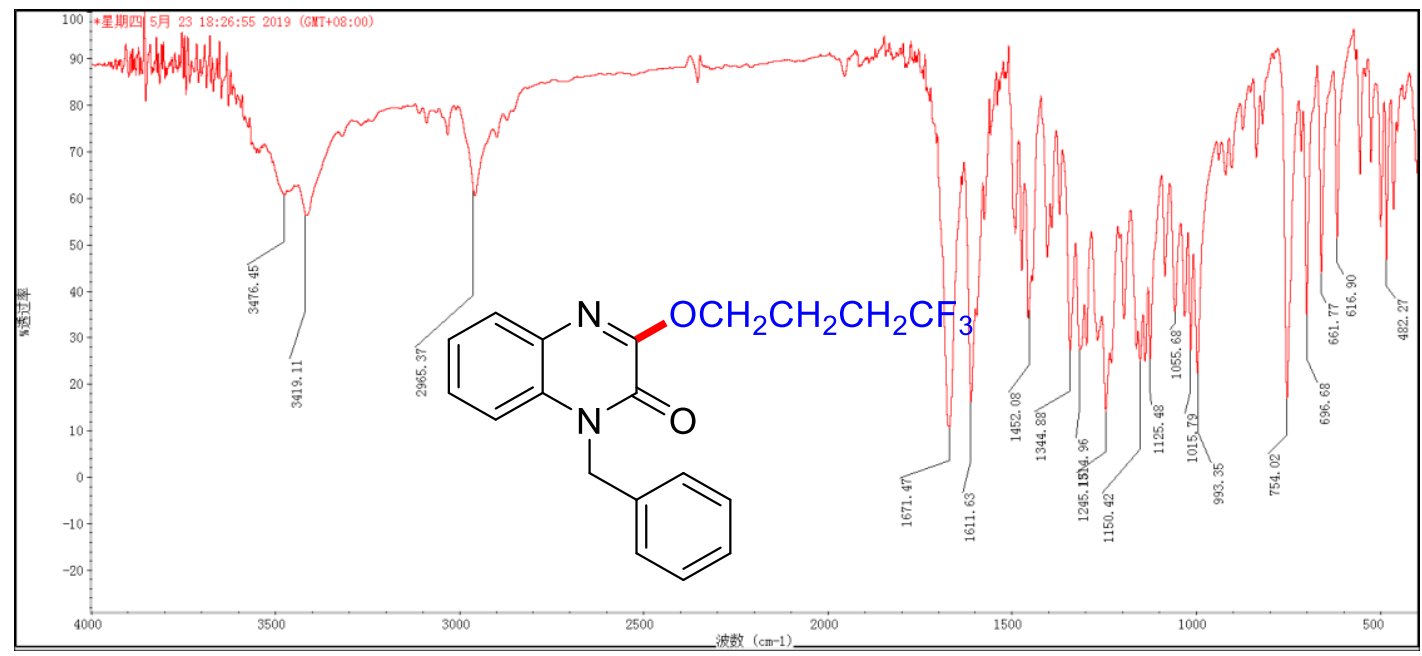

\section{9e}

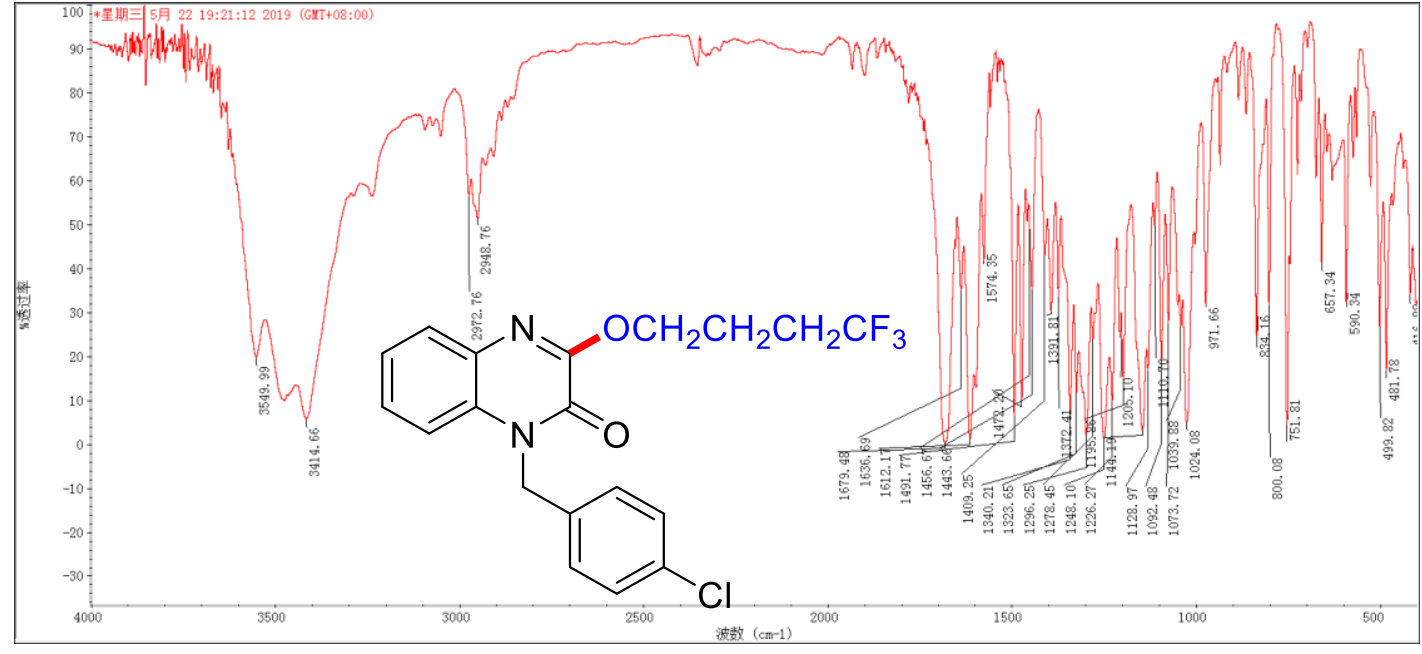

10

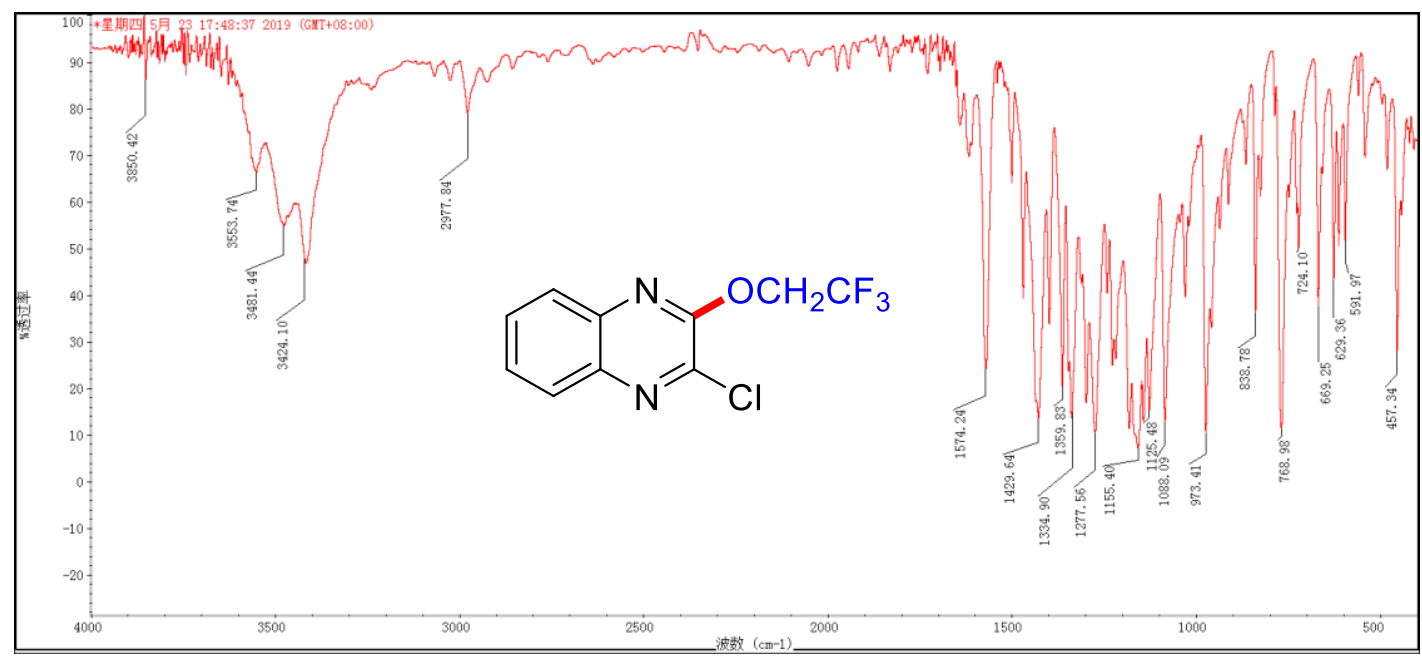


11a

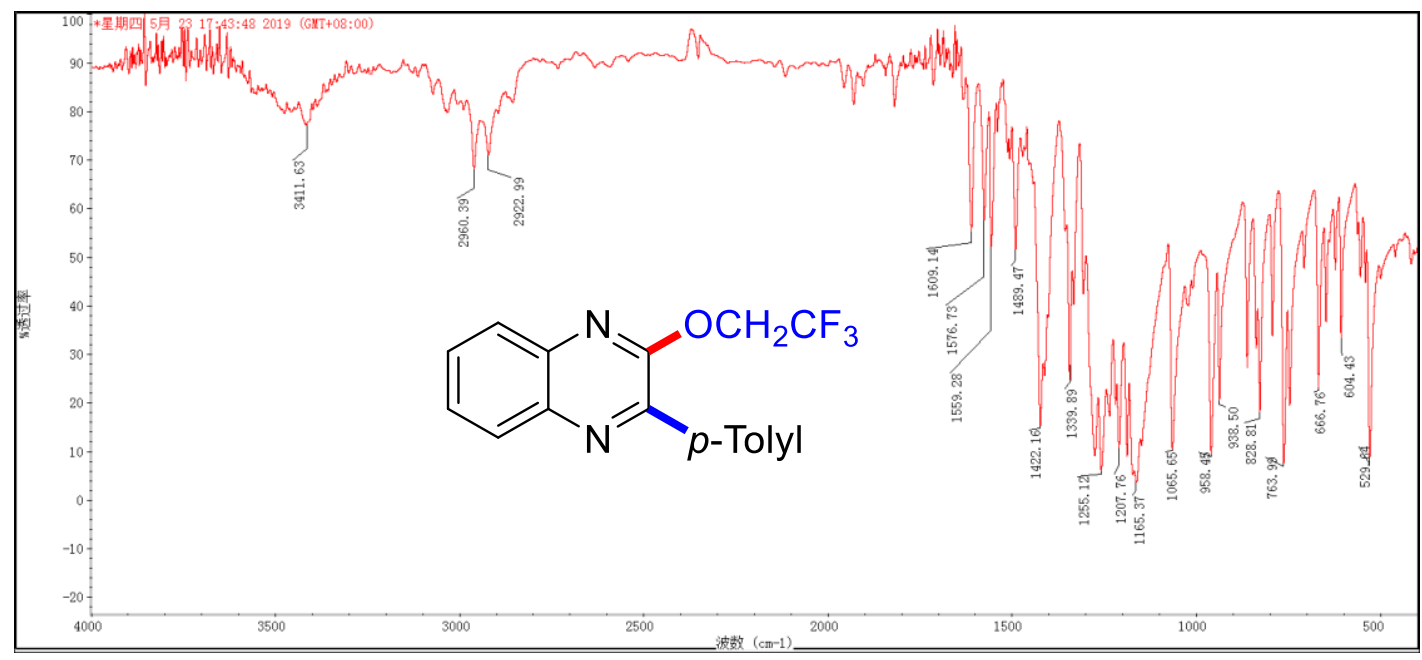

11b

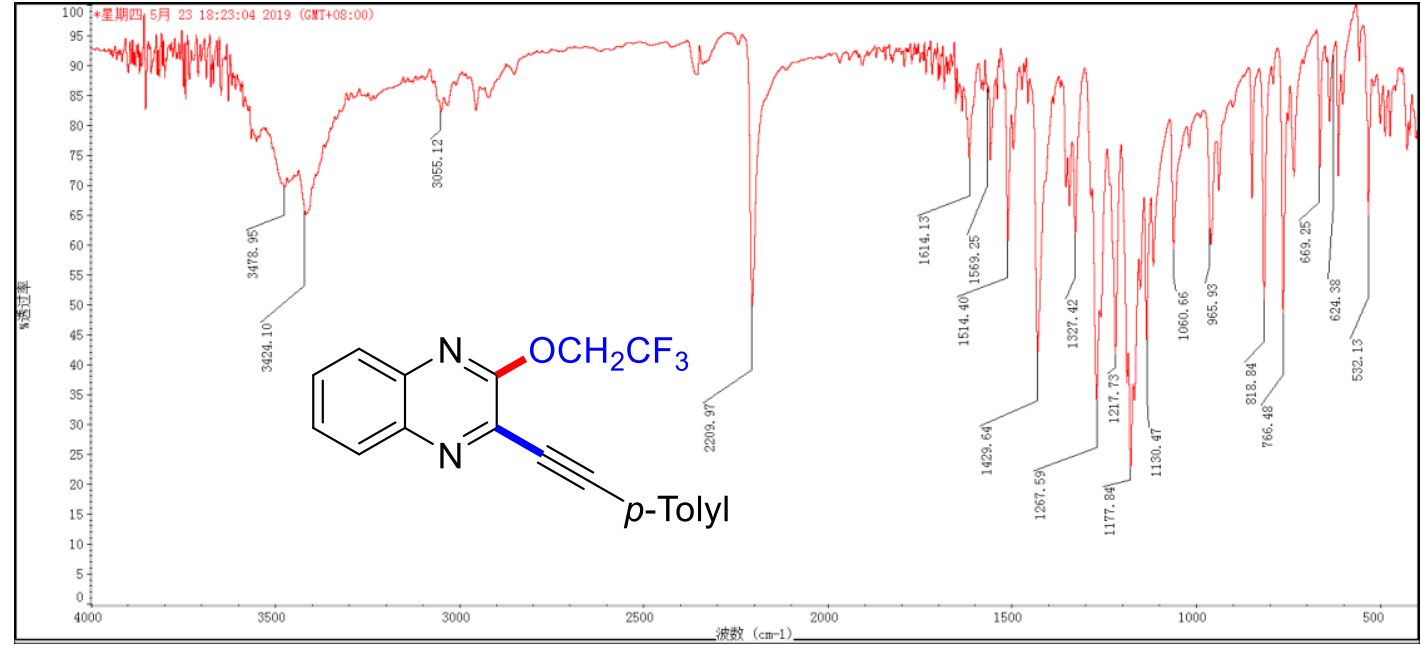

11c

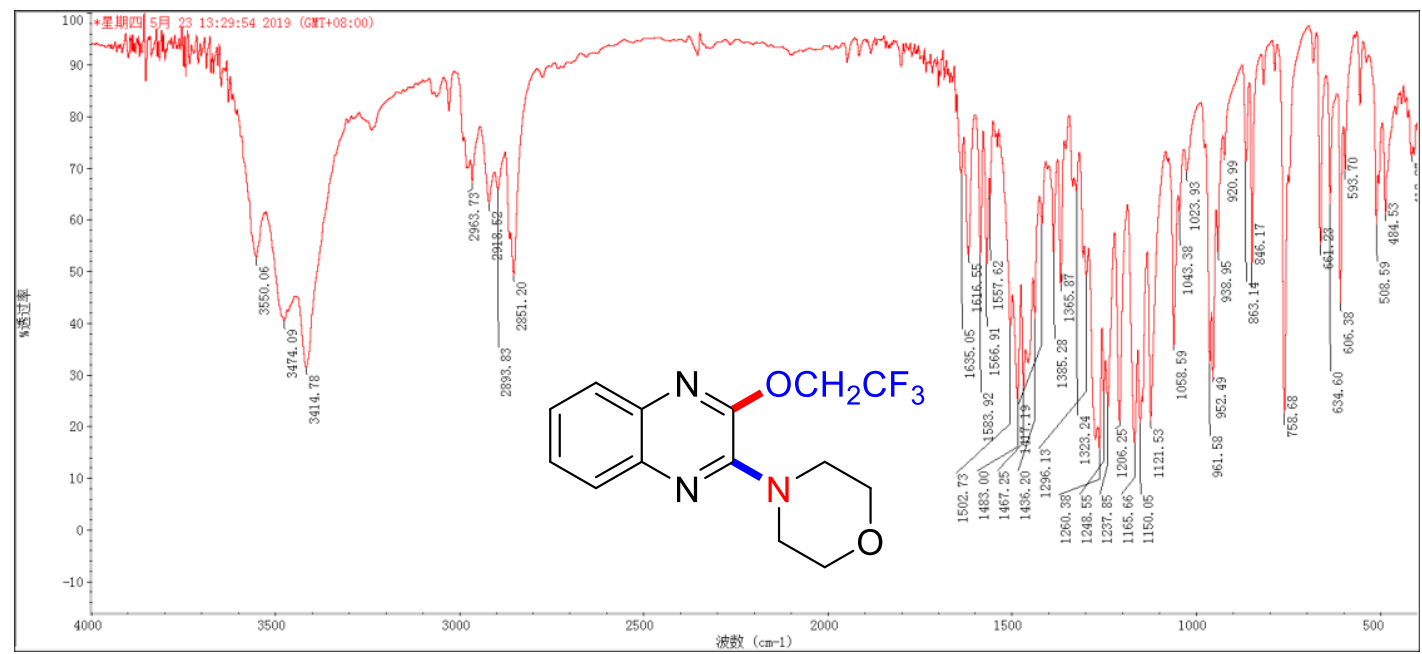


11d

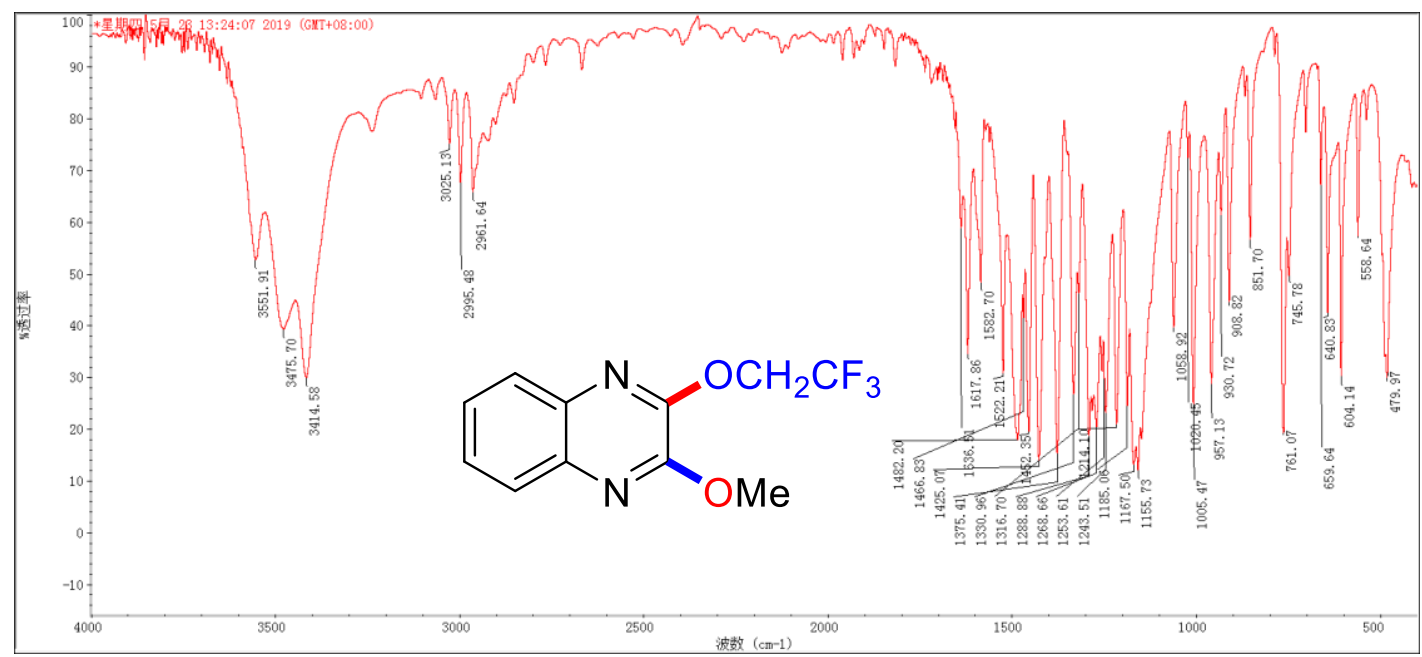

11e

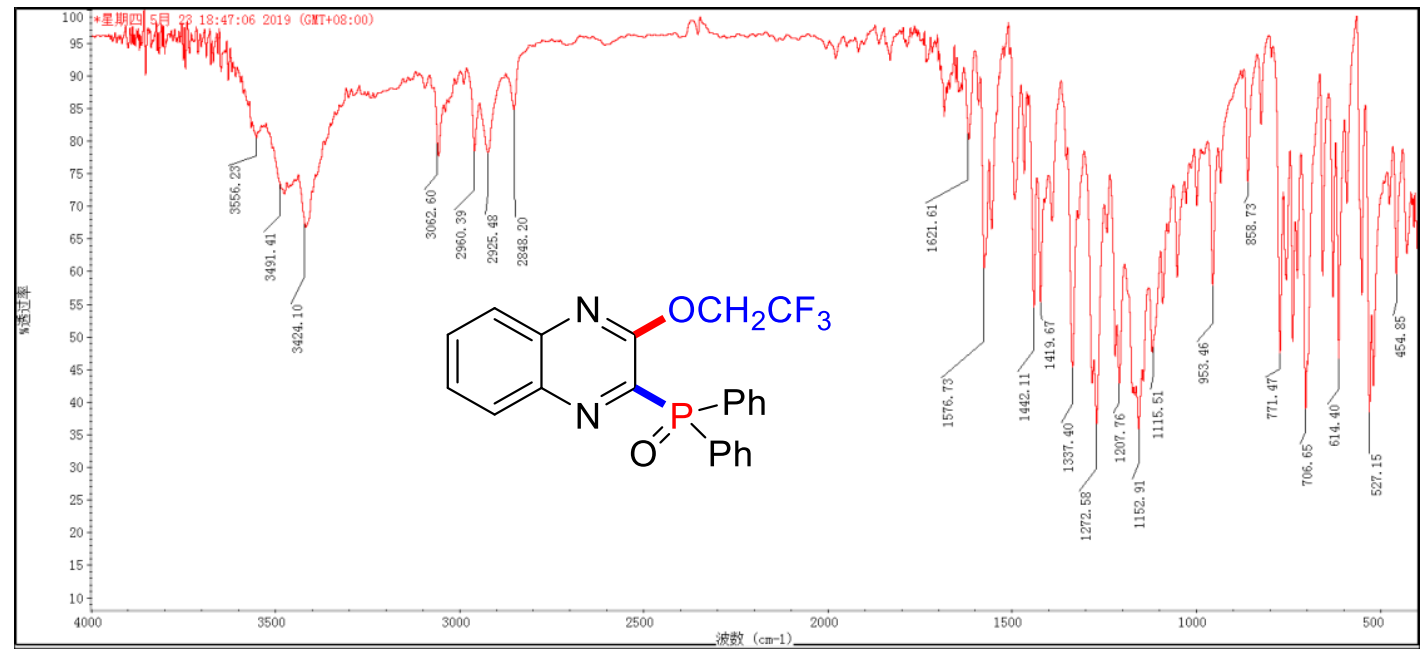

$11 f$

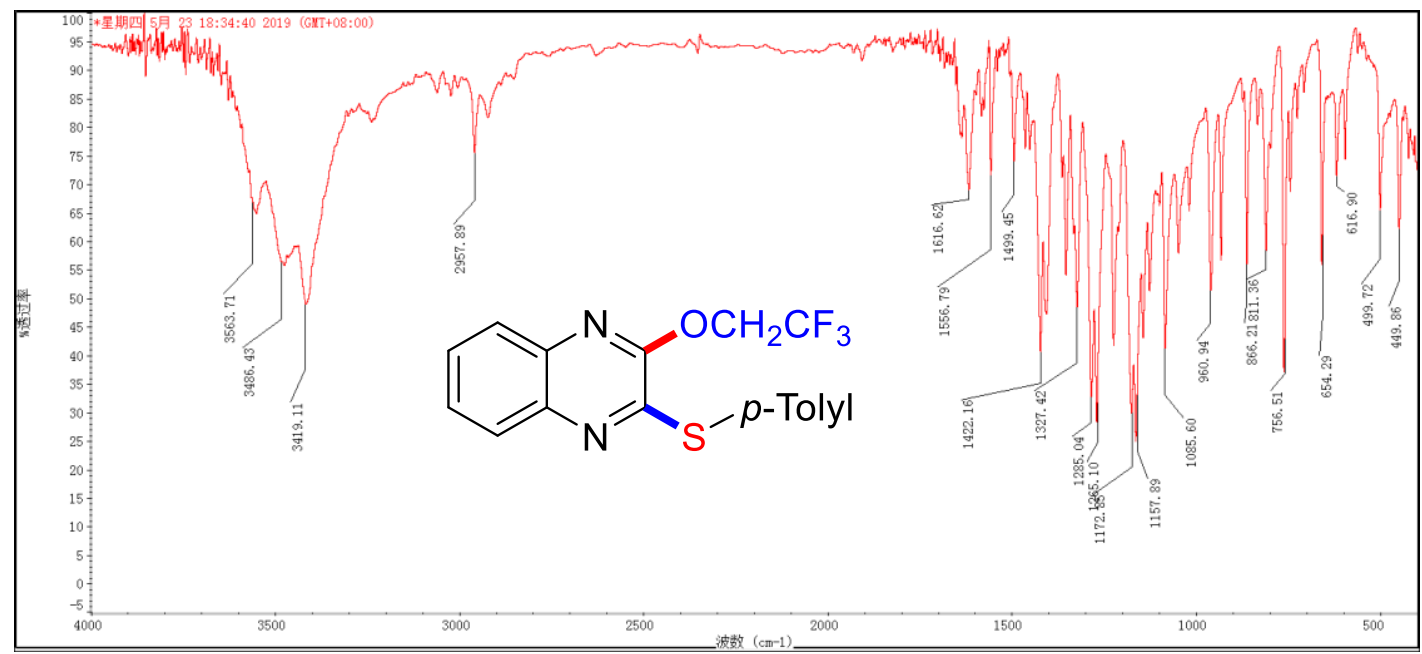




\section{$11 g$}

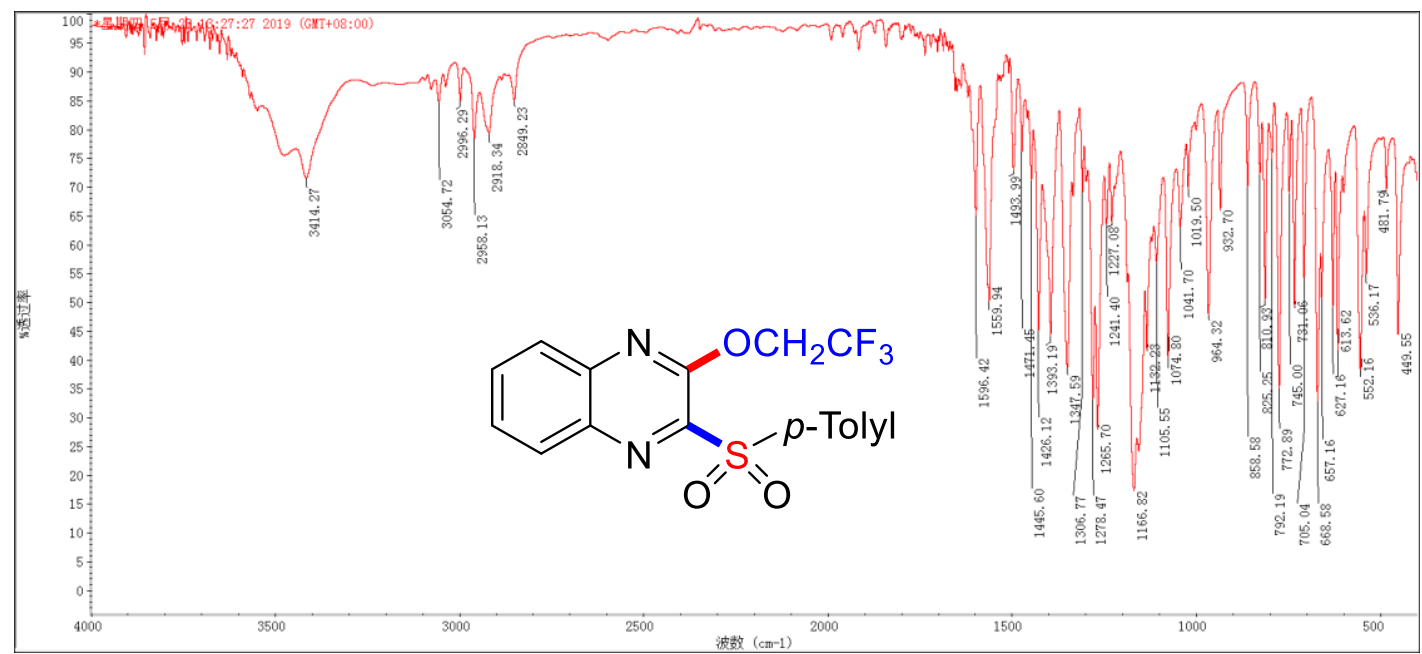

\section{$11 \mathrm{~h}$}

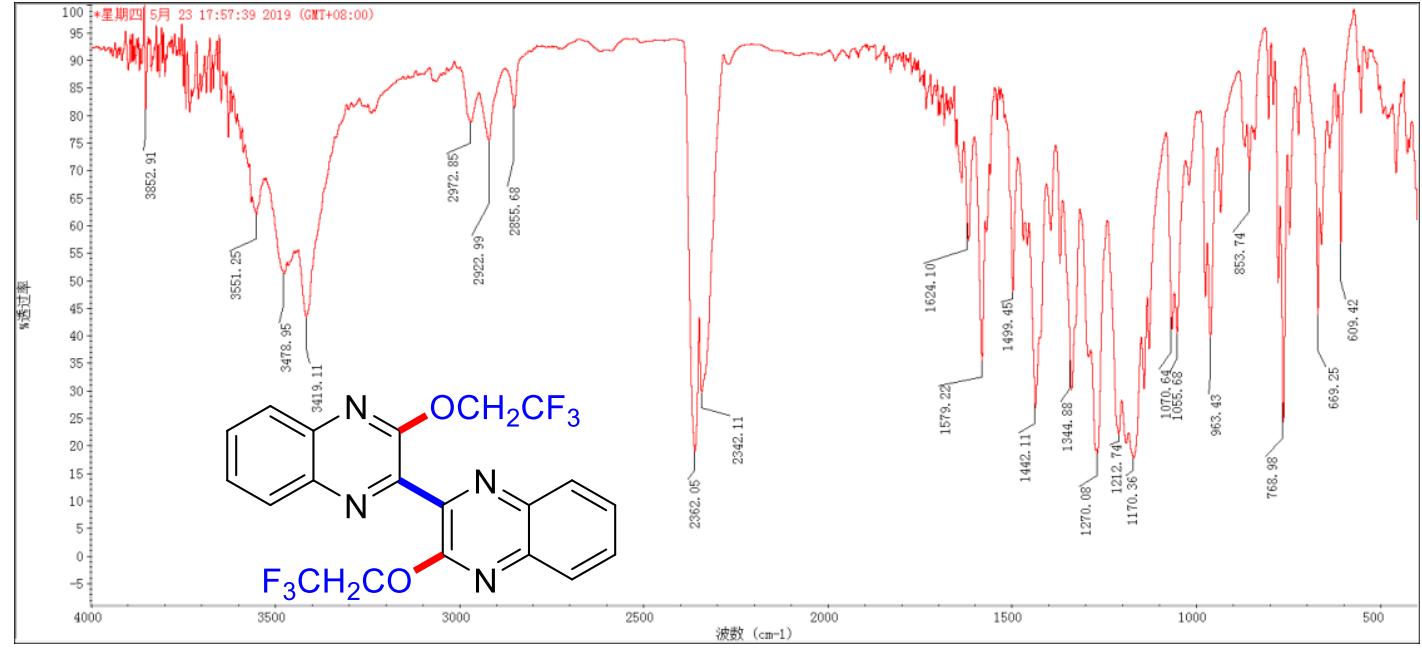

12a

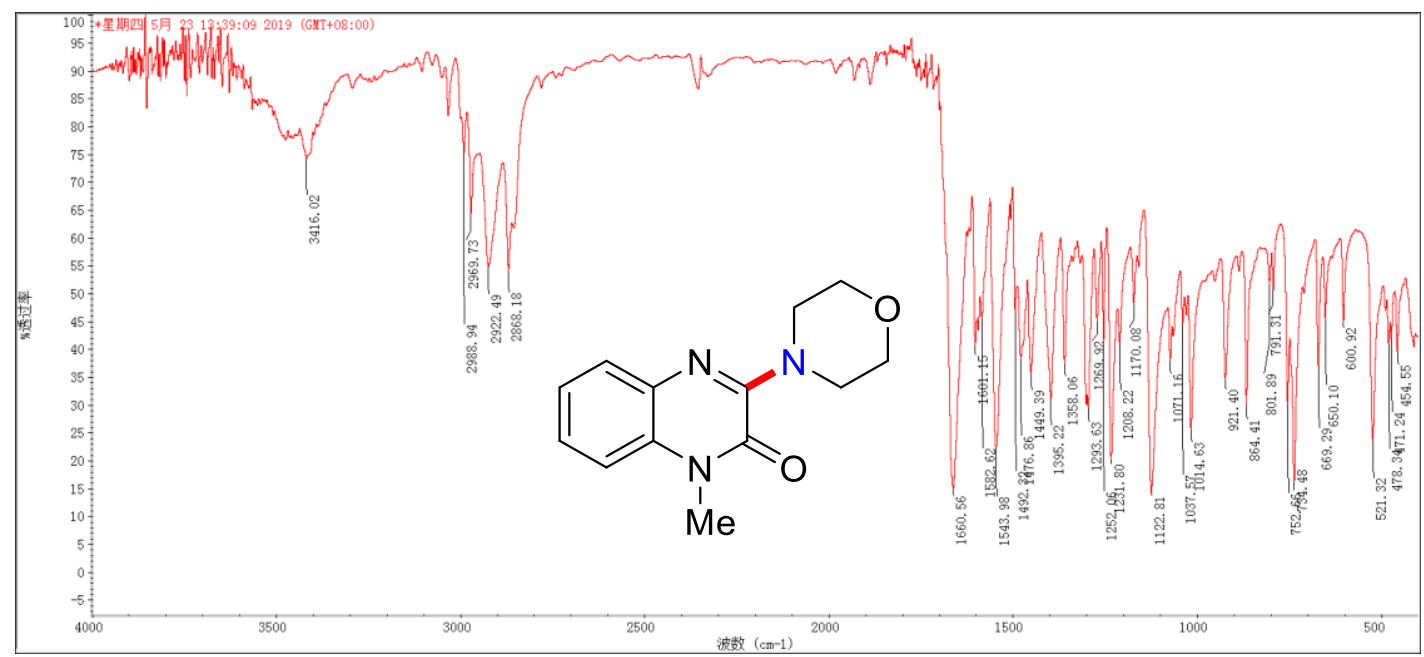




\section{2b}

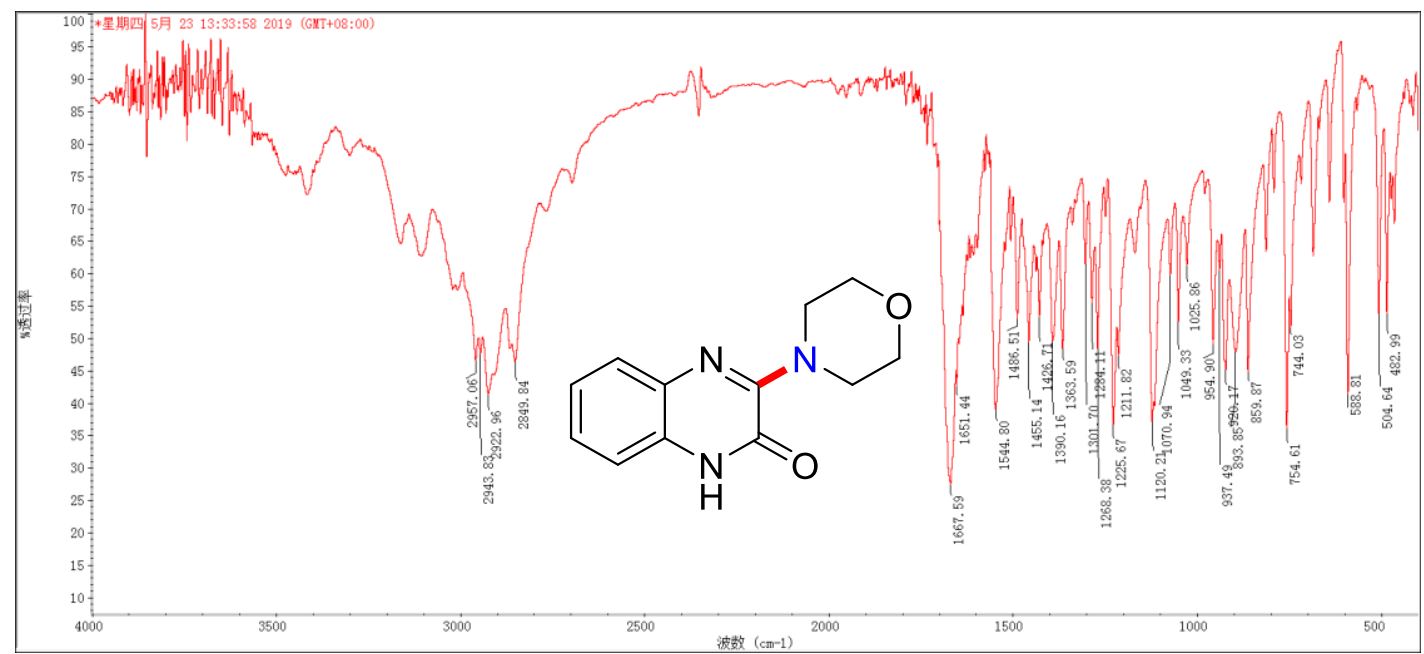

\section{3}

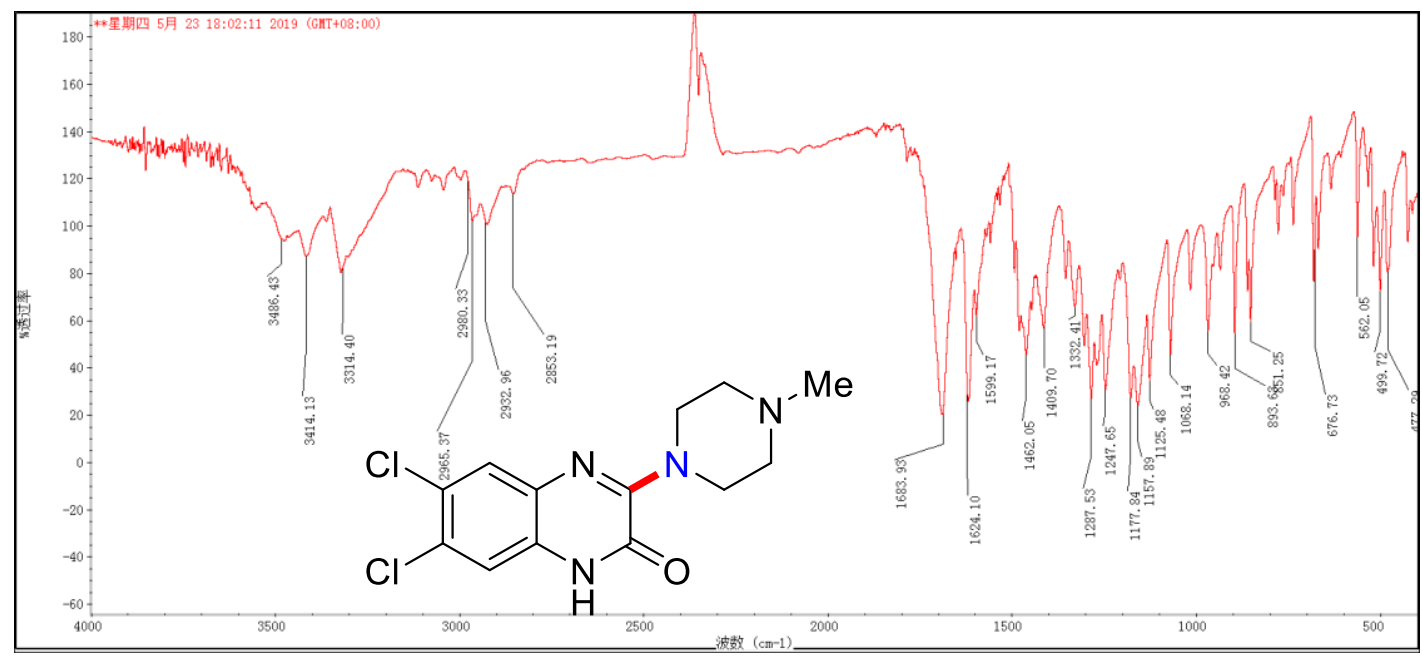

